\title{
Quantitative Molecular Composition of Heavy Petroleum Fractions: A Case Study of FCC Decant Oil
}

Haidong Li, Yahe Zhang, Chunming Xu, Suoqi Zhao, Keng H Chung, Quan Shi

State Key Laboratory of Heavy Oil Processing, Petroleum Molecular Engineering Center (PMEC), China University of Petroleum, Beijing 102249, PR China

Table S1 Contents of various molecules of saturate hydrocarbons

\begin{tabular}{|c|c|c|c|c|c|c|c|}
\hline Formula & Mass & Formula & Mass & Formula & Mass & Formula & Mass \\
\hline $\mathrm{C} 18 \mathrm{H} 38$ & 2E-05 & C20H38 & $9.59 \mathrm{E}-05$ & $\mathrm{C} 27 \mathrm{H} 46$ & 0.001086 & C39H68 & 0.00017 \\
\hline $\mathrm{C} 19 \mathrm{H} 40$ & 3.32E-05 & $\mathrm{C} 21 \mathrm{H} 40$ & 0.000139 & $\mathrm{C} 28 \mathrm{H} 48$ & 0.001447 & $\mathrm{C} 40 \mathrm{H} 70$ & 0.000237 \\
\hline $\mathrm{C} 2 \mathrm{OH} 42$ & 8.6E-05 & $\mathrm{C} 22 \mathrm{H} 42$ & 0.000222 & $\mathrm{C} 29 \mathrm{H} 50$ & 0.002694 & $\mathrm{C} 41 \mathrm{H} 72$ & 0.000129 \\
\hline $\mathrm{C} 21 \mathrm{H} 44$ & 0.000157 & $\mathrm{C} 23 \mathrm{H} 44$ & 0.000323 & $\mathrm{C} 30 \mathrm{H} 52$ & 0.00328 & $\mathrm{C} 42 \mathrm{H} 74$ & 0.000104 \\
\hline $\mathrm{C} 22 \mathrm{H} 46$ & 0.000269 & $\mathrm{C} 24 \mathrm{H} 46$ & 0.000445 & C31H54 & 0.002588 & $\mathrm{C} 43 \mathrm{H} 76$ & 0.000103 \\
\hline $\mathrm{C} 23 \mathrm{H} 48$ & 0.000474 & $\mathrm{C} 25 \mathrm{H} 48$ & 0.000673 & C32H56 & 0.002295 & $\mathrm{C} 44 \mathrm{H} 78$ & $9.05 \mathrm{E}-05$ \\
\hline $\mathrm{C} 24 \mathrm{H} 50$ & 0.000812 & $\mathrm{C} 26 \mathrm{H} 50$ & 0.001011 & C $33 \mathrm{H} 58$ & 0.002315 & $\mathrm{C} 45 \mathrm{H} 80$ & 7.93E-05 \\
\hline $\mathrm{C} 25 \mathrm{H} 52$ & 0.001229 & $\mathrm{C} 27 \mathrm{H} 52$ & 0.001225 & C34H60 & 0.002223 & $\mathrm{C} 46 \mathrm{H} 82$ & $6.45 \mathrm{E}-05$ \\
\hline C26H54 & 0.001764 & C28H54 & 0.00167 & C35H62 & 0.001961 & C47H84 & 5.3E-05 \\
\hline C27H56 & 0.002241 & C29H56 & 0.002301 & $\mathrm{C} 36 \mathrm{H} 64$ & 0.001777 & C48H86 & $5.07 \mathrm{E}-05$ \\
\hline C28H58 & 0.00173 & C30H58 & 0.002948 & C37H66 & 0.001588 & C49H88 & $3.67 \mathrm{E}-05$ \\
\hline $\mathrm{C} 29 \mathrm{H} 60$ & 0.003273 & $\mathrm{C} 31 \mathrm{H} 60$ & 0.003188 & $\mathrm{C} 38 \mathrm{H} 68$ & 0.001482 & $\mathrm{C} 50 \mathrm{H} 90$ & 2.97E-05 \\
\hline $\mathrm{C} 30 \mathrm{H} 62$ & 0.003579 & $\mathrm{C} 32 \mathrm{H} 62$ & 0.003625 & $\mathrm{C} 39 \mathrm{H} 70$ & 0.001359 & C51H92 & $2.14 \mathrm{E}-05$ \\
\hline C31H64 & 0.003771 & C33H64 & 0.004127 & $\mathrm{C} 40 \mathrm{H} 72$ & 0.001498 & C52H94 & $2.01 \mathrm{E}-05$ \\
\hline C32H66 & 0.003943 & C34H66 & 0.00418 & $\mathrm{C} 41 \mathrm{H} 74$ & 0.001151 & C53H96 & $1.41 \mathrm{E}-05$ \\
\hline C33H68 & 0.004225 & C35H68 & 0.003943 & $\mathrm{C} 42 \mathrm{H} 76$ & 0.000919 & C54H98 & $1.16 \mathrm{E}-05$ \\
\hline $\mathrm{C} 34 \mathrm{H} 70$ & 0.003969 & $\mathrm{C} 36 \mathrm{H} 70$ & 0.003777 & $\mathrm{C} 43 \mathrm{H} 78$ & 0.000872 & $\mathrm{C} 21 \mathrm{H} 30$ & $1.39 \mathrm{E}-06$ \\
\hline $\mathrm{C} 35 \mathrm{H} 72$ & 0.003884 & $\mathrm{C} 37 \mathrm{H} 72$ & 0.003708 & $\mathrm{C} 44 \mathrm{H} 80$ & 0.000818 & $\mathrm{C} 22 \mathrm{H} 32$ & $1.73 \mathrm{E}-06$ \\
\hline $\mathrm{C} 36 \mathrm{H} 74$ & 0.003721 & $\mathrm{C} 38 \mathrm{H} 74$ & 0.003553 & $\mathrm{C} 45 \mathrm{H} 82$ & 0.000702 & $\mathrm{C} 23 \mathrm{H} 34$ & 2.4E-06 \\
\hline $\mathrm{C} 37 \mathrm{H} 76$ & 0.003315 & $\mathrm{C} 39 \mathrm{H} 76$ & 0.003341 & $\mathrm{C} 46 \mathrm{H} 84$ & 0.00061 & $\mathrm{C} 24 \mathrm{H} 36$ & $4.92 \mathrm{E}-06$ \\
\hline $\mathrm{C} 38 \mathrm{H} 78$ & 0.003275 & $\mathrm{C} 40 \mathrm{H} 78$ & 0.003749 & C47H86 & 0.000517 & $\mathrm{C} 25 \mathrm{H} 38$ & 7.95E-06 \\
\hline С $39 \mathrm{H} 80$ & 0.003103 & $\mathrm{C} 41 \mathrm{H} 80$ & 0.003073 & C48H88 & 0.000447 & $\mathrm{C} 26 \mathrm{H} 40$ & $1.39 \mathrm{E}-05$ \\
\hline $\mathrm{C} 40 \mathrm{H} 82$ & 0.002812 & $\mathrm{C} 42 \mathrm{H} 82$ & 0.002341 & $\mathrm{C} 49 \mathrm{H} 90$ & 0.000334 & $\mathrm{C} 27 \mathrm{H} 42$ & $2.27 \mathrm{E}-05$ \\
\hline $\mathrm{C} 41 \mathrm{H} 84$ & 0.002567 & $\mathrm{C} 43 \mathrm{H} 84$ & 0.002217 & $\mathrm{C} 50 \mathrm{H} 92$ & 0.000261 & $\mathrm{C} 28 \mathrm{H} 44$ & $2.72 \mathrm{E}-05$ \\
\hline $\mathrm{C} 42 \mathrm{H} 86$ & 0.002207 & C44H86 & 0.002185 & C51H94 & 0.0002 & $\mathrm{C} 29 \mathrm{H} 46$ & $2.49 \mathrm{E}-05$ \\
\hline
\end{tabular}




\begin{tabular}{|c|c|c|c|c|c|c|c|}
\hline Formula & Mass & Formula & Mass & Formula & Mass & Formula & Mass \\
\hline C43H88 & 0.00201 & $\mathrm{C} 45 \mathrm{H} 88$ & 0.001923 & C52H96 & 0.000163 & $\mathrm{C} 30 \mathrm{H} 48$ & 4.45E-05 \\
\hline C44H90 & 0.001806 & $\mathrm{C} 46 \mathrm{H} 90$ & 0.001606 & C53H98 & 0.000111 & $\mathrm{C} 31 \mathrm{H} 50$ & 4.26E-05 \\
\hline C45H92 & 0.001753 & C47H92 & 0.001305 & C54H100 & 0.000108 & C $32 \mathrm{H} 52$ & $4.51 \mathrm{E}-05$ \\
\hline C46H94 & 0.00142 & C48H94 & 0.001142 & $\mathrm{C} 55 \mathrm{H} 102$ & $7.48 \mathrm{E}-05$ & C33H 54 & 4.65E-05 \\
\hline C47H96 & 0.001128 & C49H96 & 0.000911 & C56H104 & $3.76 \mathrm{E}-05$ & C34H56 & 4.43E-05 \\
\hline C48H98 & 0.00103 & $\mathrm{C} 50 \mathrm{H} 98$ & 0.000734 & C57H106 & $4.51 \mathrm{E}-05$ & C35H58 & 4.3E-05 \\
\hline C49H100 & 0.000823 & $\mathrm{C} 51 \mathrm{H} 100$ & 0.000533 & C58H108 & $3.85 \mathrm{E}-05$ & $\mathrm{C} 36 \mathrm{H} 60$ & $3.88 \mathrm{E}-05$ \\
\hline $\mathrm{C} 50 \mathrm{H} 102$ & 0.000641 & $\mathrm{C} 52 \mathrm{H} 102$ & 0.000405 & $\mathrm{C} 18 \mathrm{H} 28$ & $5.85 \mathrm{E}-06$ & C37H62 & 3.9E-05 \\
\hline C51H104 & 0.000512 & C53H104 & 0.000295 & C19H30 & $8.27 \mathrm{E}-06$ & C38H64 & 3.43E-05 \\
\hline C52H106 & 0.000395 & C54H106 & 0.000251 & $\mathrm{C} 20 \mathrm{H} 32$ & $3.87 \mathrm{E}-05$ & C39H66 & $3.41 \mathrm{E}-05$ \\
\hline C53H108 & 0.000304 & C55H108 & 0.000168 & $\mathrm{C} 21 \mathrm{H} 34$ & $3.07 \mathrm{E}-05$ & $\mathrm{C} 40 \mathrm{H} 68$ & $4.59 \mathrm{E}-05$ \\
\hline C54H110 & 0.000235 & $\mathrm{C} 56 \mathrm{H} 110$ & 0.000163 & $\mathrm{C} 22 \mathrm{H} 36$ & $4.59 \mathrm{E}-05$ & $\mathrm{C} 41 \mathrm{H} 70$ & $2.72 \mathrm{E}-05$ \\
\hline C55H112 & 0.000164 & C57H112 & $8.61 \mathrm{E}-05$ & C23H38 & 7.2E-05 & $\mathrm{C} 42 \mathrm{H} 72$ & $2.34 \mathrm{E}-05$ \\
\hline C56H114 & 0.000115 & $\mathrm{C} 16 \mathrm{H} 28$ & $1.25 \mathrm{E}-05$ & $\mathrm{C} 24 \mathrm{H} 40$ & 0.000123 & $\mathrm{C} 43 \mathrm{H} 74$ & $2.11 \mathrm{E}-05$ \\
\hline C57H116 & 0.000117 & $\mathrm{C} 17 \mathrm{H} 30$ & $1.57 \mathrm{E}-05$ & $\mathrm{C} 25 \mathrm{H} 42$ & 0.000192 & $\mathrm{C} 44 \mathrm{H} 76$ & $1.65 \mathrm{E}-05$ \\
\hline C58H118 & $8.87 \mathrm{E}-05$ & $\mathrm{C} 18 \mathrm{H} 32$ & $3.36 \mathrm{E}-05$ & $\mathrm{C} 26 \mathrm{H} 44$ & 0.000295 & $\mathrm{C} 45 \mathrm{H} 78$ & $1.78 \mathrm{E}-05$ \\
\hline $\mathrm{C} 13 \mathrm{H} 26$ & 7.1E-06 & C19H34 & $4.01 \mathrm{E}-05$ & $\mathrm{C} 27 \mathrm{H} 46$ & 0.000579 & $\mathrm{C} 46 \mathrm{H} 80$ & $1.12 \mathrm{E}-05$ \\
\hline $\mathrm{C} 14 \mathrm{H} 28$ & $6.83 \mathrm{E}-06$ & $\mathrm{C} 20 \mathrm{H} 36$ & $7.41 \mathrm{E}-05$ & $\mathrm{C} 28 \mathrm{H} 48$ & 0.000685 & $\mathrm{C} 47 \mathrm{H} 82$ & $1.1 \mathrm{E}-05$ \\
\hline $\mathrm{C} 15 \mathrm{H} 30$ & $1.49 \mathrm{E}-05$ & $\mathrm{C} 21 \mathrm{H} 38$ & 0.000111 & $\mathrm{C} 29 \mathrm{H} 50$ & 0.001599 & $\mathrm{C} 48 \mathrm{H} 84$ & $1.01 \mathrm{E}-05$ \\
\hline $\mathrm{C} 16 \mathrm{H} 32$ & $1.8 \mathrm{E}-05$ & $\mathrm{C} 22 \mathrm{H} 40$ & 0.000183 & $\mathrm{C} 30 \mathrm{H} 52$ & 0.002175 & C49H86 & $8.52 \mathrm{E}-06$ \\
\hline C17H34 & $2.62 \mathrm{E}-05$ & $\mathrm{C} 23 \mathrm{H} 42$ & 0.000308 & $\mathrm{C} 31 \mathrm{H} 54$ & 0.001579 & C50H88 & $6.03 \mathrm{E}-06$ \\
\hline C18H36 & $6.02 \mathrm{E}-05$ & $\mathrm{C} 24 \mathrm{H} 44$ & 0.000424 & $\mathrm{C} 32 \mathrm{H} 56$ & 0.001356 & C $51 \mathrm{H} 90$ & 4.73E-06 \\
\hline C19H38 & $7.41 \mathrm{E}-05$ & $\mathrm{C} 25 \mathrm{H} 46$ & 0.000652 & C33H58 & 0.001232 & $\mathrm{C} 52 \mathrm{H} 92$ & $4.52 \mathrm{E}-06$ \\
\hline $\mathrm{C} 20 \mathrm{H} 40$ & $8.47 \mathrm{E}-05$ & $\mathrm{C} 26 \mathrm{H} 48$ & 0.000908 & $\mathrm{C} 34 \mathrm{H} 60$ & 0.001083 & $\mathrm{C} 24 \mathrm{H} 34$ & $6.98 \mathrm{E}-07$ \\
\hline $\mathrm{C} 21 \mathrm{H} 42$ & 0.000111 & $\mathrm{C} 27 \mathrm{H} 50$ & 0.001203 & $\mathrm{C} 35 \mathrm{H} 62$ & 0.00097 & $\mathrm{C} 25 \mathrm{H} 36$ & $1.01 \mathrm{E}-06$ \\
\hline $\mathrm{C} 22 \mathrm{H} 44$ & 0.000174 & C28H52 & 0.001721 & C36H64 & 0.000815 & $\mathrm{C} 26 \mathrm{H} 38$ & $1.41 \mathrm{E}-06$ \\
\hline $\mathrm{C} 23 \mathrm{H} 46$ & 0.000234 & C29H54 & 0.002452 & C37H66 & 0.000747 & $\mathrm{C} 27 \mathrm{H} 40$ & 2.3E-06 \\
\hline $\mathrm{C} 24 \mathrm{H} 48$ & 0.000362 & $\mathrm{C} 30 \mathrm{H} 56$ & 0.002987 & C38H68 & 0.000679 & $\mathrm{C} 28 \mathrm{H} 42$ & $2.73 \mathrm{E}-06$ \\
\hline $\mathrm{C} 25 \mathrm{H} 50$ & 0.000517 & $\mathrm{C} 31 \mathrm{H} 58$ & 0.003253 & $\mathrm{C} 39 \mathrm{H} 70$ & 0.000631 & $\mathrm{C} 29 \mathrm{H} 44$ & $3.77 \mathrm{E}-06$ \\
\hline C26H52 & 0.000759 & $\mathrm{C} 32 \mathrm{H} 60$ & 0.003476 & $\mathrm{C} 40 \mathrm{H} 72$ & 0.000779 & $\mathrm{C} 30 \mathrm{H} 46$ & 4.37E-06 \\
\hline C27H54 & 0.000961 & $\mathrm{C} 33 \mathrm{H} 62$ & 0.003796 & $\mathrm{C} 41 \mathrm{H} 74$ & 0.00048 & $\mathrm{C} 31 \mathrm{H} 48$ & $4.68 \mathrm{E}-06$ \\
\hline C28H56 & 0.001216 & C34H64 & 0.003924 & $\mathrm{C} 42 \mathrm{H} 76$ & 0.000427 & $\mathrm{C} 32 \mathrm{H} 50$ & $4.82 \mathrm{E}-06$ \\
\hline C29H58 & 0.001571 & C35H66 & 0.003701 & $\mathrm{C} 43 \mathrm{H} 78$ & 0.000375 & C33H52 & 4.86E-06 \\
\hline $\mathrm{C} 30 \mathrm{H} 60$ & 0.001845 & C36H68 & 0.003455 & $\mathrm{C} 44 \mathrm{H} 80$ & 0.000351 & C34H54 & $4.96 \mathrm{E}-06$ \\
\hline $\mathrm{C} 31 \mathrm{H} 62$ & 0.002104 & $\mathrm{C} 37 \mathrm{H} 70$ & 0.003139 & $\mathrm{C} 45 \mathrm{H} 82$ & 0.000312 & C $35 \mathrm{H} 56$ & 4.61E-06 \\
\hline C32H64 & 0.002299 & $\mathrm{C} 38 \mathrm{H} 72$ & 0.003128 & C46H84 & 0.000262 & C $36 \mathrm{H} 58$ & 4.45E-06 \\
\hline C33H66 & 0.00254 & $\mathrm{C} 39 \mathrm{H} 74$ & 0.002837 & C47H86 & 0.000215 & $\mathrm{C} 37 \mathrm{H} 60$ & $4.78 \mathrm{E}-06$ \\
\hline C34H68 & 0.002586 & $\mathrm{C} 40 \mathrm{H} 76$ & 0.002849 & C48H88 & 0.000184 & $\mathrm{C} 38 \mathrm{H} 62$ & $3.87 \mathrm{E}-06$ \\
\hline
\end{tabular}




\begin{tabular}{|c|c|c|c|c|c|c|c|}
\hline Formula & Mass & Formula & Mass & Formula & Mass & Formula & Mass \\
\hline $\mathrm{C} 35 \mathrm{H} 70$ & 0.002559 & $\mathrm{C} 41 \mathrm{H} 78$ & 0.002275 & C49H90 & 0.000161 & C39H64 & 3.93E-06 \\
\hline $\mathrm{C} 36 \mathrm{H} 72$ & 0.002399 & $\mathrm{C} 42 \mathrm{H} 80$ & 0.002058 & $\mathrm{C} 50 \mathrm{H} 92$ & 0.000122 & C40H66 & $6.08 \mathrm{E}-06$ \\
\hline C37H74 & 0.002238 & $\mathrm{C} 43 \mathrm{H} 82$ & 0.001843 & C51H94 & $9.23 \mathrm{E}-05$ & C41H68 & $3.21 \mathrm{E}-06$ \\
\hline C38H76 & 0.002316 & C44H84 & 0.001623 & С52H96 & 7.4E-05 & $\mathrm{C} 42 \mathrm{H} 70$ & $2.68 \mathrm{E}-06$ \\
\hline C39H78 & 0.002142 & $\mathrm{C} 45 \mathrm{H} 86$ & 0.001421 & C53H98 & $4.88 \mathrm{E}-05$ & $\mathrm{C} 43 \mathrm{H} 72$ & $2.71 \mathrm{E}-06$ \\
\hline $\mathrm{C} 40 \mathrm{H} 80$ & 0.001883 & $\mathrm{C} 46 \mathrm{H} 88$ & 0.001236 & $\mathrm{C} 54 \mathrm{H} 100$ & $4.78 \mathrm{E}-05$ & $\mathrm{C} 44 \mathrm{H} 74$ & $2.49 \mathrm{E}-06$ \\
\hline C41H82 & 0.001685 & C47H90 & 0.001051 & $\mathrm{C} 55 \mathrm{H} 102$ & $2.77 \mathrm{E}-05$ & $\mathrm{C} 45 \mathrm{H} 76$ & $2.02 \mathrm{E}-06$ \\
\hline C42H84 & 0.001598 & C48H92 & 0.000932 & C56H104 & $3.26 \mathrm{E}-05$ & $\mathrm{C} 46 \mathrm{H} 78$ & $1.7 \mathrm{E}-06$ \\
\hline C43H86 & 0.001396 & С49H94 & 0.000642 & С19H28 & $2.34 \mathrm{E}-06$ & $\mathrm{C} 47 \mathrm{H} 80$ & $1.35 \mathrm{E}-06$ \\
\hline C44H88 & 0.00125 & $\mathrm{C} 50 \mathrm{H} 96$ & 0.000571 & $\mathrm{C} 20 \mathrm{H} 30$ & $5.17 \mathrm{E}-06$ & $\mathrm{C} 48 \mathrm{H} 82$ & $1.22 \mathrm{E}-06$ \\
\hline $\mathrm{C} 45 \mathrm{H} 90$ & 0.001106 & C51H98 & 0.000393 & $\mathrm{C} 21 \mathrm{H} 32$ & $4.85 \mathrm{E}-06$ & $\mathrm{C} 25 \mathrm{H} 34$ & $9.77 \mathrm{E}-08$ \\
\hline C46H92 & 0.000927 & $\mathrm{C} 52 \mathrm{H} 100$ & 0.000325 & $\mathrm{C} 22 \mathrm{H} 34$ & $1.02 \mathrm{E}-05$ & $\mathrm{C} 26 \mathrm{H} 36$ & $9.52 \mathrm{E}-08$ \\
\hline C47H94 & 0.000845 & $\mathrm{C} 53 \mathrm{H} 102$ & 0.000247 & $\mathrm{C} 23 \mathrm{H} 36$ & $1.53 \mathrm{E}-05$ & $\mathrm{C} 27 \mathrm{H} 38$ & $1.62 \mathrm{E}-07$ \\
\hline C48H96 & 0.000685 & C54H104 & 0.000212 & $\mathrm{C} 24 \mathrm{H} 38$ & $3.13 \mathrm{E}-05$ & $\mathrm{C} 28 \mathrm{H} 40$ & $1.96 \mathrm{E}-07$ \\
\hline С49H98 & 0.000569 & C55H106 & 0.000117 & $\mathrm{C} 25 \mathrm{H} 40$ & $4.35 \mathrm{E}-05$ & $\mathrm{C} 29 \mathrm{H} 42$ & $3.02 \mathrm{E}-07$ \\
\hline C50H100 & 0.000471 & $\mathrm{C} 56 \mathrm{H} 108$ & $9.4 \mathrm{E}-05$ & $\mathrm{C} 26 \mathrm{H} 42$ & 7.1E-05 & $\mathrm{C} 30 \mathrm{H} 44$ & $3.04 \mathrm{E}-07$ \\
\hline C51H102 & 0.000331 & C57H110 & $6.57 \mathrm{E}-05$ & $\mathrm{C} 27 \mathrm{H} 44$ & 0.000118 & $\mathrm{C} 31 \mathrm{H} 46$ & $3.35 \mathrm{E}-07$ \\
\hline C52H104 & 0.000286 & $\mathrm{C} 14 \mathrm{H} 22$ & $3.06 \mathrm{E}-05$ & $\mathrm{C} 28 \mathrm{H} 46$ & 0.00014 & $\mathrm{C} 32 \mathrm{H} 48$ & $3.39 \mathrm{E}-07$ \\
\hline C53H106 & 0.000178 & $\mathrm{C} 15 \mathrm{H} 24$ & $4.51 \mathrm{E}-05$ & $\mathrm{C} 29 \mathrm{H} 48$ & 0.000227 & $\mathrm{C} 33 \mathrm{H} 50$ & $4.41 \mathrm{E}-07$ \\
\hline C54H108 & 0.000133 & $\mathrm{C} 16 \mathrm{H} 26$ & 7.61E-06 & $\mathrm{C} 30 \mathrm{H} 50$ & 0.000295 & C34H52 & $4.07 \mathrm{E}-07$ \\
\hline $\mathrm{C} 55 \mathrm{H} 110$ & 0.000105 & $\mathrm{C} 18 \mathrm{H} 28$ & $9.37 \mathrm{E}-06$ & $\mathrm{C} 31 \mathrm{H} 52$ & 0.00026 & C35H54 & $3.81 \mathrm{E}-07$ \\
\hline C56H112 & $8.52 \mathrm{E}-05$ & $\mathrm{C} 19 \mathrm{H} 30$ & $2.29 \mathrm{E}-05$ & $\mathrm{C} 32 \mathrm{H} 54$ & 0.000259 & C36H56 & 3.53E-07 \\
\hline C57H114 & 4.8E-05 & $\mathrm{C} 20 \mathrm{H} 32$ & $3.54 \mathrm{E}-05$ & C33H56 & 0.00025 & C37H58 & 4.17E-07 \\
\hline C58H116 & $4.19 \mathrm{E}-05$ & $\mathrm{C} 21 \mathrm{H} 34$ & $6.35 \mathrm{E}-05$ & C34H58 & 0.000236 & $\mathrm{C} 38 \mathrm{H} 60$ & 3.43E-07 \\
\hline $\mathrm{C} 15 \mathrm{H} 28$ & $1.31 \mathrm{E}-05$ & $\mathrm{C} 22 \mathrm{H} 36$ & 0.000103 & $\mathrm{C} 35 \mathrm{H} 60$ & 0.000232 & C39H62 & $4.18 \mathrm{E}-07$ \\
\hline C16H30 & $1.9 \mathrm{E}-05$ & C23H38 & 0.000204 & $\mathrm{C} 36 \mathrm{H} 62$ & 0.000214 & $\mathrm{C} 40 \mathrm{H} 64$ & 4.52E-07 \\
\hline C17H32 & 3.4E-05 & $\mathrm{C} 24 \mathrm{H} 40$ & 0.000318 & C37H64 & 0.00018 & C41H66 & $3.11 \mathrm{E}-07$ \\
\hline C18H34 & 4.03E-05 & $\mathrm{C} 25 \mathrm{H} 42$ & 0.000437 & C38H66 & 0.000175 & $\mathrm{C} 42 \mathrm{H} 68$ & $3.28 \mathrm{E}-07$ \\
\hline
\end{tabular}

Table S2 Contents of various molecules of aromatics

\begin{tabular}{ccccccccc}
\hline Class & formula & Mass & Class & formula & Mass & Class & formula & Mass \\
\hline $\mathrm{CH}$ & $\mathrm{C} 16 \mathrm{H} 26$ & $3.02 \mathrm{E}-06$ & Non-basic N1 & $\mathrm{C} 34 \mathrm{H} 45 \mathrm{~N} 1$ & $4.21 \mathrm{E}-05$ & Basic N1O1 & C28H43N1O1 & $1.07 \mathrm{E}-07$ \\
$\mathrm{CH}$ & $\mathrm{C} 17 \mathrm{H} 28$ & $9.62 \mathrm{E}-05$ & Non-basic N1 & $\mathrm{C} 35 \mathrm{H} 47 \mathrm{~N} 1$ & $3.95 \mathrm{E}-05$ & Basic N1O1 & $\mathrm{C} 29 \mathrm{H} 45 \mathrm{~N} 1 \mathrm{O} 1$ & $1.66 \mathrm{E}-07$ \\
$\mathrm{CH}$ & $\mathrm{C} 18 \mathrm{H} 30$ & 0.000107 & Non-basic N1 & $\mathrm{C} 36 \mathrm{H} 49 \mathrm{~N} 1$ & $2.90 \mathrm{E}-05$ & Basic N1O1 & $\mathrm{C} 30 \mathrm{H} 47 \mathrm{~N} 1 \mathrm{O} 1$ & $2.19 \mathrm{E}-07$ \\
$\mathrm{CH}$ & $\mathrm{C} 19 \mathrm{H} 32$ & $9.23 \mathrm{E}-05$ & Non-basic N1 & $\mathrm{C} 37 \mathrm{H} 51 \mathrm{~N} 1$ & $2.12 \mathrm{E}-05$ & Basic N1O1 & $\mathrm{C} 31 \mathrm{H} 49 \mathrm{~N} 1 \mathrm{O} 1$ & $1.91 \mathrm{E}-07$ \\
$\mathrm{CH}$ & $\mathrm{C} 20 \mathrm{H} 34$ & $7.44 \mathrm{E}-05$ & Non-basic N1 & $\mathrm{C} 38 \mathrm{H} 53 \mathrm{~N} 1$ & $1.41 \mathrm{E}-05$ & Basic N1O1 & $\mathrm{C} 32 \mathrm{H} 51 \mathrm{~N} 1 \mathrm{O} 1$ & $3.82 \mathrm{E}-07$ \\
$\mathrm{CH}$ & $\mathrm{C} 21 \mathrm{H} 36$ & $7.44 \mathrm{E}-05$ & Non-basic N1 & $\mathrm{C} 39 \mathrm{H} 55 \mathrm{~N} 1$ & $1.56 \mathrm{E}-05$ & Basic N1O1 & $\mathrm{C} 33 \mathrm{H} 53 \mathrm{~N} 1 \mathrm{O} 1$ & $4.40 \mathrm{E}-07$ \\
\hline
\end{tabular}




\begin{tabular}{|c|c|c|c|c|c|c|c|c|}
\hline Class & formula & Mass & Class & formula & Mass & Class & formula & Mass \\
\hline $\mathrm{CH}$ & $\mathrm{C} 22 \mathrm{H} 38$ & $5.26 \mathrm{E}-05$ & Non-basic N1 & $\mathrm{C} 40 \mathrm{H} 57 \mathrm{~N} 1$ & $7.08 \mathrm{E}-06$ & Basic N1O1 & $\mathrm{C} 34 \mathrm{H} 55 \mathrm{~N} 1 \mathrm{O} 1$ & $4.63 \mathrm{E}-07$ \\
\hline $\mathrm{CH}$ & $\mathrm{C} 23 \mathrm{H} 40$ & $9.57 \mathrm{E}-05$ & Non-basic N1 & C41H59N1 & $1.85 \mathrm{E}-06$ & Basic N1O1 & $\mathrm{C} 35 \mathrm{H} 57 \mathrm{~N} 1 \mathrm{O} 1$ & $3.64 \mathrm{E}-07$ \\
\hline $\mathrm{CH}$ & $\mathrm{C} 24 \mathrm{H} 42$ & $8.66 \mathrm{E}-05$ & Non-basic N1 & $\mathrm{C} 42 \mathrm{H} 61 \mathrm{~N} 1$ & $2.55 \mathrm{E}-06$ & Basic N1O1 & C36H59N1O1 & $1.69 \mathrm{E}-07$ \\
\hline $\mathrm{CH}$ & $\mathrm{C} 25 \mathrm{H} 44$ & 0.000107 & Non-basic N1 & $\mathrm{C} 44 \mathrm{H} 65 \mathrm{~N} 1$ & $2.06 \mathrm{E}-06$ & Basic N1O1 & C37H61N1O1 & $1.57 \mathrm{E}-07$ \\
\hline $\mathrm{CH}$ & $\mathrm{C} 26 \mathrm{H} 46$ & 0.000121 & Non-basic N1 & $\mathrm{C} 20 \mathrm{H} 15 \mathrm{~N} 1$ & $5.35 \mathrm{E}-06$ & Basic N1O1 & C38H63N1O1 & $1.48 \mathrm{E}-07$ \\
\hline $\mathrm{CH}$ & $\mathrm{C} 27 \mathrm{H} 48$ & 0.000252 & Non-basic N1 & $\mathrm{C} 21 \mathrm{H} 17 \mathrm{~N} 1$ & $1.88 \mathrm{E}-05$ & Basic N1O1 & $\mathrm{C} 15 \mathrm{H} 15 \mathrm{~N} 1 \mathrm{O} 1$ & $1.29 \mathrm{E}-07$ \\
\hline $\mathrm{CH}$ & $\mathrm{C} 28 \mathrm{H} 50$ & 0.000265 & Non-basic N1 & $\mathrm{C} 22 \mathrm{H} 19 \mathrm{~N} 1$ & $3.93 \mathrm{E}-05$ & Basic N1O1 & C16H17N1O1 & $8.88 \mathrm{E}-08$ \\
\hline $\mathrm{CH}$ & $\mathrm{C} 29 \mathrm{H} 52$ & 0.000417 & Non-basic N1 & $\mathrm{C} 23 \mathrm{H} 21 \mathrm{~N} 1$ & $8.95 \mathrm{E}-05$ & Basic N1O1 & C17H19N1O1 & $6.33 \mathrm{E}-07$ \\
\hline $\mathrm{CH}$ & $\mathrm{C} 30 \mathrm{H} 54$ & 0.000522 & Non-basic N1 & $\mathrm{C} 24 \mathrm{H} 23 \mathrm{~N} 1$ & 0.000123 & Basic N1O1 & $\mathrm{C} 18 \mathrm{H} 21 \mathrm{~N} 1 \mathrm{O} 1$ & $1.84 \mathrm{E}-06$ \\
\hline $\mathrm{CH}$ & $\mathrm{C} 31 \mathrm{H} 56$ & 0.000378 & Non-basic N1 & $\mathrm{C} 25 \mathrm{H} 25 \mathrm{~N} 1$ & 0.000126 & Basic N1O1 & $\mathrm{C} 19 \mathrm{H} 23 \mathrm{~N} 1 \mathrm{O} 1$ & $1.69 \mathrm{E}-06$ \\
\hline $\mathrm{CH}$ & $\mathrm{C} 32 \mathrm{H} 58$ & 0.000334 & Non-basic N1 & $\mathrm{C} 26 \mathrm{H} 27 \mathrm{~N} 1$ & 0.000137 & Basic N1O1 & $\mathrm{C} 20 \mathrm{H} 25 \mathrm{~N} 1 \mathrm{O} 1$ & $1.41 \mathrm{E}-06$ \\
\hline $\mathrm{CH}$ & $\mathrm{C} 33 \mathrm{H} 60$ & 0.000309 & Non-basic N1 & $\mathrm{C} 27 \mathrm{H} 29 \mathrm{~N} 1$ & 0.000122 & Basic N1O1 & $\mathrm{C} 21 \mathrm{H} 27 \mathrm{~N} 1 \mathrm{O} 1$ & $6.99 \mathrm{E}-06$ \\
\hline $\mathrm{CH}$ & $\mathrm{C} 34 \mathrm{H} 62$ & 0.000285 & Non-basic N1 & $\mathrm{C} 28 \mathrm{H} 31 \mathrm{~N} 1$ & $9.43 \mathrm{E}-05$ & Basic N1O1 & $\mathrm{C} 22 \mathrm{H} 29 \mathrm{~N} 1 \mathrm{O} 1$ & $6.14 \mathrm{E}-05$ \\
\hline $\mathrm{CH}$ & C35H64 & 0.000287 & Non-basic N1 & $\mathrm{C} 29 \mathrm{H} 33 \mathrm{~N} 1$ & $6.26 \mathrm{E}-05$ & Basic N1O1 & $\mathrm{C} 23 \mathrm{H} 31 \mathrm{~N} 1 \mathrm{O} 1$ & $1.69 \mathrm{E}-06$ \\
\hline $\mathrm{CH}$ & $\mathrm{C} 36 \mathrm{H} 66$ & 0.000273 & Non-basic N1 & $\mathrm{C} 30 \mathrm{H} 35 \mathrm{~N} 1$ & 4.83E-05 & Basic N1O1 & $\mathrm{C} 24 \mathrm{H} 33 \mathrm{~N} 1 \mathrm{O} 1$ & $9.39 \mathrm{E}-07$ \\
\hline $\mathrm{CH}$ & $\mathrm{C} 16 \mathrm{H} 24$ & $1.61 \mathrm{E}-06$ & Non-basic N1 & $\mathrm{C} 31 \mathrm{H} 37 \mathrm{~N} 1$ & 4.93E-05 & Basic N1O1 & $\mathrm{C} 25 \mathrm{H} 35 \mathrm{~N} 1 \mathrm{O} 1$ & $8.35 \mathrm{E}-07$ \\
\hline $\mathrm{CH}$ & $\mathrm{C} 17 \mathrm{H} 26$ & 0.000168 & Non-basic N1 & $\mathrm{C} 32 \mathrm{H} 39 \mathrm{~N} 1$ & 2.87E-05 & Basic N1O1 & $\mathrm{C} 26 \mathrm{H} 37 \mathrm{~N} 1 \mathrm{O} 1$ & $1.41 \mathrm{E}-06$ \\
\hline $\mathrm{CH}$ & $\mathrm{C} 18 \mathrm{H} 28$ & 0.000193 & Non-basic N1 & $\mathrm{C} 33 \mathrm{H} 41 \mathrm{~N} 1$ & $2.98 \mathrm{E}-05$ & Basic N1O1 & $\mathrm{C} 27 \mathrm{H} 39 \mathrm{~N} 1 \mathrm{O} 1$ & $1.72 \mathrm{E}-06$ \\
\hline $\mathrm{CH}$ & $\mathrm{C} 19 \mathrm{H} 30$ & 0.000141 & Non-basic N1 & $\mathrm{C} 34 \mathrm{H} 43 \mathrm{~N} 1$ & $2.17 \mathrm{E}-05$ & Basic N1O1 & $\mathrm{C} 28 \mathrm{H} 41 \mathrm{~N} 1 \mathrm{O} 1$ & $1.85 \mathrm{E}-06$ \\
\hline $\mathrm{CH}$ & $\mathrm{C} 20 \mathrm{H} 32$ & 0.0001 & Non-basic N1 & $\mathrm{C} 35 \mathrm{H} 45 \mathrm{~N} 1$ & $2.21 \mathrm{E}-05$ & Basic N1O1 & $\mathrm{C} 29 \mathrm{H} 43 \mathrm{~N} 1 \mathrm{O} 1$ & $2.15 \mathrm{E}-06$ \\
\hline $\mathrm{CH}$ & $\mathrm{C} 21 \mathrm{H} 34$ & 0.000103 & Non-basic N1 & $\mathrm{C} 36 \mathrm{H} 47 \mathrm{~N} 1$ & $1.05 \mathrm{E}-05$ & Basic N1O1 & $\mathrm{C} 30 \mathrm{H} 45 \mathrm{~N} 1 \mathrm{O} 1$ & $2.99 \mathrm{E}-06$ \\
\hline $\mathrm{CH}$ & $\mathrm{C} 22 \mathrm{H} 36$ & 0.000105 & Non-basic N1 & $\mathrm{C} 37 \mathrm{H} 49 \mathrm{~N} 1$ & $7.15 \mathrm{E}-06$ & Basic N1O1 & $\mathrm{C} 31 \mathrm{H} 47 \mathrm{~N} 1 \mathrm{O} 1$ & $3.41 \mathrm{E}-06$ \\
\hline $\mathrm{CH}$ & $\mathrm{C} 23 \mathrm{H} 38$ & 0.000126 & Non-basic N1 & $\mathrm{C} 20 \mathrm{H} 13 \mathrm{~N} 1$ & $1.53 \mathrm{E}-06$ & Basic N1O1 & $\mathrm{C} 32 \mathrm{H} 49 \mathrm{~N} 1 \mathrm{O} 1$ & $4.03 \mathrm{E}-06$ \\
\hline $\mathrm{CH}$ & $\mathrm{C} 24 \mathrm{H} 40$ & 0.000133 & Non-basic N1 & $\mathrm{C} 21 \mathrm{H} 15 \mathrm{~N} 1$ & $3.06 \mathrm{E}-05$ & Basic N1O1 & C33H51N1O1 & $3.47 \mathrm{E}-06$ \\
\hline $\mathrm{CH}$ & $\mathrm{C} 25 \mathrm{H} 42$ & 0.000141 & Non-basic N1 & $\mathrm{C} 22 \mathrm{H} 17 \mathrm{~N} 1$ & 7.44E-05 & Basic N1O1 & $\mathrm{C} 34 \mathrm{H} 53 \mathrm{~N} 1 \mathrm{O} 1$ & $3.23 \mathrm{E}-06$ \\
\hline $\mathrm{CH}$ & $\mathrm{C} 26 \mathrm{H} 44$ & 0.00022 & Non-basic N1 & $\mathrm{C} 23 \mathrm{H} 19 \mathrm{~N} 1$ & 0.000122 & Basic N1O1 & $\mathrm{C} 35 \mathrm{H} 55 \mathrm{~N} 1 \mathrm{O} 1$ & $2.57 \mathrm{E}-06$ \\
\hline $\mathrm{CH}$ & $\mathrm{C} 27 \mathrm{H} 46$ & 0.000326 & Non-basic N1 & $\mathrm{C} 24 \mathrm{H} 21 \mathrm{~N} 1$ & 0.000122 & Basic N1O1 & $\mathrm{C} 36 \mathrm{H} 57 \mathrm{~N} 1 \mathrm{O} 1$ & $2.78 \mathrm{E}-06$ \\
\hline $\mathrm{CH}$ & $\mathrm{C} 28 \mathrm{H} 48$ & 0.000328 & Non-basic N1 & $\mathrm{C} 25 \mathrm{H} 23 \mathrm{~N} 1$ & 0.000122 & Basic N1O1 & $\mathrm{C} 37 \mathrm{H} 59 \mathrm{~N} 1 \mathrm{O} 1$ & $2.11 \mathrm{E}-06$ \\
\hline $\mathrm{CH}$ & $\mathrm{C} 29 \mathrm{H} 50$ & 0.000679 & Non-basic N1 & $\mathrm{C} 26 \mathrm{H} 25 \mathrm{~N} 1$ & 0.000103 & Basic N1O1 & C38H61N1O1 & $1.37 \mathrm{E}-06$ \\
\hline $\mathrm{CH}$ & $\mathrm{C} 30 \mathrm{H} 52$ & 0.000937 & Non-basic N1 & $\mathrm{C} 27 \mathrm{H} 27 \mathrm{~N} 1$ & $8.81 \mathrm{E}-05$ & Basic N1O1 & C39H63N1O1 & $1.09 \mathrm{E}-06$ \\
\hline $\mathrm{CH}$ & C31H54 & 0.000685 & Non-basic N1 & $\mathrm{C} 28 \mathrm{H} 29 \mathrm{~N} 1$ & 7.69E-05 & Basic N1O1 & C40H65N1O1 & $5.40 \mathrm{E}-07$ \\
\hline $\mathrm{CH}$ & $\mathrm{C} 32 \mathrm{H} 56$ & 0.00059 & Non-basic N1 & $\mathrm{C} 29 \mathrm{H} 31 \mathrm{~N} 1$ & $5.22 \mathrm{E}-05$ & Basic N1O1 & C41H67N1O1 & $3.01 \mathrm{E}-07$ \\
\hline $\mathrm{CH}$ & $\mathrm{C} 33 \mathrm{H} 58$ & 0.000509 & Non-basic N1 & $\mathrm{C} 30 \mathrm{H} 33 \mathrm{~N} 1$ & 4.66E-05 & Basic N1O1 & $\mathrm{C} 42 \mathrm{H} 69 \mathrm{~N} 1 \mathrm{O} 1$ & $2.16 \mathrm{E}-07$ \\
\hline $\mathrm{CH}$ & C34H60 & 0.000474 & Non-basic N1 & $\mathrm{C} 31 \mathrm{H} 35 \mathrm{~N} 1$ & $3.44 \mathrm{E}-05$ & Basic N1O1 & $\mathrm{C} 16 \mathrm{H} 15 \mathrm{~N} 1 \mathrm{O} 1$ & $1.30 \mathrm{E}-08$ \\
\hline $\mathrm{CH}$ & $\mathrm{C} 35 \mathrm{H} 62$ & 0.00045 & Non-basic N1 & $\mathrm{C} 32 \mathrm{H} 37 \mathrm{~N} 1$ & $2.49 \mathrm{E}-05$ & Basic N1O1 & $\mathrm{C} 17 \mathrm{H} 17 \mathrm{~N} 1 \mathrm{O} 1$ & $8.08 \mathrm{E}-08$ \\
\hline $\mathrm{CH}$ & C36H64 & 0.00043 & Non-basic N1 & $\mathrm{C} 33 \mathrm{H} 39 \mathrm{~N} 1$ & $2.27 \mathrm{E}-05$ & Basic N1O1 & C18H19N1O1 & $1.26 \mathrm{E}-07$ \\
\hline $\mathrm{CH}$ & C37H66 & 0.000392 & Non-basic N1 & $\mathrm{C} 34 \mathrm{H} 41 \mathrm{~N} 1$ & $1.39 \mathrm{E}-05$ & Basic N1O1 & $\mathrm{C} 19 \mathrm{H} 21 \mathrm{~N} 1 \mathrm{O} 1$ & $1.44 \mathrm{E}-06$ \\
\hline $\mathrm{CH}$ & C38H68 & 0.000416 & Non-basic N1 & $\mathrm{C} 35 \mathrm{H} 43 \mathrm{~N} 1$ & $6.43 \mathrm{E}-06$ & Basic N1O1 & $\mathrm{C} 20 \mathrm{H} 23 \mathrm{~N} 1 \mathrm{O} 1$ & $3.29 \mathrm{E}-06$ \\
\hline
\end{tabular}




\begin{tabular}{|c|c|c|c|c|c|c|c|c|}
\hline Class & formula & Mass & Class & formula & Mass & Class & formula & Mass \\
\hline $\mathrm{CH}$ & $\mathrm{C} 15 \mathrm{H} 20$ & $9.65 \mathrm{E}-07$ & Non-basic N1 & $\mathrm{C} 36 \mathrm{H} 45 \mathrm{~N} 1$ & $2.17 \mathrm{E}-06$ & Basic N1O1 & $\mathrm{C} 21 \mathrm{H} 25 \mathrm{~N} 1 \mathrm{O} 1$ & $8.30 \mathrm{E}-06$ \\
\hline $\mathrm{CH}$ & $\mathrm{C} 16 \mathrm{H} 22$ & $3.24 \mathrm{E}-06$ & Non-basic N1 & $\mathrm{C} 23 \mathrm{H} 17 \mathrm{~N} 1$ & $2.21 \mathrm{E}-05$ & Basic N1O1 & $\mathrm{C} 22 \mathrm{H} 27 \mathrm{~N} 1 \mathrm{O} 1$ & $3.35 \mathrm{E}-05$ \\
\hline $\mathrm{CH}$ & $\mathrm{C} 17 \mathrm{H} 24$ & 0.000321 & Non-basic N1 & $\mathrm{C} 24 \mathrm{H} 19 \mathrm{~N} 1$ & $6.95 \mathrm{E}-05$ & Basic N1O1 & $\mathrm{C} 23 \mathrm{H} 29 \mathrm{~N} 1 \mathrm{O} 1$ & 4.27E-06 \\
\hline $\mathrm{CH}$ & $\mathrm{C} 18 \mathrm{H} 26$ & 0.000317 & Non-basic N1 & $\mathrm{C} 25 \mathrm{H} 21 \mathrm{~N} 1$ & 0.000112 & Basic N1O1 & $\mathrm{C} 24 \mathrm{H} 31 \mathrm{~N} 1 \mathrm{O} 1$ & $2.79 \mathrm{E}-06$ \\
\hline $\mathrm{CH}$ & С19H28 & 0.000256 & Non-basic N1 & $\mathrm{C} 26 \mathrm{H} 23 \mathrm{~N} 1$ & 0.000128 & Basic N1O1 & $\mathrm{C} 25 \mathrm{H} 33 \mathrm{~N} 1 \mathrm{O} 1$ & $2.69 \mathrm{E}-06$ \\
\hline $\mathrm{CH}$ & $\mathrm{C} 20 \mathrm{H} 30$ & 0.000183 & Non-basic N1 & $\mathrm{C} 27 \mathrm{H} 25 \mathrm{~N} 1$ & $9.91 \mathrm{E}-05$ & Basic N1O1 & $\mathrm{C} 26 \mathrm{H} 35 \mathrm{~N} 1 \mathrm{O} 1$ & $3.49 \mathrm{E}-06$ \\
\hline $\mathrm{CH}$ & $\mathrm{C} 21 \mathrm{H} 32$ & 0.000151 & Non-basic N1 & $\mathrm{C} 28 \mathrm{H} 27 \mathrm{~N} 1$ & $8.25 \mathrm{E}-05$ & Basic N1O1 & $\mathrm{C} 27 \mathrm{H} 37 \mathrm{~N} 1 \mathrm{O} 1$ & $3.36 \mathrm{E}-06$ \\
\hline $\mathrm{CH}$ & $\mathrm{C} 22 \mathrm{H} 34$ & 0.000144 & Non-basic N1 & $\mathrm{C} 29 \mathrm{H} 29 \mathrm{~N} 1$ & $5.06 \mathrm{E}-05$ & Basic N1O1 & $\mathrm{C} 28 \mathrm{H} 39 \mathrm{~N} 1 \mathrm{O} 1$ & $3.59 \mathrm{E}-06$ \\
\hline $\mathrm{CH}$ & $\mathrm{C} 23 \mathrm{H} 36$ & 0.000133 & Non-basic N1 & $\mathrm{C} 30 \mathrm{H} 31 \mathrm{~N} 1$ & 4.26E-05 & Basic N1O1 & $\mathrm{C} 29 \mathrm{H} 41 \mathrm{~N} 1 \mathrm{O} 1$ & 4.75E-06 \\
\hline $\mathrm{CH}$ & $\mathrm{C} 24 \mathrm{H} 38$ & 0.000128 & Non-basic N1 & $\mathrm{C} 31 \mathrm{H} 33 \mathrm{~N} 1$ & $2.33 \mathrm{E}-05$ & Basic N1O1 & $\mathrm{C} 30 \mathrm{H} 43 \mathrm{~N} 1 \mathrm{O} 1$ & $4.56 \mathrm{E}-06$ \\
\hline $\mathrm{CH}$ & $\mathrm{C} 25 \mathrm{H} 40$ & 0.000162 & Non-basic N1 & $\mathrm{C} 32 \mathrm{H} 35 \mathrm{~N} 1$ & $1.75 \mathrm{E}-05$ & Basic N1O1 & $\mathrm{C} 31 \mathrm{H} 45 \mathrm{~N} 1 \mathrm{O} 1$ & 4.87E-06 \\
\hline $\mathrm{CH}$ & $\mathrm{C} 26 \mathrm{H} 42$ & 0.000237 & Non-basic N1 & $\mathrm{C} 33 \mathrm{H} 37 \mathrm{~N} 1$ & $1.54 \mathrm{E}-05$ & Basic N1O1 & $\mathrm{C} 32 \mathrm{H} 47 \mathrm{~N} 1 \mathrm{O} 1$ & $4.38 \mathrm{E}-06$ \\
\hline $\mathrm{CH}$ & $\mathrm{C} 27 \mathrm{H} 44$ & 0.000283 & Non-basic N1 & $\mathrm{C} 34 \mathrm{H} 39 \mathrm{~N} 1$ & $1.06 \mathrm{E}-05$ & Basic N1O1 & $\mathrm{C} 33 \mathrm{H} 49 \mathrm{~N} 1 \mathrm{O} 1$ & 4.20E-06 \\
\hline $\mathrm{CH}$ & $\mathrm{C} 28 \mathrm{H} 46$ & 0.000281 & Non-basic N1 & $\mathrm{C} 35 \mathrm{H} 41 \mathrm{~N} 1$ & $3.08 \mathrm{E}-06$ & Basic N1O1 & $\mathrm{C} 34 \mathrm{H} 51 \mathrm{~N} 1 \mathrm{O} 1$ & $3.67 \mathrm{E}-06$ \\
\hline $\mathrm{CH}$ & C29H48 & 0.000364 & Non-basic N1 & $\mathrm{C} 36 \mathrm{H} 43 \mathrm{~N} 1$ & $1.98 \mathrm{E}-06$ & Basic N1O1 & C35H53N1O1 & $3.67 \mathrm{E}-06$ \\
\hline $\mathrm{CH}$ & $\mathrm{C} 30 \mathrm{H} 50$ & 0.000451 & Non-basic N1 & $\mathrm{C} 24 \mathrm{H} 17 \mathrm{~N} 1$ & $1.68 \mathrm{E}-06$ & Basic N1O1 & $\mathrm{C} 36 \mathrm{H} 55 \mathrm{~N} 1 \mathrm{O} 1$ & $3.02 \mathrm{E}-06$ \\
\hline $\mathrm{CH}$ & $\mathrm{C} 31 \mathrm{H} 52$ & 0.000431 & Non-basic N1 & $\mathrm{C} 25 \mathrm{H} 19 \mathrm{~N} 1$ & $1.20 \mathrm{E}-06$ & Basic N1O1 & C37H57N1O1 & $2.53 \mathrm{E}-06$ \\
\hline $\mathrm{CH}$ & С $32 \mathrm{H} 54$ & 0.000416 & Non-basic N1 & $\mathrm{C} 26 \mathrm{H} 21 \mathrm{~N} 1$ & $1.32 \mathrm{E}-05$ & Basic N1O1 & C38H59N1O1 & $2.33 \mathrm{E}-06$ \\
\hline $\mathrm{CH}$ & $\mathrm{C} 33 \mathrm{H} 56$ & 0.000447 & Non-basic N1 & $\mathrm{C} 27 \mathrm{H} 23 \mathrm{~N} 1$ & $2.43 \mathrm{E}-05$ & Basic N1O1 & C39H61N1O1 & $1.55 \mathrm{E}-06$ \\
\hline $\mathrm{CH}$ & C34H58 & 0.000405 & Non-basic N1 & $\mathrm{C} 28 \mathrm{H} 25 \mathrm{~N} 1$ & $3.31 \mathrm{E}-05$ & Basic N1O1 & C40H63N1O1 & $8.48 \mathrm{E}-07$ \\
\hline $\mathrm{CH}$ & $\mathrm{C} 35 \mathrm{H} 60$ & 0.00045 & Non-basic N1 & $\mathrm{C} 29 \mathrm{H} 27 \mathrm{~N} 1$ & $2.54 \mathrm{E}-05$ & Basic N1O1 & $\mathrm{C} 16 \mathrm{H} 13 \mathrm{~N} 1 \mathrm{O} 1$ & $1.29 \mathrm{E}-08$ \\
\hline $\mathrm{CH}$ & $\mathrm{C} 36 \mathrm{H} 62$ & 0.000401 & Non-basic N1 & $\mathrm{C} 30 \mathrm{H} 29 \mathrm{~N} 1$ & $2.62 \mathrm{E}-05$ & Basic N1O1 & C17H15N1O1 & 2.34E-08 \\
\hline $\mathrm{CH}$ & C37H64 & 0.000435 & Non-basic N1 & $\mathrm{C} 31 \mathrm{H} 31 \mathrm{~N} 1$ & $1.56 \mathrm{E}-05$ & Basic N1O1 & C18H17N1O1 & 2.17E-08 \\
\hline $\mathrm{CH}$ & C38H66 & 0.000432 & Non-basic N1 & $\mathrm{C} 32 \mathrm{H} 33 \mathrm{~N} 1$ & $1.15 \mathrm{E}-05$ & Basic N1O1 & C19H19N1O1 & $1.27 \mathrm{E}-07$ \\
\hline $\mathrm{CH}$ & $\mathrm{C} 16 \mathrm{H} 20$ & 0.000107 & Non-basic N1 & $\mathrm{C} 33 \mathrm{H} 35 \mathrm{~N} 1$ & 4.96E-06 & Basic N1O1 & $\mathrm{C} 20 \mathrm{H} 21 \mathrm{~N} 1 \mathrm{O} 1$ & $3.15 \mathrm{E}-07$ \\
\hline $\mathrm{CH}$ & $\mathrm{C} 17 \mathrm{H} 22$ & 0.001028 & Non-basic N1 & $\mathrm{C} 34 \mathrm{H} 37 \mathrm{~N} 1$ & $6.46 \mathrm{E}-06$ & Basic N1O1 & $\mathrm{C} 21 \mathrm{H} 23 \mathrm{~N} 1 \mathrm{O} 1$ & $2.03 \mathrm{E}-06$ \\
\hline $\mathrm{CH}$ & $\mathrm{C} 18 \mathrm{H} 24$ & 0.000914 & Non-basic N1 & $\mathrm{C} 35 \mathrm{H} 39 \mathrm{~N} 1$ & $1.70 \mathrm{E}-06$ & Basic N1O1 & $\mathrm{C} 22 \mathrm{H} 25 \mathrm{~N} 1 \mathrm{O} 1$ & $8.02 \mathrm{E}-06$ \\
\hline $\mathrm{CH}$ & C19H26 & 0.000699 & Non-basic N1 & $\mathrm{C} 25 \mathrm{H} 17 \mathrm{~N} 1$ & $1.12 \mathrm{E}-06$ & Basic N1O1 & $\mathrm{C} 23 \mathrm{H} 27 \mathrm{~N} 1 \mathrm{O} 1$ & 3.34E-06 \\
\hline $\mathrm{CH}$ & $\mathrm{C} 20 \mathrm{H} 28$ & 0.00051 & Non-basic N1 & $\mathrm{C} 26 \mathrm{H} 19 \mathrm{~N} 1$ & $1.19 \mathrm{E}-05$ & Basic N1O1 & $\mathrm{C} 24 \mathrm{H} 29 \mathrm{~N} 1 \mathrm{O} 1$ & $3.23 \mathrm{E}-06$ \\
\hline $\mathrm{CH}$ & $\mathrm{C} 21 \mathrm{H} 30$ & 0.000431 & Non-basic N1 & $\mathrm{C} 27 \mathrm{H} 21 \mathrm{~N} 1$ & $1.38 \mathrm{E}-05$ & Basic N1O1 & $\mathrm{C} 25 \mathrm{H} 31 \mathrm{~N} 1 \mathrm{O} 1$ & $3.67 \mathrm{E}-06$ \\
\hline $\mathrm{CH}$ & $\mathrm{C} 22 \mathrm{H} 32$ & 0.000361 & Non-basic N1 & $\mathrm{C} 28 \mathrm{H} 23 \mathrm{~N} 1$ & $1.50 \mathrm{E}-05$ & Basic N1O1 & $\mathrm{C} 26 \mathrm{H} 33 \mathrm{~N} 1 \mathrm{O} 1$ & $4.50 \mathrm{E}-06$ \\
\hline $\mathrm{CH}$ & $\mathrm{C} 23 \mathrm{H} 34$ & 0.000356 & Non-basic N1 & $\mathrm{C} 29 \mathrm{H} 25 \mathrm{~N} 1$ & $1.41 \mathrm{E}-05$ & Basic N1O1 & $\mathrm{C} 27 \mathrm{H} 35 \mathrm{~N} 1 \mathrm{O} 1$ & $4.50 \mathrm{E}-06$ \\
\hline $\mathrm{CH}$ & $\mathrm{C} 24 \mathrm{H} 36$ & 0.000361 & Non-basic N1 & $\mathrm{C} 30 \mathrm{H} 27 \mathrm{~N} 1$ & $1.67 \mathrm{E}-05$ & Basic N1O1 & $\mathrm{C} 28 \mathrm{H} 37 \mathrm{~N} 1 \mathrm{O} 1$ & 4.57E-06 \\
\hline $\mathrm{CH}$ & $\mathrm{C} 25 \mathrm{H} 38$ & 0.000382 & Non-basic N1 & $\mathrm{C} 31 \mathrm{H} 29 \mathrm{~N} 1$ & $3.60 \mathrm{E}-06$ & Basic N1O1 & C29H39N1O1 & $1.05 \mathrm{E}-05$ \\
\hline $\mathrm{CH}$ & $\mathrm{C} 26 \mathrm{H} 40$ & 0.000434 & Non-basic N1 & $\mathrm{C} 32 \mathrm{H} 31 \mathrm{~N} 1$ & $2.09 \mathrm{E}-06$ & Basic N1O1 & $\mathrm{C} 30 \mathrm{H} 41 \mathrm{~N} 1 \mathrm{O} 1$ & $4.28 \mathrm{E}-06$ \\
\hline $\mathrm{CH}$ & $\mathrm{C} 27 \mathrm{H} 42$ & 0.000507 & Non-basic N1 & $\mathrm{C} 33 \mathrm{H} 33 \mathrm{~N} 1$ & $1.92 \mathrm{E}-06$ & Basic N1O1 & $\mathrm{C} 31 \mathrm{H} 43 \mathrm{~N} 1 \mathrm{O} 1$ & $4.29 \mathrm{E}-06$ \\
\hline $\mathrm{CH}$ & $\mathrm{C} 28 \mathrm{H} 44$ & 0.000538 & Non-basic N1 & $\mathrm{C} 27 \mathrm{H} 19 \mathrm{~N} 1$ & $1.23 \mathrm{E}-06$ & Basic N1O1 & $\mathrm{C} 32 \mathrm{H} 45 \mathrm{~N} 1 \mathrm{O} 1$ & 4.16E-06 \\
\hline $\mathrm{CH}$ & C29H46 & 0.00068 & Non-basic N1 & $\mathrm{C} 28 \mathrm{H} 21 \mathrm{~N} 1$ & $1.81 \mathrm{E}-06$ & Basic N1O1 & $\mathrm{C} 33 \mathrm{H} 47 \mathrm{~N} 1 \mathrm{O} 1$ & $3.53 \mathrm{E}-06$ \\
\hline
\end{tabular}




\begin{tabular}{|c|c|c|c|c|c|c|c|c|}
\hline Class & formula & Mass & Class & formula & Mass & Class & formula & Mass \\
\hline $\mathrm{CH}$ & $\mathrm{C} 30 \mathrm{H} 48$ & 0.000683 & Non-basic N1 & $\mathrm{C} 29 \mathrm{H} 23 \mathrm{~N} 1$ & 7.72E-06 & Basic N1O1 & $\mathrm{C} 34 \mathrm{H} 49 \mathrm{~N} 1 \mathrm{O} 1$ & $3.48 \mathrm{E}-06$ \\
\hline $\mathrm{CH}$ & $\mathrm{C} 31 \mathrm{H} 50$ & 0.00063 & Non-basic N1 & $\mathrm{C} 30 \mathrm{H} 25 \mathrm{~N} 1$ & $8.93 \mathrm{E}-06$ & Basic N1O1 & C35H51N1O1 & $2.85 \mathrm{E}-06$ \\
\hline $\mathrm{CH}$ & $\mathrm{C} 32 \mathrm{H} 52$ & 0.000588 & Non-basic N1 & $\mathrm{C} 31 \mathrm{H} 27 \mathrm{~N} 1$ & $7.20 \mathrm{E}-06$ & Basic N1O1 & $\mathrm{C} 36 \mathrm{H} 53 \mathrm{~N} 1 \mathrm{O} 1$ & $2.71 \mathrm{E}-06$ \\
\hline $\mathrm{CH}$ & $\mathrm{C} 33 \mathrm{H} 54$ & 0.000638 & Non-basic N1 & $\mathrm{C} 32 \mathrm{H} 29 \mathrm{~N} 1$ & 4.37E-06 & Basic N1O1 & C37H55N1O1 & $2.26 \mathrm{E}-06$ \\
\hline $\mathrm{CH}$ & C34H56 & 0.000645 & Non-basic N1 & $\mathrm{C} 33 \mathrm{H} 31 \mathrm{~N} 1$ & $2.27 \mathrm{E}-06$ & Basic N1O1 & $\mathrm{C} 38 \mathrm{H} 57 \mathrm{~N} 1 \mathrm{O} 1$ & $1.86 \mathrm{E}-06$ \\
\hline $\mathrm{CH}$ & C35H58 & 0.000628 & Non-basic N1O1 & C15H15N1O1 & 4.81E-06 & Basic N1O1 & $\mathrm{C} 39 \mathrm{H} 59 \mathrm{~N} 1 \mathrm{O} 1$ & $1.48 \mathrm{E}-06$ \\
\hline $\mathrm{CH}$ & $\mathrm{C} 36 \mathrm{H} 60$ & 0.000575 & Non-basic N1O1 & C16H17N1O1 & $6.62 \mathrm{E}-06$ & Basic N1O1 & $\mathrm{C} 40 \mathrm{H} 61 \mathrm{~N} 1 \mathrm{O} 1$ & $8.92 \mathrm{E}-07$ \\
\hline $\mathrm{CH}$ & C37H62 & 0.000605 & Non-basic N1O1 & C17H19N1O1 & $4.59 \mathrm{E}-06$ & Basic N1O1 & $\mathrm{C} 41 \mathrm{H} 63 \mathrm{~N} 1 \mathrm{O} 1$ & $2.95 \mathrm{E}-07$ \\
\hline $\mathrm{CH}$ & C38H64 & 0.000524 & Non-basic N1O1 & $\mathrm{C} 18 \mathrm{H} 21 \mathrm{~N} 1 \mathrm{O} 1$ & $7.11 \mathrm{E}-07$ & Basic N1O1 & $\mathrm{C} 42 \mathrm{H} 65 \mathrm{~N} 1 \mathrm{O} 1$ & $4.54 \mathrm{E}-07$ \\
\hline $\mathrm{CH}$ & C39H66 & 0.000525 & Non-basic N1O1 & $\mathrm{C} 19 \mathrm{H} 23 \mathrm{~N} 1 \mathrm{O} 1$ & $4.56 \mathrm{E}-07$ & Basic N1O1 & C43H67N1O1 & $1.70 \mathrm{E}-07$ \\
\hline $\mathrm{CH}$ & $\mathrm{C} 16 \mathrm{H} 18$ & 0.000519 & Non-basic N1O1 & C15H13N1O1 & $6.34 \mathrm{E}-06$ & Basic N1O1 & C44H69N1O1 & $2.46 \mathrm{E}-07$ \\
\hline $\mathrm{CH}$ & $\mathrm{C} 17 \mathrm{H} 20$ & 0.002999 & Non-basic N1O1 & C16H15N1O1 & $1.08 \mathrm{E}-05$ & Basic N1O1 & $\mathrm{C} 45 \mathrm{H} 71 \mathrm{~N} 1 \mathrm{O} 1$ & $9.18 \mathrm{E}-08$ \\
\hline $\mathrm{CH}$ & $\mathrm{C} 18 \mathrm{H} 22$ & 0.002316 & Non-basic N1O1 & C17H17N1O1 & $1.09 \mathrm{E}-05$ & Basic N1O1 & C16H11N1O1 & 8.81E-09 \\
\hline $\mathrm{CH}$ & $\mathrm{C} 19 \mathrm{H} 24$ & 0.001227 & Non-basic N1O1 & C18H19N1O1 & $7.78 \mathrm{E}-06$ & Basic N1O1 & C17H13N1O1 & $1.98 \mathrm{E}-08$ \\
\hline $\mathrm{CH}$ & $\mathrm{C} 20 \mathrm{H} 26$ & 0.000774 & Non-basic N1O1 & $\mathrm{C} 19 \mathrm{H} 21 \mathrm{~N} 1 \mathrm{O} 1$ & $2.35 \mathrm{E}-06$ & Basic N1O1 & $\mathrm{C} 18 \mathrm{H} 15 \mathrm{~N} 1 \mathrm{O} 1$ & 7.72E-08 \\
\hline $\mathrm{CH}$ & $\mathrm{C} 21 \mathrm{H} 28$ & 0.000663 & Non-basic N1O1 & $\mathrm{C} 20 \mathrm{H} 23 \mathrm{~N} 1 \mathrm{O} 1$ & $5.88 \mathrm{E}-06$ & Basic N1O1 & C19H17N1O1 & $3.69 \mathrm{E}-07$ \\
\hline $\mathrm{CH}$ & $\mathrm{C} 22 \mathrm{H} 30$ & 0.000567 & Non-basic N1O1 & $\mathrm{C} 21 \mathrm{H} 25 \mathrm{~N} 1 \mathrm{O} 1$ & $3.19 \mathrm{E}-06$ & Basic N1O1 & C20H19N1O1 & $1.54 \mathrm{E}-06$ \\
\hline $\mathrm{CH}$ & $\mathrm{C} 23 \mathrm{H} 32$ & 0.000532 & Non-basic N1O1 & $\mathrm{C} 22 \mathrm{H} 27 \mathrm{~N} 1 \mathrm{O} 1$ & $3.21 \mathrm{E}-06$ & Basic N1O1 & $\mathrm{C} 21 \mathrm{H} 21 \mathrm{~N} 1 \mathrm{O} 1$ & 4.24E-06 \\
\hline $\mathrm{CH}$ & $\mathrm{C} 24 \mathrm{H} 34$ & 0.000627 & Non-basic N1O1 & $\mathrm{C} 23 \mathrm{H} 29 \mathrm{~N} 1 \mathrm{O} 1$ & $2.99 \mathrm{E}-06$ & Basic N1O1 & $\mathrm{C} 22 \mathrm{H} 23 \mathrm{~N} 1 \mathrm{O} 1$ & $4.65 \mathrm{E}-06$ \\
\hline $\mathrm{CH}$ & $\mathrm{C} 25 \mathrm{H} 36$ & 0.000645 & Non-basic N1O1 & $\mathrm{C} 25 \mathrm{H} 33 \mathrm{~N} 1 \mathrm{O} 1$ & $1.40 \mathrm{E}-06$ & Basic N1O1 & $\mathrm{C} 23 \mathrm{H} 25 \mathrm{~N} 1 \mathrm{O} 1$ & $3.82 \mathrm{E}-06$ \\
\hline $\mathrm{CH}$ & $\mathrm{C} 26 \mathrm{H} 38$ & 0.00079 & Non-basic N1O1 & $\mathrm{C} 26 \mathrm{H} 35 \mathrm{~N} 1 \mathrm{O} 1$ & $1.62 \mathrm{E}-06$ & Basic N1O1 & $\mathrm{C} 24 \mathrm{H} 27 \mathrm{~N} 1 \mathrm{O} 1$ & $3.23 \mathrm{E}-06$ \\
\hline $\mathrm{CH}$ & $\mathrm{C} 27 \mathrm{H} 40$ & 0.000732 & Non-basic N1O1 & $\mathrm{C} 27 \mathrm{H} 37 \mathrm{~N} 1 \mathrm{O} 1$ & $3.77 \mathrm{E}-06$ & Basic N1O1 & $\mathrm{C} 25 \mathrm{H} 29 \mathrm{~N} 1 \mathrm{O} 1$ & $3.84 \mathrm{E}-06$ \\
\hline $\mathrm{CH}$ & $\mathrm{C} 28 \mathrm{H} 42$ & 0.000789 & Non-basic N1O1 & $\mathrm{C} 29 \mathrm{H} 41 \mathrm{~N} 1 \mathrm{O} 1$ & $3.05 \mathrm{E}-06$ & Basic N1O1 & C26H31N1O1 & 4.65E-06 \\
\hline $\mathrm{CH}$ & $\mathrm{C} 29 \mathrm{H} 44$ & 0.000864 & Non-basic N1O1 & $\mathrm{C} 30 \mathrm{H} 43 \mathrm{~N} 1 \mathrm{O} 1$ & $5.29 \mathrm{E}-06$ & Basic N1O1 & C27H33N1O1 & $5.67 \mathrm{E}-06$ \\
\hline $\mathrm{CH}$ & $\mathrm{C} 30 \mathrm{H} 46$ & 0.000873 & Non-basic N1O1 & $\mathrm{C} 31 \mathrm{H} 45 \mathrm{~N} 1 \mathrm{O} 1$ & $3.83 \mathrm{E}-06$ & Basic N1O1 & $\mathrm{C} 28 \mathrm{H} 35 \mathrm{~N} 1 \mathrm{O} 1$ & $6.17 \mathrm{E}-06$ \\
\hline $\mathrm{CH}$ & $\mathrm{C} 31 \mathrm{H} 48$ & 0.000827 & Non-basic N1O1 & $\mathrm{C} 32 \mathrm{H} 47 \mathrm{~N} 1 \mathrm{O} 1$ & 4.44E-06 & Basic N1O1 & C29H37N1O1 & $1.33 \mathrm{E}-05$ \\
\hline $\mathrm{CH}$ & $\mathrm{C} 32 \mathrm{H} 50$ & 0.000792 & Non-basic N1O1 & $\mathrm{C} 16 \mathrm{H} 13 \mathrm{~N} 1 \mathrm{O} 1$ & $1.08 \mathrm{E}-06$ & Basic N1O1 & $\mathrm{C} 30 \mathrm{H} 39 \mathrm{~N} 1 \mathrm{O} 1$ & $4.68 \mathrm{E}-06$ \\
\hline $\mathrm{CH}$ & C33H52 & 0.000824 & Non-basic N1O1 & $\mathrm{C} 17 \mathrm{H} 15 \mathrm{~N} 1 \mathrm{O} 1$ & $4.11 \mathrm{E}-06$ & Basic N1O1 & $\mathrm{C} 31 \mathrm{H} 41 \mathrm{~N} 1 \mathrm{O} 1$ & $4.81 \mathrm{E}-06$ \\
\hline $\mathrm{CH}$ & C34H54 & 0.00077 & Non-basic N1O1 & $\mathrm{C} 18 \mathrm{H} 17 \mathrm{~N} 1 \mathrm{O} 1$ & $8.77 \mathrm{E}-06$ & Basic N1O1 & $\mathrm{C} 32 \mathrm{H} 43 \mathrm{~N} 1 \mathrm{O} 1$ & $4.54 \mathrm{E}-06$ \\
\hline $\mathrm{CH}$ & C35H56 & 0.000757 & Non-basic N1O1 & C19H19N1O1 & $5.53 \mathrm{E}-06$ & Basic N1O1 & $\mathrm{C} 33 \mathrm{H} 45 \mathrm{~N} 1 \mathrm{O} 1$ & $3.69 \mathrm{E}-06$ \\
\hline $\mathrm{CH}$ & $\mathrm{C} 36 \mathrm{H} 58$ & 0.000717 & Non-basic N1O1 & $\mathrm{C} 20 \mathrm{H} 21 \mathrm{~N} 1 \mathrm{O} 1$ & $5.51 \mathrm{E}-06$ & Basic N1O1 & $\mathrm{C} 34 \mathrm{H} 47 \mathrm{~N} 1 \mathrm{O} 1$ & $2.71 \mathrm{E}-06$ \\
\hline $\mathrm{CH}$ & $\mathrm{C} 37 \mathrm{H} 60$ & 0.000691 & Non-basic N1O1 & $\mathrm{C} 21 \mathrm{H} 23 \mathrm{~N} 1 \mathrm{O} 1$ & $4.98 \mathrm{E}-06$ & Basic N1O1 & C35H49N1O1 & $3.44 \mathrm{E}-06$ \\
\hline $\mathrm{CH}$ & C38H62 & 0.000675 & Non-basic N1O1 & $\mathrm{C} 22 \mathrm{H} 25 \mathrm{~N} 1 \mathrm{O} 1$ & $4.16 \mathrm{E}-06$ & Basic N1O1 & C36H51N1O1 & $2.63 \mathrm{E}-06$ \\
\hline $\mathrm{CH}$ & С39H64 & 0.000653 & Non-basic N1O1 & $\mathrm{C} 23 \mathrm{H} 27 \mathrm{~N} 1 \mathrm{O} 1$ & $3.91 \mathrm{E}-06$ & Basic N1O1 & C37H53N1O1 & $2.19 \mathrm{E}-06$ \\
\hline $\mathrm{CH}$ & C16H16 & 0.000229 & Non-basic N1O1 & $\mathrm{C} 24 \mathrm{H} 29 \mathrm{~N} 1 \mathrm{O} 1$ & $3.73 \mathrm{E}-06$ & Basic N1O1 & C38H55N1O1 & $1.65 \mathrm{E}-06$ \\
\hline $\mathrm{CH}$ & $\mathrm{C} 17 \mathrm{H} 18$ & 0.002402 & Non-basic N1O1 & $\mathrm{C} 25 \mathrm{H} 31 \mathrm{~N} 1 \mathrm{O} 1$ & 4.92E-06 & Basic N1O1 & C39H57N1O1 & $9.74 \mathrm{E}-07$ \\
\hline $\mathrm{CH}$ & $\mathrm{C} 18 \mathrm{H} 20$ & 0.003858 & Non-basic N1O1 & $\mathrm{C} 26 \mathrm{H} 33 \mathrm{~N} 1 \mathrm{O} 1$ & $2.01 \mathrm{E}-06$ & Basic N1O1 & C40H59N1O1 & $1.02 \mathrm{E}-06$ \\
\hline $\mathrm{CH}$ & $\mathrm{C} 19 \mathrm{H} 22$ & 0.002691 & Non-basic N1O1 & $\mathrm{C} 27 \mathrm{H} 35 \mathrm{~N} 1 \mathrm{O} 1$ & $4.55 \mathrm{E}-06$ & Basic N1O1 & C41H61N1O1 & $6.16 \mathrm{E}-07$ \\
\hline
\end{tabular}




\begin{tabular}{|c|c|c|c|c|c|c|c|c|}
\hline Class & formula & Mass & Class & formula & Mass & Class & formula & Mass \\
\hline $\mathrm{CH}$ & $\mathrm{C} 20 \mathrm{H} 24$ & 0.001773 & Non-basic N1O1 & $\mathrm{C} 28 \mathrm{H} 37 \mathrm{~N} 1 \mathrm{O} 1$ & $2.07 \mathrm{E}-06$ & Basic N1O1 & $\mathrm{C} 42 \mathrm{H} 63 \mathrm{~N} 1 \mathrm{O} 1$ & 4.09E-07 \\
\hline $\mathrm{CH}$ & $\mathrm{C} 21 \mathrm{H} 26$ & 0.001241 & Non-basic N1O1 & C29H39N1O1 & $3.13 \mathrm{E}-06$ & Basic N1O1 & $\mathrm{C} 43 \mathrm{H} 65 \mathrm{~N} 1 \mathrm{O} 1$ & $2.32 \mathrm{E}-07$ \\
\hline $\mathrm{CH}$ & $\mathrm{C} 22 \mathrm{H} 28$ & 0.001127 & Non-basic N1O1 & $\mathrm{C} 30 \mathrm{H} 41 \mathrm{~N} 1 \mathrm{O} 1$ & $1.25 \mathrm{E}-06$ & Basic N1O1 & $\mathrm{C} 18 \mathrm{H} 13 \mathrm{~N} 1 \mathrm{O} 1$ & $1.30 \mathrm{E}-08$ \\
\hline $\mathrm{CH}$ & $\mathrm{C} 23 \mathrm{H} 30$ & 0.000887 & Non-basic N1O1 & C31H43N1O1 & $2.11 \mathrm{E}-06$ & Basic N1O1 & C19H15N1O1 & $8.69 \mathrm{E}-08$ \\
\hline $\mathrm{CH}$ & $\mathrm{C} 24 \mathrm{H} 32$ & 0.000855 & Non-basic N1O1 & $\mathrm{C} 32 \mathrm{H} 45 \mathrm{~N} 1 \mathrm{O} 1$ & $3.46 \mathrm{E}-06$ & Basic N1O1 & $\mathrm{C} 20 \mathrm{H} 17 \mathrm{~N} 1 \mathrm{O} 1$ & $2.78 \mathrm{E}-07$ \\
\hline $\mathrm{CH}$ & $\mathrm{C} 25 \mathrm{H} 34$ & 0.000865 & Non-basic N1O1 & C17H13N1O1 & $6.32 \mathrm{E}-06$ & Basic N1O1 & C21H19N1O1 & $5.74 \mathrm{E}-07$ \\
\hline $\mathrm{CH}$ & $\mathrm{C} 26 \mathrm{H} 36$ & 0.001043 & Non-basic N1O1 & C18H15N1O1 & $2.02 \mathrm{E}-05$ & Basic N1O1 & $\mathrm{C} 22 \mathrm{H} 21 \mathrm{~N} 1 \mathrm{O} 1$ & $2.62 \mathrm{E}-06$ \\
\hline $\mathrm{CH}$ & $\mathrm{C} 27 \mathrm{H} 38$ & 0.001128 & Non-basic N1O1 & C19H17N1O1 & $2.81 \mathrm{E}-05$ & Basic N1O1 & $\mathrm{C} 23 \mathrm{H} 23 \mathrm{~N} 1 \mathrm{O} 1$ & $5.71 \mathrm{E}-06$ \\
\hline $\mathrm{CH}$ & $\mathrm{C} 28 \mathrm{H} 40$ & 0.001234 & Non-basic N1O1 & $\mathrm{C} 20 \mathrm{H} 19 \mathrm{~N} 1 \mathrm{O} 1$ & $2.46 \mathrm{E}-05$ & Basic N1O1 & $\mathrm{C} 24 \mathrm{H} 25 \mathrm{~N} 1 \mathrm{O} 1$ & 7.47E-06 \\
\hline $\mathrm{CH}$ & $\mathrm{C} 29 \mathrm{H} 42$ & 0.001357 & Non-basic N1O1 & $\mathrm{C} 21 \mathrm{H} 21 \mathrm{~N} 1 \mathrm{O} 1$ & $1.77 \mathrm{E}-05$ & Basic N1O1 & $\mathrm{C} 25 \mathrm{H} 27 \mathrm{~N} 1 \mathrm{O} 1$ & 7.54E-06 \\
\hline $\mathrm{CH}$ & $\mathrm{C} 30 \mathrm{H} 44$ & 0.001198 & Non-basic N1O1 & $\mathrm{C} 22 \mathrm{H} 23 \mathrm{~N} 1 \mathrm{O} 1$ & $1.22 \mathrm{E}-05$ & Basic N1O1 & $\mathrm{C} 26 \mathrm{H} 29 \mathrm{~N} 1 \mathrm{O} 1$ & $7.87 \mathrm{E}-06$ \\
\hline $\mathrm{CH}$ & $\mathrm{C} 31 \mathrm{H} 46$ & 0.001088 & Non-basic N1O1 & $\mathrm{C} 23 \mathrm{H} 25 \mathrm{~N} 1 \mathrm{O} 1$ & $8.70 \mathrm{E}-06$ & Basic N1O1 & $\mathrm{C} 27 \mathrm{H} 31 \mathrm{~N} 1 \mathrm{O} 1$ & $7.48 \mathrm{E}-06$ \\
\hline $\mathrm{CH}$ & $\mathrm{C} 32 \mathrm{H} 48$ & 0.001008 & Non-basic N1O1 & $\mathrm{C} 24 \mathrm{H} 27 \mathrm{~N} 1 \mathrm{O} 1$ & $7.14 \mathrm{E}-06$ & Basic N1O1 & $\mathrm{C} 28 \mathrm{H} 33 \mathrm{~N} 1 \mathrm{O} 1$ & 8.23E-06 \\
\hline $\mathrm{CH}$ & $\mathrm{C} 33 \mathrm{H} 50$ & 0.001045 & Non-basic N1O1 & $\mathrm{C} 25 \mathrm{H} 29 \mathrm{~N} 1 \mathrm{O} 1$ & $6.97 \mathrm{E}-06$ & Basic N1O1 & $\mathrm{C} 29 \mathrm{H} 35 \mathrm{~N} 1 \mathrm{O} 1$ & $1.04 \mathrm{E}-05$ \\
\hline $\mathrm{CH}$ & $\mathrm{C} 34 \mathrm{H} 52$ & 0.001012 & Non-basic N1O1 & $\mathrm{C} 26 \mathrm{H} 31 \mathrm{~N} 1 \mathrm{O} 1$ & $2.20 \mathrm{E}-06$ & Basic N1O1 & $\mathrm{C} 30 \mathrm{H} 37 \mathrm{~N} 1 \mathrm{O} 1$ & $6.80 \mathrm{E}-06$ \\
\hline $\mathrm{CH}$ & C35H54 & 0.000863 & Non-basic N1O1 & $\mathrm{C} 27 \mathrm{H} 33 \mathrm{~N} 1 \mathrm{O} 1$ & $7.50 \mathrm{E}-06$ & Basic N1O1 & C31H39N1O1 & $5.54 \mathrm{E}-06$ \\
\hline $\mathrm{CH}$ & $\mathrm{C} 36 \mathrm{H} 56$ & 0.000775 & Non-basic N1O1 & $\mathrm{C} 28 \mathrm{H} 35 \mathrm{~N} 1 \mathrm{O} 1$ & $4.26 \mathrm{E}-06$ & Basic N1O1 & $\mathrm{C} 32 \mathrm{H} 41 \mathrm{~N} 1 \mathrm{O} 1$ & $5.52 \mathrm{E}-06$ \\
\hline $\mathrm{CH}$ & C37H58 & 0.000707 & Non-basic N1O1 & $\mathrm{C} 29 \mathrm{H} 37 \mathrm{~N} 1 \mathrm{O} 1$ & $2.99 \mathrm{E}-06$ & Basic N1O1 & $\mathrm{C} 33 \mathrm{H} 43 \mathrm{~N} 1 \mathrm{O} 1$ & $4.55 \mathrm{E}-06$ \\
\hline $\mathrm{CH}$ & $\mathrm{C} 38 \mathrm{H} 60$ & 0.000663 & Non-basic N1O1 & $\mathrm{C} 30 \mathrm{H} 39 \mathrm{~N} 1 \mathrm{O} 1$ & $5.12 \mathrm{E}-06$ & Basic N1O1 & $\mathrm{C} 34 \mathrm{H} 45 \mathrm{~N} 1 \mathrm{O} 1$ & $3.24 \mathrm{E}-06$ \\
\hline $\mathrm{CH}$ & C39H62 & 0.000689 & Non-basic N1O1 & C17H11N1O1 & $9.96 \mathrm{E}-07$ & Basic N1O1 & $\mathrm{C} 35 \mathrm{H} 47 \mathrm{~N} 1 \mathrm{O} 1$ & $3.21 \mathrm{E}-06$ \\
\hline $\mathrm{CH}$ & $\mathrm{C} 40 \mathrm{H} 64$ & 0.000915 & Non-basic N1O1 & C18H13N1O1 & $1.32 \mathrm{E}-05$ & Basic N1O1 & $\mathrm{C} 36 \mathrm{H} 49 \mathrm{~N} 1 \mathrm{O} 1$ & $3.29 \mathrm{E}-06$ \\
\hline $\mathrm{CH}$ & C41H66 & 0.000415 & Non-basic N1O1 & C19H15N1O1 & $2.19 \mathrm{E}-05$ & Basic N1O1 & C37H51N1O1 & $1.98 \mathrm{E}-06$ \\
\hline $\mathrm{CH}$ & $\mathrm{C} 42 \mathrm{H} 68$ & 0.000345 & Non-basic N1O1 & $\mathrm{C} 20 \mathrm{H} 17 \mathrm{~N} 1 \mathrm{O} 1$ & $3.16 \mathrm{E}-05$ & Basic N1O1 & C38H53N1O1 & $1.81 \mathrm{E}-06$ \\
\hline $\mathrm{CH}$ & $\mathrm{C} 16 \mathrm{H} 14$ & 0.001418 & Non-basic N1O1 & C21H19N1O1 & $3.62 \mathrm{E}-05$ & Basic N1O1 & C39H55N1O1 & $1.52 \mathrm{E}-06$ \\
\hline $\mathrm{CH}$ & $\mathrm{C} 17 \mathrm{H} 16$ & 0.005931 & Non-basic N1O1 & $\mathrm{C} 22 \mathrm{H} 21 \mathrm{~N} 1 \mathrm{O} 1$ & $2.70 \mathrm{E}-05$ & Basic N1O1 & $\mathrm{C} 40 \mathrm{H} 57 \mathrm{~N} 1 \mathrm{O} 1$ & $7.80 \mathrm{E}-07$ \\
\hline $\mathrm{CH}$ & $\mathrm{C} 18 \mathrm{H} 18$ & 0.009156 & Non-basic N1O1 & $\mathrm{C} 23 \mathrm{H} 23 \mathrm{~N} 1 \mathrm{O} 1$ & $2.21 \mathrm{E}-05$ & Basic N1O1 & C41H59N1O1 & $4.54 \mathrm{E}-07$ \\
\hline $\mathrm{CH}$ & $\mathrm{C} 19 \mathrm{H} 20$ & 0.004931 & Non-basic N1O1 & $\mathrm{C} 24 \mathrm{H} 25 \mathrm{~N} 1 \mathrm{O} 1$ & $1.98 \mathrm{E}-05$ & Basic N1O1 & $\mathrm{C} 42 \mathrm{H} 61 \mathrm{~N} 1 \mathrm{O} 1$ & $5.20 \mathrm{E}-07$ \\
\hline $\mathrm{CH}$ & $\mathrm{C} 20 \mathrm{H} 22$ & 0.002825 & Non-basic N1O1 & $\mathrm{C} 25 \mathrm{H} 27 \mathrm{~N} 1 \mathrm{O} 1$ & $1.07 \mathrm{E}-05$ & Basic N1O1 & C19H13N1O1 & $1.63 \mathrm{E}-08$ \\
\hline $\mathrm{CH}$ & $\mathrm{C} 21 \mathrm{H} 24$ & 0.002308 & Non-basic N1O1 & $\mathrm{C} 26 \mathrm{H} 29 \mathrm{~N} 1 \mathrm{O} 1$ & $8.39 \mathrm{E}-06$ & Basic N1O1 & $\mathrm{C} 20 \mathrm{H} 15 \mathrm{~N} 1 \mathrm{O} 1$ & $1.16 \mathrm{E}-08$ \\
\hline $\mathrm{CH}$ & $\mathrm{C} 22 \mathrm{H} 26$ & 0.001443 & Non-basic N1O1 & $\mathrm{C} 27 \mathrm{H} 31 \mathrm{~N} 1 \mathrm{O} 1$ & 4.03E-06 & Basic N1O1 & $\mathrm{C} 21 \mathrm{H} 17 \mathrm{~N} 1 \mathrm{O} 1$ & $1.76 \mathrm{E}-07$ \\
\hline $\mathrm{CH}$ & $\mathrm{C} 23 \mathrm{H} 28$ & 0.001277 & Non-basic N1O1 & $\mathrm{C} 28 \mathrm{H} 33 \mathrm{~N} 1 \mathrm{O} 1$ & $3.98 \mathrm{E}-06$ & Basic N1O1 & $\mathrm{C} 22 \mathrm{H} 19 \mathrm{~N} 1 \mathrm{O} 1$ & $4.52 \mathrm{E}-07$ \\
\hline $\mathrm{CH}$ & $\mathrm{C} 24 \mathrm{H} 30$ & 0.001289 & Non-basic N1O1 & $\mathrm{C} 20 \mathrm{H} 15 \mathrm{~N} 1 \mathrm{O} 1$ & $1.27 \mathrm{E}-05$ & Basic N1O1 & $\mathrm{C} 23 \mathrm{H} 21 \mathrm{~N} 1 \mathrm{O} 1$ & $1.12 \mathrm{E}-06$ \\
\hline $\mathrm{CH}$ & $\mathrm{C} 25 \mathrm{H} 32$ & 0.00137 & Non-basic N1O1 & $\mathrm{C} 21 \mathrm{H} 17 \mathrm{~N} 1 \mathrm{O} 1$ & $2.80 \mathrm{E}-05$ & Basic N1O1 & C24H23N1O1 & $2.28 \mathrm{E}-06$ \\
\hline $\mathrm{CH}$ & $\mathrm{C} 26 \mathrm{H} 34$ & 0.001368 & Non-basic N1O1 & $\mathrm{C} 22 \mathrm{H} 19 \mathrm{~N} 1 \mathrm{O} 1$ & $3.72 \mathrm{E}-05$ & Basic N1O1 & $\mathrm{C} 25 \mathrm{H} 25 \mathrm{~N} 1 \mathrm{O} 1$ & $3.50 \mathrm{E}-06$ \\
\hline $\mathrm{CH}$ & $\mathrm{C} 27 \mathrm{H} 36$ & 0.001412 & Non-basic N1O1 & $\mathrm{C} 23 \mathrm{H} 21 \mathrm{~N} 1 \mathrm{O} 1$ & $3.14 \mathrm{E}-05$ & Basic N1O1 & $\mathrm{C} 26 \mathrm{H} 27 \mathrm{~N} 1 \mathrm{O} 1$ & $5.41 \mathrm{E}-06$ \\
\hline $\mathrm{CH}$ & C28H38 & 0.001545 & Non-basic N1O1 & $\mathrm{C} 24 \mathrm{H} 23 \mathrm{~N} 1 \mathrm{O} 1$ & $3.04 \mathrm{E}-05$ & Basic N1O1 & $\mathrm{C} 27 \mathrm{H} 29 \mathrm{~N} 1 \mathrm{O} 1$ & $6.90 \mathrm{E}-06$ \\
\hline $\mathrm{CH}$ & $\mathrm{C} 29 \mathrm{H} 40$ & 0.001472 & Non-basic N1O1 & $\mathrm{C} 25 \mathrm{H} 25 \mathrm{~N} 1 \mathrm{O} 1$ & $2.40 \mathrm{E}-05$ & Basic N1O1 & $\mathrm{C} 28 \mathrm{H} 31 \mathrm{~N} 1 \mathrm{O} 1$ & $7.39 \mathrm{E}-06$ \\
\hline $\mathrm{CH}$ & $\mathrm{C} 30 \mathrm{H} 42$ & 0.001432 & Non-basic N1O1 & $\mathrm{C} 26 \mathrm{H} 27 \mathrm{~N} 1 \mathrm{O} 1$ & $2.14 \mathrm{E}-05$ & Basic N1O1 & C29H33N1O1 & $6.79 \mathrm{E}-06$ \\
\hline
\end{tabular}




\begin{tabular}{|c|c|c|c|c|c|c|c|c|}
\hline Class & formula & Mass & Class & formula & Mass & Class & formula & Mass \\
\hline $\mathrm{CH}$ & $\mathrm{C} 31 \mathrm{H} 44$ & 0.001333 & Non-basic N1O1 & $\mathrm{C} 27 \mathrm{H} 29 \mathrm{~N} 1 \mathrm{O} 1$ & $1.14 \mathrm{E}-05$ & Basic N1O1 & $\mathrm{C} 30 \mathrm{H} 35 \mathrm{~N} 1 \mathrm{O} 1$ & $6.47 \mathrm{E}-06$ \\
\hline $\mathrm{CH}$ & $\mathrm{C} 32 \mathrm{H} 46$ & 0.001256 & Non-basic N1O1 & $\mathrm{C} 28 \mathrm{H} 31 \mathrm{~N} 1 \mathrm{O} 1$ & $5.30 \mathrm{E}-06$ & Basic N1O1 & $\mathrm{C} 31 \mathrm{H} 37 \mathrm{~N} 1 \mathrm{O} 1$ & $6.10 \mathrm{E}-06$ \\
\hline $\mathrm{CH}$ & $\mathrm{C} 33 \mathrm{H} 48$ & 0.001133 & Non-basic N1O1 & C29H33N1O1 & 8.91E-06 & Basic N1O1 & $\mathrm{C} 32 \mathrm{H} 39 \mathrm{~N} 1 \mathrm{O} 1$ & $5.03 \mathrm{E}-06$ \\
\hline $\mathrm{CH}$ & $\mathrm{C} 34 \mathrm{H} 50$ & 0.000995 & Non-basic N1O1 & $\mathrm{C} 30 \mathrm{H} 35 \mathrm{~N} 1 \mathrm{O} 1$ & $4.88 \mathrm{E}-06$ & Basic N1O1 & $\mathrm{C} 33 \mathrm{H} 41 \mathrm{~N} 1 \mathrm{O} 1$ & 4.11E-06 \\
\hline $\mathrm{CH}$ & $\mathrm{C} 35 \mathrm{H} 52$ & 0.000885 & Non-basic N1O1 & $\mathrm{C} 31 \mathrm{H} 37 \mathrm{~N} 1 \mathrm{O} 1$ & $2.12 \mathrm{E}-06$ & Basic N1O1 & $\mathrm{C} 34 \mathrm{H} 43 \mathrm{~N} 1 \mathrm{O} 1$ & $3.57 \mathrm{E}-06$ \\
\hline $\mathrm{CH}$ & $\mathrm{C} 36 \mathrm{H} 54$ & 0.000861 & Non-basic N1O1 & C32H39N1O1 & $1.60 \mathrm{E}-06$ & Basic N1O1 & $\mathrm{C} 35 \mathrm{H} 45 \mathrm{~N} 1 \mathrm{O} 1$ & $2.74 \mathrm{E}-06$ \\
\hline $\mathrm{CH}$ & C37H56 & 0.000744 & Non-basic N1O1 & C20H13N1O1 & $4.84 \mathrm{E}-07$ & Basic N1O1 & $\mathrm{C} 36 \mathrm{H} 47 \mathrm{~N} 1 \mathrm{O} 1$ & $3.41 \mathrm{E}-06$ \\
\hline $\mathrm{CH}$ & C38H58 & 0.000681 & Non-basic N1O1 & $\mathrm{C} 21 \mathrm{H} 15 \mathrm{~N} 1 \mathrm{O} 1$ & $6.41 \mathrm{E}-06$ & Basic N1O1 & $\mathrm{C} 37 \mathrm{H} 49 \mathrm{~N} 1 \mathrm{O} 1$ & $2.43 \mathrm{E}-06$ \\
\hline $\mathrm{CH}$ & C39H60 & 0.000707 & Non-basic N1O1 & $\mathrm{C} 22 \mathrm{H} 17 \mathrm{~N} 1 \mathrm{O} 1$ & $9.59 \mathrm{E}-06$ & Basic N1O1 & $\mathrm{C} 38 \mathrm{H} 51 \mathrm{~N} 1 \mathrm{O} 1$ & $1.26 \mathrm{E}-06$ \\
\hline $\mathrm{CH}$ & $\mathrm{C} 40 \mathrm{H} 62$ & 0.000992 & Non-basic N1O1 & $\mathrm{C} 23 \mathrm{H} 19 \mathrm{~N} 1 \mathrm{O} 1$ & $1.58 \mathrm{E}-05$ & Basic N1O1 & $\mathrm{C} 39 \mathrm{H} 53 \mathrm{~N} 1 \mathrm{O} 1$ & $1.16 \mathrm{E}-06$ \\
\hline $\mathrm{CH}$ & C41H64 & 0.000422 & Non-basic N1O1 & $\mathrm{C} 24 \mathrm{H} 21 \mathrm{~N} 1 \mathrm{O} 1$ & $1.53 \mathrm{E}-05$ & Basic N1O1 & $\mathrm{C} 40 \mathrm{H} 55 \mathrm{~N} 1 \mathrm{O} 1$ & $8.49 \mathrm{E}-07$ \\
\hline $\mathrm{CH}$ & $\mathrm{C} 42 \mathrm{H} 66$ & 0.000273 & Non-basic N1O1 & $\mathrm{C} 25 \mathrm{H} 23 \mathrm{~N} 1 \mathrm{O} 1$ & $1.46 \mathrm{E}-05$ & Basic N1O1 & $\mathrm{C} 41 \mathrm{H} 57 \mathrm{~N} 1 \mathrm{O} 1$ & $6.92 \mathrm{E}-07$ \\
\hline $\mathrm{CH}$ & $\mathrm{C} 43 \mathrm{H} 68$ & 0.000228 & Non-basic N1O1 & $\mathrm{C} 26 \mathrm{H} 25 \mathrm{~N} 1 \mathrm{O} 1$ & $1.04 \mathrm{E}-05$ & Basic N1O1 & $\mathrm{C} 42 \mathrm{H} 59 \mathrm{~N} 1 \mathrm{O} 1$ & $1.92 \mathrm{E}-07$ \\
\hline $\mathrm{CH}$ & $\mathrm{C} 16 \mathrm{H} 12$ & $9.75 \mathrm{E}-05$ & Non-basic N1O1 & $\mathrm{C} 27 \mathrm{H} 27 \mathrm{~N} 1 \mathrm{O} 1$ & 7.53E-06 & Basic N1O1 & C43H61N1O1 & $3.29 \mathrm{E}-07$ \\
\hline $\mathrm{CH}$ & $\mathrm{C} 17 \mathrm{H} 14$ & 0.001365 & Non-basic N1O1 & $\mathrm{C} 28 \mathrm{H} 29 \mathrm{~N} 1 \mathrm{O} 1$ & $5.15 \mathrm{E}-06$ & Basic N1O1 & C44H63N1O1 & $1.20 \mathrm{E}-07$ \\
\hline $\mathrm{CH}$ & $\mathrm{C} 18 \mathrm{H} 16$ & 0.011021 & Non-basic N1O1 & C29H31N1O1 & $3.12 \mathrm{E}-06$ & Basic N1O1 & C45H65N1O1 & $7.50 \mathrm{E}-08$ \\
\hline $\mathrm{CH}$ & C19H18 & 0.012345 & Non-basic N1O1 & $\mathrm{C} 30 \mathrm{H} 33 \mathrm{~N} 1 \mathrm{O} 1$ & $1.84 \mathrm{E}-06$ & Basic N1O1 & $\mathrm{C} 20 \mathrm{H} 13 \mathrm{~N} 1 \mathrm{O} 1$ & $2.66 \mathrm{E}-08$ \\
\hline $\mathrm{CH}$ & $\mathrm{C} 20 \mathrm{H} 20$ & 0.008353 & Non-basic N1O1 & $\mathrm{C} 23 \mathrm{H} 17 \mathrm{~N} 1 \mathrm{O} 1$ & 3.63E-06 & Basic N1O1 & $\mathrm{C} 21 \mathrm{H} 15 \mathrm{~N} 1 \mathrm{O} 1$ & $1.71 \mathrm{E}-07$ \\
\hline $\mathrm{CH}$ & $\mathrm{C} 21 \mathrm{H} 22$ & 0.00511 & Non-basic N1O1 & $\mathrm{C} 24 \mathrm{H} 19 \mathrm{~N} 1 \mathrm{O} 1$ & 4.44E-06 & Basic N1O1 & $\mathrm{C} 22 \mathrm{H} 17 \mathrm{~N} 1 \mathrm{O} 1$ & $5.24 \mathrm{E}-07$ \\
\hline $\mathrm{CH}$ & $\mathrm{C} 22 \mathrm{H} 24$ & 0.002703 & Non-basic N1O1 & $\mathrm{C} 25 \mathrm{H} 21 \mathrm{~N} 1 \mathrm{O} 1$ & $9.85 \mathrm{E}-06$ & Basic N1O1 & C23H19N1O1 & $1.09 \mathrm{E}-06$ \\
\hline $\mathrm{CH}$ & $\mathrm{C} 23 \mathrm{H} 26$ & 0.001854 & Non-basic N1O1 & $\mathrm{C} 26 \mathrm{H} 23 \mathrm{~N} 1 \mathrm{O} 1$ & $8.34 \mathrm{E}-06$ & Basic N1O1 & $\mathrm{C} 24 \mathrm{H} 21 \mathrm{~N} 1 \mathrm{O} 1$ & $2.95 \mathrm{E}-06$ \\
\hline $\mathrm{CH}$ & $\mathrm{C} 24 \mathrm{H} 28$ & 0.001499 & Non-basic N1O1 & $\mathrm{C} 27 \mathrm{H} 25 \mathrm{~N} 1 \mathrm{O} 1$ & $5.89 \mathrm{E}-06$ & Basic N1O1 & $\mathrm{C} 25 \mathrm{H} 23 \mathrm{~N} 1 \mathrm{O} 1$ & $4.08 \mathrm{E}-06$ \\
\hline $\mathrm{CH}$ & $\mathrm{C} 25 \mathrm{H} 30$ & 0.001517 & Non-basic N1O1 & $\mathrm{C} 28 \mathrm{H} 27 \mathrm{~N} 1 \mathrm{O} 1$ & $1.49 \mathrm{E}-06$ & Basic N1O1 & $\mathrm{C} 26 \mathrm{H} 25 \mathrm{~N} 1 \mathrm{O} 1$ & 4.83E-06 \\
\hline $\mathrm{CH}$ & $\mathrm{C} 26 \mathrm{H} 32$ & 0.001585 & Non-basic N1O1 & $\mathrm{C} 29 \mathrm{H} 29 \mathrm{~N} 1 \mathrm{O} 1$ & $3.02 \mathrm{E}-06$ & Basic N1O1 & $\mathrm{C} 27 \mathrm{H} 27 \mathrm{~N} 1 \mathrm{O} 1$ & $5.02 \mathrm{E}-06$ \\
\hline $\mathrm{CH}$ & $\mathrm{C} 27 \mathrm{H} 34$ & 0.001791 & Non-basic N1O1 & $\mathrm{C} 23 \mathrm{H} 15 \mathrm{~N} 1 \mathrm{O} 1$ & $1.25 \mathrm{E}-06$ & Basic N1O1 & C28H29N1O1 & $5.26 \mathrm{E}-06$ \\
\hline $\mathrm{CH}$ & $\mathrm{C} 28 \mathrm{H} 36$ & 0.00195 & Non-basic N1O1 & $\mathrm{C} 24 \mathrm{H} 17 \mathrm{~N} 1 \mathrm{O} 1$ & $2.18 \mathrm{E}-06$ & Basic N1O1 & C29H31N1O1 & $5.25 \mathrm{E}-06$ \\
\hline $\mathrm{CH}$ & $\mathrm{C} 29 \mathrm{H} 38$ & 0.001914 & Non-basic N1O1 & $\mathrm{C} 25 \mathrm{H} 19 \mathrm{~N} 1 \mathrm{O} 1$ & $5.63 \mathrm{E}-06$ & Basic N1O1 & $\mathrm{C} 30 \mathrm{H} 33 \mathrm{~N} 1 \mathrm{O} 1$ & $5.04 \mathrm{E}-06$ \\
\hline $\mathrm{CH}$ & $\mathrm{C} 30 \mathrm{H} 40$ & 0.001632 & Non-basic N1O1 & $\mathrm{C} 26 \mathrm{H} 21 \mathrm{~N} 1 \mathrm{O} 1$ & $2.45 \mathrm{E}-06$ & Basic N1O1 & $\mathrm{C} 31 \mathrm{H} 35 \mathrm{~N} 1 \mathrm{O} 1$ & $5.04 \mathrm{E}-06$ \\
\hline $\mathrm{CH}$ & $\mathrm{C} 31 \mathrm{H} 42$ & 0.001589 & Non-basic N1O1 & $\mathrm{C} 27 \mathrm{H} 23 \mathrm{~N} 1 \mathrm{O} 1$ & $1.77 \mathrm{E}-06$ & Basic N1O1 & $\mathrm{C} 32 \mathrm{H} 37 \mathrm{~N} 1 \mathrm{O} 1$ & 4.66E-06 \\
\hline $\mathrm{CH}$ & $\mathrm{C} 32 \mathrm{H} 44$ & 0.001257 & Non-basic N1O1 & $\mathrm{C} 28 \mathrm{H} 25 \mathrm{~N} 1 \mathrm{O} 1$ & $9.18 \mathrm{E}-07$ & Basic N1O1 & C33H39N1O1 & $3.05 \mathrm{E}-06$ \\
\hline $\mathrm{CH}$ & $\mathrm{C} 33 \mathrm{H} 46$ & 0.001108 & Non-basic N1O2 & $\mathrm{C} 30 \mathrm{H} 51 \mathrm{~N} 1 \mathrm{O} 2$ & $4.78 \mathrm{E}-06$ & Basic N1O1 & $\mathrm{C} 34 \mathrm{H} 41 \mathrm{~N} 1 \mathrm{O} 1$ & $3.09 \mathrm{E}-06$ \\
\hline $\mathrm{CH}$ & $\mathrm{C} 34 \mathrm{H} 48$ & 0.001021 & Non-basic N1O2 & $\mathrm{C} 31 \mathrm{H} 53 \mathrm{~N} 1 \mathrm{O} 2$ & $2.19 \mathrm{E}-06$ & Basic N1O1 & C35H43N1O1 & $2.60 \mathrm{E}-06$ \\
\hline $\mathrm{CH}$ & $\mathrm{C} 35 \mathrm{H} 50$ & 0.000867 & Non-basic N1O2 & $\mathrm{C} 32 \mathrm{H} 55 \mathrm{~N} 1 \mathrm{O} 2$ & $5.00 \mathrm{E}-06$ & Basic N1O1 & C36H45N1O1 & $3.27 \mathrm{E}-06$ \\
\hline $\mathrm{CH}$ & $\mathrm{C} 36 \mathrm{H} 52$ & 0.000766 & Non-basic N1O2 & $\mathrm{C} 33 \mathrm{H} 57 \mathrm{~N} 1 \mathrm{O} 2$ & $9.64 \mathrm{E}-06$ & Basic N1O1 & $\mathrm{C} 37 \mathrm{H} 47 \mathrm{~N} 1 \mathrm{O} 1$ & $1.90 \mathrm{E}-06$ \\
\hline $\mathrm{CH}$ & C37H54 & 0.000699 & Non-basic N1O2 & $\mathrm{C} 34 \mathrm{H} 59 \mathrm{~N} 1 \mathrm{O} 2$ & $1.72 \mathrm{E}-06$ & Basic N1O1 & $\mathrm{C} 38 \mathrm{H} 49 \mathrm{~N} 1 \mathrm{O} 1$ & $1.33 \mathrm{E}-06$ \\
\hline $\mathrm{CH}$ & C38H56 & 0.000697 & Non-basic N1O2 & $\mathrm{C} 35 \mathrm{H} 61 \mathrm{~N} 1 \mathrm{O} 2$ & $5.03 \mathrm{E}-06$ & Basic N1O1 & C39H51N1O1 & $9.73 \mathrm{E}-07$ \\
\hline $\mathrm{CH}$ & C39H58 & 0.000811 & Non-basic N1O2 & $\mathrm{C} 36 \mathrm{H} 63 \mathrm{~N} 1 \mathrm{O} 2$ & $1.94 \mathrm{E}-06$ & Basic N1O1 & $\mathrm{C} 40 \mathrm{H} 53 \mathrm{~N} 1 \mathrm{O} 1$ & $7.45 \mathrm{E}-07$ \\
\hline $\mathrm{CH}$ & $\mathrm{C} 40 \mathrm{H} 60$ & 0.000694 & Non-basic N1O2 & $\mathrm{C} 37 \mathrm{H} 65 \mathrm{~N} 1 \mathrm{O} 2$ & $2.53 \mathrm{E}-06$ & Basic N1O1 & $\mathrm{C} 41 \mathrm{H} 55 \mathrm{~N} 1 \mathrm{O} 1$ & $4.40 \mathrm{E}-07$ \\
\hline
\end{tabular}




\begin{tabular}{|c|c|c|c|c|c|c|c|c|}
\hline Class & formula & Mass & Class & formula & Mass & Class & formula & Mass \\
\hline $\mathrm{CH}$ & C41H62 & 0.00037 & Non-basic N1O2 & $\mathrm{C} 38 \mathrm{H} 67 \mathrm{~N} 1 \mathrm{O} 2$ & $4.25 \mathrm{E}-06$ & Basic N1O1 & $\mathrm{C} 42 \mathrm{H} 57 \mathrm{~N} 1 \mathrm{O} 1$ & $2.21 \mathrm{E}-07$ \\
\hline $\mathrm{CH}$ & $\mathrm{C} 42 \mathrm{H} 64$ & 0.000271 & Non-basic N1O2 & $\mathrm{C} 27 \mathrm{H} 43 \mathrm{~N} 1 \mathrm{O} 2$ & $4.58 \mathrm{E}-06$ & Basic N1O1 & $\mathrm{C} 43 \mathrm{H} 59 \mathrm{~N} 1 \mathrm{O} 1$ & $2.17 \mathrm{E}-07$ \\
\hline $\mathrm{CH}$ & $\mathrm{C} 43 \mathrm{H} 66$ & 0.000197 & Non-basic N1O2 & $\mathrm{C} 28 \mathrm{H} 45 \mathrm{~N} 1 \mathrm{O} 2$ & $5.68 \mathrm{E}-06$ & Basic N1O1 & C44H61N1O1 & $5.72 \mathrm{E}-08$ \\
\hline $\mathrm{CH}$ & $\mathrm{C} 16 \mathrm{H} 10$ & 0.002369 & Non-basic N1O2 & $\mathrm{C} 29 \mathrm{H} 47 \mathrm{~N} 1 \mathrm{O} 2$ & $5.53 \mathrm{E}-06$ & Basic N1O1 & C21H13N1O1 & $2.77 \mathrm{E}-08$ \\
\hline $\mathrm{CH}$ & $\mathrm{C} 17 \mathrm{H} 12$ & 0.004254 & Non-basic N1O2 & $\mathrm{C} 30 \mathrm{H} 49 \mathrm{~N} 1 \mathrm{O} 2$ & $1.04 \mathrm{E}-05$ & Basic N1O1 & $\mathrm{C} 22 \mathrm{H} 15 \mathrm{~N} 1 \mathrm{O} 1$ & $4.35 \mathrm{E}-08$ \\
\hline $\mathrm{CH}$ & $\mathrm{C} 18 \mathrm{H} 14$ & 0.023914 & Non-basic N1O2 & $\mathrm{C} 31 \mathrm{H} 51 \mathrm{~N} 1 \mathrm{O} 2$ & $1.53 \mathrm{E}-05$ & Basic N1O1 & $\mathrm{C} 23 \mathrm{H} 17 \mathrm{~N} 1 \mathrm{O} 1$ & $1.44 \mathrm{E}-07$ \\
\hline $\mathrm{CH}$ & C19H16 & 0.026673 & Non-basic N1O2 & $\mathrm{C} 32 \mathrm{H} 53 \mathrm{~N} 1 \mathrm{O} 2$ & $1.66 \mathrm{E}-05$ & Basic N1O1 & $\mathrm{C} 24 \mathrm{H} 19 \mathrm{~N} 1 \mathrm{O} 1$ & $6.91 \mathrm{E}-07$ \\
\hline $\mathrm{CH}$ & $\mathrm{C} 20 \mathrm{H} 18$ & 0.01911 & Non-basic N1O2 & $\mathrm{C} 33 \mathrm{H} 55 \mathrm{~N} 1 \mathrm{O} 2$ & $2.00 \mathrm{E}-05$ & Basic N1O1 & $\mathrm{C} 25 \mathrm{H} 21 \mathrm{~N} 1 \mathrm{O} 1$ & $1.62 \mathrm{E}-06$ \\
\hline $\mathrm{CH}$ & $\mathrm{C} 21 \mathrm{H} 20$ & 0.011694 & Non-basic N1O2 & $\mathrm{C} 34 \mathrm{H} 57 \mathrm{~N} 1 \mathrm{O} 2$ & $1.90 \mathrm{E}-05$ & Basic N1O1 & $\mathrm{C} 26 \mathrm{H} 23 \mathrm{~N} 1 \mathrm{O} 1$ & 4.09E-06 \\
\hline $\mathrm{CH}$ & $\mathrm{C} 22 \mathrm{H} 22$ & 0.006766 & Non-basic N1O2 & $\mathrm{C} 35 \mathrm{H} 59 \mathrm{~N} 1 \mathrm{O} 2$ & $1.65 \mathrm{E}-05$ & Basic N1O1 & $\mathrm{C} 27 \mathrm{H} 25 \mathrm{~N} 1 \mathrm{O} 1$ & $5.99 \mathrm{E}-06$ \\
\hline $\mathrm{CH}$ & $\mathrm{C} 23 \mathrm{H} 24$ & 0.004134 & Non-basic N1O2 & $\mathrm{C} 36 \mathrm{H} 61 \mathrm{~N} 1 \mathrm{O} 2$ & $1.21 \mathrm{E}-05$ & Basic N1O1 & $\mathrm{C} 28 \mathrm{H} 27 \mathrm{~N} 1 \mathrm{O} 1$ & $6.76 \mathrm{E}-06$ \\
\hline $\mathrm{CH}$ & $\mathrm{C} 24 \mathrm{H} 26$ & 0.002979 & Non-basic N1O2 & $\mathrm{C} 37 \mathrm{H} 63 \mathrm{~N} 1 \mathrm{O} 2$ & $1.35 \mathrm{E}-05$ & Basic N1O1 & $\mathrm{C} 29 \mathrm{H} 29 \mathrm{~N} 1 \mathrm{O} 1$ & $5.73 \mathrm{E}-06$ \\
\hline $\mathrm{CH}$ & $\mathrm{C} 25 \mathrm{H} 28$ & 0.002341 & Non-basic N1O2 & $\mathrm{C} 38 \mathrm{H} 65 \mathrm{~N} 1 \mathrm{O} 2$ & $9.26 \mathrm{E}-06$ & Basic N1O1 & $\mathrm{C} 30 \mathrm{H} 31 \mathrm{~N} 1 \mathrm{O} 1$ & $5.06 \mathrm{E}-06$ \\
\hline $\mathrm{CH}$ & $\mathrm{C} 26 \mathrm{H} 30$ & 0.002135 & Non-basic N1O2 & C39H67N1O2 & $6.65 \mathrm{E}-06$ & Basic N1O1 & $\mathrm{C} 31 \mathrm{H} 33 \mathrm{~N} 1 \mathrm{O} 1$ & $5.20 \mathrm{E}-06$ \\
\hline $\mathrm{CH}$ & $\mathrm{C} 27 \mathrm{H} 32$ & 0.002015 & Non-basic N1O2 & $\mathrm{C} 40 \mathrm{H} 69 \mathrm{~N} 1 \mathrm{O} 2$ & $2.20 \mathrm{E}-06$ & Basic N1O1 & $\mathrm{C} 32 \mathrm{H} 35 \mathrm{~N} 1 \mathrm{O} 1$ & 4.24E-06 \\
\hline $\mathrm{CH}$ & $\mathrm{C} 28 \mathrm{H} 34$ & 0.001949 & Non-basic N1O2 & $\mathrm{C} 41 \mathrm{H} 71 \mathrm{~N} 1 \mathrm{O} 2$ & $8.55 \mathrm{E}-06$ & Basic N1O1 & C33H37N1O1 & $3.71 \mathrm{E}-06$ \\
\hline $\mathrm{CH}$ & $\mathrm{C} 29 \mathrm{H} 36$ & 0.001831 & Non-basic N1O2 & $\mathrm{C} 42 \mathrm{H} 73 \mathrm{~N} 1 \mathrm{O} 2$ & 2.03E-06 & Basic N1O1 & C34H39N1O1 & $2.81 \mathrm{E}-06$ \\
\hline $\mathrm{CH}$ & $\mathrm{C} 30 \mathrm{H} 38$ & 0.001655 & Non-basic N1O2 & $\mathrm{C} 14 \mathrm{H} 15 \mathrm{~N} 1 \mathrm{O} 2$ & $1.84 \mathrm{E}-06$ & Basic N1O1 & $\mathrm{C} 35 \mathrm{H} 41 \mathrm{~N} 1 \mathrm{O} 1$ & $2.75 \mathrm{E}-06$ \\
\hline $\mathrm{CH}$ & $\mathrm{C} 31 \mathrm{H} 40$ & 0.001622 & Non-basic N1O2 & $\mathrm{C} 15 \mathrm{H} 17 \mathrm{~N} 1 \mathrm{O} 2$ & $1.09 \mathrm{E}-06$ & Basic N1O1 & C36H43N1O1 & $2.55 \mathrm{E}-06$ \\
\hline $\mathrm{CH}$ & $\mathrm{C} 32 \mathrm{H} 42$ & 0.001491 & Non-basic N1O2 & $\mathrm{C} 16 \mathrm{H} 19 \mathrm{~N} 1 \mathrm{O} 2$ & $6.76 \mathrm{E}-07$ & Basic N1O1 & C37H45N1O1 & $1.45 \mathrm{E}-06$ \\
\hline $\mathrm{CH}$ & $\mathrm{C} 33 \mathrm{H} 44$ & 0.001279 & Non-basic N1O2 & $\mathrm{C} 18 \mathrm{H} 23 \mathrm{~N} 1 \mathrm{O} 2$ & $1.08 \mathrm{E}-06$ & Basic N1O1 & $\mathrm{C} 38 \mathrm{H} 47 \mathrm{~N} 1 \mathrm{O} 1$ & $1.06 \mathrm{E}-06$ \\
\hline $\mathrm{CH}$ & $\mathrm{C} 34 \mathrm{H} 46$ & 0.001142 & Non-basic N1O2 & $\mathrm{C} 19 \mathrm{H} 25 \mathrm{~N} 1 \mathrm{O} 2$ & $1.68 \mathrm{E}-06$ & Basic N1O1 & C39H49N1O1 & $6.92 \mathrm{E}-07$ \\
\hline $\mathrm{CH}$ & $\mathrm{C} 35 \mathrm{H} 48$ & 0.000926 & Non-basic N1O2 & $\mathrm{C} 20 \mathrm{H} 27 \mathrm{~N} 1 \mathrm{O} 2$ & $1.21 \mathrm{E}-06$ & Basic N1O1 & C40H51N1O1 & $5.32 \mathrm{E}-07$ \\
\hline $\mathrm{CH}$ & $\mathrm{C} 36 \mathrm{H} 50$ & 0.0008 & Non-basic N1O2 & $\mathrm{C} 21 \mathrm{H} 29 \mathrm{~N} 1 \mathrm{O} 2$ & $1.00 \mathrm{E}-06$ & Basic N1O1 & C41H53N1O1 & 4.92E-07 \\
\hline $\mathrm{CH}$ & $\mathrm{C} 37 \mathrm{H} 52$ & 0.000673 & Non-basic N1O2 & $\mathrm{C} 22 \mathrm{H} 31 \mathrm{~N} 1 \mathrm{O} 2$ & $3.97 \mathrm{E}-06$ & Basic N1O1 & $\mathrm{C} 42 \mathrm{H} 55 \mathrm{~N} 1 \mathrm{O} 1$ & $3.39 \mathrm{E}-07$ \\
\hline $\mathrm{CH}$ & C38H54 & 0.000649 & Non-basic N1O2 & $\mathrm{C} 23 \mathrm{H} 33 \mathrm{~N} 1 \mathrm{O} 2$ & $1.27 \mathrm{E}-06$ & Basic N1O1 & C43H57N1O1 & $2.06 \mathrm{E}-07$ \\
\hline $\mathrm{CH}$ & С39H56 & 0.000693 & Non-basic N1O2 & $\mathrm{C} 24 \mathrm{H} 35 \mathrm{~N} 1 \mathrm{O} 2$ & $1.49 \mathrm{E}-06$ & Basic N1O1 & C44H59N1O1 & $1.75 \mathrm{E}-07$ \\
\hline $\mathrm{CH}$ & $\mathrm{C} 40 \mathrm{H} 58$ & 0.000644 & Non-basic N1O2 & $\mathrm{C} 25 \mathrm{H} 37 \mathrm{~N} 1 \mathrm{O} 2$ & $3.97 \mathrm{E}-06$ & Basic N1O1 & C45H61N1O1 & 1.49E-07 \\
\hline $\mathrm{CH}$ & $\mathrm{C} 41 \mathrm{H} 60$ & 0.000348 & Non-basic N1O2 & $\mathrm{C} 26 \mathrm{H} 39 \mathrm{~N} 1 \mathrm{O} 2$ & $1.71 \mathrm{E}-06$ & Basic N1O1 & C46H63N1O1 & 7.16E-08 \\
\hline $\mathrm{CH}$ & C42H62 & 0.000241 & Non-basic N1O2 & $\mathrm{C} 27 \mathrm{H} 41 \mathrm{~N} 1 \mathrm{O} 2$ & $5.21 \mathrm{E}-06$ & Basic N1O1 & C47H65N1O1 & 7.26E-08 \\
\hline $\mathrm{CH}$ & C43H64 & 0.000172 & Non-basic N1O2 & $\mathrm{C} 28 \mathrm{H} 43 \mathrm{~N} 1 \mathrm{O} 2$ & $6.54 \mathrm{E}-06$ & Basic N1O1 & $\mathrm{C} 23 \mathrm{H} 15 \mathrm{~N} 1 \mathrm{O} 1$ & $5.93 \mathrm{E}-08$ \\
\hline $\mathrm{CH}$ & C44H66 & $9.16 \mathrm{E}-05$ & Non-basic N1O2 & $\mathrm{C} 29 \mathrm{H} 45 \mathrm{~N} 1 \mathrm{O} 2$ & $1.08 \mathrm{E}-05$ & Basic N1O1 & $\mathrm{C} 24 \mathrm{H} 17 \mathrm{~N} 1 \mathrm{O} 1$ & $3.05 \mathrm{E}-07$ \\
\hline $\mathrm{CH}$ & $\mathrm{C} 16 \mathrm{H} 8$ & 0.000928 & Non-basic N1O2 & $\mathrm{C} 30 \mathrm{H} 47 \mathrm{~N} 1 \mathrm{O} 2$ & $8.48 \mathrm{E}-06$ & Basic N1O1 & $\mathrm{C} 25 \mathrm{H} 19 \mathrm{~N} 1 \mathrm{O} 1$ & $5.45 \mathrm{E}-07$ \\
\hline $\mathrm{CH}$ & $\mathrm{C} 17 \mathrm{H} 10$ & $3.88 \mathrm{E}-05$ & Non-basic N1O2 & $\mathrm{C} 31 \mathrm{H} 49 \mathrm{~N} 1 \mathrm{O} 2$ & $1.13 \mathrm{E}-05$ & Basic N1O1 & $\mathrm{C} 26 \mathrm{H} 21 \mathrm{~N} 1 \mathrm{O} 1$ & 7.26E-07 \\
\hline $\mathrm{CH}$ & $\mathrm{C} 18 \mathrm{H} 12$ & 0.002506 & Non-basic N1O2 & $\mathrm{C} 32 \mathrm{H} 51 \mathrm{~N} 1 \mathrm{O} 2$ & $1.44 \mathrm{E}-05$ & Basic N1O1 & $\mathrm{C} 27 \mathrm{H} 23 \mathrm{~N} 1 \mathrm{O} 1$ & $1.33 \mathrm{E}-06$ \\
\hline $\mathrm{CH}$ & C19H14 & 0.011978 & Non-basic N1O2 & $\mathrm{C} 33 \mathrm{H} 53 \mathrm{~N} 1 \mathrm{O} 2$ & $9.63 \mathrm{E}-06$ & Basic N1O1 & $\mathrm{C} 28 \mathrm{H} 25 \mathrm{~N} 1 \mathrm{O} 1$ & $1.67 \mathrm{E}-06$ \\
\hline $\mathrm{CH}$ & C20H16 & 0.022033 & Non-basic N1O2 & $\mathrm{C} 34 \mathrm{H} 55 \mathrm{~N} 1 \mathrm{O} 2$ & $1.80 \mathrm{E}-05$ & Basic N1O1 & $\mathrm{C} 29 \mathrm{H} 27 \mathrm{~N} 1 \mathrm{O} 1$ & $2.67 \mathrm{E}-06$ \\
\hline $\mathrm{CH}$ & C21H18 & 0.022147 & Non-basic N1O2 & $\mathrm{C} 35 \mathrm{H} 57 \mathrm{~N} 1 \mathrm{O} 2$ & $1.66 \mathrm{E}-05$ & Basic N1O1 & $\mathrm{C} 30 \mathrm{H} 29 \mathrm{~N} 1 \mathrm{O} 1$ & $3.19 \mathrm{E}-06$ \\
\hline
\end{tabular}




\begin{tabular}{|c|c|c|c|c|c|c|c|c|}
\hline Class & formula & Mass & Class & formula & Mass & Class & formula & Mass \\
\hline $\mathrm{CH}$ & $\mathrm{C} 22 \mathrm{H} 20$ & 0.015402 & Non-basic N1O2 & $\mathrm{C} 36 \mathrm{H} 59 \mathrm{~N} 1 \mathrm{O} 2$ & $1.27 \mathrm{E}-05$ & Basic N1O1 & $\mathrm{C} 31 \mathrm{H} 31 \mathrm{~N} 1 \mathrm{O} 1$ & $3.76 \mathrm{E}-06$ \\
\hline $\mathrm{CH}$ & $\mathrm{C} 23 \mathrm{H} 22$ & 0.00897 & Non-basic N1O2 & $\mathrm{C} 37 \mathrm{H} 61 \mathrm{~N} 1 \mathrm{O} 2$ & $1.03 \mathrm{E}-05$ & Basic N1O1 & $\mathrm{C} 32 \mathrm{H} 33 \mathrm{~N} 1 \mathrm{O} 1$ & $3.44 \mathrm{E}-06$ \\
\hline $\mathrm{CH}$ & $\mathrm{C} 24 \mathrm{H} 24$ & 0.005535 & Non-basic N1O2 & $\mathrm{C} 38 \mathrm{H} 63 \mathrm{~N} 1 \mathrm{O} 2$ & $1.06 \mathrm{E}-05$ & Basic N1O1 & $\mathrm{C} 33 \mathrm{H} 35 \mathrm{~N} 1 \mathrm{O} 1$ & $3.39 \mathrm{E}-06$ \\
\hline $\mathrm{CH}$ & $\mathrm{C} 25 \mathrm{H} 26$ & 0.003909 & Non-basic N1O2 & $\mathrm{C} 39 \mathrm{H} 65 \mathrm{~N} 1 \mathrm{O} 2$ & $1.05 \mathrm{E}-05$ & Basic N1O1 & $\mathrm{C} 34 \mathrm{H} 37 \mathrm{~N} 1 \mathrm{O} 1$ & $2.87 \mathrm{E}-06$ \\
\hline $\mathrm{CH}$ & $\mathrm{C} 26 \mathrm{H} 28$ & 0.002845 & Non-basic N1O2 & $\mathrm{C} 40 \mathrm{H} 67 \mathrm{~N} 1 \mathrm{O} 2$ & $7.60 \mathrm{E}-06$ & Basic N1O1 & C35H39N1O1 & $2.48 \mathrm{E}-06$ \\
\hline $\mathrm{CH}$ & $\mathrm{C} 27 \mathrm{H} 30$ & 0.002424 & Non-basic N1O2 & $\mathrm{C} 14 \mathrm{H} 13 \mathrm{~N} 1 \mathrm{O} 2$ & $9.18 \mathrm{E}-07$ & Basic N1O1 & $\mathrm{C} 36 \mathrm{H} 41 \mathrm{~N} 1 \mathrm{O} 1$ & $2.12 \mathrm{E}-06$ \\
\hline $\mathrm{CH}$ & $\mathrm{C} 28 \mathrm{H} 32$ & 0.002256 & Non-basic N1O2 & $\mathrm{C} 15 \mathrm{H} 15 \mathrm{~N} 1 \mathrm{O} 2$ & 8.67E-06 & Basic N1O1 & $\mathrm{C} 37 \mathrm{H} 43 \mathrm{~N} 1 \mathrm{O} 1$ & $1.63 \mathrm{E}-06$ \\
\hline $\mathrm{CH}$ & $\mathrm{C} 29 \mathrm{H} 34$ & 0.002 & Non-basic N1O2 & $\mathrm{C} 16 \mathrm{H} 17 \mathrm{~N} 1 \mathrm{O} 2$ & $1.46 \mathrm{E}-05$ & Basic N1O1 & $\mathrm{C} 38 \mathrm{H} 45 \mathrm{~N} 1 \mathrm{O} 1$ & $1.09 \mathrm{E}-06$ \\
\hline $\mathrm{CH}$ & $\mathrm{C} 30 \mathrm{H} 36$ & 0.0017 & Non-basic N1O2 & $\mathrm{C} 17 \mathrm{H} 19 \mathrm{~N} 1 \mathrm{O} 2$ & $9.87 \mathrm{E}-06$ & Basic N1O1 & $\mathrm{C} 39 \mathrm{H} 47 \mathrm{~N} 1 \mathrm{O} 1$ & $6.46 \mathrm{E}-07$ \\
\hline $\mathrm{CH}$ & $\mathrm{C} 31 \mathrm{H} 38$ & 0.001483 & Non-basic N1O2 & $\mathrm{C} 18 \mathrm{H} 21 \mathrm{~N} 1 \mathrm{O} 2$ & $6.03 \mathrm{E}-06$ & Basic N1O1 & $\mathrm{C} 40 \mathrm{H} 49 \mathrm{~N} 1 \mathrm{O} 1$ & $6.22 \mathrm{E}-07$ \\
\hline $\mathrm{CH}$ & $\mathrm{C} 32 \mathrm{H} 40$ & 0.001264 & Non-basic N1O2 & $\mathrm{C} 19 \mathrm{H} 23 \mathrm{~N} 1 \mathrm{O} 2$ & $1.83 \mathrm{E}-06$ & Basic N1O1 & $\mathrm{C} 41 \mathrm{H} 51 \mathrm{~N} 1 \mathrm{O} 1$ & $5.06 \mathrm{E}-07$ \\
\hline $\mathrm{CH}$ & $\mathrm{C} 33 \mathrm{H} 42$ & 0.001127 & Non-basic N1O2 & $\mathrm{C} 20 \mathrm{H} 25 \mathrm{~N} 1 \mathrm{O} 2$ & $3.54 \mathrm{E}-06$ & Basic N1O1 & $\mathrm{C} 42 \mathrm{H} 53 \mathrm{~N} 1 \mathrm{O} 1$ & 4.72E-07 \\
\hline $\mathrm{CH}$ & $\mathrm{C} 34 \mathrm{H} 44$ & 0.000999 & Non-basic N1O2 & $\mathrm{C} 21 \mathrm{H} 27 \mathrm{~N} 1 \mathrm{O} 2$ & $3.55 \mathrm{E}-06$ & Basic N1O1 & $\mathrm{C} 43 \mathrm{H} 55 \mathrm{~N} 1 \mathrm{O} 1$ & $1.00 \mathrm{E}-07$ \\
\hline $\mathrm{CH}$ & $\mathrm{C} 35 \mathrm{H} 46$ & 0.000828 & Non-basic N1O2 & $\mathrm{C} 22 \mathrm{H} 29 \mathrm{~N} 1 \mathrm{O} 2$ & $9.31 \mathrm{E}-06$ & Basic N1O1 & $\mathrm{C} 44 \mathrm{H} 57 \mathrm{~N} 1 \mathrm{O} 1$ & $2.00 \mathrm{E}-07$ \\
\hline $\mathrm{CH}$ & $\mathrm{C} 36 \mathrm{H} 48$ & 0.000642 & Non-basic N1O2 & $\mathrm{C} 23 \mathrm{H} 31 \mathrm{~N} 1 \mathrm{O} 2$ & 7.99E-06 & Basic N1O1 & C45H59N1O1 & $9.50 \mathrm{E}-08$ \\
\hline $\mathrm{CH}$ & $\mathrm{C} 37 \mathrm{H} 50$ & 0.00056 & Non-basic N1O2 & $\mathrm{C} 24 \mathrm{H} 33 \mathrm{~N} 1 \mathrm{O} 2$ & $1.45 \mathrm{E}-05$ & Basic N1O1 & $\mathrm{C} 24 \mathrm{H} 15 \mathrm{~N} 1 \mathrm{O} 1$ & $2.07 \mathrm{E}-07$ \\
\hline $\mathrm{CH}$ & $\mathrm{C} 38 \mathrm{H} 52$ & 0.000586 & Non-basic N1O2 & $\mathrm{C} 25 \mathrm{H} 35 \mathrm{~N} 1 \mathrm{O} 2$ & $1.27 \mathrm{E}-05$ & Basic N1O1 & $\mathrm{C} 25 \mathrm{H} 17 \mathrm{~N} 1 \mathrm{O} 1$ & $3.03 \mathrm{E}-07$ \\
\hline $\mathrm{CH}$ & С39H54 & 0.000589 & Non-basic N1O2 & $\mathrm{C} 26 \mathrm{H} 37 \mathrm{~N} 1 \mathrm{O} 2$ & $1.98 \mathrm{E}-05$ & Basic N1O1 & C26H19N1O1 & $3.31 \mathrm{E}-07$ \\
\hline $\mathrm{CH}$ & $\mathrm{C} 40 \mathrm{H} 56$ & 0.000502 & Non-basic N1O2 & $\mathrm{C} 27 \mathrm{H} 39 \mathrm{~N} 1 \mathrm{O} 2$ & $2.28 \mathrm{E}-05$ & Basic N1O1 & $\mathrm{C} 27 \mathrm{H} 21 \mathrm{~N} 1 \mathrm{O} 1$ & $3.87 \mathrm{E}-07$ \\
\hline $\mathrm{CH}$ & $\mathrm{C} 41 \mathrm{H} 58$ & 0.000222 & Non-basic N1O2 & $\mathrm{C} 28 \mathrm{H} 41 \mathrm{~N} 1 \mathrm{O} 2$ & $3.80 \mathrm{E}-05$ & Basic N1O1 & $\mathrm{C} 28 \mathrm{H} 23 \mathrm{~N} 1 \mathrm{O} 1$ & $5.94 \mathrm{E}-07$ \\
\hline $\mathrm{CH}$ & $\mathrm{C} 42 \mathrm{H} 60$ & 0.000197 & Non-basic N1O2 & $\mathrm{C} 29 \mathrm{H} 43 \mathrm{~N} 1 \mathrm{O} 2$ & $5.17 \mathrm{E}-05$ & Basic N1O1 & $\mathrm{C} 29 \mathrm{H} 25 \mathrm{~N} 1 \mathrm{O} 1$ & $7.67 \mathrm{E}-07$ \\
\hline $\mathrm{CH}$ & $\mathrm{C} 43 \mathrm{H} 62$ & 0.000109 & Non-basic N1O2 & $\mathrm{C} 30 \mathrm{H} 45 \mathrm{~N} 1 \mathrm{O} 2$ & $6.18 \mathrm{E}-05$ & Basic N1O1 & $\mathrm{C} 30 \mathrm{H} 27 \mathrm{~N} 1 \mathrm{O} 1$ & $1.24 \mathrm{E}-06$ \\
\hline $\mathrm{CH}$ & C44H64 & $4.22 \mathrm{E}-05$ & Non-basic N1O2 & $\mathrm{C} 31 \mathrm{H} 47 \mathrm{~N} 1 \mathrm{O} 2$ & $6.90 \mathrm{E}-05$ & Basic N1O1 & C31H29N1O1 & $1.67 \mathrm{E}-06$ \\
\hline $\mathrm{CH}$ & $\mathrm{C} 17 \mathrm{H} 8$ & $6.69 \mathrm{E}-06$ & Non-basic N1O2 & $\mathrm{C} 32 \mathrm{H} 49 \mathrm{~N} 1 \mathrm{O} 2$ & $7.29 \mathrm{E}-05$ & Basic N1O1 & $\mathrm{C} 32 \mathrm{H} 31 \mathrm{~N} 1 \mathrm{O} 1$ & $2.21 \mathrm{E}-06$ \\
\hline $\mathrm{CH}$ & $\mathrm{C} 18 \mathrm{H} 10$ & $7.95 \mathrm{E}-05$ & Non-basic N1O2 & $\mathrm{C} 33 \mathrm{H} 51 \mathrm{~N} 1 \mathrm{O} 2$ & $6.47 \mathrm{E}-05$ & Basic N1O1 & C33H33N1O1 & $2.07 \mathrm{E}-06$ \\
\hline $\mathrm{CH}$ & $\mathrm{C} 19 \mathrm{H} 12$ & 0.000334 & Non-basic N1O2 & $\mathrm{C} 34 \mathrm{H} 53 \mathrm{~N} 1 \mathrm{O} 2$ & $6.81 \mathrm{E}-05$ & Basic N1O1 & C34H35N1O1 & $1.79 \mathrm{E}-06$ \\
\hline $\mathrm{CH}$ & $\mathrm{C} 20 \mathrm{H} 14$ & 0.001971 & Non-basic N1O2 & $\mathrm{C} 35 \mathrm{H} 55 \mathrm{~N} 1 \mathrm{O} 2$ & $6.53 \mathrm{E}-05$ & Basic N1O1 & $\mathrm{C} 35 \mathrm{H} 37 \mathrm{~N} 1 \mathrm{O} 1$ & $1.93 \mathrm{E}-06$ \\
\hline $\mathrm{CH}$ & $\mathrm{C} 21 \mathrm{H} 16$ & 0.005176 & Non-basic N1O2 & $\mathrm{C} 36 \mathrm{H} 57 \mathrm{~N} 1 \mathrm{O} 2$ & $5.17 \mathrm{E}-05$ & Basic N1O1 & $\mathrm{C} 36 \mathrm{H} 39 \mathrm{~N} 1 \mathrm{O} 1$ & $1.75 \mathrm{E}-06$ \\
\hline $\mathrm{CH}$ & $\mathrm{C} 22 \mathrm{H} 18$ & 0.008445 & Non-basic N1O2 & $\mathrm{C} 37 \mathrm{H} 59 \mathrm{~N} 1 \mathrm{O} 2$ & $4.78 \mathrm{E}-05$ & Basic N1O1 & $\mathrm{C} 37 \mathrm{H} 41 \mathrm{~N} 1 \mathrm{O} 1$ & $1.37 \mathrm{E}-06$ \\
\hline $\mathrm{CH}$ & $\mathrm{C} 23 \mathrm{H} 20$ & 0.009419 & Non-basic N1O2 & C38H61N1O2 & $3.40 \mathrm{E}-05$ & Basic N1O1 & $\mathrm{C} 38 \mathrm{H} 43 \mathrm{~N} 1 \mathrm{O} 1$ & $1.02 \mathrm{E}-06$ \\
\hline $\mathrm{CH}$ & $\mathrm{C} 24 \mathrm{H} 22$ & 0.007869 & Non-basic N1O2 & C39H63N1O2 & $2.93 \mathrm{E}-05$ & Basic N1O1 & C39H45N1O1 & $6.92 \mathrm{E}-07$ \\
\hline $\mathrm{CH}$ & $\mathrm{C} 25 \mathrm{H} 24$ & 0.00595 & Non-basic N1O2 & $\mathrm{C} 40 \mathrm{H} 65 \mathrm{~N} 1 \mathrm{O} 2$ & $2.21 \mathrm{E}-05$ & Basic N1O1 & $\mathrm{C} 40 \mathrm{H} 47 \mathrm{~N} 1 \mathrm{O} 1$ & 4.63E-07 \\
\hline $\mathrm{CH}$ & $\mathrm{C} 26 \mathrm{H} 26$ & 0.004127 & Non-basic N1O2 & $\mathrm{C} 41 \mathrm{H} 67 \mathrm{~N} 1 \mathrm{O} 2$ & $1.71 \mathrm{E}-05$ & Basic N1O1 & $\mathrm{C} 41 \mathrm{H} 49 \mathrm{~N} 1 \mathrm{O} 1$ & $1.15 \mathrm{E}-07$ \\
\hline $\mathrm{CH}$ & $\mathrm{C} 27 \mathrm{H} 28$ & 0.002945 & Non-basic N1O2 & $\mathrm{C} 42 \mathrm{H} 69 \mathrm{~N} 1 \mathrm{O} 2$ & $1.61 \mathrm{E}-05$ & Basic N1O1 & $\mathrm{C} 42 \mathrm{H} 51 \mathrm{~N} 1 \mathrm{O} 1$ & $7.08 \mathrm{E}-08$ \\
\hline $\mathrm{CH}$ & $\mathrm{C} 28 \mathrm{H} 30$ & 0.00233 & Non-basic N1O2 & $\mathrm{C} 43 \mathrm{H} 71 \mathrm{~N} 1 \mathrm{O} 2$ & $9.53 \mathrm{E}-06$ & Basic N1O1 & $\mathrm{C} 43 \mathrm{H} 53 \mathrm{~N} 1 \mathrm{O} 1$ & $2.19 \mathrm{E}-07$ \\
\hline $\mathrm{CH}$ & C29H32 & 0.001794 & Non-basic N1O2 & $\mathrm{C} 44 \mathrm{H} 73 \mathrm{~N} 1 \mathrm{O} 2$ & $1.11 \mathrm{E}-05$ & Basic N1O1 & $\mathrm{C} 27 \mathrm{H} 19 \mathrm{~N} 1 \mathrm{O} 1$ & $5.27 \mathrm{E}-08$ \\
\hline $\mathrm{CH}$ & $\mathrm{C} 30 \mathrm{H} 34$ & 0.001444 & Non-basic N1O2 & $\mathrm{C} 45 \mathrm{H} 75 \mathrm{~N} 1 \mathrm{O} 2$ & $1.17 \mathrm{E}-05$ & Basic N1O1 & $\mathrm{C} 28 \mathrm{H} 21 \mathrm{~N} 1 \mathrm{O} 1$ & $3.39 \mathrm{E}-07$ \\
\hline $\mathrm{CH}$ & C31H36 & 0.00129 & Non-basic N1O2 & $\mathrm{C} 46 \mathrm{H} 77 \mathrm{~N} 1 \mathrm{O} 2$ & $6.49 \mathrm{E}-06$ & Basic N1O1 & $\mathrm{C} 29 \mathrm{H} 23 \mathrm{~N} 1 \mathrm{O} 1$ & $3.88 \mathrm{E}-07$ \\
\hline
\end{tabular}




\begin{tabular}{|c|c|c|c|c|c|c|c|c|}
\hline Class & formula & Mass & Class & formula & Mass & Class & formula & Mass \\
\hline $\mathrm{CH}$ & $\mathrm{C} 32 \mathrm{H} 38$ & 0.001071 & Non-basic N1O2 & $\mathrm{C} 47 \mathrm{H} 79 \mathrm{~N} 1 \mathrm{O} 2$ & 4.97E-06 & Basic N1O1 & $\mathrm{C} 30 \mathrm{H} 25 \mathrm{~N} 1 \mathrm{O} 1$ & $7.65 \mathrm{E}-07$ \\
\hline $\mathrm{CH}$ & $\mathrm{C} 33 \mathrm{H} 40$ & 0.00097 & Non-basic N1O2 & $\mathrm{C} 48 \mathrm{H} 81 \mathrm{~N} 1 \mathrm{O} 2$ & $5.65 \mathrm{E}-06$ & Basic N1O1 & $\mathrm{C} 31 \mathrm{H} 27 \mathrm{~N} 1 \mathrm{O} 1$ & $1.38 \mathrm{E}-06$ \\
\hline $\mathrm{CH}$ & $\mathrm{C} 34 \mathrm{H} 42$ & 0.000779 & Non-basic N1O2 & C49H83N1O2 & $2.63 \mathrm{E}-06$ & Basic N1O1 & $\mathrm{C} 32 \mathrm{H} 29 \mathrm{~N} 1 \mathrm{O} 1$ & $1.25 \mathrm{E}-06$ \\
\hline $\mathrm{CH}$ & $\mathrm{C} 35 \mathrm{H} 44$ & 0.000662 & Non-basic N1O2 & $\mathrm{C} 50 \mathrm{H} 85 \mathrm{~N} 1 \mathrm{O} 2$ & $2.82 \mathrm{E}-06$ & Basic N1O1 & $\mathrm{C} 33 \mathrm{H} 31 \mathrm{~N} 1 \mathrm{O} 1$ & $1.37 \mathrm{E}-06$ \\
\hline $\mathrm{CH}$ & $\mathrm{C} 36 \mathrm{H} 46$ & 0.00055 & Non-basic N1O2 & $\mathrm{C} 14 \mathrm{H} 11 \mathrm{~N} 1 \mathrm{O} 2$ & $3.88 \mathrm{E}-06$ & Basic N1O1 & $\mathrm{C} 34 \mathrm{H} 33 \mathrm{~N} 1 \mathrm{O} 1$ & $1.36 \mathrm{E}-06$ \\
\hline $\mathrm{CH}$ & $\mathrm{C} 37 \mathrm{H} 48$ & 0.000451 & Non-basic N1O2 & $\mathrm{C} 15 \mathrm{H} 13 \mathrm{~N} 1 \mathrm{O} 2$ & $1.26 \mathrm{E}-05$ & Basic N1O1 & $\mathrm{C} 35 \mathrm{H} 35 \mathrm{~N} 1 \mathrm{O} 1$ & $1.80 \mathrm{E}-06$ \\
\hline $\mathrm{CH}$ & $\mathrm{C} 38 \mathrm{H} 50$ & 0.000456 & Non-basic N1O2 & $\mathrm{C} 16 \mathrm{H} 15 \mathrm{~N} 1 \mathrm{O} 2$ & $1.27 \mathrm{E}-05$ & Basic N1O1 & $\mathrm{C} 36 \mathrm{H} 37 \mathrm{~N} 1 \mathrm{O} 1$ & 2.04E-06 \\
\hline $\mathrm{CH}$ & C39H52 & 0.000421 & Non-basic N1O2 & $\mathrm{C} 17 \mathrm{H} 17 \mathrm{~N} 1 \mathrm{O} 2$ & $1.46 \mathrm{E}-05$ & Basic N1O1 & $\mathrm{C} 37 \mathrm{H} 39 \mathrm{~N} 1 \mathrm{O} 1$ & $2.07 \mathrm{E}-06$ \\
\hline $\mathrm{CH}$ & $\mathrm{C} 40 \mathrm{H} 54$ & 0.000291 & Non-basic N1O2 & $\mathrm{C} 18 \mathrm{H} 19 \mathrm{~N} 1 \mathrm{O} 2$ & $1.44 \mathrm{E}-05$ & Basic N1O1 & $\mathrm{C} 38 \mathrm{H} 41 \mathrm{~N} 1 \mathrm{O} 1$ & $1.29 \mathrm{E}-06$ \\
\hline $\mathrm{CH}$ & $\mathrm{C} 41 \mathrm{H} 56$ & 0.00016 & Non-basic N1O2 & $\mathrm{C} 19 \mathrm{H} 21 \mathrm{~N} 1 \mathrm{O} 2$ & $1.32 \mathrm{E}-05$ & Basic N1O1 & $\mathrm{C} 39 \mathrm{H} 43 \mathrm{~N} 1 \mathrm{O} 1$ & $8.22 \mathrm{E}-07$ \\
\hline $\mathrm{CH}$ & $\mathrm{C} 42 \mathrm{H} 58$ & 0.000103 & Non-basic N1O2 & $\mathrm{C} 20 \mathrm{H} 23 \mathrm{~N} 1 \mathrm{O} 2$ & $1.30 \mathrm{E}-05$ & Basic N1O1 & $\mathrm{C} 27 \mathrm{H} 17 \mathrm{~N} 1 \mathrm{O} 1$ & $1.11 \mathrm{E}-07$ \\
\hline $\mathrm{CH}$ & $\mathrm{C} 43 \mathrm{H} 60$ & $6.87 \mathrm{E}-05$ & Non-basic N1O2 & $\mathrm{C} 21 \mathrm{H} 25 \mathrm{~N} 1 \mathrm{O} 2$ & $1.61 \mathrm{E}-05$ & Basic N1O1 & $\mathrm{C} 28 \mathrm{H} 19 \mathrm{~N} 1 \mathrm{O} 1$ & $1.04 \mathrm{E}-06$ \\
\hline $\mathrm{CH}$ & C44H62 & $5.77 \mathrm{E}-05$ & Non-basic N1O2 & $\mathrm{C} 22 \mathrm{H} 27 \mathrm{~N} 1 \mathrm{O} 2$ & $9.35 \mathrm{E}-06$ & Basic N1O1 & $\mathrm{C} 29 \mathrm{H} 21 \mathrm{~N} 1 \mathrm{O} 1$ & $6.85 \mathrm{E}-08$ \\
\hline $\mathrm{CH}$ & $\mathrm{C} 45 \mathrm{H} 64$ & $2.94 \mathrm{E}-05$ & Non-basic N1O2 & $\mathrm{C} 23 \mathrm{H} 29 \mathrm{~N} 1 \mathrm{O} 2$ & $1.84 \mathrm{E}-05$ & Basic N1O1 & $\mathrm{C} 30 \mathrm{H} 23 \mathrm{~N} 1 \mathrm{O} 1$ & $1.06 \mathrm{E}-07$ \\
\hline $\mathrm{CH}$ & C46H66 & $2.39 \mathrm{E}-05$ & Non-basic N1O2 & $\mathrm{C} 24 \mathrm{H} 31 \mathrm{~N} 1 \mathrm{O} 2$ & $2.12 \mathrm{E}-05$ & Basic N1O1 & $\mathrm{C} 31 \mathrm{H} 25 \mathrm{~N} 1 \mathrm{O} 1$ & $2.02 \mathrm{E}-07$ \\
\hline $\mathrm{CH}$ & $\mathrm{C} 47 \mathrm{H} 68$ & $1.46 \mathrm{E}-05$ & Non-basic N1O2 & $\mathrm{C} 25 \mathrm{H} 33 \mathrm{~N} 1 \mathrm{O} 2$ & $1.90 \mathrm{E}-05$ & Basic N1O1 & $\mathrm{C} 32 \mathrm{H} 27 \mathrm{~N} 1 \mathrm{O} 1$ & $3.66 \mathrm{E}-07$ \\
\hline $\mathrm{CH}$ & $\mathrm{C} 17 \mathrm{H} 6$ & $1.20 \mathrm{E}-06$ & Non-basic N1O2 & $\mathrm{C} 26 \mathrm{H} 35 \mathrm{~N} 1 \mathrm{O} 2$ & $2.28 \mathrm{E}-05$ & Basic N1O1 & $\mathrm{C} 33 \mathrm{H} 29 \mathrm{~N} 1 \mathrm{O} 1$ & $5.08 \mathrm{E}-07$ \\
\hline $\mathrm{CH}$ & $\mathrm{C} 18 \mathrm{H} 8$ & $2.58 \mathrm{E}-05$ & Non-basic N1O2 & $\mathrm{C} 27 \mathrm{H} 37 \mathrm{~N} 1 \mathrm{O} 2$ & $2.66 \mathrm{E}-05$ & Basic N1O1 & $\mathrm{C} 34 \mathrm{H} 31 \mathrm{~N} 1 \mathrm{O} 1$ & 4.17E-07 \\
\hline $\mathrm{CH}$ & $\mathrm{C} 19 \mathrm{H} 10$ & $1.44 \mathrm{E}-05$ & Non-basic N1O2 & $\mathrm{C} 28 \mathrm{H} 39 \mathrm{~N} 1 \mathrm{O} 2$ & $2.65 \mathrm{E}-05$ & Basic N1O1 & C35H33N1O1 & $8.05 \mathrm{E}-07$ \\
\hline $\mathrm{CH}$ & $\mathrm{C} 20 \mathrm{H} 12$ & 0.004021 & Non-basic N1O2 & $\mathrm{C} 29 \mathrm{H} 41 \mathrm{~N} 1 \mathrm{O} 2$ & $3.59 \mathrm{E}-05$ & Basic N1O1 & $\mathrm{C} 36 \mathrm{H} 35 \mathrm{~N} 1 \mathrm{O} 1$ & $1.08 \mathrm{E}-06$ \\
\hline $\mathrm{CH}$ & $\mathrm{C} 21 \mathrm{H} 14$ & 0.013797 & Non-basic N1O2 & $\mathrm{C} 30 \mathrm{H} 43 \mathrm{~N} 1 \mathrm{O} 2$ & $4.68 \mathrm{E}-05$ & Basic N1O1 & $\mathrm{C} 37 \mathrm{H} 37 \mathrm{~N} 1 \mathrm{O} 1$ & $1.32 \mathrm{E}-06$ \\
\hline $\mathrm{CH}$ & $\mathrm{C} 22 \mathrm{H} 16$ & 0.023246 & Non-basic N1O2 & $\mathrm{C} 31 \mathrm{H} 45 \mathrm{~N} 1 \mathrm{O} 2$ & $4.40 \mathrm{E}-05$ & Basic N1O1 & C28H17N1O1 & $1.75 \mathrm{E}-07$ \\
\hline $\mathrm{CH}$ & $\mathrm{C} 23 \mathrm{H} 18$ & 0.024009 & Non-basic N1O2 & $\mathrm{C} 32 \mathrm{H} 47 \mathrm{~N} 1 \mathrm{O} 2$ & $4.58 \mathrm{E}-05$ & Basic N1O1 & C29H19N1O1 & 4.41E-08 \\
\hline $\mathrm{CH}$ & $\mathrm{C} 24 \mathrm{H} 20$ & 0.017969 & Non-basic N1O2 & $\mathrm{C} 33 \mathrm{H} 49 \mathrm{~N} 1 \mathrm{O} 2$ & 4.67E-05 & Basic N1O1 & $\mathrm{C} 30 \mathrm{H} 21 \mathrm{~N} 1 \mathrm{O} 1$ & 3.67E-08 \\
\hline $\mathrm{CH}$ & $\mathrm{C} 25 \mathrm{H} 22$ & 0.011909 & Non-basic N1O2 & $\mathrm{C} 34 \mathrm{H} 51 \mathrm{~N} 1 \mathrm{O} 2$ & $4.42 \mathrm{E}-05$ & Basic N1O1 & C31H23N1O1 & $2.22 \mathrm{E}-07$ \\
\hline $\mathrm{CH}$ & $\mathrm{C} 26 \mathrm{H} 24$ & 0.007293 & Non-basic N1O2 & $\mathrm{C} 35 \mathrm{H} 53 \mathrm{~N} 1 \mathrm{O} 2$ & $4.31 \mathrm{E}-05$ & Basic N1O1 & $\mathrm{C} 32 \mathrm{H} 25 \mathrm{~N} 1 \mathrm{O} 1$ & $2.08 \mathrm{E}-07$ \\
\hline $\mathrm{CH}$ & $\mathrm{C} 27 \mathrm{H} 26$ & 0.004623 & Non-basic N1O2 & $\mathrm{C} 36 \mathrm{H} 55 \mathrm{~N} 1 \mathrm{O} 2$ & $3.83 \mathrm{E}-05$ & Basic N1O1 & $\mathrm{C} 33 \mathrm{H} 27 \mathrm{~N} 1 \mathrm{O} 1$ & 3.34E-07 \\
\hline $\mathrm{CH}$ & $\mathrm{C} 28 \mathrm{H} 28$ & 0.003097 & Non-basic N1O2 & $\mathrm{C} 37 \mathrm{H} 57 \mathrm{~N} 1 \mathrm{O} 2$ & $3.86 \mathrm{E}-05$ & Basic N1O1 & $\mathrm{C} 34 \mathrm{H} 29 \mathrm{~N} 1 \mathrm{O} 1$ & $2.91 \mathrm{E}-07$ \\
\hline $\mathrm{CH}$ & $\mathrm{C} 29 \mathrm{H} 30$ & 0.002231 & Non-basic N1O2 & $\mathrm{C} 38 \mathrm{H} 59 \mathrm{~N} 1 \mathrm{O} 2$ & $3.32 \mathrm{E}-05$ & Basic N1O1 & $\mathrm{C} 35 \mathrm{H} 31 \mathrm{~N} 1 \mathrm{O} 1$ & $2.70 \mathrm{E}-07$ \\
\hline $\mathrm{CH}$ & $\mathrm{C} 30 \mathrm{H} 32$ & 0.001644 & Non-basic N1O2 & C39H61N1O2 & $2.48 \mathrm{E}-05$ & Basic N1O1 & C36H33N1O1 & $4.90 \mathrm{E}-07$ \\
\hline $\mathrm{CH}$ & $\mathrm{C} 31 \mathrm{H} 34$ & 0.001294 & Non-basic N1O2 & $\mathrm{C} 40 \mathrm{H} 63 \mathrm{~N} 1 \mathrm{O} 2$ & $2.24 \mathrm{E}-05$ & Basic N1O1 & C37H35N1O1 & $5.24 \mathrm{E}-07$ \\
\hline $\mathrm{CH}$ & $\mathrm{C} 32 \mathrm{H} 36$ & 0.00103 & Non-basic N1O2 & $\mathrm{C} 41 \mathrm{H} 65 \mathrm{~N} 1 \mathrm{O} 2$ & $1.39 \mathrm{E}-05$ & Basic N1O1 & $\mathrm{C} 38 \mathrm{H} 37 \mathrm{~N} 1 \mathrm{O} 1$ & $1.07 \mathrm{E}-06$ \\
\hline $\mathrm{CH}$ & $\mathrm{C} 33 \mathrm{H} 38$ & 0.000851 & Non-basic N1O2 & $\mathrm{C} 42 \mathrm{H} 67 \mathrm{~N} 1 \mathrm{O} 2$ & $1.20 \mathrm{E}-05$ & Basic N1O1 & C39H39N1O1 & $9.19 \mathrm{E}-07$ \\
\hline $\mathrm{CH}$ & $\mathrm{C} 34 \mathrm{H} 40$ & 0.000713 & Non-basic N1O2 & $\mathrm{C} 43 \mathrm{H} 69 \mathrm{~N} 1 \mathrm{O} 2$ & $7.65 \mathrm{E}-06$ & Basic N1O1 & $\mathrm{C} 40 \mathrm{H} 41 \mathrm{~N} 1 \mathrm{O} 1$ & $5.29 \mathrm{E}-07$ \\
\hline $\mathrm{CH}$ & $\mathrm{C} 35 \mathrm{H} 42$ & 0.000489 & Non-basic N1O2 & $\mathrm{C} 44 \mathrm{H} 71 \mathrm{~N} 1 \mathrm{O} 2$ & $5.89 \mathrm{E}-06$ & Basic N1O1 & $\mathrm{C} 41 \mathrm{H} 43 \mathrm{~N} 1 \mathrm{O} 1$ & $8.39 \mathrm{E}-07$ \\
\hline $\mathrm{CH}$ & C36H44 & 0.000438 & Non-basic N1O2 & $\mathrm{C} 45 \mathrm{H} 73 \mathrm{~N} 1 \mathrm{O} 2$ & $4.29 \mathrm{E}-06$ & Basic N1O1 & $\mathrm{C} 42 \mathrm{H} 45 \mathrm{~N} 1 \mathrm{O} 1$ & $3.13 \mathrm{E}-07$ \\
\hline $\mathrm{CH}$ & C37H46 & 0.000395 & Non-basic N1O2 & $\mathrm{C} 15 \mathrm{H} 11 \mathrm{~N} 1 \mathrm{O} 2$ & $6.85 \mathrm{E}-07$ & Basic N1O1 & $\mathrm{C} 43 \mathrm{H} 47 \mathrm{~N} 1 \mathrm{O} 1$ & $1.56 \mathrm{E}-07$ \\
\hline $\mathrm{CH}$ & C38H48 & 0.00035 & Non-basic N1O2 & $\mathrm{C} 16 \mathrm{H} 13 \mathrm{~N} 1 \mathrm{O} 2$ & $1.00 \mathrm{E}-05$ & Basic N1O1 & $\mathrm{C} 44 \mathrm{H} 49 \mathrm{~N} 1 \mathrm{O} 1$ & $3.21 \mathrm{E}-07$ \\
\hline
\end{tabular}




\begin{tabular}{|c|c|c|c|c|c|c|c|c|}
\hline Class & formula & Mass & Class & formula & Mass & Class & formula & Mass \\
\hline $\mathrm{CH}$ & $\mathrm{C} 39 \mathrm{H} 50$ & 0.000257 & Non-basic N1O2 & $\mathrm{C} 17 \mathrm{H} 15 \mathrm{~N} 1 \mathrm{O} 2$ & $1.73 \mathrm{E}-05$ & Basic N1O1 & $\mathrm{C} 32 \mathrm{H} 23 \mathrm{~N} 1 \mathrm{O} 1$ & 4.07E-08 \\
\hline $\mathrm{CH}$ & $\mathrm{C} 40 \mathrm{H} 52$ & 0.00023 & Non-basic N1O2 & $\mathrm{C} 18 \mathrm{H} 17 \mathrm{~N} 1 \mathrm{O} 2$ & $3.06 \mathrm{E}-05$ & Basic N1O1 & $\mathrm{C} 33 \mathrm{H} 25 \mathrm{~N} 1 \mathrm{O} 1$ & $4.72 \mathrm{E}-08$ \\
\hline $\mathrm{CH}$ & $\mathrm{C} 41 \mathrm{H} 54$ & 0.000126 & Non-basic N1O2 & C19H19N1O2 & $3.82 \mathrm{E}-05$ & Basic N1O1 & $\mathrm{C} 34 \mathrm{H} 27 \mathrm{~N} 1 \mathrm{O} 1$ & $8.11 \mathrm{E}-08$ \\
\hline $\mathrm{CH}$ & $\mathrm{C} 42 \mathrm{H} 56$ & $9.15 \mathrm{E}-05$ & Non-basic N1O2 & $\mathrm{C} 20 \mathrm{H} 21 \mathrm{~N} 1 \mathrm{O} 2$ & $3.66 \mathrm{E}-05$ & Basic N1O1 & $\mathrm{C} 35 \mathrm{H} 29 \mathrm{~N} 1 \mathrm{O} 1$ & $2.00 \mathrm{E}-07$ \\
\hline $\mathrm{CH}$ & $\mathrm{C} 43 \mathrm{H} 58$ & $3.24 \mathrm{E}-05$ & Non-basic N1O2 & $\mathrm{C} 21 \mathrm{H} 23 \mathrm{~N} 1 \mathrm{O} 2$ & $3.41 \mathrm{E}-05$ & Basic N1O1 & $\mathrm{C} 34 \mathrm{H} 25 \mathrm{~N} 1 \mathrm{O} 1$ & $1.39 \mathrm{E}-07$ \\
\hline $\mathrm{CH}$ & $\mathrm{C} 44 \mathrm{H} 60$ & $1.70 \mathrm{E}-05$ & Non-basic N1O2 & $\mathrm{C} 22 \mathrm{H} 25 \mathrm{~N} 1 \mathrm{O} 2$ & $3.03 \mathrm{E}-05$ & Basic N1O1 & $\mathrm{C} 35 \mathrm{H} 27 \mathrm{~N} 1 \mathrm{O} 1$ & $1.35 \mathrm{E}-06$ \\
\hline $\mathrm{CH}$ & $\mathrm{C} 45 \mathrm{H} 62$ & $1.24 \mathrm{E}-05$ & Non-basic N1O2 & $\mathrm{C} 23 \mathrm{H} 27 \mathrm{~N} 1 \mathrm{O} 2$ & $2.50 \mathrm{E}-05$ & Basic N1O1 & $\mathrm{C} 36 \mathrm{H} 29 \mathrm{~N} 1 \mathrm{O} 1$ & $9.50 \mathrm{E}-08$ \\
\hline $\mathrm{CH}$ & $\mathrm{C} 18 \mathrm{H} 6$ & $2.54 \mathrm{E}-06$ & Non-basic N1O2 & $\mathrm{C} 24 \mathrm{H} 29 \mathrm{~N} 1 \mathrm{O} 2$ & $2.89 \mathrm{E}-05$ & Basic N1O1 & $\mathrm{C} 37 \mathrm{H} 31 \mathrm{~N} 1 \mathrm{O} 1$ & $2.42 \mathrm{E}-08$ \\
\hline $\mathrm{CH}$ & C19H8 & $2.92 \mathrm{E}-06$ & Non-basic N1O2 & $\mathrm{C} 25 \mathrm{H} 31 \mathrm{~N} 1 \mathrm{O} 2$ & $4.46 \mathrm{E}-05$ & Basic N1O1 & $\mathrm{C} 38 \mathrm{H} 33 \mathrm{~N} 1 \mathrm{O} 1$ & 7.87E-08 \\
\hline $\mathrm{CH}$ & $\mathrm{C} 20 \mathrm{H} 10$ & $4.80 \mathrm{E}-05$ & Non-basic N1O2 & $\mathrm{C} 26 \mathrm{H} 33 \mathrm{~N} 1 \mathrm{O} 2$ & $2.66 \mathrm{E}-05$ & Basic N1O1 & $\mathrm{C} 34 \mathrm{H} 23 \mathrm{~N} 1 \mathrm{O} 1$ & $1.06 \mathrm{E}-07$ \\
\hline $\mathrm{CH}$ & $\mathrm{C} 21 \mathrm{H} 12$ & 0.000146 & Non-basic N1O2 & $\mathrm{C} 27 \mathrm{H} 35 \mathrm{~N} 1 \mathrm{O} 2$ & $2.85 \mathrm{E}-05$ & Basic N1O1 & $\mathrm{C} 35 \mathrm{H} 25 \mathrm{~N} 1 \mathrm{O} 1$ & $2.18 \mathrm{E}-07$ \\
\hline $\mathrm{CH}$ & $\mathrm{C} 22 \mathrm{H} 14$ & 0.001833 & Non-basic N1O2 & $\mathrm{C} 28 \mathrm{H} 37 \mathrm{~N} 1 \mathrm{O} 2$ & $3.00 \mathrm{E}-05$ & Basic N1O1 & $\mathrm{C} 36 \mathrm{H} 27 \mathrm{~N} 1 \mathrm{O} 1$ & $2.56 \mathrm{E}-08$ \\
\hline $\mathrm{CH}$ & $\mathrm{C} 23 \mathrm{H} 16$ & 0.006954 & Non-basic N1O2 & $\mathrm{C} 29 \mathrm{H} 39 \mathrm{~N} 1 \mathrm{O} 2$ & $3.29 \mathrm{E}-05$ & Basic N1O1 & $\mathrm{C} 37 \mathrm{H} 29 \mathrm{~N} 1 \mathrm{O} 1$ & $1.55 \mathrm{E}-08$ \\
\hline $\mathrm{CH}$ & $\mathrm{C} 24 \mathrm{H} 18$ & 0.013484 & Non-basic N1O2 & $\mathrm{C} 30 \mathrm{H} 41 \mathrm{~N} 1 \mathrm{O} 2$ & $3.37 \mathrm{E}-05$ & Basic N1O2 & $\mathrm{C} 14 \mathrm{H} 17 \mathrm{~N} 1 \mathrm{O} 2$ & $5.96 \mathrm{E}-06$ \\
\hline $\mathrm{CH}$ & $\mathrm{C} 25 \mathrm{H} 20$ & 0.014404 & Non-basic N1O2 & $\mathrm{C} 31 \mathrm{H} 43 \mathrm{~N} 1 \mathrm{O} 2$ & $3.19 \mathrm{E}-05$ & Basic N1O2 & $\mathrm{C} 15 \mathrm{H} 19 \mathrm{~N} 1 \mathrm{O} 2$ & $8.69 \mathrm{E}-06$ \\
\hline $\mathrm{CH}$ & $\mathrm{C} 26 \mathrm{H} 22$ & 0.011248 & Non-basic N1O2 & $\mathrm{C} 32 \mathrm{H} 45 \mathrm{~N} 1 \mathrm{O} 2$ & $3.42 \mathrm{E}-05$ & Basic N1O2 & $\mathrm{C} 16 \mathrm{H} 21 \mathrm{~N} 1 \mathrm{O} 2$ & $1.23 \mathrm{E}-05$ \\
\hline $\mathrm{CH}$ & $\mathrm{C} 27 \mathrm{H} 24$ & 0.007237 & Non-basic N1O2 & $\mathrm{C} 33 \mathrm{H} 47 \mathrm{~N} 1 \mathrm{O} 2$ & $3.54 \mathrm{E}-05$ & Basic N1O2 & $\mathrm{C} 17 \mathrm{H} 23 \mathrm{~N} 1 \mathrm{O} 2$ & $4.32 \mathrm{E}-07$ \\
\hline $\mathrm{CH}$ & $\mathrm{C} 28 \mathrm{H} 26$ & 0.004427 & Non-basic N1O2 & $\mathrm{C} 34 \mathrm{H} 49 \mathrm{~N} 1 \mathrm{O} 2$ & $2.68 \mathrm{E}-05$ & Basic N1O2 & $\mathrm{C} 18 \mathrm{H} 25 \mathrm{~N} 1 \mathrm{O} 2$ & $5.23 \mathrm{E}-08$ \\
\hline $\mathrm{CH}$ & $\mathrm{C} 29 \mathrm{H} 28$ & 0.002819 & Non-basic N1O2 & $\mathrm{C} 35 \mathrm{H} 51 \mathrm{~N} 1 \mathrm{O} 2$ & $2.87 \mathrm{E}-05$ & Basic N1O2 & $\mathrm{C} 19 \mathrm{H} 27 \mathrm{~N} 1 \mathrm{O} 2$ & $1.49 \mathrm{E}-08$ \\
\hline $\mathrm{CH}$ & $\mathrm{C} 30 \mathrm{H} 30$ & 0.0018 & Non-basic N1O2 & $\mathrm{C} 36 \mathrm{H} 53 \mathrm{~N} 1 \mathrm{O} 2$ & $2.94 \mathrm{E}-05$ & Basic N1O2 & $\mathrm{C} 20 \mathrm{H} 29 \mathrm{~N} 1 \mathrm{O} 2$ & $7.85 \mathrm{E}-08$ \\
\hline $\mathrm{CH}$ & $\mathrm{C} 31 \mathrm{H} 32$ & 0.001279 & Non-basic N1O2 & $\mathrm{C} 37 \mathrm{H} 55 \mathrm{~N} 1 \mathrm{O} 2$ & $2.38 \mathrm{E}-05$ & Basic N1O2 & $\mathrm{C} 21 \mathrm{H} 31 \mathrm{~N} 1 \mathrm{O} 2$ & $2.07 \mathrm{E}-07$ \\
\hline $\mathrm{CH}$ & $\mathrm{C} 32 \mathrm{H} 34$ & 0.000918 & Non-basic N1O2 & $\mathrm{C} 38 \mathrm{H} 57 \mathrm{~N} 1 \mathrm{O} 2$ & $2.15 \mathrm{E}-05$ & Basic N1O2 & $\mathrm{C} 22 \mathrm{H} 33 \mathrm{~N} 1 \mathrm{O} 2$ & $3.56 \mathrm{E}-07$ \\
\hline $\mathrm{CH}$ & $\mathrm{C} 33 \mathrm{H} 36$ & 0.000746 & Non-basic N1O2 & $\mathrm{C} 39 \mathrm{H} 59 \mathrm{~N} 1 \mathrm{O} 2$ & $1.81 \mathrm{E}-05$ & Basic N1O2 & $\mathrm{C} 23 \mathrm{H} 35 \mathrm{~N} 1 \mathrm{O} 2$ & $9.98 \mathrm{E}-07$ \\
\hline $\mathrm{CH}$ & C34H38 & 0.000566 & Non-basic N1O2 & $\mathrm{C} 40 \mathrm{H} 61 \mathrm{~N} 1 \mathrm{O} 2$ & $1.25 \mathrm{E}-05$ & Basic N1O2 & $\mathrm{C} 24 \mathrm{H} 37 \mathrm{~N} 1 \mathrm{O} 2$ & $2.46 \mathrm{E}-07$ \\
\hline $\mathrm{CH}$ & $\mathrm{C} 35 \mathrm{H} 40$ & 0.00047 & Non-basic N1O2 & $\mathrm{C} 41 \mathrm{H} 63 \mathrm{~N} 1 \mathrm{O} 2$ & $9.06 \mathrm{E}-06$ & Basic N1O2 & $\mathrm{C} 25 \mathrm{H} 39 \mathrm{~N} 1 \mathrm{O} 2$ & $3.65 \mathrm{E}-07$ \\
\hline $\mathrm{CH}$ & $\mathrm{C} 36 \mathrm{H} 42$ & 0.00036 & Non-basic N1O2 & $\mathrm{C} 42 \mathrm{H} 65 \mathrm{~N} 1 \mathrm{O} 2$ & $1.12 \mathrm{E}-05$ & Basic N1O2 & $\mathrm{C} 26 \mathrm{H} 41 \mathrm{~N} 1 \mathrm{O} 2$ & $3.32 \mathrm{E}-07$ \\
\hline $\mathrm{CH}$ & $\mathrm{C} 37 \mathrm{H} 44$ & 0.000264 & Non-basic N1O2 & $\mathrm{C} 43 \mathrm{H} 67 \mathrm{~N} 1 \mathrm{O} 2$ & $2.49 \mathrm{E}-06$ & Basic N1O2 & $\mathrm{C} 27 \mathrm{H} 43 \mathrm{~N} 1 \mathrm{O} 2$ & $2.79 \mathrm{E}-07$ \\
\hline $\mathrm{CH}$ & $\mathrm{C} 38 \mathrm{H} 46$ & 0.000242 & Non-basic N1O2 & $\mathrm{C} 44 \mathrm{H} 69 \mathrm{~N} 1 \mathrm{O} 2$ & $3.30 \mathrm{E}-06$ & Basic N1O2 & $\mathrm{C} 28 \mathrm{H} 45 \mathrm{~N} 1 \mathrm{O} 2$ & $4.64 \mathrm{E}-07$ \\
\hline $\mathrm{CH}$ & $\mathrm{C} 39 \mathrm{H} 48$ & 0.00019 & Non-basic N1O2 & $\mathrm{C} 45 \mathrm{H} 71 \mathrm{~N} 1 \mathrm{O} 2$ & $3.11 \mathrm{E}-06$ & Basic N1O2 & $\mathrm{C} 29 \mathrm{H} 47 \mathrm{~N} 1 \mathrm{O} 2$ & $6.80 \mathrm{E}-07$ \\
\hline $\mathrm{CH}$ & $\mathrm{C} 40 \mathrm{H} 50$ & 0.000194 & Non-basic N1O2 & $\mathrm{C} 46 \mathrm{H} 73 \mathrm{~N} 1 \mathrm{O} 2$ & 4.57E-06 & Basic N1O2 & $\mathrm{C} 30 \mathrm{H} 49 \mathrm{~N} 1 \mathrm{O} 2$ & $1.01 \mathrm{E}-06$ \\
\hline $\mathrm{CH}$ & $\mathrm{C} 41 \mathrm{H} 52$ & $8.01 \mathrm{E}-05$ & Non-basic N1O2 & $\mathrm{C} 47 \mathrm{H} 75 \mathrm{~N} 1 \mathrm{O} 2$ & $2.55 \mathrm{E}-06$ & Basic N1O2 & $\mathrm{C} 31 \mathrm{H} 51 \mathrm{~N} 1 \mathrm{O} 2$ & $1.25 \mathrm{E}-06$ \\
\hline $\mathrm{CH}$ & $\mathrm{C} 42 \mathrm{H} 54$ & $5.43 \mathrm{E}-05$ & Non-basic N1O2 & $\mathrm{C} 17 \mathrm{H} 13 \mathrm{~N} 1 \mathrm{O} 2$ & $1.03 \mathrm{E}-05$ & Basic N1O2 & $\mathrm{C} 32 \mathrm{H} 53 \mathrm{~N} 1 \mathrm{O} 2$ & $1.31 \mathrm{E}-06$ \\
\hline $\mathrm{CH}$ & $\mathrm{C} 43 \mathrm{H} 56$ & $4.45 \mathrm{E}-05$ & Non-basic N1O2 & $\mathrm{C} 18 \mathrm{H} 15 \mathrm{~N} 1 \mathrm{O} 2$ & $3.60 \mathrm{E}-05$ & Basic N1O2 & $\mathrm{C} 33 \mathrm{H} 55 \mathrm{~N} 1 \mathrm{O} 2$ & $1.28 \mathrm{E}-06$ \\
\hline $\mathrm{CH}$ & C44H58 & $1.30 \mathrm{E}-05$ & Non-basic N1O2 & $\mathrm{C} 19 \mathrm{H} 17 \mathrm{~N} 1 \mathrm{O} 2$ & 0.000101 & Basic N1O2 & $\mathrm{C} 34 \mathrm{H} 57 \mathrm{~N} 1 \mathrm{O} 2$ & $1.33 \mathrm{E}-06$ \\
\hline $\mathrm{CH}$ & $\mathrm{C} 45 \mathrm{H} 60$ & $1.54 \mathrm{E}-05$ & Non-basic N1O2 & $\mathrm{C} 20 \mathrm{H} 19 \mathrm{~N} 1 \mathrm{O} 2$ & 0.00014 & Basic $\mathrm{N} 1 \mathrm{O} 2$ & $\mathrm{C} 35 \mathrm{H} 59 \mathrm{~N} 1 \mathrm{O} 2$ & $1.22 \mathrm{E}-06$ \\
\hline $\mathrm{CH}$ & C46H62 & $3.92 \mathrm{E}-06$ & Non-basic N1O2 & $\mathrm{C} 21 \mathrm{H} 21 \mathrm{~N} 1 \mathrm{O} 2$ & 0.000108 & Basic $\mathrm{N} 1 \mathrm{O} 2$ & $\mathrm{C} 36 \mathrm{H} 61 \mathrm{~N} 1 \mathrm{O} 2$ & $9.35 \mathrm{E}-07$ \\
\hline $\mathrm{CH}$ & C49H68 & $5.29 \mathrm{E}-06$ & Non-basic N1O2 & $\mathrm{C} 22 \mathrm{H} 23 \mathrm{~N} 1 \mathrm{O} 2$ & $6.94 \mathrm{E}-05$ & Basic $\mathrm{N} 1 \mathrm{O} 2$ & $\mathrm{C} 37 \mathrm{H} 63 \mathrm{~N} 1 \mathrm{O} 2$ & $6.48 \mathrm{E}-07$ \\
\hline $\mathrm{CH}$ & C19H6 & $1.12 \mathrm{E}-06$ & Non-basic N1O2 & $\mathrm{C} 23 \mathrm{H} 25 \mathrm{~N} 1 \mathrm{O} 2$ & $6.03 \mathrm{E}-05$ & Basic $\mathrm{N}_{10} \mathrm{O}$ & $\mathrm{C} 38 \mathrm{H} 65 \mathrm{~N} 1 \mathrm{O} 2$ & $5.45 \mathrm{E}-07$ \\
\hline
\end{tabular}




\begin{tabular}{|c|c|c|c|c|c|c|c|c|}
\hline Class & formula & Mass & Class & formula & Mass & Class & formula & Mass \\
\hline $\mathrm{CH}$ & $\mathrm{C} 20 \mathrm{H} 8$ & $1.15 \mathrm{E}-05$ & Non-basic N1O2 & $\mathrm{C} 24 \mathrm{H} 27 \mathrm{~N} 1 \mathrm{O} 2$ & $4.15 \mathrm{E}-05$ & Basic N1O2 & C39H67N1O2 & $4.28 \mathrm{E}-07$ \\
\hline $\mathrm{CH}$ & $\mathrm{C} 21 \mathrm{H} 10$ & 7.02E-06 & Non-basic N1O2 & $\mathrm{C} 25 \mathrm{H} 29 \mathrm{~N} 1 \mathrm{O} 2$ & $3.51 \mathrm{E}-05$ & Basic N1O2 & $\mathrm{C} 40 \mathrm{H} 69 \mathrm{~N} 1 \mathrm{O} 2$ & $1.70 \mathrm{E}-07$ \\
\hline $\mathrm{CH}$ & $\mathrm{C} 22 \mathrm{H} 12$ & 0.001919 & Non-basic N1O2 & $\mathrm{C} 26 \mathrm{H} 31 \mathrm{~N} 1 \mathrm{O} 2$ & $3.31 \mathrm{E}-05$ & Basic N1O2 & $\mathrm{C} 41 \mathrm{H} 71 \mathrm{~N} 1 \mathrm{O} 2$ & $3.56 \mathrm{E}-07$ \\
\hline $\mathrm{CH}$ & $\mathrm{C} 23 \mathrm{H} 14$ & 0.007221 & Non-basic N1O2 & $\mathrm{C} 27 \mathrm{H} 33 \mathrm{~N} 1 \mathrm{O} 2$ & 0.000121 & Basic N1O2 & C16H19N1O2 & 4.99E-07 \\
\hline $\mathrm{CH}$ & $\mathrm{C} 24 \mathrm{H} 16$ & 0.011594 & Non-basic N1O2 & $\mathrm{C} 28 \mathrm{H} 35 \mathrm{~N} 1 \mathrm{O} 2$ & $3.36 \mathrm{E}-05$ & Basic N1O2 & $\mathrm{C} 17 \mathrm{H} 21 \mathrm{~N} 1 \mathrm{O} 2$ & 8.87E-08 \\
\hline $\mathrm{CH}$ & $\mathrm{C} 25 \mathrm{H} 18$ & 0.011512 & Non-basic N1O2 & $\mathrm{C} 29 \mathrm{H} 37 \mathrm{~N} 1 \mathrm{O} 2$ & $3.24 \mathrm{E}-05$ & Basic N1O2 & $\mathrm{C} 18 \mathrm{H} 23 \mathrm{~N} 1 \mathrm{O} 2$ & $9.52 \mathrm{E}-09$ \\
\hline $\mathrm{CH}$ & $\mathrm{C} 26 \mathrm{H} 20$ & 0.009589 & Non-basic N1O2 & $\mathrm{C} 30 \mathrm{H} 39 \mathrm{~N} 1 \mathrm{O} 2$ & $2.97 \mathrm{E}-05$ & Basic N1O2 & $\mathrm{C} 19 \mathrm{H} 25 \mathrm{~N} 1 \mathrm{O} 2$ & $1.21 \mathrm{E}-07$ \\
\hline $\mathrm{CH}$ & $\mathrm{C} 27 \mathrm{H} 22$ & 0.007003 & Non-basic N1O2 & $\mathrm{C} 31 \mathrm{H} 41 \mathrm{~N} 1 \mathrm{O} 2$ & $2.75 \mathrm{E}-05$ & Basic N1O2 & $\mathrm{C} 20 \mathrm{H} 27 \mathrm{~N} 1 \mathrm{O} 2$ & $6.02 \mathrm{E}-07$ \\
\hline $\mathrm{CH}$ & $\mathrm{C} 28 \mathrm{H} 24$ & 0.00486 & Non-basic N1O2 & $\mathrm{C} 32 \mathrm{H} 43 \mathrm{~N} 1 \mathrm{O} 2$ & $2.57 \mathrm{E}-05$ & Basic N1O2 & $\mathrm{C} 21 \mathrm{H} 29 \mathrm{~N} 1 \mathrm{O} 2$ & $2.16 \mathrm{E}-06$ \\
\hline $\mathrm{CH}$ & $\mathrm{C} 29 \mathrm{H} 26$ & 0.00304 & Non-basic N1O2 & $\mathrm{C} 33 \mathrm{H} 45 \mathrm{~N} 1 \mathrm{O} 2$ & $2.44 \mathrm{E}-05$ & Basic N1O2 & $\mathrm{C} 22 \mathrm{H} 31 \mathrm{~N} 1 \mathrm{O} 2$ & 7.97E-06 \\
\hline $\mathrm{CH}$ & $\mathrm{C} 30 \mathrm{H} 28$ & 0.001925 & Non-basic N1O2 & $\mathrm{C} 34 \mathrm{H} 47 \mathrm{~N} 1 \mathrm{O} 2$ & $3.04 \mathrm{E}-05$ & Basic N1O2 & $\mathrm{C} 23 \mathrm{H} 33 \mathrm{~N} 1 \mathrm{O} 2$ & $1.53 \mathrm{E}-05$ \\
\hline $\mathrm{CH}$ & $\mathrm{C} 31 \mathrm{H} 30$ & 0.001277 & Non-basic N1O2 & $\mathrm{C} 35 \mathrm{H} 49 \mathrm{~N} 1 \mathrm{O} 2$ & $4.21 \mathrm{E}-05$ & Basic N1O2 & $\mathrm{C} 24 \mathrm{H} 35 \mathrm{~N} 1 \mathrm{O} 2$ & $2.46 \mathrm{E}-06$ \\
\hline $\mathrm{CH}$ & $\mathrm{C} 32 \mathrm{H} 32$ & 0.00091 & Non-basic N1O2 & $\mathrm{C} 36 \mathrm{H} 51 \mathrm{~N} 1 \mathrm{O} 2$ & $4.34 \mathrm{E}-05$ & Basic N1O2 & $\mathrm{C} 25 \mathrm{H} 37 \mathrm{~N} 1 \mathrm{O} 2$ & $1.71 \mathrm{E}-06$ \\
\hline $\mathrm{CH}$ & $\mathrm{C} 33 \mathrm{H} 34$ & 0.000665 & Non-basic N1O2 & $\mathrm{C} 37 \mathrm{H} 53 \mathrm{~N} 1 \mathrm{O} 2$ & $4.19 \mathrm{E}-05$ & Basic N1O2 & $\mathrm{C} 26 \mathrm{H} 39 \mathrm{~N} 1 \mathrm{O} 2$ & $1.28 \mathrm{E}-06$ \\
\hline $\mathrm{CH}$ & $\mathrm{C} 34 \mathrm{H} 36$ & 0.00049 & Non-basic N1O2 & $\mathrm{C} 38 \mathrm{H} 55 \mathrm{~N} 1 \mathrm{O} 2$ & $3.63 \mathrm{E}-05$ & Basic N1O2 & $\mathrm{C} 27 \mathrm{H} 41 \mathrm{~N} 1 \mathrm{O} 2$ & $1.16 \mathrm{E}-06$ \\
\hline $\mathrm{CH}$ & C $35 \mathrm{H} 38$ & 0.000375 & Non-basic N1O2 & $\mathrm{C} 39 \mathrm{H} 57 \mathrm{~N} 1 \mathrm{O} 2$ & $2.71 \mathrm{E}-05$ & Basic N1O2 & $\mathrm{C} 28 \mathrm{H} 43 \mathrm{~N} 1 \mathrm{O} 2$ & $1.15 \mathrm{E}-06$ \\
\hline $\mathrm{CH}$ & $\mathrm{C} 36 \mathrm{H} 40$ & 0.000263 & Non-basic N1O2 & $\mathrm{C} 40 \mathrm{H} 59 \mathrm{~N} 1 \mathrm{O} 2$ & $2.51 \mathrm{E}-05$ & Basic N1O2 & $\mathrm{C} 29 \mathrm{H} 45 \mathrm{~N} 1 \mathrm{O} 2$ & $1.96 \mathrm{E}-06$ \\
\hline $\mathrm{CH}$ & $\mathrm{C} 37 \mathrm{H} 42$ & 0.00018 & Non-basic N1O2 & $\mathrm{C} 41 \mathrm{H} 61 \mathrm{~N} 1 \mathrm{O} 2$ & $1.08 \mathrm{E}-05$ & Basic N1O2 & $\mathrm{C} 30 \mathrm{H} 47 \mathrm{~N} 1 \mathrm{O} 2$ & $1.35 \mathrm{E}-06$ \\
\hline $\mathrm{CH}$ & $\mathrm{C} 38 \mathrm{H} 44$ & 0.000141 & Non-basic N1O2 & $\mathrm{C} 42 \mathrm{H} 63 \mathrm{~N} 1 \mathrm{O} 2$ & $1.17 \mathrm{E}-05$ & Basic N1O2 & $\mathrm{C} 31 \mathrm{H} 49 \mathrm{~N} 1 \mathrm{O} 2$ & $2.08 \mathrm{E}-06$ \\
\hline $\mathrm{CH}$ & $\mathrm{C} 39 \mathrm{H} 46$ & 0.000117 & Non-basic N1O2 & $\mathrm{C} 43 \mathrm{H} 65 \mathrm{~N} 1 \mathrm{O} 2$ & 7.11E-06 & Basic N1O2 & $\mathrm{C} 32 \mathrm{H} 51 \mathrm{~N} 1 \mathrm{O} 2$ & $1.96 \mathrm{E}-06$ \\
\hline $\mathrm{CH}$ & $\mathrm{C} 40 \mathrm{H} 48$ & $9.71 \mathrm{E}-05$ & Non-basic N1O2 & $\mathrm{C} 44 \mathrm{H} 67 \mathrm{~N} 1 \mathrm{O} 2$ & $1.05 \mathrm{E}-05$ & Basic N1O2 & $\mathrm{C} 33 \mathrm{H} 53 \mathrm{~N} 1 \mathrm{O} 2$ & $2.34 \mathrm{E}-06$ \\
\hline $\mathrm{CH}$ & $\mathrm{C} 41 \mathrm{H} 50$ & $2.50 \mathrm{E}-05$ & Non-basic N1O2 & $\mathrm{C} 45 \mathrm{H} 69 \mathrm{~N} 1 \mathrm{O} 2$ & $8.36 \mathrm{E}-06$ & Basic N1O2 & $\mathrm{C} 34 \mathrm{H} 55 \mathrm{~N} 1 \mathrm{O} 2$ & $1.42 \mathrm{E}-06$ \\
\hline $\mathrm{CH}$ & $\mathrm{C} 42 \mathrm{H} 52$ & $2.91 \mathrm{E}-05$ & Non-basic N1O2 & $\mathrm{C} 46 \mathrm{H} 71 \mathrm{~N} 1 \mathrm{O} 2$ & $5.21 \mathrm{E}-06$ & Basic N1O2 & $\mathrm{C} 35 \mathrm{H} 57 \mathrm{~N} 1 \mathrm{O} 2$ & $1.51 \mathrm{E}-06$ \\
\hline $\mathrm{CH}$ & $\mathrm{C} 43 \mathrm{H} 54$ & $1.39 \mathrm{E}-05$ & Non-basic N1O2 & $\mathrm{C} 47 \mathrm{H} 73 \mathrm{~N} 1 \mathrm{O} 2$ & $3.02 \mathrm{E}-06$ & Basic N1O2 & $\mathrm{C} 36 \mathrm{H} 59 \mathrm{~N} 1 \mathrm{O} 2$ & $1.40 \mathrm{E}-06$ \\
\hline $\mathrm{CH}$ & $\mathrm{C} 44 \mathrm{H} 56$ & $9.61 \mathrm{E}-06$ & Non-basic N1O2 & $\mathrm{C} 16 \mathrm{H} 9 \mathrm{~N} 1 \mathrm{O} 2$ & $1.78 \mathrm{E}-06$ & Basic N1O2 & $\mathrm{C} 37 \mathrm{H} 61 \mathrm{~N} 1 \mathrm{O} 2$ & $1.01 \mathrm{E}-06$ \\
\hline $\mathrm{CH}$ & C45H58 & $9.23 \mathrm{E}-06$ & Non-basic N1O2 & $\mathrm{C} 17 \mathrm{H} 11 \mathrm{~N} 1 \mathrm{O} 2$ & $2.12 \mathrm{E}-05$ & Basic N1O2 & $\mathrm{C} 38 \mathrm{H} 63 \mathrm{~N} 1 \mathrm{O} 2$ & $9.92 \mathrm{E}-07$ \\
\hline $\mathrm{CH}$ & $\mathrm{C} 46 \mathrm{H} 60$ & $6.08 \mathrm{E}-06$ & Non-basic N1O2 & $\mathrm{C} 18 \mathrm{H} 13 \mathrm{~N} 1 \mathrm{O} 2$ & $5.17 \mathrm{E}-05$ & Basic N1O2 & $\mathrm{C} 15 \mathrm{H} 15 \mathrm{~N} 1 \mathrm{O} 2$ & $6.45 \mathrm{E}-08$ \\
\hline $\mathrm{CH}$ & $\mathrm{C} 47 \mathrm{H} 62$ & 4.40E-06 & Non-basic N1O2 & $\mathrm{C} 19 \mathrm{H} 15 \mathrm{~N} 1 \mathrm{O} 2$ & $6.62 \mathrm{E}-05$ & Basic N1O2 & $\mathrm{C} 16 \mathrm{H} 17 \mathrm{~N} 1 \mathrm{O} 2$ & $2.31 \mathrm{E}-08$ \\
\hline $\mathrm{CH}$ & $\mathrm{C} 19 \mathrm{H} 4$ & $2.92 \mathrm{E}-06$ & Non-basic N1O2 & $\mathrm{C} 20 \mathrm{H} 17 \mathrm{~N} 1 \mathrm{O} 2$ & $6.95 \mathrm{E}-05$ & Basic N1O2 & $\mathrm{C} 17 \mathrm{H} 19 \mathrm{~N} 1 \mathrm{O} 2$ & $5.09 \mathrm{E}-08$ \\
\hline $\mathrm{CH}$ & $\mathrm{C} 20 \mathrm{H} 6$ & $1.76 \mathrm{E}-06$ & Non-basic N1O2 & $\mathrm{C} 21 \mathrm{H} 19 \mathrm{~N} 1 \mathrm{O} 2$ & 7.43E-05 & Basic N1O2 & $\mathrm{C} 18 \mathrm{H} 21 \mathrm{~N} 1 \mathrm{O} 2$ & $2.04 \mathrm{E}-07$ \\
\hline $\mathrm{CH}$ & $\mathrm{C} 21 \mathrm{H} 8$ & $1.54 \mathrm{E}-06$ & Non-basic N1O2 & $\mathrm{C} 22 \mathrm{H} 21 \mathrm{~N} 1 \mathrm{O} 2$ & 7.79E-05 & Basic N1O2 & $\mathrm{C} 19 \mathrm{H} 23 \mathrm{~N} 1 \mathrm{O} 2$ & $5.78 \mathrm{E}-07$ \\
\hline $\mathrm{CH}$ & $\mathrm{C} 22 \mathrm{H} 10$ & $4.90 \mathrm{E}-05$ & Non-basic N1O2 & $\mathrm{C} 23 \mathrm{H} 23 \mathrm{~N} 1 \mathrm{O} 2$ & $7.30 \mathrm{E}-05$ & Basic N1O2 & $\mathrm{C} 20 \mathrm{H} 25 \mathrm{~N} 1 \mathrm{O} 2$ & $1.67 \mathrm{E}-06$ \\
\hline $\mathrm{CH}$ & $\mathrm{C} 23 \mathrm{H} 12$ & $3.23 \mathrm{E}-05$ & Non-basic N1O2 & $\mathrm{C} 24 \mathrm{H} 25 \mathrm{~N} 1 \mathrm{O} 2$ & $6.21 \mathrm{E}-05$ & Basic N1O2 & $\mathrm{C} 21 \mathrm{H} 27 \mathrm{~N} 1 \mathrm{O} 2$ & 4.29E-06 \\
\hline $\mathrm{CH}$ & $\mathrm{C} 24 \mathrm{H} 14$ & 0.001748 & Non-basic N1O2 & $\mathrm{C} 25 \mathrm{H} 27 \mathrm{~N} 1 \mathrm{O} 2$ & $5.31 \mathrm{E}-05$ & Basic N1O2 & $\mathrm{C} 22 \mathrm{H} 29 \mathrm{~N} 1 \mathrm{O} 2$ & $1.40 \mathrm{E}-05$ \\
\hline $\mathrm{CH}$ & $\mathrm{C} 25 \mathrm{H} 16$ & 0.006341 & Non-basic N1O2 & $\mathrm{C} 26 \mathrm{H} 29 \mathrm{~N} 1 \mathrm{O} 2$ & $4.25 \mathrm{E}-05$ & Basic N1O2 & $\mathrm{C} 23 \mathrm{H} 31 \mathrm{~N} 1 \mathrm{O} 2$ & $2.26 \mathrm{E}-05$ \\
\hline $\mathrm{CH}$ & $\mathrm{C} 26 \mathrm{H} 18$ & 0.010005 & Non-basic N1O2 & $\mathrm{C} 27 \mathrm{H} 31 \mathrm{~N} 1 \mathrm{O} 2$ & $3.29 \mathrm{E}-05$ & Basic N1O2 & $\mathrm{C} 24 \mathrm{H} 33 \mathrm{~N} 1 \mathrm{O} 2$ & 4.26E-06 \\
\hline $\mathrm{CH}$ & $\mathrm{C} 27 \mathrm{H} 20$ & 0.009101 & Non-basic N1O2 & $\mathrm{C} 28 \mathrm{H} 33 \mathrm{~N} 1 \mathrm{O} 2$ & $3.29 \mathrm{E}-05$ & Basic $\mathrm{N} 1 \mathrm{O} 2$ & $\mathrm{C} 25 \mathrm{H} 35 \mathrm{~N} 1 \mathrm{O} 2$ & $3.90 \mathrm{E}-06$ \\
\hline $\mathrm{CH}$ & $\mathrm{C} 28 \mathrm{H} 22$ & 0.005986 & Non-basic N1O2 & $\mathrm{C} 29 \mathrm{H} 35 \mathrm{~N} 1 \mathrm{O} 2$ & $3.20 \mathrm{E}-05$ & Basic N1O2 & $\mathrm{C} 26 \mathrm{H} 37 \mathrm{~N} 1 \mathrm{O} 2$ & $2.96 \mathrm{E}-06$ \\
\hline
\end{tabular}




\begin{tabular}{|c|c|c|c|c|c|c|c|c|}
\hline Class & formula & Mass & Class & formula & Mass & Class & formula & Mass \\
\hline $\mathrm{CH}$ & $\mathrm{C} 29 \mathrm{H} 24$ & 0.003577 & Non-basic N1O2 & $\mathrm{C} 30 \mathrm{H} 37 \mathrm{~N} 1 \mathrm{O} 2$ & $2.78 \mathrm{E}-05$ & Basic N1O2 & $\mathrm{C} 27 \mathrm{H} 39 \mathrm{~N} 1 \mathrm{O} 2$ & $2.21 \mathrm{E}-06$ \\
\hline $\mathrm{CH}$ & $\mathrm{C} 30 \mathrm{H} 26$ & 0.00203 & Non-basic N1O2 & $\mathrm{C} 31 \mathrm{H} 39 \mathrm{~N} 1 \mathrm{O} 2$ & $2.33 \mathrm{E}-05$ & Basic N1O2 & $\mathrm{C} 28 \mathrm{H} 41 \mathrm{~N} 1 \mathrm{O} 2$ & $2.54 \mathrm{E}-06$ \\
\hline $\mathrm{CH}$ & $\mathrm{C} 31 \mathrm{H} 28$ & 0.001146 & Non-basic N1O2 & $\mathrm{C} 32 \mathrm{H} 41 \mathrm{~N} 1 \mathrm{O} 2$ & $2.41 \mathrm{E}-05$ & Basic N1O2 & $\mathrm{C} 29 \mathrm{H} 43 \mathrm{~N} 1 \mathrm{O} 2$ & $3.38 \mathrm{E}-06$ \\
\hline $\mathrm{CH}$ & $\mathrm{C} 32 \mathrm{H} 30$ & 0.000835 & Non-basic N1O2 & $\mathrm{C} 33 \mathrm{H} 43 \mathrm{~N} 1 \mathrm{O} 2$ & $2.50 \mathrm{E}-05$ & Basic N1O2 & $\mathrm{C} 30 \mathrm{H} 45 \mathrm{~N} 1 \mathrm{O} 2$ & $3.13 \mathrm{E}-06$ \\
\hline $\mathrm{CH}$ & $\mathrm{C} 33 \mathrm{H} 32$ & 0.000541 & Non-basic N1O2 & $\mathrm{C} 34 \mathrm{H} 45 \mathrm{~N} 1 \mathrm{O} 2$ & $8.68 \mathrm{E}-05$ & Basic N1O2 & $\mathrm{C} 31 \mathrm{H} 47 \mathrm{~N} 1 \mathrm{O} 2$ & 3.34E-06 \\
\hline $\mathrm{CH}$ & $\mathrm{C} 34 \mathrm{H} 34$ & 0.000375 & Non-basic N1O2 & $\mathrm{C} 35 \mathrm{H} 47 \mathrm{~N} 1 \mathrm{O} 2$ & $2.06 \mathrm{E}-05$ & Basic N1O2 & $\mathrm{C} 32 \mathrm{H} 49 \mathrm{~N} 1 \mathrm{O} 2$ & $3.94 \mathrm{E}-06$ \\
\hline $\mathrm{CH}$ & $\mathrm{C} 35 \mathrm{H} 36$ & 0.000244 & Non-basic N1O2 & $\mathrm{C} 36 \mathrm{H} 49 \mathrm{~N} 1 \mathrm{O} 2$ & $2.14 \mathrm{E}-05$ & Basic N1O2 & $\mathrm{C} 33 \mathrm{H} 51 \mathrm{~N} 1 \mathrm{O} 2$ & $3.36 \mathrm{E}-06$ \\
\hline $\mathrm{CH}$ & $\mathrm{C} 36 \mathrm{H} 38$ & 0.000181 & Non-basic N1O2 & $\mathrm{C} 37 \mathrm{H} 51 \mathrm{~N} 1 \mathrm{O} 2$ & $2.49 \mathrm{E}-05$ & Basic N1O2 & $\mathrm{C} 34 \mathrm{H} 53 \mathrm{~N} 1 \mathrm{O} 2$ & $2.78 \mathrm{E}-06$ \\
\hline $\mathrm{CH}$ & $\mathrm{C} 37 \mathrm{H} 40$ & 0.000136 & Non-basic N1O2 & $\mathrm{C} 38 \mathrm{H} 53 \mathrm{~N} 1 \mathrm{O} 2$ & $2.42 \mathrm{E}-05$ & Basic N1O2 & $\mathrm{C} 35 \mathrm{H} 55 \mathrm{~N} 1 \mathrm{O} 2$ & $3.13 \mathrm{E}-06$ \\
\hline $\mathrm{CH}$ & $\mathrm{C} 38 \mathrm{H} 42$ & 7.91E-05 & Non-basic N1O2 & $\mathrm{C} 39 \mathrm{H} 55 \mathrm{~N} 1 \mathrm{O} 2$ & $3.09 \mathrm{E}-05$ & Basic N1O2 & $\mathrm{C} 36 \mathrm{H} 57 \mathrm{~N} 1 \mathrm{O} 2$ & $2.26 \mathrm{E}-06$ \\
\hline $\mathrm{CH}$ & $\mathrm{C} 39 \mathrm{H} 44$ & $6.87 \mathrm{E}-05$ & Non-basic N1O2 & $\mathrm{C} 40 \mathrm{H} 57 \mathrm{~N} 1 \mathrm{O} 2$ & $2.69 \mathrm{E}-05$ & Basic N1O2 & $\mathrm{C} 37 \mathrm{H} 59 \mathrm{~N} 1 \mathrm{O} 2$ & $1.71 \mathrm{E}-06$ \\
\hline $\mathrm{CH}$ & $\mathrm{C} 40 \mathrm{H} 46$ & $3.19 \mathrm{E}-05$ & Non-basic N1O2 & $\mathrm{C} 41 \mathrm{H} 59 \mathrm{~N} 1 \mathrm{O} 2$ & $1.17 \mathrm{E}-05$ & Basic N1O2 & $\mathrm{C} 38 \mathrm{H} 61 \mathrm{~N} 1 \mathrm{O} 2$ & $1.30 \mathrm{E}-06$ \\
\hline $\mathrm{CH}$ & $\mathrm{C} 41 \mathrm{H} 48$ & $1.70 \mathrm{E}-05$ & Non-basic N1O2 & $\mathrm{C} 42 \mathrm{H} 61 \mathrm{~N} 1 \mathrm{O} 2$ & $2.44 \mathrm{E}-05$ & Basic N1O2 & $\mathrm{C} 17 \mathrm{H} 17 \mathrm{~N} 1 \mathrm{O} 2$ & $1.53 \mathrm{E}-08$ \\
\hline $\mathrm{CH}$ & $\mathrm{C} 42 \mathrm{H} 50$ & $1.98 \mathrm{E}-05$ & Non-basic N1O2 & $\mathrm{C} 43 \mathrm{H} 63 \mathrm{~N} 1 \mathrm{O} 2$ & $1.58 \mathrm{E}-05$ & Basic N1O2 & $\mathrm{C} 18 \mathrm{H} 19 \mathrm{~N} 1 \mathrm{O} 2$ & $9.18 \mathrm{E}-08$ \\
\hline $\mathrm{CH}$ & $\mathrm{C} 43 \mathrm{H} 52$ & 7.11E-06 & Non-basic N1O2 & $\mathrm{C} 44 \mathrm{H} 65 \mathrm{~N} 1 \mathrm{O} 2$ & $1.56 \mathrm{E}-05$ & Basic N1O2 & $\mathrm{C} 19 \mathrm{H} 21 \mathrm{~N} 1 \mathrm{O} 2$ & $2.45 \mathrm{E}-07$ \\
\hline $\mathrm{CH}$ & $\mathrm{C} 44 \mathrm{H} 54$ & $4.52 \mathrm{E}-06$ & Non-basic N1O2 & $\mathrm{C} 45 \mathrm{H} 67 \mathrm{~N} 1 \mathrm{O} 2$ & $9.60 \mathrm{E}-06$ & Basic N1O2 & $\mathrm{C} 20 \mathrm{H} 23 \mathrm{~N} 1 \mathrm{O} 2$ & $6.01 \mathrm{E}-07$ \\
\hline $\mathrm{CH}$ & $\mathrm{C} 45 \mathrm{H} 56$ & $3.04 \mathrm{E}-06$ & Non-basic N1O2 & $\mathrm{C} 19 \mathrm{H} 13 \mathrm{~N} 1 \mathrm{O} 2$ & $2.44 \mathrm{E}-06$ & Basic N1O2 & $\mathrm{C} 21 \mathrm{H} 25 \mathrm{~N} 1 \mathrm{O} 2$ & $2.99 \mathrm{E}-06$ \\
\hline $\mathrm{CH}$ & $\mathrm{C} 21 \mathrm{H} 6$ & $8.34 \mathrm{E}-06$ & Non-basic N1O2 & $\mathrm{C} 20 \mathrm{H} 15 \mathrm{~N} 1 \mathrm{O} 2$ & $9.12 \mathrm{E}-06$ & Basic N1O2 & $\mathrm{C} 22 \mathrm{H} 27 \mathrm{~N} 1 \mathrm{O} 2$ & $8.81 \mathrm{E}-06$ \\
\hline $\mathrm{CH}$ & $\mathrm{C} 22 \mathrm{H} 8$ & 5.61E-06 & Non-basic N1O2 & $\mathrm{C} 21 \mathrm{H} 17 \mathrm{~N} 1 \mathrm{O} 2$ & $2.69 \mathrm{E}-05$ & Basic N1O2 & $\mathrm{C} 23 \mathrm{H} 29 \mathrm{~N} 1 \mathrm{O} 2$ & $9.07 \mathrm{E}-06$ \\
\hline $\mathrm{CH}$ & $\mathrm{C} 23 \mathrm{H} 10$ & $7.90 \mathrm{E}-06$ & Non-basic N1O2 & $\mathrm{C} 22 \mathrm{H} 19 \mathrm{~N} 1 \mathrm{O} 2$ & $3.73 \mathrm{E}-05$ & Basic N1O2 & $\mathrm{C} 24 \mathrm{H} 31 \mathrm{~N} 1 \mathrm{O} 2$ & $3.94 \mathrm{E}-06$ \\
\hline $\mathrm{CH}$ & $\mathrm{C} 24 \mathrm{H} 12$ & $9.52 \mathrm{E}-05$ & Non-basic N1O2 & $\mathrm{C} 23 \mathrm{H} 21 \mathrm{~N} 1 \mathrm{O} 2$ & $4.62 \mathrm{E}-05$ & Basic N1O2 & $\mathrm{C} 25 \mathrm{H} 33 \mathrm{~N} 1 \mathrm{O} 2$ & 4.41E-06 \\
\hline $\mathrm{CH}$ & $\mathrm{C} 25 \mathrm{H} 14$ & 0.00039 & Non-basic N1O2 & $\mathrm{C} 24 \mathrm{H} 23 \mathrm{~N} 1 \mathrm{O} 2$ & $3.95 \mathrm{E}-05$ & Basic N1O2 & $\mathrm{C} 26 \mathrm{H} 35 \mathrm{~N} 1 \mathrm{O} 2$ & $3.68 \mathrm{E}-06$ \\
\hline $\mathrm{CH}$ & $\mathrm{C} 26 \mathrm{H} 16$ & 0.001075 & Non-basic N1O2 & $\mathrm{C} 25 \mathrm{H} 25 \mathrm{~N} 1 \mathrm{O} 2$ & $3.00 \mathrm{E}-05$ & Basic N1O2 & $\mathrm{C} 27 \mathrm{H} 37 \mathrm{~N} 1 \mathrm{O} 2$ & $3.08 \mathrm{E}-06$ \\
\hline $\mathrm{CH}$ & $\mathrm{C} 27 \mathrm{H} 18$ & 0.002129 & Non-basic N1O2 & $\mathrm{C} 26 \mathrm{H} 27 \mathrm{~N} 1 \mathrm{O} 2$ & $2.97 \mathrm{E}-05$ & Basic N1O2 & $\mathrm{C} 28 \mathrm{H} 39 \mathrm{~N} 1 \mathrm{O} 2$ & $3.57 \mathrm{E}-06$ \\
\hline $\mathrm{CH}$ & $\mathrm{C} 28 \mathrm{H} 20$ & 0.00274 & Non-basic N1O2 & $\mathrm{C} 27 \mathrm{H} 29 \mathrm{~N} 1 \mathrm{O} 2$ & $2.63 \mathrm{E}-05$ & Basic N1O2 & $\mathrm{C} 29 \mathrm{H} 41 \mathrm{~N} 1 \mathrm{O} 2$ & 4.07E-06 \\
\hline $\mathrm{CH}$ & $\mathrm{C} 29 \mathrm{H} 22$ & 0.002463 & Non-basic N1O2 & $\mathrm{C} 28 \mathrm{H} 31 \mathrm{~N} 1 \mathrm{O} 2$ & $2.18 \mathrm{E}-05$ & Basic N1O2 & $\mathrm{C} 30 \mathrm{H} 43 \mathrm{~N} 1 \mathrm{O} 2$ & $5.68 \mathrm{E}-06$ \\
\hline $\mathrm{CH}$ & $\mathrm{C} 30 \mathrm{H} 24$ & 0.001873 & Non-basic N1O2 & $\mathrm{C} 29 \mathrm{H} 33 \mathrm{~N} 1 \mathrm{O} 2$ & $1.76 \mathrm{E}-05$ & Basic N1O2 & $\mathrm{C} 31 \mathrm{H} 45 \mathrm{~N} 1 \mathrm{O} 2$ & $3.82 \mathrm{E}-06$ \\
\hline $\mathrm{CH}$ & $\mathrm{C} 31 \mathrm{H} 26$ & 0.001203 & Non-basic N1O2 & $\mathrm{C} 30 \mathrm{H} 35 \mathrm{~N} 1 \mathrm{O} 2$ & $1.90 \mathrm{E}-05$ & Basic N1O2 & $\mathrm{C} 32 \mathrm{H} 47 \mathrm{~N} 1 \mathrm{O} 2$ & $3.62 \mathrm{E}-06$ \\
\hline $\mathrm{CH}$ & $\mathrm{C} 32 \mathrm{H} 28$ & 0.000768 & Non-basic N1O2 & $\mathrm{C} 31 \mathrm{H} 37 \mathrm{~N} 1 \mathrm{O} 2$ & $1.98 \mathrm{E}-05$ & Basic N1O2 & $\mathrm{C} 33 \mathrm{H} 49 \mathrm{~N} 1 \mathrm{O} 2$ & $3.38 \mathrm{E}-06$ \\
\hline $\mathrm{CH}$ & $\mathrm{C} 33 \mathrm{H} 30$ & 0.000491 & Non-basic N1O2 & $\mathrm{C} 32 \mathrm{H} 39 \mathrm{~N} 1 \mathrm{O} 2$ & $1.19 \mathrm{E}-05$ & Basic N1O2 & $\mathrm{C} 34 \mathrm{H} 51 \mathrm{~N} 1 \mathrm{O} 2$ & $3.14 \mathrm{E}-06$ \\
\hline $\mathrm{CH}$ & $\mathrm{C} 34 \mathrm{H} 32$ & 0.000281 & Non-basic N1O2 & $\mathrm{C} 33 \mathrm{H} 41 \mathrm{~N} 1 \mathrm{O} 2$ & $1.30 \mathrm{E}-05$ & Basic N1O2 & C35H53N1O2 & $2.42 \mathrm{E}-06$ \\
\hline $\mathrm{CH}$ & $\mathrm{C} 35 \mathrm{H} 34$ & 0.000214 & Non-basic N1O2 & $\mathrm{C} 34 \mathrm{H} 43 \mathrm{~N} 1 \mathrm{O} 2$ & $9.26 \mathrm{E}-06$ & Basic N1O2 & $\mathrm{C} 36 \mathrm{H} 55 \mathrm{~N} 1 \mathrm{O} 2$ & $2.73 \mathrm{E}-06$ \\
\hline $\mathrm{CH}$ & $\mathrm{C} 36 \mathrm{H} 36$ & 0.0001 & Non-basic N1O2 & $\mathrm{C} 35 \mathrm{H} 45 \mathrm{~N} 1 \mathrm{O} 2$ & $3.69 \mathrm{E}-06$ & Basic N1O2 & $\mathrm{C} 37 \mathrm{H} 57 \mathrm{~N} 1 \mathrm{O} 2$ & $1.53 \mathrm{E}-06$ \\
\hline $\mathrm{CH}$ & C37H38 & $6.05 \mathrm{E}-05$ & Non-basic N1O2 & $\mathrm{C} 36 \mathrm{H} 47 \mathrm{~N} 1 \mathrm{O} 2$ & 7.17E-06 & Basic N1O2 & $\mathrm{C} 38 \mathrm{H} 59 \mathrm{~N} 1 \mathrm{O} 2$ & $1.49 \mathrm{E}-06$ \\
\hline $\mathrm{CH}$ & $\mathrm{C} 38 \mathrm{H} 40$ & $5.75 \mathrm{E}-05$ & Non-basic N1O2 & $\mathrm{C} 37 \mathrm{H} 49 \mathrm{~N} 1 \mathrm{O} 2$ & $1.67 \mathrm{E}-06$ & Basic N1O2 & $\mathrm{C} 39 \mathrm{H} 61 \mathrm{~N} 1 \mathrm{O} 2$ & $1.08 \mathrm{E}-06$ \\
\hline $\mathrm{CH}$ & C39H42 & $2.31 \mathrm{E}-05$ & Non-basic N1O2 & $\mathrm{C} 38 \mathrm{H} 51 \mathrm{~N} 1 \mathrm{O} 2$ & $1.23 \mathrm{E}-05$ & Basic N1O2 & $\mathrm{C} 40 \mathrm{H} 63 \mathrm{~N} 1 \mathrm{O} 2$ & $6.23 \mathrm{E}-07$ \\
\hline $\mathrm{CH}$ & $\mathrm{C} 40 \mathrm{H} 44$ & $2.66 \mathrm{E}-05$ & Non-basic N1O2 & $\mathrm{C} 21 \mathrm{H} 15 \mathrm{~N} 1 \mathrm{O} 2$ & $8.40 \mathrm{E}-07$ & Basic $\mathrm{N} 1 \mathrm{O} 2$ & $\mathrm{C} 41 \mathrm{H} 65 \mathrm{~N} 1 \mathrm{O} 2$ & $5.19 \mathrm{E}-07$ \\
\hline $\mathrm{CH}$ & $\mathrm{C} 41 \mathrm{H} 46$ & $6.35 \mathrm{E}-06$ & Non-basic N1O2 & $\mathrm{C} 22 \mathrm{H} 17 \mathrm{~N} 1 \mathrm{O} 2$ & $8.39 \mathrm{E}-06$ & Basic N1O2 & $\mathrm{C} 42 \mathrm{H} 67 \mathrm{~N} 1 \mathrm{O} 2$ & $5.15 \mathrm{E}-07$ \\
\hline
\end{tabular}




\begin{tabular}{|c|c|c|c|c|c|c|c|c|}
\hline Class & formula & Mass & Class & formula & Mass & Class & formula & Mass \\
\hline $\mathrm{CH}$ & $\mathrm{C} 42 \mathrm{H} 48$ & $1.55 \mathrm{E}-05$ & Non-basic N1O2 & $\mathrm{C} 23 \mathrm{H} 19 \mathrm{~N} 1 \mathrm{O} 2$ & $1.43 \mathrm{E}-05$ & Basic N1O2 & $\mathrm{C} 19 \mathrm{H} 19 \mathrm{~N} 1 \mathrm{O} 2$ & $9.33 \mathrm{E}-08$ \\
\hline $\mathrm{CH}$ & $\mathrm{C} 43 \mathrm{H} 50$ & $4.31 \mathrm{E}-06$ & Non-basic N1O2 & $\mathrm{C} 24 \mathrm{H} 21 \mathrm{~N} 1 \mathrm{O} 2$ & $1.86 \mathrm{E}-05$ & Basic N1O2 & $\mathrm{C} 20 \mathrm{H} 21 \mathrm{~N} 1 \mathrm{O} 2$ & $1.51 \mathrm{E}-07$ \\
\hline $\mathrm{CH}$ & $\mathrm{C} 44 \mathrm{H} 52$ & 4.03E-06 & Non-basic N1O2 & $\mathrm{C} 25 \mathrm{H} 23 \mathrm{~N} 1 \mathrm{O} 2$ & $2.13 \mathrm{E}-05$ & Basic N1O2 & $\mathrm{C} 21 \mathrm{H} 23 \mathrm{~N} 1 \mathrm{O} 2$ & $1.39 \mathrm{E}-06$ \\
\hline $\mathrm{CH}$ & $\mathrm{C} 23 \mathrm{H} 8$ & $3.66 \mathrm{E}-06$ & Non-basic N1O2 & $\mathrm{C} 26 \mathrm{H} 25 \mathrm{~N} 1 \mathrm{O} 2$ & $1.44 \mathrm{E}-05$ & Basic N1O2 & $\mathrm{C} 22 \mathrm{H} 25 \mathrm{~N} 1 \mathrm{O} 2$ & $2.90 \mathrm{E}-06$ \\
\hline $\mathrm{CH}$ & $\mathrm{C} 24 \mathrm{H} 10$ & $2.34 \mathrm{E}-05$ & Non-basic N1O2 & $\mathrm{C} 27 \mathrm{H} 27 \mathrm{~N} 1 \mathrm{O} 2$ & $1.34 \mathrm{E}-05$ & Basic N1O2 & $\mathrm{C} 23 \mathrm{H} 27 \mathrm{~N} 1 \mathrm{O} 2$ & $3.49 \mathrm{E}-06$ \\
\hline $\mathrm{CH}$ & $\mathrm{C} 25 \mathrm{H} 12$ & $1.03 \mathrm{E}-05$ & Non-basic N1O2 & $\mathrm{C} 28 \mathrm{H} 29 \mathrm{~N} 1 \mathrm{O} 2$ & $1.19 \mathrm{E}-05$ & Basic N1O2 & $\mathrm{C} 24 \mathrm{H} 29 \mathrm{~N} 1 \mathrm{O} 2$ & $6.83 \mathrm{E}-06$ \\
\hline $\mathrm{CH}$ & $\mathrm{C} 26 \mathrm{H} 14$ & 0.000608 & Non-basic N1O2 & $\mathrm{C} 29 \mathrm{H} 31 \mathrm{~N} 1 \mathrm{O} 2$ & $1.23 \mathrm{E}-05$ & Basic N1O2 & $\mathrm{C} 25 \mathrm{H} 31 \mathrm{~N} 1 \mathrm{O} 2$ & $1.43 \mathrm{E}-05$ \\
\hline $\mathrm{CH}$ & $\mathrm{C} 27 \mathrm{H} 16$ & 0.001875 & Non-basic N1O2 & $\mathrm{C} 30 \mathrm{H} 33 \mathrm{~N} 1 \mathrm{O} 2$ & $1.14 \mathrm{E}-05$ & Basic N1O2 & $\mathrm{C} 26 \mathrm{H} 33 \mathrm{~N} 1 \mathrm{O} 2$ & $1.65 \mathrm{E}-05$ \\
\hline $\mathrm{CH}$ & $\mathrm{C} 28 \mathrm{H} 18$ & 0.002328 & Non-basic N1O2 & $\mathrm{C} 31 \mathrm{H} 35 \mathrm{~N} 1 \mathrm{O} 2$ & $6.25 \mathrm{E}-06$ & Basic N1O2 & $\mathrm{C} 27 \mathrm{H} 35 \mathrm{~N} 1 \mathrm{O} 2$ & $1.24 \mathrm{E}-05$ \\
\hline $\mathrm{CH}$ & $\mathrm{C} 29 \mathrm{H} 20$ & 0.00187 & Non-basic N1O2 & $\mathrm{C} 32 \mathrm{H} 37 \mathrm{~N} 1 \mathrm{O} 2$ & $1.11 \mathrm{E}-05$ & Basic N1O2 & $\mathrm{C} 28 \mathrm{H} 37 \mathrm{~N} 1 \mathrm{O} 2$ & $7.65 \mathrm{E}-06$ \\
\hline $\mathrm{CH}$ & $\mathrm{C} 30 \mathrm{H} 22$ & 0.001231 & Non-basic N1O2 & $\mathrm{C} 33 \mathrm{H} 39 \mathrm{~N} 1 \mathrm{O} 2$ & $2.18 \mathrm{E}-06$ & Basic N1O2 & $\mathrm{C} 29 \mathrm{H} 39 \mathrm{~N} 1 \mathrm{O} 2$ & $8.95 \mathrm{E}-06$ \\
\hline $\mathrm{CH}$ & $\mathrm{C} 31 \mathrm{H} 24$ & 0.000783 & Non-basic N1O2 & $\mathrm{C} 22 \mathrm{H} 15 \mathrm{~N} 1 \mathrm{O} 2$ & $3.97 \mathrm{E}-06$ & Basic N1O2 & $\mathrm{C} 30 \mathrm{H} 41 \mathrm{~N} 1 \mathrm{O} 2$ & $1.12 \mathrm{E}-05$ \\
\hline $\mathrm{CH}$ & $\mathrm{C} 32 \mathrm{H} 26$ & 0.000488 & Non-basic N1O2 & $\mathrm{C} 23 \mathrm{H} 17 \mathrm{~N} 1 \mathrm{O} 2$ & $7.22 \mathrm{E}-06$ & Basic N1O2 & $\mathrm{C} 31 \mathrm{H} 43 \mathrm{~N} 1 \mathrm{O} 2$ & $5.74 \mathrm{E}-06$ \\
\hline $\mathrm{CH}$ & $\mathrm{C} 33 \mathrm{H} 28$ & 0.000395 & Non-basic N1O2 & $\mathrm{C} 24 \mathrm{H} 19 \mathrm{~N} 1 \mathrm{O} 2$ & $9.27 \mathrm{E}-06$ & Basic N1O2 & $\mathrm{C} 32 \mathrm{H} 45 \mathrm{~N} 1 \mathrm{O} 2$ & 4.61E-06 \\
\hline $\mathrm{CH}$ & $\mathrm{C} 34 \mathrm{H} 30$ & 0.00027 & Non-basic N1O2 & $\mathrm{C} 25 \mathrm{H} 21 \mathrm{~N} 1 \mathrm{O} 2$ & $1.30 \mathrm{E}-05$ & Basic N1O2 & $\mathrm{C} 33 \mathrm{H} 47 \mathrm{~N} 1 \mathrm{O} 2$ & $3.45 \mathrm{E}-06$ \\
\hline $\mathrm{CH}$ & $\mathrm{C} 35 \mathrm{H} 32$ & 0.000185 & Non-basic N1O2 & $\mathrm{C} 26 \mathrm{H} 23 \mathrm{~N} 1 \mathrm{O} 2$ & $1.34 \mathrm{E}-05$ & Basic N1O2 & $\mathrm{C} 34 \mathrm{H} 49 \mathrm{~N} 1 \mathrm{O} 2$ & $3.16 \mathrm{E}-06$ \\
\hline $\mathrm{CH}$ & $\mathrm{C} 36 \mathrm{H} 34$ & $8.52 \mathrm{E}-05$ & Non-basic N1O2 & $\mathrm{C} 27 \mathrm{H} 25 \mathrm{~N} 1 \mathrm{O} 2$ & $1.65 \mathrm{E}-05$ & Basic N1O2 & $\mathrm{C} 35 \mathrm{H} 51 \mathrm{~N} 1 \mathrm{O} 2$ & $2.78 \mathrm{E}-06$ \\
\hline $\mathrm{CH}$ & $\mathrm{C} 37 \mathrm{H} 36$ & $3.41 \mathrm{E}-05$ & Non-basic N1O2 & $\mathrm{C} 28 \mathrm{H} 27 \mathrm{~N} 1 \mathrm{O} 2$ & $6.12 \mathrm{E}-05$ & Basic N1O2 & $\mathrm{C} 36 \mathrm{H} 53 \mathrm{~N} 1 \mathrm{O} 2$ & $2.10 \mathrm{E}-06$ \\
\hline $\mathrm{CH}$ & $\mathrm{C} 38 \mathrm{H} 38$ & $1.32 \mathrm{E}-05$ & Non-basic N1O2 & $\mathrm{C} 29 \mathrm{H} 29 \mathrm{~N} 1 \mathrm{O} 2$ & $3.30 \mathrm{E}-06$ & Basic N1O2 & $\mathrm{C} 37 \mathrm{H} 55 \mathrm{~N} 1 \mathrm{O} 2$ & $2.00 \mathrm{E}-06$ \\
\hline $\mathrm{CH}$ & $\mathrm{C} 39 \mathrm{H} 40$ & $1.40 \mathrm{E}-05$ & Non-basic N1O2 & $\mathrm{C} 30 \mathrm{H} 31 \mathrm{~N} 1 \mathrm{O} 2$ & 4.94E-05 & Basic N1O2 & $\mathrm{C} 38 \mathrm{H} 57 \mathrm{~N} 1 \mathrm{O} 2$ & $1.34 \mathrm{E}-06$ \\
\hline $\mathrm{CH}$ & $\mathrm{C} 40 \mathrm{H} 42$ & $1.63 \mathrm{E}-05$ & Non-basic N1O2 & $\mathrm{C} 31 \mathrm{H} 33 \mathrm{~N} 1 \mathrm{O} 2$ & $3.55 \mathrm{E}-06$ & Basic N1O2 & $\mathrm{C} 39 \mathrm{H} 59 \mathrm{~N} 1 \mathrm{O} 2$ & $1.11 \mathrm{E}-06$ \\
\hline $\mathrm{CH}$ & $\mathrm{C} 41 \mathrm{H} 44$ & $5.77 \mathrm{E}-06$ & Non-basic N1O2 & $\mathrm{C} 32 \mathrm{H} 35 \mathrm{~N} 1 \mathrm{O} 2$ & 7.62E-06 & Basic N1O2 & $\mathrm{C} 40 \mathrm{H} 61 \mathrm{~N} 1 \mathrm{O} 2$ & 7.74E-07 \\
\hline $\mathrm{CH}$ & $\mathrm{C} 42 \mathrm{H} 46$ & $5.32 \mathrm{E}-06$ & Non-basic N1O2 & $\mathrm{C} 33 \mathrm{H} 37 \mathrm{~N} 1 \mathrm{O} 2$ & $2.53 \mathrm{E}-06$ & Basic N1O2 & $\mathrm{C} 18 \mathrm{H} 15 \mathrm{~N} 1 \mathrm{O} 2$ & $7.28 \mathrm{E}-09$ \\
\hline $\mathrm{CH}$ & $\mathrm{C} 43 \mathrm{H} 48$ & $3.78 \mathrm{E}-06$ & Non-basic N1O2 & $\mathrm{C} 35 \mathrm{H} 41 \mathrm{~N} 1 \mathrm{O} 2$ & $3.07 \mathrm{E}-06$ & Basic N1O2 & C19H17N1O2 & $1.90 \mathrm{E}-08$ \\
\hline $\mathrm{CH}$ & $\mathrm{C} 27 \mathrm{H} 14$ & $1.81 \mathrm{E}-05$ & Non-basic N1O2 & $\mathrm{C} 36 \mathrm{H} 43 \mathrm{~N} 1 \mathrm{O} 2$ & $2.65 \mathrm{E}-06$ & Basic N1O2 & $\mathrm{C} 20 \mathrm{H} 19 \mathrm{~N} 1 \mathrm{O} 2$ & $4.80 \mathrm{E}-07$ \\
\hline $\mathrm{CH}$ & $\mathrm{C} 28 \mathrm{H} 16$ & 0.000138 & Non-basic N1O2 & $\mathrm{C} 17 \mathrm{H} 3 \mathrm{~N} 1 \mathrm{O} 2$ & $6.92 \mathrm{E}-06$ & Basic N1O2 & $\mathrm{C} 21 \mathrm{H} 21 \mathrm{~N} 1 \mathrm{O} 2$ & $5.34 \mathrm{E}-06$ \\
\hline $\mathrm{CH}$ & $\mathrm{C} 29 \mathrm{H} 18$ & 0.000382 & Non-basic N1O2 & $\mathrm{C} 18 \mathrm{H} 5 \mathrm{~N} 1 \mathrm{O} 2$ & $6.41 \mathrm{E}-06$ & Basic N1O2 & $\mathrm{C} 22 \mathrm{H} 23 \mathrm{~N} 1 \mathrm{O} 2$ & $9.35 \mathrm{E}-06$ \\
\hline $\mathrm{CH}$ & $\mathrm{C} 30 \mathrm{H} 20$ & 0.000506 & Non-basic N1O2 & $\mathrm{C} 19 \mathrm{H} 7 \mathrm{~N} 1 \mathrm{O} 2$ & $1.47 \mathrm{E}-05$ & Basic N1O2 & $\mathrm{C} 23 \mathrm{H} 25 \mathrm{~N} 1 \mathrm{O} 2$ & 7.39E-06 \\
\hline $\mathrm{CH}$ & $\mathrm{C} 31 \mathrm{H} 22$ & 0.000442 & Non-basic N1O2 & $\mathrm{C} 20 \mathrm{H} 9 \mathrm{~N} 1 \mathrm{O} 2$ & $6.26 \mathrm{E}-05$ & Basic N1O2 & $\mathrm{C} 24 \mathrm{H} 27 \mathrm{~N} 1 \mathrm{O} 2$ & $6.38 \mathrm{E}-06$ \\
\hline $\mathrm{CH}$ & $\mathrm{C} 32 \mathrm{H} 24$ & 0.000347 & Non-basic N1O2 & $\mathrm{C} 21 \mathrm{H} 11 \mathrm{~N} 1 \mathrm{O} 2$ & 7.71E-06 & Basic N1O2 & $\mathrm{C} 25 \mathrm{H} 29 \mathrm{~N} 1 \mathrm{O} 2$ & $7.66 \mathrm{E}-06$ \\
\hline $\mathrm{CH}$ & $\mathrm{C} 33 \mathrm{H} 26$ & 0.000235 & Non-basic N1O2 & $\mathrm{C} 22 \mathrm{H} 13 \mathrm{~N} 1 \mathrm{O} 2$ & $9.94 \mathrm{E}-06$ & Basic N1O2 & $\mathrm{C} 26 \mathrm{H} 31 \mathrm{~N} 1 \mathrm{O} 2$ & 7.98E-06 \\
\hline $\mathrm{CH}$ & $\mathrm{C} 34 \mathrm{H} 28$ & 0.000145 & Non-basic N1O2 & $\mathrm{C} 23 \mathrm{H} 15 \mathrm{~N} 1 \mathrm{O} 2$ & $2.65 \mathrm{E}-06$ & Basic N1O2 & $\mathrm{C} 27 \mathrm{H} 33 \mathrm{~N} 1 \mathrm{O} 2$ & $8.30 \mathrm{E}-06$ \\
\hline $\mathrm{CH}$ & $\mathrm{C} 35 \mathrm{H} 30$ & 0.000146 & Non-basic N1O2 & $\mathrm{C} 25 \mathrm{H} 19 \mathrm{~N} 1 \mathrm{O} 2$ & $1.81 \mathrm{E}-06$ & Basic N1O2 & $\mathrm{C} 28 \mathrm{H} 35 \mathrm{~N} 1 \mathrm{O} 2$ & $8.75 \mathrm{E}-06$ \\
\hline $\mathrm{CH}$ & $\mathrm{C} 36 \mathrm{H} 32$ & $6.88 \mathrm{E}-05$ & Non-basic N1O2 & $\mathrm{C} 26 \mathrm{H} 21 \mathrm{~N} 1 \mathrm{O} 2$ & $2.21 \mathrm{E}-06$ & Basic N1O2 & $\mathrm{C} 29 \mathrm{H} 37 \mathrm{~N} 1 \mathrm{O} 2$ & $1.15 \mathrm{E}-05$ \\
\hline $\mathrm{CH}$ & C37H34 & $4.83 \mathrm{E}-05$ & Non-basic N1O2 & $\mathrm{C} 27 \mathrm{H} 23 \mathrm{~N} 1 \mathrm{O} 2$ & $2.08 \mathrm{E}-06$ & Basic $\mathrm{N} 1 \mathrm{O} 2$ & $\mathrm{C} 30 \mathrm{H} 39 \mathrm{~N} 1 \mathrm{O} 2$ & $1.17 \mathrm{E}-05$ \\
\hline $\mathrm{CH}$ & C38H36 & $2.10 \mathrm{E}-05$ & Non-basic N1O2 & $\mathrm{C} 28 \mathrm{H} 25 \mathrm{~N} 1 \mathrm{O} 2$ & $3.69 \mathrm{E}-06$ & Basic $\mathrm{N} 1 \mathrm{O} 2$ & $\mathrm{C} 31 \mathrm{H} 41 \mathrm{~N} 1 \mathrm{O} 2$ & $6.20 \mathrm{E}-06$ \\
\hline $\mathrm{CH}$ & C39H38 & $2.86 \mathrm{E}-06$ & Non-basic N1O2 & $\mathrm{C} 29 \mathrm{H} 27 \mathrm{~N} 1 \mathrm{O} 2$ & $7.41 \mathrm{E}-06$ & Basic $\mathrm{N} 1 \mathrm{O} 2$ & $\mathrm{C} 32 \mathrm{H} 43 \mathrm{~N} 1 \mathrm{O} 2$ & $5.35 \mathrm{E}-06$ \\
\hline $\mathrm{CH}$ & $\mathrm{C} 40 \mathrm{H} 40$ & $1.07 \mathrm{E}-05$ & Non-basic N1O2 & $\mathrm{C} 30 \mathrm{H} 29 \mathrm{~N} 1 \mathrm{O} 2$ & $4.50 \mathrm{E}-06$ & Basic $\mathrm{N}_{10} \mathrm{O}$ & $\mathrm{C} 33 \mathrm{H} 45 \mathrm{~N} 1 \mathrm{O} 2$ & $3.98 \mathrm{E}-06$ \\
\hline
\end{tabular}




\begin{tabular}{|c|c|c|c|c|c|c|c|c|}
\hline Class & formula & Mass & Class & formula & Mass & Class & formula & Mass \\
\hline $\mathrm{CH}$ & $\mathrm{C} 25 \mathrm{H} 8$ & $5.57 \mathrm{E}-06$ & Non-basic N1O2 & $\mathrm{C} 31 \mathrm{H} 31 \mathrm{~N} 1 \mathrm{O} 2$ & $1.57 \mathrm{E}-06$ & Basic N1O2 & $\mathrm{C} 34 \mathrm{H} 47 \mathrm{~N} 1 \mathrm{O} 2$ & $3.39 \mathrm{E}-06$ \\
\hline $\mathrm{CH}$ & $\mathrm{C} 26 \mathrm{H} 10$ & $5.56 \mathrm{E}-06$ & Non-basic $\mathrm{N} 1 \mathrm{O} 2$ & $\mathrm{C} 32 \mathrm{H} 33 \mathrm{~N} 1 \mathrm{O} 2$ & $3.63 \mathrm{E}-06$ & Basic N1O2 & $\mathrm{C} 35 \mathrm{H} 49 \mathrm{~N} 1 \mathrm{O} 2$ & $2.54 \mathrm{E}-06$ \\
\hline $\mathrm{CH}$ & $\mathrm{C} 27 \mathrm{H} 12$ & $3.93 \mathrm{E}-06$ & Non-basic $\mathrm{N} 1 \mathrm{O} 2$ & $\mathrm{C} 18 \mathrm{H} 3 \mathrm{~N} 1 \mathrm{O} 2$ & $9.83 \mathrm{E}-07$ & Basic N1O2 & $\mathrm{C} 36 \mathrm{H} 51 \mathrm{~N} 1 \mathrm{O} 2$ & $2.59 \mathrm{E}-06$ \\
\hline $\mathrm{CH}$ & $\mathrm{C} 28 \mathrm{H} 14$ & $3.80 \mathrm{E}-05$ & Non-basic $\mathrm{N} 1 \mathrm{O} 2$ & C19H5N1O2 & $9.15 \mathrm{E}-07$ & Basic N1O2 & C37H53N1O2 & $2.33 \mathrm{E}-06$ \\
\hline $\mathrm{CH}$ & $\mathrm{C} 29 \mathrm{H} 16$ & $6.74 \mathrm{E}-05$ & Non-basic $\mathrm{N} 1 \mathrm{O} 2$ & C20H7N1O2 & $4.00 \mathrm{E}-06$ & Basic N1O2 & $\mathrm{C} 38 \mathrm{H} 55 \mathrm{~N} 1 \mathrm{O} 2$ & $1.52 \mathrm{E}-06$ \\
\hline $\mathrm{CH}$ & $\mathrm{C} 30 \mathrm{H} 18$ & $7.71 \mathrm{E}-05$ & Non-basic N1O2 & C21H9N1O2 & 4.04E-06 & Basic N1O2 & $\mathrm{C} 39 \mathrm{H} 57 \mathrm{~N} 1 \mathrm{O} 2$ & $1.16 \mathrm{E}-06$ \\
\hline $\mathrm{CH}$ & $\mathrm{C} 31 \mathrm{H} 20$ & $8.44 \mathrm{E}-05$ & Non-basic $\mathrm{N} 1 \mathrm{O} 2$ & $\mathrm{C} 22 \mathrm{H} 11 \mathrm{~N} 1 \mathrm{O} 2$ & $1.38 \mathrm{E}-06$ & Basic N1O2 & $\mathrm{C} 40 \mathrm{H} 59 \mathrm{~N} 1 \mathrm{O} 2$ & 8.44E-07 \\
\hline $\mathrm{CH}$ & $\mathrm{C} 32 \mathrm{H} 22$ & $9.48 \mathrm{E}-05$ & Non-basic $\mathrm{N} 1 \mathrm{O} 2$ & $\mathrm{C} 24 \mathrm{H} 15 \mathrm{~N} 1 \mathrm{O} 2$ & $1.23 \mathrm{E}-06$ & Basic N1O2 & $\mathrm{C} 41 \mathrm{H} 61 \mathrm{~N} 1 \mathrm{O} 2$ & 4.41E-07 \\
\hline $\mathrm{CH}$ & $\mathrm{C} 33 \mathrm{H} 24$ & $6.87 \mathrm{E}-05$ & Non-basic N1O2 & $\mathrm{C} 25 \mathrm{H} 17 \mathrm{~N} 1 \mathrm{O} 2$ & $1.29 \mathrm{E}-06$ & Basic N1O2 & $\mathrm{C} 42 \mathrm{H} 63 \mathrm{~N} 1 \mathrm{O} 2$ & $4.95 \mathrm{E}-07$ \\
\hline $\mathrm{CH}$ & $\mathrm{C} 34 \mathrm{H} 26$ & $5.78 \mathrm{E}-05$ & Non-basic N1S1 & C15H19N1S1 & $3.61 \mathrm{E}-07$ & Basic N1O2 & $\mathrm{C} 43 \mathrm{H} 65 \mathrm{~N} 1 \mathrm{O} 2$ & $4.48 \mathrm{E}-07$ \\
\hline $\mathrm{CH}$ & $\mathrm{C} 35 \mathrm{H} 28$ & $4.08 \mathrm{E}-05$ & Non-basic N1S1 & $\mathrm{C} 16 \mathrm{H} 21 \mathrm{~N} 1 \mathrm{~S} 1$ & $1.87 \mathrm{E}-06$ & Basic N1O2 & $\mathrm{C} 44 \mathrm{H} 67 \mathrm{~N} 1 \mathrm{O} 2$ & $1.88 \mathrm{E}-07$ \\
\hline $\mathrm{CH}$ & $\mathrm{C} 36 \mathrm{H} 30$ & $1.82 \mathrm{E}-05$ & Non-basic N1S1 & C14H15N1S1 & $8.32 \mathrm{E}-06$ & Basic N1O2 & $\mathrm{C} 45 \mathrm{H} 69 \mathrm{~N} 1 \mathrm{O} 2$ & $9.54 \mathrm{E}-08$ \\
\hline $\mathrm{CH}$ & $\mathrm{C} 37 \mathrm{H} 32$ & $8.76 \mathrm{E}-06$ & Non-basic N1S1 & C15H17N1S1 & $3.26 \mathrm{E}-05$ & Basic N1O2 & $\mathrm{C} 18 \mathrm{H} 13 \mathrm{~N} 1 \mathrm{O} 2$ & $1.65 \mathrm{E}-08$ \\
\hline $\mathrm{CH}$ & $\mathrm{C} 28 \mathrm{H} 12$ & $6.37 \mathrm{E}-06$ & Non-basic N1S1 & C16H19N1S1 & $1.92 \mathrm{E}-05$ & Basic N1O2 & $\mathrm{C} 19 \mathrm{H} 15 \mathrm{~N} 1 \mathrm{O} 2$ & $8.01 \mathrm{E}-09$ \\
\hline $\mathrm{CH}$ & $\mathrm{C} 29 \mathrm{H} 14$ & $2.27 \mathrm{E}-06$ & Non-basic N1S1 & $\mathrm{C} 17 \mathrm{H} 21 \mathrm{~N} 1 \mathrm{~S} 1$ & $2.20 \mathrm{E}-06$ & Basic N1O2 & $\mathrm{C} 20 \mathrm{H} 17 \mathrm{~N} 1 \mathrm{O} 2$ & $2.80 \mathrm{E}-08$ \\
\hline $\mathrm{CH}$ & $\mathrm{C} 30 \mathrm{H} 16$ & 4.46E-06 & Non-basic N1S1 & C18H23N1S1 & $9.60 \mathrm{E}-06$ & Basic N1O2 & $\mathrm{C} 21 \mathrm{H} 19 \mathrm{~N} 1 \mathrm{O} 2$ & $1.79 \mathrm{E}-07$ \\
\hline $\mathrm{CH}$ & $\mathrm{C} 31 \mathrm{H} 18$ & $9.56 \mathrm{E}-06$ & Non-basic N1S1 & C19H25N1S1 & $1.71 \mathrm{E}-06$ & Basic N1O2 & $\mathrm{C} 22 \mathrm{H} 21 \mathrm{~N} 1 \mathrm{O} 2$ & $6.19 \mathrm{E}-07$ \\
\hline $\mathrm{CH}$ & $\mathrm{C} 32 \mathrm{H} 20$ & $1.76 \mathrm{E}-05$ & Non-basic N1S1 & $\mathrm{C} 20 \mathrm{H} 27 \mathrm{~N} 1 \mathrm{~S} 1$ & $3.44 \mathrm{E}-05$ & Basic N1O2 & $\mathrm{C} 23 \mathrm{H} 23 \mathrm{~N} 1 \mathrm{O} 2$ & $3.36 \mathrm{E}-06$ \\
\hline $\mathrm{CH}$ & $\mathrm{C} 33 \mathrm{H} 22$ & $1.82 \mathrm{E}-05$ & Non-basic N1S1 & $\mathrm{C} 21 \mathrm{H} 29 \mathrm{~N} 1 \mathrm{~S} 1$ & $2.65 \mathrm{E}-05$ & Basic N1O2 & $\mathrm{C} 24 \mathrm{H} 25 \mathrm{~N} 1 \mathrm{O} 2$ & $6.42 \mathrm{E}-06$ \\
\hline $\mathrm{CH}$ & $\mathrm{C} 34 \mathrm{H} 24$ & $6.07 \mathrm{E}-06$ & Non-basic N1S1 & $\mathrm{C} 22 \mathrm{H} 31 \mathrm{~N} 1 \mathrm{~S} 1$ & 0.000422 & Basic N1O2 & $\mathrm{C} 25 \mathrm{H} 27 \mathrm{~N} 1 \mathrm{O} 2$ & $8.95 \mathrm{E}-06$ \\
\hline $\mathrm{CH}$ & $\mathrm{C} 35 \mathrm{H} 26$ & $2.23 \mathrm{E}-06$ & Non-basic N1S1 & C23H33N1S1 & $1.79 \mathrm{E}-05$ & Basic N1O2 & $\mathrm{C} 26 \mathrm{H} 29 \mathrm{~N} 1 \mathrm{O} 2$ & $1.01 \mathrm{E}-05$ \\
\hline $\mathrm{CH}$ & $\mathrm{C} 36 \mathrm{H} 28$ & $5.03 \mathrm{E}-06$ & Non-basic N1S1 & $\mathrm{C} 24 \mathrm{H} 35 \mathrm{~N} 1 \mathrm{~S} 1$ & 0.000321 & Basic N1O2 & $\mathrm{C} 27 \mathrm{H} 31 \mathrm{~N} 1 \mathrm{O} 2$ & $9.36 \mathrm{E}-06$ \\
\hline $\mathrm{CH}$ & $\mathrm{C} 37 \mathrm{H} 30$ & $1.17 \mathrm{E}-05$ & Non-basic N1S1 & $\mathrm{C} 25 \mathrm{H} 37 \mathrm{~N} 1 \mathrm{~S} 1$ & $8.95 \mathrm{E}-07$ & Basic N1O2 & $\mathrm{C} 28 \mathrm{H} 33 \mathrm{~N} 1 \mathrm{O} 2$ & $1.02 \mathrm{E}-05$ \\
\hline $\mathrm{CH}$ & $\mathrm{C} 38 \mathrm{H} 32$ & $4.85 \mathrm{E}-06$ & Non-basic N1S1 & C26H39N1S1 & $5.82 \mathrm{E}-06$ & Basic N1O2 & $\mathrm{C} 29 \mathrm{H} 35 \mathrm{~N} 1 \mathrm{O} 2$ & $1.11 \mathrm{E}-05$ \\
\hline $\mathrm{CH}$ & C39H 34 & $2.68 \mathrm{E}-06$ & Non-basic N1S1 & C27H41N1S1 & $6.02 \mathrm{E}-06$ & Basic N1O2 & $\mathrm{C} 30 \mathrm{H} 37 \mathrm{~N} 1 \mathrm{O} 2$ & $9.94 \mathrm{E}-06$ \\
\hline $\mathrm{CH}$ & $\mathrm{C} 27 \mathrm{H} 8$ & $2.93 \mathrm{E}-06$ & Non-basic N1S1 & C28H43N1S1 & $1.17 \mathrm{E}-06$ & Basic N1O2 & $\mathrm{C} 31 \mathrm{H} 39 \mathrm{~N} 1 \mathrm{O} 2$ & $6.89 \mathrm{E}-06$ \\
\hline $\mathrm{CH}$ & $\mathrm{C} 28 \mathrm{H} 10$ & $2.02 \mathrm{E}-06$ & Non-basic N1S1 & C29H45N1S1 & $3.48 \mathrm{E}-06$ & Basic N1O2 & $\mathrm{C} 32 \mathrm{H} 41 \mathrm{~N} 1 \mathrm{O} 2$ & $4.96 \mathrm{E}-06$ \\
\hline $\mathrm{CH}$ & $\mathrm{C} 29 \mathrm{H} 12$ & $2.53 \mathrm{E}-06$ & Non-basic N1S1 & $\mathrm{C} 30 \mathrm{H} 47 \mathrm{~N} 1 \mathrm{~S} 1$ & $6.07 \mathrm{E}-06$ & Basic N1O2 & $\mathrm{C} 33 \mathrm{H} 43 \mathrm{~N} 1 \mathrm{O} 2$ & 4.21E-06 \\
\hline $\mathrm{S} 1$ & $\mathrm{C} 24 \mathrm{H} 38 \mathrm{~S} 1$ & $2.08 \mathrm{E}-06$ & Non-basic N1S1 & C31H49N1S1 & $2.65 \mathrm{E}-06$ & Basic N1O2 & $\mathrm{C} 34 \mathrm{H} 45 \mathrm{~N} 1 \mathrm{O} 2$ & $3.31 \mathrm{E}-06$ \\
\hline $\mathrm{S} 1$ & $\mathrm{C} 25 \mathrm{H} 40 \mathrm{~S} 1$ & 4.34E-06 & Non-basic N1S1 & C14H13N1S1 & $2.12 \mathrm{E}-06$ & Basic N1O2 & $\mathrm{C} 35 \mathrm{H} 47 \mathrm{~N} 1 \mathrm{O} 2$ & $3.03 \mathrm{E}-06$ \\
\hline $\mathrm{S} 1$ & $\mathrm{C} 26 \mathrm{H} 42 \mathrm{~S} 1$ & $3.96 \mathrm{E}-06$ & Non-basic N1S1 & C15H15N1S1 & $6.12 \mathrm{E}-06$ & Basic N1O2 & $\mathrm{C} 36 \mathrm{H} 49 \mathrm{~N} 1 \mathrm{O} 2$ & $2.78 \mathrm{E}-06$ \\
\hline $\mathrm{S} 1$ & $\mathrm{C} 27 \mathrm{H} 44 \mathrm{~S} 1$ & $1.15 \mathrm{E}-05$ & Non-basic N1S1 & C16H17N1S1 & $8.25 \mathrm{E}-06$ & Basic N1O2 & $\mathrm{C} 37 \mathrm{H} 51 \mathrm{~N} 1 \mathrm{O} 2$ & $2.51 \mathrm{E}-06$ \\
\hline $\mathrm{S} 1$ & $\mathrm{C} 28 \mathrm{H} 46 \mathrm{~S} 1$ & $6.73 \mathrm{E}-06$ & Non-basic N1S1 & C17H19N1S1 & $8.74 \mathrm{E}-07$ & Basic N1O2 & $\mathrm{C} 38 \mathrm{H} 53 \mathrm{~N} 1 \mathrm{O} 2$ & $1.27 \mathrm{E}-06$ \\
\hline S1 & $\mathrm{C} 29 \mathrm{H} 48 \mathrm{~S} 1$ & $8.37 \mathrm{E}-06$ & Non-basic N1S1 & C18H21N1S1 & $9.20 \mathrm{E}-07$ & Basic N1O2 & C39H55N1O2 & $1.01 \mathrm{E}-06$ \\
\hline $\mathrm{S} 1$ & $\mathrm{C} 30 \mathrm{H} 50 \mathrm{~S} 1$ & $3.63 \mathrm{E}-06$ & Non-basic N1S1 & C19H23N1S1 & $8.42 \mathrm{E}-07$ & Basic N1O2 & $\mathrm{C} 40 \mathrm{H} 57 \mathrm{~N} 1 \mathrm{O} 2$ & $6.95 \mathrm{E}-07$ \\
\hline $\mathrm{S} 1$ & $\mathrm{C} 31 \mathrm{H} 52 \mathrm{~S} 1$ & $8.59 \mathrm{E}-06$ & Non-basic N1S1 & $\mathrm{C} 20 \mathrm{H} 25 \mathrm{~N} 1 \mathrm{~S} 1$ & $2.33 \mathrm{E}-05$ & Basic N1O2 & $\mathrm{C} 41 \mathrm{H} 59 \mathrm{~N} 1 \mathrm{O} 2$ & $5.36 \mathrm{E}-07$ \\
\hline $\mathrm{S} 1$ & $\mathrm{C} 32 \mathrm{H} 54 \mathrm{~S} 1$ & $1.14 \mathrm{E}-05$ & Non-basic N1S1 & $\mathrm{C} 21 \mathrm{H} 27 \mathrm{~N} 1 \mathrm{~S} 1$ & $1.33 \mathrm{E}-06$ & Basic $\mathrm{N} 1 \mathrm{O} 2$ & $\mathrm{C} 42 \mathrm{H} 61 \mathrm{~N} 1 \mathrm{O} 2$ & $5.22 \mathrm{E}-07$ \\
\hline S1 & C33H56S1 & $8.11 \mathrm{E}-06$ & Non-basic N1S1 & $\mathrm{C} 22 \mathrm{H} 29 \mathrm{~N} 1 \mathrm{~S} 1$ & $1.41 \mathrm{E}-05$ & Basic N1O2 & $\mathrm{C} 43 \mathrm{H} 63 \mathrm{~N} 1 \mathrm{O} 2$ & $3.65 \mathrm{E}-07$ \\
\hline
\end{tabular}




\begin{tabular}{|c|c|c|c|c|c|c|c|c|}
\hline Class & formula & Mass & Class & formula & Mass & Class & formula & Mass \\
\hline S1 & C34H58S1 & $4.12 \mathrm{E}-06$ & Non-basic N1S1 & $\mathrm{C} 23 \mathrm{H} 31 \mathrm{~N} 1 \mathrm{~S} 1$ & $2.68 \mathrm{E}-06$ & Basic N1O2 & $\mathrm{C} 44 \mathrm{H} 65 \mathrm{~N} 1 \mathrm{O} 2$ & $2.46 \mathrm{E}-07$ \\
\hline S1 & $\mathrm{C} 35 \mathrm{H} 60 \mathrm{~S} 1$ & $2.15 \mathrm{E}-06$ & Non-basic N1S1 & C24H33N1S1 & $2.83 \mathrm{E}-05$ & Basic N1O2 & C45H67N1O2 & $1.18 \mathrm{E}-07$ \\
\hline $\mathrm{S} 1$ & $\mathrm{C} 36 \mathrm{H} 62 \mathrm{~S} 1$ & $3.17 \mathrm{E}-06$ & Non-basic N1S1 & C14H11N1S1 & $3.60 \mathrm{E}-07$ & Basic N1O2 & $\mathrm{C} 22 \mathrm{H} 19 \mathrm{~N} 1 \mathrm{O} 2$ & $1.04 \mathrm{E}-07$ \\
\hline S1 & $\mathrm{C} 21 \mathrm{H} 30 \mathrm{~S} 1$ & $2.33 \mathrm{E}-06$ & Non-basic N1S1 & C15H13N1S1 & $6.95 \mathrm{E}-07$ & Basic N1O2 & $\mathrm{C} 23 \mathrm{H} 21 \mathrm{~N} 1 \mathrm{O} 2$ & $2.95 \mathrm{E}-07$ \\
\hline $\mathrm{S} 1$ & $\mathrm{C} 22 \mathrm{H} 32 \mathrm{~S} 1$ & $1.78 \mathrm{E}-06$ & Non-basic N1S1 & C16H15N1S1 & $9.97 \mathrm{E}-07$ & Basic N1O2 & $\mathrm{C} 24 \mathrm{H} 23 \mathrm{~N} 1 \mathrm{O} 2$ & $6.86 \mathrm{E}-07$ \\
\hline $\mathrm{S} 1$ & $\mathrm{C} 23 \mathrm{H} 34 \mathrm{~S} 1$ & $2.50 \mathrm{E}-06$ & Non-basic N1S1 & C17H17N1S1 & $2.34 \mathrm{E}-06$ & Basic N1O2 & $\mathrm{C} 25 \mathrm{H} 25 \mathrm{~N} 1 \mathrm{O} 2$ & $1.94 \mathrm{E}-06$ \\
\hline S1 & $\mathrm{C} 24 \mathrm{H} 36 \mathrm{~S} 1$ & $1.05 \mathrm{E}-06$ & Non-basic N1S1 & C15H11N1S1 & $3.55 \mathrm{E}-06$ & Basic N1O2 & $\mathrm{C} 26 \mathrm{H} 27 \mathrm{~N} 1 \mathrm{O} 2$ & $3.62 \mathrm{E}-06$ \\
\hline S1 & $\mathrm{C} 25 \mathrm{H} 38 \mathrm{~S} 1$ & $4.84 \mathrm{E}-06$ & Non-basic N1S1 & C16H13N1S1 & $1.88 \mathrm{E}-06$ & Basic N1O2 & $\mathrm{C} 27 \mathrm{H} 29 \mathrm{~N} 1 \mathrm{O} 2$ & $5.57 \mathrm{E}-06$ \\
\hline S1 & $\mathrm{C} 26 \mathrm{H} 40 \mathrm{~S} 1$ & 4.41E-06 & Non-basic N1S1 & C17H15N1S1 & 4.17E-06 & Basic N1O2 & $\mathrm{C} 28 \mathrm{H} 31 \mathrm{~N} 1 \mathrm{O} 2$ & 7.09E-06 \\
\hline S1 & $\mathrm{C} 27 \mathrm{H} 42 \mathrm{~S} 1$ & $3.59 \mathrm{E}-06$ & Non-basic N1S1 & C18H17N1S1 & $3.85 \mathrm{E}-06$ & Basic N1O2 & $\mathrm{C} 29 \mathrm{H} 33 \mathrm{~N} 1 \mathrm{O} 2$ & $9.70 \mathrm{E}-06$ \\
\hline S1 & $\mathrm{C} 28 \mathrm{H} 44 \mathrm{~S} 1$ & $4.58 \mathrm{E}-06$ & Non-basic N1S1 & C15H9N1S1 & $3.31 \mathrm{E}-06$ & Basic N1O2 & $\mathrm{C} 30 \mathrm{H} 35 \mathrm{~N} 1 \mathrm{O} 2$ & $1.04 \mathrm{E}-05$ \\
\hline S1 & $\mathrm{C} 29 \mathrm{H} 46 \mathrm{~S} 1$ & $1.18 \mathrm{E}-05$ & Non-basic N1S1 & C16H11N1S1 & 7.01E-06 & Basic N1O2 & $\mathrm{C} 31 \mathrm{H} 37 \mathrm{~N} 1 \mathrm{O} 2$ & 7.87E-06 \\
\hline S1 & $\mathrm{C} 30 \mathrm{H} 48 \mathrm{~S} 1$ & $4.90 \mathrm{E}-06$ & Non-basic N1S1 & C17H13N1S1 & 3.04E-06 & Basic N1O2 & $\mathrm{C} 32 \mathrm{H} 39 \mathrm{~N} 1 \mathrm{O} 2$ & $6.00 \mathrm{E}-06$ \\
\hline S1 & C31H50S1 & $4.98 \mathrm{E}-06$ & Non-basic N1S1 & C18H15N1S1 & $2.02 \mathrm{E}-06$ & Basic N1O2 & $\mathrm{C} 33 \mathrm{H} 41 \mathrm{~N} 1 \mathrm{O} 2$ & 4.66E-06 \\
\hline $\mathrm{S} 1$ & $\mathrm{C} 32 \mathrm{H} 52 \mathrm{~S} 1$ & $3.39 \mathrm{E}-06$ & Non-basic N1S1 & C19H17N1S1 & $8.20 \mathrm{E}-07$ & Basic N1O2 & $\mathrm{C} 34 \mathrm{H} 43 \mathrm{~N} 1 \mathrm{O} 2$ & $3.32 \mathrm{E}-06$ \\
\hline $\mathrm{S} 1$ & $\mathrm{C} 33 \mathrm{H} 54 \mathrm{~S} 1$ & $6.32 \mathrm{E}-06$ & Acidic $\mathrm{O} 1$ & $\mathrm{C} 16 \mathrm{H} 14 \mathrm{O} 1$ & $1.02 \mathrm{E}-05$ & Basic N1O2 & $\mathrm{C} 35 \mathrm{H} 45 \mathrm{~N} 1 \mathrm{O} 2$ & $2.75 \mathrm{E}-06$ \\
\hline S1 & C34H56S1 & $1.33 \mathrm{E}-06$ & Acidic $\mathrm{O} 1$ & $\mathrm{C} 17 \mathrm{H} 16 \mathrm{O} 1$ & $2.05 \mathrm{E}-05$ & Basic N1O2 & $\mathrm{C} 36 \mathrm{H} 47 \mathrm{~N} 1 \mathrm{O} 2$ & $2.84 \mathrm{E}-06$ \\
\hline S1 & C35H58S1 & $1.42 \mathrm{E}-06$ & Acidic $\mathrm{O} 1$ & $\mathrm{C} 18 \mathrm{H} 18 \mathrm{O} 1$ & $1.45 \mathrm{E}-05$ & Basic N1O2 & $\mathrm{C} 37 \mathrm{H} 49 \mathrm{~N} 1 \mathrm{O} 2$ & $2.66 \mathrm{E}-06$ \\
\hline S1 & C38H64S1 & $1.49 \mathrm{E}-06$ & Acidic $\mathrm{O} 1$ & $\mathrm{C} 19 \mathrm{H} 20 \mathrm{O} 1$ & 4.74E-06 & Basic N1O2 & C38H51N1O2 & $1.50 \mathrm{E}-06$ \\
\hline S1 & C39H66S1 & $3.24 \mathrm{E}-06$ & Acidic $\mathrm{O} 1$ & $\mathrm{C} 17 \mathrm{H} 14 \mathrm{O} 1$ & $1.46 \mathrm{E}-05$ & Basic N1O2 & $\mathrm{C} 39 \mathrm{H} 53 \mathrm{~N} 1 \mathrm{O} 2$ & $6.33 \mathrm{E}-07$ \\
\hline S1 & C40H68S1 & $2.04 \mathrm{E}-06$ & Acidic $\mathrm{O} 1$ & $\mathrm{C} 18 \mathrm{H} 16 \mathrm{O} 1$ & $3.87 \mathrm{E}-05$ & Basic N1O2 & $\mathrm{C} 40 \mathrm{H} 55 \mathrm{~N} 1 \mathrm{O} 2$ & $7.82 \mathrm{E}-07$ \\
\hline S1 & $\mathrm{C} 19 \mathrm{H} 24 \mathrm{~S} 1$ & $1.76 \mathrm{E}-06$ & Acidic $\mathrm{O} 1$ & $\mathrm{C} 19 \mathrm{H} 18 \mathrm{O} 1$ & $1.76 \mathrm{E}-05$ & Basic N1O2 & $\mathrm{C} 41 \mathrm{H} 57 \mathrm{~N} 1 \mathrm{O} 2$ & 4.65E-07 \\
\hline S1 & $\mathrm{C} 20 \mathrm{H} 26 \mathrm{~S} 1$ & $2.53 \mathrm{E}-06$ & Acidic $\mathrm{O} 1$ & $\mathrm{C} 20 \mathrm{H} 20 \mathrm{O} 1$ & $5.26 \mathrm{E}-06$ & Basic N1O2 & $\mathrm{C} 42 \mathrm{H} 59 \mathrm{~N} 1 \mathrm{O} 2$ & $3.81 \mathrm{E}-07$ \\
\hline S1 & $\mathrm{C} 21 \mathrm{H} 28 \mathrm{~S} 1$ & $1.57 \mathrm{E}-06$ & Acidic $\mathrm{O} 1$ & $\mathrm{C} 21 \mathrm{H} 22 \mathrm{O} 1$ & $3.49 \mathrm{E}-06$ & Basic N1O2 & $\mathrm{C} 22 \mathrm{H} 17 \mathrm{~N} 1 \mathrm{O} 2$ & $2.18 \mathrm{E}-08$ \\
\hline S1 & $\mathrm{C} 22 \mathrm{H} 30 \mathrm{~S} 1$ & $9.32 \mathrm{E}-07$ & Acidic $\mathrm{O} 1$ & $\mathrm{C} 16 \mathrm{H} 10 \mathrm{O} 1$ & $8.24 \mathrm{E}-06$ & Basic $\mathrm{N} 1 \mathrm{O} 2$ & $\mathrm{C} 23 \mathrm{H} 19 \mathrm{~N} 1 \mathrm{O} 2$ & $9.78 \mathrm{E}-08$ \\
\hline S1 & $\mathrm{C} 23 \mathrm{H} 32 \mathrm{~S} 1$ & $1.61 \mathrm{E}-06$ & Acidic $\mathrm{O} 1$ & $\mathrm{C} 17 \mathrm{H} 12 \mathrm{O} 1$ & 0.00021 & Basic N1O2 & $\mathrm{C} 24 \mathrm{H} 21 \mathrm{~N} 1 \mathrm{O} 2$ & $1.02 \mathrm{E}-06$ \\
\hline S1 & $\mathrm{C} 24 \mathrm{H} 34 \mathrm{~S} 1$ & $8.31 \mathrm{E}-06$ & Acidic $\mathrm{O} 1$ & $\mathrm{C} 18 \mathrm{H} 14 \mathrm{O} 1$ & 0.000395 & Basic N1O2 & $\mathrm{C} 25 \mathrm{H} 23 \mathrm{~N} 1 \mathrm{O} 2$ & $3.95 \mathrm{E}-06$ \\
\hline $\mathrm{S} 1$ & $\mathrm{C} 25 \mathrm{H} 36 \mathrm{~S} 1$ & $5.14 \mathrm{E}-06$ & Acidic $\mathrm{O} 1$ & $\mathrm{C} 19 \mathrm{H} 16 \mathrm{O} 1$ & 0.000287 & Basic N1O2 & $\mathrm{C} 26 \mathrm{H} 25 \mathrm{~N} 1 \mathrm{O} 2$ & $5.82 \mathrm{E}-06$ \\
\hline S1 & $\mathrm{C} 26 \mathrm{H} 38 \mathrm{~S} 1$ & $3.99 \mathrm{E}-06$ & Acidic $\mathrm{O} 1$ & $\mathrm{C} 20 \mathrm{H} 18 \mathrm{O} 1$ & 0.000119 & Basic N1O2 & $\mathrm{C} 27 \mathrm{H} 27 \mathrm{~N} 1 \mathrm{O} 2$ & $5.34 \mathrm{E}-06$ \\
\hline $\mathrm{S} 1$ & $\mathrm{C} 27 \mathrm{H} 40 \mathrm{~S} 1$ & $4.88 \mathrm{E}-06$ & Acidic $\mathrm{O} 1$ & $\mathrm{C} 21 \mathrm{H} 20 \mathrm{O} 1$ & $3.98 \mathrm{E}-05$ & Basic N1O2 & $\mathrm{C} 28 \mathrm{H} 29 \mathrm{~N} 1 \mathrm{O} 2$ & $5.17 \mathrm{E}-06$ \\
\hline $\mathrm{S} 1$ & $\mathrm{C} 28 \mathrm{H} 42 \mathrm{~S} 1$ & $8.89 \mathrm{E}-06$ & Acidic $\mathrm{O} 1$ & $\mathrm{C} 22 \mathrm{H} 22 \mathrm{O} 1$ & $2.52 \mathrm{E}-05$ & Basic N1O2 & $\mathrm{C} 29 \mathrm{H} 31 \mathrm{~N} 1 \mathrm{O} 2$ & $5.20 \mathrm{E}-06$ \\
\hline S1 & $\mathrm{C} 29 \mathrm{H} 44 \mathrm{~S} 1$ & $5.09 \mathrm{E}-06$ & Acidic $\mathrm{O} 1$ & $\mathrm{C} 23 \mathrm{H} 24 \mathrm{O} 1$ & $3.86 \mathrm{E}-06$ & Basic N1O2 & $\mathrm{C} 30 \mathrm{H} 33 \mathrm{~N} 1 \mathrm{O} 2$ & $5.72 \mathrm{E}-06$ \\
\hline $\mathrm{S} 1$ & $\mathrm{C} 30 \mathrm{H} 46 \mathrm{~S} 1$ & $1.05 \mathrm{E}-05$ & Acidic $\mathrm{O} 1$ & $\mathrm{C} 18 \mathrm{H} 12 \mathrm{O} 1$ & $3.24 \mathrm{E}-05$ & Basic N1O2 & $\mathrm{C} 31 \mathrm{H} 35 \mathrm{~N} 1 \mathrm{O} 2$ & $5.55 \mathrm{E}-06$ \\
\hline $\mathrm{S} 1$ & $\mathrm{C} 31 \mathrm{H} 48 \mathrm{~S} 1$ & $4.85 \mathrm{E}-06$ & Acidic $\mathrm{O} 1$ & $\mathrm{C} 19 \mathrm{H} 14 \mathrm{O} 1$ & $9.19 \mathrm{E}-05$ & Basic N1O2 & $\mathrm{C} 32 \mathrm{H} 37 \mathrm{~N} 1 \mathrm{O} 2$ & $5.06 \mathrm{E}-06$ \\
\hline $\mathrm{S} 1$ & $\mathrm{C} 32 \mathrm{H} 50 \mathrm{~S} 1$ & $9.01 \mathrm{E}-06$ & Acidic $\mathrm{O} 1$ & $\mathrm{C} 20 \mathrm{H} 16 \mathrm{O} 1$ & 0.000121 & Basic N1O2 & $\mathrm{C} 33 \mathrm{H} 39 \mathrm{~N} 1 \mathrm{O} 2$ & $4.35 \mathrm{E}-06$ \\
\hline $\mathrm{S} 1$ & $\mathrm{C} 33 \mathrm{H} 52 \mathrm{~S} 1$ & $3.22 \mathrm{E}-06$ & Acidic $\mathrm{O} 1$ & $\mathrm{C} 21 \mathrm{H} 18 \mathrm{O} 1$ & $9.95 \mathrm{E}-05$ & Basic N1O2 & $\mathrm{C} 34 \mathrm{H} 41 \mathrm{~N} 1 \mathrm{O} 2$ & $3.35 \mathrm{E}-06$ \\
\hline S1 & C34H54S1 & 7.12E-06 & Acidic $\mathrm{O} 1$ & $\mathrm{C} 22 \mathrm{H} 20 \mathrm{O} 1$ & $5.48 \mathrm{E}-05$ & Basic $\mathrm{N} 1 \mathrm{O} 2$ & $\mathrm{C} 35 \mathrm{H} 43 \mathrm{~N} 1 \mathrm{O} 2$ & $2.78 \mathrm{E}-06$ \\
\hline S1 & C35H56S1 & 4.47E-06 & Acidic $\mathrm{O} 1$ & $\mathrm{C} 23 \mathrm{H} 22 \mathrm{O} 1$ & $2.79 \mathrm{E}-05$ & Basic N1O2 & $\mathrm{C} 36 \mathrm{H} 45 \mathrm{~N} 1 \mathrm{O} 2$ & $2.75 \mathrm{E}-06$ \\
\hline
\end{tabular}




\begin{tabular}{|c|c|c|c|c|c|c|c|c|}
\hline Class & formula & Mass & Class & formula & Mass & Class & formula & Mass \\
\hline $\mathrm{S} 1$ & C13H10S1 & $1.72 \mathrm{E}-05$ & Acidic $\mathrm{O} 1$ & $\mathrm{C} 24 \mathrm{H} 24 \mathrm{O} 1$ & $1.49 \mathrm{E}-05$ & Basic N1O2 & $\mathrm{C} 37 \mathrm{H} 47 \mathrm{~N} 1 \mathrm{O} 2$ & $1.73 \mathrm{E}-06$ \\
\hline $\mathrm{S} 1$ & $\mathrm{C} 14 \mathrm{H} 12 \mathrm{~S} 1$ & 0.000261 & Acidic $\mathrm{O} 1$ & $\mathrm{C} 25 \mathrm{H} 26 \mathrm{O} 1$ & $1.27 \mathrm{E}-05$ & Basic N1O2 & $\mathrm{C} 38 \mathrm{H} 49 \mathrm{~N} 1 \mathrm{O} 2$ & $7.65 \mathrm{E}-07$ \\
\hline $\mathrm{S} 1$ & $\mathrm{C} 15 \mathrm{H} 14 \mathrm{~S} 1$ & 0.000573 & Acidic $\mathrm{O} 1$ & $\mathrm{C} 26 \mathrm{H} 28 \mathrm{O} 1$ & 7.35E-06 & Basic N1O2 & $\mathrm{C} 39 \mathrm{H} 51 \mathrm{~N} 1 \mathrm{O} 2$ & 8.33E-07 \\
\hline $\mathrm{S} 1$ & C16H16S1 & 0.000617 & Acidic $\mathrm{O} 1$ & $\mathrm{C} 27 \mathrm{H} 30 \mathrm{O} 1$ & $9.72 \mathrm{E}-06$ & Basic N1O2 & $\mathrm{C} 40 \mathrm{H} 53 \mathrm{~N} 1 \mathrm{O} 2$ & $4.16 \mathrm{E}-07$ \\
\hline $\mathrm{S} 1$ & $\mathrm{C} 17 \mathrm{H} 18 \mathrm{~S} 1$ & 0.000428 & Acidic $\mathrm{O} 1$ & $\mathrm{C} 28 \mathrm{H} 32 \mathrm{O} 1$ & $1.15 \mathrm{E}-05$ & Basic N1O2 & $\mathrm{C} 41 \mathrm{H} 55 \mathrm{~N} 1 \mathrm{O} 2$ & $3.63 \mathrm{E}-07$ \\
\hline $\mathrm{S} 1$ & $\mathrm{C} 18 \mathrm{H} 20 \mathrm{~S} 1$ & 0.000253 & Acidic $\mathrm{O} 1$ & $\mathrm{C} 29 \mathrm{H} 34 \mathrm{O} 1$ & $1.14 \mathrm{E}-05$ & Basic N1O2 & $\mathrm{C} 22 \mathrm{H} 15 \mathrm{~N} 1 \mathrm{O} 2$ & $1.34 \mathrm{E}-08$ \\
\hline $\mathrm{S} 1$ & $\mathrm{C} 19 \mathrm{H} 22 \mathrm{~S} 1$ & 0.00017 & Acidic $\mathrm{O} 1$ & $\mathrm{C} 19 \mathrm{H} 12 \mathrm{O} 1$ & $1.59 \mathrm{E}-05$ & Basic N1O2 & $\mathrm{C} 23 \mathrm{H} 17 \mathrm{~N} 1 \mathrm{O} 2$ & $1.72 \mathrm{E}-08$ \\
\hline $\mathrm{S} 1$ & $\mathrm{C} 20 \mathrm{H} 24 \mathrm{~S} 1$ & 0.000145 & Acidic $\mathrm{O} 1$ & $\mathrm{C} 20 \mathrm{H} 14 \mathrm{O} 1$ & $3.84 \mathrm{E}-05$ & Basic N1O2 & $\mathrm{C} 24 \mathrm{H} 19 \mathrm{~N} 1 \mathrm{O} 2$ & $2.15 \mathrm{E}-07$ \\
\hline $\mathrm{S} 1$ & $\mathrm{C} 21 \mathrm{H} 26 \mathrm{~S} 1$ & 0.000131 & Acidic $\mathrm{O} 1$ & $\mathrm{C} 21 \mathrm{H} 16 \mathrm{O} 1$ & $5.47 \mathrm{E}-05$ & Basic N1O2 & $\mathrm{C} 25 \mathrm{H} 21 \mathrm{~N} 1 \mathrm{O} 2$ & $1.60 \mathrm{E}-07$ \\
\hline $\mathrm{S} 1$ & $\mathrm{C} 22 \mathrm{H} 28 \mathrm{~S} 1$ & 0.000128 & Acidic $\mathrm{O} 1$ & $\mathrm{C} 22 \mathrm{H} 18 \mathrm{O} 1$ & $5.41 \mathrm{E}-05$ & Basic N1O2 & $\mathrm{C} 26 \mathrm{H} 23 \mathrm{~N} 1 \mathrm{O} 2$ & $1.03 \mathrm{E}-06$ \\
\hline S1 & $\mathrm{C} 23 \mathrm{H} 30 \mathrm{~S} 1$ & 0.000133 & Acidic $\mathrm{O} 1$ & $\mathrm{C} 23 \mathrm{H} 20 \mathrm{O} 1$ & $3.44 \mathrm{E}-05$ & Basic N1O2 & $\mathrm{C} 27 \mathrm{H} 25 \mathrm{~N} 1 \mathrm{O} 2$ & $2.74 \mathrm{E}-06$ \\
\hline S1 & $\mathrm{C} 24 \mathrm{H} 32 \mathrm{~S} 1$ & 0.000136 & Acidic $\mathrm{O} 1$ & $\mathrm{C} 24 \mathrm{H} 22 \mathrm{O} 1$ & $1.70 \mathrm{E}-05$ & Basic N1O2 & $\mathrm{C} 28 \mathrm{H} 27 \mathrm{~N} 1 \mathrm{O} 2$ & $3.82 \mathrm{E}-06$ \\
\hline S1 & $\mathrm{C} 25 \mathrm{H} 34 \mathrm{~S} 1$ & 0.000144 & Acidic $\mathrm{O} 1$ & $\mathrm{C} 25 \mathrm{H} 24 \mathrm{O} 1$ & $7.58 \mathrm{E}-06$ & Basic N1O2 & $\mathrm{C} 29 \mathrm{H} 29 \mathrm{~N} 1 \mathrm{O} 2$ & 4.13E-06 \\
\hline S1 & $\mathrm{C} 26 \mathrm{H} 36 \mathrm{~S} 1$ & 0.000138 & Acidic $\mathrm{O} 1$ & $\mathrm{C} 26 \mathrm{H} 26 \mathrm{O} 1$ & $3.33 \mathrm{E}-06$ & Basic N1O2 & $\mathrm{C} 30 \mathrm{H} 31 \mathrm{~N} 1 \mathrm{O} 2$ & $5.79 \mathrm{E}-06$ \\
\hline $\mathrm{S} 1$ & $\mathrm{C} 27 \mathrm{H} 38 \mathrm{~S} 1$ & 0.000144 & Acidic $\mathrm{O} 1$ & $\mathrm{C} 20 \mathrm{H} 12 \mathrm{O} 1$ & 0.000159 & Basic N1O2 & $\mathrm{C} 31 \mathrm{H} 33 \mathrm{~N} 1 \mathrm{O} 2$ & $5.37 \mathrm{E}-06$ \\
\hline $\mathrm{S} 1$ & $\mathrm{C} 28 \mathrm{H} 40 \mathrm{~S} 1$ & 0.000145 & Acidic $\mathrm{O} 1$ & $\mathrm{C} 21 \mathrm{H} 14 \mathrm{O} 1$ & 0.000474 & Basic N1O2 & $\mathrm{C} 32 \mathrm{H} 35 \mathrm{~N} 1 \mathrm{O} 2$ & $5.10 \mathrm{E}-06$ \\
\hline $\mathrm{S} 1$ & $\mathrm{C} 29 \mathrm{H} 42 \mathrm{~S} 1$ & 0.000143 & Acidic $\mathrm{O} 1$ & $\mathrm{C} 22 \mathrm{H} 16 \mathrm{O} 1$ & 0.000463 & Basic N1O2 & $\mathrm{C} 33 \mathrm{H} 37 \mathrm{~N} 1 \mathrm{O} 2$ & 4.23E-06 \\
\hline $\mathrm{S} 1$ & $\mathrm{C} 30 \mathrm{H} 44 \mathrm{~S} 1$ & 0.000143 & Acidic $\mathrm{O} 1$ & $\mathrm{C} 23 \mathrm{H} 18 \mathrm{O} 1$ & 0.000323 & Basic N1O2 & $\mathrm{C} 34 \mathrm{H} 39 \mathrm{~N} 1 \mathrm{O} 2$ & $3.68 \mathrm{E}-06$ \\
\hline S1 & $\mathrm{C} 31 \mathrm{H} 46 \mathrm{~S} 1$ & 0.000144 & Acidic $\mathrm{O} 1$ & $\mathrm{C} 24 \mathrm{H} 20 \mathrm{O} 1$ & 0.000152 & Basic N1O2 & $\mathrm{C} 35 \mathrm{H} 41 \mathrm{~N} 1 \mathrm{O} 2$ & $3.52 \mathrm{E}-06$ \\
\hline S1 & $\mathrm{C} 32 \mathrm{H} 48 \mathrm{~S} 1$ & 0.000123 & Acidic $\mathrm{O} 1$ & $\mathrm{C} 25 \mathrm{H} 22 \mathrm{O} 1$ & $6.69 \mathrm{E}-05$ & Basic N1O2 & $\mathrm{C} 36 \mathrm{H} 43 \mathrm{~N} 1 \mathrm{O} 2$ & $2.91 \mathrm{E}-06$ \\
\hline S1 & $\mathrm{C} 33 \mathrm{H} 50 \mathrm{~S} 1$ & 0.000113 & Acidic $\mathrm{O} 1$ & $\mathrm{C} 26 \mathrm{H} 24 \mathrm{O} 1$ & 4.07E-05 & Basic N1O2 & $\mathrm{C} 37 \mathrm{H} 45 \mathrm{~N} 1 \mathrm{O} 2$ & $2.07 \mathrm{E}-06$ \\
\hline S1 & $\mathrm{C} 34 \mathrm{H} 52 \mathrm{~S} 1$ & 0.000105 & Acidic $\mathrm{O} 1$ & $\mathrm{C} 27 \mathrm{H} 26 \mathrm{O} 1$ & $1.69 \mathrm{E}-05$ & Basic N1O2 & $\mathrm{C} 38 \mathrm{H} 47 \mathrm{~N} 1 \mathrm{O} 2$ & $1.03 \mathrm{E}-06$ \\
\hline S1 & $\mathrm{C} 35 \mathrm{H} 54 \mathrm{~S} 1$ & 0.0001 & Acidic $\mathrm{O} 1$ & $\mathrm{C} 28 \mathrm{H} 28 \mathrm{O} 1$ & $4.05 \mathrm{E}-06$ & Basic N1O2 & $\mathrm{C} 23 \mathrm{H} 15 \mathrm{~N} 1 \mathrm{O} 2$ & $3.01 \mathrm{E}-08$ \\
\hline S1 & $\mathrm{C} 36 \mathrm{H} 56 \mathrm{~S} 1$ & 7.77E-05 & Acidic $\mathrm{O} 1$ & $\mathrm{C} 29 \mathrm{H} 30 \mathrm{O} 1$ & $4.04 \mathrm{E}-06$ & Basic N1O2 & $\mathrm{C} 24 \mathrm{H} 17 \mathrm{~N} 1 \mathrm{O} 2$ & $6.28 \mathrm{E}-08$ \\
\hline S1 & C37H58S1 & $8.27 \mathrm{E}-05$ & Acidic $\mathrm{O} 1$ & $\mathrm{C} 30 \mathrm{H} 32 \mathrm{O} 1$ & 4.19E-06 & Basic $\mathrm{N} 1 \mathrm{O} 2$ & $\mathrm{C} 25 \mathrm{H} 19 \mathrm{~N} 1 \mathrm{O} 2$ & $1.74 \mathrm{E}-07$ \\
\hline S1 & $\mathrm{C} 38 \mathrm{H} 60 \mathrm{~S} 1$ & $5.87 \mathrm{E}-05$ & Acidic $\mathrm{O} 1$ & $\mathrm{C} 31 \mathrm{H} 34 \mathrm{O} 1$ & $3.84 \mathrm{E}-06$ & Basic N1O2 & $\mathrm{C} 26 \mathrm{H} 21 \mathrm{~N} 1 \mathrm{O} 2$ & $4.68 \mathrm{E}-07$ \\
\hline $\mathrm{S} 1$ & $\mathrm{C} 39 \mathrm{H} 62 \mathrm{~S} 1$ & $6.09 \mathrm{E}-05$ & Acidic $\mathrm{O} 1$ & $\mathrm{C} 17 \mathrm{H} 4 \mathrm{O} 1$ & $1.11 \mathrm{E}-05$ & Basic N1O2 & $\mathrm{C} 27 \mathrm{H} 23 \mathrm{~N} 1 \mathrm{O} 2$ & $6.37 \mathrm{E}-07$ \\
\hline $\mathrm{S} 1$ & C40H64S1 & $6.51 \mathrm{E}-05$ & Acidic $\mathrm{O} 1$ & $\mathrm{C} 18 \mathrm{H} 6 \mathrm{O} 1$ & 7.57E-06 & Basic N1O2 & $\mathrm{C} 28 \mathrm{H} 25 \mathrm{~N} 1 \mathrm{O} 2$ & 9.19E-07 \\
\hline S1 & C41H66S1 & $4.13 \mathrm{E}-05$ & Acidic $\mathrm{O} 1$ & C19H8O1 & $1.33 \mathrm{E}-06$ & Basic N1O2 & $\mathrm{C} 29 \mathrm{H} 27 \mathrm{~N} 1 \mathrm{O} 2$ & $1.39 \mathrm{E}-06$ \\
\hline $\mathrm{S} 1$ & $\mathrm{C} 42 \mathrm{H} 68 \mathrm{~S} 1$ & $3.76 \mathrm{E}-05$ & Acidic $\mathrm{O} 1$ & $\mathrm{C} 20 \mathrm{H} 10 \mathrm{O} 1$ & $1.23 \mathrm{E}-06$ & Basic N1O2 & $\mathrm{C} 30 \mathrm{H} 29 \mathrm{~N} 1 \mathrm{O} 2$ & $2.83 \mathrm{E}-06$ \\
\hline $\mathrm{S} 1$ & $\mathrm{C} 43 \mathrm{H} 70 \mathrm{~S} 1$ & $3.30 \mathrm{E}-05$ & Acidic $\mathrm{O} 1$ & $\mathrm{C} 21 \mathrm{H} 12 \mathrm{O} 1$ & $1.29 \mathrm{E}-06$ & Basic N1O2 & $\mathrm{C} 31 \mathrm{H} 31 \mathrm{~N} 1 \mathrm{O} 2$ & $3.36 \mathrm{E}-06$ \\
\hline S1 & $\mathrm{C} 44 \mathrm{H} 72 \mathrm{~S} 1$ & $2.53 \mathrm{E}-05$ & Acidic $\mathrm{O} 1$ & $\mathrm{C} 22 \mathrm{H} 14 \mathrm{O} 1$ & $1.91 \mathrm{E}-05$ & Basic N1O2 & $\mathrm{C} 32 \mathrm{H} 33 \mathrm{~N} 1 \mathrm{O} 2$ & $3.31 \mathrm{E}-06$ \\
\hline $\mathrm{S} 1$ & $\mathrm{C} 45 \mathrm{H} 74 \mathrm{~S} 1$ & $2.64 \mathrm{E}-05$ & Acidic $\mathrm{O} 1$ & $\mathrm{C} 23 \mathrm{H} 16 \mathrm{O} 1$ & $6.60 \mathrm{E}-05$ & Basic N1O2 & $\mathrm{C} 33 \mathrm{H} 35 \mathrm{~N} 1 \mathrm{O} 2$ & $3.40 \mathrm{E}-06$ \\
\hline $\mathrm{S} 1$ & $\mathrm{C} 46 \mathrm{H} 76 \mathrm{~S} 1$ & $1.62 \mathrm{E}-05$ & Acidic $\mathrm{O} 1$ & $\mathrm{C} 24 \mathrm{H} 18 \mathrm{O} 1$ & $8.45 \mathrm{E}-05$ & Basic N1O2 & $\mathrm{C} 34 \mathrm{H} 37 \mathrm{~N} 1 \mathrm{O} 2$ & $3.45 \mathrm{E}-06$ \\
\hline $\mathrm{S} 1$ & $\mathrm{C} 47 \mathrm{H} 78 \mathrm{~S} 1$ & $1.55 \mathrm{E}-05$ & Acidic $\mathrm{O} 1$ & $\mathrm{C} 25 \mathrm{H} 20 \mathrm{O} 1$ & $6.42 \mathrm{E}-05$ & Basic N1O2 & $\mathrm{C} 35 \mathrm{H} 39 \mathrm{~N} 1 \mathrm{O} 2$ & $3.42 \mathrm{E}-06$ \\
\hline $\mathrm{S} 1$ & C48H80S1 & $1.65 \mathrm{E}-05$ & Acidic $\mathrm{O} 1$ & $\mathrm{C} 26 \mathrm{H} 22 \mathrm{O} 1$ & $3.48 \mathrm{E}-05$ & Basic N1O2 & $\mathrm{C} 36 \mathrm{H} 41 \mathrm{~N} 1 \mathrm{O} 2$ & $3.92 \mathrm{E}-06$ \\
\hline S1 & $\mathrm{C} 49 \mathrm{H} 82 \mathrm{~S} 1$ & $1.54 \mathrm{E}-05$ & Acidic $\mathrm{O} 1$ & $\mathrm{C} 27 \mathrm{H} 24 \mathrm{O} 1$ & $1.16 \mathrm{E}-05$ & Basic $\mathrm{N} 1 \mathrm{O} 2$ & $\mathrm{C} 37 \mathrm{H} 43 \mathrm{~N} 1 \mathrm{O} 2$ & $2.59 \mathrm{E}-06$ \\
\hline $\mathrm{S} 1$ & $\mathrm{C} 13 \mathrm{H} 8 \mathrm{~S} 1$ & $4.75 \mathrm{E}-06$ & Acidic $\mathrm{O} 1$ & $\mathrm{C} 28 \mathrm{H} 26 \mathrm{O} 1$ & 4.13E-06 & Basic N1O2 & $\mathrm{C} 38 \mathrm{H} 45 \mathrm{~N} 1 \mathrm{O} 2$ & $1.57 \mathrm{E}-06$ \\
\hline
\end{tabular}




\begin{tabular}{|c|c|c|c|c|c|c|c|c|}
\hline Class & formula & Mass & Class & formula & Mass & Class & formula & Mass \\
\hline $\mathrm{S} 1$ & C14H10S1 & $6.71 \mathrm{E}-06$ & Acidic $\mathrm{O} 1$ & $\mathrm{C} 29 \mathrm{H} 28 \mathrm{O} 1$ & 0.000209 & Basic N1O2 & $\mathrm{C} 25 \mathrm{H} 17 \mathrm{~N} 1 \mathrm{O} 2$ & 5.24E-08 \\
\hline S1 & $\mathrm{C} 15 \mathrm{H} 12 \mathrm{~S} 1$ & $2.72 \mathrm{E}-05$ & Acidic $\mathrm{O} 1$ & $\mathrm{C} 30 \mathrm{H} 30 \mathrm{O} 1$ & $2.73 \mathrm{E}-05$ & Basic N1O2 & $\mathrm{C} 26 \mathrm{H} 19 \mathrm{~N} 1 \mathrm{O} 2$ & $9.46 \mathrm{E}-08$ \\
\hline $\mathrm{S} 1$ & $\mathrm{C} 16 \mathrm{H} 14 \mathrm{~S} 1$ & 0.000147 & Acidic $\mathrm{O} 1$ & $\mathrm{C} 31 \mathrm{H} 32 \mathrm{O} 1$ & 0.000182 & Basic N1O2 & $\mathrm{C} 27 \mathrm{H} 21 \mathrm{~N} 1 \mathrm{O} 2$ & $1.45 \mathrm{E}-07$ \\
\hline $\mathrm{S} 1$ & C17H16S1 & 0.000353 & Acidic $\mathrm{O} 1$ & $\mathrm{C} 32 \mathrm{H} 34 \mathrm{O} 1$ & $1.84 \mathrm{E}-05$ & Basic N1O2 & $\mathrm{C} 28 \mathrm{H} 23 \mathrm{~N} 1 \mathrm{O} 2$ & 7.04E-07 \\
\hline $\mathrm{S} 1$ & C18H18S1 & 0.000428 & Acidic $\mathrm{O} 1$ & $\mathrm{C} 33 \mathrm{H} 36 \mathrm{O} 1$ & $2.18 \mathrm{E}-05$ & Basic N1O2 & $\mathrm{C} 29 \mathrm{H} 25 \mathrm{~N} 1 \mathrm{O} 2$ & $1.53 \mathrm{E}-06$ \\
\hline $\mathrm{S} 1$ & C19H20S1 & 0.000362 & Acidic $\mathrm{O} 1$ & $\mathrm{C} 34 \mathrm{H} 38 \mathrm{O} 1$ & $8.76 \mathrm{E}-06$ & Basic N1O2 & $\mathrm{C} 30 \mathrm{H} 27 \mathrm{~N} 1 \mathrm{O} 2$ & $1.74 \mathrm{E}-06$ \\
\hline $\mathrm{S} 1$ & $\mathrm{C} 20 \mathrm{H} 22 \mathrm{~S} 1$ & 0.000269 & Acidic $\mathrm{O} 1$ & $\mathrm{C} 35 \mathrm{H} 40 \mathrm{O} 1$ & $1.71 \mathrm{E}-05$ & Basic N1O2 & $\mathrm{C} 31 \mathrm{H} 29 \mathrm{~N} 1 \mathrm{O} 2$ & $2.45 \mathrm{E}-06$ \\
\hline $\mathrm{S} 1$ & $\mathrm{C} 21 \mathrm{H} 24 \mathrm{~S} 1$ & 0.000214 & Acidic $\mathrm{O} 1$ & $\mathrm{C} 17 \mathrm{H} 2 \mathrm{O} 1$ & 8.03E-06 & Basic N1O2 & $\mathrm{C} 32 \mathrm{H} 31 \mathrm{~N} 1 \mathrm{O} 2$ & $2.68 \mathrm{E}-06$ \\
\hline $\mathrm{S} 1$ & $\mathrm{C} 22 \mathrm{H} 26 \mathrm{~S} 1$ & 0.000183 & Acidic $\mathrm{O} 1$ & $\mathrm{C} 18 \mathrm{H} 4 \mathrm{O} 1$ & 4.93E-06 & Basic N1O2 & $\mathrm{C} 33 \mathrm{H} 33 \mathrm{~N} 1 \mathrm{O} 2$ & $2.55 \mathrm{E}-06$ \\
\hline $\mathrm{S} 1$ & $\mathrm{C} 23 \mathrm{H} 28 \mathrm{~S} 1$ & 0.000164 & Acidic $\mathrm{O} 1$ & $\mathrm{C} 19 \mathrm{H} 6 \mathrm{O} 1$ & $1.99 \mathrm{E}-05$ & Basic N1O2 & $\mathrm{C} 34 \mathrm{H} 35 \mathrm{~N} 1 \mathrm{O} 2$ & $2.48 \mathrm{E}-06$ \\
\hline $\mathrm{S} 1$ & $\mathrm{C} 24 \mathrm{H} 30 \mathrm{~S} 1$ & 0.000153 & Acidic O1 & $\mathrm{C} 20 \mathrm{H} 8 \mathrm{O} 1$ & $2.42 \mathrm{E}-05$ & Basic N1O2 & $\mathrm{C} 35 \mathrm{H} 37 \mathrm{~N} 1 \mathrm{O} 2$ & $3.41 \mathrm{E}-06$ \\
\hline $\mathrm{S} 1$ & $\mathrm{C} 25 \mathrm{H} 32 \mathrm{~S} 1$ & 0.000148 & Acidic $\mathrm{O} 1$ & $\mathrm{C} 21 \mathrm{H} 10 \mathrm{O} 1$ & $7.05 \mathrm{E}-05$ & Basic N1O2 & $\mathrm{C} 28 \mathrm{H} 21 \mathrm{~N} 1 \mathrm{O} 2$ & $3.27 \mathrm{E}-08$ \\
\hline $\mathrm{S} 1$ & $\mathrm{C} 26 \mathrm{H} 34 \mathrm{~S} 1$ & 0.000147 & Acidic $\mathrm{O} 1$ & $\mathrm{C} 22 \mathrm{H} 12 \mathrm{O} 1$ & $5.58 \mathrm{E}-05$ & Basic N1O2 & $\mathrm{C} 29 \mathrm{H} 23 \mathrm{~N} 1 \mathrm{O} 2$ & $9.35 \mathrm{E}-08$ \\
\hline $\mathrm{S} 1$ & $\mathrm{C} 27 \mathrm{H} 36 \mathrm{~S} 1$ & 0.00016 & Acidic $\mathrm{O} 1$ & $\mathrm{C} 23 \mathrm{H} 14 \mathrm{O} 1$ & 0.000159 & Basic N1O2 & $\mathrm{C} 30 \mathrm{H} 25 \mathrm{~N} 1 \mathrm{O} 2$ & $1.83 \mathrm{E}-07$ \\
\hline $\mathrm{S} 1$ & $\mathrm{C} 28 \mathrm{H} 38 \mathrm{~S} 1$ & 0.000158 & Acidic $\mathrm{O} 1$ & $\mathrm{C} 24 \mathrm{H} 16 \mathrm{O} 1$ & 0.000188 & Basic N1O2 & $\mathrm{C} 31 \mathrm{H} 27 \mathrm{~N} 1 \mathrm{O} 2$ & $9.52 \mathrm{E}-07$ \\
\hline $\mathrm{S} 1$ & $\mathrm{C} 29 \mathrm{H} 40 \mathrm{~S} 1$ & 0.000163 & Acidic $\mathrm{O} 1$ & $\mathrm{C} 25 \mathrm{H} 18 \mathrm{O} 1$ & 0.000148 & Basic N1O2 & $\mathrm{C} 32 \mathrm{H} 29 \mathrm{~N} 1 \mathrm{O} 2$ & $7.72 \mathrm{E}-07$ \\
\hline S1 & $\mathrm{C} 30 \mathrm{H} 42 \mathrm{~S} 1$ & 0.00015 & Acidic $\mathrm{O} 1$ & $\mathrm{C} 26 \mathrm{H} 20 \mathrm{O} 1$ & $6.75 \mathrm{E}-05$ & Basic N1O2 & $\mathrm{C} 33 \mathrm{H} 31 \mathrm{~N} 1 \mathrm{O} 2$ & $1.16 \mathrm{E}-06$ \\
\hline S1 & $\mathrm{C} 31 \mathrm{H} 44 \mathrm{~S} 1$ & 0.000128 & Acidic $\mathrm{O} 1$ & $\mathrm{C} 27 \mathrm{H} 22 \mathrm{O} 1$ & $3.72 \mathrm{E}-05$ & Basic N1O2 & $\mathrm{C} 34 \mathrm{H} 33 \mathrm{~N} 1 \mathrm{O} 2$ & $1.24 \mathrm{E}-06$ \\
\hline $\mathrm{S} 1$ & $\mathrm{C} 32 \mathrm{H} 46 \mathrm{~S} 1$ & 0.000123 & Acidic $\mathrm{O} 1$ & $\mathrm{C} 28 \mathrm{H} 24 \mathrm{O} 1$ & $8.86 \mathrm{E}-06$ & Basic N1O2 & $\mathrm{C} 35 \mathrm{H} 35 \mathrm{~N} 1 \mathrm{O} 2$ & $1.87 \mathrm{E}-06$ \\
\hline $\mathrm{S} 1$ & $\mathrm{C} 33 \mathrm{H} 48 \mathrm{~S} 1$ & 0.000107 & Acidic $\mathrm{O} 1$ & $\mathrm{C} 29 \mathrm{H} 26 \mathrm{O} 1$ & $5.29 \mathrm{E}-06$ & Basic N1O2 & $\mathrm{C} 36 \mathrm{H} 37 \mathrm{~N} 1 \mathrm{O} 2$ & $2.46 \mathrm{E}-06$ \\
\hline S1 & C34H50S1 & 0.000108 & Acidic $\mathrm{O} 1$ & $\mathrm{C} 24 \mathrm{H} 14 \mathrm{O} 1$ & $2.15 \mathrm{E}-05$ & Basic N1O2 & $\mathrm{C} 37 \mathrm{H} 39 \mathrm{~N} 1 \mathrm{O} 2$ & $3.22 \mathrm{E}-06$ \\
\hline S1 & $\mathrm{C} 35 \mathrm{H} 52 \mathrm{~S} 1$ & $8.84 \mathrm{E}-05$ & Acidic $\mathrm{O} 1$ & $\mathrm{C} 25 \mathrm{H} 16 \mathrm{O} 1$ & $9.55 \mathrm{E}-05$ & Basic N1O2 & $\mathrm{C} 38 \mathrm{H} 41 \mathrm{~N} 1 \mathrm{O} 2$ & $2.57 \mathrm{E}-06$ \\
\hline S1 & $\mathrm{C} 36 \mathrm{H} 54 \mathrm{~S} 1$ & $8.45 \mathrm{E}-05$ & Acidic $\mathrm{O} 1$ & $\mathrm{C} 26 \mathrm{H} 18 \mathrm{O} 1$ & 0.000119 & Basic N1O2 & $\mathrm{C} 28 \mathrm{H} 19 \mathrm{~N} 1 \mathrm{O} 2$ & $1.23 \mathrm{E}-07$ \\
\hline S1 & $\mathrm{C} 37 \mathrm{H} 56 \mathrm{~S} 1$ & $6.79 \mathrm{E}-05$ & Acidic $\mathrm{O} 1$ & $\mathrm{C} 27 \mathrm{H} 20 \mathrm{O} 1$ & $7.49 \mathrm{E}-05$ & Basic N1O2 & $\mathrm{C} 29 \mathrm{H} 21 \mathrm{~N} 1 \mathrm{O} 2$ & $6.25 \mathrm{E}-07$ \\
\hline S1 & C38H58S1 & $6.69 \mathrm{E}-05$ & Acidic $\mathrm{O} 1$ & $\mathrm{C} 28 \mathrm{H} 22 \mathrm{O} 1$ & $4.65 \mathrm{E}-05$ & Basic N1O2 & $\mathrm{C} 30 \mathrm{H} 23 \mathrm{~N} 1 \mathrm{O} 2$ & $2.05 \mathrm{E}-07$ \\
\hline S1 & C39H60S1 & $6.89 \mathrm{E}-05$ & Acidic $\mathrm{O} 1$ & $\mathrm{C} 29 \mathrm{H} 24 \mathrm{O} 1$ & $1.78 \mathrm{E}-05$ & Basic N1O2 & $\mathrm{C} 31 \mathrm{H} 25 \mathrm{~N} 1 \mathrm{O} 2$ & $2.41 \mathrm{E}-07$ \\
\hline S1 & $\mathrm{C} 40 \mathrm{H} 62 \mathrm{~S} 1$ & 4.77E-05 & Acidic O1 & $\mathrm{C} 26 \mathrm{H} 16 \mathrm{O} 1$ & $1.46 \mathrm{E}-05$ & Basic N1O2 & $\mathrm{C} 32 \mathrm{H} 27 \mathrm{~N} 1 \mathrm{O} 2$ & $2.40 \mathrm{E}-07$ \\
\hline S1 & $\mathrm{C} 41 \mathrm{H} 64 \mathrm{~S} 1$ & 4.64E-05 & Acidic O1 & $\mathrm{C} 27 \mathrm{H} 18 \mathrm{O} 1$ & $2.67 \mathrm{E}-05$ & Basic N1O2 & $\mathrm{C} 33 \mathrm{H} 29 \mathrm{~N} 1 \mathrm{O} 2$ & $4.25 \mathrm{E}-07$ \\
\hline S1 & $\mathrm{C} 42 \mathrm{H} 66 \mathrm{~S} 1$ & $3.89 \mathrm{E}-05$ & Acidic $\mathrm{O} 1$ & $\mathrm{C} 28 \mathrm{H} 20 \mathrm{O} 1$ & $2.59 \mathrm{E}-05$ & Neutral O1 & $\mathrm{C} 15 \mathrm{H} 20 \mathrm{O} 1$ & $1.86 \mathrm{E}-06$ \\
\hline $\mathrm{S} 1$ & C43H68S1 & $4.48 \mathrm{E}-05$ & Acidic $\mathrm{O} 1$ & $\mathrm{C} 29 \mathrm{H} 22 \mathrm{O} 1$ & $2.40 \mathrm{E}-05$ & Neutral O1 & $\mathrm{C} 16 \mathrm{H} 22 \mathrm{O} 1$ & 7.07E-07 \\
\hline S1 & $\mathrm{C} 44 \mathrm{H} 70 \mathrm{~S} 1$ & $3.26 \mathrm{E}-05$ & Acidic $\mathrm{O} 1$ & $\mathrm{C} 30 \mathrm{H} 24 \mathrm{O} 1$ & $7.91 \mathrm{E}-06$ & Neutral O1 & $\mathrm{C} 17 \mathrm{H} 24 \mathrm{O} 1$ & $9.85 \mathrm{E}-07$ \\
\hline S1 & $\mathrm{C} 45 \mathrm{H} 72 \mathrm{~S} 1$ & $2.83 \mathrm{E}-05$ & Acidic $\mathrm{O} 1$ & $\mathrm{C} 26 \mathrm{H} 14 \mathrm{O} 1$ & $1.68 \mathrm{E}-05$ & Neutral O1 & $\mathrm{C} 18 \mathrm{H} 26 \mathrm{O} 1$ & $1.88 \mathrm{E}-07$ \\
\hline S1 & $\mathrm{C} 46 \mathrm{H} 74 \mathrm{~S} 1$ & $2.27 \mathrm{E}-05$ & Acidic $\mathrm{O} 1$ & $\mathrm{C} 27 \mathrm{H} 16 \mathrm{O} 1$ & $4.89 \mathrm{E}-05$ & Neutral O1 & $\mathrm{C} 15 \mathrm{H} 18 \mathrm{O} 1$ & $3.13 \mathrm{E}-06$ \\
\hline S1 & $\mathrm{C} 47 \mathrm{H} 76 \mathrm{~S} 1$ & $1.36 \mathrm{E}-05$ & Acidic $\mathrm{O} 1$ & $\mathrm{C} 28 \mathrm{H} 18 \mathrm{O} 1$ & $2.99 \mathrm{E}-05$ & Neutral O1 & $\mathrm{C} 16 \mathrm{H} 20 \mathrm{O} 1$ & $6.07 \mathrm{E}-07$ \\
\hline S1 & $\mathrm{C} 48 \mathrm{H} 78 \mathrm{~S} 1$ & $1.60 \mathrm{E}-05$ & Acidic $\mathrm{O} 1$ & $\mathrm{C} 29 \mathrm{H} 20 \mathrm{O} 1$ & $4.57 \mathrm{E}-06$ & Neutral O1 & $\mathrm{C} 17 \mathrm{H} 22 \mathrm{O} 1$ & $6.57 \mathrm{E}-07$ \\
\hline S1 & C49H80S1 & $9.15 \mathrm{E}-06$ & Acidic $\mathrm{O} 1$ & $\mathrm{C} 30 \mathrm{H} 22 \mathrm{O} 1$ & $3.27 \mathrm{E}-06$ & Neutral O1 & $\mathrm{C} 15 \mathrm{H} 16 \mathrm{O} 1$ & $1.27 \mathrm{E}-05$ \\
\hline S1 & $\mathrm{C} 50 \mathrm{H} 82 \mathrm{~S} 1$ & $1.21 \mathrm{E}-05$ & Acidic O1 & $\mathrm{C} 29 \mathrm{H} 18 \mathrm{O} 1$ & $1.04 \mathrm{E}-05$ & Neutral O1 & $\mathrm{C} 16 \mathrm{H} 18 \mathrm{O} 1$ & $2.23 \mathrm{E}-06$ \\
\hline S1 & $\mathrm{C} 51 \mathrm{H} 84 \mathrm{~S} 1$ & $6.76 \mathrm{E}-06$ & Acidic $\mathrm{O} 1$ & $\mathrm{C} 30 \mathrm{H} 20 \mathrm{O} 1$ & $1.49 \mathrm{E}-05$ & Neutral O1 & $\mathrm{C} 17 \mathrm{H} 20 \mathrm{O} 1$ & $8.23 \mathrm{E}-07$ \\
\hline
\end{tabular}




\begin{tabular}{|c|c|c|c|c|c|c|c|c|}
\hline Class & formula & Mass & Class & formula & Mass & Class & formula & Mass \\
\hline $\mathrm{S} 1$ & $\mathrm{C} 52 \mathrm{H} 86 \mathrm{~S} 1$ & $3.50 \mathrm{E}-06$ & Acidic $\mathrm{O} 1$ & $\mathrm{C} 31 \mathrm{H} 22 \mathrm{O} 1$ & $4.07 \mathrm{E}-06$ & Neutral O1 & $\mathrm{C} 18 \mathrm{H} 22 \mathrm{O} 1$ & $8.58 \mathrm{E}-07$ \\
\hline $\mathrm{S} 1$ & C53H88S1 & $2.65 \mathrm{E}-06$ & Acidic $\mathrm{O} 1$ & $\mathrm{C} 32 \mathrm{H} 24 \mathrm{O} 1$ & $6.09 \mathrm{E}-06$ & Neutral O1 & $\mathrm{C} 19 \mathrm{H} 24 \mathrm{O} 1$ & $1.63 \mathrm{E}-06$ \\
\hline $\mathrm{S} 1$ & C54H90S1 & $2.26 \mathrm{E}-06$ & Acidic $\mathrm{O} 1$ & $\mathrm{C} 30 \mathrm{H} 18 \mathrm{O} 1$ & $1.52 \mathrm{E}-05$ & Neutral O1 & $\mathrm{C} 16 \mathrm{H} 16 \mathrm{O} 1$ & $1.04 \mathrm{E}-05$ \\
\hline $\mathrm{S} 1$ & $\mathrm{C} 55 \mathrm{H} 92 \mathrm{~S} 1$ & $2.26 \mathrm{E}-06$ & Acidic $\mathrm{O} 1$ & $\mathrm{C} 31 \mathrm{H} 20 \mathrm{O} 1$ & $3.28 \mathrm{E}-06$ & Neutral O1 & $\mathrm{C} 17 \mathrm{H} 18 \mathrm{O} 1$ & $3.48 \mathrm{E}-05$ \\
\hline $\mathrm{S} 1$ & C14H8S1 & $3.60 \mathrm{E}-06$ & Acidic $\mathrm{O} 1$ & $\mathrm{C} 32 \mathrm{H} 22 \mathrm{O} 1$ & $4.03 \mathrm{E}-06$ & Neutral O1 & $\mathrm{C} 18 \mathrm{H} 20 \mathrm{O} 1$ & $3.96 \mathrm{E}-05$ \\
\hline $\mathrm{S} 1$ & C15H10S1 & $6.96 \mathrm{E}-05$ & Acidic $\mathrm{O} 1$ & $\mathrm{C} 33 \mathrm{H} 24 \mathrm{O} 1$ & $3.43 \mathrm{E}-06$ & Neutral O1 & $\mathrm{C} 19 \mathrm{H} 22 \mathrm{O} 1$ & $8.32 \mathrm{E}-06$ \\
\hline $\mathrm{S} 1$ & $\mathrm{C} 16 \mathrm{H} 12 \mathrm{~S} 1$ & 0.000273 & Acidic $\mathrm{O} 1$ & $\mathrm{C} 30 \mathrm{H} 16 \mathrm{O} 1$ & $3.07 \mathrm{E}-06$ & Neutral O1 & $\mathrm{C} 20 \mathrm{H} 24 \mathrm{O} 1$ & $3.68 \mathrm{E}-06$ \\
\hline $\mathrm{S} 1$ & $\mathrm{C} 17 \mathrm{H} 14 \mathrm{~S} 1$ & 0.000465 & Acidic $\mathrm{O} 1$ & $\mathrm{C} 31 \mathrm{H} 18 \mathrm{O} 1$ & $4.15 \mathrm{E}-06$ & Neutral O1 & $\mathrm{C} 21 \mathrm{H} 26 \mathrm{O} 1$ & $3.80 \mathrm{E}-06$ \\
\hline $\mathrm{S} 1$ & C18H16S1 & 0.000399 & Acidic $\mathrm{O} 1$ & $\mathrm{C} 32 \mathrm{H} 20 \mathrm{O} 1$ & $3.50 \mathrm{E}-06$ & Neutral O1 & $\mathrm{C} 22 \mathrm{H} 28 \mathrm{O} 1$ & $1.13 \mathrm{E}-05$ \\
\hline $\mathrm{S} 1$ & C19H18S1 & 0.000301 & Acidic O2 & $\mathrm{C} 15 \mathrm{H} 16 \mathrm{O} 2$ & $2.27 \mathrm{E}-05$ & Neutral O1 & $\mathrm{C} 23 \mathrm{H} 30 \mathrm{O} 1$ & $1.53 \mathrm{E}-06$ \\
\hline $\mathrm{S} 1$ & $\mathrm{C} 20 \mathrm{H} 20 \mathrm{~S} 1$ & 0.000255 & Acidic O2 & $\mathrm{C} 16 \mathrm{H} 18 \mathrm{O} 2$ & $5.26 \mathrm{E}-06$ & Neutral O1 & $\mathrm{C} 24 \mathrm{H} 32 \mathrm{O} 1$ & $1.52 \mathrm{E}-06$ \\
\hline $\mathrm{S} 1$ & $\mathrm{C} 21 \mathrm{H} 22 \mathrm{~S} 1$ & 0.000217 & Acidic $\mathrm{O} 2$ & $\mathrm{C} 17 \mathrm{H} 20 \mathrm{O} 2$ & $4.27 \mathrm{E}-06$ & Neutral O1 & $\mathrm{C} 25 \mathrm{H} 34 \mathrm{O} 1$ & $9.96 \mathrm{E}-07$ \\
\hline $\mathrm{S} 1$ & $\mathrm{C} 22 \mathrm{H} 24 \mathrm{~S} 1$ & 0.00021 & Acidic $\mathrm{O} 2$ & $\mathrm{C} 18 \mathrm{H} 22 \mathrm{O} 2$ & $2.24 \mathrm{E}-06$ & Neutral O1 & $\mathrm{C} 26 \mathrm{H} 36 \mathrm{O} 1$ & $1.49 \mathrm{E}-06$ \\
\hline $\mathrm{S} 1$ & $\mathrm{C} 23 \mathrm{H} 26 \mathrm{~S} 1$ & 0.000192 & Acidic $\mathrm{O} 2$ & $\mathrm{C} 15 \mathrm{H} 14 \mathrm{O} 2$ & $1.05 \mathrm{E}-05$ & Neutral O1 & $\mathrm{C} 27 \mathrm{H} 38 \mathrm{O} 1$ & $2.70 \mathrm{E}-06$ \\
\hline $\mathrm{S} 1$ & $\mathrm{C} 24 \mathrm{H} 28 \mathrm{~S} 1$ & 0.000188 & Acidic $\mathrm{O} 2$ & $\mathrm{C} 16 \mathrm{H} 16 \mathrm{O} 2$ & $2.40 \mathrm{E}-05$ & Neutral O1 & $\mathrm{C} 28 \mathrm{H} 40 \mathrm{O} 1$ & $3.74 \mathrm{E}-06$ \\
\hline $\mathrm{S} 1$ & $\mathrm{C} 25 \mathrm{H} 30 \mathrm{~S} 1$ & 0.000172 & Acidic $\mathrm{O} 2$ & $\mathrm{C} 17 \mathrm{H} 18 \mathrm{O} 2$ & $2.42 \mathrm{E}-05$ & Neutral O1 & $\mathrm{C} 29 \mathrm{H} 42 \mathrm{O} 1$ & $3.30 \mathrm{E}-06$ \\
\hline $\mathrm{S} 1$ & $\mathrm{C} 26 \mathrm{H} 32 \mathrm{~S} 1$ & 0.000162 & Acidic $\mathrm{O} 2$ & $\mathrm{C} 18 \mathrm{H} 20 \mathrm{O} 2$ & $3.10 \mathrm{E}-05$ & Neutral O1 & $\mathrm{C} 30 \mathrm{H} 44 \mathrm{O} 1$ & $7.40 \mathrm{E}-06$ \\
\hline $\mathrm{S} 1$ & $\mathrm{C} 27 \mathrm{H} 34 \mathrm{~S} 1$ & 0.000156 & Acidic $\mathrm{O} 2$ & $\mathrm{C} 19 \mathrm{H} 22 \mathrm{O} 2$ & $8.02 \mathrm{E}-06$ & Neutral O1 & $\mathrm{C} 31 \mathrm{H} 46 \mathrm{O} 1$ & $2.67 \mathrm{E}-06$ \\
\hline $\mathrm{S} 1$ & $\mathrm{C} 28 \mathrm{H} 36 \mathrm{~S} 1$ & 0.000143 & Acidic $\mathrm{O} 2$ & $\mathrm{C} 20 \mathrm{H} 24 \mathrm{O} 2$ & 4.62E-06 & Neutral O1 & $\mathrm{C} 32 \mathrm{H} 48 \mathrm{O} 1$ & $3.21 \mathrm{E}-06$ \\
\hline $\mathrm{S} 1$ & $\mathrm{C} 29 \mathrm{H} 38 \mathrm{~S} 1$ & 0.000133 & Acidic $\mathrm{O} 2$ & $\mathrm{C} 21 \mathrm{H} 26 \mathrm{O} 2$ & $3.05 \mathrm{E}-06$ & Neutral O1 & $\mathrm{C} 33 \mathrm{H} 50 \mathrm{O} 1$ & $1.32 \mathrm{E}-06$ \\
\hline $\mathrm{S} 1$ & $\mathrm{C} 30 \mathrm{H} 40 \mathrm{~S} 1$ & 0.000131 & Acidic $\mathrm{O} 2$ & $\mathrm{C} 22 \mathrm{H} 28 \mathrm{O} 2$ & $4.66 \mathrm{E}-06$ & Neutral O1 & $\mathrm{C} 34 \mathrm{H} 52 \mathrm{O} 1$ & $6.87 \mathrm{E}-07$ \\
\hline $\mathrm{S} 1$ & $\mathrm{C} 31 \mathrm{H} 42 \mathrm{~S} 1$ & 0.000138 & Acidic $\mathrm{O} 2$ & $\mathrm{C} 15 \mathrm{H} 12 \mathrm{O} 2$ & $1.41 \mathrm{E}-05$ & Neutral O1 & $\mathrm{C} 35 \mathrm{H} 54 \mathrm{O} 1$ & $1.40 \mathrm{E}-06$ \\
\hline $\mathrm{S} 1$ & $\mathrm{C} 32 \mathrm{H} 44 \mathrm{~S} 1$ & 0.000111 & Acidic $\mathrm{O} 2$ & $\mathrm{C} 16 \mathrm{H} 14 \mathrm{O} 2$ & $3.92 \mathrm{E}-05$ & Neutral O1 & $\mathrm{C} 15 \mathrm{H} 12 \mathrm{O} 1$ & $9.74 \mathrm{E}-07$ \\
\hline S1 & $\mathrm{C} 33 \mathrm{H} 46 \mathrm{~S} 1$ & $9.74 \mathrm{E}-05$ & Acidic $\mathrm{O} 2$ & $\mathrm{C} 17 \mathrm{H} 16 \mathrm{O} 2$ & $3.58 \mathrm{E}-05$ & Neutral O1 & $\mathrm{C} 16 \mathrm{H} 14 \mathrm{O} 1$ & $5.69 \mathrm{E}-05$ \\
\hline S1 & $\mathrm{C} 34 \mathrm{H} 48 \mathrm{~S} 1$ & $9.56 \mathrm{E}-05$ & Acidic $\mathrm{O} 2$ & $\mathrm{C} 18 \mathrm{H} 18 \mathrm{O} 2$ & $2.94 \mathrm{E}-05$ & Neutral O1 & $\mathrm{C} 17 \mathrm{H} 16 \mathrm{O} 1$ & 0.000475 \\
\hline S1 & C35H50S1 & $7.66 \mathrm{E}-05$ & Acidic $\mathrm{O} 2$ & $\mathrm{C} 19 \mathrm{H} 20 \mathrm{O} 2$ & $2.12 \mathrm{E}-05$ & Neutral O1 & $\mathrm{C} 18 \mathrm{H} 18 \mathrm{O} 1$ & 0.000475 \\
\hline $\mathrm{S} 1$ & $\mathrm{C} 36 \mathrm{H} 52 \mathrm{~S} 1$ & $7.46 \mathrm{E}-05$ & Acidic $\mathrm{O} 2$ & $\mathrm{C} 20 \mathrm{H} 22 \mathrm{O} 2$ & $2.35 \mathrm{E}-05$ & Neutral O1 & $\mathrm{C} 19 \mathrm{H} 20 \mathrm{O} 1$ & 0.000355 \\
\hline $\mathrm{S} 1$ & $\mathrm{C} 37 \mathrm{H} 54 \mathrm{~S} 1$ & $7.72 \mathrm{E}-05$ & Acidic $\mathrm{O} 2$ & $\mathrm{C} 21 \mathrm{H} 24 \mathrm{O} 2$ & $6.77 \mathrm{E}-06$ & Neutral O1 & $\mathrm{C} 20 \mathrm{H} 22 \mathrm{O} 1$ & 0.000222 \\
\hline $\mathrm{S} 1$ & C38H56S1 & $6.30 \mathrm{E}-05$ & Acidic $\mathrm{O} 2$ & $\mathrm{C} 22 \mathrm{H} 26 \mathrm{O} 2$ & $9.55 \mathrm{E}-06$ & Neutral O1 & $\mathrm{C} 21 \mathrm{H} 24 \mathrm{O} 1$ & 0.000127 \\
\hline $\mathrm{S} 1$ & C39H58S1 & $5.80 \mathrm{E}-05$ & Acidic $\mathrm{O} 2$ & $\mathrm{C} 16 \mathrm{H} 12 \mathrm{O} 2$ & $2.42 \mathrm{E}-05$ & Neutral O1 & $\mathrm{C} 22 \mathrm{H} 26 \mathrm{O} 1$ & 7.74E-05 \\
\hline $\mathrm{S} 1$ & C40H60S1 & $5.54 \mathrm{E}-05$ & Acidic $\mathrm{O} 2$ & $\mathrm{C} 17 \mathrm{H} 14 \mathrm{O} 2$ & 5.54E-05 & Neutral O1 & $\mathrm{C} 23 \mathrm{H} 28 \mathrm{O} 1$ & $5.20 \mathrm{E}-05$ \\
\hline $\mathrm{S} 1$ & $\mathrm{C} 41 \mathrm{H} 62 \mathrm{~S} 1$ & $4.14 \mathrm{E}-05$ & Acidic $\mathrm{O} 2$ & $\mathrm{C} 18 \mathrm{H} 16 \mathrm{O} 2$ & $8.03 \mathrm{E}-05$ & Neutral O1 & $\mathrm{C} 24 \mathrm{H} 30 \mathrm{O} 1$ & $3.05 \mathrm{E}-05$ \\
\hline $\mathrm{S} 1$ & $\mathrm{C} 42 \mathrm{H} 64 \mathrm{~S} 1$ & $4.05 \mathrm{E}-05$ & Acidic $\mathrm{O} 2$ & $\mathrm{C} 19 \mathrm{H} 18 \mathrm{O} 2$ & $8.03 \mathrm{E}-05$ & Neutral O1 & $\mathrm{C} 25 \mathrm{H} 32 \mathrm{O} 1$ & $2.99 \mathrm{E}-05$ \\
\hline S1 & C43H66S1 & $3.78 \mathrm{E}-05$ & Acidic $\mathrm{O} 2$ & $\mathrm{C} 20 \mathrm{H} 20 \mathrm{O} 2$ & $6.16 \mathrm{E}-05$ & Neutral O1 & $\mathrm{C} 26 \mathrm{H} 34 \mathrm{O} 1$ & $1.67 \mathrm{E}-05$ \\
\hline S1 & C44H68S1 & $2.45 \mathrm{E}-05$ & Acidic $\mathrm{O} 2$ & $\mathrm{C} 21 \mathrm{H} 22 \mathrm{O} 2$ & $3.56 \mathrm{E}-05$ & Neutral O1 & $\mathrm{C} 27 \mathrm{H} 36 \mathrm{O} 1$ & $2.67 \mathrm{E}-05$ \\
\hline S1 & $\mathrm{C} 45 \mathrm{H} 70 \mathrm{~S} 1$ & $2.25 \mathrm{E}-05$ & Acidic $\mathrm{O} 2$ & $\mathrm{C} 22 \mathrm{H} 24 \mathrm{O} 2$ & $1.36 \mathrm{E}-05$ & Neutral O1 & $\mathrm{C} 28 \mathrm{H} 38 \mathrm{O} 1$ & $2.71 \mathrm{E}-05$ \\
\hline S1 & $\mathrm{C} 46 \mathrm{H} 72 \mathrm{~S} 1$ & $2.21 \mathrm{E}-05$ & Acidic $\mathrm{O} 2$ & $\mathrm{C} 23 \mathrm{H} 26 \mathrm{O} 2$ & $7.46 \mathrm{E}-06$ & Neutral O1 & $\mathrm{C} 29 \mathrm{H} 40 \mathrm{O} 1$ & 2.94E-05 \\
\hline S1 & $\mathrm{C} 47 \mathrm{H} 74 \mathrm{~S} 1$ & $1.30 \mathrm{E}-05$ & Acidic $\mathrm{O} 2$ & $\mathrm{C} 15 \mathrm{H} 8 \mathrm{O} 2$ & 8.91E-06 & Neutral O1 & $\mathrm{C} 30 \mathrm{H} 42 \mathrm{O} 1$ & $1.97 \mathrm{E}-05$ \\
\hline
\end{tabular}




\begin{tabular}{|c|c|c|c|c|c|c|c|c|}
\hline Class & formula & Mass & Class & formula & Mass & Class & formula & Mass \\
\hline $\mathrm{S} 1$ & $\mathrm{C} 48 \mathrm{H} 76 \mathrm{~S} 1$ & $1.37 \mathrm{E}-05$ & Acidic $\mathrm{O} 2$ & $\mathrm{C} 16 \mathrm{H} 10 \mathrm{O} 2$ & $1.55 \mathrm{E}-05$ & Neutral O1 & $\mathrm{C} 31 \mathrm{H} 44 \mathrm{O} 1$ & $2.00 \mathrm{E}-05$ \\
\hline $\mathrm{S} 1$ & $\mathrm{C} 49 \mathrm{H} 78 \mathrm{~S} 1$ & $1.97 \mathrm{E}-05$ & Acidic $\mathrm{O} 2$ & $\mathrm{C} 17 \mathrm{H} 12 \mathrm{O} 2$ & $8.05 \mathrm{E}-05$ & Neutral O1 & $\mathrm{C} 32 \mathrm{H} 46 \mathrm{O} 1$ & $1.64 \mathrm{E}-05$ \\
\hline $\mathrm{S} 1$ & C50H80S1 & $2.53 \mathrm{E}-06$ & Acidic $\mathrm{O} 2$ & $\mathrm{C} 18 \mathrm{H} 14 \mathrm{O} 2$ & 0.000205 & Neutral O1 & $\mathrm{C} 33 \mathrm{H} 48 \mathrm{O} 1$ & $1.20 \mathrm{E}-05$ \\
\hline $\mathrm{S} 1$ & $\mathrm{C} 51 \mathrm{H} 82 \mathrm{~S} 1$ & $6.21 \mathrm{E}-06$ & Acidic $\mathrm{O} 2$ & $\mathrm{C} 19 \mathrm{H} 16 \mathrm{O} 2$ & 0.00021 & Neutral O1 & $\mathrm{C} 34 \mathrm{H} 50 \mathrm{O} 1$ & $5.21 \mathrm{E}-06$ \\
\hline $\mathrm{S} 1$ & $\mathrm{C} 52 \mathrm{H} 84 \mathrm{~S} 1$ & $5.00 \mathrm{E}-06$ & Acidic $\mathrm{O} 2$ & $\mathrm{C} 20 \mathrm{H} 18 \mathrm{O} 2$ & 0.000199 & Neutral O1 & C35H52O1 & $7.62 \mathrm{E}-06$ \\
\hline $\mathrm{S} 1$ & C53H86S1 & $3.13 \mathrm{E}-06$ & Acidic $\mathrm{O} 2$ & $\mathrm{C} 21 \mathrm{H} 20 \mathrm{O} 2$ & 0.000127 & Neutral O1 & $\mathrm{C} 36 \mathrm{H} 54 \mathrm{O} 1$ & $6.67 \mathrm{E}-06$ \\
\hline $\mathrm{S} 1$ & C14H6S1 & $2.47 \mathrm{E}-06$ & Acidic $\mathrm{O} 2$ & $\mathrm{C} 22 \mathrm{H} 22 \mathrm{O} 2$ & $8.67 \mathrm{E}-05$ & Neutral O1 & $\mathrm{C} 37 \mathrm{H} 56 \mathrm{O} 1$ & $1.15 \mathrm{E}-05$ \\
\hline $\mathrm{S} 1$ & C15H8S1 & $5.35 \mathrm{E}-06$ & Acidic $\mathrm{O} 2$ & $\mathrm{C} 23 \mathrm{H} 24 \mathrm{O} 2$ & $5.35 \mathrm{E}-05$ & Neutral O1 & C38H58O1 & $5.63 \mathrm{E}-06$ \\
\hline $\mathrm{S} 1$ & C16H10S1 & 0.000234 & Acidic $\mathrm{O} 2$ & $\mathrm{C} 24 \mathrm{H} 26 \mathrm{O} 2$ & $2.85 \mathrm{E}-05$ & Neutral O1 & $\mathrm{C} 39 \mathrm{H} 60 \mathrm{O} 1$ & $2.81 \mathrm{E}-06$ \\
\hline $\mathrm{S} 1$ & $\mathrm{C} 17 \mathrm{H} 12 \mathrm{~S} 1$ & 0.001289 & Acidic O2 & $\mathrm{C} 25 \mathrm{H} 28 \mathrm{O} 2$ & $2.48 \mathrm{E}-05$ & Neutral O1 & $\mathrm{C} 40 \mathrm{H} 62 \mathrm{O} 1$ & $3.02 \mathrm{E}-06$ \\
\hline $\mathrm{S} 1$ & $\mathrm{C} 18 \mathrm{H} 14 \mathrm{~S} 1$ & 0.002629 & Acidic O2 & $\mathrm{C} 26 \mathrm{H} 30 \mathrm{O} 2$ & $1.56 \mathrm{E}-05$ & Neutral O1 & $\mathrm{C} 16 \mathrm{H} 12 \mathrm{O} 1$ & $3.31 \mathrm{E}-07$ \\
\hline $\mathrm{S} 1$ & C19H16S1 & 0.002951 & Acidic $\mathrm{O} 2$ & $\mathrm{C} 27 \mathrm{H} 32 \mathrm{O} 2$ & $9.70 \mathrm{E}-06$ & Neutral O1 & $\mathrm{C} 17 \mathrm{H} 14 \mathrm{O} 1$ & $4.22 \mathrm{E}-05$ \\
\hline $\mathrm{S} 1$ & C20H18S1 & 0.002032 & Acidic $\mathrm{O} 2$ & $\mathrm{C} 28 \mathrm{H} 34 \mathrm{O} 2$ & $1.95 \mathrm{E}-05$ & Neutral O1 & $\mathrm{C} 18 \mathrm{H} 16 \mathrm{O} 1$ & 0.000134 \\
\hline $\mathrm{S} 1$ & $\mathrm{C} 21 \mathrm{H} 20 \mathrm{~S} 1$ & 0.001084 & Acidic $\mathrm{O} 2$ & $\mathrm{C} 29 \mathrm{H} 36 \mathrm{O} 2$ & $1.25 \mathrm{E}-05$ & Neutral O1 & $\mathrm{C} 19 \mathrm{H} 18 \mathrm{O} 1$ & 0.000274 \\
\hline $\mathrm{S} 1$ & $\mathrm{C} 22 \mathrm{H} 22 \mathrm{~S} 1$ & 0.000564 & Acidic $\mathrm{O} 2$ & $\mathrm{C} 30 \mathrm{H} 38 \mathrm{O} 2$ & $3.37 \mathrm{E}-05$ & Neutral O1 & $\mathrm{C} 20 \mathrm{H} 20 \mathrm{O} 1$ & 0.0004 \\
\hline $\mathrm{S} 1$ & $\mathrm{C} 23 \mathrm{H} 24 \mathrm{~S} 1$ & 0.000368 & Acidic $\mathrm{O} 2$ & $\mathrm{C} 31 \mathrm{H} 40 \mathrm{O} 2$ & $4.25 \mathrm{E}-06$ & Neutral O1 & $\mathrm{C} 21 \mathrm{H} 22 \mathrm{O} 1$ & 0.000314 \\
\hline $\mathrm{S} 1$ & $\mathrm{C} 24 \mathrm{H} 26 \mathrm{~S} 1$ & 0.000284 & Acidic $\mathrm{O} 2$ & $\mathrm{C} 32 \mathrm{H} 42 \mathrm{O} 2$ & $4.32 \mathrm{E}-06$ & Neutral O1 & $\mathrm{C} 22 \mathrm{H} 24 \mathrm{O} 1$ & 0.000235 \\
\hline $\mathrm{S} 1$ & $\mathrm{C} 25 \mathrm{H} 28 \mathrm{~S} 1$ & 0.000223 & Acidic $\mathrm{O} 2$ & $\mathrm{C} 17 \mathrm{H} 10 \mathrm{O} 2$ & $2.82 \mathrm{E}-05$ & Neutral O1 & $\mathrm{C} 23 \mathrm{H} 26 \mathrm{O} 1$ & 0.000153 \\
\hline $\mathrm{S} 1$ & $\mathrm{C} 26 \mathrm{H} 30 \mathrm{~S} 1$ & 0.000194 & Acidic $\mathrm{O} 2$ & $\mathrm{C} 18 \mathrm{H} 12 \mathrm{O} 2$ & 0.000119 & Neutral O1 & $\mathrm{C} 24 \mathrm{H} 28 \mathrm{O} 1$ & 0.000105 \\
\hline $\mathrm{S} 1$ & $\mathrm{C} 27 \mathrm{H} 32 \mathrm{~S} 1$ & 0.000182 & Acidic $\mathrm{O} 2$ & $\mathrm{C} 19 \mathrm{H} 14 \mathrm{O} 2$ & 0.00038 & Neutral O1 & $\mathrm{C} 25 \mathrm{H} 30 \mathrm{O} 1$ & $6.10 \mathrm{E}-05$ \\
\hline $\mathrm{S} 1$ & $\mathrm{C} 28 \mathrm{H} 34 \mathrm{~S} 1$ & 0.000178 & Acidic $\mathrm{O} 2$ & $\mathrm{C} 20 \mathrm{H} 16 \mathrm{O} 2$ & 0.000741 & Neutral O1 & $\mathrm{C} 26 \mathrm{H} 32 \mathrm{O} 1$ & 4.94E-05 \\
\hline $\mathrm{S} 1$ & $\mathrm{C} 29 \mathrm{H} 36 \mathrm{~S} 1$ & 0.000173 & Acidic $\mathrm{O} 2$ & $\mathrm{C} 21 \mathrm{H} 18 \mathrm{O} 2$ & 0.000668 & Neutral O1 & $\mathrm{C} 27 \mathrm{H} 34 \mathrm{O} 1$ & $6.07 \mathrm{E}-05$ \\
\hline S1 & $\mathrm{C} 30 \mathrm{H} 38 \mathrm{~S} 1$ & 0.000155 & Acidic $\mathrm{O} 2$ & $\mathrm{C} 22 \mathrm{H} 20 \mathrm{O} 2$ & 0.00036 & Neutral O1 & $\mathrm{C} 28 \mathrm{H} 36 \mathrm{O} 1$ & 4.34E-05 \\
\hline S1 & $\mathrm{C} 31 \mathrm{H} 40 \mathrm{~S} 1$ & 0.000141 & Acidic $\mathrm{O} 2$ & $\mathrm{C} 23 \mathrm{H} 22 \mathrm{O} 2$ & 0.000176 & Neutral O1 & $\mathrm{C} 29 \mathrm{H} 38 \mathrm{O} 1$ & $5.18 \mathrm{E}-05$ \\
\hline S1 & $\mathrm{C} 32 \mathrm{H} 42 \mathrm{~S} 1$ & 0.000131 & Acidic $\mathrm{O} 2$ & $\mathrm{C} 24 \mathrm{H} 24 \mathrm{O} 2$ & 0.000103 & Neutral O1 & $\mathrm{C} 30 \mathrm{H} 40 \mathrm{O} 1$ & $3.52 \mathrm{E}-05$ \\
\hline S1 & $\mathrm{C} 33 \mathrm{H} 44 \mathrm{~S} 1$ & 0.000117 & Acidic $\mathrm{O} 2$ & $\mathrm{C} 25 \mathrm{H} 26 \mathrm{O} 2$ & $4.77 \mathrm{E}-05$ & Neutral O1 & $\mathrm{C} 31 \mathrm{H} 42 \mathrm{O} 1$ & $4.62 \mathrm{E}-05$ \\
\hline S1 & $\mathrm{C} 34 \mathrm{H} 46 \mathrm{~S} 1$ & $9.95 \mathrm{E}-05$ & Acidic $\mathrm{O} 2$ & $\mathrm{C} 26 \mathrm{H} 28 \mathrm{O} 2$ & $4.65 \mathrm{E}-05$ & Neutral O1 & $\mathrm{C} 32 \mathrm{H} 44 \mathrm{O} 1$ & $3.55 \mathrm{E}-05$ \\
\hline S1 & $\mathrm{C} 35 \mathrm{H} 48 \mathrm{~S} 1$ & 0.0001 & Acidic $\mathrm{O} 2$ & $\mathrm{C} 27 \mathrm{H} 30 \mathrm{O} 2$ & $2.44 \mathrm{E}-05$ & Neutral O1 & $\mathrm{C} 33 \mathrm{H} 46 \mathrm{O} 1$ & $2.40 \mathrm{E}-05$ \\
\hline S1 & $\mathrm{C} 36 \mathrm{H} 50 \mathrm{~S} 1$ & $7.31 \mathrm{E}-05$ & Acidic $\mathrm{O} 2$ & $\mathrm{C} 28 \mathrm{H} 32 \mathrm{O} 2$ & $1.55 \mathrm{E}-05$ & Neutral O1 & $\mathrm{C} 34 \mathrm{H} 48 \mathrm{O} 1$ & $2.00 \mathrm{E}-05$ \\
\hline S1 & $\mathrm{C} 37 \mathrm{H} 52 \mathrm{~S} 1$ & $8.34 \mathrm{E}-05$ & Acidic $\mathrm{O} 2$ & $\mathrm{C} 30 \mathrm{H} 36 \mathrm{O} 2$ & $7.78 \mathrm{E}-06$ & Neutral O1 & $\mathrm{C} 35 \mathrm{H} 50 \mathrm{O} 1$ & $1.36 \mathrm{E}-05$ \\
\hline S1 & $\mathrm{C} 38 \mathrm{H} 54 \mathrm{~S} 1$ & $6.69 \mathrm{E}-05$ & Acidic $\mathrm{O} 2$ & $\mathrm{C} 31 \mathrm{H} 38 \mathrm{O} 2$ & $8.49 \mathrm{E}-06$ & Neutral O1 & $\mathrm{C} 36 \mathrm{H} 52 \mathrm{O} 1$ & $8.84 \mathrm{E}-06$ \\
\hline S1 & C39H56S1 & $5.15 \mathrm{E}-05$ & Acidic $\mathrm{O} 2$ & $\mathrm{C} 32 \mathrm{H} 40 \mathrm{O} 2$ & $4.82 \mathrm{E}-06$ & Neutral O1 & $\mathrm{C} 37 \mathrm{H} 54 \mathrm{O} 1$ & $9.46 \mathrm{E}-06$ \\
\hline S1 & C40H58S1 & $5.52 \mathrm{E}-05$ & Acidic $\mathrm{O} 2$ & $\mathrm{C} 33 \mathrm{H} 42 \mathrm{O} 2$ & $7.80 \mathrm{E}-06$ & Neutral O1 & $\mathrm{C} 38 \mathrm{H} 56 \mathrm{O} 1$ & $2.88 \mathrm{E}-06$ \\
\hline S1 & C41H60S1 & $4.91 \mathrm{E}-05$ & Acidic $\mathrm{O} 2$ & $\mathrm{C} 19 \mathrm{H} 12 \mathrm{O} 2$ & $4.26 \mathrm{E}-05$ & Neutral O1 & $\mathrm{C} 39 \mathrm{H} 58 \mathrm{O} 1$ & $3.83 \mathrm{E}-06$ \\
\hline S1 & $\mathrm{C} 42 \mathrm{H} 62 \mathrm{~S} 1$ & $4.52 \mathrm{E}-05$ & Acidic $\mathrm{O} 2$ & $\mathrm{C} 20 \mathrm{H} 14 \mathrm{O} 2$ & 0.000115 & Neutral O1 & $\mathrm{C} 40 \mathrm{H} 60 \mathrm{O} 1$ & $2.90 \mathrm{E}-06$ \\
\hline S1 & C43H64S1 & $3.93 \mathrm{E}-05$ & Acidic $\mathrm{O} 2$ & $\mathrm{C} 21 \mathrm{H} 16 \mathrm{O} 2$ & 0.000175 & Neutral O1 & $\mathrm{C} 41 \mathrm{H} 62 \mathrm{O} 1$ & $1.91 \mathrm{E}-06$ \\
\hline S1 & C44H66S1 & $2.92 \mathrm{E}-05$ & Acidic O2 & $\mathrm{C} 22 \mathrm{H} 18 \mathrm{O} 2$ & 0.000219 & Neutral O1 & $\mathrm{C} 42 \mathrm{H} 64 \mathrm{O} 1$ & $1.19 \mathrm{E}-06$ \\
\hline S1 & $\mathrm{C} 45 \mathrm{H} 68 \mathrm{~S} 1$ & $2.15 \mathrm{E}-05$ & Acidic $\mathrm{O} 2$ & $\mathrm{C} 23 \mathrm{H} 20 \mathrm{O} 2$ & 0.000204 & Neutral O1 & $\mathrm{C} 17 \mathrm{H} 12 \mathrm{O} 1$ & $2.04 \mathrm{E}-05$ \\
\hline
\end{tabular}




\begin{tabular}{|c|c|c|c|c|c|c|c|c|}
\hline Class & formula & Mass & Class & formula & Mass & Class & formula & Mass \\
\hline S1 & $\mathrm{C} 46 \mathrm{H} 70 \mathrm{~S} 1$ & $1.84 \mathrm{E}-05$ & Acidic $\mathrm{O} 2$ & $\mathrm{C} 24 \mathrm{H} 22 \mathrm{O} 2$ & 0.000121 & Neutral O1 & $\mathrm{C} 18 \mathrm{H} 14 \mathrm{O} 1$ & 0.000126 \\
\hline S1 & $\mathrm{C} 47 \mathrm{H} 72 \mathrm{~S} 1$ & $1.55 \mathrm{E}-05$ & Acidic $\mathrm{O} 2$ & $\mathrm{C} 25 \mathrm{H} 24 \mathrm{O} 2$ & $8.06 \mathrm{E}-05$ & Neutral O1 & C19H16O1 & 0.000297 \\
\hline S1 & $\mathrm{C} 48 \mathrm{H} 74 \mathrm{~S} 1$ & $3.31 \mathrm{E}-06$ & Acidic $\mathrm{O} 2$ & $\mathrm{C} 26 \mathrm{H} 26 \mathrm{O} 2$ & 4.44E-05 & Neutral O1 & $\mathrm{C} 20 \mathrm{H} 18 \mathrm{O} 1$ & 0.000438 \\
\hline S1 & $\mathrm{C} 49 \mathrm{H} 76 \mathrm{~S} 1$ & $1.29 \mathrm{E}-05$ & Acidic $\mathrm{O} 2$ & $\mathrm{C} 27 \mathrm{H} 28 \mathrm{O} 2$ & $1.64 \mathrm{E}-05$ & Neutral O1 & $\mathrm{C} 21 \mathrm{H} 20 \mathrm{O} 1$ & 0.000413 \\
\hline S1 & $\mathrm{C} 50 \mathrm{H} 78 \mathrm{~S} 1$ & $2.16 \mathrm{E}-06$ & Acidic $\mathrm{O} 2$ & $\mathrm{C} 28 \mathrm{H} 30 \mathrm{O} 2$ & $1.80 \mathrm{E}-05$ & Neutral O1 & $\mathrm{C} 22 \mathrm{H} 22 \mathrm{O} 1$ & 0.000288 \\
\hline S1 & C51H80S1 & $5.65 \mathrm{E}-06$ & Acidic $\mathrm{O} 2$ & $\mathrm{C} 29 \mathrm{H} 32 \mathrm{O} 2$ & $1.71 \mathrm{E}-05$ & Neutral O1 & $\mathrm{C} 23 \mathrm{H} 24 \mathrm{O} 1$ & 0.000173 \\
\hline S1 & $\mathrm{C} 52 \mathrm{H} 82 \mathrm{~S} 1$ & $2.16 \mathrm{E}-06$ & Acidic $\mathrm{O} 2$ & $\mathrm{C} 30 \mathrm{H} 34 \mathrm{O} 2$ & $3.44 \mathrm{E}-06$ & Neutral O1 & $\mathrm{C} 24 \mathrm{H} 26 \mathrm{O} 1$ & 0.000152 \\
\hline S1 & $\mathrm{C} 53 \mathrm{H} 84 \mathrm{~S} 1$ & 2.71E-06 & Acidic $\mathrm{O} 2$ & $\mathrm{C} 31 \mathrm{H} 36 \mathrm{O} 2$ & $8.53 \mathrm{E}-06$ & Neutral O1 & $\mathrm{C} 25 \mathrm{H} 28 \mathrm{O} 1$ & 0.000104 \\
\hline S1 & $\mathrm{C} 54 \mathrm{H} 86 \mathrm{~S} 1$ & $5.20 \mathrm{E}-06$ & Acidic $\mathrm{O} 2$ & $\mathrm{C} 33 \mathrm{H} 40 \mathrm{O} 2$ & $8.57 \mathrm{E}-06$ & Neutral O1 & $\mathrm{C} 26 \mathrm{H} 30 \mathrm{O} 1$ & $8.13 \mathrm{E}-05$ \\
\hline S1 & $\mathrm{C} 55 \mathrm{H} 88 \mathrm{~S} 1$ & $2.95 \mathrm{E}-06$ & Acidic $\mathrm{O} 2$ & $\mathrm{C} 34 \mathrm{H} 42 \mathrm{O} 2$ & 4.17E-06 & Neutral O1 & $\mathrm{C} 27 \mathrm{H} 32 \mathrm{O} 1$ & $7.31 \mathrm{E}-05$ \\
\hline S1 & C56H90S1 & $5.59 \mathrm{E}-06$ & Acidic $\mathrm{O} 2$ & $\mathrm{C} 19 \mathrm{H} 10 \mathrm{O} 2$ & $1.76 \mathrm{E}-05$ & Neutral O1 & $\mathrm{C} 28 \mathrm{H} 34 \mathrm{O} 1$ & 7.77E-05 \\
\hline S1 & C15H6S1 & $8.84 \mathrm{E}-07$ & Acidic $\mathrm{O} 2$ & $\mathrm{C} 20 \mathrm{H} 12 \mathrm{O} 2$ & $7.30 \mathrm{E}-05$ & Neutral O1 & $\mathrm{C} 29 \mathrm{H} 36 \mathrm{O} 1$ & $5.68 \mathrm{E}-05$ \\
\hline S1 & C16H8S1 & $1.49 \mathrm{E}-05$ & Acidic $\mathrm{O} 2$ & $\mathrm{C} 21 \mathrm{H} 14 \mathrm{O} 2$ & 0.00019 & Neutral O1 & $\mathrm{C} 30 \mathrm{H} 38 \mathrm{O} 1$ & $5.27 \mathrm{E}-05$ \\
\hline S1 & C17H10S1 & $2.43 \mathrm{E}-05$ & Acidic $\mathrm{O} 2$ & $\mathrm{C} 22 \mathrm{H} 16 \mathrm{O} 2$ & 0.000393 & Neutral O1 & $\mathrm{C} 31 \mathrm{H} 40 \mathrm{O} 1$ & $3.65 \mathrm{E}-05$ \\
\hline S1 & $\mathrm{C} 18 \mathrm{H} 12 \mathrm{~S} 1$ & $3.76 \mathrm{E}-05$ & Acidic $\mathrm{O} 2$ & $\mathrm{C} 23 \mathrm{H} 18 \mathrm{O} 2$ & 0.000478 & Neutral O1 & $\mathrm{C} 32 \mathrm{H} 42 \mathrm{O} 1$ & $2.92 \mathrm{E}-05$ \\
\hline S1 & $\mathrm{C} 19 \mathrm{H} 14 \mathrm{~S} 1$ & 0.000204 & Acidic $\mathrm{O} 2$ & $\mathrm{C} 24 \mathrm{H} 20 \mathrm{O} 2$ & 0.000301 & Neutral O1 & $\mathrm{C} 33 \mathrm{H} 44 \mathrm{O} 1$ & $2.55 \mathrm{E}-05$ \\
\hline S1 & $\mathrm{C} 20 \mathrm{H} 16 \mathrm{~S} 1$ & 0.000551 & Acidic $\mathrm{O} 2$ & $\mathrm{C} 25 \mathrm{H} 22 \mathrm{O} 2$ & 0.000186 & Neutral O1 & $\mathrm{C} 34 \mathrm{H} 46 \mathrm{O} 1$ & $1.58 \mathrm{E}-05$ \\
\hline S1 & $\mathrm{C} 21 \mathrm{H} 18 \mathrm{~S} 1$ & 0.000831 & Acidic $\mathrm{O} 2$ & $\mathrm{C} 26 \mathrm{H} 24 \mathrm{O} 2$ & 0.000102 & Neutral O1 & $\mathrm{C} 35 \mathrm{H} 48 \mathrm{O} 1$ & $8.17 \mathrm{E}-06$ \\
\hline S1 & $\mathrm{C} 22 \mathrm{H} 20 \mathrm{~S} 1$ & 0.000791 & Acidic $\mathrm{O} 2$ & $\mathrm{C} 27 \mathrm{H} 26 \mathrm{O} 2$ & $5.11 \mathrm{E}-05$ & Neutral O1 & $\mathrm{C} 36 \mathrm{H} 50 \mathrm{O} 1$ & $9.40 \mathrm{E}-06$ \\
\hline S1 & $\mathrm{C} 23 \mathrm{H} 22 \mathrm{~S} 1$ & 0.00061 & Acidic $\mathrm{O} 2$ & $\mathrm{C} 28 \mathrm{H} 28 \mathrm{O} 2$ & $2.40 \mathrm{E}-05$ & Neutral O1 & $\mathrm{C} 37 \mathrm{H} 52 \mathrm{O} 1$ & $3.33 \mathrm{E}-06$ \\
\hline S1 & $\mathrm{C} 24 \mathrm{H} 24 \mathrm{~S} 1$ & 0.000438 & Acidic $\mathrm{O} 2$ & $\mathrm{C} 29 \mathrm{H} 30 \mathrm{O} 2$ & $1.38 \mathrm{E}-05$ & Neutral O1 & $\mathrm{C} 38 \mathrm{H} 54 \mathrm{O} 1$ & $8.04 \mathrm{E}-06$ \\
\hline S1 & $\mathrm{C} 25 \mathrm{H} 26 \mathrm{~S} 1$ & 0.000327 & Acidic $\mathrm{O} 2$ & $\mathrm{C} 30 \mathrm{H} 32 \mathrm{O} 2$ & $5.14 \mathrm{E}-06$ & Neutral O1 & $\mathrm{C} 39 \mathrm{H} 56 \mathrm{O} 1$ & $3.55 \mathrm{E}-06$ \\
\hline S1 & $\mathrm{C} 26 \mathrm{H} 28 \mathrm{~S} 1$ & 0.000257 & Acidic $\mathrm{O} 2$ & $\mathrm{C} 31 \mathrm{H} 34 \mathrm{O} 2$ & $4.59 \mathrm{E}-06$ & Neutral O1 & $\mathrm{C} 40 \mathrm{H} 58 \mathrm{O} 1$ & $2.93 \mathrm{E}-06$ \\
\hline S1 & $\mathrm{C} 27 \mathrm{H} 30 \mathrm{~S} 1$ & 0.000208 & Acidic $\mathrm{O} 2$ & $\mathrm{C} 20 \mathrm{H} 10 \mathrm{O} 2$ & $2.32 \mathrm{E}-05$ & Neutral O1 & $\mathrm{C} 41 \mathrm{H} 60 \mathrm{O} 1$ & $1.07 \mathrm{E}-06$ \\
\hline S1 & $\mathrm{C} 28 \mathrm{H} 32 \mathrm{~S} 1$ & 0.00019 & Acidic $\mathrm{O} 2$ & $\mathrm{C} 21 \mathrm{H} 12 \mathrm{O} 2$ & $4.60 \mathrm{E}-05$ & Neutral O1 & $\mathrm{C} 42 \mathrm{H} 62 \mathrm{O} 1$ & $6.71 \mathrm{E}-07$ \\
\hline $\mathrm{S} 1$ & $\mathrm{C} 29 \mathrm{H} 34 \mathrm{~S} 1$ & 0.000173 & Acidic $\mathrm{O} 2$ & $\mathrm{C} 22 \mathrm{H} 14 \mathrm{O} 2$ & 0.000176 & Neutral O1 & $\mathrm{C} 43 \mathrm{H} 64 \mathrm{O} 1$ & $1.19 \mathrm{E}-06$ \\
\hline S1 & $\mathrm{C} 30 \mathrm{H} 36 \mathrm{~S} 1$ & 0.000163 & Acidic $\mathrm{O} 2$ & $\mathrm{C} 23 \mathrm{H} 16 \mathrm{O} 2$ & 0.000271 & Neutral O1 & $\mathrm{C} 18 \mathrm{H} 12 \mathrm{O} 1$ & $4.09 \mathrm{E}-05$ \\
\hline S1 & $\mathrm{C} 31 \mathrm{H} 38 \mathrm{~S} 1$ & 0.000155 & Acidic $\mathrm{O} 2$ & $\mathrm{C} 24 \mathrm{H} 18 \mathrm{O} 2$ & 0.000318 & Neutral O1 & $\mathrm{C} 19 \mathrm{H} 14 \mathrm{O} 1$ & 0.000227 \\
\hline S1 & $\mathrm{C} 32 \mathrm{H} 40 \mathrm{~S} 1$ & 0.000144 & Acidic $\mathrm{O} 2$ & $\mathrm{C} 25 \mathrm{H} 20 \mathrm{O} 2$ & 0.000246 & Neutral O1 & $\mathrm{C} 20 \mathrm{H} 16 \mathrm{O} 1$ & 0.000629 \\
\hline S1 & $\mathrm{C} 33 \mathrm{H} 42 \mathrm{~S} 1$ & 0.000132 & Acidic $\mathrm{O} 2$ & $\mathrm{C} 26 \mathrm{H} 22 \mathrm{O} 2$ & 0.000161 & Neutral O1 & $\mathrm{C} 21 \mathrm{H} 18 \mathrm{O} 1$ & 0.001128 \\
\hline S1 & $\mathrm{C} 34 \mathrm{H} 44 \mathrm{~S} 1$ & 0.000106 & Acidic $\mathrm{O} 2$ & $\mathrm{C} 27 \mathrm{H} 24 \mathrm{O} 2$ & $6.96 \mathrm{E}-05$ & Neutral O1 & $\mathrm{C} 22 \mathrm{H} 20 \mathrm{O} 1$ & 0.00127 \\
\hline S1 & $\mathrm{C} 35 \mathrm{H} 46 \mathrm{~S} 1$ & 0.00011 & Acidic $\mathrm{O} 2$ & $\mathrm{C} 28 \mathrm{H} 26 \mathrm{O} 2$ & $4.14 \mathrm{E}-05$ & Neutral O1 & $\mathrm{C} 23 \mathrm{H} 22 \mathrm{O} 1$ & 0.000965 \\
\hline S1 & $\mathrm{C} 36 \mathrm{H} 48 \mathrm{~S} 1$ & 7.16E-05 & Acidic $\mathrm{O} 2$ & $\mathrm{C} 29 \mathrm{H} 28 \mathrm{O} 2$ & $2.94 \mathrm{E}-05$ & Neutral O1 & $\mathrm{C} 24 \mathrm{H} 24 \mathrm{O} 1$ & 0.000558 \\
\hline S1 & C37H50S1 & $8.85 \mathrm{E}-05$ & Acidic $\mathrm{O} 2$ & $\mathrm{C} 30 \mathrm{H} 30 \mathrm{O} 2$ & $3.58 \mathrm{E}-05$ & Neutral O1 & $\mathrm{C} 25 \mathrm{H} 26 \mathrm{O} 1$ & 0.000275 \\
\hline S1 & $\mathrm{C} 38 \mathrm{H} 52 \mathrm{~S} 1$ & $7.42 \mathrm{E}-05$ & Acidic $\mathrm{O} 2$ & $\mathrm{C} 31 \mathrm{H} 32 \mathrm{O} 2$ & 0.00029 & Neutral O1 & $\mathrm{C} 26 \mathrm{H} 28 \mathrm{O} 1$ & 0.000154 \\
\hline S1 & $\mathrm{C} 39 \mathrm{H} 54 \mathrm{~S} 1$ & $7.27 \mathrm{E}-05$ & Acidic $\mathrm{O} 2$ & $\mathrm{C} 32 \mathrm{H} 34 \mathrm{O} 2$ & $4.37 \mathrm{E}-05$ & Neutral O1 & $\mathrm{C} 27 \mathrm{H} 30 \mathrm{O} 1$ & 0.000122 \\
\hline S1 & $\mathrm{C} 40 \mathrm{H} 56 \mathrm{~S} 1$ & $6.68 \mathrm{E}-05$ & Acidic $\mathrm{O} 2$ & $\mathrm{C} 33 \mathrm{H} 36 \mathrm{O} 2$ & 0.000188 & Neutral O1 & $\mathrm{C} 28 \mathrm{H} 32 \mathrm{O} 1$ & $9.77 \mathrm{E}-05$ \\
\hline S1 & C41H58S1 & $5.16 \mathrm{E}-05$ & Acidic $\mathrm{O} 2$ & $\mathrm{C} 34 \mathrm{H} 38 \mathrm{O} 2$ & $1.23 \mathrm{E}-05$ & Neutral O1 & $\mathrm{C} 29 \mathrm{H} 34 \mathrm{O} 1$ & $6.07 \mathrm{E}-05$ \\
\hline
\end{tabular}




\begin{tabular}{|c|c|c|c|c|c|c|c|c|}
\hline Class & formula & Mass & Class & formula & Mass & Class & formula & Mass \\
\hline $\mathrm{S} 1$ & $\mathrm{C} 42 \mathrm{H} 60 \mathrm{~S} 1$ & 4.67E-05 & Acidic $\mathrm{O} 2$ & $\mathrm{C} 35 \mathrm{H} 40 \mathrm{O} 2$ & $1.19 \mathrm{E}-05$ & Neutral O1 & $\mathrm{C} 30 \mathrm{H} 36 \mathrm{O} 1$ & $4.76 \mathrm{E}-05$ \\
\hline $\mathrm{S} 1$ & $\mathrm{C} 43 \mathrm{H} 62 \mathrm{~S} 1$ & $5.00 \mathrm{E}-05$ & Acidic $\mathrm{O} 2$ & $\mathrm{C} 18 \mathrm{H} 4 \mathrm{O} 2$ & $9.05 \mathrm{E}-06$ & Neutral O1 & $\mathrm{C} 31 \mathrm{H} 38 \mathrm{O} 1$ & $3.51 \mathrm{E}-05$ \\
\hline $\mathrm{S} 1$ & C44H64S1 & $3.47 \mathrm{E}-05$ & Acidic $\mathrm{O} 2$ & $\mathrm{C} 19 \mathrm{H} 6 \mathrm{O} 2$ & $1.19 \mathrm{E}-05$ & Neutral O1 & $\mathrm{C} 32 \mathrm{H} 40 \mathrm{O} 1$ & $3.87 \mathrm{E}-05$ \\
\hline $\mathrm{S} 1$ & C45H66S1 & $4.11 \mathrm{E}-05$ & Acidic $\mathrm{O} 2$ & $\mathrm{C} 20 \mathrm{H} 8 \mathrm{O} 2$ & $1.54 \mathrm{E}-05$ & Neutral O1 & $\mathrm{C} 33 \mathrm{H} 42 \mathrm{O} 1$ & $1.14 \mathrm{E}-05$ \\
\hline $\mathrm{S} 1$ & $\mathrm{C} 46 \mathrm{H} 68 \mathrm{~S} 1$ & $2.64 \mathrm{E}-05$ & Acidic $\mathrm{O} 2$ & $\mathrm{C} 21 \mathrm{H} 10 \mathrm{O} 2$ & $1.83 \mathrm{E}-05$ & Neutral O1 & $\mathrm{C} 34 \mathrm{H} 44 \mathrm{O} 1$ & $2.18 \mathrm{E}-05$ \\
\hline $\mathrm{S} 1$ & $\mathrm{C} 47 \mathrm{H} 70 \mathrm{~S} 1$ & $2.31 \mathrm{E}-05$ & Acidic $\mathrm{O} 2$ & $\mathrm{C} 22 \mathrm{H} 12 \mathrm{O} 2$ & 0.00025 & Neutral O1 & $\mathrm{C} 35 \mathrm{H} 46 \mathrm{O} 1$ & $1.09 \mathrm{E}-05$ \\
\hline $\mathrm{S} 1$ & $\mathrm{C} 48 \mathrm{H} 72 \mathrm{~S} 1$ & $2.46 \mathrm{E}-05$ & Acidic $\mathrm{O} 2$ & $\mathrm{C} 23 \mathrm{H} 14 \mathrm{O} 2$ & 0.000148 & Neutral O1 & $\mathrm{C} 36 \mathrm{H} 48 \mathrm{O} 1$ & $9.35 \mathrm{E}-06$ \\
\hline $\mathrm{S} 1$ & $\mathrm{C} 49 \mathrm{H} 74 \mathrm{~S} 1$ & $1.00 \mathrm{E}-05$ & Acidic $\mathrm{O} 2$ & $\mathrm{C} 24 \mathrm{H} 16 \mathrm{O} 2$ & 0.000384 & Neutral O1 & $\mathrm{C} 37 \mathrm{H} 50 \mathrm{O} 1$ & $6.63 \mathrm{E}-06$ \\
\hline $\mathrm{S} 1$ & $\mathrm{C} 50 \mathrm{H} 76 \mathrm{~S} 1$ & $1.69 \mathrm{E}-05$ & Acidic $\mathrm{O} 2$ & $\mathrm{C} 25 \mathrm{H} 18 \mathrm{O} 2$ & 0.000523 & Neutral O1 & C38H52O1 & $2.86 \mathrm{E}-06$ \\
\hline $\mathrm{S} 1$ & $\mathrm{C} 51 \mathrm{H} 78 \mathrm{~S} 1$ & $1.30 \mathrm{E}-05$ & Acidic O2 & $\mathrm{C} 26 \mathrm{H} 20 \mathrm{O} 2$ & 0.000302 & Neutral O1 & $\mathrm{C} 39 \mathrm{H} 54 \mathrm{O} 1$ & $6.23 \mathrm{E}-06$ \\
\hline $\mathrm{S} 1$ & C52H80S1 & $2.86 \mathrm{E}-06$ & Acidic O2 & $\mathrm{C} 27 \mathrm{H} 22 \mathrm{O} 2$ & 0.000157 & Neutral O1 & $\mathrm{C} 18 \mathrm{H} 10 \mathrm{O} 1$ & $2.62 \mathrm{E}-07$ \\
\hline $\mathrm{S} 1$ & $\mathrm{C} 53 \mathrm{H} 82 \mathrm{~S} 1$ & $1.62 \mathrm{E}-05$ & Acidic $\mathrm{O} 2$ & $\mathrm{C} 28 \mathrm{H} 24 \mathrm{O} 2$ & $7.52 \mathrm{E}-05$ & Neutral O1 & $\mathrm{C} 19 \mathrm{H} 12 \mathrm{O} 1$ & $6.74 \mathrm{E}-06$ \\
\hline $\mathrm{S} 1$ & C54H84S1 & $5.45 \mathrm{E}-06$ & Acidic $\mathrm{O} 2$ & $\mathrm{C} 29 \mathrm{H} 26 \mathrm{O} 2$ & $4.21 \mathrm{E}-05$ & Neutral O1 & $\mathrm{C} 20 \mathrm{H} 14 \mathrm{O} 1$ & $6.89 \mathrm{E}-05$ \\
\hline $\mathrm{S} 1$ & C55H86S1 & $2.41 \mathrm{E}-06$ & Acidic $\mathrm{O} 2$ & $\mathrm{C} 30 \mathrm{H} 28 \mathrm{O} 2$ & $1.54 \mathrm{E}-05$ & Neutral O1 & $\mathrm{C} 21 \mathrm{H} 16 \mathrm{O} 1$ & 0.000179 \\
\hline $\mathrm{S} 1$ & $\mathrm{C} 56 \mathrm{H} 88 \mathrm{~S} 1$ & $2.59 \mathrm{E}-06$ & Acidic $\mathrm{O} 2$ & $\mathrm{C} 31 \mathrm{H} 30 \mathrm{O} 2$ & $4.76 \mathrm{E}-06$ & Neutral O1 & $\mathrm{C} 22 \mathrm{H} 18 \mathrm{O} 1$ & 0.00032 \\
\hline $\mathrm{S} 1$ & C16H6S1 & $3.00 \mathrm{E}-06$ & Acidic $\mathrm{O} 2$ & $\mathrm{C} 32 \mathrm{H} 32 \mathrm{O} 2$ & $5.27 \mathrm{E}-06$ & Neutral O1 & $\mathrm{C} 23 \mathrm{H} 20 \mathrm{O} 1$ & 0.000484 \\
\hline $\mathrm{S} 1$ & C17H8S1 & $3.82 \mathrm{E}-07$ & Acidic $\mathrm{O} 2$ & $\mathrm{C} 33 \mathrm{H} 34 \mathrm{O} 2$ & $2.07 \mathrm{E}-05$ & Neutral O1 & $\mathrm{C} 24 \mathrm{H} 22 \mathrm{O} 1$ & 0.000594 \\
\hline $\mathrm{S} 1$ & C18H10S1 & $5.81 \mathrm{E}-05$ & Acidic $\mathrm{O} 2$ & $\mathrm{C} 19 \mathrm{H} 4 \mathrm{O} 2$ & $2.69 \mathrm{E}-06$ & Neutral O1 & $\mathrm{C} 25 \mathrm{H} 24 \mathrm{O} 1$ & 0.000471 \\
\hline $\mathrm{S} 1$ & $\mathrm{C} 19 \mathrm{H} 12 \mathrm{~S} 1$ & 0.000242 & Acidic $\mathrm{O} 2$ & $\mathrm{C} 20 \mathrm{H} 6 \mathrm{O} 2$ & $3.81 \mathrm{E}-05$ & Neutral O1 & $\mathrm{C} 26 \mathrm{H} 26 \mathrm{O} 1$ & 0.000318 \\
\hline $\mathrm{S} 1$ & $\mathrm{C} 20 \mathrm{H} 14 \mathrm{~S} 1$ & 0.00051 & Acidic $\mathrm{O} 2$ & $\mathrm{C} 21 \mathrm{H} 8 \mathrm{O} 2$ & $1.35 \mathrm{E}-05$ & Neutral O1 & $\mathrm{C} 27 \mathrm{H} 28 \mathrm{O} 1$ & 0.000211 \\
\hline $\mathrm{S} 1$ & $\mathrm{C} 21 \mathrm{H} 16 \mathrm{~S} 1$ & 0.000755 & Acidic $\mathrm{O} 2$ & $\mathrm{C} 22 \mathrm{H} 10 \mathrm{O} 2$ & $2.24 \mathrm{E}-05$ & Neutral O1 & $\mathrm{C} 28 \mathrm{H} 30 \mathrm{O} 1$ & 0.000133 \\
\hline $\mathrm{S} 1$ & $\mathrm{C} 22 \mathrm{H} 18 \mathrm{~S} 1$ & 0.000806 & Acidic $\mathrm{O} 2$ & $\mathrm{C} 23 \mathrm{H} 12 \mathrm{O} 2$ & $3.54 \mathrm{E}-05$ & Neutral O1 & $\mathrm{C} 29 \mathrm{H} 32 \mathrm{O} 1$ & 7.23E-05 \\
\hline $\mathrm{S} 1$ & $\mathrm{C} 23 \mathrm{H} 20 \mathrm{~S} 1$ & 0.000718 & Acidic $\mathrm{O} 2$ & $\mathrm{C} 24 \mathrm{H} 14 \mathrm{O} 2$ & 0.000129 & Neutral O1 & $\mathrm{C} 30 \mathrm{H} 34 \mathrm{O} 1$ & $5.67 \mathrm{E}-05$ \\
\hline S1 & $\mathrm{C} 24 \mathrm{H} 22 \mathrm{~S} 1$ & 0.000515 & Acidic $\mathrm{O} 2$ & $\mathrm{C} 25 \mathrm{H} 16 \mathrm{O} 2$ & 0.000204 & Neutral O1 & $\mathrm{C} 31 \mathrm{H} 36 \mathrm{O} 1$ & $2.40 \mathrm{E}-05$ \\
\hline S1 & $\mathrm{C} 25 \mathrm{H} 24 \mathrm{~S} 1$ & 0.000402 & Acidic $\mathrm{O} 2$ & $\mathrm{C} 26 \mathrm{H} 18 \mathrm{O} 2$ & 0.000197 & Neutral O1 & $\mathrm{C} 32 \mathrm{H} 38 \mathrm{O} 1$ & $3.12 \mathrm{E}-05$ \\
\hline S1 & $\mathrm{C} 26 \mathrm{H} 26 \mathrm{~S} 1$ & 0.00029 & Acidic $\mathrm{O} 2$ & $\mathrm{C} 27 \mathrm{H} 20 \mathrm{O} 2$ & 0.000175 & Neutral O1 & $\mathrm{C} 33 \mathrm{H} 40 \mathrm{O} 1$ & $2.23 \mathrm{E}-05$ \\
\hline $\mathrm{S} 1$ & $\mathrm{C} 27 \mathrm{H} 28 \mathrm{~S} 1$ & 0.00024 & Acidic $\mathrm{O} 2$ & $\mathrm{C} 28 \mathrm{H} 22 \mathrm{O} 2$ & 0.000112 & Neutral O1 & $\mathrm{C} 34 \mathrm{H} 42 \mathrm{O} 1$ & $1.42 \mathrm{E}-05$ \\
\hline $\mathrm{S} 1$ & $\mathrm{C} 28 \mathrm{H} 30 \mathrm{~S} 1$ & 0.0002 & Acidic $\mathrm{O} 2$ & $\mathrm{C} 29 \mathrm{H} 24 \mathrm{O} 2$ & $7.09 \mathrm{E}-05$ & Neutral O1 & $\mathrm{C} 35 \mathrm{H} 44 \mathrm{O} 1$ & $5.22 \mathrm{E}-06$ \\
\hline $\mathrm{S} 1$ & $\mathrm{C} 29 \mathrm{H} 32 \mathrm{~S} 1$ & 0.000181 & Acidic $\mathrm{O} 2$ & $\mathrm{C} 30 \mathrm{H} 26 \mathrm{O} 2$ & $1.56 \mathrm{E}-05$ & Neutral O1 & $\mathrm{C} 36 \mathrm{H} 46 \mathrm{O} 1$ & $5.52 \mathrm{E}-06$ \\
\hline $\mathrm{S} 1$ & $\mathrm{C} 30 \mathrm{H} 34 \mathrm{~S} 1$ & 0.000144 & Acidic $\mathrm{O} 2$ & $\mathrm{C} 31 \mathrm{H} 28 \mathrm{O} 2$ & $8.62 \mathrm{E}-06$ & Neutral O1 & $\mathrm{C} 37 \mathrm{H} 48 \mathrm{O} 1$ & $5.84 \mathrm{E}-06$ \\
\hline $\mathrm{S} 1$ & $\mathrm{C} 31 \mathrm{H} 36 \mathrm{~S} 1$ & 0.000139 & Acidic $\mathrm{O} 2$ & $\mathrm{C} 20 \mathrm{H} 4 \mathrm{O} 2$ & $2.41 \mathrm{E}-06$ & Neutral O1 & $\mathrm{C} 38 \mathrm{H} 50 \mathrm{O} 1$ & $2.58 \mathrm{E}-06$ \\
\hline $\mathrm{S} 1$ & $\mathrm{C} 32 \mathrm{H} 38 \mathrm{~S} 1$ & 0.000131 & Acidic $\mathrm{O} 2$ & $\mathrm{C} 21 \mathrm{H} 6 \mathrm{O} 2$ & $3.00 \mathrm{E}-06$ & Neutral O1 & $\mathrm{C} 39 \mathrm{H} 52 \mathrm{O} 1$ & 2.44E-06 \\
\hline $\mathrm{S} 1$ & $\mathrm{C} 33 \mathrm{H} 40 \mathrm{~S} 1$ & 0.000116 & Acidic $\mathrm{O} 2$ & $\mathrm{C} 22 \mathrm{H} 8 \mathrm{O} 2$ & $5.79 \mathrm{E}-06$ & Neutral O1 & $\mathrm{C} 40 \mathrm{H} 54 \mathrm{O} 1$ & $9.11 \mathrm{E}-07$ \\
\hline S1 & $\mathrm{C} 34 \mathrm{H} 42 \mathrm{~S} 1$ & $9.99 \mathrm{E}-05$ & Acidic $\mathrm{O} 2$ & $\mathrm{C} 23 \mathrm{H} 10 \mathrm{O} 2$ & $5.83 \mathrm{E}-06$ & Neutral O1 & $\mathrm{C} 41 \mathrm{H} 56 \mathrm{O} 1$ & $1.11 \mathrm{E}-06$ \\
\hline S1 & $\mathrm{C} 35 \mathrm{H} 44 \mathrm{~S} 1$ & $8.37 \mathrm{E}-05$ & Acidic $\mathrm{O} 2$ & $\mathrm{C} 24 \mathrm{H} 12 \mathrm{O} 2$ & $6.09 \mathrm{E}-06$ & Neutral O1 & $\mathrm{C} 42 \mathrm{H} 58 \mathrm{O} 1$ & $1.31 \mathrm{E}-06$ \\
\hline S1 & $\mathrm{C} 36 \mathrm{H} 46 \mathrm{~S} 1$ & 7.91E-05 & Acidic $\mathrm{O} 2$ & $\mathrm{C} 25 \mathrm{H} 14 \mathrm{O} 2$ & $1.49 \mathrm{E}-05$ & Neutral O1 & C18H8O1 & $2.21 \mathrm{E}-07$ \\
\hline S1 & $\mathrm{C} 37 \mathrm{H} 48 \mathrm{~S} 1$ & $7.44 \mathrm{E}-05$ & Acidic $\mathrm{O} 2$ & $\mathrm{C} 26 \mathrm{H} 16 \mathrm{O} 2$ & $3.00 \mathrm{E}-05$ & Neutral O1 & $\mathrm{C} 19 \mathrm{H} 10 \mathrm{O} 1$ & $1.02 \mathrm{E}-06$ \\
\hline S1 & C38H50S1 & $6.34 \mathrm{E}-05$ & Acidic $\mathrm{O} 2$ & $\mathrm{C} 27 \mathrm{H} 18 \mathrm{O} 2$ & $5.48 \mathrm{E}-05$ & Neutral O1 & $\mathrm{C} 20 \mathrm{H} 12 \mathrm{O} 1$ & 7.77E-06 \\
\hline
\end{tabular}




\begin{tabular}{|c|c|c|c|c|c|c|c|c|}
\hline Class & formula & Mass & Class & formula & Mass & Class & formula & Mass \\
\hline $\mathrm{S} 1$ & $\mathrm{C} 39 \mathrm{H} 52 \mathrm{~S} 1$ & $6.03 \mathrm{E}-05$ & Acidic $\mathrm{O} 2$ & $\mathrm{C} 28 \mathrm{H} 20 \mathrm{O} 2$ & $3.87 \mathrm{E}-05$ & Neutral O1 & $\mathrm{C} 21 \mathrm{H} 14 \mathrm{O} 1$ & 0.000102 \\
\hline $\mathrm{S} 1$ & $\mathrm{C} 40 \mathrm{H} 54 \mathrm{~S} 1$ & $5.82 \mathrm{E}-05$ & Acidic $\mathrm{O} 2$ & $\mathrm{C} 29 \mathrm{H} 22 \mathrm{O} 2$ & 4.64E-05 & Neutral O1 & $\mathrm{C} 22 \mathrm{H} 16 \mathrm{O} 1$ & 0.000353 \\
\hline $\mathrm{S} 1$ & C41H56S1 & $3.94 \mathrm{E}-05$ & Acidic $\mathrm{O} 2$ & $\mathrm{C} 30 \mathrm{H} 24 \mathrm{O} 2$ & $2.99 \mathrm{E}-05$ & Neutral O1 & $\mathrm{C} 23 \mathrm{H} 18 \mathrm{O} 1$ & 0.000738 \\
\hline $\mathrm{S} 1$ & $\mathrm{C} 42 \mathrm{H} 58 \mathrm{~S} 1$ & $4.02 \mathrm{E}-05$ & Acidic $\mathrm{O} 2$ & $\mathrm{C} 31 \mathrm{H} 26 \mathrm{O} 2$ & $4.50 \mathrm{E}-06$ & Neutral O1 & $\mathrm{C} 24 \mathrm{H} 20 \mathrm{O} 1$ & 0.000994 \\
\hline $\mathrm{S} 1$ & C43H60S1 & $4.25 \mathrm{E}-05$ & Acidic $\mathrm{O} 2$ & $\mathrm{C} 22 \mathrm{H} 6 \mathrm{O} 2$ & $2.43 \mathrm{E}-05$ & Neutral O1 & $\mathrm{C} 25 \mathrm{H} 22 \mathrm{O} 1$ & 0.000781 \\
\hline $\mathrm{S} 1$ & $\mathrm{C} 44 \mathrm{H} 62 \mathrm{~S} 1$ & $2.52 \mathrm{E}-05$ & Acidic $\mathrm{O} 2$ & $\mathrm{C} 23 \mathrm{H} 8 \mathrm{O} 2$ & $2.88 \mathrm{E}-06$ & Neutral O1 & $\mathrm{C} 26 \mathrm{H} 24 \mathrm{O} 1$ & 0.000479 \\
\hline $\mathrm{S} 1$ & C45H64S1 & $3.43 \mathrm{E}-05$ & Acidic $\mathrm{O} 2$ & $\mathrm{C} 24 \mathrm{H} 10 \mathrm{O} 2$ & $2.98 \mathrm{E}-06$ & Neutral O1 & $\mathrm{C} 27 \mathrm{H} 26 \mathrm{O} 1$ & 0.000329 \\
\hline $\mathrm{S} 1$ & C46H66S1 & $2.34 \mathrm{E}-05$ & Acidic $\mathrm{O} 2$ & $\mathrm{C} 25 \mathrm{H} 12 \mathrm{O} 2$ & $3.11 \mathrm{E}-06$ & Neutral O1 & $\mathrm{C} 28 \mathrm{H} 28 \mathrm{O} 1$ & 0.000215 \\
\hline $\mathrm{S} 1$ & C47H68S1 & $2.33 \mathrm{E}-05$ & Acidic $\mathrm{O} 2$ & $\mathrm{C} 26 \mathrm{H} 14 \mathrm{O} 2$ & $2.03 \mathrm{E}-05$ & Neutral O1 & $\mathrm{C} 29 \mathrm{H} 30 \mathrm{O} 1$ & 0.000126 \\
\hline $\mathrm{S} 1$ & $\mathrm{C} 48 \mathrm{H} 70 \mathrm{~S} 1$ & $1.79 \mathrm{E}-05$ & Acidic O2 & $\mathrm{C} 27 \mathrm{H} 16 \mathrm{O} 2$ & $4.23 \mathrm{E}-05$ & Neutral O1 & $\mathrm{C} 30 \mathrm{H} 32 \mathrm{O} 1$ & $7.42 \mathrm{E}-05$ \\
\hline $\mathrm{S} 1$ & $\mathrm{C} 49 \mathrm{H} 72 \mathrm{~S} 1$ & $1.37 \mathrm{E}-05$ & Acidic O2 & $\mathrm{C} 28 \mathrm{H} 18 \mathrm{O} 2$ & 7.77E-05 & Neutral O1 & $\mathrm{C} 31 \mathrm{H} 34 \mathrm{O} 1$ & $5.19 \mathrm{E}-05$ \\
\hline $\mathrm{S} 1$ & $\mathrm{C} 50 \mathrm{H} 74 \mathrm{~S} 1$ & $1.17 \mathrm{E}-05$ & Acidic $\mathrm{O} 2$ & $\mathrm{C} 29 \mathrm{H} 20 \mathrm{O} 2$ & $7.34 \mathrm{E}-05$ & Neutral O1 & $\mathrm{C} 32 \mathrm{H} 36 \mathrm{O} 1$ & $3.34 \mathrm{E}-05$ \\
\hline $\mathrm{S} 1$ & C51H76S1 & $3.95 \mathrm{E}-06$ & Acidic $\mathrm{O} 2$ & $\mathrm{C} 30 \mathrm{H} 22 \mathrm{O} 2$ & 0.000173 & Neutral O1 & $\mathrm{C} 33 \mathrm{H} 38 \mathrm{O} 1$ & $1.75 \mathrm{E}-05$ \\
\hline $\mathrm{S} 1$ & $\mathrm{C} 52 \mathrm{H} 78 \mathrm{~S} 1$ & $7.41 \mathrm{E}-06$ & Acidic $\mathrm{O} 2$ & $\mathrm{C} 31 \mathrm{H} 24 \mathrm{O} 2$ & $3.51 \mathrm{E}-05$ & Neutral O1 & $\mathrm{C} 34 \mathrm{H} 40 \mathrm{O} 1$ & $1.88 \mathrm{E}-05$ \\
\hline $\mathrm{S} 1$ & C18H8S1 & $5.60 \mathrm{E}-06$ & Acidic $\mathrm{O} 2$ & $\mathrm{C} 32 \mathrm{H} 26 \mathrm{O} 2$ & $1.60 \mathrm{E}-05$ & Neutral O1 & $\mathrm{C} 35 \mathrm{H} 42 \mathrm{O} 1$ & $6.75 \mathrm{E}-06$ \\
\hline $\mathrm{S} 1$ & C19H10S1 & $3.96 \mathrm{E}-06$ & Acidic $\mathrm{O} 2$ & $\mathrm{C} 33 \mathrm{H} 28 \mathrm{O} 2$ & $9.82 \mathrm{E}-06$ & Neutral O1 & $\mathrm{C} 36 \mathrm{H} 44 \mathrm{O} 1$ & $6.58 \mathrm{E}-06$ \\
\hline $\mathrm{S} 1$ & $\mathrm{C} 20 \mathrm{H} 12 \mathrm{~S} 1$ & $9.25 \mathrm{E}-05$ & Acidic $\mathrm{O} 2$ & $\mathrm{C} 27 \mathrm{H} 14 \mathrm{O} 2$ & $5.11 \mathrm{E}-06$ & Neutral O1 & $\mathrm{C} 37 \mathrm{H} 46 \mathrm{O} 1$ & $2.62 \mathrm{E}-06$ \\
\hline $\mathrm{S} 1$ & $\mathrm{C} 21 \mathrm{H} 14 \mathrm{~S} 1$ & 0.000389 & Acidic $\mathrm{O} 2$ & $\mathrm{C} 28 \mathrm{H} 16 \mathrm{O} 2$ & $7.25 \mathrm{E}-06$ & Neutral O1 & $\mathrm{C} 38 \mathrm{H} 48 \mathrm{O} 1$ & $3.98 \mathrm{E}-06$ \\
\hline $\mathrm{S} 1$ & $\mathrm{C} 22 \mathrm{H} 16 \mathrm{~S} 1$ & 0.000798 & Acidic $\mathrm{O} 2$ & $\mathrm{C} 29 \mathrm{H} 18 \mathrm{O} 2$ & $3.06 \mathrm{E}-05$ & Neutral O1 & $\mathrm{C} 21 \mathrm{H} 12 \mathrm{O} 1$ & $8.50 \mathrm{E}-05$ \\
\hline $\mathrm{S} 1$ & $\mathrm{C} 23 \mathrm{H} 18 \mathrm{~S} 1$ & 0.000906 & Acidic $\mathrm{O} 2$ & $\mathrm{C} 30 \mathrm{H} 20 \mathrm{O} 2$ & $4.09 \mathrm{E}-05$ & Neutral O1 & $\mathrm{C} 22 \mathrm{H} 14 \mathrm{O} 1$ & 0.000263 \\
\hline $\mathrm{S} 1$ & $\mathrm{C} 24 \mathrm{H} 20 \mathrm{~S} 1$ & 0.00073 & Acidic $\mathrm{O} 2$ & $\mathrm{C} 31 \mathrm{H} 22 \mathrm{O} 2$ & $3.72 \mathrm{E}-05$ & Neutral O1 & $\mathrm{C} 23 \mathrm{H} 16 \mathrm{O} 1$ & 0.000381 \\
\hline $\mathrm{S} 1$ & $\mathrm{C} 25 \mathrm{H} 22 \mathrm{~S} 1$ & 0.00047 & Acidic $\mathrm{O} 2$ & $\mathrm{C} 32 \mathrm{H} 24 \mathrm{O} 2$ & $1.53 \mathrm{E}-05$ & Neutral O1 & $\mathrm{C} 24 \mathrm{H} 18 \mathrm{O} 1$ & 0.000526 \\
\hline $\mathrm{S} 1$ & $\mathrm{C} 26 \mathrm{H} 24 \mathrm{~S} 1$ & 0.000337 & Acidic $\mathrm{O} 2$ & $\mathrm{C} 33 \mathrm{H} 26 \mathrm{O} 2$ & $4.46 \mathrm{E}-06$ & Neutral O1 & $\mathrm{C} 25 \mathrm{H} 20 \mathrm{O} 1$ & 0.000651 \\
\hline S1 & $\mathrm{C} 27 \mathrm{H} 26 \mathrm{~S} 1$ & 0.000215 & Acidic $\mathrm{O} 2$ & $\mathrm{C} 34 \mathrm{H} 28 \mathrm{O} 2$ & $5.45 \mathrm{E}-06$ & Neutral O1 & $\mathrm{C} 26 \mathrm{H} 22 \mathrm{O} 1$ & 0.000626 \\
\hline S1 & $\mathrm{C} 28 \mathrm{H} 28 \mathrm{~S} 1$ & 0.000193 & Acidic $\mathrm{O} 2$ & $\mathrm{C} 30 \mathrm{H} 18 \mathrm{O} 2$ & $3.02 \mathrm{E}-06$ & Neutral O1 & $\mathrm{C} 27 \mathrm{H} 24 \mathrm{O} 1$ & 0.000522 \\
\hline S1 & C29H30S1 & 0.000136 & Acidic $\mathrm{O} 2$ & $\mathrm{C} 31 \mathrm{H} 20 \mathrm{O} 2$ & $1.49 \mathrm{E}-05$ & Neutral O1 & $\mathrm{C} 28 \mathrm{H} 26 \mathrm{O} 1$ & 0.000364 \\
\hline $\mathrm{S} 1$ & $\mathrm{C} 30 \mathrm{H} 32 \mathrm{~S} 1$ & 0.000117 & Acidic $\mathrm{O} 2$ & $\mathrm{C} 32 \mathrm{H} 22 \mathrm{O} 2$ & $9.54 \mathrm{E}-06$ & Neutral O1 & $\mathrm{C} 29 \mathrm{H} 28 \mathrm{O} 1$ & 0.000204 \\
\hline $\mathrm{S} 1$ & $\mathrm{C} 31 \mathrm{H} 34 \mathrm{~S} 1$ & $9.79 \mathrm{E}-05$ & Acidic $\mathrm{O} 2$ & $\mathrm{C} 33 \mathrm{H} 24 \mathrm{O} 2$ & $5.59 \mathrm{E}-06$ & Neutral O1 & $\mathrm{C} 30 \mathrm{H} 30 \mathrm{O} 1$ & 0.000107 \\
\hline $\mathrm{S} 1$ & $\mathrm{C} 32 \mathrm{H} 36 \mathrm{~S} 1$ & 0.000102 & Basic N1 & $\mathrm{C} 24 \mathrm{H} 41 \mathrm{~N} 1$ & $1.60 \mathrm{E}-08$ & Neutral O1 & $\mathrm{C} 31 \mathrm{H} 32 \mathrm{O} 1$ & $4.53 \mathrm{E}-05$ \\
\hline $\mathrm{S} 1$ & $\mathrm{C} 33 \mathrm{H} 38 \mathrm{~S} 1$ & $8.53 \mathrm{E}-05$ & Basic N1 & $\mathrm{C} 25 \mathrm{H} 43 \mathrm{~N} 1$ & $1.19 \mathrm{E}-08$ & Neutral O1 & $\mathrm{C} 32 \mathrm{H} 34 \mathrm{O} 1$ & $4.05 \mathrm{E}-05$ \\
\hline S1 & $\mathrm{C} 34 \mathrm{H} 40 \mathrm{~S} 1$ & $7.28 \mathrm{E}-05$ & Basic N1 & $\mathrm{C} 26 \mathrm{H} 45 \mathrm{~N} 1$ & $1.13 \mathrm{E}-08$ & Neutral O1 & $\mathrm{C} 33 \mathrm{H} 36 \mathrm{O} 1$ & $1.29 \mathrm{E}-05$ \\
\hline $\mathrm{S} 1$ & $\mathrm{C} 35 \mathrm{H} 42 \mathrm{~S} 1$ & $6.45 \mathrm{E}-05$ & Basic N1 & $\mathrm{C} 28 \mathrm{H} 49 \mathrm{~N} 1$ & $3.62 \mathrm{E}-08$ & Neutral O1 & $\mathrm{C} 34 \mathrm{H} 38 \mathrm{O} 1$ & $3.55 \mathrm{E}-06$ \\
\hline $\mathrm{S} 1$ & $\mathrm{C} 36 \mathrm{H} 44 \mathrm{~S} 1$ & $6.16 \mathrm{E}-05$ & Basic N1 & $\mathrm{C} 29 \mathrm{H} 51 \mathrm{~N} 1$ & $1.86 \mathrm{E}-08$ & Neutral O1 & $\mathrm{C} 35 \mathrm{H} 40 \mathrm{O} 1$ & $6.80 \mathrm{E}-06$ \\
\hline S1 & $\mathrm{C} 37 \mathrm{H} 46 \mathrm{~S} 1$ & $5.16 \mathrm{E}-05$ & Basic N1 & C30H53N1 & $1.53 \mathrm{E}-07$ & Neutral O1 & $\mathrm{C} 36 \mathrm{H} 42 \mathrm{O} 1$ & $1.08 \mathrm{E}-06$ \\
\hline S1 & $\mathrm{C} 38 \mathrm{H} 48 \mathrm{~S} 1$ & 4.67E-05 & Basic N1 & $\mathrm{C} 31 \mathrm{H} 55 \mathrm{~N} 1$ & $7.32 \mathrm{E}-08$ & Neutral O1 & $\mathrm{C} 37 \mathrm{H} 44 \mathrm{O} 1$ & $7.82 \mathrm{E}-06$ \\
\hline S1 & C39H50S1 & $4.39 \mathrm{E}-05$ & Basic N1 & $\mathrm{C} 32 \mathrm{H} 57 \mathrm{~N} 1$ & 7.77E-08 & Neutral O1 & $\mathrm{C} 38 \mathrm{H} 46 \mathrm{O} 1$ & $5.39 \mathrm{E}-06$ \\
\hline S1 & $\mathrm{C} 40 \mathrm{H} 52 \mathrm{~S} 1$ & $3.93 \mathrm{E}-05$ & Basic N1 & C33H59N1 & $3.76 \mathrm{E}-08$ & Neutral O1 & $\mathrm{C} 39 \mathrm{H} 48 \mathrm{O} 1$ & $1.57 \mathrm{E}-06$ \\
\hline S1 & $\mathrm{C} 41 \mathrm{H} 54 \mathrm{~S} 1$ & $3.56 \mathrm{E}-05$ & Basic N1 & $\mathrm{C} 34 \mathrm{H} 61 \mathrm{~N} 1$ & $1.08 \mathrm{E}-07$ & Neutral O1 & $\mathrm{C} 40 \mathrm{H} 50 \mathrm{O} 1$ & $2.66 \mathrm{E}-06$ \\
\hline
\end{tabular}




\begin{tabular}{|c|c|c|c|c|c|c|c|c|}
\hline Class & formula & Mass & Class & formula & Mass & Class & formula & Mass \\
\hline $\mathrm{S} 1$ & $\mathrm{C} 42 \mathrm{H} 56 \mathrm{~S} 1$ & $3.82 \mathrm{E}-05$ & Basic N1 & $\mathrm{C} 35 \mathrm{H} 63 \mathrm{~N} 1$ & $2.52 \mathrm{E}-07$ & Neutral O1 & $\mathrm{C} 41 \mathrm{H} 52 \mathrm{O} 1$ & $1.31 \mathrm{E}-06$ \\
\hline $\mathrm{S} 1$ & C43H58S1 & $2.54 \mathrm{E}-05$ & Basic N1 & $\mathrm{C} 36 \mathrm{H} 65 \mathrm{~N} 1$ & $2.39 \mathrm{E}-07$ & Neutral O1 & $\mathrm{C} 42 \mathrm{H} 54 \mathrm{O} 1$ & $1.96 \mathrm{E}-06$ \\
\hline $\mathrm{S} 1$ & C44H60S1 & $2.83 \mathrm{E}-05$ & Basic N1 & C37H67N1 & $5.14 \mathrm{E}-08$ & Neutral O1 & $\mathrm{C} 20 \mathrm{H} 8 \mathrm{O} 1$ & $1.91 \mathrm{E}-07$ \\
\hline $\mathrm{S} 1$ & $\mathrm{C} 45 \mathrm{H} 62 \mathrm{~S} 1$ & $1.45 \mathrm{E}-05$ & Basic N1 & C38H69N1 & $1.56 \mathrm{E}-07$ & Neutral O1 & $\mathrm{C} 21 \mathrm{H} 10 \mathrm{O} 1$ & $9.09 \mathrm{E}-06$ \\
\hline $\mathrm{S} 1$ & $\mathrm{C} 46 \mathrm{H} 64 \mathrm{~S} 1$ & $1.18 \mathrm{E}-05$ & Basic N1 & $\mathrm{C} 39 \mathrm{H} 71 \mathrm{~N} 1$ & $2.37 \mathrm{E}-07$ & Neutral O1 & $\mathrm{C} 22 \mathrm{H} 12 \mathrm{O} 1$ & $3.13 \mathrm{E}-06$ \\
\hline $\mathrm{S} 1$ & C47H66S1 & $9.61 \mathrm{E}-06$ & Basic N1 & $\mathrm{C} 40 \mathrm{H} 73 \mathrm{~N} 1$ & $3.55 \mathrm{E}-07$ & Neutral O1 & $\mathrm{C} 23 \mathrm{H} 14 \mathrm{O} 1$ & 7.07E-05 \\
\hline $\mathrm{S} 1$ & C48H68S1 & $1.35 \mathrm{E}-05$ & Basic N1 & $\mathrm{C} 41 \mathrm{H} 75 \mathrm{~N} 1$ & $1.72 \mathrm{E}-07$ & Neutral O1 & $\mathrm{C} 24 \mathrm{H} 16 \mathrm{O} 1$ & 0.000286 \\
\hline $\mathrm{S} 1$ & $\mathrm{C} 49 \mathrm{H} 70 \mathrm{~S} 1$ & $1.10 \mathrm{E}-05$ & Basic N1 & $\mathrm{C} 42 \mathrm{H} 77 \mathrm{~N} 1$ & $1.75 \mathrm{E}-07$ & Neutral O1 & $\mathrm{C} 25 \mathrm{H} 18 \mathrm{O} 1$ & 0.000504 \\
\hline $\mathrm{S} 1$ & $\mathrm{C} 50 \mathrm{H} 72 \mathrm{~S} 1$ & $3.80 \mathrm{E}-06$ & Basic N1 & $\mathrm{C} 43 \mathrm{H} 79 \mathrm{~N} 1$ & $1.33 \mathrm{E}-07$ & Neutral O1 & $\mathrm{C} 26 \mathrm{H} 20 \mathrm{O} 1$ & 0.000457 \\
\hline $\mathrm{S} 1$ & $\mathrm{C} 51 \mathrm{H} 74 \mathrm{~S} 1$ & $2.00 \mathrm{E}-06$ & Basic N1 & $\mathrm{C} 44 \mathrm{H} 81 \mathrm{~N} 1$ & $2.77 \mathrm{E}-07$ & Neutral O1 & $\mathrm{C} 27 \mathrm{H} 22 \mathrm{O} 1$ & 0.000375 \\
\hline $\mathrm{S} 1$ & $\mathrm{C} 52 \mathrm{H} 76 \mathrm{~S} 1$ & $2.78 \mathrm{E}-06$ & Basic N1 & $\mathrm{C} 45 \mathrm{H} 83 \mathrm{~N} 1$ & $5.24 \mathrm{E}-07$ & Neutral O1 & $\mathrm{C} 28 \mathrm{H} 24 \mathrm{O} 1$ & 0.000337 \\
\hline $\mathrm{S} 1$ & $\mathrm{C} 53 \mathrm{H} 78 \mathrm{~S} 1$ & $3.10 \mathrm{E}-06$ & Basic N1 & $\mathrm{C} 46 \mathrm{H} 85 \mathrm{~N} 1$ & $1.52 \mathrm{E}-07$ & Neutral O1 & $\mathrm{C} 29 \mathrm{H} 26 \mathrm{O} 1$ & 0.000245 \\
\hline $\mathrm{S} 1$ & C54H80S1 & $4.50 \mathrm{E}-06$ & Basic N1 & C47H87N1 & $9.03 \mathrm{E}-08$ & Neutral O1 & $\mathrm{C} 30 \mathrm{H} 28 \mathrm{O} 1$ & 0.000139 \\
\hline $\mathrm{S} 1$ & C20H10S1 & $7.34 \mathrm{E}-06$ & Basic N1 & C48H89N1 & $7.28 \mathrm{E}-08$ & Neutral O1 & $\mathrm{C} 31 \mathrm{H} 30 \mathrm{O} 1$ & $7.85 \mathrm{E}-05$ \\
\hline $\mathrm{S} 1$ & $\mathrm{C} 21 \mathrm{H} 12 \mathrm{~S} 1$ & 4.33E-05 & Basic N1 & $\mathrm{C} 49 \mathrm{H} 91 \mathrm{~N} 1$ & $1.43 \mathrm{E}-07$ & Neutral O1 & $\mathrm{C} 32 \mathrm{H} 32 \mathrm{O} 1$ & $4.58 \mathrm{E}-05$ \\
\hline $\mathrm{S} 1$ & $\mathrm{C} 22 \mathrm{H} 14 \mathrm{~S} 1$ & 0.00012 & Basic N1 & $\mathrm{C} 23 \mathrm{H} 37 \mathrm{~N} 1$ & $1.19 \mathrm{E}-08$ & Neutral O1 & $\mathrm{C} 33 \mathrm{H} 34 \mathrm{O} 1$ & $2.84 \mathrm{E}-05$ \\
\hline $\mathrm{S} 1$ & $\mathrm{C} 23 \mathrm{H} 16 \mathrm{~S} 1$ & 0.000242 & Basic N1 & C24H39N1 & $4.95 \mathrm{E}-08$ & Neutral O1 & $\mathrm{C} 34 \mathrm{H} 36 \mathrm{O} 1$ & $6.73 \mathrm{E}-06$ \\
\hline $\mathrm{S} 1$ & $\mathrm{C} 24 \mathrm{H} 18 \mathrm{~S} 1$ & 0.000362 & Basic N1 & $\mathrm{C} 25 \mathrm{H} 41 \mathrm{~N} 1$ & $4.16 \mathrm{E}-08$ & Neutral O1 & $\mathrm{C} 35 \mathrm{H} 38 \mathrm{O} 1$ & 4.27E-06 \\
\hline $\mathrm{S} 1$ & $\mathrm{C} 25 \mathrm{H} 20 \mathrm{~S} 1$ & 0.000389 & Basic N1 & $\mathrm{C} 26 \mathrm{H} 43 \mathrm{~N} 1$ & 4.44E-08 & Neutral O1 & $\mathrm{C} 36 \mathrm{H} 40 \mathrm{O} 1$ & $1.67 \mathrm{E}-05$ \\
\hline $\mathrm{S} 1$ & $\mathrm{C} 26 \mathrm{H} 22 \mathrm{~S} 1$ & 0.000364 & Basic N1 & $\mathrm{C} 27 \mathrm{H} 45 \mathrm{~N} 1$ & $6.75 \mathrm{E}-08$ & Neutral O1 & $\mathrm{C} 37 \mathrm{H} 42 \mathrm{O} 1$ & $2.25 \mathrm{E}-06$ \\
\hline $\mathrm{S} 1$ & $\mathrm{C} 27 \mathrm{H} 24 \mathrm{~S} 1$ & 0.000289 & Basic N1 & $\mathrm{C} 28 \mathrm{H} 47 \mathrm{~N} 1$ & $7.55 \mathrm{E}-08$ & Neutral O1 & $\mathrm{C} 38 \mathrm{H} 44 \mathrm{O} 1$ & $2.26 \mathrm{E}-06$ \\
\hline $\mathrm{S} 1$ & $\mathrm{C} 28 \mathrm{H} 26 \mathrm{~S} 1$ & 0.000207 & Basic N1 & $\mathrm{C} 29 \mathrm{H} 49 \mathrm{~N} 1$ & $1.08 \mathrm{E}-07$ & Neutral O1 & $\mathrm{C} 39 \mathrm{H} 46 \mathrm{O} 1$ & $1.75 \mathrm{E}-06$ \\
\hline $\mathrm{S} 1$ & $\mathrm{C} 29 \mathrm{H} 28 \mathrm{~S} 1$ & 0.000169 & Basic N1 & C30H51N1 & $1.89 \mathrm{E}-07$ & Neutral O1 & $\mathrm{C} 40 \mathrm{H} 48 \mathrm{O} 1$ & $1.38 \mathrm{E}-06$ \\
\hline S1 & $\mathrm{C} 30 \mathrm{H} 30 \mathrm{~S} 1$ & 0.000115 & Basic N1 & C31H53N1 & $3.78 \mathrm{E}-07$ & Neutral O1 & $\mathrm{C} 41 \mathrm{H} 50 \mathrm{O} 1$ & $3.44 \mathrm{E}-06$ \\
\hline S1 & $\mathrm{C} 31 \mathrm{H} 32 \mathrm{~S} 1$ & 0.000105 & Basic N1 & $\mathrm{C} 32 \mathrm{H} 55 \mathrm{~N} 1$ & $2.97 \mathrm{E}-07$ & Neutral O1 & $\mathrm{C} 23 \mathrm{H} 12 \mathrm{O} 1$ & $3.07 \mathrm{E}-05$ \\
\hline S1 & $\mathrm{C} 32 \mathrm{H} 34 \mathrm{~S} 1$ & $9.02 \mathrm{E}-05$ & Basic N1 & $\mathrm{C} 33 \mathrm{H} 57 \mathrm{~N} 1$ & $4.15 \mathrm{E}-07$ & Neutral O1 & $\mathrm{C} 24 \mathrm{H} 14 \mathrm{O} 1$ & 0.000148 \\
\hline $\mathrm{S} 1$ & $\mathrm{C} 33 \mathrm{H} 36 \mathrm{~S} 1$ & $7.42 \mathrm{E}-05$ & Basic N1 & C34H59N1 & $5.20 \mathrm{E}-07$ & Neutral O1 & $\mathrm{C} 25 \mathrm{H} 16 \mathrm{O} 1$ & 0.000209 \\
\hline $\mathrm{S} 1$ & $\mathrm{C} 34 \mathrm{H} 38 \mathrm{~S} 1$ & $6.42 \mathrm{E}-05$ & Basic N1 & $\mathrm{C} 35 \mathrm{H} 61 \mathrm{~N} 1$ & $7.28 \mathrm{E}-07$ & Neutral O1 & $\mathrm{C} 26 \mathrm{H} 18 \mathrm{O} 1$ & 0.000225 \\
\hline $\mathrm{S} 1$ & $\mathrm{C} 35 \mathrm{H} 40 \mathrm{~S} 1$ & $5.10 \mathrm{E}-05$ & Basic N1 & C36H63N1 & $7.11 \mathrm{E}-07$ & Neutral O1 & $\mathrm{C} 27 \mathrm{H} 20 \mathrm{O} 1$ & 0.000304 \\
\hline $\mathrm{S} 1$ & $\mathrm{C} 36 \mathrm{H} 42 \mathrm{~S} 1$ & $5.64 \mathrm{E}-05$ & Basic N1 & C37H65N1 & $8.92 \mathrm{E}-07$ & Neutral O1 & $\mathrm{C} 28 \mathrm{H} 22 \mathrm{O} 1$ & 0.000369 \\
\hline $\mathrm{S} 1$ & $\mathrm{C} 37 \mathrm{H} 44 \mathrm{~S} 1$ & 4.37E- 05 & Basic N1 & $\mathrm{C} 38 \mathrm{H} 67 \mathrm{~N} 1$ & $1.15 \mathrm{E}-06$ & Neutral O1 & $\mathrm{C} 29 \mathrm{H} 24 \mathrm{O} 1$ & 0.000316 \\
\hline $\mathrm{S} 1$ & $\mathrm{C} 38 \mathrm{H} 46 \mathrm{~S} 1$ & $4.75 \mathrm{E}-05$ & Basic N1 & C39H69N1 & $1.01 \mathrm{E}-06$ & Neutral O1 & $\mathrm{C} 30 \mathrm{H} 26 \mathrm{O} 1$ & 0.00021 \\
\hline $\mathrm{S} 1$ & $\mathrm{C} 39 \mathrm{H} 48 \mathrm{~S} 1$ & $4.31 \mathrm{E}-05$ & Basic N1 & $\mathrm{C} 40 \mathrm{H} 71 \mathrm{~N} 1$ & $2.44 \mathrm{E}-06$ & Neutral O1 & $\mathrm{C} 31 \mathrm{H} 28 \mathrm{O} 1$ & 0.000139 \\
\hline S1 & C40H50S1 & $3.53 \mathrm{E}-05$ & Basic N1 & $\mathrm{C} 41 \mathrm{H} 73 \mathrm{~N} 1$ & $7.89 \mathrm{E}-07$ & Neutral O1 & $\mathrm{C} 32 \mathrm{H} 30 \mathrm{O} 1$ & $7.23 \mathrm{E}-05$ \\
\hline S1 & $\mathrm{C} 41 \mathrm{H} 52 \mathrm{~S} 1$ & $2.46 \mathrm{E}-05$ & Basic N1 & $\mathrm{C} 42 \mathrm{H} 75 \mathrm{~N} 1$ & $5.27 \mathrm{E}-07$ & Neutral O1 & $\mathrm{C} 33 \mathrm{H} 32 \mathrm{O} 1$ & $3.60 \mathrm{E}-05$ \\
\hline S1 & $\mathrm{C} 42 \mathrm{H} 54 \mathrm{~S} 1$ & $2.35 \mathrm{E}-05$ & Basic N1 & $\mathrm{C} 43 \mathrm{H} 77 \mathrm{~N} 1$ & $7.68 \mathrm{E}-07$ & Neutral O1 & $\mathrm{C} 34 \mathrm{H} 34 \mathrm{O} 1$ & $1.60 \mathrm{E}-05$ \\
\hline S1 & $\mathrm{C} 43 \mathrm{H} 56 \mathrm{~S} 1$ & $2.35 \mathrm{E}-05$ & Basic N1 & C44H79N1 & $4.66 \mathrm{E}-07$ & Neutral O1 & $\mathrm{C} 35 \mathrm{H} 36 \mathrm{O} 1$ & 7.62E-06 \\
\hline S1 & C44H58S1 & $1.94 \mathrm{E}-05$ & Basic N1 & $\mathrm{C} 45 \mathrm{H} 81 \mathrm{~N} 1$ & $9.07 \mathrm{E}-07$ & Neutral O1 & $\mathrm{C} 36 \mathrm{H} 38 \mathrm{O} 1$ & $6.06 \mathrm{E}-06$ \\
\hline
\end{tabular}




\begin{tabular}{|c|c|c|c|c|c|c|c|c|}
\hline Class & formula & Mass & Class & formula & Mass & Class & formula & Mass \\
\hline S1 & C45H60S1 & $1.05 \mathrm{E}-05$ & Basic N1 & $\mathrm{C} 46 \mathrm{H} 83 \mathrm{~N} 1$ & $2.83 \mathrm{E}-07$ & Neutral O1 & $\mathrm{C} 37 \mathrm{H} 40 \mathrm{O} 1$ & $3.00 \mathrm{E}-06$ \\
\hline S1 & $\mathrm{C} 46 \mathrm{H} 62 \mathrm{~S} 1$ & $7.26 \mathrm{E}-06$ & Basic N1 & C47H85N1 & $1.79 \mathrm{E}-07$ & Neutral O1 & $\mathrm{C} 38 \mathrm{H} 42 \mathrm{O} 1$ & $3.30 \mathrm{E}-06$ \\
\hline S1 & C47H64S1 & $1.05 \mathrm{E}-05$ & Basic N1 & $\mathrm{C} 48 \mathrm{H} 87 \mathrm{~N} 1$ & $1.36 \mathrm{E}-07$ & Neutral O1 & $\mathrm{C} 39 \mathrm{H} 44 \mathrm{O} 1$ & $6.41 \mathrm{E}-07$ \\
\hline S1 & C48H66S1 & $6.71 \mathrm{E}-06$ & Basic N1 & $\mathrm{C} 20 \mathrm{H} 29 \mathrm{~N} 1$ & $1.82 \mathrm{E}-08$ & Neutral O1 & $\mathrm{C} 22 \mathrm{H} 8 \mathrm{O} 1$ & $3.25 \mathrm{E}-07$ \\
\hline S1 & $\mathrm{C} 49 \mathrm{H} 68 \mathrm{~S} 1$ & $1.33 \mathrm{E}-05$ & Basic N1 & $\mathrm{C} 21 \mathrm{H} 31 \mathrm{~N} 1$ & $8.01 \mathrm{E}-08$ & Neutral O1 & $\mathrm{C} 23 \mathrm{H} 10 \mathrm{O} 1$ & $5.18 \mathrm{E}-07$ \\
\hline S1 & $\mathrm{C} 50 \mathrm{H} 70 \mathrm{~S} 1$ & $2.09 \mathrm{E}-06$ & Basic N1 & $\mathrm{C} 22 \mathrm{H} 33 \mathrm{~N} 1$ & $3.45 \mathrm{E}-08$ & Neutral O1 & $\mathrm{C} 24 \mathrm{H} 12 \mathrm{O} 1$ & $1.16 \mathrm{E}-06$ \\
\hline S1 & $\mathrm{C} 20 \mathrm{H} 8 \mathrm{~S} 1$ & $8.78 \mathrm{E}-07$ & Basic N1 & $\mathrm{C} 23 \mathrm{H} 35 \mathrm{~N} 1$ & $1.89 \mathrm{E}-07$ & Neutral O1 & $\mathrm{C} 25 \mathrm{H} 14 \mathrm{O} 1$ & $1.84 \mathrm{E}-05$ \\
\hline S1 & $\mathrm{C} 21 \mathrm{H} 10 \mathrm{~S} 1$ & $9.43 \mathrm{E}-07$ & Basic N1 & $\mathrm{C} 24 \mathrm{H} 37 \mathrm{~N} 1$ & $2.87 \mathrm{E}-07$ & Neutral O1 & $\mathrm{C} 26 \mathrm{H} 16 \mathrm{O} 1$ & $7.40 \mathrm{E}-05$ \\
\hline S1 & $\mathrm{C} 22 \mathrm{H} 12 \mathrm{~S} 1$ & $2.81 \mathrm{E}-05$ & Basic N1 & $\mathrm{C} 25 \mathrm{H} 39 \mathrm{~N} 1$ & 4.07E-07 & Neutral O1 & $\mathrm{C} 27 \mathrm{H} 18 \mathrm{O} 1$ & 0.000127 \\
\hline S1 & $\mathrm{C} 23 \mathrm{H} 14 \mathrm{~S} 1$ & 0.000115 & Basic N1 & $\mathrm{C} 26 \mathrm{H} 41 \mathrm{~N} 1$ & $9.74 \mathrm{E}-07$ & Neutral O1 & $\mathrm{C} 28 \mathrm{H} 20 \mathrm{O} 1$ & 0.000191 \\
\hline S1 & $\mathrm{C} 24 \mathrm{H} 16 \mathrm{~S} 1$ & 0.000263 & Basic N1 & $\mathrm{C} 27 \mathrm{H} 43 \mathrm{~N} 1$ & $1.38 \mathrm{E}-06$ & Neutral O1 & $\mathrm{C} 29 \mathrm{H} 22 \mathrm{O} 1$ & 0.000234 \\
\hline S1 & $\mathrm{C} 25 \mathrm{H} 18 \mathrm{~S} 1$ & 0.000339 & Basic N1 & $\mathrm{C} 28 \mathrm{H} 45 \mathrm{~N} 1$ & $2.14 \mathrm{E}-06$ & Neutral O1 & $\mathrm{C} 30 \mathrm{H} 24 \mathrm{O} 1$ & 0.000251 \\
\hline S1 & $\mathrm{C} 26 \mathrm{H} 20 \mathrm{~S} 1$ & 0.000337 & Basic N1 & $\mathrm{C} 29 \mathrm{H} 47 \mathrm{~N} 1$ & $3.00 \mathrm{E}-06$ & Neutral O1 & $\mathrm{C} 31 \mathrm{H} 26 \mathrm{O} 1$ & 0.000208 \\
\hline S1 & $\mathrm{C} 27 \mathrm{H} 22 \mathrm{~S} 1$ & 0.000308 & Basic N1 & $\mathrm{C} 30 \mathrm{H} 49 \mathrm{~N} 1$ & $3.97 \mathrm{E}-06$ & Neutral O1 & $\mathrm{C} 32 \mathrm{H} 28 \mathrm{O} 1$ & 0.000143 \\
\hline S1 & $\mathrm{C} 28 \mathrm{H} 24 \mathrm{~S} 1$ & 0.000233 & Basic N1 & $\mathrm{C} 31 \mathrm{H} 51 \mathrm{~N} 1$ & $5.31 \mathrm{E}-06$ & Neutral O1 & $\mathrm{C} 33 \mathrm{H} 30 \mathrm{O} 1$ & $8.09 \mathrm{E}-05$ \\
\hline S1 & $\mathrm{C} 29 \mathrm{H} 26 \mathrm{~S} 1$ & 0.000187 & Basic N1 & $\mathrm{C} 32 \mathrm{H} 53 \mathrm{~N} 1$ & $6.34 \mathrm{E}-06$ & Neutral O1 & $\mathrm{C} 34 \mathrm{H} 32 \mathrm{O} 1$ & $2.46 \mathrm{E}-05$ \\
\hline S1 & $\mathrm{C} 30 \mathrm{H} 28 \mathrm{~S} 1$ & 0.00014 & Basic N1 & $\mathrm{C} 33 \mathrm{H} 55 \mathrm{~N} 1$ & $7.20 \mathrm{E}-06$ & Neutral O1 & $\mathrm{C} 35 \mathrm{H} 34 \mathrm{O} 1$ & $8.76 \mathrm{E}-06$ \\
\hline S1 & $\mathrm{C} 31 \mathrm{H} 30 \mathrm{~S} 1$ & 0.000125 & Basic N1 & $\mathrm{C} 34 \mathrm{H} 57 \mathrm{~N} 1$ & $7.65 \mathrm{E}-06$ & Neutral O1 & $\mathrm{C} 36 \mathrm{H} 36 \mathrm{O} 1$ & $5.50 \mathrm{E}-06$ \\
\hline S1 & $\mathrm{C} 32 \mathrm{H} 32 \mathrm{~S} 1$ & $9.44 \mathrm{E}-05$ & Basic N1 & $\mathrm{C} 35 \mathrm{H} 59 \mathrm{~N} 1$ & $7.60 \mathrm{E}-06$ & Neutral O1 & $\mathrm{C} 37 \mathrm{H} 38 \mathrm{O} 1$ & $2.63 \mathrm{E}-06$ \\
\hline S1 & $\mathrm{C} 33 \mathrm{H} 34 \mathrm{~S} 1$ & 7.73E-05 & Basic N1 & $\mathrm{C} 36 \mathrm{H} 61 \mathrm{~N} 1$ & $8.26 \mathrm{E}-06$ & Neutral O1 & $\mathrm{C} 38 \mathrm{H} 40 \mathrm{O} 1$ & $2.10 \mathrm{E}-06$ \\
\hline S1 & $\mathrm{C} 34 \mathrm{H} 36 \mathrm{~S} 1$ & 6.77E-05 & Basic N1 & $\mathrm{C} 37 \mathrm{H} 63 \mathrm{~N} 1$ & $8.65 \mathrm{E}-06$ & Neutral O1 & $\mathrm{C} 39 \mathrm{H} 42 \mathrm{O} 1$ & $1.70 \mathrm{E}-06$ \\
\hline S1 & $\mathrm{C} 35 \mathrm{H} 38 \mathrm{~S} 1$ & $6.07 \mathrm{E}-05$ & Basic N1 & $\mathrm{C} 38 \mathrm{H} 65 \mathrm{~N} 1$ & $8.31 \mathrm{E}-06$ & Neutral O1 & $\mathrm{C} 40 \mathrm{H} 44 \mathrm{O} 1$ & $2.64 \mathrm{E}-06$ \\
\hline S1 & $\mathrm{C} 36 \mathrm{H} 40 \mathrm{~S} 1$ & $4.01 \mathrm{E}-05$ & Basic N1 & $\mathrm{C} 39 \mathrm{H} 67 \mathrm{~N} 1$ & $7.48 \mathrm{E}-06$ & Neutral O1 & $\mathrm{C} 27 \mathrm{H} 16 \mathrm{O} 1$ & $8.54 \mathrm{E}-06$ \\
\hline S1 & $\mathrm{C} 37 \mathrm{H} 42 \mathrm{~S} 1$ & $3.92 \mathrm{E}-05$ & Basic N1 & $\mathrm{C} 40 \mathrm{H} 69 \mathrm{~N} 1$ & $7.62 \mathrm{E}-06$ & Neutral O1 & $\mathrm{C} 28 \mathrm{H} 18 \mathrm{O} 1$ & $8.82 \mathrm{E}-05$ \\
\hline S1 & $\mathrm{C} 38 \mathrm{H} 44 \mathrm{~S} 1$ & $3.68 \mathrm{E}-05$ & Basic N1 & $\mathrm{C} 41 \mathrm{H} 71 \mathrm{~N} 1$ & $4.89 \mathrm{E}-06$ & Neutral O1 & $\mathrm{C} 29 \mathrm{H} 20 \mathrm{O} 1$ & 0.000151 \\
\hline S1 & $\mathrm{C} 39 \mathrm{H} 46 \mathrm{~S} 1$ & $3.62 \mathrm{E}-05$ & Basic N1 & $\mathrm{C} 42 \mathrm{H} 73 \mathrm{~N} 1$ & $3.94 \mathrm{E}-06$ & Neutral O1 & $\mathrm{C} 30 \mathrm{H} 22 \mathrm{O} 1$ & 0.000168 \\
\hline S1 & $\mathrm{C} 40 \mathrm{H} 48 \mathrm{~S} 1$ & $2.50 \mathrm{E}-05$ & Basic N1 & $\mathrm{C} 43 \mathrm{H} 75 \mathrm{~N} 1$ & $3.07 \mathrm{E}-06$ & Neutral O1 & $\mathrm{C} 31 \mathrm{H} 24 \mathrm{O} 1$ & 0.000137 \\
\hline S1 & C41H50S1 & $2.63 \mathrm{E}-05$ & Basic N1 & $\mathrm{C} 44 \mathrm{H} 77 \mathrm{~N} 1$ & $2.22 \mathrm{E}-06$ & Neutral O1 & $\mathrm{C} 32 \mathrm{H} 26 \mathrm{O} 1$ & 0.000118 \\
\hline S1 & $\mathrm{C} 42 \mathrm{H} 52 \mathrm{~S} 1$ & $2.83 \mathrm{E}-05$ & Basic N1 & $\mathrm{C} 45 \mathrm{H} 79 \mathrm{~N} 1$ & $1.99 \mathrm{E}-06$ & Neutral O1 & $\mathrm{C} 33 \mathrm{H} 28 \mathrm{O} 1$ & $7.80 \mathrm{E}-05$ \\
\hline S1 & $\mathrm{C} 43 \mathrm{H} 54 \mathrm{~S} 1$ & $1.87 \mathrm{E}-05$ & Basic N1 & $\mathrm{C} 46 \mathrm{H} 81 \mathrm{~N} 1$ & $1.23 \mathrm{E}-06$ & Neutral O1 & $\mathrm{C} 34 \mathrm{H} 30 \mathrm{O} 1$ & $3.50 \mathrm{E}-05$ \\
\hline S1 & C44H56S1 & $1.39 \mathrm{E}-05$ & Basic N1 & C47H83N1 & $6.70 \mathrm{E}-07$ & Neutral O1 & $\mathrm{C} 35 \mathrm{H} 32 \mathrm{O} 1$ & $2.21 \mathrm{E}-05$ \\
\hline S1 & $\mathrm{C} 45 \mathrm{H} 58 \mathrm{~S} 1$ & $1.58 \mathrm{E}-05$ & Basic N1 & $\mathrm{C} 15 \mathrm{H} 17 \mathrm{~N} 1$ & $5.45 \mathrm{E}-08$ & Neutral O1 & $\mathrm{C} 36 \mathrm{H} 34 \mathrm{O} 1$ & $7.06 \mathrm{E}-06$ \\
\hline S1 & $\mathrm{C} 46 \mathrm{H} 60 \mathrm{~S} 1$ & $6.81 \mathrm{E}-06$ & Basic N1 & $\mathrm{C} 16 \mathrm{H} 19 \mathrm{~N} 1$ & 4.77E-09 & Neutral O1 & $\mathrm{C} 37 \mathrm{H} 36 \mathrm{O} 1$ & $6.98 \mathrm{E}-06$ \\
\hline S1 & C47H62S1 & $4.82 \mathrm{E}-06$ & Basic N1 & $\mathrm{C} 17 \mathrm{H} 21 \mathrm{~N} 1$ & $2.14 \mathrm{E}-08$ & Neutral O1 & $\mathrm{C} 38 \mathrm{H} 38 \mathrm{O} 1$ & $2.67 \mathrm{E}-06$ \\
\hline S1 & C48H64S1 & $3.27 \mathrm{E}-06$ & Basic N1 & $\mathrm{C} 18 \mathrm{H} 23 \mathrm{~N} 1$ & $9.44 \mathrm{E}-07$ & Neutral O1 & $\mathrm{C} 39 \mathrm{H} 40 \mathrm{O} 1$ & $2.59 \mathrm{E}-06$ \\
\hline S1 & C49H66S1 & $4.11 \mathrm{E}-06$ & Basic N1 & $\mathrm{C} 19 \mathrm{H} 25 \mathrm{~N} 1$ & $1.35 \mathrm{E}-06$ & Neutral O1 & $\mathrm{C} 26 \mathrm{H} 12 \mathrm{O} 1$ & $4.38 \mathrm{E}-07$ \\
\hline S1 & $\mathrm{C} 50 \mathrm{H} 68 \mathrm{~S} 1$ & $2.51 \mathrm{E}-06$ & Basic N1 & $\mathrm{C} 20 \mathrm{H} 27 \mathrm{~N} 1$ & $2.96 \mathrm{E}-06$ & Neutral O1 & $\mathrm{C} 27 \mathrm{H} 14 \mathrm{O} 1$ & $6.81 \mathrm{E}-06$ \\
\hline S1 & C51H70S1 & $2.30 \mathrm{E}-06$ & Basic N1 & $\mathrm{C} 21 \mathrm{H} 29 \mathrm{~N} 1$ & 4.63E-06 & Neutral O1 & $\mathrm{C} 28 \mathrm{H} 16 \mathrm{O} 1$ & $3.96 \mathrm{E}-05$ \\
\hline
\end{tabular}




\begin{tabular}{|c|c|c|c|c|c|c|c|c|}
\hline Class & formula & Mass & Class & formula & Mass & Class & formula & Mass \\
\hline $\mathrm{S} 1$ & $\mathrm{C} 24 \mathrm{H} 14 \mathrm{~S} 1$ & $1.27 \mathrm{E}-05$ & Basic N1 & $\mathrm{C} 22 \mathrm{H} 31 \mathrm{~N} 1$ & $1.43 \mathrm{E}-06$ & Neutral O1 & $\mathrm{C} 29 \mathrm{H} 18 \mathrm{O} 1$ & $6.06 \mathrm{E}-05$ \\
\hline S1 & $\mathrm{C} 25 \mathrm{H} 16 \mathrm{~S} 1$ & $5.20 \mathrm{E}-05$ & Basic N1 & $\mathrm{C} 23 \mathrm{H} 33 \mathrm{~N} 1$ & $1.85 \mathrm{E}-06$ & Neutral O1 & $\mathrm{C} 30 \mathrm{H} 20 \mathrm{O} 1$ & $6.44 \mathrm{E}-05$ \\
\hline $\mathrm{S} 1$ & $\mathrm{C} 26 \mathrm{H} 18 \mathrm{~S} 1$ & 0.00012 & Basic N1 & $\mathrm{C} 24 \mathrm{H} 35 \mathrm{~N} 1$ & $3.52 \mathrm{E}-06$ & Neutral O1 & $\mathrm{C} 31 \mathrm{H} 22 \mathrm{O} 1$ & $7.09 \mathrm{E}-05$ \\
\hline $\mathrm{S} 1$ & $\mathrm{C} 27 \mathrm{H} 20 \mathrm{~S} 1$ & 0.000168 & Basic N1 & $\mathrm{C} 25 \mathrm{H} 37 \mathrm{~N} 1$ & $3.26 \mathrm{E}-06$ & Neutral O1 & $\mathrm{C} 32 \mathrm{H} 24 \mathrm{O} 1$ & $8.62 \mathrm{E}-05$ \\
\hline $\mathrm{S} 1$ & $\mathrm{C} 28 \mathrm{H} 22 \mathrm{~S} 1$ & 0.000174 & Basic N1 & $\mathrm{C} 26 \mathrm{H} 39 \mathrm{~N} 1$ & 4.84E-06 & Neutral O1 & $\mathrm{C} 33 \mathrm{H} 26 \mathrm{O} 1$ & $7.39 \mathrm{E}-05$ \\
\hline $\mathrm{S} 1$ & $\mathrm{C} 29 \mathrm{H} 24 \mathrm{~S} 1$ & 0.000155 & Basic N1 & $\mathrm{C} 27 \mathrm{H} 41 \mathrm{~N} 1$ & $6.62 \mathrm{E}-06$ & Neutral O1 & $\mathrm{C} 34 \mathrm{H} 28 \mathrm{O} 1$ & $4.43 \mathrm{E}-05$ \\
\hline $\mathrm{S} 1$ & $\mathrm{C} 30 \mathrm{H} 26 \mathrm{~S} 1$ & 0.000145 & Basic N1 & $\mathrm{C} 28 \mathrm{H} 43 \mathrm{~N} 1$ & $7.70 \mathrm{E}-06$ & Neutral O1 & $\mathrm{C} 35 \mathrm{H} 30 \mathrm{O} 1$ & $2.28 \mathrm{E}-05$ \\
\hline $\mathrm{S} 1$ & $\mathrm{C} 31 \mathrm{H} 28 \mathrm{~S} 1$ & 0.000107 & Basic N1 & $\mathrm{C} 29 \mathrm{H} 45 \mathrm{~N} 1$ & $8.21 \mathrm{E}-06$ & Neutral O1 & $\mathrm{C} 36 \mathrm{H} 32 \mathrm{O} 1$ & $1.23 \mathrm{E}-05$ \\
\hline $\mathrm{S} 1$ & $\mathrm{C} 32 \mathrm{H} 30 \mathrm{~S} 1$ & $8.35 \mathrm{E}-05$ & Basic N1 & $\mathrm{C} 30 \mathrm{H} 47 \mathrm{~N} 1$ & $1.03 \mathrm{E}-05$ & Neutral O1 & $\mathrm{C} 37 \mathrm{H} 34 \mathrm{O} 1$ & $3.47 \mathrm{E}-06$ \\
\hline $\mathrm{S} 1$ & $\mathrm{C} 33 \mathrm{H} 32 \mathrm{~S} 1$ & $7.14 \mathrm{E}-05$ & Basic N1 & $\mathrm{C} 31 \mathrm{H} 49 \mathrm{~N} 1$ & $1.29 \mathrm{E}-05$ & Neutral O1 & $\mathrm{C} 38 \mathrm{H} 36 \mathrm{O} 1$ & $2.54 \mathrm{E}-06$ \\
\hline $\mathrm{S} 1$ & $\mathrm{C} 34 \mathrm{H} 34 \mathrm{~S} 1$ & $5.34 \mathrm{E}-05$ & Basic N1 & $\mathrm{C} 32 \mathrm{H} 51 \mathrm{~N} 1$ & $1.51 \mathrm{E}-05$ & Neutral O1 & $\mathrm{C} 39 \mathrm{H} 38 \mathrm{O} 1$ & $1.70 \mathrm{E}-06$ \\
\hline $\mathrm{S} 1$ & $\mathrm{C} 35 \mathrm{H} 36 \mathrm{~S} 1$ & $4.18 \mathrm{E}-05$ & Basic N1 & $\mathrm{C} 33 \mathrm{H} 53 \mathrm{~N} 1$ & $1.61 \mathrm{E}-05$ & Neutral O1 & $\mathrm{C} 29 \mathrm{H} 16 \mathrm{O} 1$ & $5.70 \mathrm{E}-07$ \\
\hline $\mathrm{S} 1$ & $\mathrm{C} 36 \mathrm{H} 38 \mathrm{~S} 1$ & $3.88 \mathrm{E}-05$ & Basic N1 & $\mathrm{C} 34 \mathrm{H} 55 \mathrm{~N} 1$ & $1.62 \mathrm{E}-05$ & Neutral O1 & $\mathrm{C} 30 \mathrm{H} 18 \mathrm{O} 1$ & 4.83E-06 \\
\hline $\mathrm{S} 1$ & $\mathrm{C} 37 \mathrm{H} 40 \mathrm{~S} 1$ & $2.92 \mathrm{E}-05$ & Basic N1 & $\mathrm{C} 35 \mathrm{H} 57 \mathrm{~N} 1$ & $1.70 \mathrm{E}-05$ & Neutral O1 & $\mathrm{C} 31 \mathrm{H} 20 \mathrm{O} 1$ & $1.23 \mathrm{E}-05$ \\
\hline $\mathrm{S} 1$ & $\mathrm{C} 38 \mathrm{H} 42 \mathrm{~S} 1$ & $2.72 \mathrm{E}-05$ & Basic N1 & $\mathrm{C} 36 \mathrm{H} 59 \mathrm{~N} 1$ & $1.79 \mathrm{E}-05$ & Neutral O1 & $\mathrm{C} 32 \mathrm{H} 22 \mathrm{O} 1$ & $1.57 \mathrm{E}-05$ \\
\hline $\mathrm{S} 1$ & $\mathrm{C} 39 \mathrm{H} 44 \mathrm{~S} 1$ & $2.48 \mathrm{E}-05$ & Basic N1 & C37H61N1 & $1.75 \mathrm{E}-05$ & Neutral O1 & $\mathrm{C} 33 \mathrm{H} 24 \mathrm{O} 1$ & $2.19 \mathrm{E}-05$ \\
\hline $\mathrm{S} 1$ & $\mathrm{C} 40 \mathrm{H} 46 \mathrm{~S} 1$ & $2.62 \mathrm{E}-05$ & Basic N1 & $\mathrm{C} 38 \mathrm{H} 63 \mathrm{~N} 1$ & $1.57 \mathrm{E}-05$ & Neutral O1 & $\mathrm{C} 34 \mathrm{H} 26 \mathrm{O} 1$ & $3.56 \mathrm{E}-05$ \\
\hline $\mathrm{S} 1$ & $\mathrm{C} 41 \mathrm{H} 48 \mathrm{~S} 1$ & $1.89 \mathrm{E}-05$ & Basic N1 & $\mathrm{C} 39 \mathrm{H} 65 \mathrm{~N} 1$ & $1.37 \mathrm{E}-05$ & Neutral O1 & $\mathrm{C} 35 \mathrm{H} 28 \mathrm{O} 1$ & $2.58 \mathrm{E}-05$ \\
\hline $\mathrm{S} 1$ & $\mathrm{C} 42 \mathrm{H} 50 \mathrm{~S} 1$ & $1.13 \mathrm{E}-05$ & Basic N1 & $\mathrm{C} 40 \mathrm{H} 67 \mathrm{~N} 1$ & $1.32 \mathrm{E}-05$ & Neutral O1 & $\mathrm{C} 36 \mathrm{H} 30 \mathrm{O} 1$ & $1.02 \mathrm{E}-05$ \\
\hline $\mathrm{S} 1$ & $\mathrm{C} 43 \mathrm{H} 52 \mathrm{~S} 1$ & $1.49 \mathrm{E}-05$ & Basic N1 & C41H69N1 & $9.87 \mathrm{E}-06$ & Neutral O1 & $\mathrm{C} 37 \mathrm{H} 32 \mathrm{O} 1$ & $2.98 \mathrm{E}-06$ \\
\hline $\mathrm{S} 1$ & C44H54S1 & $8.70 \mathrm{E}-06$ & Basic N1 & $\mathrm{C} 42 \mathrm{H} 71 \mathrm{~N} 1$ & $7.83 \mathrm{E}-06$ & Neutral O1 & $\mathrm{C} 38 \mathrm{H} 34 \mathrm{O} 1$ & $4.65 \mathrm{E}-06$ \\
\hline $\mathrm{S} 1$ & C45H56S1 & $2.49 \mathrm{E}-06$ & Basic N1 & $\mathrm{C} 43 \mathrm{H} 73 \mathrm{~N} 1$ & $5.83 \mathrm{E}-06$ & Neutral O1 & $\mathrm{C} 39 \mathrm{H} 36 \mathrm{O} 1$ & $2.34 \mathrm{E}-06$ \\
\hline S1 & C46H58S1 & $7.41 \mathrm{E}-06$ & Basic N1 & $\mathrm{C} 44 \mathrm{H} 75 \mathrm{~N} 1$ & 4.44E-06 & Neutral O1 & $\mathrm{C} 40 \mathrm{H} 38 \mathrm{O} 1$ & $1.18 \mathrm{E}-06$ \\
\hline S1 & C47H60S1 & $5.61 \mathrm{E}-06$ & Basic N1 & $\mathrm{C} 45 \mathrm{H} 77 \mathrm{~N} 1$ & $4.12 \mathrm{E}-06$ & Neutral O1 & $\mathrm{C} 30 \mathrm{H} 16 \mathrm{O} 1$ & $4.68 \mathrm{E}-07$ \\
\hline S1 & $\mathrm{C} 48 \mathrm{H} 62 \mathrm{~S} 1$ & $4.96 \mathrm{E}-06$ & Basic N1 & $\mathrm{C} 46 \mathrm{H} 79 \mathrm{~N} 1$ & $2.79 \mathrm{E}-06$ & Neutral O1 & $\mathrm{C} 31 \mathrm{H} 18 \mathrm{O} 1$ & $6.74 \mathrm{E}-07$ \\
\hline S1 & $\mathrm{C} 24 \mathrm{H} 12 \mathrm{~S} 1$ & $1.32 \mathrm{E}-06$ & Basic N1 & $\mathrm{C} 47 \mathrm{H} 81 \mathrm{~N} 1$ & $1.59 \mathrm{E}-06$ & Neutral O1 & $\mathrm{C} 32 \mathrm{H} 20 \mathrm{O} 1$ & $2.13 \mathrm{E}-06$ \\
\hline $\mathrm{S} 1$ & $\mathrm{C} 25 \mathrm{H} 14 \mathrm{~S} 1$ & $1.68 \mathrm{E}-05$ & Basic N1 & $\mathrm{C} 48 \mathrm{H} 83 \mathrm{~N} 1$ & 8.95E-07 & Neutral O1 & $\mathrm{C} 33 \mathrm{H} 22 \mathrm{O} 1$ & $5.06 \mathrm{E}-06$ \\
\hline S1 & $\mathrm{C} 26 \mathrm{H} 16 \mathrm{~S} 1$ & $4.40 \mathrm{E}-05$ & Basic N1 & $\mathrm{C} 49 \mathrm{H} 85 \mathrm{~N} 1$ & $9.67 \mathrm{E}-07$ & Neutral O1 & $\mathrm{C} 34 \mathrm{H} 24 \mathrm{O} 1$ & $7.62 \mathrm{E}-06$ \\
\hline S1 & $\mathrm{C} 27 \mathrm{H} 18 \mathrm{~S} 1$ & $7.70 \mathrm{E}-05$ & Basic N1 & $\mathrm{C} 50 \mathrm{H} 87 \mathrm{~N} 1$ & $4.21 \mathrm{E}-07$ & Neutral O1 & $\mathrm{C} 35 \mathrm{H} 26 \mathrm{O} 1$ & $8.60 \mathrm{E}-06$ \\
\hline S1 & $\mathrm{C} 28 \mathrm{H} 20 \mathrm{~S} 1$ & $8.63 \mathrm{E}-05$ & Basic N1 & $\mathrm{C} 16 \mathrm{H} 17 \mathrm{~N} 1$ & $9.63 \mathrm{E}-08$ & Neutral O1 & $\mathrm{C} 36 \mathrm{H} 28 \mathrm{O} 1$ & 8.44E-06 \\
\hline S1 & $\mathrm{C} 29 \mathrm{H} 22 \mathrm{~S} 1$ & 0.0001 & Basic N1 & C17H19N1 & $1.84 \mathrm{E}-07$ & Neutral O1 & $\mathrm{C} 37 \mathrm{H} 30 \mathrm{O} 1$ & $5.60 \mathrm{E}-06$ \\
\hline S1 & $\mathrm{C} 30 \mathrm{H} 24 \mathrm{~S} 1$ & $8.95 \mathrm{E}-05$ & Basic N1 & $\mathrm{C} 18 \mathrm{H} 21 \mathrm{~N} 1$ & $6.14 \mathrm{E}-07$ & Neutral O1 & $\mathrm{C} 38 \mathrm{H} 32 \mathrm{O} 1$ & $4.12 \mathrm{E}-06$ \\
\hline S1 & $\mathrm{C} 31 \mathrm{H} 26 \mathrm{~S} 1$ & $9.59 \mathrm{E}-05$ & Basic N1 & $\mathrm{C} 19 \mathrm{H} 23 \mathrm{~N} 1$ & $1.43 \mathrm{E}-06$ & Neutral O1 & $\mathrm{C} 39 \mathrm{H} 34 \mathrm{O} 1$ & $3.63 \mathrm{E}-06$ \\
\hline S1 & $\mathrm{C} 32 \mathrm{H} 28 \mathrm{~S} 1$ & $7.29 \mathrm{E}-05$ & Basic N1 & $\mathrm{C} 20 \mathrm{H} 25 \mathrm{~N} 1$ & $2.33 \mathrm{E}-06$ & Neutral O1 & $\mathrm{C} 40 \mathrm{H} 36 \mathrm{O} 1$ & $4.23 \mathrm{E}-06$ \\
\hline S1 & $\mathrm{C} 33 \mathrm{H} 30 \mathrm{~S} 1$ & $5.75 \mathrm{E}-05$ & Basic N1 & $\mathrm{C} 21 \mathrm{H} 27 \mathrm{~N} 1$ & $5.84 \mathrm{E}-05$ & Neutral O1 & $\mathrm{C} 33 \mathrm{H} 20 \mathrm{O} 1$ & $2.34 \mathrm{E}-06$ \\
\hline S1 & $\mathrm{C} 34 \mathrm{H} 32 \mathrm{~S} 1$ & $4.73 \mathrm{E}-05$ & Basic N1 & $\mathrm{C} 22 \mathrm{H} 29 \mathrm{~N} 1$ & $4.91 \mathrm{E}-06$ & Neutral O1 & $\mathrm{C} 34 \mathrm{H} 22 \mathrm{O} 1$ & $1.41 \mathrm{E}-06$ \\
\hline S1 & $\mathrm{C} 35 \mathrm{H} 34 \mathrm{~S} 1$ & $3.93 \mathrm{E}-05$ & Basic N1 & $\mathrm{C} 23 \mathrm{H} 31 \mathrm{~N} 1$ & $6.89 \mathrm{E}-06$ & Neutral O1 & $\mathrm{C} 35 \mathrm{H} 24 \mathrm{O} 1$ & $3.17 \mathrm{E}-06$ \\
\hline S1 & $\mathrm{C} 36 \mathrm{H} 36 \mathrm{~S} 1$ & $3.21 \mathrm{E}-05$ & Basic N1 & $\mathrm{C} 24 \mathrm{H} 33 \mathrm{~N} 1$ & $8.40 \mathrm{E}-06$ & Neutral O1 & $\mathrm{C} 36 \mathrm{H} 26 \mathrm{O} 1$ & $5.12 \mathrm{E}-06$ \\
\hline
\end{tabular}




\begin{tabular}{|c|c|c|c|c|c|c|c|c|}
\hline Class & formula & Mass & Class & formula & Mass & Class & formula & Mass \\
\hline $\mathrm{S} 1$ & C37H38S1 & $2.45 \mathrm{E}-05$ & Basic N1 & $\mathrm{C} 25 \mathrm{H} 35 \mathrm{~N} 1$ & 8.91E-06 & Neutral O1 & $\mathrm{C} 37 \mathrm{H} 28 \mathrm{O} 1$ & $1.56 \mathrm{E}-06$ \\
\hline S1 & $\mathrm{C} 38 \mathrm{H} 40 \mathrm{~S} 1$ & $1.90 \mathrm{E}-05$ & Basic N1 & $\mathrm{C} 26 \mathrm{H} 37 \mathrm{~N} 1$ & $1.10 \mathrm{E}-05$ & Neutral O1 & $\mathrm{C} 38 \mathrm{H} 30 \mathrm{O} 1$ & $4.75 \mathrm{E}-06$ \\
\hline $\mathrm{S} 1$ & $\mathrm{C} 39 \mathrm{H} 42 \mathrm{~S} 1$ & $1.35 \mathrm{E}-05$ & Basic N1 & $\mathrm{C} 27 \mathrm{H} 39 \mathrm{~N} 1$ & $1.41 \mathrm{E}-05$ & Neutral O1 & $\mathrm{C} 39 \mathrm{H} 32 \mathrm{O} 1$ & $3.23 \mathrm{E}-06$ \\
\hline $\mathrm{S} 1$ & $\mathrm{C} 40 \mathrm{H} 44 \mathrm{~S} 1$ & $1.63 \mathrm{E}-05$ & Basic N1 & $\mathrm{C} 28 \mathrm{H} 41 \mathrm{~N} 1$ & $1.56 \mathrm{E}-05$ & Neutral O1 & $\mathrm{C} 40 \mathrm{H} 34 \mathrm{O} 1$ & $2.06 \mathrm{E}-06$ \\
\hline $\mathrm{S} 1$ & $\mathrm{C} 41 \mathrm{H} 46 \mathrm{~S} 1$ & $1.41 \mathrm{E}-05$ & Basic N1 & $\mathrm{C} 29 \mathrm{H} 43 \mathrm{~N} 1$ & $1.70 \mathrm{E}-05$ & Neutral O1 & $\mathrm{C} 41 \mathrm{H} 36 \mathrm{O} 1$ & $1.38 \mathrm{E}-06$ \\
\hline $\mathrm{S} 1$ & $\mathrm{C} 42 \mathrm{H} 48 \mathrm{~S} 1$ & $1.24 \mathrm{E}-05$ & Basic N1 & $\mathrm{C} 30 \mathrm{H} 45 \mathrm{~N} 1$ & $1.98 \mathrm{E}-05$ & Neutral O1 & $\mathrm{C} 42 \mathrm{H} 38 \mathrm{O} 1$ & $1.75 \mathrm{E}-06$ \\
\hline $\mathrm{S} 1$ & C43H50S1 & $2.47 \mathrm{E}-06$ & Basic N1 & $\mathrm{C} 31 \mathrm{H} 47 \mathrm{~N} 1$ & $2.23 \mathrm{E}-05$ & Neutral O1 & $\mathrm{C} 43 \mathrm{H} 40 \mathrm{O} 1$ & $1.63 \mathrm{E}-06$ \\
\hline $\mathrm{S} 1$ & C44H52S1 & $6.60 \mathrm{E}-06$ & Basic N1 & $\mathrm{C} 32 \mathrm{H} 49 \mathrm{~N} 1$ & $2.34 \mathrm{E}-05$ & Neutral O1 & $\mathrm{C} 44 \mathrm{H} 42 \mathrm{O} 1$ & $1.75 \mathrm{E}-06$ \\
\hline $\mathrm{S} 1$ & $\mathrm{C} 45 \mathrm{H} 54 \mathrm{~S} 1$ & $1.80 \mathrm{E}-06$ & Basic N1 & $\mathrm{C} 33 \mathrm{H} 51 \mathrm{~N} 1$ & $2.47 \mathrm{E}-05$ & Neutral O1 & $\mathrm{C} 45 \mathrm{H} 44 \mathrm{O} 1$ & $1.49 \mathrm{E}-06$ \\
\hline $\mathrm{S} 1$ & C46H56S1 & 4.84E-06 & Basic N1 & $\mathrm{C} 34 \mathrm{H} 53 \mathrm{~N} 1$ & $2.42 \mathrm{E}-05$ & Neutral O1 & $\mathrm{C} 30 \mathrm{H} 12 \mathrm{O} 1$ & $5.15 \mathrm{E}-07$ \\
\hline $\mathrm{S} 1$ & $\mathrm{C} 47 \mathrm{H} 58 \mathrm{~S} 1$ & $4.66 \mathrm{E}-06$ & Basic N1 & $\mathrm{C} 35 \mathrm{H} 55 \mathrm{~N} 1$ & $2.35 \mathrm{E}-05$ & Neutral O1 & $\mathrm{C} 31 \mathrm{H} 14 \mathrm{O} 1$ & $9.44 \mathrm{E}-07$ \\
\hline $\mathrm{S} 1$ & C48H60S1 & $1.79 \mathrm{E}-06$ & Basic N1 & $\mathrm{C} 36 \mathrm{H} 57 \mathrm{~N} 1$ & $2.34 \mathrm{E}-05$ & Neutral O1 & $\mathrm{C} 32 \mathrm{H} 16 \mathrm{O} 1$ & $5.45 \mathrm{E}-07$ \\
\hline $\mathrm{S} 1$ & $\mathrm{C} 27 \mathrm{H} 16 \mathrm{~S} 1$ & $1.99 \mathrm{E}-05$ & Basic N1 & $\mathrm{C} 37 \mathrm{H} 59 \mathrm{~N} 1$ & $2.39 \mathrm{E}-05$ & Neutral O1 & $\mathrm{C} 34 \mathrm{H} 20 \mathrm{O} 1$ & $6.93 \mathrm{E}-07$ \\
\hline $\mathrm{S} 1$ & $\mathrm{C} 28 \mathrm{H} 18 \mathrm{~S} 1$ & $3.80 \mathrm{E}-05$ & Basic N1 & $\mathrm{C} 38 \mathrm{H} 61 \mathrm{~N} 1$ & $2.12 \mathrm{E}-05$ & Neutral O1 & $\mathrm{C} 35 \mathrm{H} 22 \mathrm{O} 1$ & $2.94 \mathrm{E}-06$ \\
\hline $\mathrm{S} 1$ & $\mathrm{C} 29 \mathrm{H} 20 \mathrm{~S} 1$ & $6.47 \mathrm{E}-05$ & Basic N1 & $\mathrm{C} 39 \mathrm{H} 63 \mathrm{~N} 1$ & $1.82 \mathrm{E}-05$ & Neutral O1 & $\mathrm{C} 36 \mathrm{H} 24 \mathrm{O} 1$ & $6.92 \mathrm{E}-07$ \\
\hline $\mathrm{S} 1$ & $\mathrm{C} 30 \mathrm{H} 22 \mathrm{~S} 1$ & $6.53 \mathrm{E}-05$ & Basic N1 & $\mathrm{C} 40 \mathrm{H} 65 \mathrm{~N} 1$ & $1.70 \mathrm{E}-05$ & Neutral O1 & $\mathrm{C} 37 \mathrm{H} 26 \mathrm{O} 1$ & $3.89 \mathrm{E}-06$ \\
\hline $\mathrm{S} 1$ & $\mathrm{C} 31 \mathrm{H} 24 \mathrm{~S} 1$ & $6.04 \mathrm{E}-05$ & Basic N1 & C41H67N1 & $1.36 \mathrm{E}-05$ & Neutral O1 & $\mathrm{C} 38 \mathrm{H} 28 \mathrm{O} 1$ & $9.37 \mathrm{E}-07$ \\
\hline $\mathrm{S} 1$ & $\mathrm{C} 32 \mathrm{H} 26 \mathrm{~S} 1$ & $5.80 \mathrm{E}-05$ & Basic N1 & $\mathrm{C} 42 \mathrm{H} 69 \mathrm{~N} 1$ & $1.04 \mathrm{E}-05$ & Neutral O1 & $\mathrm{C} 39 \mathrm{H} 30 \mathrm{O} 1$ & $4.92 \mathrm{E}-06$ \\
\hline $\mathrm{S} 1$ & $\mathrm{C} 33 \mathrm{H} 28 \mathrm{~S} 1$ & $5.73 \mathrm{E}-05$ & Basic N1 & $\mathrm{C} 43 \mathrm{H} 71 \mathrm{~N} 1$ & 8.04E-06 & Neutral O1 & $\mathrm{C} 40 \mathrm{H} 32 \mathrm{O} 1$ & $6.39 \mathrm{E}-06$ \\
\hline $\mathrm{S} 1$ & $\mathrm{C} 34 \mathrm{H} 30 \mathrm{~S} 1$ & $4.35 \mathrm{E}-05$ & Basic N1 & $\mathrm{C} 44 \mathrm{H} 73 \mathrm{~N} 1$ & $6.67 \mathrm{E}-06$ & Neutral O1 & $\mathrm{C} 41 \mathrm{H} 34 \mathrm{O} 1$ & 7.71E-06 \\
\hline $\mathrm{S} 1$ & $\mathrm{C} 35 \mathrm{H} 32 \mathrm{~S} 1$ & $3.35 \mathrm{E}-05$ & Basic N1 & $\mathrm{C} 45 \mathrm{H} 75 \mathrm{~N} 1$ & $5.17 \mathrm{E}-06$ & Neutral O1 & $\mathrm{C} 42 \mathrm{H} 36 \mathrm{O} 1$ & $4.62 \mathrm{E}-06$ \\
\hline $\mathrm{S} 1$ & $\mathrm{C} 36 \mathrm{H} 34 \mathrm{~S} 1$ & $3.23 \mathrm{E}-05$ & Basic N1 & $\mathrm{C} 46 \mathrm{H} 77 \mathrm{~N} 1$ & $3.90 \mathrm{E}-06$ & Neutral O1 & $\mathrm{C} 43 \mathrm{H} 38 \mathrm{O} 1$ & $1.61 \mathrm{E}-06$ \\
\hline S1 & $\mathrm{C} 37 \mathrm{H} 36 \mathrm{~S} 1$ & $2.32 \mathrm{E}-05$ & Basic N1 & $\mathrm{C} 47 \mathrm{H} 79 \mathrm{~N} 1$ & $2.65 \mathrm{E}-06$ & Neutral O1 & $\mathrm{C} 44 \mathrm{H} 40 \mathrm{O} 1$ & $7.70 \mathrm{E}-07$ \\
\hline S1 & $\mathrm{C} 38 \mathrm{H} 38 \mathrm{~S} 1$ & $1.94 \mathrm{E}-05$ & Basic N1 & $\mathrm{C} 48 \mathrm{H} 81 \mathrm{~N} 1$ & $1.79 \mathrm{E}-06$ & Neutral O2 & $\mathrm{C} 15 \mathrm{H} 20 \mathrm{O} 2$ & $3.98 \mathrm{E}-06$ \\
\hline S1 & $\mathrm{C} 39 \mathrm{H} 40 \mathrm{~S} 1$ & $1.52 \mathrm{E}-05$ & Basic N1 & $\mathrm{C} 49 \mathrm{H} 83 \mathrm{~N} 1$ & $1.33 \mathrm{E}-06$ & Neutral O2 & $\mathrm{C} 16 \mathrm{H} 22 \mathrm{O} 2$ & $6.31 \mathrm{E}-06$ \\
\hline S1 & $\mathrm{C} 40 \mathrm{H} 42 \mathrm{~S} 1$ & $6.54 \mathrm{E}-06$ & Basic N1 & $\mathrm{C} 50 \mathrm{H} 85 \mathrm{~N} 1$ & $1.03 \mathrm{E}-06$ & Neutral O2 & $\mathrm{C} 17 \mathrm{H} 24 \mathrm{O} 2$ & $6.71 \mathrm{E}-07$ \\
\hline $\mathrm{S} 1$ & $\mathrm{C} 41 \mathrm{H} 44 \mathrm{~S} 1$ & $1.05 \mathrm{E}-05$ & Basic N1 & $\mathrm{C} 16 \mathrm{H} 15 \mathrm{~N} 1$ & $3.37 \mathrm{E}-07$ & Neutral O2 & $\mathrm{C} 18 \mathrm{H} 26 \mathrm{O} 2$ & $4.91 \mathrm{E}-07$ \\
\hline S1 & $\mathrm{C} 28 \mathrm{H} 16 \mathrm{~S} 1$ & $5.80 \mathrm{E}-06$ & Basic N1 & $\mathrm{C} 17 \mathrm{H} 17 \mathrm{~N} 1$ & $1.80 \mathrm{E}-06$ & Neutral O2 & $\mathrm{C} 19 \mathrm{H} 28 \mathrm{O} 2$ & $2.88 \mathrm{E}-07$ \\
\hline S1 & $\mathrm{C} 29 \mathrm{H} 18 \mathrm{~S} 1$ & $1.02 \mathrm{E}-05$ & Basic N1 & C18H19N1 & $2.68 \mathrm{E}-06$ & Neutral O2 & $\mathrm{C} 20 \mathrm{H} 30 \mathrm{O} 2$ & $8.14 \mathrm{E}-07$ \\
\hline S1 & $\mathrm{C} 30 \mathrm{H} 20 \mathrm{~S} 1$ & $2.00 \mathrm{E}-05$ & Basic N1 & $\mathrm{C} 19 \mathrm{H} 21 \mathrm{~N} 1$ & $3.52 \mathrm{E}-06$ & Neutral O2 & $\mathrm{C} 14 \mathrm{H} 16 \mathrm{O} 2$ & $3.47 \mathrm{E}-06$ \\
\hline S1 & $\mathrm{C} 31 \mathrm{H} 22 \mathrm{~S} 1$ & $3.63 \mathrm{E}-05$ & Basic N1 & $\mathrm{C} 20 \mathrm{H} 23 \mathrm{~N} 1$ & $5.99 \mathrm{E}-06$ & Neutral O2 & $\mathrm{C} 15 \mathrm{H} 18 \mathrm{O} 2$ & $5.99 \mathrm{E}-06$ \\
\hline S1 & $\mathrm{C} 32 \mathrm{H} 24 \mathrm{~S} 1$ & $4.62 \mathrm{E}-05$ & Basic N1 & $\mathrm{C} 21 \mathrm{H} 25 \mathrm{~N} 1$ & $5.28 \mathrm{E}-05$ & Neutral O2 & $\mathrm{C} 16 \mathrm{H} 20 \mathrm{O} 2$ & $1.16 \mathrm{E}-05$ \\
\hline S1 & $\mathrm{C} 33 \mathrm{H} 26 \mathrm{~S} 1$ & $5.07 \mathrm{E}-05$ & Basic N1 & $\mathrm{C} 22 \mathrm{H} 27 \mathrm{~N} 1$ & $9.83 \mathrm{E}-06$ & Neutral O2 & $\mathrm{C} 17 \mathrm{H} 22 \mathrm{O} 2$ & $1.59 \mathrm{E}-06$ \\
\hline S1 & $\mathrm{C} 34 \mathrm{H} 28 \mathrm{~S} 1$ & 5.17E-05 & Basic N1 & $\mathrm{C} 23 \mathrm{H} 29 \mathrm{~N} 1$ & $1.29 \mathrm{E}-05$ & Neutral O2 & $\mathrm{C} 18 \mathrm{H} 24 \mathrm{O} 2$ & $1.18 \mathrm{E}-06$ \\
\hline S1 & $\mathrm{C} 35 \mathrm{H} 30 \mathrm{~S} 1$ & 3.67E-05 & Basic N1 & $\mathrm{C} 24 \mathrm{H} 31 \mathrm{~N} 1$ & $1.74 \mathrm{E}-05$ & Neutral O2 & $\mathrm{C} 19 \mathrm{H} 26 \mathrm{O} 2$ & $9.60 \mathrm{E}-07$ \\
\hline S1 & $\mathrm{C} 36 \mathrm{H} 32 \mathrm{~S} 1$ & $3.32 \mathrm{E}-05$ & Basic N1 & $\mathrm{C} 25 \mathrm{H} 33 \mathrm{~N} 1$ & $2.02 \mathrm{E}-05$ & Neutral O2 & $\mathrm{C} 20 \mathrm{H} 28 \mathrm{O} 2$ & $7.32 \mathrm{E}-07$ \\
\hline S1 & $\mathrm{C} 37 \mathrm{H} 34 \mathrm{~S} 1$ & $2.50 \mathrm{E}-05$ & Basic N1 & $\mathrm{C} 26 \mathrm{H} 35 \mathrm{~N} 1$ & $2.38 \mathrm{E}-05$ & Neutral O2 & $\mathrm{C} 21 \mathrm{H} 30 \mathrm{O} 2$ & $3.82 \mathrm{E}-07$ \\
\hline S1 & $\mathrm{C} 38 \mathrm{H} 36 \mathrm{~S} 1$ & $2.36 \mathrm{E}-05$ & Basic N1 & $\mathrm{C} 27 \mathrm{H} 37 \mathrm{~N} 1$ & 3.07E-05 & Neutral O2 & $\mathrm{C} 13 \mathrm{H} 12 \mathrm{O} 2$ & $1.29 \mathrm{E}-07$ \\
\hline
\end{tabular}




\begin{tabular}{|c|c|c|c|c|c|c|c|c|}
\hline Class & formula & Mass & Class & formula & Mass & Class & formula & Mass \\
\hline $\mathrm{S} 1$ & C39H38S1 & $1.85 \mathrm{E}-05$ & Basic N1 & $\mathrm{C} 28 \mathrm{H} 39 \mathrm{~N} 1$ & $3.56 \mathrm{E}-05$ & Neutral O2 & $\mathrm{C} 14 \mathrm{H} 14 \mathrm{O} 2$ & $4.26 \mathrm{E}-07$ \\
\hline S1 & $\mathrm{C} 40 \mathrm{H} 40 \mathrm{~S} 1$ & $1.13 \mathrm{E}-05$ & Basic N1 & $\mathrm{C} 29 \mathrm{H} 41 \mathrm{~N} 1$ & 3.67E-05 & Neutral O2 & $\mathrm{C} 15 \mathrm{H} 16 \mathrm{O} 2$ & $3.91 \mathrm{E}-05$ \\
\hline $\mathrm{S} 1$ & $\mathrm{C} 41 \mathrm{H} 42 \mathrm{~S} 1$ & $6.49 \mathrm{E}-06$ & Basic N1 & $\mathrm{C} 30 \mathrm{H} 43 \mathrm{~N} 1$ & $3.94 \mathrm{E}-05$ & Neutral O2 & $\mathrm{C} 16 \mathrm{H} 18 \mathrm{O} 2$ & 0.001875 \\
\hline $\mathrm{S} 1$ & $\mathrm{C} 42 \mathrm{H} 44 \mathrm{~S} 1$ & $6.16 \mathrm{E}-06$ & Basic N1 & $\mathrm{C} 31 \mathrm{H} 45 \mathrm{~N} 1$ & $4.56 \mathrm{E}-05$ & Neutral O2 & $\mathrm{C} 17 \mathrm{H} 20 \mathrm{O} 2$ & $3.18 \mathrm{E}-06$ \\
\hline $\mathrm{S} 1$ & $\mathrm{C} 43 \mathrm{H} 46 \mathrm{~S} 1$ & $2.34 \mathrm{E}-06$ & Basic N1 & $\mathrm{C} 32 \mathrm{H} 47 \mathrm{~N} 1$ & $5.05 \mathrm{E}-05$ & Neutral O2 & $\mathrm{C} 14 \mathrm{H} 12 \mathrm{O} 2$ & $1.80 \mathrm{E}-07$ \\
\hline $\mathrm{S} 1$ & $\mathrm{C} 44 \mathrm{H} 48 \mathrm{~S} 1$ & $2.62 \mathrm{E}-06$ & Basic N1 & $\mathrm{C} 33 \mathrm{H} 49 \mathrm{~N} 1$ & 4.91E-05 & Neutral O2 & $\mathrm{C} 15 \mathrm{H} 14 \mathrm{O} 2$ & $1.45 \mathrm{E}-05$ \\
\hline $\mathrm{S} 1$ & $\mathrm{C} 30 \mathrm{H} 18 \mathrm{~S} 1$ & $3.35 \mathrm{E}-06$ & Basic N1 & C34H51N1 & $4.65 \mathrm{E}-05$ & Neutral O2 & $\mathrm{C} 16 \mathrm{H} 16 \mathrm{O} 2$ & 0.000274 \\
\hline $\mathrm{S} 1$ & $\mathrm{C} 31 \mathrm{H} 20 \mathrm{~S} 1$ & $6.78 \mathrm{E}-06$ & Basic N1 & $\mathrm{C} 35 \mathrm{H} 53 \mathrm{~N} 1$ & $4.20 \mathrm{E}-05$ & Neutral O2 & $\mathrm{C} 17 \mathrm{H} 18 \mathrm{O} 2$ & $1.24 \mathrm{E}-05$ \\
\hline $\mathrm{S} 1$ & $\mathrm{C} 32 \mathrm{H} 22 \mathrm{~S} 1$ & $1.27 \mathrm{E}-05$ & Basic N1 & $\mathrm{C} 36 \mathrm{H} 55 \mathrm{~N} 1$ & $3.87 \mathrm{E}-05$ & Neutral O2 & $\mathrm{C} 18 \mathrm{H} 20 \mathrm{O} 2$ & $5.01 \mathrm{E}-06$ \\
\hline $\mathrm{S} 1$ & $\mathrm{C} 33 \mathrm{H} 24 \mathrm{~S} 1$ & $1.87 \mathrm{E}-05$ & Basic N1 & $\mathrm{C} 37 \mathrm{H} 57 \mathrm{~N} 1$ & $3.62 \mathrm{E}-05$ & Neutral O2 & $\mathrm{C} 19 \mathrm{H} 22 \mathrm{O} 2$ & $5.25 \mathrm{E}-06$ \\
\hline $\mathrm{S} 1$ & $\mathrm{C} 34 \mathrm{H} 26 \mathrm{~S} 1$ & $2.64 \mathrm{E}-05$ & Basic N1 & C38H59N1 & $3.05 \mathrm{E}-05$ & Neutral O2 & $\mathrm{C} 20 \mathrm{H} 24 \mathrm{O} 2$ & 4.73E-06 \\
\hline $\mathrm{S} 1$ & $\mathrm{C} 35 \mathrm{H} 28 \mathrm{~S} 1$ & $2.59 \mathrm{E}-05$ & Basic N1 & C39H61N1 & $2.67 \mathrm{E}-05$ & Neutral O2 & $\mathrm{C} 21 \mathrm{H} 26 \mathrm{O} 2$ & $3.59 \mathrm{E}-06$ \\
\hline $\mathrm{S} 1$ & $\mathrm{C} 36 \mathrm{H} 30 \mathrm{~S} 1$ & $3.75 \mathrm{E}-05$ & Basic N1 & C40H63N1 & $2.25 \mathrm{E}-05$ & Neutral O2 & $\mathrm{C} 22 \mathrm{H} 28 \mathrm{O} 2$ & 4.47E-06 \\
\hline $\mathrm{S} 1$ & $\mathrm{C} 37 \mathrm{H} 32 \mathrm{~S} 1$ & $2.27 \mathrm{E}-05$ & Basic N1 & $\mathrm{C} 41 \mathrm{H} 65 \mathrm{~N} 1$ & $1.69 \mathrm{E}-05$ & Neutral O2 & $\mathrm{C} 23 \mathrm{H} 30 \mathrm{O} 2$ & $5.32 \mathrm{E}-06$ \\
\hline $\mathrm{S} 1$ & $\mathrm{C} 38 \mathrm{H} 34 \mathrm{~S} 1$ & $1.86 \mathrm{E}-05$ & Basic N1 & $\mathrm{C} 42 \mathrm{H} 67 \mathrm{~N} 1$ & $1.26 \mathrm{E}-05$ & Neutral O2 & $\mathrm{C} 24 \mathrm{H} 32 \mathrm{O} 2$ & 7.07E-06 \\
\hline $\mathrm{S} 1$ & $\mathrm{C} 39 \mathrm{H} 36 \mathrm{~S} 1$ & $2.11 \mathrm{E}-05$ & Basic N1 & $\mathrm{C} 43 \mathrm{H} 69 \mathrm{~N} 1$ & $1.07 \mathrm{E}-05$ & Neutral O2 & $\mathrm{C} 25 \mathrm{H} 34 \mathrm{O} 2$ & $3.51 \mathrm{E}-06$ \\
\hline $\mathrm{S} 1$ & $\mathrm{C} 40 \mathrm{H} 38 \mathrm{~S} 1$ & $1.36 \mathrm{E}-05$ & Basic N1 & $\mathrm{C} 44 \mathrm{H} 71 \mathrm{~N} 1$ & $7.89 \mathrm{E}-06$ & Neutral O2 & $\mathrm{C} 26 \mathrm{H} 36 \mathrm{O} 2$ & $4.53 \mathrm{E}-06$ \\
\hline $\mathrm{S} 1$ & $\mathrm{C} 41 \mathrm{H} 40 \mathrm{~S} 1$ & $1.17 \mathrm{E}-05$ & Basic N1 & $\mathrm{C} 45 \mathrm{H} 73 \mathrm{~N} 1$ & $6.88 \mathrm{E}-06$ & Neutral O2 & $\mathrm{C} 27 \mathrm{H} 38 \mathrm{O} 2$ & $3.10 \mathrm{E}-06$ \\
\hline $\mathrm{S} 1$ & $\mathrm{C} 42 \mathrm{H} 42 \mathrm{~S} 1$ & $6.81 \mathrm{E}-06$ & Basic N1 & $\mathrm{C} 46 \mathrm{H} 75 \mathrm{~N} 1$ & $6.10 \mathrm{E}-06$ & Neutral O2 & $\mathrm{C} 28 \mathrm{H} 40 \mathrm{O} 2$ & $6.89 \mathrm{E}-06$ \\
\hline $\mathrm{S} 1$ & $\mathrm{C} 43 \mathrm{H} 44 \mathrm{~S} 1$ & $4.16 \mathrm{E}-06$ & Basic N1 & $\mathrm{C} 47 \mathrm{H} 77 \mathrm{~N} 1$ & $3.51 \mathrm{E}-06$ & Neutral O2 & $\mathrm{C} 29 \mathrm{H} 42 \mathrm{O} 2$ & $1.34 \mathrm{E}-05$ \\
\hline $\mathrm{S} 1$ & $\mathrm{C} 44 \mathrm{H} 46 \mathrm{~S} 1$ & $3.76 \mathrm{E}-06$ & Basic N1 & C48H79N1 & $3.20 \mathrm{E}-06$ & Neutral O2 & $\mathrm{C} 30 \mathrm{H} 44 \mathrm{O} 2$ & $1.20 \mathrm{E}-05$ \\
\hline $\mathrm{S} 1$ & $\mathrm{C} 45 \mathrm{H} 48 \mathrm{~S} 1$ & $3.42 \mathrm{E}-06$ & Basic N1 & C49H81N1 & $2.59 \mathrm{E}-06$ & Neutral O2 & $\mathrm{C} 31 \mathrm{H} 46 \mathrm{O} 2$ & $6.50 \mathrm{E}-06$ \\
\hline S1 & C46H50S1 & $4.54 \mathrm{E}-06$ & Basic N1 & C50H83N1 & $1.28 \mathrm{E}-06$ & Neutral O2 & $\mathrm{C} 32 \mathrm{H} 48 \mathrm{O} 2$ & $3.97 \mathrm{E}-06$ \\
\hline S1 & $\mathrm{C} 32 \mathrm{H} 20 \mathrm{~S} 1$ & $1.42 \mathrm{E}-06$ & Basic N1 & C51H85N1 & $1.25 \mathrm{E}-06$ & Neutral O2 & $\mathrm{C} 33 \mathrm{H} 50 \mathrm{O} 2$ & 7.44E-07 \\
\hline S1 & $\mathrm{C} 33 \mathrm{H} 22 \mathrm{~S} 1$ & $4.96 \mathrm{E}-06$ & Basic N1 & $\mathrm{C} 52 \mathrm{H} 87 \mathrm{~N} 1$ & $1.05 \mathrm{E}-06$ & Neutral O2 & $\mathrm{C} 34 \mathrm{H} 52 \mathrm{O} 2$ & $1.15 \mathrm{E}-06$ \\
\hline S1 & $\mathrm{C} 34 \mathrm{H} 24 \mathrm{~S} 1$ & $3.58 \mathrm{E}-06$ & Basic N1 & C53H89N1 & $6.85 \mathrm{E}-07$ & Neutral O2 & $\mathrm{C} 35 \mathrm{H} 54 \mathrm{O} 2$ & $1.09 \mathrm{E}-06$ \\
\hline $\mathrm{S} 1$ & $\mathrm{C} 35 \mathrm{H} 26 \mathrm{~S} 1$ & $1.19 \mathrm{E}-05$ & Basic N1 & C54H91N1 & $5.65 \mathrm{E}-07$ & Neutral O2 & $\mathrm{C} 36 \mathrm{H} 56 \mathrm{O} 2$ & $1.38 \mathrm{E}-06$ \\
\hline $\mathrm{S} 1$ & $\mathrm{C} 36 \mathrm{H} 28 \mathrm{~S} 1$ & $1.39 \mathrm{E}-05$ & Basic N1 & $\mathrm{C} 16 \mathrm{H} 13 \mathrm{~N} 1$ & 7.29E-09 & Neutral O2 & $\mathrm{C} 37 \mathrm{H} 58 \mathrm{O} 2$ & 8.61E-07 \\
\hline S1 & $\mathrm{C} 37 \mathrm{H} 30 \mathrm{~S} 1$ & $2.10 \mathrm{E}-05$ & Basic N1 & $\mathrm{C} 17 \mathrm{H} 15 \mathrm{~N} 1$ & $6.45 \mathrm{E}-07$ & Neutral O2 & $\mathrm{C} 15 \mathrm{H} 12 \mathrm{O} 2$ & $3.81 \mathrm{E}-06$ \\
\hline S1 & $\mathrm{C} 38 \mathrm{H} 32 \mathrm{~S} 1$ & $1.70 \mathrm{E}-05$ & Basic N1 & $\mathrm{C} 18 \mathrm{H} 17 \mathrm{~N} 1$ & $3.96 \mathrm{E}-06$ & Neutral O2 & $\mathrm{C} 16 \mathrm{H} 14 \mathrm{O} 2$ & $2.18 \mathrm{E}-05$ \\
\hline S1 & $\mathrm{C} 39 \mathrm{H} 34 \mathrm{~S} 1$ & $1.94 \mathrm{E}-05$ & Basic N1 & C19H19N1 & $1.06 \mathrm{E}-05$ & Neutral O2 & $\mathrm{C} 17 \mathrm{H} 16 \mathrm{O} 2$ & $9.24 \mathrm{E}-05$ \\
\hline $\mathrm{S} 1$ & $\mathrm{C} 40 \mathrm{H} 36 \mathrm{~S} 1$ & $2.13 \mathrm{E}-05$ & Basic N1 & $\mathrm{C} 20 \mathrm{H} 21 \mathrm{~N} 1$ & $2.29 \mathrm{E}-05$ & Neutral O2 & $\mathrm{C} 18 \mathrm{H} 18 \mathrm{O} 2$ & 0.000159 \\
\hline $\mathrm{S} 1$ & $\mathrm{C} 41 \mathrm{H} 38 \mathrm{~S} 1$ & $9.44 \mathrm{E}-06$ & Basic N1 & $\mathrm{C} 21 \mathrm{H} 23 \mathrm{~N} 1$ & $4.21 \mathrm{E}-05$ & Neutral O2 & $\mathrm{C} 19 \mathrm{H} 20 \mathrm{O} 2$ & 0.000177 \\
\hline S1 & $\mathrm{C} 42 \mathrm{H} 40 \mathrm{~S} 1$ & $1.19 \mathrm{E}-05$ & Basic N1 & $\mathrm{C} 22 \mathrm{H} 25 \mathrm{~N} 1$ & $2.57 \mathrm{E}-05$ & Neutral O2 & $\mathrm{C} 20 \mathrm{H} 22 \mathrm{O} 2$ & 0.000124 \\
\hline S1 & $\mathrm{C} 35 \mathrm{H} 24 \mathrm{~S} 1$ & $7.58 \mathrm{E}-06$ & Basic N1 & $\mathrm{C} 23 \mathrm{H} 27 \mathrm{~N} 1$ & $2.63 \mathrm{E}-05$ & Neutral O2 & $\mathrm{C} 21 \mathrm{H} 24 \mathrm{O} 2$ & $5.95 \mathrm{E}-05$ \\
\hline S1 & $\mathrm{C} 36 \mathrm{H} 26 \mathrm{~S} 1$ & $8.80 \mathrm{E}-06$ & Basic N1 & $\mathrm{C} 24 \mathrm{H} 29 \mathrm{~N} 1$ & $2.98 \mathrm{E}-05$ & Neutral O2 & $\mathrm{C} 22 \mathrm{H} 26 \mathrm{O} 2$ & $3.40 \mathrm{E}-05$ \\
\hline S1 & $\mathrm{C} 37 \mathrm{H} 28 \mathrm{~S} 1$ & $1.55 \mathrm{E}-05$ & Basic N1 & $\mathrm{C} 25 \mathrm{H} 31 \mathrm{~N} 1$ & $3.21 \mathrm{E}-05$ & Neutral O2 & $\mathrm{C} 23 \mathrm{H} 28 \mathrm{O} 2$ & $2.33 \mathrm{E}-05$ \\
\hline S1 & $\mathrm{C} 38 \mathrm{H} 30 \mathrm{~S} 1$ & $2.55 \mathrm{E}-05$ & Basic N1 & $\mathrm{C} 26 \mathrm{H} 33 \mathrm{~N} 1$ & $3.61 \mathrm{E}-05$ & Neutral O2 & $\mathrm{C} 24 \mathrm{H} 30 \mathrm{O} 2$ & $1.06 \mathrm{E}-05$ \\
\hline
\end{tabular}




\begin{tabular}{|c|c|c|c|c|c|c|c|c|}
\hline Class & formula & Mass & Class & formula & Mass & Class & formula & Mass \\
\hline $\mathrm{S} 1$ & $\mathrm{C} 39 \mathrm{H} 32 \mathrm{~S} 1$ & $2.28 \mathrm{E}-05$ & Basic N1 & $\mathrm{C} 27 \mathrm{H} 35 \mathrm{~N} 1$ & $4.37 \mathrm{E}-05$ & Neutral O2 & $\mathrm{C} 25 \mathrm{H} 32 \mathrm{O} 2$ & $1.33 \mathrm{E}-05$ \\
\hline S1 & $\mathrm{C} 40 \mathrm{H} 34 \mathrm{~S} 1$ & $2.12 \mathrm{E}-05$ & Basic N1 & $\mathrm{C} 28 \mathrm{H} 37 \mathrm{~N} 1$ & $5.38 \mathrm{E}-05$ & Neutral O2 & $\mathrm{C} 26 \mathrm{H} 34 \mathrm{O} 2$ & $1.60 \mathrm{E}-05$ \\
\hline $\mathrm{S} 1$ & $\mathrm{C} 41 \mathrm{H} 36 \mathrm{~S} 1$ & $1.53 \mathrm{E}-05$ & Basic N1 & $\mathrm{C} 29 \mathrm{H} 39 \mathrm{~N} 1$ & $5.11 \mathrm{E}-05$ & Neutral O2 & $\mathrm{C} 27 \mathrm{H} 36 \mathrm{O} 2$ & $1.56 \mathrm{E}-05$ \\
\hline $\mathrm{S} 1$ & $\mathrm{C} 42 \mathrm{H} 38 \mathrm{~S} 1$ & $1.34 \mathrm{E}-05$ & Basic N1 & $\mathrm{C} 30 \mathrm{H} 41 \mathrm{~N} 1$ & $5.19 \mathrm{E}-05$ & Neutral O2 & $\mathrm{C} 28 \mathrm{H} 38 \mathrm{O} 2$ & $1.06 \mathrm{E}-05$ \\
\hline $\mathrm{S} 1$ & $\mathrm{C} 43 \mathrm{H} 40 \mathrm{~S} 1$ & $6.21 \mathrm{E}-06$ & Basic N1 & $\mathrm{C} 31 \mathrm{H} 43 \mathrm{~N} 1$ & $5.84 \mathrm{E}-05$ & Neutral O2 & $\mathrm{C} 29 \mathrm{H} 40 \mathrm{O} 2$ & $1.30 \mathrm{E}-05$ \\
\hline $\mathrm{S} 1$ & $\mathrm{C} 44 \mathrm{H} 42 \mathrm{~S} 1$ & $3.36 \mathrm{E}-06$ & Basic N1 & $\mathrm{C} 32 \mathrm{H} 45 \mathrm{~N} 1$ & $6.89 \mathrm{E}-05$ & Neutral O2 & $\mathrm{C} 30 \mathrm{H} 42 \mathrm{O} 2$ & $1.22 \mathrm{E}-05$ \\
\hline $\mathrm{S} 1$ & $\mathrm{C} 35 \mathrm{H} 22 \mathrm{~S} 1$ & $2.16 \mathrm{E}-06$ & Basic N1 & $\mathrm{C} 33 \mathrm{H} 47 \mathrm{~N} 1$ & $6.84 \mathrm{E}-05$ & Neutral O2 & $\mathrm{C} 31 \mathrm{H} 44 \mathrm{O} 2$ & $6.28 \mathrm{E}-06$ \\
\hline $\mathrm{S} 1$ & $\mathrm{C} 36 \mathrm{H} 24 \mathrm{~S} 1$ & $2.06 \mathrm{E}-06$ & Basic N1 & $\mathrm{C} 34 \mathrm{H} 49 \mathrm{~N} 1$ & $6.25 \mathrm{E}-05$ & Neutral O2 & $\mathrm{C} 32 \mathrm{H} 46 \mathrm{O} 2$ & $1.01 \mathrm{E}-05$ \\
\hline $\mathrm{S} 1$ & $\mathrm{C} 37 \mathrm{H} 26 \mathrm{~S} 1$ & $1.68 \mathrm{E}-06$ & Basic N1 & $\mathrm{C} 35 \mathrm{H} 51 \mathrm{~N} 1$ & 4.46E-05 & Neutral O2 & $\mathrm{C} 33 \mathrm{H} 48 \mathrm{O} 2$ & $8.22 \mathrm{E}-06$ \\
\hline $\mathrm{S} 1$ & $\mathrm{C} 38 \mathrm{H} 28 \mathrm{~S} 1$ & $1.22 \mathrm{E}-05$ & Basic N1 & $\mathrm{C} 36 \mathrm{H} 53 \mathrm{~N} 1$ & 4.19E-05 & Neutral O2 & $\mathrm{C} 34 \mathrm{H} 50 \mathrm{O} 2$ & $3.26 \mathrm{E}-06$ \\
\hline $\mathrm{S} 1$ & $\mathrm{C} 39 \mathrm{H} 30 \mathrm{~S} 1$ & $1.67 \mathrm{E}-05$ & Basic N1 & $\mathrm{C} 37 \mathrm{H} 55 \mathrm{~N} 1$ & $4.04 \mathrm{E}-05$ & Neutral O2 & $\mathrm{C} 35 \mathrm{H} 52 \mathrm{O} 2$ & $1.07 \mathrm{E}-06$ \\
\hline $\mathrm{S} 1$ & $\mathrm{C} 40 \mathrm{H} 32 \mathrm{~S} 1$ & $1.34 \mathrm{E}-05$ & Basic N1 & $\mathrm{C} 38 \mathrm{H} 57 \mathrm{~N} 1$ & $3.64 \mathrm{E}-05$ & Neutral O2 & $\mathrm{C} 36 \mathrm{H} 54 \mathrm{O} 2$ & $1.92 \mathrm{E}-06$ \\
\hline $\mathrm{S} 1$ & $\mathrm{C} 41 \mathrm{H} 34 \mathrm{~S} 1$ & $2.36 \mathrm{E}-05$ & Basic N1 & $\mathrm{C} 39 \mathrm{H} 59 \mathrm{~N} 1$ & $3.02 \mathrm{E}-05$ & Neutral O2 & $\mathrm{C} 37 \mathrm{H} 56 \mathrm{O} 2$ & $2.87 \mathrm{E}-06$ \\
\hline $\mathrm{S} 1$ & $\mathrm{C} 42 \mathrm{H} 36 \mathrm{~S} 1$ & $1.19 \mathrm{E}-05$ & Basic N1 & $\mathrm{C} 40 \mathrm{H} 61 \mathrm{~N} 1$ & $2.35 \mathrm{E}-05$ & Neutral O2 & $\mathrm{C} 15 \mathrm{H} 10 \mathrm{O} 2$ & $1.04 \mathrm{E}-06$ \\
\hline $\mathrm{S} 1$ & $\mathrm{C} 43 \mathrm{H} 38 \mathrm{~S} 1$ & $9.98 \mathrm{E}-06$ & Basic N1 & C41H63N1 & $1.80 \mathrm{E}-05$ & Neutral O2 & $\mathrm{C} 16 \mathrm{H} 12 \mathrm{O} 2$ & $1.49 \mathrm{E}-06$ \\
\hline $\mathrm{S} 1$ & $\mathrm{C} 44 \mathrm{H} 40 \mathrm{~S} 1$ & $7.53 \mathrm{E}-06$ & Basic N1 & $\mathrm{C} 42 \mathrm{H} 65 \mathrm{~N} 1$ & $1.42 \mathrm{E}-05$ & Neutral O2 & $\mathrm{C} 17 \mathrm{H} 14 \mathrm{O} 2$ & $1.09 \mathrm{E}-05$ \\
\hline $\mathrm{S} 1$ & $\mathrm{C} 45 \mathrm{H} 42 \mathrm{~S} 1$ & $2.69 \mathrm{E}-06$ & Basic N1 & $\mathrm{C} 43 \mathrm{H} 67 \mathrm{~N} 1$ & $1.12 \mathrm{E}-05$ & Neutral O2 & $\mathrm{C} 18 \mathrm{H} 16 \mathrm{O} 2$ & $5.75 \mathrm{E}-05$ \\
\hline $\mathrm{S} 1$ & $\mathrm{C} 46 \mathrm{H} 44 \mathrm{~S} 1$ & $1.73 \mathrm{E}-06$ & Basic N1 & C44H69N1 & $8.79 \mathrm{E}-06$ & Neutral O2 & $\mathrm{C} 19 \mathrm{H} 18 \mathrm{O} 2$ & 0.000131 \\
\hline $\mathrm{S} 1$ & $\mathrm{C} 47 \mathrm{H} 46 \mathrm{~S} 1$ & $2.06 \mathrm{E}-06$ & Basic N1 & $\mathrm{C} 45 \mathrm{H} 71 \mathrm{~N} 1$ & $6.61 \mathrm{E}-06$ & Neutral O2 & $\mathrm{C} 20 \mathrm{H} 20 \mathrm{O} 2$ & 0.000198 \\
\hline $\mathrm{S} 1$ & $\mathrm{C} 48 \mathrm{H} 48 \mathrm{~S} 1$ & $1.57 \mathrm{E}-06$ & Basic N1 & $\mathrm{C} 46 \mathrm{H} 73 \mathrm{~N} 1$ & $6.25 \mathrm{E}-06$ & Neutral O2 & $\mathrm{C} 21 \mathrm{H} 22 \mathrm{O} 2$ & 0.000211 \\
\hline $\mathrm{S} 1$ & $\mathrm{C} 37 \mathrm{H} 24 \mathrm{~S} 1$ & $1.67 \mathrm{E}-06$ & Basic N1 & $\mathrm{C} 47 \mathrm{H} 75 \mathrm{~N} 1$ & $4.56 \mathrm{E}-06$ & Neutral O2 & $\mathrm{C} 22 \mathrm{H} 24 \mathrm{O} 2$ & 0.000169 \\
\hline $\mathrm{S} 1$ & $\mathrm{C} 38 \mathrm{H} 26 \mathrm{~S} 1$ & $6.18 \mathrm{E}-06$ & Basic N1 & $\mathrm{C} 48 \mathrm{H} 77 \mathrm{~N} 1$ & $3.60 \mathrm{E}-06$ & Neutral O2 & $\mathrm{C} 23 \mathrm{H} 26 \mathrm{O} 2$ & 0.000109 \\
\hline S1 & $\mathrm{C} 39 \mathrm{H} 28 \mathrm{~S} 1$ & $4.32 \mathrm{E}-06$ & Basic N1 & $\mathrm{C} 49 \mathrm{H} 79 \mathrm{~N} 1$ & $2.37 \mathrm{E}-06$ & Neutral O2 & $\mathrm{C} 24 \mathrm{H} 28 \mathrm{O} 2$ & $5.03 \mathrm{E}-05$ \\
\hline S1 & $\mathrm{C} 40 \mathrm{H} 30 \mathrm{~S} 1$ & $1.48 \mathrm{E}-05$ & Basic N1 & $\mathrm{C} 50 \mathrm{H} 81 \mathrm{~N} 1$ & $1.97 \mathrm{E}-06$ & Neutral O2 & $\mathrm{C} 25 \mathrm{H} 30 \mathrm{O} 2$ & $3.36 \mathrm{E}-05$ \\
\hline S1 & $\mathrm{C} 41 \mathrm{H} 32 \mathrm{~S} 1$ & $1.43 \mathrm{E}-05$ & Basic N1 & $\mathrm{C} 17 \mathrm{H} 13 \mathrm{~N} 1$ & $4.13 \mathrm{E}-07$ & Neutral O2 & $\mathrm{C} 26 \mathrm{H} 32 \mathrm{O} 2$ & $3.13 \mathrm{E}-05$ \\
\hline S1 & $\mathrm{C} 42 \mathrm{H} 34 \mathrm{~S} 1$ & $1.68 \mathrm{E}-05$ & Basic N1 & $\mathrm{C} 18 \mathrm{H} 15 \mathrm{~N} 1$ & $4.63 \mathrm{E}-06$ & Neutral O2 & $\mathrm{C} 27 \mathrm{H} 34 \mathrm{O} 2$ & $2.86 \mathrm{E}-05$ \\
\hline $\mathrm{S} 1$ & $\mathrm{C} 43 \mathrm{H} 36 \mathrm{~S} 1$ & $8.77 \mathrm{E}-06$ & Basic N1 & $\mathrm{C} 19 \mathrm{H} 17 \mathrm{~N} 1$ & $1.54 \mathrm{E}-05$ & Neutral O2 & $\mathrm{C} 28 \mathrm{H} 36 \mathrm{O} 2$ & $2.76 \mathrm{E}-05$ \\
\hline $\mathrm{S} 1$ & $\mathrm{C} 44 \mathrm{H} 38 \mathrm{~S} 1$ & $7.23 \mathrm{E}-06$ & Basic N1 & $\mathrm{C} 20 \mathrm{H} 19 \mathrm{~N} 1$ & $2.57 \mathrm{E}-05$ & Neutral O2 & $\mathrm{C} 29 \mathrm{H} 38 \mathrm{O} 2$ & $2.30 \mathrm{E}-05$ \\
\hline S1 & $\mathrm{C} 45 \mathrm{H} 40 \mathrm{~S} 1$ & 4.33E-06 & Basic N1 & $\mathrm{C} 21 \mathrm{H} 21 \mathrm{~N} 1$ & $3.30 \mathrm{E}-05$ & Neutral O2 & $\mathrm{C} 30 \mathrm{H} 40 \mathrm{O} 2$ & $2.31 \mathrm{E}-05$ \\
\hline S1 & $\mathrm{C} 46 \mathrm{H} 42 \mathrm{~S} 1$ & $4.26 \mathrm{E}-06$ & Basic N1 & $\mathrm{C} 22 \mathrm{H} 23 \mathrm{~N} 1$ & $3.25 \mathrm{E}-05$ & Neutral O2 & $\mathrm{C} 31 \mathrm{H} 42 \mathrm{O} 2$ & $1.86 \mathrm{E}-05$ \\
\hline S1 & $\mathrm{C} 39 \mathrm{H} 26 \mathrm{~S} 1$ & $2.92 \mathrm{E}-06$ & Basic N1 & $\mathrm{C} 23 \mathrm{H} 25 \mathrm{~N} 1$ & $3.91 \mathrm{E}-05$ & Neutral O2 & $\mathrm{C} 32 \mathrm{H} 44 \mathrm{O} 2$ & $1.83 \mathrm{E}-05$ \\
\hline S1 & $\mathrm{C} 40 \mathrm{H} 28 \mathrm{~S} 1$ & 4.62E-06 & Basic N1 & $\mathrm{C} 24 \mathrm{H} 27 \mathrm{~N} 1$ & $5.03 \mathrm{E}-05$ & Neutral O2 & $\mathrm{C} 33 \mathrm{H} 46 \mathrm{O} 2$ & $5.89 \mathrm{E}-06$ \\
\hline $\mathrm{S} 1$ & C41H30S1 & $5.40 \mathrm{E}-06$ & Basic N1 & $\mathrm{C} 25 \mathrm{H} 29 \mathrm{~N} 1$ & $5.18 \mathrm{E}-05$ & Neutral O2 & $\mathrm{C} 34 \mathrm{H} 48 \mathrm{O} 2$ & $6.19 \mathrm{E}-06$ \\
\hline S1 & $\mathrm{C} 42 \mathrm{H} 32 \mathrm{~S} 1$ & $1.06 \mathrm{E}-05$ & Basic N1 & $\mathrm{C} 26 \mathrm{H} 31 \mathrm{~N} 1$ & $5.53 \mathrm{E}-05$ & Neutral O2 & $\mathrm{C} 35 \mathrm{H} 50 \mathrm{O} 2$ & $2.59 \mathrm{E}-06$ \\
\hline S1 & $\mathrm{C} 43 \mathrm{H} 34 \mathrm{~S} 1$ & $9.04 \mathrm{E}-06$ & Basic N1 & $\mathrm{C} 27 \mathrm{H} 33 \mathrm{~N} 1$ & $6.49 \mathrm{E}-05$ & Neutral O2 & $\mathrm{C} 36 \mathrm{H} 52 \mathrm{O} 2$ & $4.59 \mathrm{E}-06$ \\
\hline S1 & $\mathrm{C} 44 \mathrm{H} 36 \mathrm{~S} 1$ & $6.14 \mathrm{E}-06$ & Basic N1 & $\mathrm{C} 28 \mathrm{H} 35 \mathrm{~N} 1$ & $7.49 \mathrm{E}-05$ & Neutral O2 & $\mathrm{C} 37 \mathrm{H} 54 \mathrm{O} 2$ & $1.01 \mathrm{E}-06$ \\
\hline S1 & $\mathrm{C} 45 \mathrm{H} 38 \mathrm{~S} 1$ & $5.36 \mathrm{E}-06$ & Basic N1 & $\mathrm{C} 29 \mathrm{H} 37 \mathrm{~N} 1$ & $6.43 \mathrm{E}-05$ & Neutral O2 & $\mathrm{C} 38 \mathrm{H} 56 \mathrm{O} 2$ & $1.06 \mathrm{E}-06$ \\
\hline S1 & $\mathrm{C} 46 \mathrm{H} 40 \mathrm{~S} 1$ & $2.32 \mathrm{E}-06$ & Basic N1 & $\mathrm{C} 30 \mathrm{H} 39 \mathrm{~N} 1$ & $6.39 \mathrm{E}-05$ & Neutral O2 & $\mathrm{C} 16 \mathrm{H} 10 \mathrm{O} 2$ & $6.37 \mathrm{E}-07$ \\
\hline
\end{tabular}




\begin{tabular}{|c|c|c|c|c|c|c|c|c|}
\hline Class & formula & Mass & Class & formula & Mass & Class & formula & Mass \\
\hline $\mathrm{S} 1$ & $\mathrm{C} 47 \mathrm{H} 42 \mathrm{~S} 1$ & $5.02 \mathrm{E}-06$ & Basic N1 & $\mathrm{C} 31 \mathrm{H} 41 \mathrm{~N} 1$ & $6.95 \mathrm{E}-05$ & Neutral O2 & $\mathrm{C} 17 \mathrm{H} 12 \mathrm{O} 2$ & $9.22 \mathrm{E}-07$ \\
\hline $\mathrm{S} 1$ & $\mathrm{C} 48 \mathrm{H} 44 \mathrm{~S} 1$ & $2.49 \mathrm{E}-06$ & Basic N1 & $\mathrm{C} 32 \mathrm{H} 43 \mathrm{~N} 1$ & $8.82 \mathrm{E}-05$ & Neutral O2 & $\mathrm{C} 18 \mathrm{H} 14 \mathrm{O} 2$ & $3.03 \mathrm{E}-05$ \\
\hline $\mathrm{S} 1$ & $\mathrm{C} 42 \mathrm{H} 30 \mathrm{~S} 1$ & $1.75 \mathrm{E}-06$ & Basic N1 & $\mathrm{C} 33 \mathrm{H} 45 \mathrm{~N} 1$ & $9.00 \mathrm{E}-05$ & Neutral O2 & $\mathrm{C} 19 \mathrm{H} 16 \mathrm{O} 2$ & 0.000202 \\
\hline $\mathrm{S} 1$ & $\mathrm{C} 43 \mathrm{H} 32 \mathrm{~S} 1$ & $6.74 \mathrm{E}-06$ & Basic N1 & $\mathrm{C} 34 \mathrm{H} 47 \mathrm{~N} 1$ & $7.99 \mathrm{E}-05$ & Neutral O2 & $\mathrm{C} 20 \mathrm{H} 18 \mathrm{O} 2$ & 0.00047 \\
\hline $\mathrm{S} 1$ & $\mathrm{C} 44 \mathrm{H} 34 \mathrm{~S} 1$ & $1.83 \mathrm{E}-06$ & Basic N1 & $\mathrm{C} 35 \mathrm{H} 49 \mathrm{~N} 1$ & $5.26 \mathrm{E}-05$ & Neutral O2 & $\mathrm{C} 21 \mathrm{H} 20 \mathrm{O} 2$ & 0.000483 \\
\hline $\mathrm{S} 1$ & $\mathrm{C} 45 \mathrm{H} 36 \mathrm{~S} 1$ & $1.23 \mathrm{E}-05$ & Basic N1 & $\mathrm{C} 36 \mathrm{H} 51 \mathrm{~N} 1$ & $4.65 \mathrm{E}-05$ & Neutral O2 & $\mathrm{C} 22 \mathrm{H} 22 \mathrm{O} 2$ & 0.000366 \\
\hline $\mathrm{S} 1$ & $\mathrm{C} 46 \mathrm{H} 38 \mathrm{~S} 1$ & 4.15E-06 & Basic N1 & $\mathrm{C} 37 \mathrm{H} 53 \mathrm{~N} 1$ & $4.11 \mathrm{E}-05$ & Neutral O2 & $\mathrm{C} 23 \mathrm{H} 24 \mathrm{O} 2$ & 0.000359 \\
\hline $\mathrm{S} 1$ & $\mathrm{C} 47 \mathrm{H} 40 \mathrm{~S} 1$ & $1.26 \mathrm{E}-05$ & Basic N1 & $\mathrm{C} 38 \mathrm{H} 55 \mathrm{~N} 1$ & $3.76 \mathrm{E}-05$ & Neutral O2 & $\mathrm{C} 24 \mathrm{H} 26 \mathrm{O} 2$ & 0.000145 \\
\hline $\mathrm{S} 1$ & $\mathrm{C} 48 \mathrm{H} 42 \mathrm{~S} 1$ & $3.26 \mathrm{E}-06$ & Basic N1 & $\mathrm{C} 39 \mathrm{H} 57 \mathrm{~N} 1$ & $3.37 \mathrm{E}-05$ & Neutral O2 & $\mathrm{C} 25 \mathrm{H} 28 \mathrm{O} 2$ & $9.60 \mathrm{E}-05$ \\
\hline $\mathrm{S} 1$ & $\mathrm{C} 43 \mathrm{H} 30 \mathrm{~S} 1$ & $6.36 \mathrm{E}-06$ & Basic N1 & $\mathrm{C} 40 \mathrm{H} 59 \mathrm{~N} 1$ & $2.50 \mathrm{E}-05$ & Neutral O2 & $\mathrm{C} 26 \mathrm{H} 30 \mathrm{O} 2$ & 8.97E-05 \\
\hline $\mathrm{S} 1$ & $\mathrm{C} 44 \mathrm{H} 32 \mathrm{~S} 1$ & $1.88 \mathrm{E}-06$ & Basic N1 & $\mathrm{C} 41 \mathrm{H} 61 \mathrm{~N} 1$ & $1.86 \mathrm{E}-05$ & Neutral O2 & $\mathrm{C} 27 \mathrm{H} 32 \mathrm{O} 2$ & $7.36 \mathrm{E}-05$ \\
\hline $\mathrm{S} 1$ & $\mathrm{C} 45 \mathrm{H} 34 \mathrm{~S} 1$ & $9.20 \mathrm{E}-06$ & Basic N1 & $\mathrm{C} 42 \mathrm{H} 63 \mathrm{~N} 1$ & $1.51 \mathrm{E}-05$ & Neutral O2 & $\mathrm{C} 28 \mathrm{H} 34 \mathrm{O} 2$ & $5.37 \mathrm{E}-05$ \\
\hline $\mathrm{S} 1$ & $\mathrm{C} 46 \mathrm{H} 36 \mathrm{~S} 1$ & $1.03 \mathrm{E}-05$ & Basic N1 & C43H65N1 & $1.11 \mathrm{E}-05$ & Neutral O2 & $\mathrm{C} 29 \mathrm{H} 36 \mathrm{O} 2$ & $3.18 \mathrm{E}-05$ \\
\hline $\mathrm{S} 1$ & $\mathrm{C} 47 \mathrm{H} 38 \mathrm{~S} 1$ & $8.44 \mathrm{E}-06$ & Basic N1 & $\mathrm{C} 44 \mathrm{H} 67 \mathrm{~N} 1$ & $9.45 \mathrm{E}-06$ & Neutral O2 & $\mathrm{C} 30 \mathrm{H} 38 \mathrm{O} 2$ & $3.10 \mathrm{E}-05$ \\
\hline $\mathrm{S} 1$ & $\mathrm{C} 48 \mathrm{H} 40 \mathrm{~S} 1$ & $8.89 \mathrm{E}-06$ & Basic N1 & $\mathrm{C} 45 \mathrm{H} 69 \mathrm{~N} 1$ & $8.02 \mathrm{E}-06$ & Neutral O2 & $\mathrm{C} 31 \mathrm{H} 40 \mathrm{O} 2$ & $1.72 \mathrm{E}-05$ \\
\hline $\mathrm{S} 1$ & $\mathrm{C} 49 \mathrm{H} 42 \mathrm{~S} 1$ & $3.73 \mathrm{E}-06$ & Basic N1 & $\mathrm{C} 46 \mathrm{H} 71 \mathrm{~N} 1$ & $5.62 \mathrm{E}-06$ & Neutral O2 & $\mathrm{C} 32 \mathrm{H} 42 \mathrm{O} 2$ & $1.60 \mathrm{E}-05$ \\
\hline $\mathrm{S} 1$ & $\mathrm{C} 50 \mathrm{H} 44 \mathrm{~S} 1$ & $5.17 \mathrm{E}-06$ & Basic N1 & $\mathrm{C} 47 \mathrm{H} 73 \mathrm{~N} 1$ & $5.01 \mathrm{E}-06$ & Neutral O2 & $\mathrm{C} 33 \mathrm{H} 44 \mathrm{O} 2$ & $1.16 \mathrm{E}-05$ \\
\hline $\mathrm{S} 1$ & C51H46S1 & $5.66 \mathrm{E}-06$ & Basic N1 & $\mathrm{C} 48 \mathrm{H} 75 \mathrm{~N} 1$ & $4.35 \mathrm{E}-06$ & Neutral O2 & $\mathrm{C} 34 \mathrm{H} 46 \mathrm{O} 2$ & $8.06 \mathrm{E}-06$ \\
\hline $\mathrm{S} 1$ & $\mathrm{C} 52 \mathrm{H} 48 \mathrm{~S} 1$ & $3.08 \mathrm{E}-06$ & Basic N1 & $\mathrm{C} 49 \mathrm{H} 77 \mathrm{~N} 1$ & $2.70 \mathrm{E}-06$ & Neutral O2 & $\mathrm{C} 35 \mathrm{H} 48 \mathrm{O} 2$ & $4.19 \mathrm{E}-06$ \\
\hline $\mathrm{S} 1$ & C53H50S1 & $2.46 \mathrm{E}-06$ & Basic N1 & $\mathrm{C} 50 \mathrm{H} 79 \mathrm{~N} 1$ & $1.94 \mathrm{E}-06$ & Neutral O2 & $\mathrm{C} 36 \mathrm{H} 50 \mathrm{O} 2$ & $1.98 \mathrm{E}-06$ \\
\hline $\mathrm{S} 1$ & C54H52S1 & $2.00 \mathrm{E}-06$ & Basic N1 & C51H81N1 & $1.50 \mathrm{E}-06$ & Neutral O2 & $\mathrm{C} 37 \mathrm{H} 52 \mathrm{O} 2$ & $3.61 \mathrm{E}-06$ \\
\hline $\mathrm{S} 1$ & $\mathrm{C} 46 \mathrm{H} 34 \mathrm{~S} 1$ & $3.96 \mathrm{E}-06$ & Basic N1 & $\mathrm{C} 52 \mathrm{H} 83 \mathrm{~N} 1$ & $1.39 \mathrm{E}-06$ & Neutral O2 & $\mathrm{C} 38 \mathrm{H} 54 \mathrm{O} 2$ & $8.05 \mathrm{E}-07$ \\
\hline $\mathrm{S} 1$ & C47H36S1 & $4.38 \mathrm{E}-06$ & Basic N1 & $\mathrm{C} 53 \mathrm{H} 85 \mathrm{~N} 1$ & $8.91 \mathrm{E}-07$ & Neutral O2 & $\mathrm{C} 39 \mathrm{H} 56 \mathrm{O} 2$ & $2.98 \mathrm{E}-06$ \\
\hline S1 & $\mathrm{C} 48 \mathrm{H} 38 \mathrm{~S} 1$ & $1.19 \mathrm{E}-05$ & Basic N1 & $\mathrm{C} 17 \mathrm{H} 11 \mathrm{~N} 1$ & $2.85 \mathrm{E}-08$ & Neutral O2 & $\mathrm{C} 16 \mathrm{H} 8 \mathrm{O} 2$ & $7.82 \mathrm{E}-07$ \\
\hline S1 & C49H40S1 & $1.33 \mathrm{E}-05$ & Basic N1 & $\mathrm{C} 18 \mathrm{H} 13 \mathrm{~N} 1$ & $2.98 \mathrm{E}-06$ & Neutral O2 & $\mathrm{C} 17 \mathrm{H} 10 \mathrm{O} 2$ & $2.18 \mathrm{E}-07$ \\
\hline S1 & $\mathrm{C} 46 \mathrm{H} 32 \mathrm{~S} 1$ & $6.41 \mathrm{E}-06$ & Basic N1 & $\mathrm{C} 19 \mathrm{H} 15 \mathrm{~N} 1$ & $1.68 \mathrm{E}-05$ & Neutral O2 & $\mathrm{C} 18 \mathrm{H} 12 \mathrm{O} 2$ & $1.76 \mathrm{E}-05$ \\
\hline $\mathrm{S} 1$ & $\mathrm{C} 47 \mathrm{H} 34 \mathrm{~S} 1$ & $6.87 \mathrm{E}-06$ & Basic N1 & $\mathrm{C} 20 \mathrm{H} 17 \mathrm{~N} 1$ & $3.17 \mathrm{E}-05$ & Neutral O2 & $\mathrm{C} 19 \mathrm{H} 14 \mathrm{O} 2$ & $9.50 \mathrm{E}-05$ \\
\hline $\mathrm{S} 1$ & $\mathrm{C} 48 \mathrm{H} 36 \mathrm{~S} 1$ & $6.51 \mathrm{E}-06$ & Basic N1 & $\mathrm{C} 21 \mathrm{H} 19 \mathrm{~N} 1$ & $4.22 \mathrm{E}-05$ & Neutral O2 & $\mathrm{C} 20 \mathrm{H} 16 \mathrm{O} 2$ & 0.000254 \\
\hline $\mathrm{S} 1$ & C49H38S1 & $9.32 \mathrm{E}-06$ & Basic N1 & $\mathrm{C} 22 \mathrm{H} 21 \mathrm{~N} 1$ & $3.93 \mathrm{E}-05$ & Neutral O2 & $\mathrm{C} 21 \mathrm{H} 18 \mathrm{O} 2$ & 0.000567 \\
\hline $\mathrm{S} 1$ & $\mathrm{C} 50 \mathrm{H} 40 \mathrm{~S} 1$ & $1.64 \mathrm{E}-05$ & Basic N1 & $\mathrm{C} 23 \mathrm{H} 23 \mathrm{~N} 1$ & $4.11 \mathrm{E}-05$ & Neutral O2 & $\mathrm{C} 22 \mathrm{H} 20 \mathrm{O} 2$ & 0.001035 \\
\hline $\mathrm{S} 1$ & $\mathrm{C} 51 \mathrm{H} 42 \mathrm{~S} 1$ & 5.32E-06 & Basic N1 & $\mathrm{C} 24 \mathrm{H} 25 \mathrm{~N} 1$ & 5.17E-05 & Neutral O2 & $\mathrm{C} 23 \mathrm{H} 22 \mathrm{O} 2$ & 0.001101 \\
\hline $\mathrm{S} 1$ & $\mathrm{C} 52 \mathrm{H} 44 \mathrm{~S} 1$ & $9.05 \mathrm{E}-06$ & Basic N1 & $\mathrm{C} 25 \mathrm{H} 27 \mathrm{~N} 1$ & $5.71 \mathrm{E}-05$ & Neutral O2 & $\mathrm{C} 24 \mathrm{H} 24 \mathrm{O} 2$ & 0.000594 \\
\hline $\mathrm{S} 1$ & $\mathrm{C} 53 \mathrm{H} 46 \mathrm{~S} 1$ & $9.31 \mathrm{E}-06$ & Basic N1 & $\mathrm{C} 26 \mathrm{H} 29 \mathrm{~N} 1$ & $6.07 \mathrm{E}-05$ & Neutral O2 & $\mathrm{C} 25 \mathrm{H} 26 \mathrm{O} 2$ & 0.000281 \\
\hline S1 & C54H48S1 & $4.92 \mathrm{E}-06$ & Basic N1 & $\mathrm{C} 27 \mathrm{H} 31 \mathrm{~N} 1$ & $6.92 \mathrm{E}-05$ & Neutral O2 & $\mathrm{C} 26 \mathrm{H} 28 \mathrm{O} 2$ & 0.00017 \\
\hline S1 & C55H50S1 & $2.54 \mathrm{E}-06$ & Basic N1 & $\mathrm{C} 28 \mathrm{H} 33 \mathrm{~N} 1$ & $8.04 \mathrm{E}-05$ & Neutral O2 & $\mathrm{C} 27 \mathrm{H} 30 \mathrm{O} 2$ & $9.70 \mathrm{E}-05$ \\
\hline S1 & $\mathrm{C} 56 \mathrm{H} 52 \mathrm{~S} 1$ & $2.43 \mathrm{E}-06$ & Basic N1 & $\mathrm{C} 29 \mathrm{H} 35 \mathrm{~N} 1$ & $7.04 \mathrm{E}-05$ & Neutral O2 & $\mathrm{C} 28 \mathrm{H} 32 \mathrm{O} 2$ & $5.90 \mathrm{E}-05$ \\
\hline S1 & $\mathrm{C} 48 \mathrm{H} 34 \mathrm{~S} 1$ & $2.42 \mathrm{E}-06$ & Basic N1 & $\mathrm{C} 30 \mathrm{H} 37 \mathrm{~N} 1$ & $6.54 \mathrm{E}-05$ & Neutral O2 & $\mathrm{C} 29 \mathrm{H} 34 \mathrm{O} 2$ & $3.87 \mathrm{E}-05$ \\
\hline S1 & $\mathrm{C} 49 \mathrm{H} 36 \mathrm{~S} 1$ & $4.90 \mathrm{E}-06$ & Basic N1 & $\mathrm{C} 31 \mathrm{H} 39 \mathrm{~N} 1$ & $6.38 \mathrm{E}-05$ & Neutral O2 & $\mathrm{C} 30 \mathrm{H} 36 \mathrm{O} 2$ & $3.28 \mathrm{E}-05$ \\
\hline
\end{tabular}




\begin{tabular}{|c|c|c|c|c|c|c|c|c|}
\hline Class & formula & Mass & Class & formula & Mass & Class & formula & Mass \\
\hline $\mathrm{S} 1$ & $\mathrm{C} 50 \mathrm{H} 38 \mathrm{~S} 1$ & $6.53 \mathrm{E}-06$ & Basic N1 & $\mathrm{C} 32 \mathrm{H} 41 \mathrm{~N} 1$ & $6.70 \mathrm{E}-05$ & Neutral O2 & $\mathrm{C} 31 \mathrm{H} 38 \mathrm{O} 2$ & $2.79 \mathrm{E}-05$ \\
\hline S1 & C51H40S1 & $6.95 \mathrm{E}-06$ & Basic N1 & $\mathrm{C} 33 \mathrm{H} 43 \mathrm{~N} 1$ & $6.31 \mathrm{E}-05$ & Neutral O2 & $\mathrm{C} 32 \mathrm{H} 40 \mathrm{O} 2$ & $1.86 \mathrm{E}-05$ \\
\hline $\mathrm{S} 1$ & $\mathrm{C} 52 \mathrm{H} 42 \mathrm{~S} 1$ & $7.93 \mathrm{E}-06$ & Basic N1 & $\mathrm{C} 34 \mathrm{H} 45 \mathrm{~N} 1$ & $5.56 \mathrm{E}-05$ & Neutral O2 & $\mathrm{C} 33 \mathrm{H} 42 \mathrm{O} 2$ & $1.02 \mathrm{E}-05$ \\
\hline $\mathrm{S} 1$ & $\mathrm{C} 53 \mathrm{H} 44 \mathrm{~S} 1$ & $9.11 \mathrm{E}-06$ & Basic N1 & $\mathrm{C} 35 \mathrm{H} 47 \mathrm{~N} 1$ & 4.72E-05 & Neutral O2 & $\mathrm{C} 34 \mathrm{H} 44 \mathrm{O} 2$ & $9.92 \mathrm{E}-06$ \\
\hline $\mathrm{S} 1$ & $\mathrm{C} 54 \mathrm{H} 46 \mathrm{~S} 1$ & $7.41 \mathrm{E}-06$ & Basic N1 & $\mathrm{C} 36 \mathrm{H} 49 \mathrm{~N} 1$ & 4.32E-05 & Neutral O2 & $\mathrm{C} 35 \mathrm{H} 46 \mathrm{O} 2$ & $1.02 \mathrm{E}-05$ \\
\hline $\mathrm{S} 1$ & $\mathrm{C} 55 \mathrm{H} 48 \mathrm{~S} 1$ & $8.04 \mathrm{E}-06$ & Basic N1 & $\mathrm{C} 37 \mathrm{H} 51 \mathrm{~N} 1$ & $3.95 \mathrm{E}-05$ & Neutral O2 & $\mathrm{C} 36 \mathrm{H} 48 \mathrm{O} 2$ & $1.39 \mathrm{E}-06$ \\
\hline $\mathrm{S} 1$ & $\mathrm{C} 49 \mathrm{H} 34 \mathrm{~S} 1$ & $2.80 \mathrm{E}-06$ & Basic N1 & $\mathrm{C} 38 \mathrm{H} 53 \mathrm{~N} 1$ & $3.51 \mathrm{E}-05$ & Neutral O2 & $\mathrm{C} 37 \mathrm{H} 50 \mathrm{O} 2$ & $4.01 \mathrm{E}-06$ \\
\hline $\mathrm{S} 1$ & $\mathrm{C} 50 \mathrm{H} 36 \mathrm{~S} 1$ & $9.74 \mathrm{E}-06$ & Basic N1 & $\mathrm{C} 39 \mathrm{H} 55 \mathrm{~N} 1$ & $2.96 \mathrm{E}-05$ & Neutral O2 & $\mathrm{C} 38 \mathrm{H} 52 \mathrm{O} 2$ & $5.16 \mathrm{E}-07$ \\
\hline $\mathrm{S} 1$ & $\mathrm{C} 51 \mathrm{H} 38 \mathrm{~S} 1$ & $2.97 \mathrm{E}-06$ & Basic N1 & $\mathrm{C} 40 \mathrm{H} 57 \mathrm{~N} 1$ & $2.24 \mathrm{E}-05$ & Neutral O2 & $\mathrm{C} 39 \mathrm{H} 54 \mathrm{O} 2$ & $4.41 \mathrm{E}-06$ \\
\hline $\mathrm{S} 1$ & $\mathrm{C} 52 \mathrm{H} 40 \mathrm{~S} 1$ & $1.21 \mathrm{E}-05$ & Basic N1 & C41H59N1 & $1.69 \mathrm{E}-05$ & Neutral O2 & $\mathrm{C} 40 \mathrm{H} 56 \mathrm{O} 2$ & $1.74 \mathrm{E}-06$ \\
\hline $\mathrm{S} 1$ & $\mathrm{C} 53 \mathrm{H} 42 \mathrm{~S} 1$ & $1.56 \mathrm{E}-05$ & Basic N1 & $\mathrm{C} 42 \mathrm{H} 61 \mathrm{~N} 1$ & $1.33 \mathrm{E}-05$ & Neutral O2 & $\mathrm{C} 41 \mathrm{H} 58 \mathrm{O} 2$ & $3.35 \mathrm{E}-06$ \\
\hline $\mathrm{S} 1$ & $\mathrm{C} 54 \mathrm{H} 44 \mathrm{~S} 1$ & $1.20 \mathrm{E}-05$ & Basic N1 & C43H63N1 & $1.09 \mathrm{E}-05$ & Neutral O2 & $\mathrm{C} 19 \mathrm{H} 12 \mathrm{O} 2$ & $1.12 \mathrm{E}-06$ \\
\hline $\mathrm{S} 1$ & $\mathrm{C} 55 \mathrm{H} 46 \mathrm{~S} 1$ & $4.56 \mathrm{E}-06$ & Basic N1 & C44H65N1 & $8.90 \mathrm{E}-06$ & Neutral O2 & $\mathrm{C} 20 \mathrm{H} 14 \mathrm{O} 2$ & $5.94 \mathrm{E}-05$ \\
\hline $\mathrm{S} 1$ & $\mathrm{C} 56 \mathrm{H} 48 \mathrm{~S} 1$ & $7.84 \mathrm{E}-06$ & Basic N1 & $\mathrm{C} 45 \mathrm{H} 67 \mathrm{~N} 1$ & $7.18 \mathrm{E}-06$ & Neutral O2 & $\mathrm{C} 21 \mathrm{H} 16 \mathrm{O} 2$ & 0.000112 \\
\hline $\mathrm{S} 1$ & C57H50S1 & $2.21 \mathrm{E}-06$ & Basic N1 & $\mathrm{C} 46 \mathrm{H} 69 \mathrm{~N} 1$ & $5.76 \mathrm{E}-06$ & Neutral O2 & $\mathrm{C} 22 \mathrm{H} 18 \mathrm{O} 2$ & 0.000208 \\
\hline $\mathrm{S} 1$ & C58H52S1 & $2.86 \mathrm{E}-06$ & Basic N1 & $\mathrm{C} 47 \mathrm{H} 71 \mathrm{~N} 1$ & 4.73E-06 & Neutral O2 & $\mathrm{C} 23 \mathrm{H} 20 \mathrm{O} 2$ & 0.000354 \\
\hline $\mathrm{S} 1$ & $\mathrm{C} 52 \mathrm{H} 38 \mathrm{~S} 1$ & $1.01 \mathrm{E}-05$ & Basic N1 & $\mathrm{C} 48 \mathrm{H} 73 \mathrm{~N} 1$ & $3.57 \mathrm{E}-06$ & Neutral O2 & $\mathrm{C} 24 \mathrm{H} 22 \mathrm{O} 2$ & 0.000428 \\
\hline $\mathrm{S} 1$ & $\mathrm{C} 53 \mathrm{H} 40 \mathrm{~S} 1$ & $1.17 \mathrm{E}-05$ & Basic N1 & $\mathrm{C} 49 \mathrm{H} 75 \mathrm{~N} 1$ & $2.48 \mathrm{E}-06$ & Neutral O2 & $\mathrm{C} 25 \mathrm{H} 24 \mathrm{O} 2$ & 0.000391 \\
\hline $\mathrm{S} 1$ & $\mathrm{C} 54 \mathrm{H} 42 \mathrm{~S} 1$ & $6.62 \mathrm{E}-06$ & Basic N1 & C50H77N1 & $2.45 \mathrm{E}-06$ & Neutral O2 & $\mathrm{C} 26 \mathrm{H} 26 \mathrm{O} 2$ & 0.000321 \\
\hline S2 & C15H10S2 & $1.49 \mathrm{E}-05$ & Basic N1 & C51H79N1 & $1.57 \mathrm{E}-06$ & Neutral O2 & $\mathrm{C} 27 \mathrm{H} 28 \mathrm{O} 2$ & 0.000195 \\
\hline S2 & $\mathrm{C} 16 \mathrm{H} 12 \mathrm{~S} 2$ & $3.85 \mathrm{E}-05$ & Basic N1 & C52H81N1 & $1.21 \mathrm{E}-06$ & Neutral O2 & $\mathrm{C} 28 \mathrm{H} 30 \mathrm{O} 2$ & 0.000105 \\
\hline S2 & $\mathrm{C} 17 \mathrm{H} 14 \mathrm{~S} 2$ & $5.12 \mathrm{E}-05$ & Basic N1 & C53H83N1 & $9.12 \mathrm{E}-07$ & Neutral O2 & $\mathrm{C} 29 \mathrm{H} 32 \mathrm{O} 2$ & $4.22 \mathrm{E}-05$ \\
\hline S2 & C18H16S2 & $4.04 \mathrm{E}-05$ & Basic N1 & C54H85N1 & $6.08 \mathrm{E}-07$ & Neutral O2 & $\mathrm{C} 30 \mathrm{H} 34 \mathrm{O} 2$ & $4.41 \mathrm{E}-05$ \\
\hline S2 & C19H18S2 & $2.67 \mathrm{E}-05$ & Basic N1 & C55H87N1 & $8.44 \mathrm{E}-07$ & Neutral O2 & $\mathrm{C} 31 \mathrm{H} 36 \mathrm{O} 2$ & $2.13 \mathrm{E}-05$ \\
\hline S2 & $\mathrm{C} 20 \mathrm{H} 20 \mathrm{~S} 2$ & $1.56 \mathrm{E}-05$ & Basic N1 & $\mathrm{C} 19 \mathrm{H} 13 \mathrm{~N} 1$ & $4.04 \mathrm{E}-07$ & Neutral O2 & $\mathrm{C} 32 \mathrm{H} 38 \mathrm{O} 2$ & $9.53 \mathrm{E}-06$ \\
\hline S2 & $\mathrm{C} 21 \mathrm{H} 22 \mathrm{~S} 2$ & $9.29 \mathrm{E}-06$ & Basic N1 & $\mathrm{C} 20 \mathrm{H} 15 \mathrm{~N} 1$ & $6.10 \mathrm{E}-06$ & Neutral O2 & $\mathrm{C} 33 \mathrm{H} 40 \mathrm{O} 2$ & $8.80 \mathrm{E}-06$ \\
\hline S2 & $\mathrm{C} 22 \mathrm{H} 24 \mathrm{~S} 2$ & $2.73 \mathrm{E}-06$ & Basic N1 & $\mathrm{C} 21 \mathrm{H} 17 \mathrm{~N} 1$ & $1.77 \mathrm{E}-05$ & Neutral O2 & $\mathrm{C} 34 \mathrm{H} 42 \mathrm{O} 2$ & $2.16 \mathrm{E}-06$ \\
\hline S2 & $\mathrm{C} 23 \mathrm{H} 26 \mathrm{~S} 2$ & $1.75 \mathrm{E}-06$ & Basic N1 & $\mathrm{C} 22 \mathrm{H} 19 \mathrm{~N} 1$ & $3.38 \mathrm{E}-05$ & Neutral O2 & $\mathrm{C} 35 \mathrm{H} 44 \mathrm{O} 2$ & $3.97 \mathrm{E}-06$ \\
\hline S2 & $\mathrm{C} 25 \mathrm{H} 30 \mathrm{~S} 2$ & $2.92 \mathrm{E}-06$ & Basic N1 & $\mathrm{C} 23 \mathrm{H} 21 \mathrm{~N} 1$ & 4.87E-05 & Neutral O2 & $\mathrm{C} 36 \mathrm{H} 46 \mathrm{O} 2$ & $1.89 \mathrm{E}-06$ \\
\hline S2 & $\mathrm{C} 26 \mathrm{H} 32 \mathrm{~S} 2$ & $1.28 \mathrm{E}-06$ & Basic N1 & $\mathrm{C} 24 \mathrm{H} 23 \mathrm{~N} 1$ & $5.89 \mathrm{E}-05$ & Neutral O2 & $\mathrm{C} 37 \mathrm{H} 48 \mathrm{O} 2$ & $2.10 \mathrm{E}-06$ \\
\hline S2 & $\mathrm{C} 18 \mathrm{H} 14 \mathrm{~S} 2$ & $2.74 \mathrm{E}-06$ & Basic N1 & $\mathrm{C} 25 \mathrm{H} 25 \mathrm{~N} 1$ & $5.92 \mathrm{E}-05$ & Neutral O2 & $\mathrm{C} 38 \mathrm{H} 50 \mathrm{O} 2$ & $9.68 \mathrm{E}-07$ \\
\hline S2 & C19H16S2 & $4.24 \mathrm{E}-06$ & Basic N1 & $\mathrm{C} 26 \mathrm{H} 27 \mathrm{~N} 1$ & $5.57 \mathrm{E}-05$ & Neutral O2 & $\mathrm{C} 39 \mathrm{H} 52 \mathrm{O} 2$ & $3.85 \mathrm{E}-06$ \\
\hline S2 & $\mathrm{C} 20 \mathrm{H} 18 \mathrm{~S} 2$ & $8.36 \mathrm{E}-06$ & Basic N1 & $\mathrm{C} 27 \mathrm{H} 29 \mathrm{~N} 1$ & $5.95 \mathrm{E}-05$ & Neutral O2 & $\mathrm{C} 40 \mathrm{H} 54 \mathrm{O} 2$ & $2.05 \mathrm{E}-06$ \\
\hline S2 & $\mathrm{C} 21 \mathrm{H} 20 \mathrm{~S} 2$ & $7.64 \mathrm{E}-06$ & Basic N1 & $\mathrm{C} 28 \mathrm{H} 31 \mathrm{~N} 1$ & $6.60 \mathrm{E}-05$ & Neutral O2 & $\mathrm{C} 41 \mathrm{H} 56 \mathrm{O} 2$ & $1.61 \mathrm{E}-06$ \\
\hline S2 & $\mathrm{C} 22 \mathrm{H} 22 \mathrm{~S} 2$ & $2.73 \mathrm{E}-06$ & Basic N1 & $\mathrm{C} 29 \mathrm{H} 33 \mathrm{~N} 1$ & $5.93 \mathrm{E}-05$ & Neutral O2 & $\mathrm{C} 20 \mathrm{H} 12 \mathrm{O} 2$ & $2.53 \mathrm{E}-06$ \\
\hline S2 & $\mathrm{C} 23 \mathrm{H} 24 \mathrm{~S} 2$ & $1.21 \mathrm{E}-06$ & Basic N1 & $\mathrm{C} 30 \mathrm{H} 35 \mathrm{~N} 1$ & $5.40 \mathrm{E}-05$ & Neutral O2 & $\mathrm{C} 21 \mathrm{H} 14 \mathrm{O} 2$ & 4.09E-05 \\
\hline S2 & $\mathrm{C} 24 \mathrm{H} 26 \mathrm{~S} 2$ & $1.57 \mathrm{E}-06$ & Basic N1 & $\mathrm{C} 31 \mathrm{H} 37 \mathrm{~N} 1$ & $5.37 \mathrm{E}-05$ & Neutral O2 & $\mathrm{C} 22 \mathrm{H} 16 \mathrm{O} 2$ & 0.000403 \\
\hline S2 & $\mathrm{C} 17 \mathrm{H} 10 \mathrm{~S} 2$ & $2.60 \mathrm{E}-06$ & Basic N1 & $\mathrm{C} 32 \mathrm{H} 39 \mathrm{~N} 1$ & $5.55 \mathrm{E}-05$ & Neutral O2 & $\mathrm{C} 23 \mathrm{H} 18 \mathrm{O} 2$ & 0.00147 \\
\hline
\end{tabular}




\begin{tabular}{|c|c|c|c|c|c|c|c|c|}
\hline Class & formula & Mass & Class & formula & Mass & Class & formula & Mass \\
\hline S2 & $\mathrm{C} 18 \mathrm{H} 12 \mathrm{~S} 2$ & $7.56 \mathrm{E}-06$ & Basic N1 & $\mathrm{C} 33 \mathrm{H} 41 \mathrm{~N} 1$ & $5.14 \mathrm{E}-05$ & Neutral O2 & $\mathrm{C} 24 \mathrm{H} 20 \mathrm{O} 2$ & 0.002007 \\
\hline S2 & C19H14S2 & $8.29 \mathrm{E}-06$ & Basic N1 & $\mathrm{C} 34 \mathrm{H} 43 \mathrm{~N} 1$ & $4.38 \mathrm{E}-05$ & Neutral O2 & $\mathrm{C} 25 \mathrm{H} 22 \mathrm{O} 2$ & 0.001413 \\
\hline S2 & C20H16S2 & $9.92 \mathrm{E}-06$ & Basic N1 & $\mathrm{C} 35 \mathrm{H} 45 \mathrm{~N} 1$ & $3.62 \mathrm{E}-05$ & Neutral O2 & $\mathrm{C} 26 \mathrm{H} 24 \mathrm{O} 2$ & 0.000818 \\
\hline S2 & $\mathrm{C} 21 \mathrm{H} 18 \mathrm{~S} 2$ & $2.72 \mathrm{E}-06$ & Basic N1 & $\mathrm{C} 36 \mathrm{H} 47 \mathrm{~N} 1$ & $3.00 \mathrm{E}-05$ & Neutral O2 & $\mathrm{C} 27 \mathrm{H} 26 \mathrm{O} 2$ & 0.000429 \\
\hline S2 & $\mathrm{C} 22 \mathrm{H} 20 \mathrm{~S} 2$ & $2.21 \mathrm{E}-06$ & Basic N1 & $\mathrm{C} 37 \mathrm{H} 49 \mathrm{~N} 1$ & $2.68 \mathrm{E}-05$ & Neutral O2 & $\mathrm{C} 28 \mathrm{H} 28 \mathrm{O} 2$ & 0.000214 \\
\hline S2 & $\mathrm{C} 23 \mathrm{H} 22 \mathrm{~S} 2$ & $4.58 \mathrm{E}-06$ & Basic N1 & $\mathrm{C} 38 \mathrm{H} 51 \mathrm{~N} 1$ & $2.51 \mathrm{E}-05$ & Neutral O2 & $\mathrm{C} 29 \mathrm{H} 30 \mathrm{O} 2$ & 0.000125 \\
\hline S2 & C18H10S2 & $9.97 \mathrm{E}-07$ & Basic N1 & $\mathrm{C} 39 \mathrm{H} 53 \mathrm{~N} 1$ & $2.12 \mathrm{E}-05$ & Neutral O2 & $\mathrm{C} 30 \mathrm{H} 32 \mathrm{O} 2$ & $7.08 \mathrm{E}-05$ \\
\hline S2 & $\mathrm{C} 19 \mathrm{H} 12 \mathrm{~S} 2$ & $1.62 \mathrm{E}-05$ & Basic N1 & $\mathrm{C} 40 \mathrm{H} 55 \mathrm{~N} 1$ & $1.69 \mathrm{E}-05$ & Neutral O2 & $\mathrm{C} 31 \mathrm{H} 34 \mathrm{O} 2$ & $3.74 \mathrm{E}-05$ \\
\hline S2 & $\mathrm{C} 20 \mathrm{H} 14 \mathrm{~S} 2$ & $4.09 \mathrm{E}-05$ & Basic N1 & $\mathrm{C} 41 \mathrm{H} 57 \mathrm{~N} 1$ & $1.36 \mathrm{E}-05$ & Neutral O2 & $\mathrm{C} 32 \mathrm{H} 36 \mathrm{O} 2$ & $2.28 \mathrm{E}-05$ \\
\hline S2 & $\mathrm{C} 21 \mathrm{H} 16 \mathrm{~S} 2$ & $5.76 \mathrm{E}-05$ & Basic N1 & $\mathrm{C} 42 \mathrm{H} 59 \mathrm{~N} 1$ & $1.15 \mathrm{E}-05$ & Neutral O2 & $\mathrm{C} 33 \mathrm{H} 38 \mathrm{O} 2$ & $5.51 \mathrm{E}-06$ \\
\hline S2 & $\mathrm{C} 22 \mathrm{H} 18 \mathrm{~S} 2$ & $6.26 \mathrm{E}-05$ & Basic N1 & $\mathrm{C} 43 \mathrm{H} 61 \mathrm{~N} 1$ & $9.39 \mathrm{E}-06$ & Neutral O2 & $\mathrm{C} 34 \mathrm{H} 40 \mathrm{O} 2$ & $9.51 \mathrm{E}-06$ \\
\hline S2 & $\mathrm{C} 23 \mathrm{H} 20 \mathrm{~S} 2$ & 4.57E-05 & Basic N1 & C44H63N1 & $8.10 \mathrm{E}-06$ & Neutral O2 & $\mathrm{C} 35 \mathrm{H} 42 \mathrm{O} 2$ & $4.00 \mathrm{E}-06$ \\
\hline S2 & $\mathrm{C} 24 \mathrm{H} 22 \mathrm{~S} 2$ & $2.99 \mathrm{E}-05$ & Basic N1 & C45H65N1 & $6.60 \mathrm{E}-06$ & Neutral O2 & $\mathrm{C} 36 \mathrm{H} 44 \mathrm{O} 2$ & $2.58 \mathrm{E}-06$ \\
\hline S2 & $\mathrm{C} 25 \mathrm{H} 24 \mathrm{~S} 2$ & $1.89 \mathrm{E}-05$ & Basic N1 & $\mathrm{C} 46 \mathrm{H} 67 \mathrm{~N} 1$ & $4.58 \mathrm{E}-06$ & Neutral O2 & $\mathrm{C} 37 \mathrm{H} 46 \mathrm{O} 2$ & $2.44 \mathrm{E}-06$ \\
\hline S2 & $\mathrm{C} 26 \mathrm{H} 26 \mathrm{~S} 2$ & $1.47 \mathrm{E}-05$ & Basic N1 & $\mathrm{C} 47 \mathrm{H} 69 \mathrm{~N} 1$ & $4.26 \mathrm{E}-06$ & Neutral O2 & $\mathrm{C} 38 \mathrm{H} 48 \mathrm{O} 2$ & $2.70 \mathrm{E}-06$ \\
\hline S2 & $\mathrm{C} 27 \mathrm{H} 28 \mathrm{~S} 2$ & $8.24 \mathrm{E}-06$ & Basic N1 & $\mathrm{C} 48 \mathrm{H} 71 \mathrm{~N} 1$ & $3.03 \mathrm{E}-06$ & Neutral O2 & $\mathrm{C} 20 \mathrm{H} 10 \mathrm{O} 2$ & $5.95 \mathrm{E}-07$ \\
\hline S2 & $\mathrm{C} 28 \mathrm{H} 30 \mathrm{~S} 2$ & $7.62 \mathrm{E}-06$ & Basic N1 & $\mathrm{C} 49 \mathrm{H} 73 \mathrm{~N} 1$ & $2.08 \mathrm{E}-06$ & Neutral O2 & $\mathrm{C} 21 \mathrm{H} 12 \mathrm{O} 2$ & $6.53 \mathrm{E}-05$ \\
\hline S2 & $\mathrm{C} 29 \mathrm{H} 32 \mathrm{~S} 2$ & $3.73 \mathrm{E}-06$ & Basic N1 & $\mathrm{C} 19 \mathrm{H} 11 \mathrm{~N} 1$ & $3.65 \mathrm{E}-08$ & Neutral O2 & $\mathrm{C} 22 \mathrm{H} 14 \mathrm{O} 2$ & 0.000302 \\
\hline S2 & $\mathrm{C} 30 \mathrm{H} 34 \mathrm{~S} 2$ & $1.76 \mathrm{E}-06$ & Basic N1 & $\mathrm{C} 20 \mathrm{H} 13 \mathrm{~N} 1$ & $2.66 \mathrm{E}-06$ & Neutral O2 & $\mathrm{C} 23 \mathrm{H} 16 \mathrm{O} 2$ & 0.000515 \\
\hline S2 & $\mathrm{C} 32 \mathrm{H} 38 \mathrm{~S} 2$ & $4.08 \mathrm{E}-06$ & Basic N1 & $\mathrm{C} 21 \mathrm{H} 15 \mathrm{~N} 1$ & $1.40 \mathrm{E}-05$ & Neutral O2 & $\mathrm{C} 24 \mathrm{H} 18 \mathrm{O} 2$ & 0.000513 \\
\hline S2 & $\mathrm{C} 33 \mathrm{H} 40 \mathrm{~S} 2$ & $3.66 \mathrm{E}-06$ & Basic N1 & $\mathrm{C} 22 \mathrm{H} 17 \mathrm{~N} 1$ & $3.25 \mathrm{E}-05$ & Neutral O2 & $\mathrm{C} 25 \mathrm{H} 20 \mathrm{O} 2$ & 0.00051 \\
\hline S2 & $\mathrm{C} 34 \mathrm{H} 42 \mathrm{~S} 2$ & $1.29 \mathrm{E}-06$ & Basic N1 & $\mathrm{C} 23 \mathrm{H} 19 \mathrm{~N} 1$ & $5.24 \mathrm{E}-05$ & Neutral O2 & $\mathrm{C} 26 \mathrm{H} 22 \mathrm{O} 2$ & 0.00059 \\
\hline S2 & $\mathrm{C} 22 \mathrm{H} 16 \mathrm{~S} 2$ & $6.41 \mathrm{E}-06$ & Basic N1 & $\mathrm{C} 24 \mathrm{H} 21 \mathrm{~N} 1$ & $8.47 \mathrm{E}-05$ & Neutral O2 & $\mathrm{C} 27 \mathrm{H} 24 \mathrm{O} 2$ & 0.000529 \\
\hline S2 & $\mathrm{C} 23 \mathrm{H} 18 \mathrm{~S} 2$ & $6.92 \mathrm{E}-06$ & Basic N1 & $\mathrm{C} 25 \mathrm{H} 23 \mathrm{~N} 1$ & $6.33 \mathrm{E}-05$ & Neutral O2 & $\mathrm{C} 28 \mathrm{H} 26 \mathrm{O} 2$ & 0.000326 \\
\hline S2 & $\mathrm{C} 24 \mathrm{H} 20 \mathrm{~S} 2$ & $1.40 \mathrm{E}-05$ & Basic N1 & $\mathrm{C} 26 \mathrm{H} 25 \mathrm{~N} 1$ & $5.62 \mathrm{E}-05$ & Neutral O2 & $\mathrm{C} 29 \mathrm{H} 28 \mathrm{O} 2$ & 0.000174 \\
\hline S2 & $\mathrm{C} 25 \mathrm{H} 22 \mathrm{~S} 2$ & $1.56 \mathrm{E}-05$ & Basic N1 & $\mathrm{C} 27 \mathrm{H} 27 \mathrm{~N} 1$ & $5.66 \mathrm{E}-05$ & Neutral O2 & $\mathrm{C} 30 \mathrm{H} 30 \mathrm{O} 2$ & $9.75 \mathrm{E}-05$ \\
\hline S2 & $\mathrm{C} 26 \mathrm{H} 24 \mathrm{~S} 2$ & $1.18 \mathrm{E}-05$ & Basic N1 & $\mathrm{C} 28 \mathrm{H} 29 \mathrm{~N} 1$ & $5.87 \mathrm{E}-05$ & Neutral O2 & $\mathrm{C} 31 \mathrm{H} 32 \mathrm{O} 2$ & $5.03 \mathrm{E}-05$ \\
\hline $\mathrm{S} 2$ & $\mathrm{C} 27 \mathrm{H} 26 \mathrm{~S} 2$ & $1.28 \mathrm{E}-05$ & Basic N1 & $\mathrm{C} 29 \mathrm{H} 31 \mathrm{~N} 1$ & $5.25 \mathrm{E}-05$ & Neutral O2 & $\mathrm{C} 32 \mathrm{H} 34 \mathrm{O} 2$ & $2.53 \mathrm{E}-05$ \\
\hline $\mathrm{S} 2$ & $\mathrm{C} 28 \mathrm{H} 28 \mathrm{~S} 2$ & $7.91 \mathrm{E}-06$ & Basic N1 & $\mathrm{C} 30 \mathrm{H} 33 \mathrm{~N} 1$ & $4.68 \mathrm{E}-05$ & Neutral O2 & $\mathrm{C} 33 \mathrm{H} 36 \mathrm{O} 2$ & $9.96 \mathrm{E}-06$ \\
\hline S2 & $\mathrm{C} 29 \mathrm{H} 30 \mathrm{~S} 2$ & $8.08 \mathrm{E}-06$ & Basic N1 & $\mathrm{C} 31 \mathrm{H} 35 \mathrm{~N} 1$ & $4.36 \mathrm{E}-05$ & Neutral O2 & $\mathrm{C} 34 \mathrm{H} 38 \mathrm{O} 2$ & $1.08 \mathrm{E}-05$ \\
\hline S2 & $\mathrm{C} 30 \mathrm{H} 32 \mathrm{~S} 2$ & $5.81 \mathrm{E}-06$ & Basic N1 & $\mathrm{C} 32 \mathrm{H} 37 \mathrm{~N} 1$ & 4.43E-05 & Neutral O2 & $\mathrm{C} 35 \mathrm{H} 40 \mathrm{O} 2$ & $3.60 \mathrm{E}-06$ \\
\hline S2 & $\mathrm{C} 22 \mathrm{H} 14 \mathrm{~S} 2$ & $6.76 \mathrm{E}-06$ & Basic N1 & C33H39N1 & 4.03E-05 & Neutral O2 & $\mathrm{C} 36 \mathrm{H} 42 \mathrm{O} 2$ & $1.50 \mathrm{E}-06$ \\
\hline S2 & $\mathrm{C} 23 \mathrm{H} 16 \mathrm{~S} 2$ & $1.15 \mathrm{E}-05$ & Basic N1 & C34H41N1 & $3.41 \mathrm{E}-05$ & Neutral O2 & $\mathrm{C} 37 \mathrm{H} 44 \mathrm{O} 2$ & $2.52 \mathrm{E}-06$ \\
\hline S2 & $\mathrm{C} 24 \mathrm{H} 18 \mathrm{~S} 2$ & $1.30 \mathrm{E}-05$ & Basic N1 & $\mathrm{C} 35 \mathrm{H} 43 \mathrm{~N} 1$ & $2.79 \mathrm{E}-05$ & Neutral O2 & $\mathrm{C} 38 \mathrm{H} 46 \mathrm{O} 2$ & $1.12 \mathrm{E}-06$ \\
\hline S2 & $\mathrm{C} 25 \mathrm{H} 20 \mathrm{~S} 2$ & 7.83E-06 & Basic N1 & $\mathrm{C} 36 \mathrm{H} 45 \mathrm{~N} 1$ & $2.38 \mathrm{E}-05$ & Neutral O2 & $\mathrm{C} 20 \mathrm{H} 8 \mathrm{O} 2$ & $4.54 \mathrm{E}-07$ \\
\hline S2 & $\mathrm{C} 26 \mathrm{H} 22 \mathrm{~S} 2$ & $1.02 \mathrm{E}-05$ & Basic N1 & $\mathrm{C} 37 \mathrm{H} 47 \mathrm{~N} 1$ & $2.18 \mathrm{E}-05$ & Neutral O2 & $\mathrm{C} 21 \mathrm{H} 10 \mathrm{O} 2$ & $1.19 \mathrm{E}-06$ \\
\hline S2 & $\mathrm{C} 27 \mathrm{H} 24 \mathrm{~S} 2$ & $3.85 \mathrm{E}-06$ & Basic N1 & $\mathrm{C} 38 \mathrm{H} 49 \mathrm{~N} 1$ & $1.85 \mathrm{E}-05$ & Neutral O2 & $\mathrm{C} 22 \mathrm{H} 12 \mathrm{O} 2$ & $5.42 \mathrm{E}-06$ \\
\hline S2 & $\mathrm{C} 28 \mathrm{H} 26 \mathrm{~S} 2$ & $1.80 \mathrm{E}-06$ & Basic N1 & $\mathrm{C} 39 \mathrm{H} 51 \mathrm{~N} 1$ & $1.62 \mathrm{E}-05$ & Neutral O2 & $\mathrm{C} 23 \mathrm{H} 14 \mathrm{O} 2$ & $1.50 \mathrm{E}-05$ \\
\hline
\end{tabular}




\begin{tabular}{|c|c|c|c|c|c|c|c|c|}
\hline Class & formula & Mass & Class & formula & Mass & Class & formula & Mass \\
\hline S2 & $\mathrm{C} 29 \mathrm{H} 28 \mathrm{~S} 2$ & $5.25 \mathrm{E}-06$ & Basic N1 & $\mathrm{C} 40 \mathrm{H} 53 \mathrm{~N} 1$ & $1.32 \mathrm{E}-05$ & Neutral O2 & $\mathrm{C} 24 \mathrm{H} 16 \mathrm{O} 2$ & 0.000164 \\
\hline S2 & $\mathrm{C} 30 \mathrm{H} 30 \mathrm{~S} 2$ & $5.31 \mathrm{E}-06$ & Basic N1 & $\mathrm{C} 41 \mathrm{H} 55 \mathrm{~N} 1$ & $1.08 \mathrm{E}-05$ & Neutral O2 & $\mathrm{C} 25 \mathrm{H} 18 \mathrm{O} 2$ & 0.000434 \\
\hline S2 & $\mathrm{C} 31 \mathrm{H} 32 \mathrm{~S} 2$ & $1.31 \mathrm{E}-06$ & Basic N1 & $\mathrm{C} 42 \mathrm{H} 57 \mathrm{~N} 1$ & $9.71 \mathrm{E}-06$ & Neutral O2 & $\mathrm{C} 26 \mathrm{H} 20 \mathrm{O} 2$ & 0.000597 \\
\hline $\mathrm{S} 2$ & $\mathrm{C} 32 \mathrm{H} 34 \mathrm{~S} 2$ & $1.34 \mathrm{E}-06$ & Basic N1 & $\mathrm{C} 43 \mathrm{H} 59 \mathrm{~N} 1$ & 7.75E-06 & Neutral O2 & $\mathrm{C} 27 \mathrm{H} 22 \mathrm{O} 2$ & 0.000493 \\
\hline S2 & $\mathrm{C} 23 \mathrm{H} 14 \mathrm{~S} 2$ & $3.87 \mathrm{E}-06$ & Basic N1 & C44H61N1 & $5.82 \mathrm{E}-06$ & Neutral O2 & $\mathrm{C} 28 \mathrm{H} 24 \mathrm{O} 2$ & 0.000293 \\
\hline S2 & $\mathrm{C} 24 \mathrm{H} 16 \mathrm{~S} 2$ & $1.23 \mathrm{E}-05$ & Basic N1 & $\mathrm{C} 45 \mathrm{H} 63 \mathrm{~N} 1$ & $5.07 \mathrm{E}-06$ & Neutral O2 & $\mathrm{C} 29 \mathrm{H} 26 \mathrm{O} 2$ & 0.000188 \\
\hline S2 & $\mathrm{C} 25 \mathrm{H} 18 \mathrm{~S} 2$ & $1.86 \mathrm{E}-05$ & Basic N1 & $\mathrm{C} 46 \mathrm{H} 65 \mathrm{~N} 1$ & 4.02E-06 & Neutral O2 & $\mathrm{C} 30 \mathrm{H} 28 \mathrm{O} 2$ & 0.000124 \\
\hline S2 & $\mathrm{C} 26 \mathrm{H} 20 \mathrm{~S} 2$ & $2.10 \mathrm{E}-05$ & Basic N1 & $\mathrm{C} 21 \mathrm{H} 13 \mathrm{~N} 1$ & $1.87 \mathrm{E}-06$ & Neutral O2 & $\mathrm{C} 31 \mathrm{H} 30 \mathrm{O} 2$ & $7.55 \mathrm{E}-05$ \\
\hline S2 & $\mathrm{C} 27 \mathrm{H} 22 \mathrm{~S} 2$ & $1.34 \mathrm{E}-05$ & Basic N1 & $\mathrm{C} 22 \mathrm{H} 15 \mathrm{~N} 1$ & $1.11 \mathrm{E}-05$ & Neutral O2 & $\mathrm{C} 32 \mathrm{H} 32 \mathrm{O} 2$ & $2.46 \mathrm{E}-05$ \\
\hline S2 & $\mathrm{C} 28 \mathrm{H} 24 \mathrm{~S} 2$ & $1.38 \mathrm{E}-05$ & Basic N1 & $\mathrm{C} 23 \mathrm{H} 17 \mathrm{~N} 1$ & $3.34 \mathrm{E}-05$ & Neutral O2 & $\mathrm{C} 33 \mathrm{H} 34 \mathrm{O} 2$ & $1.28 \mathrm{E}-05$ \\
\hline S2 & $\mathrm{C} 29 \mathrm{H} 26 \mathrm{~S} 2$ & $1.62 \mathrm{E}-06$ & Basic N1 & $\mathrm{C} 24 \mathrm{H} 19 \mathrm{~N} 1$ & $4.96 \mathrm{E}-05$ & Neutral O2 & $\mathrm{C} 34 \mathrm{H} 36 \mathrm{O} 2$ & $9.63 \mathrm{E}-07$ \\
\hline S2 & $\mathrm{C} 25 \mathrm{H} 16 \mathrm{~S} 2$ & $1.12 \mathrm{E}-06$ & Basic N1 & $\mathrm{C} 25 \mathrm{H} 21 \mathrm{~N} 1$ & 4.97E-05 & Neutral O2 & $\mathrm{C} 35 \mathrm{H} 38 \mathrm{O} 2$ & $3.11 \mathrm{E}-06$ \\
\hline S2 & $\mathrm{C} 26 \mathrm{H} 18 \mathrm{~S} 2$ & $4.16 \mathrm{E}-06$ & Basic N1 & $\mathrm{C} 26 \mathrm{H} 23 \mathrm{~N} 1$ & 4.43E-05 & Neutral O2 & $\mathrm{C} 36 \mathrm{H} 40 \mathrm{O} 2$ & $2.13 \mathrm{E}-06$ \\
\hline S2 & $\mathrm{C} 27 \mathrm{H} 20 \mathrm{~S} 2$ & $4.00 \mathrm{E}-06$ & Basic N1 & $\mathrm{C} 27 \mathrm{H} 25 \mathrm{~N} 1$ & 4.44E-05 & Neutral O2 & $\mathrm{C} 37 \mathrm{H} 42 \mathrm{O} 2$ & $1.74 \mathrm{E}-06$ \\
\hline S2 & $\mathrm{C} 28 \mathrm{H} 22 \mathrm{~S} 2$ & $1.03 \mathrm{E}-05$ & Basic N1 & $\mathrm{C} 28 \mathrm{H} 27 \mathrm{~N} 1$ & $4.45 \mathrm{E}-05$ & Neutral O2 & $\mathrm{C} 38 \mathrm{H} 44 \mathrm{O} 2$ & $1.18 \mathrm{E}-05$ \\
\hline S2 & $\mathrm{C} 29 \mathrm{H} 24 \mathrm{~S} 2$ & $1.05 \mathrm{E}-05$ & Basic N1 & $\mathrm{C} 29 \mathrm{H} 29 \mathrm{~N} 1$ & 4.13E-05 & Neutral O2 & $\mathrm{C} 39 \mathrm{H} 46 \mathrm{O} 2$ & $8.62 \mathrm{E}-07$ \\
\hline S2 & $\mathrm{C} 30 \mathrm{H} 26 \mathrm{~S} 2$ & $9.07 \mathrm{E}-06$ & Basic N1 & $\mathrm{C} 30 \mathrm{H} 31 \mathrm{~N} 1$ & $3.42 \mathrm{E}-05$ & Neutral O2 & $\mathrm{C} 23 \mathrm{H} 12 \mathrm{O} 2$ & 4.47E-06 \\
\hline S2 & $\mathrm{C} 31 \mathrm{H} 28 \mathrm{~S} 2$ & $5.17 \mathrm{E}-06$ & Basic N1 & $\mathrm{C} 31 \mathrm{H} 33 \mathrm{~N} 1$ & $2.99 \mathrm{E}-05$ & Neutral O2 & $\mathrm{C} 24 \mathrm{H} 14 \mathrm{O} 2$ & $4.85 \mathrm{E}-05$ \\
\hline S2 & $\mathrm{C} 32 \mathrm{H} 30 \mathrm{~S} 2$ & $1.70 \mathrm{E}-06$ & Basic N1 & $\mathrm{C} 32 \mathrm{H} 35 \mathrm{~N} 1$ & $2.90 \mathrm{E}-05$ & Neutral O2 & $\mathrm{C} 25 \mathrm{H} 16 \mathrm{O} 2$ & $9.58 \mathrm{E}-05$ \\
\hline S2 & $\mathrm{C} 33 \mathrm{H} 32 \mathrm{~S} 2$ & $1.58 \mathrm{E}-06$ & Basic N1 & $\mathrm{C} 33 \mathrm{H} 37 \mathrm{~N} 1$ & $2.69 \mathrm{E}-05$ & Neutral O2 & $\mathrm{C} 26 \mathrm{H} 18 \mathrm{O} 2$ & 0.000165 \\
\hline S2 & $\mathrm{C} 34 \mathrm{H} 34 \mathrm{~S} 2$ & $1.59 \mathrm{E}-06$ & Basic N1 & $\mathrm{C} 34 \mathrm{H} 39 \mathrm{~N} 1$ & $2.17 \mathrm{E}-05$ & Neutral O2 & $\mathrm{C} 27 \mathrm{H} 20 \mathrm{O} 2$ & 0.000349 \\
\hline S2 & $\mathrm{C} 27 \mathrm{H} 18 \mathrm{~S} 2$ & $1.25 \mathrm{E}-06$ & Basic N1 & $\mathrm{C} 35 \mathrm{H} 41 \mathrm{~N} 1$ & $1.94 \mathrm{E}-05$ & Neutral O2 & $\mathrm{C} 28 \mathrm{H} 22 \mathrm{O} 2$ & 0.000477 \\
\hline S2 & $\mathrm{C} 28 \mathrm{H} 20 \mathrm{~S} 2$ & $2.01 \mathrm{E}-06$ & Basic N1 & $\mathrm{C} 36 \mathrm{H} 43 \mathrm{~N} 1$ & $1.57 \mathrm{E}-05$ & Neutral O2 & $\mathrm{C} 29 \mathrm{H} 24 \mathrm{O} 2$ & 0.00041 \\
\hline S2 & $\mathrm{C} 29 \mathrm{H} 22 \mathrm{~S} 2$ & $2.83 \mathrm{E}-06$ & Basic N1 & $\mathrm{C} 37 \mathrm{H} 45 \mathrm{~N} 1$ & $1.36 \mathrm{E}-05$ & Neutral O2 & $\mathrm{C} 30 \mathrm{H} 26 \mathrm{O} 2$ & 0.000288 \\
\hline S2 & $\mathrm{C} 30 \mathrm{H} 24 \mathrm{~S} 2$ & $3.89 \mathrm{E}-06$ & Basic N1 & $\mathrm{C} 38 \mathrm{H} 47 \mathrm{~N} 1$ & $1.33 \mathrm{E}-05$ & Neutral O2 & $\mathrm{C} 31 \mathrm{H} 28 \mathrm{O} 2$ & 0.000156 \\
\hline S2 & $\mathrm{C} 31 \mathrm{H} 26 \mathrm{~S} 2$ & $1.56 \mathrm{E}-06$ & Basic N1 & $\mathrm{C} 39 \mathrm{H} 49 \mathrm{~N} 1$ & $1.19 \mathrm{E}-05$ & Neutral O2 & $\mathrm{C} 32 \mathrm{H} 30 \mathrm{O} 2$ & $7.52 \mathrm{E}-05$ \\
\hline S2 & $\mathrm{C} 32 \mathrm{H} 28 \mathrm{~S} 2$ & $2.14 \mathrm{E}-06$ & Basic N1 & $\mathrm{C} 40 \mathrm{H} 51 \mathrm{~N} 1$ & $9.68 \mathrm{E}-06$ & Neutral O2 & $\mathrm{C} 33 \mathrm{H} 32 \mathrm{O} 2$ & $2.66 \mathrm{E}-05$ \\
\hline S2 & $\mathrm{C} 29 \mathrm{H} 20 \mathrm{~S} 2$ & $1.44 \mathrm{E}-06$ & Basic N1 & $\mathrm{C} 41 \mathrm{H} 53 \mathrm{~N} 1$ & $8.22 \mathrm{E}-06$ & Neutral O2 & $\mathrm{C} 34 \mathrm{H} 34 \mathrm{O} 2$ & $1.72 \mathrm{E}-05$ \\
\hline S2 & $\mathrm{C} 30 \mathrm{H} 22 \mathrm{~S} 2$ & $3.29 \mathrm{E}-06$ & Basic N1 & $\mathrm{C} 42 \mathrm{H} 55 \mathrm{~N} 1$ & $6.99 \mathrm{E}-06$ & Neutral O2 & $\mathrm{C} 35 \mathrm{H} 36 \mathrm{O} 2$ & $2.57 \mathrm{E}-06$ \\
\hline S2 & $\mathrm{C} 31 \mathrm{H} 24 \mathrm{~S} 2$ & $1.78 \mathrm{E}-06$ & Basic N1 & $\mathrm{C} 43 \mathrm{H} 57 \mathrm{~N} 1$ & $5.62 \mathrm{E}-06$ & Neutral O2 & $\mathrm{C} 36 \mathrm{H} 38 \mathrm{O} 2$ & 4.01E-06 \\
\hline S2 & $\mathrm{C} 32 \mathrm{H} 26 \mathrm{~S} 2$ & $2.96 \mathrm{E}-06$ & Basic N1 & $\mathrm{C} 44 \mathrm{H} 59 \mathrm{~N} 1$ & $4.36 \mathrm{E}-06$ & Neutral O2 & $\mathrm{C} 37 \mathrm{H} 40 \mathrm{O} 2$ & 4.15E-06 \\
\hline Non-basic N1 & $\mathrm{C} 33 \mathrm{H} 55 \mathrm{~N} 1$ & 7.04E-06 & Basic N1 & $\mathrm{C} 22 \mathrm{H} 13 \mathrm{~N} 1$ & 8.64E-08 & Neutral O2 & $\mathrm{C} 38 \mathrm{H} 42 \mathrm{O} 2$ & $1.20 \mathrm{E}-06$ \\
\hline Non-basic N1 & $\mathrm{C} 34 \mathrm{H} 57 \mathrm{~N} 1$ & 4.63E-06 & Basic N1 & $\mathrm{C} 23 \mathrm{H} 15 \mathrm{~N} 1$ & $1.39 \mathrm{E}-06$ & Neutral O2 & $\mathrm{C} 39 \mathrm{H} 44 \mathrm{O} 2$ & $8.67 \mathrm{E}-07$ \\
\hline Non-basic N1 & C35H59N1 & $3.54 \mathrm{E}-06$ & Basic N1 & $\mathrm{C} 24 \mathrm{H} 17 \mathrm{~N} 1$ & $6.34 \mathrm{E}-06$ & Neutral O2 & $\mathrm{C} 24 \mathrm{H} 12 \mathrm{O} 2$ & $1.67 \mathrm{E}-05$ \\
\hline Non-basic N1 & $\mathrm{C} 36 \mathrm{H} 61 \mathrm{~N} 1$ & $4.85 \mathrm{E}-06$ & Basic N1 & $\mathrm{C} 25 \mathrm{H} 19 \mathrm{~N} 1$ & $1.56 \mathrm{E}-05$ & Neutral O2 & $\mathrm{C} 25 \mathrm{H} 14 \mathrm{O} 2$ & 0.000273 \\
\hline Non-basic N1 & $\mathrm{C} 37 \mathrm{H} 63 \mathrm{~N} 1$ & 1.69E-06 & Basic N1 & $\mathrm{C} 26 \mathrm{H} 21 \mathrm{~N} 1$ & $2.04 \mathrm{E}-05$ & Neutral O2 & $\mathrm{C} 26 \mathrm{H} 16 \mathrm{O} 2$ & 0.000639 \\
\hline Non-basic N1 & $\mathrm{C} 38 \mathrm{H} 65 \mathrm{~N} 1$ & $1.93 \mathrm{E}-06$ & Basic N1 & $\mathrm{C} 27 \mathrm{H} 23 \mathrm{~N} 1$ & $2.27 \mathrm{E}-05$ & Neutral O2 & $\mathrm{C} 27 \mathrm{H} 18 \mathrm{O} 2$ & 0.000852 \\
\hline Non-basic N1 & $\mathrm{C} 29 \mathrm{H} 45 \mathrm{~N} 1$ & $1.33 \mathrm{E}-06$ & Basic N1 & $\mathrm{C} 28 \mathrm{H} 25 \mathrm{~N} 1$ & $2.46 \mathrm{E}-05$ & Neutral O2 & $\mathrm{C} 28 \mathrm{H} 20 \mathrm{O} 2$ & 0.000661 \\
\hline
\end{tabular}




\begin{tabular}{|c|c|c|c|c|c|c|c|c|}
\hline Class & formula & Mass & Class & formula & Mass & Class & formula & Mass \\
\hline Non-basic N1 & $\mathrm{C} 30 \mathrm{H} 47 \mathrm{~N} 1$ & $2.22 \mathrm{E}-06$ & Basic N1 & $\mathrm{C} 29 \mathrm{H} 27 \mathrm{~N} 1$ & $2.29 \mathrm{E}-05$ & Neutral O2 & $\mathrm{C} 29 \mathrm{H} 22 \mathrm{O} 2$ & 0.000404 \\
\hline Non-basic N1 & $\mathrm{C} 31 \mathrm{H} 49 \mathrm{~N} 1$ & $1.73 \mathrm{E}-06$ & Basic N1 & $\mathrm{C} 30 \mathrm{H} 29 \mathrm{~N} 1$ & $1.97 \mathrm{E}-05$ & Neutral O2 & $\mathrm{C} 30 \mathrm{H} 24 \mathrm{O} 2$ & 0.000307 \\
\hline Non-basic N1 & $\mathrm{C} 32 \mathrm{H} 51 \mathrm{~N} 1$ & $1.29 \mathrm{E}-06$ & Basic N1 & $\mathrm{C} 31 \mathrm{H} 31 \mathrm{~N} 1$ & $1.77 \mathrm{E}-05$ & Neutral O2 & $\mathrm{C} 31 \mathrm{H} 26 \mathrm{O} 2$ & 0.000275 \\
\hline Non-basic N1 & $\mathrm{C} 33 \mathrm{H} 53 \mathrm{~N} 1$ & 3.19E-06 & Basic N1 & $\mathrm{C} 32 \mathrm{H} 33 \mathrm{~N} 1$ & $1.73 \mathrm{E}-05$ & Neutral O2 & $\mathrm{C} 32 \mathrm{H} 28 \mathrm{O} 2$ & 0.000189 \\
\hline Non-basic N1 & $\mathrm{C} 34 \mathrm{H} 55 \mathrm{~N} 1$ & $2.10 \mathrm{E}-06$ & Basic N1 & $\mathrm{C} 33 \mathrm{H} 35 \mathrm{~N} 1$ & $1.70 \mathrm{E}-05$ & Neutral O2 & $\mathrm{C} 33 \mathrm{H} 30 \mathrm{O} 2$ & $8.89 \mathrm{E}-05$ \\
\hline Non-basic N1 & $\mathrm{C} 35 \mathrm{H} 57 \mathrm{~N} 1$ & $2.12 \mathrm{E}-06$ & Basic N1 & $\mathrm{C} 34 \mathrm{H} 37 \mathrm{~N} 1$ & $1.48 \mathrm{E}-05$ & Neutral O2 & $\mathrm{C} 34 \mathrm{H} 32 \mathrm{O} 2$ & $3.99 \mathrm{E}-05$ \\
\hline Non-basic N1 & C36H59N1 & $2.01 \mathrm{E}-06$ & Basic N1 & C35H39N1 & $1.21 \mathrm{E}-05$ & Neutral O2 & $\mathrm{C} 35 \mathrm{H} 34 \mathrm{O} 2$ & $1.51 \mathrm{E}-05$ \\
\hline Non-basic N1 & $\mathrm{C} 15 \mathrm{H} 15 \mathrm{~N} 1$ & $1.01 \mathrm{E}-05$ & Basic N1 & $\mathrm{C} 36 \mathrm{H} 41 \mathrm{~N} 1$ & $9.72 \mathrm{E}-06$ & Neutral O2 & $\mathrm{C} 36 \mathrm{H} 36 \mathrm{O} 2$ & $3.21 \mathrm{E}-06$ \\
\hline Non-basic N1 & $\mathrm{C} 16 \mathrm{H} 17 \mathrm{~N} 1$ & $9.15 \mathrm{E}-05$ & Basic N1 & $\mathrm{C} 37 \mathrm{H} 43 \mathrm{~N} 1$ & $9.83 \mathrm{E}-06$ & Neutral O2 & $\mathrm{C} 37 \mathrm{H} 38 \mathrm{O} 2$ & $4.38 \mathrm{E}-06$ \\
\hline Non-basic N1 & $\mathrm{C} 17 \mathrm{H} 19 \mathrm{~N} 1$ & $8.77 \mathrm{E}-05$ & Basic N1 & $\mathrm{C} 38 \mathrm{H} 45 \mathrm{~N} 1$ & $9.13 \mathrm{E}-06$ & Neutral O2 & $\mathrm{C} 38 \mathrm{H} 40 \mathrm{O} 2$ & $1.87 \mathrm{E}-06$ \\
\hline Non-basic N1 & $\mathrm{C} 18 \mathrm{H} 21 \mathrm{~N} 1$ & $5.32 \mathrm{E}-05$ & Basic N1 & $\mathrm{C} 39 \mathrm{H} 47 \mathrm{~N} 1$ & $8.39 \mathrm{E}-06$ & Neutral O2 & $\mathrm{C} 39 \mathrm{H} 42 \mathrm{O} 2$ & $8.20 \mathrm{E}-07$ \\
\hline Non-basic N1 & $\mathrm{C} 19 \mathrm{H} 23 \mathrm{~N} 1$ & $3.87 \mathrm{E}-05$ & Basic N1 & $\mathrm{C} 40 \mathrm{H} 49 \mathrm{~N} 1$ & $6.36 \mathrm{E}-06$ & Neutral O2 & $\mathrm{C} 25 \mathrm{H} 12 \mathrm{O} 2$ & $7.85 \mathrm{E}-07$ \\
\hline Non-basic N1 & $\mathrm{C} 20 \mathrm{H} 25 \mathrm{~N} 1$ & $3.56 \mathrm{E}-05$ & Basic N1 & $\mathrm{C} 41 \mathrm{H} 51 \mathrm{~N} 1$ & $5.71 \mathrm{E}-06$ & Neutral O2 & $\mathrm{C} 26 \mathrm{H} 14 \mathrm{O} 2$ & $2.25 \mathrm{E}-06$ \\
\hline Non-basic N1 & $\mathrm{C} 21 \mathrm{H} 27 \mathrm{~N} 1$ & 3.99E-05 & Basic N1 & $\mathrm{C} 42 \mathrm{H} 53 \mathrm{~N} 1$ & 4.74E-06 & Neutral O2 & $\mathrm{C} 27 \mathrm{H} 16 \mathrm{O} 2$ & $2.40 \mathrm{E}-05$ \\
\hline Non-basic N1 & $\mathrm{C} 22 \mathrm{H} 29 \mathrm{~N} 1$ & $5.21 \mathrm{E}-05$ & Basic N1 & $\mathrm{C} 43 \mathrm{H} 55 \mathrm{~N} 1$ & $3.60 \mathrm{E}-06$ & Neutral O2 & $\mathrm{C} 28 \mathrm{H} 18 \mathrm{O} 2$ & 0.000113 \\
\hline Non-basic N1 & $\mathrm{C} 23 \mathrm{H} 31 \mathrm{~N} 1$ & 7.09E-05 & Basic N1 & $\mathrm{C} 24 \mathrm{H} 15 \mathrm{~N} 1$ & $1.94 \mathrm{E}-06$ & Neutral O2 & $\mathrm{C} 29 \mathrm{H} 20 \mathrm{O} 2$ & 0.000198 \\
\hline Non-basic N1 & $\mathrm{C} 24 \mathrm{H} 33 \mathrm{~N} 1$ & $8.54 \mathrm{E}-05$ & Basic N1 & $\mathrm{C} 25 \mathrm{H} 17 \mathrm{~N} 1$ & $7.35 \mathrm{E}-06$ & Neutral O2 & $\mathrm{C} 30 \mathrm{H} 22 \mathrm{O} 2$ & 0.00024 \\
\hline Non-basic N1 & $\mathrm{C} 25 \mathrm{H} 35 \mathrm{~N} 1$ & $9.14 \mathrm{E}-05$ & Basic N1 & $\mathrm{C} 26 \mathrm{H} 19 \mathrm{~N} 1$ & $1.03 \mathrm{E}-05$ & Neutral O2 & $\mathrm{C} 31 \mathrm{H} 24 \mathrm{O} 2$ & 0.000216 \\
\hline Non-basic N1 & $\mathrm{C} 26 \mathrm{H} 37 \mathrm{~N} 1$ & 0.00011 & Basic N1 & $\mathrm{C} 27 \mathrm{H} 21 \mathrm{~N} 1$ & $1.10 \mathrm{E}-05$ & Neutral O2 & $\mathrm{C} 32 \mathrm{H} 26 \mathrm{O} 2$ & 0.000159 \\
\hline Non-basic N1 & $\mathrm{C} 27 \mathrm{H} 39 \mathrm{~N} 1$ & 0.000128 & Basic N1 & $\mathrm{C} 28 \mathrm{H} 23 \mathrm{~N} 1$ & $1.18 \mathrm{E}-05$ & Neutral O2 & $\mathrm{C} 33 \mathrm{H} 28 \mathrm{O} 2$ & $9.55 \mathrm{E}-05$ \\
\hline Non-basic N1 & $\mathrm{C} 28 \mathrm{H} 41 \mathrm{~N} 1$ & 0.00013 & Basic N1 & $\mathrm{C} 29 \mathrm{H} 25 \mathrm{~N} 1$ & $1.20 \mathrm{E}-05$ & Neutral O2 & $\mathrm{C} 34 \mathrm{H} 30 \mathrm{O} 2$ & $5.44 \mathrm{E}-05$ \\
\hline Non-basic N1 & $\mathrm{C} 29 \mathrm{H} 43 \mathrm{~N} 1$ & 0.000154 & Basic N1 & $\mathrm{C} 30 \mathrm{H} 27 \mathrm{~N} 1$ & $1.06 \mathrm{E}-05$ & Neutral O2 & $\mathrm{C} 35 \mathrm{H} 32 \mathrm{O} 2$ & $1.85 \mathrm{E}-05$ \\
\hline Non-basic N1 & $\mathrm{C} 30 \mathrm{H} 45 \mathrm{~N} 1$ & 0.000161 & Basic N1 & C31H29N1 & $1.05 \mathrm{E}-05$ & Neutral O2 & $\mathrm{C} 36 \mathrm{H} 34 \mathrm{O} 2$ & 8.13E-06 \\
\hline Non-basic N1 & $\mathrm{C} 31 \mathrm{H} 47 \mathrm{~N} 1$ & 0.00014 & Basic N1 & $\mathrm{C} 32 \mathrm{H} 31 \mathrm{~N} 1$ & $1.02 \mathrm{E}-05$ & Neutral O2 & $\mathrm{C} 37 \mathrm{H} 36 \mathrm{O} 2$ & 2.95E-06 \\
\hline Non-basic N1 & $\mathrm{C} 32 \mathrm{H} 49 \mathrm{~N} 1$ & 0.000147 & Basic N1 & C33H33N1 & $1.08 \mathrm{E}-05$ & Neutral O2 & $\mathrm{C} 38 \mathrm{H} 38 \mathrm{O} 2$ & $1.86 \mathrm{E}-06$ \\
\hline Non-basic N1 & C33H51N1 & 0.000135 & Basic N1 & C34H35N1 & $9.81 \mathrm{E}-06$ & Neutral O2 & $\mathrm{C} 39 \mathrm{H} 40 \mathrm{O} 2$ & $1.87 \mathrm{E}-06$ \\
\hline Non-basic N1 & $\mathrm{C} 34 \mathrm{H} 53 \mathrm{~N} 1$ & 0.000109 & Basic N1 & $\mathrm{C} 35 \mathrm{H} 37 \mathrm{~N} 1$ & $8.33 \mathrm{E}-06$ & Neutral O2 & $\mathrm{C} 40 \mathrm{H} 42 \mathrm{O} 2$ & $1.33 \mathrm{E}-06$ \\
\hline Non-basic N1 & $\mathrm{C} 35 \mathrm{H} 55 \mathrm{~N} 1$ & 7.24E-05 & Basic N1 & $\mathrm{C} 36 \mathrm{H} 39 \mathrm{~N} 1$ & $6.82 \mathrm{E}-06$ & Neutral O2 & $\mathrm{C} 41 \mathrm{H} 44 \mathrm{O} 2$ & $9.77 \mathrm{E}-07$ \\
\hline Non-basic N1 & $\mathrm{C} 36 \mathrm{H} 57 \mathrm{~N} 1$ & $5.92 \mathrm{E}-05$ & Basic N1 & $\mathrm{C} 37 \mathrm{H} 41 \mathrm{~N} 1$ & $6.54 \mathrm{E}-06$ & Neutral O2 & $\mathrm{C} 26 \mathrm{H} 12 \mathrm{O} 2$ & $1.08 \mathrm{E}-05$ \\
\hline Non-basic N1 & C37H59N1 & 4.43E-05 & Basic N1 & $\mathrm{C} 38 \mathrm{H} 43 \mathrm{~N} 1$ & $6.00 \mathrm{E}-06$ & Neutral O2 & $\mathrm{C} 27 \mathrm{H} 14 \mathrm{O} 2$ & 0.000172 \\
\hline Non-basic N1 & $\mathrm{C} 38 \mathrm{H} 61 \mathrm{~N} 1$ & $3.03 \mathrm{E}-05$ & Basic N1 & $\mathrm{C} 39 \mathrm{H} 45 \mathrm{~N} 1$ & $5.58 \mathrm{E}-06$ & Neutral O2 & $\mathrm{C} 28 \mathrm{H} 16 \mathrm{O} 2$ & 0.000348 \\
\hline Non-basic N1 & $\mathrm{C} 39 \mathrm{H} 63 \mathrm{~N} 1$ & $2.06 \mathrm{E}-05$ & Basic N1 & $\mathrm{C} 40 \mathrm{H} 47 \mathrm{~N} 1$ & $4.68 \mathrm{E}-06$ & Neutral O2 & $\mathrm{C} 29 \mathrm{H} 18 \mathrm{O} 2$ & 0.000319 \\
\hline Non-basic N1 & $\mathrm{C} 40 \mathrm{H} 65 \mathrm{~N} 1$ & $1.97 \mathrm{E}-05$ & Basic N1 & $\mathrm{C} 41 \mathrm{H} 49 \mathrm{~N} 1$ & $4.28 \mathrm{E}-06$ & Neutral O2 & $\mathrm{C} 30 \mathrm{H} 20 \mathrm{O} 2$ & 0.000192 \\
\hline Non-basic N1 & C41H67N1 & $4.21 \mathrm{E}-06$ & Basic N1 & C42H51N1 & $2.84 \mathrm{E}-06$ & Neutral O2 & $\mathrm{C} 31 \mathrm{H} 22 \mathrm{O} 2$ & 0.000124 \\
\hline Non-basic N1 & C42H69N1 & $4.53 \mathrm{E}-06$ & Basic N1 & $\mathrm{C} 43 \mathrm{H} 53 \mathrm{~N} 1$ & $2.61 \mathrm{E}-06$ & Neutral O2 & $\mathrm{C} 32 \mathrm{H} 24 \mathrm{O} 2$ & 0.000114 \\
\hline Non-basic N1 & $\mathrm{C} 43 \mathrm{H} 71 \mathrm{~N} 1$ & $2.67 \mathrm{E}-06$ & Basic N1 & $\mathrm{C} 44 \mathrm{H} 55 \mathrm{~N} 1$ & $1.82 \mathrm{E}-06$ & Neutral O2 & $\mathrm{C} 33 \mathrm{H} 26 \mathrm{O} 2$ & $9.13 \mathrm{E}-05$ \\
\hline Non-basic N1 & $\mathrm{C} 16 \mathrm{H} 15 \mathrm{~N} 1$ & $6.11 \mathrm{E}-06$ & Basic N1 & C27H19N1 & $4.08 \mathrm{E}-06$ & Neutral O2 & $\mathrm{C} 34 \mathrm{H} 28 \mathrm{O} 2$ & 4.23E-05 \\
\hline Non-basic N1 & $\mathrm{C} 17 \mathrm{H} 17 \mathrm{~N} 1$ & $4.00 \mathrm{E}-05$ & Basic N1 & $\mathrm{C} 28 \mathrm{H} 21 \mathrm{~N} 1$ & $5.41 \mathrm{E}-06$ & Neutral O2 & $\mathrm{C} 35 \mathrm{H} 30 \mathrm{O} 2$ & $2.73 \mathrm{E}-05$ \\
\hline
\end{tabular}




\begin{tabular}{|c|c|c|c|c|c|c|c|c|}
\hline Class & formula & Mass & Class & formula & Mass & Class & formula & Mass \\
\hline Non-basic N1 & C18H19N1 & $5.48 \mathrm{E}-05$ & Basic N1 & $\mathrm{C} 29 \mathrm{H} 23 \mathrm{~N} 1$ & $6.04 \mathrm{E}-06$ & Neutral O2 & $\mathrm{C} 36 \mathrm{H} 32 \mathrm{O} 2$ & $1.62 \mathrm{E}-05$ \\
\hline Non-basic N1 & $\mathrm{C} 19 \mathrm{H} 21 \mathrm{~N} 1$ & $5.23 \mathrm{E}-05$ & Basic N1 & $\mathrm{C} 30 \mathrm{H} 25 \mathrm{~N} 1$ & $5.63 \mathrm{E}-06$ & Neutral O2 & $\mathrm{C} 37 \mathrm{H} 34 \mathrm{O} 2$ & $3.12 \mathrm{E}-06$ \\
\hline Non-basic N1 & $\mathrm{C} 20 \mathrm{H} 23 \mathrm{~N} 1$ & $5.19 \mathrm{E}-05$ & Basic N1 & $\mathrm{C} 31 \mathrm{H} 27 \mathrm{~N} 1$ & $5.56 \mathrm{E}-06$ & Neutral O2 & $\mathrm{C} 38 \mathrm{H} 36 \mathrm{O} 2$ & 4.22E-06 \\
\hline Non-basic N1 & $\mathrm{C} 21 \mathrm{H} 25 \mathrm{~N} 1$ & $5.13 \mathrm{E}-05$ & Basic N1 & $\mathrm{C} 32 \mathrm{H} 29 \mathrm{~N} 1$ & $5.40 \mathrm{E}-06$ & Neutral O2 & $\mathrm{C} 39 \mathrm{H} 38 \mathrm{O} 2$ & $4.89 \mathrm{E}-06$ \\
\hline Non-basic N1 & $\mathrm{C} 22 \mathrm{H} 27 \mathrm{~N} 1$ & $5.83 \mathrm{E}-05$ & Basic N1 & $\mathrm{C} 33 \mathrm{H} 31 \mathrm{~N} 1$ & $6.36 \mathrm{E}-06$ & Neutral O2 & $\mathrm{C} 25 \mathrm{H} 8 \mathrm{O} 2$ & $3.55 \mathrm{E}-07$ \\
\hline Non-basic N1 & $\mathrm{C} 23 \mathrm{H} 29 \mathrm{~N} 1$ & $6.74 \mathrm{E}-05$ & Basic N1 & C34H33N1 & $6.23 \mathrm{E}-06$ & Neutral O2 & $\mathrm{C} 26 \mathrm{H} 10 \mathrm{O} 2$ & $4.00 \mathrm{E}-07$ \\
\hline Non-basic N1 & $\mathrm{C} 24 \mathrm{H} 31 \mathrm{~N} 1$ & 7.37E-05 & Basic N1 & $\mathrm{C} 35 \mathrm{H} 35 \mathrm{~N} 1$ & $5.40 \mathrm{E}-06$ & Neutral O2 & $\mathrm{C} 27 \mathrm{H} 12 \mathrm{O} 2$ & $1.15 \mathrm{E}-06$ \\
\hline Non-basic N1 & $\mathrm{C} 25 \mathrm{H} 33 \mathrm{~N} 1$ & 8.27E-05 & Basic N1 & $\mathrm{C} 36 \mathrm{H} 37 \mathrm{~N} 1$ & 4.93E-06 & Neutral O2 & $\mathrm{C} 28 \mathrm{H} 14 \mathrm{O} 2$ & $5.88 \mathrm{E}-07$ \\
\hline Non-basic N1 & $\mathrm{C} 26 \mathrm{H} 35 \mathrm{~N} 1$ & $8.00 \mathrm{E}-05$ & Basic N1 & $\mathrm{C} 37 \mathrm{H} 39 \mathrm{~N} 1$ & $4.48 \mathrm{E}-06$ & Neutral O2 & $\mathrm{C} 29 \mathrm{H} 16 \mathrm{O} 2$ & $5.10 \mathrm{E}-06$ \\
\hline Non-basic N1 & $\mathrm{C} 27 \mathrm{H} 37 \mathrm{~N} 1$ & $9.88 \mathrm{E}-05$ & Basic N1 & $\mathrm{C} 38 \mathrm{H} 41 \mathrm{~N} 1$ & 4.37E-06 & Neutral O2 & $\mathrm{C} 30 \mathrm{H} 18 \mathrm{O} 2$ & $3.87 \mathrm{E}-05$ \\
\hline Non-basic N1 & $\mathrm{C} 28 \mathrm{H} 39 \mathrm{~N} 1$ & $8.70 \mathrm{E}-05$ & Basic N1 & $\mathrm{C} 39 \mathrm{H} 43 \mathrm{~N} 1$ & $3.55 \mathrm{E}-06$ & Neutral O2 & $\mathrm{C} 31 \mathrm{H} 20 \mathrm{O} 2$ & $3.84 \mathrm{E}-05$ \\
\hline Non-basic N1 & $\mathrm{C} 29 \mathrm{H} 41 \mathrm{~N} 1$ & $8.95 \mathrm{E}-05$ & Basic N1 & $\mathrm{C} 40 \mathrm{H} 45 \mathrm{~N} 1$ & $3.13 \mathrm{E}-06$ & Neutral O2 & $\mathrm{C} 32 \mathrm{H} 22 \mathrm{O} 2$ & $8.21 \mathrm{E}-05$ \\
\hline Non-basic N1 & $\mathrm{C} 30 \mathrm{H} 43 \mathrm{~N} 1$ & $8.27 \mathrm{E}-05$ & Basic N1 & $\mathrm{C} 41 \mathrm{H} 47 \mathrm{~N} 1$ & $2.30 \mathrm{E}-06$ & Neutral O2 & $\mathrm{C} 33 \mathrm{H} 24 \mathrm{O} 2$ & 4.74E-05 \\
\hline Non-basic N1 & $\mathrm{C} 31 \mathrm{H} 45 \mathrm{~N} 1$ & $8.14 \mathrm{E}-05$ & Basic N1 & $\mathrm{C} 42 \mathrm{H} 49 \mathrm{~N} 1$ & $2.15 \mathrm{E}-06$ & Neutral O2 & $\mathrm{C} 34 \mathrm{H} 26 \mathrm{O} 2$ & $3.19 \mathrm{E}-05$ \\
\hline Non-basic N1 & $\mathrm{C} 32 \mathrm{H} 47 \mathrm{~N} 1$ & $7.63 \mathrm{E}-05$ & Basic N1 & $\mathrm{C} 43 \mathrm{H} 51 \mathrm{~N} 1$ & $1.49 \mathrm{E}-06$ & Neutral O2 & $\mathrm{C} 35 \mathrm{H} 28 \mathrm{O} 2$ & $2.34 \mathrm{E}-05$ \\
\hline Non-basic N1 & $\mathrm{C} 33 \mathrm{H} 49 \mathrm{~N} 1$ & $7.69 \mathrm{E}-05$ & Basic N1 & $\mathrm{C} 44 \mathrm{H} 53 \mathrm{~N} 1$ & $1.20 \mathrm{E}-06$ & Neutral O2 & $\mathrm{C} 36 \mathrm{H} 30 \mathrm{O} 2$ & $2.01 \mathrm{E}-05$ \\
\hline Non-basic N1 & $\mathrm{C} 34 \mathrm{H} 51 \mathrm{~N} 1$ & $6.16 \mathrm{E}-05$ & Basic N1 & $\mathrm{C} 29 \mathrm{H} 21 \mathrm{~N} 1$ & $1.49 \mathrm{E}-06$ & Neutral O2 & $\mathrm{C} 37 \mathrm{H} 32 \mathrm{O} 2$ & $5.70 \mathrm{E}-06$ \\
\hline Non-basic N1 & $\mathrm{C} 35 \mathrm{H} 53 \mathrm{~N} 1$ & $5.18 \mathrm{E}-05$ & Basic N1 & $\mathrm{C} 30 \mathrm{H} 23 \mathrm{~N} 1$ & $1.93 \mathrm{E}-06$ & Neutral O2 & $\mathrm{C} 38 \mathrm{H} 34 \mathrm{O} 2$ & 4.41E-06 \\
\hline Non-basic N1 & $\mathrm{C} 36 \mathrm{H} 55 \mathrm{~N} 1$ & $4.00 \mathrm{E}-05$ & Basic N1 & $\mathrm{C} 31 \mathrm{H} 25 \mathrm{~N} 1$ & $1.95 \mathrm{E}-06$ & Neutral O2 & $\mathrm{C} 39 \mathrm{H} 36 \mathrm{O} 2$ & 4.04E-06 \\
\hline Non-basic N1 & $\mathrm{C} 37 \mathrm{H} 57 \mathrm{~N} 1$ & $3.32 \mathrm{E}-05$ & Basic N1 & $\mathrm{C} 32 \mathrm{H} 27 \mathrm{~N} 1$ & $2.62 \mathrm{E}-06$ & Neutral O2 & $\mathrm{C} 40 \mathrm{H} 38 \mathrm{O} 2$ & $2.00 \mathrm{E}-06$ \\
\hline Non-basic N1 & C38H59N1 & $2.21 \mathrm{E}-05$ & Basic N1 & $\mathrm{C} 33 \mathrm{H} 29 \mathrm{~N} 1$ & $3.53 \mathrm{E}-06$ & Neutral O2 & $\mathrm{C} 41 \mathrm{H} 40 \mathrm{O} 2$ & $1.15 \mathrm{E}-06$ \\
\hline Non-basic N1 & $\mathrm{C} 39 \mathrm{H} 61 \mathrm{~N} 1$ & $1.47 \mathrm{E}-05$ & Basic N1 & $\mathrm{C} 34 \mathrm{H} 31 \mathrm{~N} 1$ & $3.51 \mathrm{E}-06$ & Neutral O2 & $\mathrm{C} 31 \mathrm{H} 18 \mathrm{O} 2$ & $8.22 \mathrm{E}-07$ \\
\hline Non-basic N1 & $\mathrm{C} 40 \mathrm{H} 63 \mathrm{~N} 1$ & $6.79 \mathrm{E}-06$ & Basic N1 & $\mathrm{C} 35 \mathrm{H} 33 \mathrm{~N} 1$ & $3.17 \mathrm{E}-06$ & Neutral O2 & $\mathrm{C} 32 \mathrm{H} 20 \mathrm{O} 2$ & $1.21 \mathrm{E}-06$ \\
\hline Non-basic N1 & C41H65N1 & 4.64E-06 & Basic N1 & $\mathrm{C} 36 \mathrm{H} 35 \mathrm{~N} 1$ & $3.49 \mathrm{E}-06$ & Neutral O2 & $\mathrm{C} 33 \mathrm{H} 22 \mathrm{O} 2$ & $6.66 \mathrm{E}-06$ \\
\hline Non-basic N1 & $\mathrm{C} 42 \mathrm{H} 67 \mathrm{~N} 1$ & $4.79 \mathrm{E}-06$ & Basic N1 & $\mathrm{C} 37 \mathrm{H} 37 \mathrm{~N} 1$ & $2.61 \mathrm{E}-06$ & Neutral O2 & $\mathrm{C} 34 \mathrm{H} 24 \mathrm{O} 2$ & $9.95 \mathrm{E}-06$ \\
\hline Non-basic N1 & $\mathrm{C} 17 \mathrm{H} 15 \mathrm{~N} 1$ & $2.02 \mathrm{E}-06$ & Basic N1 & $\mathrm{C} 38 \mathrm{H} 39 \mathrm{~N} 1$ & $2.40 \mathrm{E}-06$ & Neutral O2 & $\mathrm{C} 35 \mathrm{H} 26 \mathrm{O} 2$ & $1.07 \mathrm{E}-05$ \\
\hline Non-basic N1 & $\mathrm{C} 18 \mathrm{H} 17 \mathrm{~N} 1$ & $2.39 \mathrm{E}-06$ & Basic N1 & $\mathrm{C} 39 \mathrm{H} 41 \mathrm{~N} 1$ & $2.22 \mathrm{E}-06$ & Neutral O2 & $\mathrm{C} 36 \mathrm{H} 28 \mathrm{O} 2$ & $1.34 \mathrm{E}-05$ \\
\hline Non-basic N1 & C19H19N1 & $6.29 \mathrm{E}-06$ & Basic N1 & $\mathrm{C} 40 \mathrm{H} 43 \mathrm{~N} 1$ & $2.32 \mathrm{E}-06$ & Neutral O2 & $\mathrm{C} 37 \mathrm{H} 30 \mathrm{O} 2$ & $1.15 \mathrm{E}-05$ \\
\hline Non-basic N1 & $\mathrm{C} 20 \mathrm{H} 21 \mathrm{~N} 1$ & $1.35 \mathrm{E}-05$ & Basic N1 & $\mathrm{C} 33 \mathrm{H} 27 \mathrm{~N} 1$ & $1.03 \mathrm{E}-06$ & Neutral O2 & $\mathrm{C} 38 \mathrm{H} 32 \mathrm{O} 2$ & $1.17 \mathrm{E}-05$ \\
\hline Non-basic N1 & $\mathrm{C} 21 \mathrm{H} 23 \mathrm{~N} 1$ & $2.19 \mathrm{E}-05$ & Basic N1 & $\mathrm{C} 34 \mathrm{H} 29 \mathrm{~N} 1$ & $1.38 \mathrm{E}-06$ & Neutral O2 & $\mathrm{C} 39 \mathrm{H} 34 \mathrm{O} 2$ & $4.92 \mathrm{E}-06$ \\
\hline Non-basic N1 & $\mathrm{C} 22 \mathrm{H} 25 \mathrm{~N} 1$ & $2.58 \mathrm{E}-05$ & Basic N1 & $\mathrm{C} 35 \mathrm{H} 31 \mathrm{~N} 1$ & $1.43 \mathrm{E}-06$ & Neutral O2 & $\mathrm{C} 40 \mathrm{H} 36 \mathrm{O} 2$ & $7.32 \mathrm{E}-07$ \\
\hline Non-basic N1 & $\mathrm{C} 23 \mathrm{H} 27 \mathrm{~N} 1$ & $3.82 \mathrm{E}-05$ & Basic N1 & $\mathrm{C} 36 \mathrm{H} 33 \mathrm{~N} 1$ & $1.43 \mathrm{E}-06$ & Neutral O2 & $\mathrm{C} 41 \mathrm{H} 38 \mathrm{O} 2$ & $1.08 \mathrm{E}-06$ \\
\hline Non-basic N1 & $\mathrm{C} 24 \mathrm{H} 29 \mathrm{~N} 1$ & $4.34 \mathrm{E}-05$ & Basic N1 & $\mathrm{C} 37 \mathrm{H} 35 \mathrm{~N} 1$ & $2.11 \mathrm{E}-06$ & Neutral O2 & $\mathrm{C} 34 \mathrm{H} 22 \mathrm{O} 2$ & $2.58 \mathrm{E}-06$ \\
\hline Non-basic N1 & $\mathrm{C} 25 \mathrm{H} 31 \mathrm{~N} 1$ & $5.00 \mathrm{E}-05$ & Basic N1 & $\mathrm{C} 38 \mathrm{H} 35 \mathrm{~N} 1$ & $9.17 \mathrm{E}-07$ & Neutral O2 & $\mathrm{C} 35 \mathrm{H} 24 \mathrm{O} 2$ & $4.70 \mathrm{E}-06$ \\
\hline Non-basic N1 & $\mathrm{C} 26 \mathrm{H} 33 \mathrm{~N} 1$ & $5.09 \mathrm{E}-05$ & Basic N1 & $\mathrm{C} 39 \mathrm{H} 37 \mathrm{~N} 1$ & $1.34 \mathrm{E}-06$ & Neutral O2 & $\mathrm{C} 36 \mathrm{H} 26 \mathrm{O} 2$ & 7.16E-06 \\
\hline Non-basic N1 & $\mathrm{C} 27 \mathrm{H} 35 \mathrm{~N} 1$ & 4.87E-05 & Basic N1 & $\mathrm{C} 31 \mathrm{H} 19 \mathrm{~N} 1$ & $3.05 \mathrm{E}-08$ & Neutral O2 & $\mathrm{C} 37 \mathrm{H} 28 \mathrm{O} 2$ & $3.36 \mathrm{E}-06$ \\
\hline Non-basic N1 & $\mathrm{C} 28 \mathrm{H} 37 \mathrm{~N} 1$ & $5.23 \mathrm{E}-05$ & Basic N1 & $\mathrm{C} 32 \mathrm{H} 21 \mathrm{~N} 1$ & $2.07 \mathrm{E}-08$ & Neutral O2 & $\mathrm{C} 38 \mathrm{H} 30 \mathrm{O} 2$ & $5.83 \mathrm{E}-06$ \\
\hline Non-basic N1 & $\mathrm{C} 29 \mathrm{H} 39 \mathrm{~N} 1$ & 4.40E-05 & Basic N1 & $\mathrm{C} 33 \mathrm{H} 23 \mathrm{~N} 1$ & 7.04E-08 & Neutral O2 & $\mathrm{C} 39 \mathrm{H} 32 \mathrm{O} 2$ & $1.95 \mathrm{E}-06$ \\
\hline
\end{tabular}




\begin{tabular}{|c|c|c|c|c|c|c|c|c|}
\hline Class & formula & Mass & Class & formula & Mass & Class & formula & Mass \\
\hline Non-basic N1 & $\mathrm{C} 30 \mathrm{H} 41 \mathrm{~N} 1$ & 4.24E-05 & Basic N1 & $\mathrm{C} 34 \mathrm{H} 25 \mathrm{~N} 1$ & $3.84 \mathrm{E}-07$ & Neutral O2 & $\mathrm{C} 40 \mathrm{H} 34 \mathrm{O} 2$ & $6.92 \mathrm{E}-06$ \\
\hline Non-basic N1 & $\mathrm{C} 31 \mathrm{H} 43 \mathrm{~N} 1$ & $5.09 \mathrm{E}-05$ & Basic N1 & $\mathrm{C} 35 \mathrm{H} 27 \mathrm{~N} 1$ & $1.70 \mathrm{E}-07$ & Neutral O2 & $\mathrm{C} 41 \mathrm{H} 36 \mathrm{O} 2$ & $6.30 \mathrm{E}-06$ \\
\hline Non-basic N1 & $\mathrm{C} 32 \mathrm{H} 45 \mathrm{~N} 1$ & 4.49E-05 & Basic N1 & $\mathrm{C} 39 \mathrm{H} 33 \mathrm{~N} 1$ & $6.39 \mathrm{E}-07$ & Neutral O2 & $\mathrm{C} 42 \mathrm{H} 38 \mathrm{O} 2$ & $2.62 \mathrm{E}-06$ \\
\hline Non-basic N1 & $\mathrm{C} 33 \mathrm{H} 47 \mathrm{~N} 1$ & $3.67 \mathrm{E}-05$ & Basic N1 & $\mathrm{C} 40 \mathrm{H} 35 \mathrm{~N} 1$ & $6.92 \mathrm{E}-07$ & Neutral O2 & $\mathrm{C} 43 \mathrm{H} 40 \mathrm{O} 2$ & $1.16 \mathrm{E}-06$ \\
\hline Non-basic N1 & $\mathrm{C} 34 \mathrm{H} 49 \mathrm{~N} 1$ & $2.74 \mathrm{E}-05$ & Basic N1 & $\mathrm{C} 42 \mathrm{H} 37 \mathrm{~N} 1$ & $6.70 \mathrm{E}-07$ & Neutral O2 & $\mathrm{C} 44 \mathrm{H} 42 \mathrm{O} 2$ & $2.04 \mathrm{E}-06$ \\
\hline Non-basic N1 & $\mathrm{C} 35 \mathrm{H} 51 \mathrm{~N} 1$ & $2.74 \mathrm{E}-05$ & Basic N1 & $\mathrm{C} 43 \mathrm{H} 39 \mathrm{~N} 1$ & $5.25 \mathrm{E}-07$ & Neutral O2 & $\mathrm{C} 34 \mathrm{H} 20 \mathrm{O} 2$ & $2.65 \mathrm{E}-06$ \\
\hline Non-basic N1 & $\mathrm{C} 36 \mathrm{H} 53 \mathrm{~N} 1$ & $2.31 \mathrm{E}-05$ & Basic N1 & $\mathrm{C} 36 \mathrm{H} 23 \mathrm{~N} 1$ & 4.03E-08 & Neutral O2 & $\mathrm{C} 35 \mathrm{H} 22 \mathrm{O} 2$ & $5.54 \mathrm{E}-07$ \\
\hline Non-basic N1 & $\mathrm{C} 37 \mathrm{H} 55 \mathrm{~N} 1$ & $2.30 \mathrm{E}-05$ & Basic N1 & $\mathrm{C} 37 \mathrm{H} 25 \mathrm{~N} 1$ & $7.36 \mathrm{E}-08$ & Neutral O2 & $\mathrm{C} 36 \mathrm{H} 24 \mathrm{O} 2$ & $1.10 \mathrm{E}-06$ \\
\hline Non-basic N1 & $\mathrm{C} 38 \mathrm{H} 57 \mathrm{~N} 1$ & $1.03 \mathrm{E}-05$ & Basic N1 & $\mathrm{C} 38 \mathrm{H} 27 \mathrm{~N} 1$ & $1.27 \mathrm{E}-07$ & Neutral O2 & $\mathrm{C} 37 \mathrm{H} 26 \mathrm{O} 2$ & $3.54 \mathrm{E}-06$ \\
\hline Non-basic N1 & C39H59N1 & $1.67 \mathrm{E}-06$ & Basic N1 & $\mathrm{C} 39 \mathrm{H} 29 \mathrm{~N} 1$ & $2.90 \mathrm{E}-07$ & Neutral O2 & $\mathrm{C} 38 \mathrm{H} 28 \mathrm{O} 2$ & $4.55 \mathrm{E}-06$ \\
\hline Non-basic N1 & C16H11N1 & $2.14 \mathrm{E}-06$ & Basic N1 & $\mathrm{C} 40 \mathrm{H} 31 \mathrm{~N} 1$ & $3.56 \mathrm{E}-07$ & Neutral O2 & $\mathrm{C} 39 \mathrm{H} 30 \mathrm{O} 2$ & $5.25 \mathrm{E}-06$ \\
\hline Non-basic N1 & $\mathrm{C} 17 \mathrm{H} 13 \mathrm{~N} 1$ & 0.000239 & Basic N1 & $\mathrm{C} 39 \mathrm{H} 27 \mathrm{~N} 1$ & $7.00 \mathrm{E}-08$ & Neutral O2 & $\mathrm{C} 40 \mathrm{H} 32 \mathrm{O} 2$ & 4.03E-06 \\
\hline Non-basic N1 & $\mathrm{C} 18 \mathrm{H} 15 \mathrm{~N} 1$ & 0.001001 & Basic N1 & $\mathrm{C} 40 \mathrm{H} 29 \mathrm{~N} 1$ & $1.65 \mathrm{E}-07$ & Neutral O2 & $\mathrm{C} 41 \mathrm{H} 34 \mathrm{O} 2$ & $2.87 \mathrm{E}-06$ \\
\hline Non-basic N1 & C19H17N1 & 0.001361 & Basic N1 & $\mathrm{C} 41 \mathrm{H} 31 \mathrm{~N} 1$ & $4.04 \mathrm{E}-07$ & Neutral O2 & $\mathrm{C} 42 \mathrm{H} 36 \mathrm{O} 2$ & $1.36 \mathrm{E}-06$ \\
\hline Non-basic N1 & $\mathrm{C} 20 \mathrm{H} 19 \mathrm{~N} 1$ & 0.001127 & Basic N1 & $\mathrm{C} 42 \mathrm{H} 33 \mathrm{~N} 1$ & $2.28 \mathrm{E}-07$ & Neutral O2 & $\mathrm{C} 43 \mathrm{H} 38 \mathrm{O} 2$ & $2.61 \mathrm{E}-06$ \\
\hline Non-basic N1 & $\mathrm{C} 21 \mathrm{H} 21 \mathrm{~N} 1$ & 0.00075 & Basic N1 & $\mathrm{C} 43 \mathrm{H} 35 \mathrm{~N} 1$ & $3.33 \mathrm{E}-07$ & Neutral O2 & $\mathrm{C} 44 \mathrm{H} 40 \mathrm{O} 2$ & $3.80 \mathrm{E}-06$ \\
\hline Non-basic N1 & $\mathrm{C} 22 \mathrm{H} 23 \mathrm{~N} 1$ & 0.0005 & Basic N1 & $\mathrm{C} 44 \mathrm{H} 37 \mathrm{~N} 1$ & $4.10 \mathrm{E}-07$ & Neutral O2 & $\mathrm{C} 45 \mathrm{H} 42 \mathrm{O} 2$ & $9.47 \mathrm{E}-07$ \\
\hline Non-basic N1 & $\mathrm{C} 23 \mathrm{H} 25 \mathrm{~N} 1$ & 0.000365 & Basic N1 & $\mathrm{C} 39 \mathrm{H} 25 \mathrm{~N} 1$ & $5.74 \mathrm{E}-08$ & Neutral O2 & $\mathrm{C} 46 \mathrm{H} 44 \mathrm{O} 2$ & $2.12 \mathrm{E}-06$ \\
\hline Non-basic N1 & $\mathrm{C} 24 \mathrm{H} 27 \mathrm{~N} 1$ & 0.000302 & Basic N1 & $\mathrm{C} 40 \mathrm{H} 27 \mathrm{~N} 1$ & $1.78 \mathrm{E}-07$ & Neutral O2 & $\mathrm{C} 47 \mathrm{H} 46 \mathrm{O} 2$ & $2.67 \mathrm{E}-06$ \\
\hline Non-basic N1 & $\mathrm{C} 25 \mathrm{H} 29 \mathrm{~N} 1$ & 0.000257 & Basic N1 & $\mathrm{C} 41 \mathrm{H} 29 \mathrm{~N} 1$ & $3.30 \mathrm{E}-07$ & Neutral O2 & $\mathrm{C} 48 \mathrm{H} 48 \mathrm{O} 2$ & $7.58 \mathrm{E}-07$ \\
\hline Non-basic N1 & $\mathrm{C} 26 \mathrm{H} 31 \mathrm{~N} 1$ & 0.000246 & Basic N1 & $\mathrm{C} 42 \mathrm{H} 31 \mathrm{~N} 1$ & $9.93 \mathrm{E}-08$ & Neutral O2 & $\mathrm{C} 49 \mathrm{H} 50 \mathrm{O} 2$ & $1.15 \mathrm{E}-06$ \\
\hline Non-basic N1 & $\mathrm{C} 27 \mathrm{H} 33 \mathrm{~N} 1$ & 0.000227 & Basic N1 & $\mathrm{C} 43 \mathrm{H} 33 \mathrm{~N} 1$ & $3.67 \mathrm{E}-07$ & Neutral O2 & $\mathrm{C} 37 \mathrm{H} 24 \mathrm{O} 2$ & 5.64E-07 \\
\hline Non-basic N1 & $\mathrm{C} 28 \mathrm{H} 35 \mathrm{~N} 1$ & 0.000199 & Basic N1O1 & $\mathrm{C} 30 \mathrm{H} 51 \mathrm{~N} 1 \mathrm{O} 1$ & $4.29 \mathrm{E}-08$ & Neutral O2 & $\mathrm{C} 38 \mathrm{H} 26 \mathrm{O} 2$ & $2.62 \mathrm{E}-06$ \\
\hline Non-basic N1 & $\mathrm{C} 29 \mathrm{H} 37 \mathrm{~N} 1$ & 0.000167 & Basic N1O1 & C31H53N1O1 & $2.51 \mathrm{E}-08$ & Neutral O2 & $\mathrm{C} 39 \mathrm{H} 28 \mathrm{O} 2$ & $1.54 \mathrm{E}-06$ \\
\hline Non-basic N1 & $\mathrm{C} 30 \mathrm{H} 39 \mathrm{~N} 1$ & 0.000148 & Basic N1O1 & $\mathrm{C} 32 \mathrm{H} 55 \mathrm{~N} 1 \mathrm{O} 1$ & $1.38 \mathrm{E}-08$ & Neutral O2 & $\mathrm{C} 40 \mathrm{H} 30 \mathrm{O} 2$ & $2.97 \mathrm{E}-06$ \\
\hline Non-basic N1 & $\mathrm{C} 31 \mathrm{H} 41 \mathrm{~N} 1$ & 0.000144 & Basic N1O1 & $\mathrm{C} 28 \mathrm{H} 45 \mathrm{~N} 1 \mathrm{O} 1$ & $1.33 \mathrm{E}-08$ & Neutral O2 & $\mathrm{C} 38 \mathrm{H} 24 \mathrm{O} 2$ & $1.24 \mathrm{E}-06$ \\
\hline Non-basic N1 & $\mathrm{C} 32 \mathrm{H} 43 \mathrm{~N} 1$ & 0.000123 & Basic N1O1 & $\mathrm{C} 29 \mathrm{H} 47 \mathrm{~N} 1 \mathrm{O} 1$ & $1.52 \mathrm{E}-08$ & Neutral O2 & $\mathrm{C} 39 \mathrm{H} 26 \mathrm{O} 2$ & $7.25 \mathrm{E}-07$ \\
\hline Non-basic N1 & $\mathrm{C} 33 \mathrm{H} 45 \mathrm{~N} 1$ & $9.51 \mathrm{E}-05$ & Basic N1O1 & $\mathrm{C} 30 \mathrm{H} 49 \mathrm{~N} 1 \mathrm{O} 1$ & $8.27 \mathrm{E}-08$ & Neutral O2 & $\mathrm{C} 40 \mathrm{H} 28 \mathrm{O} 2$ & $9.96 \mathrm{E}-07$ \\
\hline Non-basic N1 & $\mathrm{C} 34 \mathrm{H} 47 \mathrm{~N} 1$ & $6.84 \mathrm{E}-05$ & Basic N1O1 & $\mathrm{C} 31 \mathrm{H} 51 \mathrm{~N} 1 \mathrm{O} 1$ & $1.46 \mathrm{E}-07$ & Neutral O2 & $\mathrm{C} 41 \mathrm{H} 30 \mathrm{O} 2$ & $3.05 \mathrm{E}-06$ \\
\hline Non-basic N1 & $\mathrm{C} 35 \mathrm{H} 49 \mathrm{~N} 1$ & $6.25 \mathrm{E}-05$ & Basic N1O1 & $\mathrm{C} 32 \mathrm{H} 53 \mathrm{~N} 1 \mathrm{O} 1$ & $5.47 \mathrm{E}-08$ & Neutral O2 & $\mathrm{C} 42 \mathrm{H} 32 \mathrm{O} 2$ & $3.01 \mathrm{E}-06$ \\
\hline Non-basic N1 & $\mathrm{C} 36 \mathrm{H} 51 \mathrm{~N} 1$ & 4.36E-05 & Basic N1O1 & $\mathrm{C} 33 \mathrm{H} 55 \mathrm{~N} 1 \mathrm{O} 1$ & $6.43 \mathrm{E}-08$ & Neutral O2 & $\mathrm{C} 43 \mathrm{H} 34 \mathrm{O} 2$ & $2.62 \mathrm{E}-06$ \\
\hline Non-basic N1 & $\mathrm{C} 37 \mathrm{H} 53 \mathrm{~N} 1$ & $3.81 \mathrm{E}-05$ & Basic N1O1 & $\mathrm{C} 34 \mathrm{H} 57 \mathrm{~N} 1 \mathrm{O} 1$ & $1.63 \mathrm{E}-07$ & Neutral O2 & $\mathrm{C} 44 \mathrm{H} 36 \mathrm{O} 2$ & $3.06 \mathrm{E}-06$ \\
\hline Non-basic N1 & $\mathrm{C} 38 \mathrm{H} 55 \mathrm{~N} 1$ & $2.49 \mathrm{E}-05$ & Basic N1O1 & C35H59N1O1 & $9.21 \mathrm{E}-08$ & Neutral O2 & $\mathrm{C} 45 \mathrm{H} 38 \mathrm{O} 2$ & $6.74 \mathrm{E}-06$ \\
\hline Non-basic N1 & $\mathrm{C} 39 \mathrm{H} 57 \mathrm{~N} 1$ & $9.66 \mathrm{E}-06$ & Basic N1O1 & $\mathrm{C} 36 \mathrm{H} 61 \mathrm{~N} 1 \mathrm{O} 1$ & $1.06 \mathrm{E}-07$ & Neutral O2 & $\mathrm{C} 41 \mathrm{H} 28 \mathrm{O} 2$ & $2.50 \mathrm{E}-06$ \\
\hline Non-basic N1 & $\mathrm{C} 40 \mathrm{H} 59 \mathrm{~N} 1$ & $1.46 \mathrm{E}-05$ & Basic N1O1 & C37H63N1O1 & $1.03 \mathrm{E}-07$ & Neutral O2 & $\mathrm{C} 42 \mathrm{H} 30 \mathrm{O} 2$ & $2.44 \mathrm{E}-06$ \\
\hline Non-basic N1 & $\mathrm{C} 18 \mathrm{H} 13 \mathrm{~N} 1$ & $3.57 \mathrm{E}-06$ & Basic N1O1 & $\mathrm{C} 39 \mathrm{H} 67 \mathrm{~N} 1 \mathrm{O} 1$ & 4.79E-08 & Neutral O2 & $\mathrm{C} 43 \mathrm{H} 32 \mathrm{O} 2$ & 4.95E-06 \\
\hline Non-basic N1 & $\mathrm{C} 19 \mathrm{H} 15 \mathrm{~N} 1$ & $7.21 \mathrm{E}-05$ & Basic N1O1 & $\mathrm{C} 40 \mathrm{H} 69 \mathrm{~N} 1 \mathrm{O} 1$ & $1.78 \mathrm{E}-08$ & Neutral O2 & $\mathrm{C} 44 \mathrm{H} 34 \mathrm{O} 2$ & 4.94E-06 \\
\hline Non-basic N1 & $\mathrm{C} 20 \mathrm{H} 17 \mathrm{~N} 1$ & 0.000289 & Basic N1O1 & $\mathrm{C} 14 \mathrm{H} 15 \mathrm{~N} 1 \mathrm{O} 1$ & $1.49 \mathrm{E}-07$ & Neutral O2 & $\mathrm{C} 42 \mathrm{H} 28 \mathrm{O} 2$ & $1.63 \mathrm{E}-06$ \\
\hline
\end{tabular}




\begin{tabular}{|c|c|c|c|c|c|c|c|c|}
\hline Class & formula & Mass & Class & formula & Mass & Class & formula & Mass \\
\hline Non-basic N1 & $\mathrm{C} 21 \mathrm{H} 19 \mathrm{~N} 1$ & 0.000464 & Basic N1O1 & $\mathrm{C} 15 \mathrm{H} 17 \mathrm{~N} 1 \mathrm{O} 1$ & $1.23 \mathrm{E}-05$ & Neutral O2 & $\mathrm{C} 43 \mathrm{H} 30 \mathrm{O} 2$ & $2.64 \mathrm{E}-06$ \\
\hline Non-basic N1 & $\mathrm{C} 22 \mathrm{H} 21 \mathrm{~N} 1$ & 0.000474 & Basic N1O1 & C16H19N1O1 & $1.09 \mathrm{E}-06$ & Neutral O2 & $\mathrm{C} 44 \mathrm{H} 32 \mathrm{O} 2$ & $2.15 \mathrm{E}-06$ \\
\hline Non-basic N1 & $\mathrm{C} 23 \mathrm{H} 23 \mathrm{~N} 1$ & 0.000436 & Basic N1O1 & $\mathrm{C} 17 \mathrm{H} 21 \mathrm{~N} 1 \mathrm{O} 1$ & $4.24 \mathrm{E}-08$ & Neutral O2 & $\mathrm{C} 45 \mathrm{H} 34 \mathrm{O} 2$ & $4.40 \mathrm{E}-06$ \\
\hline Non-basic N1 & $\mathrm{C} 24 \mathrm{H} 25 \mathrm{~N} 1$ & 0.000367 & Basic N1O1 & $\mathrm{C} 18 \mathrm{H} 23 \mathrm{~N} 1 \mathrm{O} 1$ & $4.34 \mathrm{E}-08$ & Neutral O2 & $\mathrm{C} 46 \mathrm{H} 36 \mathrm{O} 2$ & $6.82 \mathrm{E}-06$ \\
\hline Non-basic N1 & $\mathrm{C} 25 \mathrm{H} 27 \mathrm{~N} 1$ & 0.000256 & Basic N1O1 & $\mathrm{C} 19 \mathrm{H} 25 \mathrm{~N} 1 \mathrm{O} 1$ & $2.62 \mathrm{E}-07$ & Neutral O2 & $\mathrm{C} 47 \mathrm{H} 38 \mathrm{O} 2$ & 4.97E-06 \\
\hline Non-basic N1 & $\mathrm{C} 26 \mathrm{H} 29 \mathrm{~N} 1$ & 0.000233 & Basic N1O1 & $\mathrm{C} 20 \mathrm{H} 27 \mathrm{~N} 1 \mathrm{O} 1$ & $2.08 \mathrm{E}-07$ & Neutral O2 & $\mathrm{C} 48 \mathrm{H} 40 \mathrm{O} 2$ & $9.75 \mathrm{E}-07$ \\
\hline Non-basic N1 & $\mathrm{C} 27 \mathrm{H} 31 \mathrm{~N} 1$ & 0.000183 & Basic N1O1 & $\mathrm{C} 21 \mathrm{H} 29 \mathrm{~N} 1 \mathrm{O} 1$ & $1.93 \mathrm{E}-07$ & Neutral O2 & $\mathrm{C} 43 \mathrm{H} 28 \mathrm{O} 2$ & $8.08 \mathrm{E}-07$ \\
\hline Non-basic N1 & $\mathrm{C} 28 \mathrm{H} 33 \mathrm{~N} 1$ & 0.000151 & Basic N1O1 & $\mathrm{C} 22 \mathrm{H} 31 \mathrm{~N} 1 \mathrm{O} 1$ & $1.45 \mathrm{E}-05$ & Neutral O2 & $\mathrm{C} 44 \mathrm{H} 30 \mathrm{O} 2$ & $1.14 \mathrm{E}-06$ \\
\hline Non-basic N1 & $\mathrm{C} 29 \mathrm{H} 35 \mathrm{~N} 1$ & 0.000113 & Basic N1O1 & $\mathrm{C} 23 \mathrm{H} 33 \mathrm{~N} 1 \mathrm{O} 1$ & $2.51 \mathrm{E}-07$ & Neutral O2 & $\mathrm{C} 45 \mathrm{H} 32 \mathrm{O} 2$ & $1.45 \mathrm{E}-06$ \\
\hline Non-basic N1 & $\mathrm{C} 30 \mathrm{H} 37 \mathrm{~N} 1$ & $9.84 \mathrm{E}-05$ & Basic N1O1 & $\mathrm{C} 24 \mathrm{H} 35 \mathrm{~N} 1 \mathrm{O} 1$ & $7.09 \mathrm{E}-08$ & Neutral O2 & $\mathrm{C} 46 \mathrm{H} 34 \mathrm{O} 2$ & $2.54 \mathrm{E}-06$ \\
\hline Non-basic N1 & $\mathrm{C} 31 \mathrm{H} 39 \mathrm{~N} 1$ & 8.44E-05 & Basic N1O1 & $\mathrm{C} 25 \mathrm{H} 37 \mathrm{~N} 1 \mathrm{O} 1$ & $1.21 \mathrm{E}-07$ & Neutral O2 & $\mathrm{C} 47 \mathrm{H} 36 \mathrm{O} 2$ & $2.11 \mathrm{E}-06$ \\
\hline Non-basic N1 & $\mathrm{C} 32 \mathrm{H} 41 \mathrm{~N} 1$ & 7.34E-05 & Basic N1O1 & $\mathrm{C} 26 \mathrm{H} 39 \mathrm{~N} 1 \mathrm{O} 1$ & $5.66 \mathrm{E}-08$ & Neutral O2 & $\mathrm{C} 48 \mathrm{H} 38 \mathrm{O} 2$ & $1.75 \mathrm{E}-06$ \\
\hline Non-basic N1 & $\mathrm{C} 33 \mathrm{H} 43 \mathrm{~N} 1$ & $7.48 \mathrm{E}-05$ & Basic N1O1 & $\mathrm{C} 27 \mathrm{H} 41 \mathrm{~N} 1 \mathrm{O} 1$ & $8.11 \mathrm{E}-08$ & & & \\
\hline
\end{tabular}

Table S3 Contents of various molecules of resins

\begin{tabular}{|c|c|c|c|c|c|c|c|c|}
\hline Class & Formula & Mass & Class & Formula & Mass & Class & Formula & Mass \\
\hline $\mathrm{CH}$ & $\mathrm{C} 15 \mathrm{H} 24$ & $5.40 \mathrm{E}-05$ & Non-basic N1O2 & $\mathrm{C} 41 \mathrm{H} 43 \mathrm{~N} 1 \mathrm{O} 2$ & $7.30 \mathrm{E}-06$ & Basic N1 & $\mathrm{C} 24 \mathrm{H} 25 \mathrm{~N} 1$ & 0.00039 \\
\hline $\mathrm{CH}$ & $\mathrm{C} 16 \mathrm{H} 26$ & 0.000619 & Non-basic N1O2 & $\mathrm{C} 42 \mathrm{H} 45 \mathrm{~N} 1 \mathrm{O} 2$ & $5.44 \mathrm{E}-06$ & Basic N1 & $\mathrm{C} 25 \mathrm{H} 27 \mathrm{~N} 1$ & 0.000516 \\
\hline $\mathrm{CH}$ & $\mathrm{C} 17 \mathrm{H} 28$ & 0.003833 & Non-basic N1O2 & $\mathrm{C} 43 \mathrm{H} 47 \mathrm{~N} 1 \mathrm{O} 2$ & $5.57 \mathrm{E}-06$ & Basic N1 & $\mathrm{C} 26 \mathrm{H} 29 \mathrm{~N} 1$ & 0.000461 \\
\hline $\mathrm{CH}$ & $\mathrm{C} 18 \mathrm{H} 30$ & 0.004739 & Non-basic N1O2 & $\mathrm{C} 44 \mathrm{H} 49 \mathrm{~N} 1 \mathrm{O} 2$ & $4.66 \mathrm{E}-06$ & Basic N1 & $\mathrm{C} 27 \mathrm{H} 31 \mathrm{~N} 1$ & 0.000554 \\
\hline $\mathrm{CH}$ & $\mathrm{C} 19 \mathrm{H} 32$ & 0.004012 & Non-basic N1O2 & $\mathrm{C} 45 \mathrm{H} 51 \mathrm{~N} 1 \mathrm{O} 2$ & $5.78 \mathrm{E}-06$ & Basic N1 & $\mathrm{C} 28 \mathrm{H} 33 \mathrm{~N} 1$ & 0.000497 \\
\hline $\mathrm{CH}$ & $\mathrm{C} 20 \mathrm{H} 34$ & 0.004259 & Non-basic N1O2 & $\mathrm{C} 46 \mathrm{H} 53 \mathrm{~N} 1 \mathrm{O} 2$ & $9.88 \mathrm{E}-06$ & Basic N1 & $\mathrm{C} 29 \mathrm{H} 35 \mathrm{~N} 1$ & 0.000454 \\
\hline $\mathrm{CH}$ & $\mathrm{C} 21 \mathrm{H} 36$ & 0.003711 & Non-basic N1O2 & $\mathrm{C} 47 \mathrm{H} 55 \mathrm{~N} 1 \mathrm{O} 2$ & $1.59 \mathrm{E}-05$ & Basic N1 & $\mathrm{C} 30 \mathrm{H} 37 \mathrm{~N} 1$ & 0.000453 \\
\hline $\mathrm{CH}$ & C22H38 & 0.002772 & Non-basic N1O2 & $\mathrm{C} 48 \mathrm{H} 57 \mathrm{~N} 1 \mathrm{O} 2$ & $6.41 \mathrm{E}-06$ & Basic N1 & C31H39N1 & 0.000458 \\
\hline $\mathrm{CH}$ & $\mathrm{C} 23 \mathrm{H} 40$ & 0.001986 & Non-basic N1O2 & $\mathrm{C} 49 \mathrm{H} 59 \mathrm{~N} 1 \mathrm{O} 2$ & $6.70 \mathrm{E}-06$ & Basic N1 & $\mathrm{C} 32 \mathrm{H} 41 \mathrm{~N} 1$ & 0.00038 \\
\hline $\mathrm{CH}$ & $\mathrm{C} 24 \mathrm{H} 42$ & 0.001119 & Non-basic N1O2 & C50H61N1O2 & $6.84 \mathrm{E}-06$ & Basic N1 & $\mathrm{C} 33 \mathrm{H} 43 \mathrm{~N} 1$ & 0.000377 \\
\hline $\mathrm{CH}$ & $\mathrm{C} 25 \mathrm{H} 44$ & 0.000973 & Non-basic $\mathrm{N} 1 \mathrm{O} 2$ & $\mathrm{C} 51 \mathrm{H} 63 \mathrm{~N} 1 \mathrm{O} 2$ & $1.04 \mathrm{E}-05$ & Basic N1 & $\mathrm{C} 34 \mathrm{H} 45 \mathrm{~N} 1$ & 0.000304 \\
\hline $\mathrm{CH}$ & $\mathrm{C} 26 \mathrm{H} 46$ & 0.000343 & Non-basic $\mathrm{N} 1 \mathrm{O} 2$ & $\mathrm{C} 52 \mathrm{H} 65 \mathrm{~N} 1 \mathrm{O} 2$ & $8.33 \mathrm{E}-06$ & Basic N1 & $\mathrm{C} 35 \mathrm{H} 47 \mathrm{~N} 1$ & 0.00033 \\
\hline $\mathrm{CH}$ & $\mathrm{C} 27 \mathrm{H} 48$ & 0.000253 & Non-basic N1O2 & $\mathrm{C} 53 \mathrm{H} 67 \mathrm{~N} 1 \mathrm{O} 2$ & $4.16 \mathrm{E}-06$ & Basic N1 & $\mathrm{C} 36 \mathrm{H} 49 \mathrm{~N} 1$ & 0.000241 \\
\hline $\mathrm{CH}$ & $\mathrm{C} 28 \mathrm{H} 50$ & $6.32 \mathrm{E}-05$ & Non-basic N1O2 & $\mathrm{C} 28 \mathrm{H} 15 \mathrm{~N} 1 \mathrm{O} 2$ & $1.44 \mathrm{E}-06$ & Basic N1 & $\mathrm{C} 37 \mathrm{H} 51 \mathrm{~N} 1$ & 0.000236 \\
\hline $\mathrm{CH}$ & $\mathrm{C} 15 \mathrm{H} 22$ & 0.000472 & Non-basic N1O2 & $\mathrm{C} 29 \mathrm{H} 17 \mathrm{~N} 1 \mathrm{O} 2$ & $1.39 \mathrm{E}-05$ & Basic N1 & $\mathrm{C} 38 \mathrm{H} 53 \mathrm{~N} 1$ & 0.000175 \\
\hline $\mathrm{CH}$ & $\mathrm{C} 16 \mathrm{H} 24$ & 0.000573 & Non-basic N1O2 & $\mathrm{C} 30 \mathrm{H} 19 \mathrm{~N} 1 \mathrm{O} 2$ & $2.18 \mathrm{E}-05$ & Basic N1 & $\mathrm{C} 39 \mathrm{H} 55 \mathrm{~N} 1$ & 0.000179 \\
\hline $\mathrm{CH}$ & $\mathrm{C} 17 \mathrm{H} 26$ & 0.016309 & Non-basic N1O2 & $\mathrm{C} 31 \mathrm{H} 21 \mathrm{~N} 1 \mathrm{O} 2$ & $3.89 \mathrm{E}-05$ & Basic N1 & $\mathrm{C} 40 \mathrm{H} 57 \mathrm{~N} 1$ & 0.000125 \\
\hline $\mathrm{CH}$ & $\mathrm{C} 18 \mathrm{H} 28$ & 0.007213 & Non-basic N1O2 & $\mathrm{C} 32 \mathrm{H} 23 \mathrm{~N} 1 \mathrm{O} 2$ & $4.68 \mathrm{E}-05$ & Basic N1 & $\mathrm{C} 41 \mathrm{H} 59 \mathrm{~N} 1$ & 0.00011 \\
\hline $\mathrm{CH}$ & $\mathrm{C} 19 \mathrm{H} 30$ & 0.007782 & Non-basic N1O2 & $\mathrm{C} 33 \mathrm{H} 25 \mathrm{~N} 1 \mathrm{O} 2$ & $5.27 \mathrm{E}-05$ & Basic N1 & $\mathrm{C} 42 \mathrm{H} 61 \mathrm{~N} 1$ & $8.03 \mathrm{E}-05$ \\
\hline $\mathrm{CH}$ & $\mathrm{C} 20 \mathrm{H} 32$ & 0.011666 & Non-basic N1O2 & $\mathrm{C} 34 \mathrm{H} 27 \mathrm{~N} 1 \mathrm{O} 2$ & 4.91E-05 & Basic N1 & $\mathrm{C} 43 \mathrm{H} 63 \mathrm{~N} 1$ & $4.83 \mathrm{E}-05$ \\
\hline $\mathrm{CH}$ & $\mathrm{C} 21 \mathrm{H} 34$ & 0.007353 & Non-basic $\mathrm{N} 1 \mathrm{O} 2$ & $\mathrm{C} 35 \mathrm{H} 29 \mathrm{~N} 1 \mathrm{O} 2$ & $3.21 \mathrm{E}-05$ & Basic N1 & $\mathrm{C} 44 \mathrm{H} 65 \mathrm{~N} 1$ & 4.59E- 05 \\
\hline
\end{tabular}




\begin{tabular}{|c|c|c|c|c|c|c|c|c|}
\hline Class & Formula & Mass & Class & Formula & Mass & Class & Formula & Mass \\
\hline $\mathrm{CH}$ & $\mathrm{C} 22 \mathrm{H} 36$ & 0.00556 & Non-basic N1O2 & $\mathrm{C} 36 \mathrm{H} 31 \mathrm{~N} 1 \mathrm{O} 2$ & $2.07 \mathrm{E}-05$ & Basic N1 & $\mathrm{C} 45 \mathrm{H} 67 \mathrm{~N} 1$ & $2.43 \mathrm{E}-05$ \\
\hline $\mathrm{CH}$ & $\mathrm{C} 23 \mathrm{H} 38$ & 0.003336 & Non-basic N1O2 & $\mathrm{C} 37 \mathrm{H} 33 \mathrm{~N} 1 \mathrm{O} 2$ & $1.37 \mathrm{E}-05$ & Basic N1 & $\mathrm{C} 46 \mathrm{H} 69 \mathrm{~N} 1$ & $2.04 \mathrm{E}-05$ \\
\hline $\mathrm{CH}$ & $\mathrm{C} 24 \mathrm{H} 40$ & 0.002057 & Non-basic N1O2 & $\mathrm{C} 38 \mathrm{H} 35 \mathrm{~N} 1 \mathrm{O} 2$ & $1.41 \mathrm{E}-05$ & Basic N1 & $\mathrm{C} 48 \mathrm{H} 73 \mathrm{~N} 1$ & $6.19 \mathrm{E}-06$ \\
\hline $\mathrm{CH}$ & $\mathrm{C} 25 \mathrm{H} 42$ & 0.001175 & Non-basic $\mathrm{N} 1 \mathrm{O} 2$ & $\mathrm{C} 39 \mathrm{H} 37 \mathrm{~N} 1 \mathrm{O} 2$ & $3.46 \mathrm{E}-06$ & Basic N1 & $\mathrm{C} 49 \mathrm{H} 75 \mathrm{~N} 1$ & $8.49 \mathrm{E}-06$ \\
\hline $\mathrm{CH}$ & $\mathrm{C} 26 \mathrm{H} 44$ & 0.000794 & Non-basic N1O2 & $\mathrm{C} 40 \mathrm{H} 39 \mathrm{~N} 1 \mathrm{O} 2$ & $6.53 \mathrm{E}-06$ & Basic N1 & $\mathrm{C} 50 \mathrm{H} 77 \mathrm{~N} 1$ & $8.66 \mathrm{E}-06$ \\
\hline $\mathrm{CH}$ & $\mathrm{C} 27 \mathrm{H} 46$ & 0.000914 & Non-basic N1O2 & $\mathrm{C} 41 \mathrm{H} 41 \mathrm{~N} 1 \mathrm{O} 2$ & $6.55 \mathrm{E}-06$ & Basic N1 & $\mathrm{C} 51 \mathrm{H} 79 \mathrm{~N} 1$ & $1.08 \mathrm{E}-05$ \\
\hline $\mathrm{CH}$ & $\mathrm{C} 28 \mathrm{H} 48$ & 0.000185 & Non-basic N1O2 & $\mathrm{C} 43 \mathrm{H} 45 \mathrm{~N} 1 \mathrm{O} 2$ & $3.66 \mathrm{E}-06$ & Basic N1 & $\mathrm{C} 52 \mathrm{H} 81 \mathrm{~N} 1$ & $2.80 \mathrm{E}-06$ \\
\hline $\mathrm{CH}$ & $\mathrm{C} 29 \mathrm{H} 50$ & 0.000179 & Non-basic N1O2 & $\mathrm{C} 45 \mathrm{H} 49 \mathrm{~N} 1 \mathrm{O} 2$ & $4.01 \mathrm{E}-06$ & Basic N1 & $\mathrm{C} 53 \mathrm{H} 83 \mathrm{~N} 1$ & $3.79 \mathrm{E}-06$ \\
\hline $\mathrm{CH}$ & $\mathrm{C} 15 \mathrm{H} 20$ & 0.000198 & Non-basic N1O2 & $\mathrm{C} 46 \mathrm{H} 51 \mathrm{~N} 1 \mathrm{O} 2$ & $1.94 \mathrm{E}-06$ & Basic N1 & $\mathrm{C} 54 \mathrm{H} 85 \mathrm{~N} 1$ & $2.02 \mathrm{E}-06$ \\
\hline $\mathrm{CH}$ & $\mathrm{C} 16 \mathrm{H} 22$ & 0.000361 & Non-basic N1O2 & $\mathrm{C} 47 \mathrm{H} 53 \mathrm{~N} 1 \mathrm{O} 2$ & $3.41 \mathrm{E}-06$ & Basic N1 & $\mathrm{C} 55 \mathrm{H} 87 \mathrm{~N} 1$ & $2.11 \mathrm{E}-06$ \\
\hline $\mathrm{CH}$ & $\mathrm{C} 17 \mathrm{H} 24$ & 0.005276 & Non-basic N1O2 & $\mathrm{C} 48 \mathrm{H} 55 \mathrm{~N} 1 \mathrm{O} 2$ & $2.05 \mathrm{E}-06$ & Basic N1 & C19H13N1 & $3.86 \mathrm{E}-07$ \\
\hline $\mathrm{CH}$ & $\mathrm{C} 18 \mathrm{H} 26$ & 0.006526 & Non-basic $\mathrm{N} 1 \mathrm{O} 2$ & $\mathrm{C} 29 \mathrm{H} 15 \mathrm{~N} 1 \mathrm{O} 2$ & $1.22 \mathrm{E}-06$ & Basic N1 & $\mathrm{C} 20 \mathrm{H} 15 \mathrm{~N} 1$ & 7.47E-06 \\
\hline $\mathrm{CH}$ & $\mathrm{C} 19 \mathrm{H} 28$ & 0.00921 & Non-basic $\mathrm{N} 1 \mathrm{O} 2$ & $\mathrm{C} 30 \mathrm{H} 17 \mathrm{~N} 1 \mathrm{O} 2$ & $1.95 \mathrm{E}-06$ & Basic N1 & $\mathrm{C} 21 \mathrm{H} 17 \mathrm{~N} 1$ & $1.94 \mathrm{E}-05$ \\
\hline $\mathrm{CH}$ & $\mathrm{C} 20 \mathrm{H} 30$ & 0.011626 & Non-basic $\mathrm{N} 1 \mathrm{O} 2$ & $\mathrm{C} 31 \mathrm{H} 19 \mathrm{~N} 1 \mathrm{O} 2$ & $6.98 \mathrm{E}-06$ & Basic N1 & $\mathrm{C} 22 \mathrm{H} 19 \mathrm{~N} 1$ & $5.15 \mathrm{E}-05$ \\
\hline $\mathrm{CH}$ & $\mathrm{C} 21 \mathrm{H} 32$ & 0.008686 & Non-basic N1O2 & $\mathrm{C} 32 \mathrm{H} 21 \mathrm{~N} 1 \mathrm{O} 2$ & $1.81 \mathrm{E}-05$ & Basic N1 & $\mathrm{C} 23 \mathrm{H} 21 \mathrm{~N} 1$ & 0.000118 \\
\hline $\mathrm{CH}$ & $\mathrm{C} 22 \mathrm{H} 34$ & 0.006668 & Non-basic N1O2 & $\mathrm{C} 33 \mathrm{H} 23 \mathrm{~N} 1 \mathrm{O} 2$ & $2.30 \mathrm{E}-05$ & Basic N1 & $\mathrm{C} 24 \mathrm{H} 23 \mathrm{~N} 1$ & 0.000217 \\
\hline $\mathrm{CH}$ & $\mathrm{C} 23 \mathrm{H} 36$ & 0.003666 & Non-basic N1O2 & $\mathrm{C} 34 \mathrm{H} 25 \mathrm{~N} 1 \mathrm{O} 2$ & $1.61 \mathrm{E}-05$ & Basic N1 & $\mathrm{C} 25 \mathrm{H} 25 \mathrm{~N} 1$ & 0.00029 \\
\hline $\mathrm{CH}$ & $\mathrm{C} 24 \mathrm{H} 38$ & 0.001954 & Non-basic N1O2 & $\mathrm{C} 35 \mathrm{H} 27 \mathrm{~N} 1 \mathrm{O} 2$ & $2.46 \mathrm{E}-05$ & Basic N1 & $\mathrm{C} 26 \mathrm{H} 27 \mathrm{~N} 1$ & 0.000315 \\
\hline $\mathrm{CH}$ & $\mathrm{C} 25 \mathrm{H} 40$ & 0.001439 & Non-basic N1O2 & $\mathrm{C} 36 \mathrm{H} 29 \mathrm{~N} 1 \mathrm{O} 2$ & $3.68 \mathrm{E}-05$ & Basic N1 & $\mathrm{C} 27 \mathrm{H} 29 \mathrm{~N} 1$ & 0.000324 \\
\hline $\mathrm{CH}$ & $\mathrm{C} 26 \mathrm{H} 42$ & 0.00112 & Non-basic N1O2 & $\mathrm{C} 37 \mathrm{H} 31 \mathrm{~N} 1 \mathrm{O} 2$ & $2.67 \mathrm{E}-05$ & Basic N1 & $\mathrm{C} 28 \mathrm{H} 31 \mathrm{~N} 1$ & 0.000378 \\
\hline $\mathrm{CH}$ & $\mathrm{C} 27 \mathrm{H} 44$ & 0.002988 & Non-basic N1O2 & $\mathrm{C} 38 \mathrm{H} 33 \mathrm{~N} 1 \mathrm{O} 2$ & $1.58 \mathrm{E}-05$ & Basic N1 & $\mathrm{C} 29 \mathrm{H} 33 \mathrm{~N} 1$ & 0.000358 \\
\hline $\mathrm{CH}$ & $\mathrm{C} 28 \mathrm{H} 46$ & 0.0007 & Non-basic N1O2 & $\mathrm{C} 39 \mathrm{H} 35 \mathrm{~N} 1 \mathrm{O} 2$ & $1.84 \mathrm{E}-05$ & Basic N1 & $\mathrm{C} 30 \mathrm{H} 35 \mathrm{~N} 1$ & 0.000364 \\
\hline $\mathrm{CH}$ & $\mathrm{C} 29 \mathrm{H} 48$ & 0.000966 & Non-basic N1O2 & $\mathrm{C} 40 \mathrm{H} 37 \mathrm{~N} 1 \mathrm{O} 2$ & 4.92E-06 & Basic N1 & $\mathrm{C} 31 \mathrm{H} 37 \mathrm{~N} 1$ & 0.000313 \\
\hline $\mathrm{CH}$ & $\mathrm{C} 30 \mathrm{H} 50$ & 0.000471 & Non-basic $\mathrm{N} 1 \mathrm{O} 2$ & $\mathrm{C} 41 \mathrm{H} 39 \mathrm{~N} 1 \mathrm{O} 2$ & 8.37E-06 & Basic N1 & $\mathrm{C} 32 \mathrm{H} 39 \mathrm{~N} 1$ & 0.000279 \\
\hline $\mathrm{CH}$ & $\mathrm{C} 16 \mathrm{H} 20$ & 0.000203 & Non-basic N1O2 & $\mathrm{C} 42 \mathrm{H} 41 \mathrm{~N} 1 \mathrm{O} 2$ & 8.57E-06 & Basic N1 & $\mathrm{C} 33 \mathrm{H} 41 \mathrm{~N} 1$ & 0.000254 \\
\hline $\mathrm{CH}$ & $\mathrm{C} 17 \mathrm{H} 22$ & 0.002246 & Non-basic N1O2 & $\mathrm{C} 43 \mathrm{H} 43 \mathrm{~N} 1 \mathrm{O} 2$ & $8.78 \mathrm{E}-06$ & Basic N1 & $\mathrm{C} 34 \mathrm{H} 43 \mathrm{~N} 1$ & 0.000232 \\
\hline $\mathrm{CH}$ & $\mathrm{C} 18 \mathrm{H} 24$ & 0.004233 & Non-basic N1O2 & $\mathrm{C} 44 \mathrm{H} 45 \mathrm{~N} 1 \mathrm{O} 2$ & $8.98 \mathrm{E}-06$ & Basic N1 & $\mathrm{C} 35 \mathrm{H} 45 \mathrm{~N} 1$ & 0.000207 \\
\hline $\mathrm{CH}$ & $\mathrm{C} 19 \mathrm{H} 26$ & 0.00609 & Non-basic N1O2 & $\mathrm{C} 45 \mathrm{H} 47 \mathrm{~N} 1 \mathrm{O} 2$ & $1.89 \mathrm{E}-06$ & Basic N1 & $\mathrm{C} 36 \mathrm{H} 47 \mathrm{~N} 1$ & 0.000153 \\
\hline $\mathrm{CH}$ & $\mathrm{C} 20 \mathrm{H} 28$ & 0.006104 & Non-basic N1O2 & $\mathrm{C} 46 \mathrm{H} 49 \mathrm{~N} 1 \mathrm{O} 2$ & $7.80 \mathrm{E}-06$ & Basic N1 & $\mathrm{C} 37 \mathrm{H} 49 \mathrm{~N} 1$ & 0.00018 \\
\hline $\mathrm{CH}$ & $\mathrm{C} 21 \mathrm{H} 30$ & 0.004689 & Non-basic N1O2 & $\mathrm{C} 47 \mathrm{H} 51 \mathrm{~N} 1 \mathrm{O} 2$ & $1.77 \mathrm{E}-06$ & Basic N1 & $\mathrm{C} 38 \mathrm{H} 51 \mathrm{~N} 1$ & 0.000202 \\
\hline $\mathrm{CH}$ & $\mathrm{C} 22 \mathrm{H} 32$ & 0.003635 & Non-basic N1O2 & $\mathrm{C} 48 \mathrm{H} 53 \mathrm{~N} 1 \mathrm{O} 2$ & $4.50 \mathrm{E}-06$ & Basic N1 & $\mathrm{C} 39 \mathrm{H} 53 \mathrm{~N} 1$ & 0.000102 \\
\hline $\mathrm{CH}$ & $\mathrm{C} 23 \mathrm{H} 34$ & 0.002268 & Non-basic N1O2 & $\mathrm{C} 31 \mathrm{H} 17 \mathrm{~N} 1 \mathrm{O} 2$ & $1.26 \mathrm{E}-06$ & Basic N1 & $\mathrm{C} 40 \mathrm{H} 55 \mathrm{~N} 1$ & $9.30 \mathrm{E}-05$ \\
\hline $\mathrm{CH}$ & $\mathrm{C} 24 \mathrm{H} 36$ & 0.001635 & Non-basic N1O2 & $\mathrm{C} 32 \mathrm{H} 19 \mathrm{~N} 1 \mathrm{O} 2$ & $7.21 \mathrm{E}-06$ & Basic N1 & $\mathrm{C} 41 \mathrm{H} 57 \mathrm{~N} 1$ & 7.66E- 05 \\
\hline $\mathrm{CH}$ & $\mathrm{C} 25 \mathrm{H} 38$ & 0.001185 & Non-basic N1O2 & $\mathrm{C} 33 \mathrm{H} 21 \mathrm{~N} 1 \mathrm{O} 2$ & 7.74E-06 & Basic N1 & $\mathrm{C} 42 \mathrm{H} 59 \mathrm{~N} 1$ & $5.20 \mathrm{E}-05$ \\
\hline $\mathrm{CH}$ & $\mathrm{C} 26 \mathrm{H} 40$ & 0.000837 & Non-basic N1O2 & $\mathrm{C} 34 \mathrm{H} 23 \mathrm{~N} 1 \mathrm{O} 2$ & $1.37 \mathrm{E}-05$ & Basic N1 & $\mathrm{C} 43 \mathrm{H} 61 \mathrm{~N} 1$ & $3.16 \mathrm{E}-05$ \\
\hline $\mathrm{CH}$ & $\mathrm{C} 27 \mathrm{H} 42$ & 0.000989 & Non-basic N1O2 & $\mathrm{C} 35 \mathrm{H} 25 \mathrm{~N} 1 \mathrm{O} 2$ & $1.10 \mathrm{E}-05$ & Basic N1 & $\mathrm{C} 44 \mathrm{H} 63 \mathrm{~N} 1$ & $2.92 \mathrm{E}-05$ \\
\hline $\mathrm{CH}$ & $\mathrm{C} 28 \mathrm{H} 44$ & 0.000344 & Non-basic N1O2 & $\mathrm{C} 36 \mathrm{H} 27 \mathrm{~N} 1 \mathrm{O} 2$ & $1.91 \mathrm{E}-05$ & Basic N1 & $\mathrm{C} 45 \mathrm{H} 65 \mathrm{~N} 1$ & $2.22 \mathrm{E}-05$ \\
\hline $\mathrm{CH}$ & $\mathrm{C} 29 \mathrm{H} 46$ & 0.00064 & Non-basic N1O2 & $\mathrm{C} 37 \mathrm{H} 29 \mathrm{~N} 1 \mathrm{O} 2$ & $2.12 \mathrm{E}-05$ & Basic N1 & $\mathrm{C} 46 \mathrm{H} 67 \mathrm{~N} 1$ & $1.49 \mathrm{E}-05$ \\
\hline
\end{tabular}




\begin{tabular}{|c|c|c|c|c|c|c|c|c|}
\hline Class & Formula & Mass & Class & Formula & Mass & Class & Formula & Mass \\
\hline $\mathrm{CH}$ & $\mathrm{C} 30 \mathrm{H} 48$ & 0.000836 & Non-basic N1O2 & $\mathrm{C} 38 \mathrm{H} 31 \mathrm{~N} 1 \mathrm{O} 2$ & $2.10 \mathrm{E}-05$ & Basic N1 & C47H69N1 & $8.87 \mathrm{E}-06$ \\
\hline $\mathrm{CH}$ & $\mathrm{C} 31 \mathrm{H} 50$ & $9.33 \mathrm{E}-05$ & Non-basic N1O2 & $\mathrm{C} 39 \mathrm{H} 33 \mathrm{~N} 1 \mathrm{O} 2$ & $1.36 \mathrm{E}-05$ & Basic N1 & $\mathrm{C} 48 \mathrm{H} 71 \mathrm{~N} 1$ & $1.21 \mathrm{E}-05$ \\
\hline $\mathrm{CH}$ & $\mathrm{C} 32 \mathrm{H} 52$ & $5.21 \mathrm{E}-05$ & Non-basic N1O2 & $\mathrm{C} 40 \mathrm{H} 35 \mathrm{~N} 1 \mathrm{O} 2$ & $2.03 \mathrm{E}-05$ & Basic N1 & $\mathrm{C} 49 \mathrm{H} 73 \mathrm{~N} 1$ & $1.85 \mathrm{E}-06$ \\
\hline $\mathrm{CH}$ & C16H18 & $5.01 \mathrm{E}-05$ & Non-basic $\mathrm{N} 1 \mathrm{O} 2$ & $\mathrm{C} 41 \mathrm{H} 37 \mathrm{~N} 1 \mathrm{O} 2$ & $1.83 \mathrm{E}-05$ & Basic N1 & C19H11N1 & $2.76 \mathrm{E}-06$ \\
\hline $\mathrm{CH}$ & $\mathrm{C} 17 \mathrm{H} 20$ & 0.001132 & Non-basic N1O2 & $\mathrm{C} 42 \mathrm{H} 39 \mathrm{~N} 1 \mathrm{O} 2$ & $5.13 \mathrm{E}-06$ & Basic N1 & $\mathrm{C} 20 \mathrm{H} 13 \mathrm{~N} 1$ & $1.39 \mathrm{E}-05$ \\
\hline $\mathrm{CH}$ & $\mathrm{C} 18 \mathrm{H} 22$ & 0.002786 & Non-basic N1O2 & $\mathrm{C} 43 \mathrm{H} 41 \mathrm{~N} 1 \mathrm{O} 2$ & $6.64 \mathrm{E}-06$ & Basic N1 & $\mathrm{C} 21 \mathrm{H} 15 \mathrm{~N} 1$ & $2.43 \mathrm{E}-05$ \\
\hline $\mathrm{CH}$ & $\mathrm{C} 19 \mathrm{H} 24$ & 0.004059 & Non-basic N1O2 & $\mathrm{C} 44 \mathrm{H} 43 \mathrm{~N} 1 \mathrm{O} 2$ & $3.49 \mathrm{E}-06$ & Basic N1 & $\mathrm{C} 22 \mathrm{H} 17 \mathrm{~N} 1$ & $4.07 \mathrm{E}-05$ \\
\hline $\mathrm{CH}$ & $\mathrm{C} 20 \mathrm{H} 26$ & 0.004025 & Non-basic N1O2 & $\mathrm{C} 45 \mathrm{H} 45 \mathrm{~N} 1 \mathrm{O} 2$ & $3.83 \mathrm{E}-06$ & Basic N1 & $\mathrm{C} 23 \mathrm{H} 19 \mathrm{~N} 1$ & $5.87 \mathrm{E}-05$ \\
\hline $\mathrm{CH}$ & $\mathrm{C} 21 \mathrm{H} 28$ & 0.002774 & Non-basic N1O2 & $\mathrm{C} 46 \mathrm{H} 47 \mathrm{~N} 1 \mathrm{O} 2$ & $6.29 \mathrm{E}-06$ & Basic N1 & $\mathrm{C} 24 \mathrm{H} 21 \mathrm{~N} 1$ & $8.10 \mathrm{E}-05$ \\
\hline $\mathrm{CH}$ & $\mathrm{C} 22 \mathrm{H} 30$ & 0.002117 & Non-basic N1O2 & $\mathrm{C} 34 \mathrm{H} 21 \mathrm{~N} 1 \mathrm{O} 2$ & $1.60 \mathrm{E}-06$ & Basic N1 & $\mathrm{C} 25 \mathrm{H} 23 \mathrm{~N} 1$ & 0.000111 \\
\hline $\mathrm{CH}$ & $\mathrm{C} 23 \mathrm{H} 32$ & 0.001375 & Non-basic N1O2 & $\mathrm{C} 35 \mathrm{H} 23 \mathrm{~N} 1 \mathrm{O} 2$ & $1.09 \mathrm{E}-05$ & Basic N1 & $\mathrm{C} 26 \mathrm{H} 25 \mathrm{~N} 1$ & 0.000106 \\
\hline $\mathrm{CH}$ & $\mathrm{C} 24 \mathrm{H} 34$ & 0.000999 & Non-basic $\mathrm{N} 1 \mathrm{O} 2$ & $\mathrm{C} 36 \mathrm{H} 25 \mathrm{~N} 1 \mathrm{O} 2$ & 4.13E-06 & Basic N1 & $\mathrm{C} 27 \mathrm{H} 27 \mathrm{~N} 1$ & 0.000175 \\
\hline $\mathrm{CH}$ & $\mathrm{C} 25 \mathrm{H} 36$ & 0.000554 & Non-basic $\mathrm{N} 1 \mathrm{O} 2$ & $\mathrm{C} 37 \mathrm{H} 27 \mathrm{~N} 1 \mathrm{O} 2$ & $1.72 \mathrm{E}-05$ & Basic N1 & $\mathrm{C} 28 \mathrm{H} 29 \mathrm{~N} 1$ & 0.000202 \\
\hline $\mathrm{CH}$ & $\mathrm{C} 26 \mathrm{H} 38$ & 0.000491 & Non-basic $\mathrm{N} 1 \mathrm{O} 2$ & $\mathrm{C} 38 \mathrm{H} 29 \mathrm{~N} 1 \mathrm{O} 2$ & $1.88 \mathrm{E}-05$ & Basic N1 & $\mathrm{C} 29 \mathrm{H} 31 \mathrm{~N} 1$ & 0.000203 \\
\hline $\mathrm{CH}$ & $\mathrm{C} 27 \mathrm{H} 40$ & 0.000413 & Non-basic N1O2 & $\mathrm{C} 39 \mathrm{H} 31 \mathrm{~N} 1 \mathrm{O} 2$ & $2.26 \mathrm{E}-05$ & Basic N1 & $\mathrm{C} 30 \mathrm{H} 33 \mathrm{~N} 1$ & 0.000224 \\
\hline $\mathrm{CH}$ & $\mathrm{C} 28 \mathrm{H} 42$ & 0.000167 & Non-basic N1O2 & $\mathrm{C} 40 \mathrm{H} 33 \mathrm{~N} 1 \mathrm{O} 2$ & $1.87 \mathrm{E}-05$ & Basic N1 & $\mathrm{C} 31 \mathrm{H} 35 \mathrm{~N} 1$ & 0.000186 \\
\hline $\mathrm{CH}$ & $\mathrm{C} 29 \mathrm{H} 44$ & $7.21 \mathrm{E}-05$ & Non-basic N1O2 & $\mathrm{C} 41 \mathrm{H} 35 \mathrm{~N} 1 \mathrm{O} 2$ & $1.83 \mathrm{E}-05$ & Basic N1 & $\mathrm{C} 32 \mathrm{H} 37 \mathrm{~N} 1$ & 0.0002 \\
\hline $\mathrm{CH}$ & $\mathrm{C} 30 \mathrm{H} 46$ & $9.39 \mathrm{E}-05$ & Non-basic N1O2 & $\mathrm{C} 42 \mathrm{H} 37 \mathrm{~N} 1 \mathrm{O} 2$ & $1.26 \mathrm{E}-05$ & Basic N1 & $\mathrm{C} 33 \mathrm{H} 39 \mathrm{~N} 1$ & 0.000127 \\
\hline $\mathrm{CH}$ & C16H16 & 0.000211 & Non-basic N1O2 & $\mathrm{C} 43 \mathrm{H} 39 \mathrm{~N} 1 \mathrm{O} 2$ & $5.52 \mathrm{E}-06$ & Basic N1 & $\mathrm{C} 34 \mathrm{H} 41 \mathrm{~N} 1$ & 0.000152 \\
\hline $\mathrm{CH}$ & $\mathrm{C} 17 \mathrm{H} 18$ & 0.000816 & Non-basic N1O2 & $\mathrm{C} 44 \mathrm{H} 41 \mathrm{~N} 1 \mathrm{O} 2$ & $1.23 \mathrm{E}-05$ & Basic N1 & $\mathrm{C} 35 \mathrm{H} 43 \mathrm{~N} 1$ & 0.000142 \\
\hline $\mathrm{CH}$ & $\mathrm{C} 18 \mathrm{H} 20$ & 0.001908 & Non-basic N1O2 & $\mathrm{C} 45 \mathrm{H} 43 \mathrm{~N} 1 \mathrm{O} 2$ & $2.99 \mathrm{E}-06$ & Basic N1 & $\mathrm{C} 36 \mathrm{H} 45 \mathrm{~N} 1$ & 0.000103 \\
\hline $\mathrm{CH}$ & $\mathrm{C} 19 \mathrm{H} 22$ & 0.001939 & Non-basic N1O2 & $\mathrm{C} 46 \mathrm{H} 45 \mathrm{~N} 1 \mathrm{O} 2$ & $3.65 \mathrm{E}-06$ & Basic N1 & $\mathrm{C} 37 \mathrm{H} 47 \mathrm{~N} 1$ & 7.21E- 05 \\
\hline $\mathrm{CH}$ & $\mathrm{C} 20 \mathrm{H} 24$ & $2.10 \mathrm{E}-05$ & Non-basic N1O2 & $\mathrm{C} 36 \mathrm{H} 23 \mathrm{~N} 1 \mathrm{O} 2$ & $1.32 \mathrm{E}-06$ & Basic N1 & $\mathrm{C} 38 \mathrm{H} 49 \mathrm{~N} 1$ & $7.72 \mathrm{E}-05$ \\
\hline $\mathrm{CH}$ & $\mathrm{C} 20 \mathrm{H} 24$ & 0.001693 & Non-basic $\mathrm{N} 1 \mathrm{O} 2$ & $\mathrm{C} 37 \mathrm{H} 25 \mathrm{~N} 1 \mathrm{O} 2$ & $5.35 \mathrm{E}-06$ & Basic N1 & $\mathrm{C} 39 \mathrm{H} 51 \mathrm{~N} 1$ & 7.22E- 05 \\
\hline $\mathrm{CH}$ & $\mathrm{C} 21 \mathrm{H} 26$ & 0.001211 & Non-basic N1O2 & $\mathrm{C} 38 \mathrm{H} 27 \mathrm{~N} 1 \mathrm{O} 2$ & $1.11 \mathrm{E}-05$ & Basic N1 & $\mathrm{C} 40 \mathrm{H} 53 \mathrm{~N} 1$ & $6.25 \mathrm{E}-05$ \\
\hline $\mathrm{CH}$ & $\mathrm{C} 22 \mathrm{H} 28$ & 0.001117 & Non-basic N1O2 & $\mathrm{C} 39 \mathrm{H} 29 \mathrm{~N} 1 \mathrm{O} 2$ & $9.51 \mathrm{E}-06$ & Basic N1 & $\mathrm{C} 41 \mathrm{H} 55 \mathrm{~N} 1$ & 4.77E- 05 \\
\hline $\mathrm{CH}$ & $\mathrm{C} 23 \mathrm{H} 30$ & 0.000724 & Non-basic N1O2 & $\mathrm{C} 40 \mathrm{H} 31 \mathrm{~N} 1 \mathrm{O} 2$ & $1.98 \mathrm{E}-05$ & Basic N1 & $\mathrm{C} 42 \mathrm{H} 57 \mathrm{~N} 1$ & $2.56 \mathrm{E}-05$ \\
\hline $\mathrm{CH}$ & $\mathrm{C} 24 \mathrm{H} 32$ & 0.000586 & Non-basic N1O2 & $\mathrm{C} 41 \mathrm{H} 33 \mathrm{~N} 1 \mathrm{O} 2$ & $1.64 \mathrm{E}-05$ & Basic N1 & $\mathrm{C} 43 \mathrm{H} 59 \mathrm{~N} 1$ & $3.95 \mathrm{E}-05$ \\
\hline $\mathrm{CH}$ & $\mathrm{C} 25 \mathrm{H} 34$ & 0.000398 & Non-basic N1O2 & $\mathrm{C} 42 \mathrm{H} 35 \mathrm{~N} 1 \mathrm{O} 2$ & $2.50 \mathrm{E}-05$ & Basic N1 & C44H61N1 & $2.31 \mathrm{E}-05$ \\
\hline $\mathrm{CH}$ & $\mathrm{C} 26 \mathrm{H} 36$ & 0.000359 & Non-basic N1O2 & $\mathrm{C} 43 \mathrm{H} 37 \mathrm{~N} 1 \mathrm{O} 2$ & $1.65 \mathrm{E}-05$ & Basic N1 & $\mathrm{C} 45 \mathrm{H} 63 \mathrm{~N} 1$ & 4.66E- 05 \\
\hline $\mathrm{CH}$ & $\mathrm{C} 27 \mathrm{H} 38$ & 0.000162 & Non-basic N1O2 & $\mathrm{C} 44 \mathrm{H} 39 \mathrm{~N} 1 \mathrm{O} 2$ & $1.37 \mathrm{E}-05$ & Basic N1 & $\mathrm{C} 46 \mathrm{H} 65 \mathrm{~N} 1$ & $1.13 \mathrm{E}-05$ \\
\hline $\mathrm{CH}$ & $\mathrm{C} 28 \mathrm{H} 40$ & $5.32 \mathrm{E}-05$ & Non-basic N1O2 & $\mathrm{C} 45 \mathrm{H} 41 \mathrm{~N} 1 \mathrm{O} 2$ & $1.69 \mathrm{E}-05$ & Basic N1 & $\mathrm{C} 21 \mathrm{H} 13 \mathrm{~N} 1$ & $5.82 \mathrm{E}-07$ \\
\hline $\mathrm{CH}$ & $\mathrm{C} 29 \mathrm{H} 42$ & 0.000199 & Non-basic N1O2 & $\mathrm{C} 46 \mathrm{H} 43 \mathrm{~N} 1 \mathrm{O} 2$ & $6.80 \mathrm{E}-06$ & Basic N1 & $\mathrm{C} 22 \mathrm{H} 15 \mathrm{~N} 1$ & $3.51 \mathrm{E}-06$ \\
\hline $\mathrm{CH}$ & $\mathrm{C} 30 \mathrm{H} 44$ & $8.27 \mathrm{E}-05$ & Non-basic N1O2 & $\mathrm{C} 47 \mathrm{H} 45 \mathrm{~N} 1 \mathrm{O} 2$ & $1.28 \mathrm{E}-05$ & Basic N1 & $\mathrm{C} 23 \mathrm{H} 17 \mathrm{~N} 1$ & $1.28 \mathrm{E}-05$ \\
\hline $\mathrm{CH}$ & $\mathrm{C} 16 \mathrm{H} 14$ & 0.000131 & Non-basic N1O2 & $\mathrm{C} 48 \mathrm{H} 47 \mathrm{~N} 1 \mathrm{O} 2$ & $3.30 \mathrm{E}-06$ & Basic N1 & $\mathrm{C} 24 \mathrm{H} 19 \mathrm{~N} 1$ & $2.83 \mathrm{E}-05$ \\
\hline $\mathrm{CH}$ & $\mathrm{C} 17 \mathrm{H} 16$ & 0.000985 & Non-basic N1O2 & $\mathrm{C} 49 \mathrm{H} 49 \mathrm{~N} 1 \mathrm{O} 2$ & $8.68 \mathrm{E}-06$ & Basic N1 & $\mathrm{C} 25 \mathrm{H} 21 \mathrm{~N} 1$ & $5.71 \mathrm{E}-05$ \\
\hline $\mathrm{CH}$ & $\mathrm{C} 18 \mathrm{H} 18$ & 0.002321 & Non-basic N1O2 & $\mathrm{C} 50 \mathrm{H} 51 \mathrm{~N} 1 \mathrm{O} 2$ & $2.21 \mathrm{E}-06$ & Basic N1 & $\mathrm{C} 26 \mathrm{H} 23 \mathrm{~N} 1$ & $5.82 \mathrm{E}-05$ \\
\hline $\mathrm{CH}$ & $\mathrm{C} 19 \mathrm{H} 20$ & 0.001721 & Non-basic N1O2 & $\mathrm{C} 37 \mathrm{H} 23 \mathrm{~N} 1 \mathrm{O} 2$ & $1.61 \mathrm{E}-06$ & Basic N1 & $\mathrm{C} 27 \mathrm{H} 25 \mathrm{~N} 1$ & $7.21 \mathrm{E}-05$ \\
\hline
\end{tabular}




\begin{tabular}{|c|c|c|c|c|c|c|c|c|}
\hline Class & Formula & Mass & Class & Formula & Mass & Class & Formula & Mass \\
\hline $\mathrm{CH}$ & $\mathrm{C} 20 \mathrm{H} 22$ & 0.001179 & Non-basic N1O2 & $\mathrm{C} 38 \mathrm{H} 25 \mathrm{~N} 1 \mathrm{O} 2$ & $2.76 \mathrm{E}-06$ & Basic N1 & $\mathrm{C} 28 \mathrm{H} 27 \mathrm{~N} 1$ & $9.12 \mathrm{E}-05$ \\
\hline $\mathrm{CH}$ & $\mathrm{C} 21 \mathrm{H} 24$ & 0.000937 & Non-basic N1O2 & $\mathrm{C} 39 \mathrm{H} 27 \mathrm{~N} 1 \mathrm{O} 2$ & $1.35 \mathrm{E}-05$ & Basic N1 & $\mathrm{C} 29 \mathrm{H} 29 \mathrm{~N} 1$ & $7.88 \mathrm{E}-05$ \\
\hline $\mathrm{CH}$ & $\mathrm{C} 22 \mathrm{H} 26$ & 0.000689 & Non-basic N1O2 & $\mathrm{C} 40 \mathrm{H} 29 \mathrm{~N} 1 \mathrm{O} 2$ & $1.28 \mathrm{E}-05$ & Basic N1 & $\mathrm{C} 30 \mathrm{H} 31 \mathrm{~N} 1$ & $7.74 \mathrm{E}-05$ \\
\hline $\mathrm{CH}$ & $\mathrm{C} 23 \mathrm{H} 28$ & 0.000452 & Non-basic N1O2 & $\mathrm{C} 41 \mathrm{H} 31 \mathrm{~N} 1 \mathrm{O} 2$ & $1.59 \mathrm{E}-05$ & Basic N1 & C31H33N1 & 0.000114 \\
\hline $\mathrm{CH}$ & $\mathrm{C} 24 \mathrm{H} 30$ & 0.000258 & Non-basic N1O2 & $\mathrm{C} 42 \mathrm{H} 33 \mathrm{~N} 1 \mathrm{O} 2$ & $1.13 \mathrm{E}-05$ & Basic N1 & $\mathrm{C} 32 \mathrm{H} 35 \mathrm{~N} 1$ & $7.87 \mathrm{E}-05$ \\
\hline $\mathrm{CH}$ & $\mathrm{C} 25 \mathrm{H} 32$ & 0.000209 & Non-basic N1O2 & $\mathrm{C} 43 \mathrm{H} 35 \mathrm{~N} 1 \mathrm{O} 2$ & $2.78 \mathrm{E}-05$ & Basic N1 & $\mathrm{C} 33 \mathrm{H} 37 \mathrm{~N} 1$ & $9.23 \mathrm{E}-05$ \\
\hline $\mathrm{CH}$ & $\mathrm{C} 26 \mathrm{H} 34$ & 0.000296 & Non-basic $\mathrm{N} 1 \mathrm{O} 2$ & $\mathrm{C} 44 \mathrm{H} 37 \mathrm{~N} 1 \mathrm{O} 2$ & $2.62 \mathrm{E}-05$ & Basic N1 & $\mathrm{C} 34 \mathrm{H} 39 \mathrm{~N} 1$ & $8.02 \mathrm{E}-05$ \\
\hline $\mathrm{CH}$ & $\mathrm{C} 27 \mathrm{H} 36$ & 0.000141 & Non-basic N1O2 & $\mathrm{C} 45 \mathrm{H} 39 \mathrm{~N} 1 \mathrm{O} 2$ & $1.38 \mathrm{E}-05$ & Basic N1 & $\mathrm{C} 35 \mathrm{H} 41 \mathrm{~N} 1$ & $7.94 \mathrm{E}-05$ \\
\hline $\mathrm{CH}$ & $\mathrm{C} 16 \mathrm{H} 12$ & $5.91 \mathrm{E}-05$ & Non-basic $\mathrm{N} 1 \mathrm{O} 2$ & $\mathrm{C} 46 \mathrm{H} 41 \mathrm{~N} 1 \mathrm{O} 2$ & $1.63 \mathrm{E}-05$ & Basic N1 & $\mathrm{C} 36 \mathrm{H} 43 \mathrm{~N} 1$ & $6.59 \mathrm{E}-05$ \\
\hline $\mathrm{CH}$ & $\mathrm{C} 17 \mathrm{H} 14$ & $3.43 \mathrm{E}-05$ & Non-basic $\mathrm{N} 1 \mathrm{O} 2$ & $\mathrm{C} 47 \mathrm{H} 43 \mathrm{~N} 1 \mathrm{O} 2$ & $1.91 \mathrm{E}-06$ & Basic N1 & $\mathrm{C} 37 \mathrm{H} 45 \mathrm{~N} 1$ & 4.97E-05 \\
\hline $\mathrm{CH}$ & $\mathrm{C} 18 \mathrm{H} 16$ & 0.001627 & Non-basic $\mathrm{N} 1 \mathrm{O} 2$ & $\mathrm{C} 48 \mathrm{H} 45 \mathrm{~N} 1 \mathrm{O} 2$ & $1.16 \mathrm{E}-05$ & Basic N1 & $\mathrm{C} 38 \mathrm{H} 47 \mathrm{~N} 1$ & $5.45 \mathrm{E}-05$ \\
\hline $\mathrm{CH}$ & C19H18 & 0.002407 & Non-basic N1O2 & $\mathrm{C} 49 \mathrm{H} 47 \mathrm{~N} 1 \mathrm{O} 2$ & $3.91 \mathrm{E}-06$ & Basic N1 & C39H49N1 & $4.20 \mathrm{E}-05$ \\
\hline $\mathrm{CH}$ & $\mathrm{C} 20 \mathrm{H} 20$ & 0.001979 & Non-basic N1O2 & $\mathrm{C} 39 \mathrm{H} 25 \mathrm{~N} 1 \mathrm{O} 2$ & $2.65 \mathrm{E}-06$ & Basic N1 & $\mathrm{C} 40 \mathrm{H} 51 \mathrm{~N} 1$ & $3.97 \mathrm{E}-05$ \\
\hline $\mathrm{CH}$ & $\mathrm{C} 21 \mathrm{H} 22$ & 0.001141 & Non-basic $\mathrm{N} 1 \mathrm{O} 2$ & $\mathrm{C} 40 \mathrm{H} 27 \mathrm{~N} 1 \mathrm{O} 2$ & $8.51 \mathrm{E}-06$ & Basic N1 & $\mathrm{C} 41 \mathrm{H} 53 \mathrm{~N} 1$ & $2.76 \mathrm{E}-05$ \\
\hline $\mathrm{CH}$ & $\mathrm{C} 22 \mathrm{H} 24$ & 0.000571 & Non-basic N1O2 & $\mathrm{C} 41 \mathrm{H} 29 \mathrm{~N} 1 \mathrm{O} 2$ & $4.11 \mathrm{E}-06$ & Basic N1 & $\mathrm{C} 42 \mathrm{H} 55 \mathrm{~N} 1$ & $1.44 \mathrm{E}-05$ \\
\hline $\mathrm{CH}$ & $\mathrm{C} 23 \mathrm{H} 26$ & 0.000326 & Non-basic N1O2 & $\mathrm{C} 42 \mathrm{H} 31 \mathrm{~N} 1 \mathrm{O} 2$ & $1.30 \mathrm{E}-05$ & Basic N1 & $\mathrm{C} 43 \mathrm{H} 57 \mathrm{~N} 1$ & $1.23 \mathrm{E}-05$ \\
\hline $\mathrm{CH}$ & $\mathrm{C} 24 \mathrm{H} 28$ & 0.000125 & Non-basic N1O2 & $\mathrm{C} 43 \mathrm{H} 33 \mathrm{~N} 1 \mathrm{O} 2$ & $1.35 \mathrm{E}-05$ & Basic N1 & C44H59N1 & $1.32 \mathrm{E}-05$ \\
\hline $\mathrm{CH}$ & $\mathrm{C} 25 \mathrm{H} 30$ & 0.000241 & Non-basic N1O2 & $\mathrm{C} 44 \mathrm{H} 35 \mathrm{~N} 1 \mathrm{O} 2$ & $1.97 \mathrm{E}-05$ & Basic N1 & $\mathrm{C} 22 \mathrm{H} 13 \mathrm{~N} 1$ & $1.62 \mathrm{E}-06$ \\
\hline $\mathrm{CH}$ & $\mathrm{C} 26 \mathrm{H} 32$ & $5.91 \mathrm{E}-05$ & Non-basic $\mathrm{N} 1 \mathrm{O} 2$ & $\mathrm{C} 45 \mathrm{H} 37 \mathrm{~N} 1 \mathrm{O} 2$ & $2.68 \mathrm{E}-05$ & Basic N1 & $\mathrm{C} 23 \mathrm{H} 15 \mathrm{~N} 1$ & $6.59 \mathrm{E}-06$ \\
\hline $\mathrm{CH}$ & $\mathrm{C} 27 \mathrm{H} 34$ & $5.68 \mathrm{E}-05$ & Non-basic N1O2 & $\mathrm{C} 46 \mathrm{H} 39 \mathrm{~N} 1 \mathrm{O} 2$ & $9.59 \mathrm{E}-06$ & Basic N1 & $\mathrm{C} 24 \mathrm{H} 17 \mathrm{~N} 1$ & $7.18 \mathrm{E}-06$ \\
\hline $\mathrm{CH}$ & $\mathrm{C} 16 \mathrm{H} 10$ & $5.19 \mathrm{E}-05$ & Non-basic N1O2 & $\mathrm{C} 47 \mathrm{H} 41 \mathrm{~N} 1 \mathrm{O} 2$ & $9.68 \mathrm{E}-06$ & Basic N1 & $\mathrm{C} 25 \mathrm{H} 19 \mathrm{~N} 1$ & $1.38 \mathrm{E}-05$ \\
\hline $\mathrm{CH}$ & $\mathrm{C} 17 \mathrm{H} 12$ & 0.000433 & Non-basic N1O2 & $\mathrm{C} 48 \mathrm{H} 43 \mathrm{~N} 1 \mathrm{O} 2$ & $6.51 \mathrm{E}-06$ & Basic N1 & $\mathrm{C} 26 \mathrm{H} 21 \mathrm{~N} 1$ & $2.08 \mathrm{E}-05$ \\
\hline $\mathrm{CH}$ & $\mathrm{C} 18 \mathrm{H} 14$ & 0.005525 & Non-basic N1O2 & $\mathrm{C} 49 \mathrm{H} 45 \mathrm{~N} 1 \mathrm{O} 2$ & $3.40 \mathrm{E}-06$ & Basic N1 & $\mathrm{C} 27 \mathrm{H} 23 \mathrm{~N} 1$ & $2.47 \mathrm{E}-05$ \\
\hline $\mathrm{CH}$ & C19H16 & 0.007345 & Non-basic N1O2 & $\mathrm{C} 50 \mathrm{H} 47 \mathrm{~N} 1 \mathrm{O} 2$ & $4.31 \mathrm{E}-06$ & Basic N1 & $\mathrm{C} 28 \mathrm{H} 25 \mathrm{~N} 1$ & $3.37 \mathrm{E}-05$ \\
\hline $\mathrm{CH}$ & $\mathrm{C} 20 \mathrm{H} 18$ & 0.004208 & Non-basic N1O2 & $\mathrm{C} 51 \mathrm{H} 49 \mathrm{~N} 1 \mathrm{O} 2$ & $5.05 \mathrm{E}-06$ & Basic N1 & $\mathrm{C} 29 \mathrm{H} 27 \mathrm{~N} 1$ & $3.42 \mathrm{E}-05$ \\
\hline $\mathrm{CH}$ & $\mathrm{C} 21 \mathrm{H} 20$ & 0.00199 & Non-basic N1O2 & $\mathrm{C} 40 \mathrm{H} 25 \mathrm{~N} 1 \mathrm{O} 2$ & $2.76 \mathrm{E}-06$ & Basic N1 & $\mathrm{C} 30 \mathrm{H} 29 \mathrm{~N} 1$ & $3.31 \mathrm{E}-05$ \\
\hline $\mathrm{CH}$ & $\mathrm{C} 22 \mathrm{H} 22$ & 0.001091 & Non-basic N1O2 & $\mathrm{C} 41 \mathrm{H} 27 \mathrm{~N} 1 \mathrm{O} 2$ & $3.03 \mathrm{E}-06$ & Basic N1 & $\mathrm{C} 31 \mathrm{H} 31 \mathrm{~N} 1$ & $3.62 \mathrm{E}-05$ \\
\hline $\mathrm{CH}$ & $\mathrm{C} 23 \mathrm{H} 24$ & 0.000465 & Non-basic N1O2 & $\mathrm{C} 42 \mathrm{H} 29 \mathrm{~N} 1 \mathrm{O} 2$ & $2.82 \mathrm{E}-06$ & Basic N1 & $\mathrm{C} 32 \mathrm{H} 33 \mathrm{~N} 1$ & $3.79 \mathrm{E}-05$ \\
\hline $\mathrm{CH}$ & $\mathrm{C} 24 \mathrm{H} 26$ & $4.82 \mathrm{E}-05$ & Non-basic N1O2 & $\mathrm{C} 43 \mathrm{H} 31 \mathrm{~N} 1 \mathrm{O} 2$ & $1.66 \mathrm{E}-05$ & Basic N1 & $\mathrm{C} 33 \mathrm{H} 35 \mathrm{~N} 1$ & $6.84 \mathrm{E}-05$ \\
\hline $\mathrm{CH}$ & $\mathrm{C} 25 \mathrm{H} 28$ & 0.000114 & Non-basic N1O2 & $\mathrm{C} 44 \mathrm{H} 33 \mathrm{~N} 1 \mathrm{O} 2$ & $1.30 \mathrm{E}-05$ & Basic N1 & $\mathrm{C} 34 \mathrm{H} 37 \mathrm{~N} 1$ & $4.10 \mathrm{E}-05$ \\
\hline $\mathrm{CH}$ & $\mathrm{C} 26 \mathrm{H} 30$ & $9.33 \mathrm{E}-05$ & Non-basic N1O2 & $\mathrm{C} 45 \mathrm{H} 35 \mathrm{~N} 1 \mathrm{O} 2$ & $2.16 \mathrm{E}-05$ & Basic N1 & $\mathrm{C} 35 \mathrm{H} 39 \mathrm{~N} 1$ & $2.39 \mathrm{E}-05$ \\
\hline $\mathrm{CH}$ & $\mathrm{C} 27 \mathrm{H} 32$ & $5.04 \mathrm{E}-05$ & Non-basic N1O2 & $\mathrm{C} 46 \mathrm{H} 37 \mathrm{~N} 1 \mathrm{O} 2$ & $1.94 \mathrm{E}-05$ & Basic N1 & $\mathrm{C} 36 \mathrm{H} 41 \mathrm{~N} 1$ & $2.86 \mathrm{E}-05$ \\
\hline $\mathrm{CH}$ & $\mathrm{C} 28 \mathrm{H} 34$ & $5.09 \mathrm{E}-05$ & Non-basic N1O2 & $\mathrm{C} 47 \mathrm{H} 39 \mathrm{~N} 1 \mathrm{O} 2$ & $2.30 \mathrm{E}-05$ & Basic N1 & $\mathrm{C} 37 \mathrm{H} 43 \mathrm{~N} 1$ & $3.06 \mathrm{E}-05$ \\
\hline $\mathrm{CH}$ & C16H8 & $3.14 \mathrm{E}-05$ & Non-basic $\mathrm{N} 1 \mathrm{O} 2$ & $\mathrm{C} 48 \mathrm{H} 41 \mathrm{~N} 1 \mathrm{O} 2$ & $1.23 \mathrm{E}-05$ & Basic N1 & $\mathrm{C} 38 \mathrm{H} 45 \mathrm{~N} 1$ & $2.92 \mathrm{E}-05$ \\
\hline $\mathrm{CH}$ & C17H10 & 0.000185 & Non-basic N1O2 & $\mathrm{C} 49 \mathrm{H} 43 \mathrm{~N} 1 \mathrm{O} 2$ & $9.95 \mathrm{E}-06$ & Basic N1 & $\mathrm{C} 39 \mathrm{H} 47 \mathrm{~N} 1$ & $2.16 \mathrm{E}-05$ \\
\hline $\mathrm{CH}$ & $\mathrm{C} 18 \mathrm{H} 12$ & 0.001001 & Non-basic N1O2 & $\mathrm{C} 41 \mathrm{H} 25 \mathrm{~N} 1 \mathrm{O} 2$ & $1.92 \mathrm{E}-06$ & Basic N1 & $\mathrm{C} 40 \mathrm{H} 49 \mathrm{~N} 1$ & $3.74 \mathrm{E}-05$ \\
\hline $\mathrm{CH}$ & C19H14 & 0.002849 & Non-basic N1O2 & $\mathrm{C} 42 \mathrm{H} 27 \mathrm{~N} 1 \mathrm{O} 2$ & $1.22 \mathrm{E}-05$ & Basic N1 & $\mathrm{C} 41 \mathrm{H} 51 \mathrm{~N} 1$ & $6.85 \mathrm{E}-06$ \\
\hline $\mathrm{CH}$ & $\mathrm{C} 20 \mathrm{H} 16$ & 0.004098 & Non-basic $\mathrm{N} 1 \mathrm{O} 2$ & $\mathrm{C} 43 \mathrm{H} 29 \mathrm{~N} 1 \mathrm{O} 2$ & $1.94 \mathrm{E}-06$ & Basic N1 & $\mathrm{C} 42 \mathrm{H} 53 \mathrm{~N} 1$ & $1.68 \mathrm{E}-05$ \\
\hline
\end{tabular}




\begin{tabular}{|c|c|c|c|c|c|c|c|c|}
\hline Class & Formula & Mass & Class & Formula & Mass & Class & Formula & Mass \\
\hline $\mathrm{CH}$ & $\mathrm{C} 21 \mathrm{H} 18$ & 0.002645 & Non-basic N1O2 & $\mathrm{C} 44 \mathrm{H} 31 \mathrm{~N} 1 \mathrm{O} 2$ & $7.76 \mathrm{E}-06$ & Basic N1 & $\mathrm{C} 43 \mathrm{H} 55 \mathrm{~N} 1$ & $9.96 \mathrm{E}-06$ \\
\hline $\mathrm{CH}$ & $\mathrm{C} 22 \mathrm{H} 20$ & 0.001108 & Non-basic N1O2 & $\mathrm{C} 45 \mathrm{H} 33 \mathrm{~N} 1 \mathrm{O} 2$ & $9.19 \mathrm{E}-06$ & Basic N1 & $\mathrm{C} 24 \mathrm{H} 15 \mathrm{~N} 1$ & $6.72 \mathrm{E}-07$ \\
\hline $\mathrm{CH}$ & $\mathrm{C} 23 \mathrm{H} 22$ & 0.00062 & Non-basic N1O2 & $\mathrm{C} 46 \mathrm{H} 35 \mathrm{~N} 1 \mathrm{O} 2$ & $9.04 \mathrm{E}-06$ & Basic N1 & $\mathrm{C} 25 \mathrm{H} 17 \mathrm{~N} 1$ & $1.80 \mathrm{E}-06$ \\
\hline $\mathrm{CH}$ & $\mathrm{C} 24 \mathrm{H} 24$ & 0.00012 & Non-basic N1O2 & $\mathrm{C} 47 \mathrm{H} 37 \mathrm{~N} 1 \mathrm{O} 2$ & $2.62 \mathrm{E}-05$ & Basic N1 & C26H19N1 & 4.40E-06 \\
\hline $\mathrm{CH}$ & $\mathrm{C} 25 \mathrm{H} 26$ & $9.78 \mathrm{E}-05$ & Non-basic N1O2 & $\mathrm{C} 48 \mathrm{H} 39 \mathrm{~N} 1 \mathrm{O} 2$ & 7.02E-06 & Basic N1 & $\mathrm{C} 27 \mathrm{H} 21 \mathrm{~N} 1$ & $7.34 \mathrm{E}-06$ \\
\hline $\mathrm{CH}$ & $\mathrm{C} 17 \mathrm{H} 8$ & $1.62 \mathrm{E}-05$ & Non-basic N1O2 & $\mathrm{C} 49 \mathrm{H} 41 \mathrm{~N} 1 \mathrm{O} 2$ & $1.59 \mathrm{E}-05$ & Basic N1 & $\mathrm{C} 28 \mathrm{H} 23 \mathrm{~N} 1$ & $1.18 \mathrm{E}-05$ \\
\hline $\mathrm{CH}$ & $\mathrm{C} 18 \mathrm{H} 10$ & $8.38 \mathrm{E}-05$ & Non-basic $\mathrm{N} 1 \mathrm{O} 2$ & $\mathrm{C} 45 \mathrm{H} 31 \mathrm{~N} 1 \mathrm{O} 2$ & $8.23 \mathrm{E}-06$ & Basic N1 & $\mathrm{C} 29 \mathrm{H} 25 \mathrm{~N} 1$ & $6.14 \mathrm{E}-06$ \\
\hline $\mathrm{CH}$ & $\mathrm{C} 19 \mathrm{H} 12$ & 0.000106 & Non-basic N1O2 & $\mathrm{C} 46 \mathrm{H} 33 \mathrm{~N} 1 \mathrm{O} 2$ & $1.27 \mathrm{E}-05$ & Basic N1 & $\mathrm{C} 30 \mathrm{H} 27 \mathrm{~N} 1$ & $9.58 \mathrm{E}-06$ \\
\hline $\mathrm{CH}$ & $\mathrm{C} 20 \mathrm{H} 14$ & 0.00037 & Non-basic $\mathrm{N} 1 \mathrm{O} 2$ & $\mathrm{C} 47 \mathrm{H} 35 \mathrm{~N} 1 \mathrm{O} 2$ & $1.55 \mathrm{E}-05$ & Basic N1 & $\mathrm{C} 31 \mathrm{H} 29 \mathrm{~N} 1$ & $1.66 \mathrm{E}-05$ \\
\hline $\mathrm{CH}$ & $\mathrm{C} 21 \mathrm{H} 16$ & 0.000664 & Non-basic $\mathrm{N} 1 \mathrm{O} 2$ & $\mathrm{C} 48 \mathrm{H} 37 \mathrm{~N} 1 \mathrm{O} 2$ & $1.23 \mathrm{E}-05$ & Basic N1 & $\mathrm{C} 32 \mathrm{H} 31 \mathrm{~N} 1$ & $1.74 \mathrm{E}-05$ \\
\hline $\mathrm{CH}$ & $\mathrm{C} 22 \mathrm{H} 18$ & 0.000428 & Non-basic $\mathrm{N} 1 \mathrm{O} 2$ & $\mathrm{C} 49 \mathrm{H} 39 \mathrm{~N} 1 \mathrm{O} 2$ & $1.36 \mathrm{E}-05$ & Basic N1 & $\mathrm{C} 33 \mathrm{H} 33 \mathrm{~N} 1$ & $1.43 \mathrm{E}-05$ \\
\hline $\mathrm{CH}$ & $\mathrm{C} 23 \mathrm{H} 20$ & 0.000246 & Non-basic N1O2 & $\mathrm{C} 47 \mathrm{H} 33 \mathrm{~N} 1 \mathrm{O} 2$ & $4.02 \mathrm{E}-06$ & Basic N1 & $\mathrm{C} 34 \mathrm{H} 35 \mathrm{~N} 1$ & $1.93 \mathrm{E}-05$ \\
\hline $\mathrm{CH}$ & $\mathrm{C} 24 \mathrm{H} 22$ & 0.000131 & Non-basic N1O2 & $\mathrm{C} 48 \mathrm{H} 35 \mathrm{~N} 1 \mathrm{O} 2$ & $1.94 \mathrm{E}-06$ & Basic N1 & $\mathrm{C} 35 \mathrm{H} 37 \mathrm{~N} 1$ & $1.26 \mathrm{E}-05$ \\
\hline $\mathrm{CH}$ & $\mathrm{C} 25 \mathrm{H} 24$ & $5.28 \mathrm{E}-05$ & Non-basic $\mathrm{N} 1 \mathrm{O} 2$ & $\mathrm{C} 49 \mathrm{H} 37 \mathrm{~N} 1 \mathrm{O} 2$ & $6.58 \mathrm{E}-06$ & Basic N1 & $\mathrm{C} 36 \mathrm{H} 39 \mathrm{~N} 1$ & $2.18 \mathrm{E}-05$ \\
\hline $\mathrm{CH}$ & C19H10 & $3.13 \mathrm{E}-05$ & Non-basic N1O3 & $\mathrm{C} 22 \mathrm{H} 35 \mathrm{~N} 1 \mathrm{O} 3$ & $1.86 \mathrm{E}-06$ & Basic N1 & $\mathrm{C} 37 \mathrm{H} 41 \mathrm{~N} 1$ & $8.41 \mathrm{E}-06$ \\
\hline $\mathrm{CH}$ & $\mathrm{C} 20 \mathrm{H} 12$ & 0.001191 & Non-basic N1O3 & $\mathrm{C} 23 \mathrm{H} 37 \mathrm{~N} 1 \mathrm{O} 3$ & $1.12 \mathrm{E}-06$ & Basic N1 & $\mathrm{C} 38 \mathrm{H} 43 \mathrm{~N} 1$ & $1.41 \mathrm{E}-05$ \\
\hline $\mathrm{CH}$ & $\mathrm{C} 21 \mathrm{H} 14$ & 0.000679 & Non-basic N1O3 & $\mathrm{C} 24 \mathrm{H} 39 \mathrm{~N} 1 \mathrm{O} 3$ & $3.47 \mathrm{E}-06$ & Basic N1 & $\mathrm{C} 39 \mathrm{H} 45 \mathrm{~N} 1$ & $1.21 \mathrm{E}-05$ \\
\hline $\mathrm{CH}$ & $\mathrm{C} 22 \mathrm{H} 16$ & 0.000989 & Non-basic N1O3 & $\mathrm{C} 25 \mathrm{H} 41 \mathrm{~N} 1 \mathrm{O} 3$ & $4.86 \mathrm{E}-06$ & Basic N1 & $\mathrm{C} 40 \mathrm{H} 47 \mathrm{~N} 1$ & $9.75 \mathrm{E}-06$ \\
\hline $\mathrm{CH}$ & $\mathrm{C} 23 \mathrm{H} 18$ & 0.001021 & Non-basic N1O3 & $\mathrm{C} 26 \mathrm{H} 43 \mathrm{~N} 1 \mathrm{O} 3$ & $5.08 \mathrm{E}-06$ & Basic N1 & $\mathrm{C} 41 \mathrm{H} 49 \mathrm{~N} 1$ & $3.36 \mathrm{E}-06$ \\
\hline $\mathrm{CH}$ & $\mathrm{C} 24 \mathrm{H} 20$ & 0.000575 & Non-basic N1O3 & $\mathrm{C} 27 \mathrm{H} 45 \mathrm{~N} 1 \mathrm{O} 3$ & $6.64 \mathrm{E}-06$ & Basic N1 & $\mathrm{C} 42 \mathrm{H} 51 \mathrm{~N} 1$ & $9.00 \mathrm{E}-06$ \\
\hline $\mathrm{CH}$ & $\mathrm{C} 25 \mathrm{H} 22$ & $8.64 \mathrm{E}-05$ & Non-basic N1O3 & $\mathrm{C} 28 \mathrm{H} 47 \mathrm{~N} 1 \mathrm{O} 3$ & $2.58 \mathrm{E}-06$ & Basic N1 & $\mathrm{C} 43 \mathrm{H} 53 \mathrm{~N} 1$ & $1.85 \mathrm{E}-06$ \\
\hline $\mathrm{CH}$ & $\mathrm{C} 26 \mathrm{H} 24$ & 0.000109 & Non-basic N1O3 & $\mathrm{C} 29 \mathrm{H} 49 \mathrm{~N} 1 \mathrm{O} 3$ & $3.52 \mathrm{E}-06$ & Basic N1 & $\mathrm{C} 44 \mathrm{H} 55 \mathrm{~N} 1$ & $2.62 \mathrm{E}-06$ \\
\hline $\mathrm{CH}$ & $\mathrm{C} 21 \mathrm{H} 12$ & 0.000188 & Non-basic N1O3 & $\mathrm{C} 30 \mathrm{H} 51 \mathrm{~N} 1 \mathrm{O} 3$ & $5.50 \mathrm{E}-06$ & Basic N1 & $\mathrm{C} 45 \mathrm{H} 57 \mathrm{~N} 1$ & $5.12 \mathrm{E}-06$ \\
\hline $\mathrm{CH}$ & $\mathrm{C} 22 \mathrm{H} 14$ & 0.000174 & Non-basic N1O3 & C31H53N1O3 & $3.52 \mathrm{E}-06$ & Basic N1 & $\mathrm{C} 27 \mathrm{H} 19 \mathrm{~N} 1$ & $2.13 \mathrm{E}-07$ \\
\hline $\mathrm{CH}$ & $\mathrm{C} 23 \mathrm{H} 16$ & 0.000414 & Non-basic N1O3 & $\mathrm{C} 32 \mathrm{H} 55 \mathrm{~N} 1 \mathrm{O} 3$ & $9.06 \mathrm{E}-06$ & Basic N1 & $\mathrm{C} 28 \mathrm{H} 21 \mathrm{~N} 1$ & $3.04 \mathrm{E}-06$ \\
\hline $\mathrm{CH}$ & $\mathrm{C} 24 \mathrm{H} 18$ & 0.00073 & Non-basic N1O3 & $\mathrm{C} 33 \mathrm{H} 57 \mathrm{~N} 1 \mathrm{O} 3$ & $6.91 \mathrm{E}-06$ & Basic N1 & $\mathrm{C} 29 \mathrm{H} 23 \mathrm{~N} 1$ & $3.90 \mathrm{E}-06$ \\
\hline $\mathrm{CH}$ & $\mathrm{C} 25 \mathrm{H} 20$ & 0.000423 & Non-basic N1O3 & $\mathrm{C} 13 \mathrm{H} 15 \mathrm{~N} 1 \mathrm{O} 3$ & $3.91 \mathrm{E}-06$ & Basic N1 & $\mathrm{C} 30 \mathrm{H} 25 \mathrm{~N} 1$ & $3.69 \mathrm{E}-06$ \\
\hline $\mathrm{CH}$ & $\mathrm{C} 26 \mathrm{H} 22$ & $9.62 \mathrm{E}-05$ & Non-basic N1O3 & $\mathrm{C} 14 \mathrm{H} 17 \mathrm{~N} 1 \mathrm{O} 3$ & $9.67 \mathrm{E}-06$ & Basic N1 & $\mathrm{C} 31 \mathrm{H} 27 \mathrm{~N} 1$ & $5.04 \mathrm{E}-06$ \\
\hline $\mathrm{CH}$ & $\mathrm{C} 27 \mathrm{H} 24$ & 0.000114 & Non-basic N1O3 & $\mathrm{C} 15 \mathrm{H} 19 \mathrm{~N} 1 \mathrm{O} 3$ & $4.75 \mathrm{E}-06$ & Basic N1 & $\mathrm{C} 32 \mathrm{H} 29 \mathrm{~N} 1$ & $2.48 \mathrm{E}-06$ \\
\hline $\mathrm{CH}$ & $\mathrm{C} 21 \mathrm{H} 10$ & $1.85 \mathrm{E}-05$ & Non-basic N1O3 & $\mathrm{C} 16 \mathrm{H} 21 \mathrm{~N} 1 \mathrm{O} 3$ & $1.13 \mathrm{E}-06$ & Basic N1 & $\mathrm{C} 33 \mathrm{H} 31 \mathrm{~N} 1$ & $3.54 \mathrm{E}-06$ \\
\hline $\mathrm{CH}$ & $\mathrm{C} 21 \mathrm{H} 10$ & $3.57 \mathrm{E}-05$ & Non-basic N1O3 & $\mathrm{C} 17 \mathrm{H} 23 \mathrm{~N} 1 \mathrm{O} 3$ & $3.44 \mathrm{E}-06$ & Basic N1 & $\mathrm{C} 34 \mathrm{H} 33 \mathrm{~N} 1$ & $2.65 \mathrm{E}-06$ \\
\hline $\mathrm{CH}$ & $\mathrm{C} 22 \mathrm{H} 12$ & 0.000228 & Non-basic N1O3 & $\mathrm{C} 18 \mathrm{H} 25 \mathrm{~N} 1 \mathrm{O} 3$ & $4.46 \mathrm{E}-06$ & Basic N1 & $\mathrm{C} 35 \mathrm{H} 35 \mathrm{~N} 1$ & $6.40 \mathrm{E}-06$ \\
\hline $\mathrm{CH}$ & $\mathrm{C} 24 \mathrm{H} 16$ & 0.000258 & Non-basic N1O3 & $\mathrm{C} 19 \mathrm{H} 27 \mathrm{~N} 1 \mathrm{O} 3$ & $1.82 \mathrm{E}-06$ & Basic N1 & $\mathrm{C} 36 \mathrm{H} 37 \mathrm{~N} 1$ & $1.03 \mathrm{E}-05$ \\
\hline $\mathrm{CH}$ & $\mathrm{C} 25 \mathrm{H} 18$ & 0.000573 & Non-basic N1O3 & $\mathrm{C} 20 \mathrm{H} 29 \mathrm{~N} 1 \mathrm{O} 3$ & 3.97E-06 & Basic N1 & $\mathrm{C} 37 \mathrm{H} 39 \mathrm{~N} 1$ & $2.75 \mathrm{E}-06$ \\
\hline $\mathrm{CH}$ & $\mathrm{C} 26 \mathrm{H} 20$ & 0.00087 & Non-basic N1O3 & $\mathrm{C} 21 \mathrm{H} 31 \mathrm{~N} 1 \mathrm{O} 3$ & $4.14 \mathrm{E}-06$ & Basic N1 & $\mathrm{C} 38 \mathrm{H} 41 \mathrm{~N} 1$ & $3.90 \mathrm{E}-06$ \\
\hline $\mathrm{CH}$ & $\mathrm{C} 27 \mathrm{H} 22$ & 0.000419 & Non-basic N1O3 & $\mathrm{C} 22 \mathrm{H} 33 \mathrm{~N} 1 \mathrm{O} 3$ & $1.15 \mathrm{E}-05$ & Basic N1 & $\mathrm{C} 39 \mathrm{H} 43 \mathrm{~N} 1$ & $4.04 \mathrm{E}-06$ \\
\hline $\mathrm{CH}$ & $\mathrm{C} 28 \mathrm{H} 24$ & 0.000246 & Non-basic N1O3 & $\mathrm{C} 23 \mathrm{H} 35 \mathrm{~N} 1 \mathrm{O} 3$ & $5.93 \mathrm{E}-06$ & Basic N1 & $\mathrm{C} 40 \mathrm{H} 45 \mathrm{~N} 1$ & $3.53 \mathrm{E}-06$ \\
\hline $\mathrm{CH}$ & $\mathrm{C} 23 \mathrm{H} 12$ & $3.60 \mathrm{E}-05$ & Non-basic N1O3 & $\mathrm{C} 24 \mathrm{H} 37 \mathrm{~N} 1 \mathrm{O} 3$ & $5.51 \mathrm{E}-06$ & Basic N1 & $\mathrm{C} 41 \mathrm{H} 47 \mathrm{~N} 1$ & $3.51 \mathrm{E}-06$ \\
\hline
\end{tabular}




\begin{tabular}{|c|c|c|c|c|c|c|c|c|}
\hline Class & Formula & Mass & Class & Formula & Mass & Class & Formula & Mass \\
\hline $\mathrm{CH}$ & $\mathrm{C} 24 \mathrm{H} 14$ & 0.000167 & Non-basic N1O3 & $\mathrm{C} 25 \mathrm{H} 39 \mathrm{~N} 1 \mathrm{O} 3$ & $7.18 \mathrm{E}-06$ & Basic N1 & $\mathrm{C} 42 \mathrm{H} 49 \mathrm{~N} 1$ & $2.96 \mathrm{E}-06$ \\
\hline $\mathrm{CH}$ & $\mathrm{C} 25 \mathrm{H} 16$ & 0.000949 & Non-basic N1O3 & $\mathrm{C} 26 \mathrm{H} 41 \mathrm{~N} 1 \mathrm{O} 3$ & $1.26 \mathrm{E}-05$ & Basic N1 & $\mathrm{C} 43 \mathrm{H} 51 \mathrm{~N} 1$ & $3.08 \mathrm{E}-06$ \\
\hline $\mathrm{CH}$ & $\mathrm{C} 26 \mathrm{H} 18$ & 0.00138 & Non-basic N1O3 & $\mathrm{C} 27 \mathrm{H} 43 \mathrm{~N} 1 \mathrm{O} 3$ & $1.30 \mathrm{E}-05$ & Basic N1 & C44H53N1 & $3.81 \mathrm{E}-07$ \\
\hline $\mathrm{CH}$ & $\mathrm{C} 27 \mathrm{H} 20$ & 0.001296 & Non-basic N1O3 & $\mathrm{C} 28 \mathrm{H} 45 \mathrm{~N} 1 \mathrm{O} 3$ & $2.34 \mathrm{E}-06$ & Basic N1 & $\mathrm{C} 29 \mathrm{H} 21 \mathrm{~N} 1$ & $9.14 \mathrm{E}-07$ \\
\hline $\mathrm{CH}$ & $\mathrm{C} 28 \mathrm{H} 22$ & 0.000774 & Non-basic N1O3 & $\mathrm{C} 29 \mathrm{H} 47 \mathrm{~N} 1 \mathrm{O} 3$ & $4.95 \mathrm{E}-06$ & Basic N1 & $\mathrm{C} 30 \mathrm{H} 23 \mathrm{~N} 1$ & $1.26 \mathrm{E}-06$ \\
\hline $\mathrm{CH}$ & $\mathrm{C} 29 \mathrm{H} 24$ & 0.000277 & Non-basic N1O3 & $\mathrm{C} 31 \mathrm{H} 51 \mathrm{~N} 1 \mathrm{O} 3$ & $8.63 \mathrm{E}-06$ & Basic N1 & $\mathrm{C} 31 \mathrm{H} 25 \mathrm{~N} 1$ & $3.40 \mathrm{E}-06$ \\
\hline $\mathrm{CH}$ & $\mathrm{C} 26 \mathrm{H} 16$ & 0.000244 & Non-basic N1O3 & $\mathrm{C} 32 \mathrm{H} 53 \mathrm{~N} 1 \mathrm{O} 3$ & $5.13 \mathrm{E}-06$ & Basic N1 & $\mathrm{C} 32 \mathrm{H} 27 \mathrm{~N} 1$ & $1.72 \mathrm{E}-06$ \\
\hline $\mathrm{CH}$ & $\mathrm{C} 27 \mathrm{H} 18$ & 0.000324 & Non-basic N1O3 & $\mathrm{C} 33 \mathrm{H} 55 \mathrm{~N} 1 \mathrm{O} 3$ & $7.96 \mathrm{E}-06$ & Basic N1 & $\mathrm{C} 33 \mathrm{H} 29 \mathrm{~N} 1$ & $1.67 \mathrm{E}-06$ \\
\hline $\mathrm{CH}$ & $\mathrm{C} 28 \mathrm{H} 20$ & 0.000575 & Non-basic N1O3 & $\mathrm{C} 34 \mathrm{H} 57 \mathrm{~N} 1 \mathrm{O} 3$ & $1.72 \mathrm{E}-06$ & Basic N1 & $\mathrm{C} 34 \mathrm{H} 31 \mathrm{~N} 1$ & $2.57 \mathrm{E}-06$ \\
\hline $\mathrm{CH}$ & $\mathrm{C} 29 \mathrm{H} 22$ & 0.000399 & Non-basic N1O3 & $\mathrm{C} 35 \mathrm{H} 59 \mathrm{~N} 1 \mathrm{O} 3$ & $6.42 \mathrm{E}-06$ & Basic N1 & $\mathrm{C} 35 \mathrm{H} 33 \mathrm{~N} 1$ & $9.78 \mathrm{E}-07$ \\
\hline $\mathrm{CH}$ & $\mathrm{C} 27 \mathrm{H} 16$ & 0.000119 & Non-basic N1O3 & $\mathrm{C} 36 \mathrm{H} 61 \mathrm{~N} 1 \mathrm{O} 3$ & $1.85 \mathrm{E}-06$ & Basic N1 & $\mathrm{C} 36 \mathrm{H} 35 \mathrm{~N} 1$ & $1.13 \mathrm{E}-06$ \\
\hline $\mathrm{CH}$ & $\mathrm{C} 28 \mathrm{H} 18$ & 0.000717 & Non-basic N1O3 & $\mathrm{C} 13 \mathrm{H} 13 \mathrm{~N} 1 \mathrm{O} 3$ & $1.92 \mathrm{E}-06$ & Basic N1 & $\mathrm{C} 37 \mathrm{H} 37 \mathrm{~N} 1$ & $3.65 \mathrm{E}-06$ \\
\hline $\mathrm{CH}$ & $\mathrm{C} 29 \mathrm{H} 20$ & 0.000538 & Non-basic N1O3 & $\mathrm{C} 15 \mathrm{H} 17 \mathrm{~N} 1 \mathrm{O} 3$ & $1.68 \mathrm{E}-06$ & Basic N1 & $\mathrm{C} 38 \mathrm{H} 39 \mathrm{~N} 1$ & $1.95 \mathrm{E}-06$ \\
\hline $\mathrm{CH}$ & $\mathrm{C} 30 \mathrm{H} 22$ & 0.000329 & Non-basic N1O3 & $\mathrm{C} 16 \mathrm{H} 19 \mathrm{~N} 1 \mathrm{O} 3$ & $1.99 \mathrm{E}-06$ & Basic N1 & $\mathrm{C} 39 \mathrm{H} 41 \mathrm{~N} 1$ & $2.62 \mathrm{E}-07$ \\
\hline $\mathrm{CH}$ & $\mathrm{C} 21 \mathrm{H} 2$ & 0.000431 & Non-basic N1O3 & $\mathrm{C} 18 \mathrm{H} 23 \mathrm{~N} 1 \mathrm{O} 3$ & $3.66 \mathrm{E}-06$ & Basic N1 & $\mathrm{C} 40 \mathrm{H} 43 \mathrm{~N} 1$ & $1.68 \mathrm{E}-05$ \\
\hline $\mathrm{CH}$ & $\mathrm{C} 22 \mathrm{H} 4$ & 0.000356 & Non-basic N1O3 & $\mathrm{C} 19 \mathrm{H} 25 \mathrm{~N} 1 \mathrm{O} 3$ & $2.87 \mathrm{E}-06$ & Basic N1 & $\mathrm{C} 33 \mathrm{H} 27 \mathrm{~N} 1$ & $2.39 \mathrm{E}-07$ \\
\hline $\mathrm{CH}$ & $\mathrm{C} 23 \mathrm{H} 6$ & 0.000122 & Non-basic N1O3 & $\mathrm{C} 20 \mathrm{H} 27 \mathrm{~N} 1 \mathrm{O} 3$ & $7.16 \mathrm{E}-06$ & Basic N1 & $\mathrm{C} 34 \mathrm{H} 29 \mathrm{~N} 1$ & $7.91 \mathrm{E}-06$ \\
\hline $\mathrm{CH}$ & $\mathrm{C} 24 \mathrm{H} 8$ & $3.55 \mathrm{E}-05$ & Non-basic N1O3 & $\mathrm{C} 22 \mathrm{H} 31 \mathrm{~N} 1 \mathrm{O} 3$ & $1.44 \mathrm{E}-05$ & Basic N1 & $\mathrm{C} 35 \mathrm{H} 31 \mathrm{~N} 1$ & 3.53E-07 \\
\hline $\mathrm{CH}$ & $\mathrm{C} 25 \mathrm{H} 10$ & $5.03 \mathrm{E}-05$ & Non-basic N1O3 & $\mathrm{C} 23 \mathrm{H} 33 \mathrm{~N} 1 \mathrm{O} 3$ & $9.84 \mathrm{E}-06$ & Basic N1 & $\mathrm{C} 36 \mathrm{H} 33 \mathrm{~N} 1$ & $1.47 \mathrm{E}-06$ \\
\hline $\mathrm{CH}$ & $\mathrm{C} 21 \mathrm{H} 0$ & 0.00097 & Non-basic N1O3 & $\mathrm{C} 24 \mathrm{H} 35 \mathrm{~N} 1 \mathrm{O} 3$ & $6.27 \mathrm{E}-06$ & Basic N1 & $\mathrm{C} 37 \mathrm{H} 35 \mathrm{~N} 1$ & $1.17 \mathrm{E}-06$ \\
\hline $\mathrm{CH}$ & $\mathrm{C} 22 \mathrm{H} 2$ & 0.00036 & Non-basic N1O3 & $\mathrm{C} 25 \mathrm{H} 37 \mathrm{~N} 1 \mathrm{O} 3$ & $7.26 \mathrm{E}-06$ & Basic N1 & $\mathrm{C} 38 \mathrm{H} 35 \mathrm{~N} 1$ & $1.07 \mathrm{E}-06$ \\
\hline $\mathrm{CH}$ & $\mathrm{C} 22 \mathrm{H} 0$ & $6.50 \mathrm{E}-05$ & Non-basic N1O3 & $\mathrm{C} 26 \mathrm{H} 39 \mathrm{~N} 1 \mathrm{O} 3$ & 4.57E-06 & Basic N1 & $\mathrm{C} 39 \mathrm{H} 37 \mathrm{~N} 1$ & $1.44 \mathrm{E}-06$ \\
\hline $\mathrm{CH}$ & $\mathrm{C} 23 \mathrm{H} 2$ & 0.000479 & Non-basic N1O3 & $\mathrm{C} 27 \mathrm{H} 41 \mathrm{~N} 1 \mathrm{O} 3$ & $9.69 \mathrm{E}-06$ & Basic N1 & $\mathrm{C} 31 \mathrm{H} 19 \mathrm{~N} 1$ & $8.87 \mathrm{E}-07$ \\
\hline $\mathrm{CH}$ & $\mathrm{C} 24 \mathrm{H} 4$ & 0.000212 & Non-basic N1O3 & $\mathrm{C} 28 \mathrm{H} 43 \mathrm{~N} 1 \mathrm{O} 3$ & $1.29 \mathrm{E}-05$ & Basic N1 & $\mathrm{C} 32 \mathrm{H} 21 \mathrm{~N} 1$ & 7.66E- 07 \\
\hline $\mathrm{CH}$ & $\mathrm{C} 25 \mathrm{H} 4$ & $3.80 \mathrm{E}-05$ & Non-basic N1O3 & $\mathrm{C} 29 \mathrm{H} 45 \mathrm{~N} 1 \mathrm{O} 3$ & $1.74 \mathrm{E}-05$ & Basic N1 & $\mathrm{C} 33 \mathrm{H} 23 \mathrm{~N} 1$ & $3.15 \mathrm{E}-07$ \\
\hline $\mathrm{CH}$ & $\mathrm{C} 26 \mathrm{H} 6$ & 4.05E-05 & Non-basic N1O3 & $\mathrm{C} 30 \mathrm{H} 47 \mathrm{~N} 1 \mathrm{O} 3$ & $1.49 \mathrm{E}-05$ & Basic N1 & $\mathrm{C} 34 \mathrm{H} 25 \mathrm{~N} 1$ & $4.31 \mathrm{E}-06$ \\
\hline S1 & C13H10S1 & $5.48 \mathrm{E}-07$ & Non-basic N1O3 & $\mathrm{C} 31 \mathrm{H} 49 \mathrm{~N} 1 \mathrm{O} 3$ & $2.81 \mathrm{E}-05$ & Basic N1 & $\mathrm{C} 35 \mathrm{H} 27 \mathrm{~N} 1$ & $1.31 \mathrm{E}-06$ \\
\hline S1 & $\mathrm{C} 14 \mathrm{H} 12 \mathrm{~S} 1$ & $1.12 \mathrm{E}-06$ & Non-basic N1O3 & $\mathrm{C} 32 \mathrm{H} 51 \mathrm{~N} 1 \mathrm{O} 3$ & $1.44 \mathrm{E}-05$ & Basic N1 & $\mathrm{C} 39 \mathrm{H} 33 \mathrm{~N} 1$ & $5.02 \mathrm{E}-06$ \\
\hline S1 & $\mathrm{C} 15 \mathrm{H} 14 \mathrm{~S} 1$ & $8.44 \mathrm{E}-06$ & Non-basic N1O3 & $\mathrm{C} 33 \mathrm{H} 53 \mathrm{~N} 1 \mathrm{O} 3$ & $1.27 \mathrm{E}-05$ & Basic N1 & $\mathrm{C} 40 \mathrm{H} 35 \mathrm{~N} 1$ & $2.85 \mathrm{E}-06$ \\
\hline S1 & $\mathrm{C} 16 \mathrm{H} 16 \mathrm{~S} 1$ & $1.22 \mathrm{E}-05$ & Non-basic N1O3 & $\mathrm{C} 34 \mathrm{H} 55 \mathrm{~N} 1 \mathrm{O} 3$ & $1.20 \mathrm{E}-05$ & Basic N1 & $\mathrm{C} 42 \mathrm{H} 37 \mathrm{~N} 1$ & $1.51 \mathrm{E}-06$ \\
\hline S1 & C17H18S1 & $1.20 \mathrm{E}-05$ & Non-basic N1O3 & $\mathrm{C} 35 \mathrm{H} 57 \mathrm{~N} 1 \mathrm{O} 3$ & $2.29 \mathrm{E}-06$ & Basic N1 & $\mathrm{C} 43 \mathrm{H} 39 \mathrm{~N} 1$ & 4.84E- 06 \\
\hline S1 & $\mathrm{C} 16 \mathrm{H} 14 \mathrm{~S} 1$ & $2.64 \mathrm{E}-06$ & Non-basic N1O3 & $\mathrm{C} 36 \mathrm{H} 59 \mathrm{~N} 1 \mathrm{O} 3$ & $9.28 \mathrm{E}-06$ & Basic N1 & $\mathrm{C} 34 \mathrm{H} 19 \mathrm{~N} 1$ & $2.15 \mathrm{E}-07$ \\
\hline S1 & $\mathrm{C} 17 \mathrm{H} 16 \mathrm{~S} 1$ & $3.68 \mathrm{E}-06$ & Non-basic N1O3 & $\mathrm{C} 37 \mathrm{H} 61 \mathrm{~N} 1 \mathrm{O} 3$ & $1.16 \mathrm{E}-05$ & Basic N1 & $\mathrm{C} 35 \mathrm{H} 21 \mathrm{~N} 1$ & $2.35 \mathrm{E}-06$ \\
\hline S1 & C18H18S1 & $8.83 \mathrm{E}-06$ & Non-basic N1O3 & $\mathrm{C} 13 \mathrm{H} 11 \mathrm{~N} 1 \mathrm{O} 3$ & $1.26 \mathrm{E}-06$ & Basic N1 & $\mathrm{C} 36 \mathrm{H} 23 \mathrm{~N} 1$ & $6.65 \mathrm{E}-06$ \\
\hline S1 & $\mathrm{C} 19 \mathrm{H} 20 \mathrm{~S} 1$ & 2.39E-06 & Non-basic N1O3 & $\mathrm{C} 14 \mathrm{H} 13 \mathrm{~N} 1 \mathrm{O} 3$ & $7.35 \mathrm{E}-06$ & Basic N1 & $\mathrm{C} 37 \mathrm{H} 25 \mathrm{~N} 1$ & $1.06 \mathrm{E}-05$ \\
\hline S1 & $\mathrm{C} 16 \mathrm{H} 12 \mathrm{~S} 1$ & 4.07E-06 & Non-basic N1O3 & $\mathrm{C} 15 \mathrm{H} 15 \mathrm{~N} 1 \mathrm{O} 3$ & $6.47 \mathrm{E}-06$ & Basic N1 & $\mathrm{C} 38 \mathrm{H} 27 \mathrm{~N} 1$ & $4.79 \mathrm{E}-06$ \\
\hline S1 & $\mathrm{C} 17 \mathrm{H} 14 \mathrm{~S} 1$ & $1.94 \mathrm{E}-05$ & Non-basic N1O3 & $\mathrm{C} 16 \mathrm{H} 17 \mathrm{~N} 1 \mathrm{O} 3$ & $1.03 \mathrm{E}-05$ & Basic N1 & $\mathrm{C} 39 \mathrm{H} 29 \mathrm{~N} 1$ & $3.54 \mathrm{E}-05$ \\
\hline S1 & $\mathrm{C} 18 \mathrm{H} 16 \mathrm{~S} 1$ & $1.76 \mathrm{E}-05$ & Non-basic N1O3 & $\mathrm{C} 17 \mathrm{H} 19 \mathrm{~N} 1 \mathrm{O} 3$ & $1.07 \mathrm{E}-05$ & Basic N1 & $\mathrm{C} 40 \mathrm{H} 31 \mathrm{~N} 1$ & $4.35 \mathrm{E}-06$ \\
\hline
\end{tabular}




\begin{tabular}{|c|c|c|c|c|c|c|c|c|}
\hline Class & Formula & Mass & Class & Formula & Mass & Class & Formula & Mass \\
\hline S1 & C19H18S1 & $3.47 \mathrm{E}-06$ & Non-basic N1O3 & $\mathrm{C} 18 \mathrm{H} 21 \mathrm{~N} 1 \mathrm{O} 3$ & $2.80 \mathrm{E}-06$ & Basic N1 & $\mathrm{C} 39 \mathrm{H} 27 \mathrm{~N} 1$ & $1.15 \mathrm{E}-06$ \\
\hline S1 & $\mathrm{C} 20 \mathrm{H} 20 \mathrm{~S} 1$ & $1.20 \mathrm{E}-05$ & Non-basic N1O3 & C19H23N1O3 & $7.80 \mathrm{E}-06$ & Basic N1 & $\mathrm{C} 40 \mathrm{H} 29 \mathrm{~N} 1$ & $3.42 \mathrm{E}-06$ \\
\hline S1 & $\mathrm{C} 21 \mathrm{H} 22 \mathrm{~S} 1$ & 2.07E-05 & Non-basic N1O3 & $\mathrm{C} 20 \mathrm{H} 25 \mathrm{~N} 1 \mathrm{O} 3$ & $4.56 \mathrm{E}-06$ & Basic N1 & $\mathrm{C} 41 \mathrm{H} 31 \mathrm{~N} 1$ & $2.70 \mathrm{E}-06$ \\
\hline S1 & $\mathrm{C} 22 \mathrm{H} 24 \mathrm{~S} 1$ & 7.27E-06 & Non-basic N1O3 & $\mathrm{C} 21 \mathrm{H} 27 \mathrm{~N} 1 \mathrm{O} 3$ & 7.01E-06 & Basic N1 & $\mathrm{C} 42 \mathrm{H} 33 \mathrm{~N} 1$ & $3.63 \mathrm{E}-06$ \\
\hline S1 & $\mathrm{C} 23 \mathrm{H} 26 \mathrm{~S} 1$ & $1.96 \mathrm{E}-06$ & Non-basic N1O3 & $\mathrm{C} 22 \mathrm{H} 29 \mathrm{~N} 1 \mathrm{O} 3$ & $1.29 \mathrm{E}-05$ & Basic N1 & $\mathrm{C} 43 \mathrm{H} 35 \mathrm{~N} 1$ & $3.47 \mathrm{E}-06$ \\
\hline S1 & $\mathrm{C} 24 \mathrm{H} 28 \mathrm{~S} 1$ & $1.61 \mathrm{E}-06$ & Non-basic N1O3 & $\mathrm{C} 23 \mathrm{H} 31 \mathrm{~N} 1 \mathrm{O} 3$ & $1.51 \mathrm{E}-05$ & Basic N1 & $\mathrm{C} 44 \mathrm{H} 37 \mathrm{~N} 1$ & $3.10 \mathrm{E}-06$ \\
\hline S1 & $\mathrm{C} 25 \mathrm{H} 30 \mathrm{~S} 1$ & $1.82 \mathrm{E}-06$ & Non-basic N1O3 & $\mathrm{C} 24 \mathrm{H} 33 \mathrm{~N} 1 \mathrm{O} 3$ & $1.77 \mathrm{E}-05$ & Basic N1 & $\mathrm{C} 36 \mathrm{H} 19 \mathrm{~N} 1$ & $2.94 \mathrm{E}-06$ \\
\hline S1 & $\mathrm{C} 26 \mathrm{H} 32 \mathrm{~S} 1$ & $6.24 \mathrm{E}-06$ & Non-basic N1O3 & $\mathrm{C} 25 \mathrm{H} 35 \mathrm{~N} 1 \mathrm{O} 3$ & $1.54 \mathrm{E}-05$ & Basic N1 & $\mathrm{C} 37 \mathrm{H} 21 \mathrm{~N} 1$ & $3.12 \mathrm{E}-06$ \\
\hline S1 & $\mathrm{C} 27 \mathrm{H} 34 \mathrm{~S} 1$ & $6.66 \mathrm{E}-06$ & Non-basic N1O3 & $\mathrm{C} 26 \mathrm{H} 37 \mathrm{~N} 1 \mathrm{O} 3$ & $2.38 \mathrm{E}-05$ & Basic N1 & $\mathrm{C} 38 \mathrm{H} 23 \mathrm{~N} 1$ & $8.46 \mathrm{E}-06$ \\
\hline S1 & $\mathrm{C} 28 \mathrm{H} 36 \mathrm{~S} 1$ & $4.80 \mathrm{E}-06$ & Non-basic N1O3 & $\mathrm{C} 27 \mathrm{H} 39 \mathrm{~N} 1 \mathrm{O} 3$ & $2.48 \mathrm{E}-05$ & Basic N1 & $\mathrm{C} 39 \mathrm{H} 25 \mathrm{~N} 1$ & $3.76 \mathrm{E}-06$ \\
\hline S1 & C29H38S1 & 4.83E-06 & Non-basic N1O3 & $\mathrm{C} 28 \mathrm{H} 41 \mathrm{~N} 1 \mathrm{O} 3$ & $3.69 \mathrm{E}-05$ & Basic N1 & $\mathrm{C} 40 \mathrm{H} 27 \mathrm{~N} 1$ & $3.24 \mathrm{E}-06$ \\
\hline S1 & $\mathrm{C} 30 \mathrm{H} 40 \mathrm{~S} 1$ & 4.95E-06 & Non-basic N1O3 & $\mathrm{C} 29 \mathrm{H} 43 \mathrm{~N} 1 \mathrm{O} 3$ & $3.73 \mathrm{E}-05$ & Basic N1 & $\mathrm{C} 41 \mathrm{H} 29 \mathrm{~N} 1$ & $1.58 \mathrm{E}-06$ \\
\hline S1 & $\mathrm{C} 31 \mathrm{H} 42 \mathrm{~S} 1$ & $2.25 \mathrm{E}-06$ & Non-basic N1O3 & $\mathrm{C} 30 \mathrm{H} 45 \mathrm{~N} 1 \mathrm{O} 3$ & $3.04 \mathrm{E}-05$ & Basic N1 & $\mathrm{C} 42 \mathrm{H} 31 \mathrm{~N} 1$ & $3.48 \mathrm{E}-07$ \\
\hline S1 & $\mathrm{C} 32 \mathrm{H} 44 \mathrm{~S} 1$ & $8.19 \mathrm{E}-06$ & Non-basic N1O3 & $\mathrm{C} 31 \mathrm{H} 47 \mathrm{~N} 1 \mathrm{O} 3$ & $3.21 \mathrm{E}-05$ & Basic N1 & $\mathrm{C} 43 \mathrm{H} 33 \mathrm{~N} 1$ & $3.68 \mathrm{E}-07$ \\
\hline S1 & $\mathrm{C} 33 \mathrm{H} 46 \mathrm{~S} 1$ & $1.37 \mathrm{E}-05$ & Non-basic N1O3 & $\mathrm{C} 32 \mathrm{H} 49 \mathrm{~N} 1 \mathrm{O} 3$ & $3.83 \mathrm{E}-05$ & Basic N1 & $\mathrm{C} 38 \mathrm{H} 21 \mathrm{~N} 1$ & $5.60 \mathrm{E}-06$ \\
\hline S1 & $\mathrm{C} 34 \mathrm{H} 48 \mathrm{~S} 1$ & $1.32 \mathrm{E}-05$ & Non-basic N1O3 & C33H51N1O3 & $2.56 \mathrm{E}-05$ & Basic N1 & $\mathrm{C} 39 \mathrm{H} 23 \mathrm{~N} 1$ & $1.31 \mathrm{E}-05$ \\
\hline S1 & $\mathrm{C} 35 \mathrm{H} 50 \mathrm{~S} 1$ & $5.16 \mathrm{E}-06$ & Non-basic N1O3 & C34H53N1O3 & $2.80 \mathrm{E}-05$ & Basic N1 & $\mathrm{C} 40 \mathrm{H} 25 \mathrm{~N} 1$ & 4.41E-05 \\
\hline S1 & $\mathrm{C} 36 \mathrm{H} 52 \mathrm{~S} 1$ & $1.42 \mathrm{E}-05$ & Non-basic N1O3 & C35H55N1O3 & $2.93 \mathrm{E}-05$ & Basic N1 & $\mathrm{C} 41 \mathrm{H} 27 \mathrm{~N} 1$ & $2.25 \mathrm{E}-05$ \\
\hline S1 & $\mathrm{C} 37 \mathrm{H} 54 \mathrm{~S} 1$ & 3.79E-06 & Non-basic N1O3 & $\mathrm{C} 36 \mathrm{H} 57 \mathrm{~N} 1 \mathrm{O} 3$ & $9.85 \mathrm{E}-06$ & Basic N1 & $\mathrm{C} 42 \mathrm{H} 29 \mathrm{~N} 1$ & $1.50 \mathrm{E}-05$ \\
\hline S1 & C15H8S1 & $1.32 \mathrm{E}-06$ & Non-basic N1O3 & C37H59N1O3 & $1.06 \mathrm{E}-05$ & Basic N1 & $\mathrm{C} 43 \mathrm{H} 31 \mathrm{~N} 1$ & $6.41 \mathrm{E}-06$ \\
\hline S1 & C16H10S1 & $2.80 \mathrm{E}-06$ & Non-basic N1O3 & C38H61N1O3 & $4.89 \mathrm{E}-06$ & Basic N1 & $\mathrm{C} 44 \mathrm{H} 33 \mathrm{~N} 1$ & $3.46 \mathrm{E}-06$ \\
\hline S1 & $\mathrm{C} 17 \mathrm{H} 12 \mathrm{~S} 1$ & 4.80E-05 & Non-basic N1O3 & C39H63N1O3 & $7.21 \mathrm{E}-06$ & Basic N1 & $\mathrm{C} 39 \mathrm{H} 21 \mathrm{~N} 1$ & $1.49 \mathrm{E}-06$ \\
\hline S1 & $\mathrm{C} 18 \mathrm{H} 14 \mathrm{~S} 1$ & 0.000119 & Non-basic N1O3 & C14H11N1O3 & $3.36 \mathrm{E}-06$ & Basic N1 & $\mathrm{C} 40 \mathrm{H} 23 \mathrm{~N} 1$ & $3.81 \mathrm{E}-07$ \\
\hline S1 & C19H16S1 & 0.000157 & Non-basic N1O3 & C15H13N1O3 & $3.46 \mathrm{E}-06$ & Basic N1 & $\mathrm{C} 41 \mathrm{H} 25 \mathrm{~N} 1$ & $3.13 \mathrm{E}-06$ \\
\hline S1 & $\mathrm{C} 20 \mathrm{H} 18 \mathrm{~S} 1$ & 0.000128 & Non-basic N1O3 & C16H15N1O3 & $8.26 \mathrm{E}-06$ & Basic N1 & $\mathrm{C} 42 \mathrm{H} 27 \mathrm{~N} 1$ & $2.01 \mathrm{E}-06$ \\
\hline S1 & $\mathrm{C} 21 \mathrm{H} 20 \mathrm{~S} 1$ & $6.02 \mathrm{E}-05$ & Non-basic N1O3 & $\mathrm{C} 17 \mathrm{H} 17 \mathrm{~N} 1 \mathrm{O} 3$ & $1.90 \mathrm{E}-05$ & Basic N1 & $\mathrm{C} 43 \mathrm{H} 29 \mathrm{~N} 1$ & $3.22 \mathrm{E}-06$ \\
\hline S1 & $\mathrm{C} 22 \mathrm{H} 22 \mathrm{~S} 1$ & $2.46 \mathrm{E}-05$ & Non-basic N1O3 & C18H19N1O3 & $1.98 \mathrm{E}-05$ & Basic N1 & $\mathrm{C} 44 \mathrm{H} 31 \mathrm{~N} 1$ & $2.90 \mathrm{E}-06$ \\
\hline S1 & $\mathrm{C} 23 \mathrm{H} 24 \mathrm{~S} 1$ & $1.12 \mathrm{E}-05$ & Non-basic N1O3 & $\mathrm{C} 19 \mathrm{H} 21 \mathrm{~N} 1 \mathrm{O} 3$ & $1.97 \mathrm{E}-05$ & Basic N1 & $\mathrm{C} 45 \mathrm{H} 33 \mathrm{~N} 1$ & 3.37E-06 \\
\hline S1 & $\mathrm{C} 24 \mathrm{H} 26 \mathrm{~S} 1$ & $1.08 \mathrm{E}-05$ & Non-basic N1O3 & $\mathrm{C} 20 \mathrm{H} 23 \mathrm{~N} 1 \mathrm{O} 3$ & $1.60 \mathrm{E}-05$ & Basic N1 & $\mathrm{C} 40 \mathrm{H} 21 \mathrm{~N} 1$ & $4.83 \mathrm{E}-07$ \\
\hline S1 & $\mathrm{C} 25 \mathrm{H} 28 \mathrm{~S} 1$ & 4.65E-06 & Non-basic N1O3 & $\mathrm{C} 21 \mathrm{H} 25 \mathrm{~N} 1 \mathrm{O} 3$ & $1.85 \mathrm{E}-05$ & Basic N1 & $\mathrm{C} 41 \mathrm{H} 23 \mathrm{~N} 1$ & 4.77E-06 \\
\hline S1 & $\mathrm{C} 26 \mathrm{H} 30 \mathrm{~S} 1$ & 4.35E-06 & Non-basic N1O3 & $\mathrm{C} 22 \mathrm{H} 27 \mathrm{~N} 1 \mathrm{O} 3$ & $2.19 \mathrm{E}-05$ & Basic N1 & $\mathrm{C} 42 \mathrm{H} 25 \mathrm{~N} 1$ & $6.46 \mathrm{E}-06$ \\
\hline S1 & $\mathrm{C} 27 \mathrm{H} 32 \mathrm{~S} 1$ & $1.11 \mathrm{E}-05$ & Non-basic N1O3 & $\mathrm{C} 23 \mathrm{H} 29 \mathrm{~N} 1 \mathrm{O} 3$ & $2.76 \mathrm{E}-05$ & Basic N1 & $\mathrm{C} 43 \mathrm{H} 27 \mathrm{~N} 1$ & $8.52 \mathrm{E}-06$ \\
\hline S1 & $\mathrm{C} 28 \mathrm{H} 34 \mathrm{~S} 1$ & $1.22 \mathrm{E}-05$ & Non-basic N1O3 & $\mathrm{C} 24 \mathrm{H} 31 \mathrm{~N} 1 \mathrm{O} 3$ & $2.02 \mathrm{E}-05$ & Basic N1 & $\mathrm{C} 44 \mathrm{H} 29 \mathrm{~N} 1$ & $5.55 \mathrm{E}-06$ \\
\hline S1 & $\mathrm{C} 29 \mathrm{H} 36 \mathrm{~S} 1$ & $1.80 \mathrm{E}-05$ & Non-basic N1O3 & $\mathrm{C} 25 \mathrm{H} 33 \mathrm{~N} 1 \mathrm{O} 3$ & $2.08 \mathrm{E}-05$ & Basic N1 & $\mathrm{C} 45 \mathrm{H} 31 \mathrm{~N} 1$ & $4.39 \mathrm{E}-06$ \\
\hline S1 & $\mathrm{C} 30 \mathrm{H} 38 \mathrm{~S} 1$ & $1.51 \mathrm{E}-05$ & Non-basic N1O3 & $\mathrm{C} 26 \mathrm{H} 35 \mathrm{~N} 1 \mathrm{O} 3$ & $2.28 \mathrm{E}-05$ & Basic N1 & $\mathrm{C} 46 \mathrm{H} 33 \mathrm{~N} 1$ & $6.12 \mathrm{E}-07$ \\
\hline S1 & $\mathrm{C} 31 \mathrm{H} 40 \mathrm{~S} 1$ & $9.81 \mathrm{E}-06$ & Non-basic N1O3 & $\mathrm{C} 27 \mathrm{H} 37 \mathrm{~N} 1 \mathrm{O} 3$ & $2.92 \mathrm{E}-05$ & Basic N1 & $\mathrm{C} 47 \mathrm{H} 35 \mathrm{~N} 1$ & $2.79 \mathrm{E}-06$ \\
\hline S1 & $\mathrm{C} 32 \mathrm{H} 42 \mathrm{~S} 1$ & $1.10 \mathrm{E}-05$ & Non-basic N1O3 & $\mathrm{C} 28 \mathrm{H} 39 \mathrm{~N} 1 \mathrm{O} 3$ & $3.20 \mathrm{E}-05$ & Basic N1 & $\mathrm{C} 42 \mathrm{H} 23 \mathrm{~N} 1$ & $9.57 \mathrm{E}-07$ \\
\hline S1 & $\mathrm{C} 33 \mathrm{H} 44 \mathrm{~S} 1$ & $3.77 \mathrm{E}-06$ & Non-basic N1O3 & $\mathrm{C} 29 \mathrm{H} 41 \mathrm{~N} 1 \mathrm{O} 3$ & $3.08 \mathrm{E}-05$ & Basic N1 & $\mathrm{C} 43 \mathrm{H} 25 \mathrm{~N} 1$ & $2.38 \mathrm{E}-06$ \\
\hline
\end{tabular}




\begin{tabular}{|c|c|c|c|c|c|c|c|c|}
\hline Class & Formula & Mass & Class & Formula & Mass & Class & Formula & Mass \\
\hline S1 & $\mathrm{C} 34 \mathrm{H} 46 \mathrm{~S} 1$ & $2.67 \mathrm{E}-06$ & Non-basic N1O3 & $\mathrm{C} 30 \mathrm{H} 43 \mathrm{~N} 1 \mathrm{O} 3$ & $3.47 \mathrm{E}-05$ & Basic N1 & $\mathrm{C} 44 \mathrm{H} 27 \mathrm{~N} 1$ & $7.91 \mathrm{E}-06$ \\
\hline S1 & $\mathrm{C} 35 \mathrm{H} 48 \mathrm{~S} 1$ & $1.64 \mathrm{E}-05$ & Non-basic N1O3 & $\mathrm{C} 31 \mathrm{H} 45 \mathrm{~N} 1 \mathrm{O} 3$ & $3.78 \mathrm{E}-05$ & Basic N1 & $\mathrm{C} 45 \mathrm{H} 29 \mathrm{~N} 1$ & $9.53 \mathrm{E}-06$ \\
\hline S1 & $\mathrm{C} 36 \mathrm{H} 50 \mathrm{~S} 1$ & $6.79 \mathrm{E}-06$ & Non-basic N1O3 & $\mathrm{C} 32 \mathrm{H} 47 \mathrm{~N} 1 \mathrm{O} 3$ & $3.68 \mathrm{E}-05$ & Basic N1 & $\mathrm{C} 46 \mathrm{H} 31 \mathrm{~N} 1$ & $5.14 \mathrm{E}-06$ \\
\hline S1 & $\mathrm{C} 37 \mathrm{H} 52 \mathrm{~S} 1$ & $2.04 \mathrm{E}-05$ & Non-basic N1O3 & C33H49N1O3 & $2.32 \mathrm{E}-05$ & Basic N1 & $\mathrm{C} 47 \mathrm{H} 33 \mathrm{~N} 1$ & $3.99 \mathrm{E}-05$ \\
\hline S1 & $\mathrm{C} 38 \mathrm{H} 54 \mathrm{~S} 1$ & $9.34 \mathrm{E}-06$ & Non-basic N1O3 & $\mathrm{C} 34 \mathrm{H} 51 \mathrm{~N} 1 \mathrm{O} 3$ & $3.06 \mathrm{E}-05$ & Basic N1 & $\mathrm{C} 48 \mathrm{H} 35 \mathrm{~N} 1$ & $1.92 \mathrm{E}-06$ \\
\hline S1 & $\mathrm{C} 39 \mathrm{H} 56 \mathrm{~S} 1$ & $5.17 \mathrm{E}-06$ & Non-basic N1O3 & $\mathrm{C} 35 \mathrm{H} 53 \mathrm{~N} 1 \mathrm{O} 3$ & $3.13 \mathrm{E}-05$ & Basic N1 & $\mathrm{C} 49 \mathrm{H} 37 \mathrm{~N} 1$ & $1.56 \mathrm{E}-06$ \\
\hline S1 & $\mathrm{C} 40 \mathrm{H} 58 \mathrm{~S} 1$ & $5.40 \mathrm{E}-06$ & Non-basic N1O3 & $\mathrm{C} 36 \mathrm{H} 55 \mathrm{~N} 1 \mathrm{O} 3$ & $1.96 \mathrm{E}-05$ & Basic N1O1 & $\mathrm{C} 18 \mathrm{H} 31 \mathrm{~N} 1 \mathrm{O} 1$ & $3.19 \mathrm{E}-07$ \\
\hline S1 & C17H10S1 & $1.09 \mathrm{E}-06$ & Non-basic N1O3 & C37H57N1O3 & $1.88 \mathrm{E}-05$ & Basic N1O1 & C19H33N1O1 & $9.92 \mathrm{E}-08$ \\
\hline S1 & $\mathrm{C} 18 \mathrm{H} 12 \mathrm{~S} 1$ & $2.74 \mathrm{E}-06$ & Non-basic N1O3 & $\mathrm{C} 38 \mathrm{H} 59 \mathrm{~N} 1 \mathrm{O} 3$ & $8.49 \mathrm{E}-06$ & Basic N1O1 & $\mathrm{C} 20 \mathrm{H} 35 \mathrm{~N} 1 \mathrm{O} 1$ & $1.25 \mathrm{E}-07$ \\
\hline S1 & $\mathrm{C} 19 \mathrm{H} 14 \mathrm{~S} 1$ & $2.14 \mathrm{E}-05$ & Non-basic N1O3 & C39H61N1O3 & $3.75 \mathrm{E}-06$ & Basic N1O1 & $\mathrm{C} 21 \mathrm{H} 37 \mathrm{~N} 1 \mathrm{O} 1$ & $1.50 \mathrm{E}-06$ \\
\hline S1 & $\mathrm{C} 20 \mathrm{H} 16 \mathrm{~S} 1$ & 4.41E-05 & Non-basic N1O3 & $\mathrm{C} 40 \mathrm{H} 63 \mathrm{~N} 1 \mathrm{O} 3$ & $3.25 \mathrm{E}-06$ & Basic N1O1 & $\mathrm{C} 22 \mathrm{H} 39 \mathrm{~N} 1 \mathrm{O} 1$ & $8.31 \mathrm{E}-07$ \\
\hline S1 & $\mathrm{C} 21 \mathrm{H} 18 \mathrm{~S} 1$ & $6.08 \mathrm{E}-05$ & Non-basic N1O3 & $\mathrm{C} 41 \mathrm{H} 65 \mathrm{~N} 1 \mathrm{O} 3$ & $2.84 \mathrm{E}-06$ & Basic N1O1 & $\mathrm{C} 23 \mathrm{H} 41 \mathrm{~N} 1 \mathrm{O} 1$ & $1.45 \mathrm{E}-06$ \\
\hline S1 & $\mathrm{C} 22 \mathrm{H} 20 \mathrm{~S} 1$ & $6.06 \mathrm{E}-05$ & Non-basic N1O3 & C15H11N1O3 & $5.50 \mathrm{E}-06$ & Basic N1O1 & $\mathrm{C} 24 \mathrm{H} 43 \mathrm{~N} 1 \mathrm{O} 1$ & $2.45 \mathrm{E}-06$ \\
\hline S1 & $\mathrm{C} 23 \mathrm{H} 22 \mathrm{~S} 1$ & $5.13 \mathrm{E}-05$ & Non-basic N1O3 & $\mathrm{C} 16 \mathrm{H} 13 \mathrm{~N} 1 \mathrm{O} 3$ & $1.56 \mathrm{E}-05$ & Basic N1O1 & $\mathrm{C} 25 \mathrm{H} 45 \mathrm{~N} 1 \mathrm{O} 1$ & $1.97 \mathrm{E}-06$ \\
\hline S1 & $\mathrm{C} 24 \mathrm{H} 24 \mathrm{~S} 1$ & $1.77 \mathrm{E}-05$ & Non-basic N1O3 & $\mathrm{C} 17 \mathrm{H} 15 \mathrm{~N} 1 \mathrm{O} 3$ & $2.70 \mathrm{E}-05$ & Basic N1O1 & $\mathrm{C} 26 \mathrm{H} 47 \mathrm{~N} 1 \mathrm{O} 1$ & 7.00E-06 \\
\hline S1 & $\mathrm{C} 25 \mathrm{H} 26 \mathrm{~S} 1$ & $8.03 \mathrm{E}-06$ & Non-basic N1O3 & $\mathrm{C} 18 \mathrm{H} 17 \mathrm{~N} 1 \mathrm{O} 3$ & $5.87 \mathrm{E}-05$ & Basic N1O1 & $\mathrm{C} 27 \mathrm{H} 49 \mathrm{~N} 1 \mathrm{O} 1$ & 4.03E-06 \\
\hline S1 & $\mathrm{C} 26 \mathrm{H} 28 \mathrm{~S} 1$ & 7.14E-06 & Non-basic N1O3 & C19H19N1O3 & $6.74 \mathrm{E}-05$ & Basic N1O1 & $\mathrm{C} 28 \mathrm{H} 51 \mathrm{~N} 1 \mathrm{O} 1$ & $9.92 \mathrm{E}-06$ \\
\hline S1 & $\mathrm{C} 27 \mathrm{H} 30 \mathrm{~S} 1$ & $1.74 \mathrm{E}-05$ & Non-basic N1O3 & $\mathrm{C} 20 \mathrm{H} 21 \mathrm{~N} 1 \mathrm{O} 3$ & $6.16 \mathrm{E}-05$ & Basic N1O1 & $\mathrm{C} 29 \mathrm{H} 53 \mathrm{~N} 1 \mathrm{O} 1$ & $1.03 \mathrm{E}-05$ \\
\hline S1 & $\mathrm{C} 28 \mathrm{H} 32 \mathrm{~S} 1$ & $9.67 \mathrm{E}-06$ & Non-basic N1O3 & $\mathrm{C} 21 \mathrm{H} 23 \mathrm{~N} 1 \mathrm{O} 3$ & $4.29 \mathrm{E}-05$ & Basic N1O1 & $\mathrm{C} 30 \mathrm{H} 55 \mathrm{~N} 1 \mathrm{O} 1$ & $2.55 \mathrm{E}-05$ \\
\hline S1 & $\mathrm{C} 29 \mathrm{H} 34 \mathrm{~S} 1$ & $1.85 \mathrm{E}-05$ & Non-basic N1O3 & $\mathrm{C} 22 \mathrm{H} 25 \mathrm{~N} 1 \mathrm{O} 3$ & $4.00 \mathrm{E}-05$ & Basic N1O1 & $\mathrm{C} 31 \mathrm{H} 57 \mathrm{~N} 1 \mathrm{O} 1$ & $1.01 \mathrm{E}-05$ \\
\hline S1 & $\mathrm{C} 30 \mathrm{H} 36 \mathrm{~S} 1$ & $1.11 \mathrm{E}-05$ & Non-basic N1O3 & $\mathrm{C} 23 \mathrm{H} 27 \mathrm{~N} 1 \mathrm{O} 3$ & $2.24 \mathrm{E}-05$ & Basic N1O1 & C33H61N1O1 & $1.67 \mathrm{E}-06$ \\
\hline S1 & $\mathrm{C} 31 \mathrm{H} 38 \mathrm{~S} 1$ & $1.50 \mathrm{E}-05$ & Non-basic N1O3 & $\mathrm{C} 24 \mathrm{H} 29 \mathrm{~N} 1 \mathrm{O} 3$ & $2.44 \mathrm{E}-05$ & Basic N1O1 & $\mathrm{C} 34 \mathrm{H} 63 \mathrm{~N} 1 \mathrm{O} 1$ & $4.69 \mathrm{E}-05$ \\
\hline S1 & $\mathrm{C} 32 \mathrm{H} 40 \mathrm{~S} 1$ & $1.29 \mathrm{E}-05$ & Non-basic N1O3 & $\mathrm{C} 25 \mathrm{H} 31 \mathrm{~N} 1 \mathrm{O} 3$ & $2.49 \mathrm{E}-05$ & Basic N1O1 & $\mathrm{C} 35 \mathrm{H} 65 \mathrm{~N} 1 \mathrm{O} 1$ & $5.32 \mathrm{E}-06$ \\
\hline S1 & $\mathrm{C} 33 \mathrm{H} 42 \mathrm{~S} 1$ & $1.17 \mathrm{E}-05$ & Non-basic N1O3 & $\mathrm{C} 26 \mathrm{H} 33 \mathrm{~N} 1 \mathrm{O} 3$ & $2.98 \mathrm{E}-05$ & Basic N1O1 & $\mathrm{C} 36 \mathrm{H} 67 \mathrm{~N} 1 \mathrm{O} 1$ & $2.77 \mathrm{E}-05$ \\
\hline S1 & $\mathrm{C} 34 \mathrm{H} 44 \mathrm{~S} 1$ & $2.78 \mathrm{E}-05$ & Non-basic N1O3 & $\mathrm{C} 27 \mathrm{H} 35 \mathrm{~N} 1 \mathrm{O} 3$ & $2.67 \mathrm{E}-05$ & Basic N1O1 & C37H69N1O1 & $2.59 \mathrm{E}-06$ \\
\hline S1 & $\mathrm{C} 35 \mathrm{H} 46 \mathrm{~S} 1$ & $2.80 \mathrm{E}-05$ & Non-basic N1O3 & $\mathrm{C} 28 \mathrm{H} 37 \mathrm{~N} 1 \mathrm{O} 3$ & $4.40 \mathrm{E}-05$ & Basic N1O1 & $\mathrm{C} 16 \mathrm{H} 25 \mathrm{~N} 1 \mathrm{O} 1$ & $2.46 \mathrm{E}-07$ \\
\hline S1 & $\mathrm{C} 36 \mathrm{H} 48 \mathrm{~S} 1$ & $3.85 \mathrm{E}-05$ & Non-basic N1O3 & $\mathrm{C} 29 \mathrm{H} 39 \mathrm{~N} 1 \mathrm{O} 3$ & $3.27 \mathrm{E}-05$ & Basic N1O1 & $\mathrm{C} 17 \mathrm{H} 27 \mathrm{~N} 1 \mathrm{O} 1$ & $1.40 \mathrm{E}-07$ \\
\hline S1 & C37H50S1 & $1.94 \mathrm{E}-05$ & Non-basic N1O3 & $\mathrm{C} 30 \mathrm{H} 41 \mathrm{~N} 1 \mathrm{O} 3$ & $3.64 \mathrm{E}-05$ & Basic N1O1 & $\mathrm{C} 18 \mathrm{H} 29 \mathrm{~N} 1 \mathrm{O} 1$ & $1.04 \mathrm{E}-06$ \\
\hline S1 & $\mathrm{C} 38 \mathrm{H} 52 \mathrm{~S} 1$ & $2.93 \mathrm{E}-05$ & Non-basic N1O3 & $\mathrm{C} 31 \mathrm{H} 43 \mathrm{~N} 1 \mathrm{O} 3$ & $3.02 \mathrm{E}-05$ & Basic N1O1 & $\mathrm{C} 19 \mathrm{H} 31 \mathrm{~N} 1 \mathrm{O} 1$ & $9.99 \mathrm{E}-07$ \\
\hline S1 & $\mathrm{C} 39 \mathrm{H} 54 \mathrm{~S} 1$ & 7.92E-06 & Non-basic N1O3 & $\mathrm{C} 32 \mathrm{H} 45 \mathrm{~N} 1 \mathrm{O} 3$ & $3.62 \mathrm{E}-05$ & Basic N1O1 & $\mathrm{C} 20 \mathrm{H} 33 \mathrm{~N} 1 \mathrm{O} 1$ & $2.94 \mathrm{E}-06$ \\
\hline S1 & $\mathrm{C} 40 \mathrm{H} 56 \mathrm{~S} 1$ & $6.71 \mathrm{E}-06$ & Non-basic N1O3 & $\mathrm{C} 33 \mathrm{H} 47 \mathrm{~N} 1 \mathrm{O} 3$ & $3.47 \mathrm{E}-05$ & Basic N1O1 & $\mathrm{C} 21 \mathrm{H} 35 \mathrm{~N} 1 \mathrm{O} 1$ & $2.00 \mathrm{E}-06$ \\
\hline S1 & C18H10S1 & $5.66 \mathrm{E}-06$ & Non-basic N1O3 & $\mathrm{C} 34 \mathrm{H} 49 \mathrm{~N} 1 \mathrm{O} 3$ & $3.01 \mathrm{E}-05$ & Basic N1O1 & $\mathrm{C} 22 \mathrm{H} 37 \mathrm{~N} 1 \mathrm{O} 1$ & $4.25 \mathrm{E}-06$ \\
\hline S1 & $\mathrm{C} 19 \mathrm{H} 12 \mathrm{~S} 1$ & $5.82 \mathrm{E}-05$ & Non-basic N1O3 & $\mathrm{C} 35 \mathrm{H} 51 \mathrm{~N} 1 \mathrm{O} 3$ & $2.71 \mathrm{E}-05$ & Basic N1O1 & $\mathrm{C} 23 \mathrm{H} 39 \mathrm{~N} 1 \mathrm{O} 1$ & $6.75 \mathrm{E}-06$ \\
\hline S1 & $\mathrm{C} 20 \mathrm{H} 14 \mathrm{~S} 1$ & 0.000118 & Non-basic N1O3 & $\mathrm{C} 36 \mathrm{H} 53 \mathrm{~N} 1 \mathrm{O} 3$ & $2.35 \mathrm{E}-05$ & Basic N1O1 & $\mathrm{C} 24 \mathrm{H} 41 \mathrm{~N} 1 \mathrm{O} 1$ & $8.82 \mathrm{E}-06$ \\
\hline $\mathrm{S} 1$ & $\mathrm{C} 21 \mathrm{H} 16 \mathrm{~S} 1$ & 0.000157 & Non-basic N1O3 & $\mathrm{C} 37 \mathrm{H} 55 \mathrm{~N} 1 \mathrm{O} 3$ & $2.29 \mathrm{E}-05$ & Basic N1O1 & $\mathrm{C} 25 \mathrm{H} 43 \mathrm{~N} 1 \mathrm{O} 1$ & $5.65 \mathrm{E}-06$ \\
\hline S1 & $\mathrm{C} 22 \mathrm{H} 18 \mathrm{~S} 1$ & 0.000122 & Non-basic N1O3 & $\mathrm{C} 38 \mathrm{H} 57 \mathrm{~N} 1 \mathrm{O} 3$ & $9.42 \mathrm{E}-06$ & Basic N1O1 & $\mathrm{C} 26 \mathrm{H} 45 \mathrm{~N} 1 \mathrm{O} 1$ & $9.55 \mathrm{E}-06$ \\
\hline S1 & $\mathrm{C} 23 \mathrm{H} 20 \mathrm{~S} 1$ & $9.08 \mathrm{E}-05$ & Non-basic N1O3 & $\mathrm{C} 39 \mathrm{H} 59 \mathrm{~N} 1 \mathrm{O} 3$ & $7.16 \mathrm{E}-06$ & Basic N1O1 & $\mathrm{C} 27 \mathrm{H} 47 \mathrm{~N} 1 \mathrm{O} 1$ & $1.74 \mathrm{E}-05$ \\
\hline $\mathrm{S} 1$ & $\mathrm{C} 24 \mathrm{H} 22 \mathrm{~S} 1$ & $5.99 \mathrm{E}-05$ & Non-basic N1O3 & $\mathrm{C} 40 \mathrm{H} 61 \mathrm{~N} 1 \mathrm{O} 3$ & $9.04 \mathrm{E}-06$ & Basic N1O1 & $\mathrm{C} 28 \mathrm{H} 49 \mathrm{~N} 1 \mathrm{O} 1$ & $2.05 \mathrm{E}-05$ \\
\hline
\end{tabular}




\begin{tabular}{|c|c|c|c|c|c|c|c|c|}
\hline Class & Formula & Mass & Class & Formula & Mass & Class & Formula & Mass \\
\hline S1 & $\mathrm{C} 25 \mathrm{H} 24 \mathrm{~S} 1$ & $1.98 \mathrm{E}-05$ & Non-basic N1O3 & C15H9N1O3 & $1.18 \mathrm{E}-06$ & Basic N1O1 & C29H51N1O1 & $1.61 \mathrm{E}-05$ \\
\hline S1 & $\mathrm{C} 26 \mathrm{H} 26 \mathrm{~S} 1$ & $1.78 \mathrm{E}-05$ & Non-basic N1O3 & C16H11N1O3 & $1.76 \mathrm{E}-05$ & Basic N1O1 & C30H53N1O1 & $1.65 \mathrm{E}-05$ \\
\hline S1 & $\mathrm{C} 27 \mathrm{H} 28 \mathrm{~S} 1$ & $9.47 \mathrm{E}-06$ & Non-basic N1O3 & C17H13N1O3 & 4.64E-05 & Basic N1O1 & $\mathrm{C} 31 \mathrm{H} 55 \mathrm{~N} 1 \mathrm{O} 1$ & $1.50 \mathrm{E}-05$ \\
\hline S1 & $\mathrm{C} 28 \mathrm{H} 30 \mathrm{~S} 1$ & $1.32 \mathrm{E}-05$ & Non-basic N1O3 & C18H15N1O3 & 0.00014 & Basic N1O1 & C32H57N1O1 & $1.46 \mathrm{E}-05$ \\
\hline S1 & $\mathrm{C} 29 \mathrm{H} 32 \mathrm{~S} 1$ & $1.13 \mathrm{E}-05$ & Non-basic N1O3 & C19H17N1O3 & 0.000237 & Basic N1O1 & C33H59N1O1 & $1.64 \mathrm{E}-05$ \\
\hline S1 & $\mathrm{C} 30 \mathrm{H} 34 \mathrm{~S} 1$ & $2.03 \mathrm{E}-05$ & Non-basic N1O3 & $\mathrm{C} 20 \mathrm{H} 19 \mathrm{~N} 1 \mathrm{O} 3$ & 0.000248 & Basic N1O1 & C34H61N1O1 & $1.36 \mathrm{E}-05$ \\
\hline S1 & $\mathrm{C} 31 \mathrm{H} 36 \mathrm{~S} 1$ & $1.64 \mathrm{E}-05$ & Non-basic N1O3 & $\mathrm{C} 21 \mathrm{H} 21 \mathrm{~N} 1 \mathrm{O} 3$ & 0.000163 & Basic N1O1 & C35H63N1O1 & $1.14 \mathrm{E}-05$ \\
\hline S1 & $\mathrm{C} 32 \mathrm{H} 38 \mathrm{~S} 1$ & $1.14 \mathrm{E}-05$ & Non-basic N1O3 & $\mathrm{C} 22 \mathrm{H} 23 \mathrm{~N} 1 \mathrm{O} 3$ & 0.000101 & Basic N1O1 & C36H65N1O1 & $1.20 \mathrm{E}-05$ \\
\hline S1 & $\mathrm{C} 33 \mathrm{H} 40 \mathrm{~S} 1$ & $1.38 \mathrm{E}-05$ & Non-basic N1O3 & $\mathrm{C} 23 \mathrm{H} 25 \mathrm{~N} 1 \mathrm{O} 3$ & $6.83 \mathrm{E}-05$ & Basic N1O1 & C37H67N1O1 & 7.02E-06 \\
\hline S1 & $\mathrm{C} 34 \mathrm{H} 42 \mathrm{~S} 1$ & $3.06 \mathrm{E}-05$ & Non-basic N1O3 & $\mathrm{C} 24 \mathrm{H} 27 \mathrm{~N} 1 \mathrm{O} 3$ & $5.83 \mathrm{E}-05$ & Basic N1O1 & C38H69N1O1 & $2.61 \mathrm{E}-06$ \\
\hline S1 & $\mathrm{C} 35 \mathrm{H} 44 \mathrm{~S} 1$ & $2.57 \mathrm{E}-05$ & Non-basic N1O3 & $\mathrm{C} 25 \mathrm{H} 29 \mathrm{~N} 1 \mathrm{O} 3$ & $3.72 \mathrm{E}-05$ & Basic N1O1 & C39H71N1O1 & $6.15 \mathrm{E}-06$ \\
\hline S1 & $\mathrm{C} 36 \mathrm{H} 46 \mathrm{~S} 1$ & $3.47 \mathrm{E}-05$ & Non-basic N1O3 & $\mathrm{C} 26 \mathrm{H} 31 \mathrm{~N} 1 \mathrm{O} 3$ & $3.52 \mathrm{E}-05$ & Basic N1O1 & $\mathrm{C} 18 \mathrm{H} 27 \mathrm{~N} 1 \mathrm{O} 1$ & $2.67 \mathrm{E}-07$ \\
\hline S1 & $\mathrm{C} 37 \mathrm{H} 48 \mathrm{~S} 1$ & $3.11 \mathrm{E}-05$ & Non-basic N1O3 & $\mathrm{C} 27 \mathrm{H} 33 \mathrm{~N} 1 \mathrm{O} 3$ & $3.96 \mathrm{E}-05$ & Basic N1O1 & C19H29N1O1 & $5.87 \mathrm{E}-07$ \\
\hline S1 & $\mathrm{C} 38 \mathrm{H} 50 \mathrm{~S} 1$ & $1.88 \mathrm{E}-05$ & Non-basic N1O3 & $\mathrm{C} 28 \mathrm{H} 35 \mathrm{~N} 1 \mathrm{O} 3$ & $4.05 \mathrm{E}-05$ & Basic N1O1 & $\mathrm{C} 20 \mathrm{H} 31 \mathrm{~N} 1 \mathrm{O} 1$ & $2.99 \mathrm{E}-06$ \\
\hline S1 & $\mathrm{C} 39 \mathrm{H} 52 \mathrm{~S} 1$ & $2.35 \mathrm{E}-05$ & Non-basic N1O3 & $\mathrm{C} 29 \mathrm{H} 37 \mathrm{~N} 1 \mathrm{O} 3$ & 4.36E-05 & Basic N1O1 & $\mathrm{C} 21 \mathrm{H} 33 \mathrm{~N} 1 \mathrm{O} 1$ & $3.62 \mathrm{E}-06$ \\
\hline $\mathrm{S} 1$ & $\mathrm{C} 40 \mathrm{H} 54 \mathrm{~S} 1$ & $1.49 \mathrm{E}-05$ & Non-basic N1O3 & $\mathrm{C} 30 \mathrm{H} 39 \mathrm{~N} 1 \mathrm{O} 3$ & $3.54 \mathrm{E}-05$ & Basic N1O1 & $\mathrm{C} 22 \mathrm{H} 35 \mathrm{~N} 1 \mathrm{O} 1$ & $8.58 \mathrm{E}-06$ \\
\hline S1 & $\mathrm{C} 41 \mathrm{H} 56 \mathrm{~S} 1$ & $1.84 \mathrm{E}-05$ & Non-basic N1O3 & $\mathrm{C} 31 \mathrm{H} 41 \mathrm{~N} 1 \mathrm{O} 3$ & $3.89 \mathrm{E}-05$ & Basic N1O1 & $\mathrm{C} 23 \mathrm{H} 37 \mathrm{~N} 1 \mathrm{O} 1$ & $2.38 \mathrm{E}-05$ \\
\hline S1 & $\mathrm{C} 42 \mathrm{H} 58 \mathrm{~S} 1$ & 7.64E-06 & Non-basic N1O3 & $\mathrm{C} 32 \mathrm{H} 43 \mathrm{~N} 1 \mathrm{O} 3$ & $4.05 \mathrm{E}-05$ & Basic N1O1 & $\mathrm{C} 24 \mathrm{H} 39 \mathrm{~N} 1 \mathrm{O} 1$ & $1.15 \mathrm{E}-05$ \\
\hline S1 & $\mathrm{C} 20 \mathrm{H} 12 \mathrm{~S} 1$ & $5.07 \mathrm{E}-06$ & Non-basic N1O3 & $\mathrm{C} 33 \mathrm{H} 45 \mathrm{~N} 1 \mathrm{O} 3$ & 4.99E-05 & Basic N1O1 & $\mathrm{C} 25 \mathrm{H} 41 \mathrm{~N} 1 \mathrm{O} 1$ & $1.61 \mathrm{E}-05$ \\
\hline S1 & $\mathrm{C} 21 \mathrm{H} 14 \mathrm{~S} 1$ & $7.25 \mathrm{E}-05$ & Non-basic N1O3 & $\mathrm{C} 34 \mathrm{H} 47 \mathrm{~N} 1 \mathrm{O} 3$ & $3.32 \mathrm{E}-05$ & Basic N1O1 & $\mathrm{C} 26 \mathrm{H} 43 \mathrm{~N} 1 \mathrm{O} 1$ & $1.51 \mathrm{E}-05$ \\
\hline S1 & $\mathrm{C} 22 \mathrm{H} 16 \mathrm{~S} 1$ & 0.000167 & Non-basic N1O3 & $\mathrm{C} 35 \mathrm{H} 49 \mathrm{~N} 1 \mathrm{O} 3$ & 2.99E-05 & Basic N1O1 & $\mathrm{C} 27 \mathrm{H} 45 \mathrm{~N} 1 \mathrm{O} 1$ & $2.21 \mathrm{E}-05$ \\
\hline S1 & $\mathrm{C} 23 \mathrm{H} 18 \mathrm{~S} 1$ & 0.000186 & Non-basic N1O3 & $\mathrm{C} 36 \mathrm{H} 51 \mathrm{~N} 1 \mathrm{O} 3$ & $3.02 \mathrm{E}-05$ & Basic N1O1 & $\mathrm{C} 28 \mathrm{H} 47 \mathrm{~N} 1 \mathrm{O} 1$ & $1.88 \mathrm{E}-05$ \\
\hline S1 & $\mathrm{C} 24 \mathrm{H} 20 \mathrm{~S} 1$ & 0.000121 & Non-basic N1O3 & C37H53N1O3 & $1.81 \mathrm{E}-05$ & Basic N1O1 & $\mathrm{C} 29 \mathrm{H} 49 \mathrm{~N} 1 \mathrm{O} 1$ & $2.69 \mathrm{E}-05$ \\
\hline S1 & $\mathrm{C} 25 \mathrm{H} 22 \mathrm{~S} 1$ & $8.12 \mathrm{E}-05$ & Non-basic N1O3 & C38H55N1O3 & $1.66 \mathrm{E}-05$ & Basic N1O1 & C30H51N1O1 & $3.42 \mathrm{E}-05$ \\
\hline S1 & $\mathrm{C} 26 \mathrm{H} 24 \mathrm{~S} 1$ & $4.28 \mathrm{E}-05$ & Non-basic N1O3 & $\mathrm{C} 39 \mathrm{H} 57 \mathrm{~N} 1 \mathrm{O} 3$ & 4.91E-06 & Basic N1O1 & $\mathrm{C} 31 \mathrm{H} 53 \mathrm{~N} 1 \mathrm{O} 1$ & $3.80 \mathrm{E}-05$ \\
\hline S1 & $\mathrm{C} 27 \mathrm{H} 26 \mathrm{~S} 1$ & $1.32 \mathrm{E}-05$ & Non-basic N1O3 & $\mathrm{C} 40 \mathrm{H} 59 \mathrm{~N} 1 \mathrm{O} 3$ & $8.18 \mathrm{E}-06$ & Basic N1O1 & $\mathrm{C} 32 \mathrm{H} 55 \mathrm{~N} 1 \mathrm{O} 1$ & $2.09 \mathrm{E}-05$ \\
\hline S1 & $\mathrm{C} 28 \mathrm{H} 28 \mathrm{~S} 1$ & $1.59 \mathrm{E}-05$ & Non-basic N1O3 & $\mathrm{C} 41 \mathrm{H} 61 \mathrm{~N} 1 \mathrm{O} 3$ & $2.02 \mathrm{E}-06$ & Basic N1O1 & $\mathrm{C} 33 \mathrm{H} 57 \mathrm{~N} 1 \mathrm{O} 1$ & 4.49E-05 \\
\hline S1 & $\mathrm{C} 29 \mathrm{H} 30 \mathrm{~S} 1$ & $4.23 \mathrm{E}-06$ & Non-basic N1O3 & $\mathrm{C} 42 \mathrm{H} 63 \mathrm{~N} 1 \mathrm{O} 3$ & 4.60E-06 & Basic N1O1 & $\mathrm{C} 18 \mathrm{H} 25 \mathrm{~N} 1 \mathrm{O} 1$ & $1.35 \mathrm{E}-07$ \\
\hline S1 & $\mathrm{C} 30 \mathrm{H} 32 \mathrm{~S} 1$ & $1.44 \mathrm{E}-05$ & Non-basic N1O3 & C16H9N1O3 & $6.65 \mathrm{E}-06$ & Basic N1O1 & $\mathrm{C} 19 \mathrm{H} 27 \mathrm{~N} 1 \mathrm{O} 1$ & $1.30 \mathrm{E}-06$ \\
\hline S1 & $\mathrm{C} 31 \mathrm{H} 34 \mathrm{~S} 1$ & $2.69 \mathrm{E}-05$ & Non-basic N1O3 & $\mathrm{C} 17 \mathrm{H} 11 \mathrm{~N} 1 \mathrm{O} 3$ & $2.45 \mathrm{E}-05$ & Basic N1O1 & $\mathrm{C} 20 \mathrm{H} 29 \mathrm{~N} 1 \mathrm{O} 1$ & $2.91 \mathrm{E}-06$ \\
\hline S1 & $\mathrm{C} 32 \mathrm{H} 36 \mathrm{~S} 1$ & $1.32 \mathrm{E}-05$ & Non-basic N1O3 & $\mathrm{C} 18 \mathrm{H} 13 \mathrm{~N} 1 \mathrm{O} 3$ & $5.99 \mathrm{E}-05$ & Basic N1O1 & $\mathrm{C} 21 \mathrm{H} 31 \mathrm{~N} 1 \mathrm{O} 1$ & $7.81 \mathrm{E}-06$ \\
\hline S1 & $\mathrm{C} 33 \mathrm{H} 38 \mathrm{~S} 1$ & $8.21 \mathrm{E}-06$ & Non-basic N1O3 & C19H15N1O3 & 0.000124 & Basic N1O1 & $\mathrm{C} 22 \mathrm{H} 33 \mathrm{~N} 1 \mathrm{O} 1$ & $1.23 \mathrm{E}-05$ \\
\hline S1 & $\mathrm{C} 34 \mathrm{H} 40 \mathrm{~S} 1$ & $9.33 \mathrm{E}-06$ & Non-basic N1O3 & $\mathrm{C} 20 \mathrm{H} 17 \mathrm{~N} 1 \mathrm{O} 3$ & 0.000157 & Basic N1O1 & $\mathrm{C} 23 \mathrm{H} 35 \mathrm{~N} 1 \mathrm{O} 1$ & $1.07 \mathrm{E}-05$ \\
\hline S1 & $\mathrm{C} 35 \mathrm{H} 42 \mathrm{~S} 1$ & $2.78 \mathrm{E}-05$ & Non-basic N1O3 & $\mathrm{C} 21 \mathrm{H} 19 \mathrm{~N} 1 \mathrm{O} 3$ & 0.000172 & Basic N1O1 & $\mathrm{C} 24 \mathrm{H} 37 \mathrm{~N} 1 \mathrm{O} 1$ & $1.71 \mathrm{E}-05$ \\
\hline $\mathrm{S} 1$ & $\mathrm{C} 36 \mathrm{H} 44 \mathrm{~S} 1$ & $2.00 \mathrm{E}-05$ & Non-basic N1O3 & $\mathrm{C} 22 \mathrm{H} 21 \mathrm{~N} 1 \mathrm{O} 3$ & 0.000175 & Basic N1O1 & $\mathrm{C} 25 \mathrm{H} 39 \mathrm{~N} 1 \mathrm{O} 1$ & $2.23 \mathrm{E}-05$ \\
\hline $\mathrm{S} 1$ & $\mathrm{C} 37 \mathrm{H} 46 \mathrm{~S} 1$ & $2.20 \mathrm{E}-05$ & Non-basic N1O3 & $\mathrm{C} 23 \mathrm{H} 23 \mathrm{~N} 1 \mathrm{O} 3$ & 0.000148 & Basic N1O1 & $\mathrm{C} 26 \mathrm{H} 41 \mathrm{~N} 1 \mathrm{O} 1$ & $2.22 \mathrm{E}-05$ \\
\hline S1 & $\mathrm{C} 38 \mathrm{H} 48 \mathrm{~S} 1$ & $2.91 \mathrm{E}-05$ & Non-basic N1O3 & $\mathrm{C} 24 \mathrm{H} 25 \mathrm{~N} 1 \mathrm{O} 3$ & $9.45 \mathrm{E}-05$ & Basic N1O1 & $\mathrm{C} 27 \mathrm{H} 43 \mathrm{~N} 1 \mathrm{O} 1$ & $1.89 \mathrm{E}-05$ \\
\hline S1 & C39H50S1 & $2.81 \mathrm{E}-05$ & Non-basic N1O3 & $\mathrm{C} 25 \mathrm{H} 27 \mathrm{~N} 1 \mathrm{O} 3$ & $8.03 \mathrm{E}-05$ & Basic N1O1 & $\mathrm{C} 28 \mathrm{H} 45 \mathrm{~N} 1 \mathrm{O} 1$ & $2.52 \mathrm{E}-05$ \\
\hline
\end{tabular}




\begin{tabular}{|c|c|c|c|c|c|c|c|c|}
\hline Class & Formula & Mass & Class & Formula & Mass & Class & Formula & Mass \\
\hline S1 & $\mathrm{C} 40 \mathrm{H} 52 \mathrm{~S} 1$ & $2.39 \mathrm{E}-05$ & Non-basic N1O3 & $\mathrm{C} 26 \mathrm{H} 29 \mathrm{~N} 1 \mathrm{O} 3$ & $5.84 \mathrm{E}-05$ & Basic N1O1 & $\mathrm{C} 29 \mathrm{H} 47 \mathrm{~N} 1 \mathrm{O} 1$ & $3.18 \mathrm{E}-05$ \\
\hline S1 & $\mathrm{C} 41 \mathrm{H} 54 \mathrm{~S} 1$ & $2.06 \mathrm{E}-05$ & Non-basic N1O3 & $\mathrm{C} 27 \mathrm{H} 31 \mathrm{~N} 1 \mathrm{O} 3$ & $4.62 \mathrm{E}-05$ & Basic N1O1 & $\mathrm{C} 30 \mathrm{H} 49 \mathrm{~N} 1 \mathrm{O} 1$ & $2.98 \mathrm{E}-05$ \\
\hline S1 & $\mathrm{C} 42 \mathrm{H} 56 \mathrm{~S} 1$ & $2.26 \mathrm{E}-05$ & Non-basic N1O3 & $\mathrm{C} 28 \mathrm{H} 33 \mathrm{~N} 1 \mathrm{O} 3$ & $4.84 \mathrm{E}-05$ & Basic N1O1 & $\mathrm{C} 31 \mathrm{H} 51 \mathrm{~N} 1 \mathrm{O} 1$ & $3.57 \mathrm{E}-05$ \\
\hline S1 & C43H58S1 & $2.05 \mathrm{E}-05$ & Non-basic N1O3 & $\mathrm{C} 29 \mathrm{H} 35 \mathrm{~N} 1 \mathrm{O} 3$ & $5.38 \mathrm{E}-05$ & Basic N1O1 & $\mathrm{C} 32 \mathrm{H} 53 \mathrm{~N} 1 \mathrm{O} 1$ & $3.09 \mathrm{E}-05$ \\
\hline S1 & $\mathrm{C} 21 \mathrm{H} 12 \mathrm{~S} 1$ & $8.23 \mathrm{E}-06$ & Non-basic N1O3 & $\mathrm{C} 30 \mathrm{H} 37 \mathrm{~N} 1 \mathrm{O} 3$ & $4.35 \mathrm{E}-05$ & Basic N1O1 & C33H55N1O1 & $2.73 \mathrm{E}-05$ \\
\hline S1 & $\mathrm{C} 22 \mathrm{H} 14 \mathrm{~S} 1$ & $1.27 \mathrm{E}-05$ & Non-basic N1O3 & $\mathrm{C} 31 \mathrm{H} 39 \mathrm{~N} 1 \mathrm{O} 3$ & $4.03 \mathrm{E}-05$ & Basic N1O1 & $\mathrm{C} 34 \mathrm{H} 57 \mathrm{~N} 1 \mathrm{O} 1$ & $2.36 \mathrm{E}-05$ \\
\hline S1 & $\mathrm{C} 23 \mathrm{H} 16 \mathrm{~S} 1$ & $5.06 \mathrm{E}-05$ & Non-basic N1O3 & $\mathrm{C} 32 \mathrm{H} 41 \mathrm{~N} 1 \mathrm{O} 3$ & $4.72 \mathrm{E}-05$ & Basic N1O1 & $\mathrm{C} 35 \mathrm{H} 59 \mathrm{~N} 1 \mathrm{O} 1$ & $2.71 \mathrm{E}-05$ \\
\hline S1 & $\mathrm{C} 24 \mathrm{H} 18 \mathrm{~S} 1$ & $8.65 \mathrm{E}-05$ & Non-basic N1O3 & $\mathrm{C} 33 \mathrm{H} 43 \mathrm{~N} 1 \mathrm{O} 3$ & $3.66 \mathrm{E}-05$ & Basic N1O1 & $\mathrm{C} 36 \mathrm{H} 61 \mathrm{~N} 1 \mathrm{O} 1$ & $2.03 \mathrm{E}-05$ \\
\hline S1 & $\mathrm{C} 25 \mathrm{H} 20 \mathrm{~S} 1$ & $6.91 \mathrm{E}-05$ & Non-basic N1O3 & $\mathrm{C} 34 \mathrm{H} 45 \mathrm{~N} 1 \mathrm{O} 3$ & $4.09 \mathrm{E}-05$ & Basic N1O1 & $\mathrm{C} 37 \mathrm{H} 63 \mathrm{~N} 1 \mathrm{O} 1$ & $1.26 \mathrm{E}-05$ \\
\hline S1 & $\mathrm{C} 26 \mathrm{H} 22 \mathrm{~S} 1$ & $5.68 \mathrm{E}-05$ & Non-basic N1O3 & $\mathrm{C} 35 \mathrm{H} 47 \mathrm{~N} 1 \mathrm{O} 3$ & $4.92 \mathrm{E}-05$ & Basic N1O1 & C38H65N1O1 & $1.24 \mathrm{E}-05$ \\
\hline S1 & $\mathrm{C} 27 \mathrm{H} 24 \mathrm{~S} 1$ & $4.06 \mathrm{E}-05$ & Non-basic N1O3 & $\mathrm{C} 36 \mathrm{H} 49 \mathrm{~N} 1 \mathrm{O} 3$ & $4.14 \mathrm{E}-05$ & Basic N1O1 & C39H67N1O1 & $1.47 \mathrm{E}-05$ \\
\hline S1 & $\mathrm{C} 28 \mathrm{H} 26 \mathrm{~S} 1$ & $2.34 \mathrm{E}-05$ & Non-basic N1O3 & C37H51N1O3 & $4.14 \mathrm{E}-05$ & Basic N1O1 & C40H69N1O1 & $3.93 \mathrm{E}-06$ \\
\hline S1 & $\mathrm{C} 29 \mathrm{H} 28 \mathrm{~S} 1$ & $6.46 \mathrm{E}-06$ & Non-basic N1O3 & $\mathrm{C} 38 \mathrm{H} 53 \mathrm{~N} 1 \mathrm{O} 3$ & $3.45 \mathrm{E}-05$ & Basic N1O1 & $\mathrm{C} 14 \mathrm{H} 15 \mathrm{~N} 1 \mathrm{O} 1$ & $9.05 \mathrm{E}-08$ \\
\hline S1 & $\mathrm{C} 30 \mathrm{H} 30 \mathrm{~S} 1$ & $9.58 \mathrm{E}-06$ & Non-basic N1O3 & C39H55N1O3 & $1.58 \mathrm{E}-05$ & Basic N1O1 & $\mathrm{C} 15 \mathrm{H} 17 \mathrm{~N} 1 \mathrm{O} 1$ & $1.23 \mathrm{E}-06$ \\
\hline S1 & $\mathrm{C} 31 \mathrm{H} 32 \mathrm{~S} 1$ & $1.69 \mathrm{E}-05$ & Non-basic N1O3 & $\mathrm{C} 40 \mathrm{H} 57 \mathrm{~N} 1 \mathrm{O} 3$ & $2.19 \mathrm{E}-05$ & Basic N1O1 & $\mathrm{C} 16 \mathrm{H} 19 \mathrm{~N} 1 \mathrm{O} 1$ & $1.60 \mathrm{E}-06$ \\
\hline S1 & $\mathrm{C} 32 \mathrm{H} 34 \mathrm{~S} 1$ & $1.44 \mathrm{E}-05$ & Non-basic N1O3 & $\mathrm{C} 41 \mathrm{H} 59 \mathrm{~N} 1 \mathrm{O} 3$ & $1.26 \mathrm{E}-05$ & Basic N1O1 & $\mathrm{C} 17 \mathrm{H} 21 \mathrm{~N} 1 \mathrm{O} 1$ & $6.31 \mathrm{E}-07$ \\
\hline S1 & $\mathrm{C} 33 \mathrm{H} 36 \mathrm{~S} 1$ & $1.41 \mathrm{E}-05$ & Non-basic N1O3 & $\mathrm{C} 42 \mathrm{H} 61 \mathrm{~N} 1 \mathrm{O} 3$ & $1.23 \mathrm{E}-05$ & Basic N1O1 & $\mathrm{C} 18 \mathrm{H} 23 \mathrm{~N} 1 \mathrm{O} 1$ & $1.84 \mathrm{E}-06$ \\
\hline S1 & $\mathrm{C} 34 \mathrm{H} 38 \mathrm{~S} 1$ & $7.18 \mathrm{E}-06$ & Non-basic N1O3 & C17H9N1O3 & $6.62 \mathrm{E}-07$ & Basic N1O1 & $\mathrm{C} 19 \mathrm{H} 25 \mathrm{~N} 1 \mathrm{O} 1$ & $2.58 \mathrm{E}-06$ \\
\hline S1 & $\mathrm{C} 35 \mathrm{H} 40 \mathrm{~S} 1$ & $2.03 \mathrm{E}-05$ & Non-basic N1O3 & $\mathrm{C} 18 \mathrm{H} 11 \mathrm{~N} 1 \mathrm{O} 3$ & $2.03 \mathrm{E}-05$ & Basic N1O1 & $\mathrm{C} 20 \mathrm{H} 27 \mathrm{~N} 1 \mathrm{O} 1$ & $5.42 \mathrm{E}-06$ \\
\hline S1 & $\mathrm{C} 36 \mathrm{H} 42 \mathrm{~S} 1$ & $1.97 \mathrm{E}-05$ & Non-basic N1O3 & C19H13N1O3 & $5.39 \mathrm{E}-05$ & Basic N1O1 & $\mathrm{C} 21 \mathrm{H} 29 \mathrm{~N} 1 \mathrm{O} 1$ & $9.18 \mathrm{E}-06$ \\
\hline S1 & $\mathrm{C} 37 \mathrm{H} 44 \mathrm{~S} 1$ & $1.63 \mathrm{E}-05$ & Non-basic N1O3 & $\mathrm{C} 20 \mathrm{H} 15 \mathrm{~N} 1 \mathrm{O} 3$ & 0.000125 & Basic N1O1 & $\mathrm{C} 22 \mathrm{H} 31 \mathrm{~N} 1 \mathrm{O} 1$ & $1.13 \mathrm{E}-05$ \\
\hline S1 & $\mathrm{C} 38 \mathrm{H} 46 \mathrm{~S} 1$ & $3.36 \mathrm{E}-05$ & Non-basic N1O3 & $\mathrm{C} 21 \mathrm{H} 17 \mathrm{~N} 1 \mathrm{O} 3$ & 0.000226 & Basic N1O1 & $\mathrm{C} 23 \mathrm{H} 33 \mathrm{~N} 1 \mathrm{O} 1$ & $1.43 \mathrm{E}-05$ \\
\hline S1 & $\mathrm{C} 39 \mathrm{H} 48 \mathrm{~S} 1$ & $1.27 \mathrm{E}-05$ & Non-basic N1O3 & $\mathrm{C} 22 \mathrm{H} 19 \mathrm{~N} 1 \mathrm{O} 3$ & 0.000286 & Basic N1O1 & $\mathrm{C} 24 \mathrm{H} 35 \mathrm{~N} 1 \mathrm{O} 1$ & $3.92 \mathrm{E}-05$ \\
\hline S1 & C40H50S1 & $2.23 \mathrm{E}-05$ & Non-basic N1O3 & $\mathrm{C} 23 \mathrm{H} 21 \mathrm{~N} 1 \mathrm{O} 3$ & 0.000258 & Basic N1O1 & $\mathrm{C} 25 \mathrm{H} 37 \mathrm{~N} 1 \mathrm{O} 1$ & 4.53E-05 \\
\hline S1 & $\mathrm{C} 41 \mathrm{H} 52 \mathrm{~S} 1$ & $3.01 \mathrm{E}-05$ & Non-basic N1O3 & $\mathrm{C} 24 \mathrm{H} 23 \mathrm{~N} 1 \mathrm{O} 3$ & 0.000178 & Basic N1O1 & $\mathrm{C} 26 \mathrm{H} 39 \mathrm{~N} 1 \mathrm{O} 1$ & $3.40 \mathrm{E}-05$ \\
\hline S1 & $\mathrm{C} 42 \mathrm{H} 54 \mathrm{~S} 1$ & $1.24 \mathrm{E}-05$ & Non-basic N1O3 & $\mathrm{C} 25 \mathrm{H} 25 \mathrm{~N} 1 \mathrm{O} 3$ & 0.000124 & Basic N1O1 & $\mathrm{C} 27 \mathrm{H} 41 \mathrm{~N} 1 \mathrm{O} 1$ & $5.35 \mathrm{E}-05$ \\
\hline S1 & $\mathrm{C} 43 \mathrm{H} 56 \mathrm{~S} 1$ & $1.78 \mathrm{E}-05$ & Non-basic N1O3 & $\mathrm{C} 26 \mathrm{H} 27 \mathrm{~N} 1 \mathrm{O} 3$ & $8.29 \mathrm{E}-05$ & Basic N1O1 & $\mathrm{C} 28 \mathrm{H} 43 \mathrm{~N} 1 \mathrm{O} 1$ & $3.06 \mathrm{E}-05$ \\
\hline S1 & C44H58S1 & $1.26 \mathrm{E}-05$ & Non-basic N1O3 & $\mathrm{C} 27 \mathrm{H} 29 \mathrm{~N} 1 \mathrm{O} 3$ & $7.91 \mathrm{E}-05$ & Basic N1O1 & $\mathrm{C} 29 \mathrm{H} 45 \mathrm{~N} 1 \mathrm{O} 1$ & $5.00 \mathrm{E}-05$ \\
\hline S1 & C45H60S1 & $1.77 \mathrm{E}-05$ & Non-basic N1O3 & $\mathrm{C} 28 \mathrm{H} 31 \mathrm{~N} 1 \mathrm{O} 3$ & $6.09 \mathrm{E}-05$ & Basic N1O1 & $\mathrm{C} 30 \mathrm{H} 47 \mathrm{~N} 1 \mathrm{O} 1$ & $3.92 \mathrm{E}-05$ \\
\hline S1 & $\mathrm{C} 22 \mathrm{H} 12 \mathrm{~S} 1$ & $9.18 \mathrm{E}-06$ & Non-basic N1O3 & $\mathrm{C} 29 \mathrm{H} 33 \mathrm{~N} 1 \mathrm{O} 3$ & $5.05 \mathrm{E}-05$ & Basic N1O1 & $\mathrm{C} 31 \mathrm{H} 49 \mathrm{~N} 1 \mathrm{O} 1$ & $3.86 \mathrm{E}-05$ \\
\hline S1 & $\mathrm{C} 23 \mathrm{H} 14 \mathrm{~S} 1$ & $9.88 \mathrm{E}-05$ & Non-basic N1O3 & $\mathrm{C} 30 \mathrm{H} 35 \mathrm{~N} 1 \mathrm{O} 3$ & $5.23 \mathrm{E}-05$ & Basic N1O1 & $\mathrm{C} 32 \mathrm{H} 51 \mathrm{~N} 1 \mathrm{O} 1$ & $3.23 \mathrm{E}-05$ \\
\hline S1 & $\mathrm{C} 24 \mathrm{H} 16 \mathrm{~S} 1$ & 0.000193 & Non-basic N1O3 & $\mathrm{C} 31 \mathrm{H} 37 \mathrm{~N} 1 \mathrm{O} 3$ & $3.77 \mathrm{E}-05$ & Basic N1O1 & $\mathrm{C} 33 \mathrm{H} 53 \mathrm{~N} 1 \mathrm{O} 1$ & $3.46 \mathrm{E}-05$ \\
\hline S1 & $\mathrm{C} 25 \mathrm{H} 18 \mathrm{~S} 1$ & 0.000254 & Non-basic N1O3 & $\mathrm{C} 32 \mathrm{H} 39 \mathrm{~N} 1 \mathrm{O} 3$ & $3.32 \mathrm{E}-05$ & Basic N1O1 & $\mathrm{C} 34 \mathrm{H} 55 \mathrm{~N} 1 \mathrm{O} 1$ & $3.95 \mathrm{E}-05$ \\
\hline S1 & $\mathrm{C} 26 \mathrm{H} 20 \mathrm{~S} 1$ & 0.000234 & Non-basic N1O3 & $\mathrm{C} 33 \mathrm{H} 41 \mathrm{~N} 1 \mathrm{O} 3$ & $2.92 \mathrm{E}-05$ & Basic N1O1 & $\mathrm{C} 35 \mathrm{H} 57 \mathrm{~N} 1 \mathrm{O} 1$ & 4.42E-05 \\
\hline S1 & $\mathrm{C} 27 \mathrm{H} 22 \mathrm{~S} 1$ & 0.000138 & Non-basic N1O3 & $\mathrm{C} 34 \mathrm{H} 43 \mathrm{~N} 1 \mathrm{O} 3$ & $3.41 \mathrm{E}-05$ & Basic N1O1 & $\mathrm{C} 36 \mathrm{H} 59 \mathrm{~N} 1 \mathrm{O} 1$ & $3.66 \mathrm{E}-05$ \\
\hline S1 & $\mathrm{C} 28 \mathrm{H} 24 \mathrm{~S} 1$ & 8.37E-05 & Non-basic N1O3 & $\mathrm{C} 35 \mathrm{H} 45 \mathrm{~N} 1 \mathrm{O} 3$ & $3.81 \mathrm{E}-05$ & Basic N1O1 & $\mathrm{C} 37 \mathrm{H} 61 \mathrm{~N} 1 \mathrm{O} 1$ & $3.26 \mathrm{E}-05$ \\
\hline S1 & $\mathrm{C} 29 \mathrm{H} 26 \mathrm{~S} 1$ & $3.29 \mathrm{E}-05$ & Non-basic N1O3 & $\mathrm{C} 36 \mathrm{H} 47 \mathrm{~N} 1 \mathrm{O} 3$ & $5.09 \mathrm{E}-05$ & Basic N1O1 & $\mathrm{C} 38 \mathrm{H} 63 \mathrm{~N} 1 \mathrm{O} 1$ & $2.91 \mathrm{E}-05$ \\
\hline $\mathrm{S} 1$ & $\mathrm{C} 30 \mathrm{H} 28 \mathrm{~S} 1$ & $1.98 \mathrm{E}-05$ & Non-basic N1O3 & $\mathrm{C} 37 \mathrm{H} 49 \mathrm{~N} 1 \mathrm{O} 3$ & $4.34 \mathrm{E}-05$ & Basic N1O1 & $\mathrm{C} 15 \mathrm{H} 15 \mathrm{~N} 1 \mathrm{O} 1$ & 4.55E-07 \\
\hline
\end{tabular}




\begin{tabular}{|c|c|c|c|c|c|c|c|c|}
\hline Class & Formula & Mass & Class & Formula & Mass & Class & Formula & Mass \\
\hline S1 & $\mathrm{C} 31 \mathrm{H} 30 \mathrm{~S} 1$ & $4.58 \mathrm{E}-06$ & Non-basic N1O3 & $\mathrm{C} 38 \mathrm{H} 51 \mathrm{~N} 1 \mathrm{O} 3$ & $5.23 \mathrm{E}-05$ & Basic N1O1 & C16H17N1O1 & $1.25 \mathrm{E}-06$ \\
\hline S1 & $\mathrm{C} 32 \mathrm{H} 32 \mathrm{~S} 1$ & $7.54 \mathrm{E}-06$ & Non-basic N1O3 & C39H53N1O3 & $3.84 \mathrm{E}-05$ & Basic N1O1 & C17H19N1O1 & $1.07 \mathrm{E}-06$ \\
\hline S1 & $\mathrm{C} 33 \mathrm{H} 34 \mathrm{~S} 1$ & $7.96 \mathrm{E}-06$ & Non-basic N1O3 & $\mathrm{C} 40 \mathrm{H} 55 \mathrm{~N} 1 \mathrm{O} 3$ & $2.97 \mathrm{E}-05$ & Basic N1O1 & $\mathrm{C} 18 \mathrm{H} 21 \mathrm{~N} 1 \mathrm{O} 1$ & $7.94 \mathrm{E}-06$ \\
\hline S1 & $\mathrm{C} 34 \mathrm{H} 36 \mathrm{~S} 1$ & $8.04 \mathrm{E}-06$ & Non-basic N1O3 & C41H57N1O3 & $1.84 \mathrm{E}-05$ & Basic N1O1 & $\mathrm{C} 19 \mathrm{H} 23 \mathrm{~N} 1 \mathrm{O} 1$ & $5.84 \mathrm{E}-06$ \\
\hline S1 & $\mathrm{C} 35 \mathrm{H} 38 \mathrm{~S} 1$ & $1.72 \mathrm{E}-05$ & Non-basic N1O3 & $\mathrm{C} 42 \mathrm{H} 59 \mathrm{~N} 1 \mathrm{O} 3$ & 4.89E-06 & Basic N1O1 & $\mathrm{C} 20 \mathrm{H} 25 \mathrm{~N} 1 \mathrm{O} 1$ & $1.23 \mathrm{E}-05$ \\
\hline S1 & $\mathrm{C} 36 \mathrm{H} 40 \mathrm{~S} 1$ & $3.23 \mathrm{E}-06$ & Non-basic N1O3 & $\mathrm{C} 43 \mathrm{H} 61 \mathrm{~N} 1 \mathrm{O} 3$ & $5.35 \mathrm{E}-06$ & Basic N1O1 & $\mathrm{C} 21 \mathrm{H} 27 \mathrm{~N} 1 \mathrm{O} 1$ & $1.45 \mathrm{E}-05$ \\
\hline S1 & $\mathrm{C} 37 \mathrm{H} 42 \mathrm{~S} 1$ & $6.91 \mathrm{E}-06$ & Non-basic N1O3 & $\mathrm{C} 44 \mathrm{H} 63 \mathrm{~N} 1 \mathrm{O} 3$ & $4.86 \mathrm{E}-06$ & Basic N1O1 & $\mathrm{C} 22 \mathrm{H} 29 \mathrm{~N} 1 \mathrm{O} 1$ & $1.57 \mathrm{E}-05$ \\
\hline S1 & $\mathrm{C} 38 \mathrm{H} 44 \mathrm{~S} 1$ & $2.00 \mathrm{E}-05$ & Non-basic N1O3 & $\mathrm{C} 45 \mathrm{H} 65 \mathrm{~N} 1 \mathrm{O} 3$ & $4.71 \mathrm{E}-06$ & Basic N1O1 & $\mathrm{C} 23 \mathrm{H} 31 \mathrm{~N} 1 \mathrm{O} 1$ & $2.31 \mathrm{E}-05$ \\
\hline S1 & $\mathrm{C} 39 \mathrm{H} 46 \mathrm{~S} 1$ & $1.18 \mathrm{E}-05$ & Non-basic N1O3 & $\mathrm{C} 46 \mathrm{H} 67 \mathrm{~N} 1 \mathrm{O} 3$ & $5.20 \mathrm{E}-06$ & Basic N1O1 & $\mathrm{C} 24 \mathrm{H} 33 \mathrm{~N} 1 \mathrm{O} 1$ & $2.61 \mathrm{E}-05$ \\
\hline S1 & $\mathrm{C} 40 \mathrm{H} 48 \mathrm{~S} 1$ & $1.81 \mathrm{E}-05$ & Non-basic N1O3 & C18H9N1O3 & $8.67 \mathrm{E}-07$ & Basic N1O1 & $\mathrm{C} 25 \mathrm{H} 35 \mathrm{~N} 1 \mathrm{O} 1$ & $2.98 \mathrm{E}-05$ \\
\hline S1 & C41H50S1 & $5.81 \mathrm{E}-06$ & Non-basic N1O3 & C19H11N1O3 & $2.16 \mathrm{E}-05$ & Basic N1O1 & $\mathrm{C} 26 \mathrm{H} 37 \mathrm{~N} 1 \mathrm{O} 1$ & 4.62E-05 \\
\hline S1 & $\mathrm{C} 42 \mathrm{H} 52 \mathrm{~S} 1$ & $2.50 \mathrm{E}-05$ & Non-basic N1O3 & $\mathrm{C} 20 \mathrm{H} 13 \mathrm{~N} 1 \mathrm{O} 3$ & $5.00 \mathrm{E}-05$ & Basic N1O1 & $\mathrm{C} 27 \mathrm{H} 39 \mathrm{~N} 1 \mathrm{O} 1$ & $6.39 \mathrm{E}-05$ \\
\hline S1 & $\mathrm{C} 43 \mathrm{H} 54 \mathrm{~S} 1$ & $2.59 \mathrm{E}-05$ & Non-basic N1O3 & $\mathrm{C} 21 \mathrm{H} 15 \mathrm{~N} 1 \mathrm{O} 3$ & 0.000108 & Basic N1O1 & $\mathrm{C} 28 \mathrm{H} 41 \mathrm{~N} 1 \mathrm{O} 1$ & $4.61 \mathrm{E}-05$ \\
\hline S1 & C44H56S1 & $2.80 \mathrm{E}-05$ & Non-basic N1O3 & $\mathrm{C} 22 \mathrm{H} 17 \mathrm{~N} 1 \mathrm{O} 3$ & 0.000188 & Basic N1O1 & $\mathrm{C} 29 \mathrm{H} 43 \mathrm{~N} 1 \mathrm{O} 1$ & $4.21 \mathrm{E}-05$ \\
\hline S1 & $\mathrm{C} 45 \mathrm{H} 58 \mathrm{~S} 1$ & $1.19 \mathrm{E}-05$ & Non-basic N1O3 & $\mathrm{C} 23 \mathrm{H} 19 \mathrm{~N} 1 \mathrm{O} 3$ & 0.000255 & Basic N1O1 & $\mathrm{C} 30 \mathrm{H} 45 \mathrm{~N} 1 \mathrm{O} 1$ & 4.64E-05 \\
\hline S1 & $\mathrm{C} 46 \mathrm{H} 60 \mathrm{~S} 1$ & $1.21 \mathrm{E}-05$ & Non-basic N1O3 & $\mathrm{C} 24 \mathrm{H} 21 \mathrm{~N} 1 \mathrm{O} 3$ & 0.000238 & Basic N1O1 & $\mathrm{C} 31 \mathrm{H} 47 \mathrm{~N} 1 \mathrm{O} 1$ & 4.96E-05 \\
\hline S1 & $\mathrm{C} 47 \mathrm{H} 62 \mathrm{~S} 1$ & $3.71 \mathrm{E}-06$ & Non-basic N1O3 & $\mathrm{C} 25 \mathrm{H} 23 \mathrm{~N} 1 \mathrm{O} 3$ & 0.000177 & Basic N1O1 & $\mathrm{C} 32 \mathrm{H} 49 \mathrm{~N} 1 \mathrm{O} 1$ & $5.67 \mathrm{E}-05$ \\
\hline S1 & C48H64S1 & $3.93 \mathrm{E}-06$ & Non-basic N1O3 & $\mathrm{C} 26 \mathrm{H} 25 \mathrm{~N} 1 \mathrm{O} 3$ & 0.000125 & Basic N1O1 & $\mathrm{C} 33 \mathrm{H} 51 \mathrm{~N} 1 \mathrm{O} 1$ & $4.54 \mathrm{E}-05$ \\
\hline S1 & $\mathrm{C} 24 \mathrm{H} 14 \mathrm{~S} 1$ & $4.73 \mathrm{E}-05$ & Non-basic N1O3 & $\mathrm{C} 27 \mathrm{H} 27 \mathrm{~N} 1 \mathrm{O} 3$ & $8.22 \mathrm{E}-05$ & Basic N1O1 & $\mathrm{C} 34 \mathrm{H} 53 \mathrm{~N} 1 \mathrm{O} 1$ & 4.32E-05 \\
\hline S1 & $\mathrm{C} 25 \mathrm{H} 16 \mathrm{~S} 1$ & 0.000162 & Non-basic N1O3 & $\mathrm{C} 28 \mathrm{H} 29 \mathrm{~N} 1 \mathrm{O} 3$ & $7.28 \mathrm{E}-05$ & Basic N1O1 & $\mathrm{C} 35 \mathrm{H} 55 \mathrm{~N} 1 \mathrm{O} 1$ & 4.49E-05 \\
\hline S1 & $\mathrm{C} 26 \mathrm{H} 18 \mathrm{~S} 1$ & 0.00023 & Non-basic N1O3 & $\mathrm{C} 29 \mathrm{H} 31 \mathrm{~N} 1 \mathrm{O} 3$ & $5.49 \mathrm{E}-05$ & Basic N1O1 & $\mathrm{C} 36 \mathrm{H} 57 \mathrm{~N} 1 \mathrm{O} 1$ & $2.88 \mathrm{E}-05$ \\
\hline S1 & $\mathrm{C} 27 \mathrm{H} 20 \mathrm{~S} 1$ & 0.0002 & Non-basic N1O3 & $\mathrm{C} 30 \mathrm{H} 33 \mathrm{~N} 1 \mathrm{O} 3$ & $5.01 \mathrm{E}-05$ & Basic N1O1 & $\mathrm{C} 37 \mathrm{H} 59 \mathrm{~N} 1 \mathrm{O} 1$ & $3.25 \mathrm{E}-05$ \\
\hline S1 & $\mathrm{C} 28 \mathrm{H} 22 \mathrm{~S} 1$ & 0.000116 & Non-basic N1O3 & $\mathrm{C} 31 \mathrm{H} 35 \mathrm{~N} 1 \mathrm{O} 3$ & $3.69 \mathrm{E}-05$ & Basic N1O1 & C38H61N1O1 & $3.15 \mathrm{E}-05$ \\
\hline S1 & $\mathrm{C} 29 \mathrm{H} 24 \mathrm{~S} 1$ & $5.88 \mathrm{E}-05$ & Non-basic N1O3 & C32H37N1O3 & $2.97 \mathrm{E}-05$ & Basic N1O1 & C39H63N1O1 & $2.58 \mathrm{E}-05$ \\
\hline S1 & $\mathrm{C} 30 \mathrm{H} 26 \mathrm{~S} 1$ & $3.02 \mathrm{E}-05$ & Non-basic N1O3 & $\mathrm{C} 33 \mathrm{H} 39 \mathrm{~N} 1 \mathrm{O} 3$ & $1.98 \mathrm{E}-05$ & Basic N1O1 & C40H65N1O1 & $2.16 \mathrm{E}-05$ \\
\hline S1 & $\mathrm{C} 31 \mathrm{H} 28 \mathrm{~S} 1$ & $2.41 \mathrm{E}-06$ & Non-basic N1O3 & $\mathrm{C} 34 \mathrm{H} 41 \mathrm{~N} 1 \mathrm{O} 3$ & $2.86 \mathrm{E}-05$ & Basic N1O1 & C41H67N1O1 & $1.64 \mathrm{E}-05$ \\
\hline S1 & $\mathrm{C} 32 \mathrm{H} 30 \mathrm{~S} 1$ & $5.95 \mathrm{E}-06$ & Non-basic N1O3 & $\mathrm{C} 35 \mathrm{H} 43 \mathrm{~N} 1 \mathrm{O} 3$ & $2.41 \mathrm{E}-05$ & Basic N1O1 & $\mathrm{C} 42 \mathrm{H} 69 \mathrm{~N} 1 \mathrm{O} 1$ & $1.56 \mathrm{E}-06$ \\
\hline S1 & $\mathrm{C} 33 \mathrm{H} 32 \mathrm{~S} 1$ & $3.82 \mathrm{E}-06$ & Non-basic N1O3 & $\mathrm{C} 36 \mathrm{H} 45 \mathrm{~N} 1 \mathrm{O} 3$ & $2.39 \mathrm{E}-05$ & Basic N1O1 & $\mathrm{C} 15 \mathrm{H} 13 \mathrm{~N} 1 \mathrm{O} 1$ & 2.92E-07 \\
\hline S1 & $\mathrm{C} 34 \mathrm{H} 34 \mathrm{~S} 1$ & $1.10 \mathrm{E}-05$ & Non-basic N1O3 & $\mathrm{C} 37 \mathrm{H} 47 \mathrm{~N} 1 \mathrm{O} 3$ & $2.48 \mathrm{E}-05$ & Basic N1O1 & C16H15N1O1 & $1.40 \mathrm{E}-06$ \\
\hline S1 & $\mathrm{C} 36 \mathrm{H} 38 \mathrm{~S} 1$ & $3.32 \mathrm{E}-06$ & Non-basic N1O3 & $\mathrm{C} 38 \mathrm{H} 49 \mathrm{~N} 1 \mathrm{O} 3$ & $2.73 \mathrm{E}-05$ & Basic N1O1 & C17H17N1O1 & $1.69 \mathrm{E}-06$ \\
\hline S1 & $\mathrm{C} 37 \mathrm{H} 40 \mathrm{~S} 1$ & $3.76 \mathrm{E}-06$ & Non-basic N1O3 & C39H51N1O3 & $3.40 \mathrm{E}-05$ & Basic N1O1 & C18H19N1O1 & 4.16E-06 \\
\hline S1 & $\mathrm{C} 38 \mathrm{H} 42 \mathrm{~S} 1$ & $1.16 \mathrm{E}-05$ & Non-basic N1O3 & $\mathrm{C} 40 \mathrm{H} 53 \mathrm{~N} 1 \mathrm{O} 3$ & $2.38 \mathrm{E}-05$ & Basic N1O1 & $\mathrm{C} 19 \mathrm{H} 21 \mathrm{~N} 1 \mathrm{O} 1$ & $7.54 \mathrm{E}-06$ \\
\hline S1 & $\mathrm{C} 39 \mathrm{H} 44 \mathrm{~S} 1$ & $3.71 \mathrm{E}-06$ & Non-basic N1O3 & $\mathrm{C} 41 \mathrm{H} 55 \mathrm{~N} 1 \mathrm{O} 3$ & $1.71 \mathrm{E}-05$ & Basic N1O1 & $\mathrm{C} 20 \mathrm{H} 23 \mathrm{~N} 1 \mathrm{O} 1$ & $8.70 \mathrm{E}-06$ \\
\hline S1 & $\mathrm{C} 40 \mathrm{H} 46 \mathrm{~S} 1$ & $3.73 \mathrm{E}-06$ & Non-basic N1O3 & $\mathrm{C} 42 \mathrm{H} 57 \mathrm{~N} 1 \mathrm{O} 3$ & 7.70E-06 & Basic N1O1 & $\mathrm{C} 21 \mathrm{H} 25 \mathrm{~N} 1 \mathrm{O} 1$ & $2.08 \mathrm{E}-05$ \\
\hline S1 & $\mathrm{C} 41 \mathrm{H} 48 \mathrm{~S} 1$ & $6.80 \mathrm{E}-06$ & Non-basic N1O3 & $\mathrm{C} 43 \mathrm{H} 59 \mathrm{~N} 1 \mathrm{O} 3$ & $1.48 \mathrm{E}-05$ & Basic N1O1 & $\mathrm{C} 22 \mathrm{H} 27 \mathrm{~N} 1 \mathrm{O} 1$ & $2.09 \mathrm{E}-05$ \\
\hline S1 & $\mathrm{C} 42 \mathrm{H} 50 \mathrm{~S} 1$ & $1.73 \mathrm{E}-05$ & Non-basic N1O3 & $\mathrm{C} 44 \mathrm{H} 61 \mathrm{~N} 1 \mathrm{O} 3$ & 7.33E-06 & Basic N1O1 & $\mathrm{C} 23 \mathrm{H} 29 \mathrm{~N} 1 \mathrm{O} 1$ & $1.90 \mathrm{E}-05$ \\
\hline S1 & $\mathrm{C} 43 \mathrm{H} 52 \mathrm{~S} 1$ & $1.73 \mathrm{E}-05$ & Non-basic N1O3 & $\mathrm{C} 45 \mathrm{H} 63 \mathrm{~N} 1 \mathrm{O} 3$ & $3.47 \mathrm{E}-06$ & Basic N1O1 & $\mathrm{C} 24 \mathrm{H} 31 \mathrm{~N} 1 \mathrm{O} 1$ & $3.11 \mathrm{E}-05$ \\
\hline $\mathrm{S} 1$ & $\mathrm{C} 44 \mathrm{H} 54 \mathrm{~S} 1$ & $2.08 \mathrm{E}-05$ & Non-basic N1O3 & $\mathrm{C} 46 \mathrm{H} 65 \mathrm{~N} 1 \mathrm{O} 3$ & $6.30 \mathrm{E}-06$ & Basic N1O1 & $\mathrm{C} 25 \mathrm{H} 33 \mathrm{~N} 1 \mathrm{O} 1$ & 4.39E-05 \\
\hline
\end{tabular}




\begin{tabular}{|c|c|c|c|c|c|c|c|c|}
\hline Class & Formula & Mass & Class & Formula & Mass & Class & Formula & Mass \\
\hline S1 & $\mathrm{C} 45 \mathrm{H} 56 \mathrm{~S} 1$ & $1.71 \mathrm{E}-05$ & Non-basic N1O3 & C47H67N1O3 & $3.70 \mathrm{E}-06$ & Basic N1O1 & $\mathrm{C} 26 \mathrm{H} 35 \mathrm{~N} 1 \mathrm{O} 1$ & $5.91 \mathrm{E}-05$ \\
\hline S1 & $\mathrm{C} 46 \mathrm{H} 58 \mathrm{~S} 1$ & 7.37E-06 & Non-basic N1O3 & C19H9N1O3 & $9.32 \mathrm{E}-07$ & Basic N1O1 & $\mathrm{C} 27 \mathrm{H} 37 \mathrm{~N} 1 \mathrm{O} 1$ & $4.86 \mathrm{E}-05$ \\
\hline S1 & C47H60S1 & $1.52 \mathrm{E}-05$ & Non-basic N1O3 & $\mathrm{C} 20 \mathrm{H} 11 \mathrm{~N} 1 \mathrm{O} 3$ & $6.47 \mathrm{E}-06$ & Basic N1O1 & $\mathrm{C} 28 \mathrm{H} 39 \mathrm{~N} 1 \mathrm{O} 1$ & $3.70 \mathrm{E}-05$ \\
\hline S1 & $\mathrm{C} 25 \mathrm{H} 14 \mathrm{~S} 1$ & $3.26 \mathrm{E}-05$ & Non-basic N1O3 & $\mathrm{C} 21 \mathrm{H} 13 \mathrm{~N} 1 \mathrm{O} 3$ & $3.77 \mathrm{E}-05$ & Basic N1O1 & $\mathrm{C} 29 \mathrm{H} 41 \mathrm{~N} 1 \mathrm{O} 1$ & $5.01 \mathrm{E}-05$ \\
\hline S1 & $\mathrm{C} 26 \mathrm{H} 16 \mathrm{~S} 1$ & 7.33E-05 & Non-basic N1O3 & $\mathrm{C} 22 \mathrm{H} 15 \mathrm{~N} 1 \mathrm{O} 3$ & $8.75 \mathrm{E}-05$ & Basic N1O1 & $\mathrm{C} 30 \mathrm{H} 43 \mathrm{~N} 1 \mathrm{O} 1$ & 4.93E-05 \\
\hline S1 & $\mathrm{C} 27 \mathrm{H} 18 \mathrm{~S} 1$ & 0.000105 & Non-basic N1O3 & $\mathrm{C} 23 \mathrm{H} 17 \mathrm{~N} 1 \mathrm{O} 3$ & 0.000152 & Basic N1O1 & $\mathrm{C} 31 \mathrm{H} 45 \mathrm{~N} 1 \mathrm{O} 1$ & $5.37 \mathrm{E}-05$ \\
\hline S1 & $\mathrm{C} 28 \mathrm{H} 20 \mathrm{~S} 1$ & 0.000116 & Non-basic N1O3 & $\mathrm{C} 24 \mathrm{H} 19 \mathrm{~N} 1 \mathrm{O} 3$ & 0.000184 & Basic N1O1 & $\mathrm{C} 32 \mathrm{H} 47 \mathrm{~N} 1 \mathrm{O} 1$ & $4.86 \mathrm{E}-05$ \\
\hline S1 & $\mathrm{C} 29 \mathrm{H} 22 \mathrm{~S} 1$ & $8.70 \mathrm{E}-05$ & Non-basic N1O3 & $\mathrm{C} 25 \mathrm{H} 21 \mathrm{~N} 1 \mathrm{O} 3$ & 0.000154 & Basic N1O1 & $\mathrm{C} 33 \mathrm{H} 49 \mathrm{~N} 1 \mathrm{O} 1$ & 4.79E-05 \\
\hline S1 & $\mathrm{C} 30 \mathrm{H} 24 \mathrm{~S} 1$ & $5.95 \mathrm{E}-05$ & Non-basic N1O3 & $\mathrm{C} 26 \mathrm{H} 23 \mathrm{~N} 1 \mathrm{O} 3$ & 0.000142 & Basic N1O1 & $\mathrm{C} 34 \mathrm{H} 51 \mathrm{~N} 1 \mathrm{O} 1$ & $3.35 \mathrm{E}-05$ \\
\hline S1 & $\mathrm{C} 31 \mathrm{H} 26 \mathrm{~S} 1$ & $3.09 \mathrm{E}-05$ & Non-basic N1O3 & $\mathrm{C} 27 \mathrm{H} 25 \mathrm{~N} 1 \mathrm{O} 3$ & 0.000112 & Basic N1O1 & C35H53N1O1 & 4.95E-05 \\
\hline S1 & $\mathrm{C} 32 \mathrm{H} 28 \mathrm{~S} 1$ & $1.86 \mathrm{E}-05$ & Non-basic N1O3 & $\mathrm{C} 28 \mathrm{H} 27 \mathrm{~N} 1 \mathrm{O} 3$ & $7.19 \mathrm{E}-05$ & Basic N1O1 & $\mathrm{C} 36 \mathrm{H} 55 \mathrm{~N} 1 \mathrm{O} 1$ & $3.48 \mathrm{E}-05$ \\
\hline S1 & $\mathrm{C} 33 \mathrm{H} 30 \mathrm{~S} 1$ & $1.13 \mathrm{E}-05$ & Non-basic N1O3 & $\mathrm{C} 29 \mathrm{H} 29 \mathrm{~N} 1 \mathrm{O} 3$ & $4.85 \mathrm{E}-05$ & Basic N1O1 & $\mathrm{C} 37 \mathrm{H} 57 \mathrm{~N} 1 \mathrm{O} 1$ & $3.10 \mathrm{E}-05$ \\
\hline S1 & $\mathrm{C} 34 \mathrm{H} 32 \mathrm{~S} 1$ & $1.46 \mathrm{E}-05$ & Non-basic N1O3 & $\mathrm{C} 30 \mathrm{H} 31 \mathrm{~N} 1 \mathrm{O} 3$ & $5.22 \mathrm{E}-05$ & Basic N1O1 & C38H59N1O1 & $2.29 \mathrm{E}-05$ \\
\hline S1 & $\mathrm{C} 36 \mathrm{H} 36 \mathrm{~S} 1$ & $6.48 \mathrm{E}-06$ & Non-basic N1O3 & $\mathrm{C} 31 \mathrm{H} 33 \mathrm{~N} 1 \mathrm{O} 3$ & $3.34 \mathrm{E}-05$ & Basic N1O1 & C39H61N1O1 & $2.13 \mathrm{E}-05$ \\
\hline S1 & $\mathrm{C} 37 \mathrm{H} 38 \mathrm{~S} 1$ & $7.50 \mathrm{E}-06$ & Non-basic N1O3 & $\mathrm{C} 32 \mathrm{H} 35 \mathrm{~N} 1 \mathrm{O} 3$ & $2.63 \mathrm{E}-05$ & Basic N1O1 & $\mathrm{C} 40 \mathrm{H} 63 \mathrm{~N} 1 \mathrm{O} 1$ & $1.72 \mathrm{E}-05$ \\
\hline S1 & $\mathrm{C} 38 \mathrm{H} 40 \mathrm{~S} 1$ & $5.27 \mathrm{E}-06$ & Non-basic N1O3 & $\mathrm{C} 33 \mathrm{H} 37 \mathrm{~N} 1 \mathrm{O} 3$ & $1.48 \mathrm{E}-05$ & Basic N1O1 & $\mathrm{C} 16 \mathrm{H} 13 \mathrm{~N} 1 \mathrm{O} 1$ & $8.11 \mathrm{E}-07$ \\
\hline S1 & $\mathrm{C} 39 \mathrm{H} 42 \mathrm{~S} 1$ & $5.59 \mathrm{E}-06$ & Non-basic N1O3 & $\mathrm{C} 34 \mathrm{H} 39 \mathrm{~N} 1 \mathrm{O} 3$ & $2.71 \mathrm{E}-05$ & Basic N1O1 & $\mathrm{C} 17 \mathrm{H} 15 \mathrm{~N} 1 \mathrm{O} 1$ & $1.79 \mathrm{E}-05$ \\
\hline S1 & $\mathrm{C} 40 \mathrm{H} 44 \mathrm{~S} 1$ & $3.15 \mathrm{E}-06$ & Non-basic N1O3 & $\mathrm{C} 35 \mathrm{H} 41 \mathrm{~N} 1 \mathrm{O} 3$ & $1.07 \mathrm{E}-05$ & Basic N1O1 & $\mathrm{C} 18 \mathrm{H} 17 \mathrm{~N} 1 \mathrm{O} 1$ & $9.08 \mathrm{E}-06$ \\
\hline S1 & $\mathrm{C} 41 \mathrm{H} 46 \mathrm{~S} 1$ & $5.78 \mathrm{E}-06$ & Non-basic N1O3 & $\mathrm{C} 36 \mathrm{H} 43 \mathrm{~N} 1 \mathrm{O} 3$ & $2.49 \mathrm{E}-05$ & Basic N1O1 & C19H19N1O1 & $8.75 \mathrm{E}-06$ \\
\hline S1 & $\mathrm{C} 42 \mathrm{H} 48 \mathrm{~S} 1$ & $3.05 \mathrm{E}-06$ & Non-basic N1O3 & $\mathrm{C} 37 \mathrm{H} 45 \mathrm{~N} 1 \mathrm{O} 3$ & $2.00 \mathrm{E}-05$ & Basic N1O1 & $\mathrm{C} 20 \mathrm{H} 21 \mathrm{~N} 1 \mathrm{O} 1$ & $1.52 \mathrm{E}-05$ \\
\hline S1 & $\mathrm{C} 43 \mathrm{H} 50 \mathrm{~S} 1$ & $9.81 \mathrm{E}-06$ & Non-basic N1O3 & $\mathrm{C} 38 \mathrm{H} 47 \mathrm{~N} 1 \mathrm{O} 3$ & $3.04 \mathrm{E}-05$ & Basic N1O1 & $\mathrm{C} 21 \mathrm{H} 23 \mathrm{~N} 1 \mathrm{O} 1$ & $1.48 \mathrm{E}-05$ \\
\hline S1 & $\mathrm{C} 44 \mathrm{H} 52 \mathrm{~S} 1$ & $6.40 \mathrm{E}-06$ & Non-basic N1O3 & C39H49N1O3 & $3.59 \mathrm{E}-05$ & Basic N1O1 & $\mathrm{C} 22 \mathrm{H} 25 \mathrm{~N} 1 \mathrm{O} 1$ & $2.34 \mathrm{E}-05$ \\
\hline S1 & $\mathrm{C} 45 \mathrm{H} 54 \mathrm{~S} 1$ & $1.55 \mathrm{E}-05$ & Non-basic N1O3 & $\mathrm{C} 40 \mathrm{H} 51 \mathrm{~N} 1 \mathrm{O} 3$ & 4.04E-05 & Basic N1O1 & $\mathrm{C} 23 \mathrm{H} 27 \mathrm{~N} 1 \mathrm{O} 1$ & $2.62 \mathrm{E}-05$ \\
\hline S1 & C46H56S1 & $3.30 \mathrm{E}-06$ & Non-basic N1O3 & C41H53N1O3 & $2.74 \mathrm{E}-05$ & Basic N1O1 & $\mathrm{C} 24 \mathrm{H} 29 \mathrm{~N} 1 \mathrm{O} 1$ & $4.75 \mathrm{E}-05$ \\
\hline S1 & C47H58S1 & $4.18 \mathrm{E}-06$ & Non-basic N1O3 & $\mathrm{C} 42 \mathrm{H} 55 \mathrm{~N} 1 \mathrm{O} 3$ & $2.09 \mathrm{E}-05$ & Basic N1O1 & $\mathrm{C} 25 \mathrm{H} 31 \mathrm{~N} 1 \mathrm{O} 1$ & 4.23E-05 \\
\hline S1 & C48H60S1 & $3.81 \mathrm{E}-06$ & Non-basic N1O3 & $\mathrm{C} 43 \mathrm{H} 57 \mathrm{~N} 1 \mathrm{O} 3$ & $1.76 \mathrm{E}-05$ & Basic N1O1 & $\mathrm{C} 26 \mathrm{H} 33 \mathrm{~N} 1 \mathrm{O} 1$ & $4.30 \mathrm{E}-05$ \\
\hline S1 & $\mathrm{C} 49 \mathrm{H} 62 \mathrm{~S} 1$ & $1.25 \mathrm{E}-05$ & Non-basic N1O3 & $\mathrm{C} 44 \mathrm{H} 59 \mathrm{~N} 1 \mathrm{O} 3$ & $1.49 \mathrm{E}-05$ & Basic N1O1 & $\mathrm{C} 27 \mathrm{H} 35 \mathrm{~N} 1 \mathrm{O} 1$ & 4.09E-05 \\
\hline S1 & C50H64S1 & $6.87 \mathrm{E}-06$ & Non-basic N1O3 & $\mathrm{C} 45 \mathrm{H} 61 \mathrm{~N} 1 \mathrm{O} 3$ & $4.85 \mathrm{E}-06$ & Basic N1O1 & $\mathrm{C} 28 \mathrm{H} 37 \mathrm{~N} 1 \mathrm{O} 1$ & $4.56 \mathrm{E}-05$ \\
\hline S1 & C51H66S1 & $3.83 \mathrm{E}-06$ & Non-basic N1O3 & $\mathrm{C} 46 \mathrm{H} 63 \mathrm{~N} 1 \mathrm{O} 3$ & $5.13 \mathrm{E}-06$ & Basic N1O1 & $\mathrm{C} 29 \mathrm{H} 39 \mathrm{~N} 1 \mathrm{O} 1$ & $5.38 \mathrm{E}-05$ \\
\hline S1 & $\mathrm{C} 26 \mathrm{H} 14 \mathrm{~S} 1$ & $6.17 \mathrm{E}-06$ & Non-basic N1O3 & C47H65N1O3 & $9.97 \mathrm{E}-06$ & Basic N1O1 & $\mathrm{C} 30 \mathrm{H} 41 \mathrm{~N} 1 \mathrm{O} 1$ & $8.35 \mathrm{E}-05$ \\
\hline S1 & $\mathrm{C} 27 \mathrm{H} 16 \mathrm{~S} 1$ & $6.91 \mathrm{E}-05$ & Non-basic N1O3 & $\mathrm{C} 48 \mathrm{H} 67 \mathrm{~N} 1 \mathrm{O} 3$ & $6.04 \mathrm{E}-06$ & Basic N1O1 & $\mathrm{C} 31 \mathrm{H} 43 \mathrm{~N} 1 \mathrm{O} 1$ & $8.11 \mathrm{E}-05$ \\
\hline S1 & C28H18S1 & 0.00014 & Non-basic N1O3 & C49H69N1O3 & $9.36 \mathrm{E}-06$ & Basic N1O1 & $\mathrm{C} 32 \mathrm{H} 45 \mathrm{~N} 1 \mathrm{O} 1$ & $5.07 \mathrm{E}-05$ \\
\hline S1 & $\mathrm{C} 29 \mathrm{H} 20 \mathrm{~S} 1$ & 0.00017 & Non-basic N1O3 & $\mathrm{C} 50 \mathrm{H} 71 \mathrm{~N} 1 \mathrm{O} 3$ & $3.55 \mathrm{E}-06$ & Basic N1O1 & $\mathrm{C} 33 \mathrm{H} 47 \mathrm{~N} 1 \mathrm{O} 1$ & $5.41 \mathrm{E}-05$ \\
\hline S1 & $\mathrm{C} 30 \mathrm{H} 22 \mathrm{~S} 1$ & 0.000134 & Non-basic N1O3 & $\mathrm{C} 21 \mathrm{H} 11 \mathrm{~N} 1 \mathrm{O} 3$ & $6.96 \mathrm{E}-06$ & Basic N1O1 & $\mathrm{C} 34 \mathrm{H} 49 \mathrm{~N} 1 \mathrm{O} 1$ & $5.79 \mathrm{E}-05$ \\
\hline S1 & $\mathrm{C} 31 \mathrm{H} 24 \mathrm{~S} 1$ & 0.000111 & Non-basic N1O3 & $\mathrm{C} 22 \mathrm{H} 13 \mathrm{~N} 1 \mathrm{O} 3$ & $4.83 \mathrm{E}-05$ & Basic N1O1 & $\mathrm{C} 35 \mathrm{H} 51 \mathrm{~N} 1 \mathrm{O} 1$ & $4.02 \mathrm{E}-05$ \\
\hline S1 & $\mathrm{C} 32 \mathrm{H} 26 \mathrm{~S} 1$ & $6.23 \mathrm{E}-05$ & Non-basic N1O3 & $\mathrm{C} 23 \mathrm{H} 15 \mathrm{~N} 1 \mathrm{O} 3$ & $8.57 \mathrm{E}-05$ & Basic N1O1 & $\mathrm{C} 36 \mathrm{H} 53 \mathrm{~N} 1 \mathrm{O} 1$ & $3.26 \mathrm{E}-05$ \\
\hline S1 & $\mathrm{C} 33 \mathrm{H} 28 \mathrm{~S} 1$ & $2.77 \mathrm{E}-05$ & Non-basic N1O3 & $\mathrm{C} 24 \mathrm{H} 17 \mathrm{~N} 1 \mathrm{O} 3$ & 0.000143 & Basic N1O1 & $\mathrm{C} 37 \mathrm{H} 55 \mathrm{~N} 1 \mathrm{O} 1$ & 4.57E-05 \\
\hline $\mathrm{S} 1$ & $\mathrm{C} 34 \mathrm{H} 30 \mathrm{~S} 1$ & $9.22 \mathrm{E}-06$ & Non-basic N1O3 & $\mathrm{C} 25 \mathrm{H} 19 \mathrm{~N} 1 \mathrm{O} 3$ & 0.000141 & Basic N1O1 & $\mathrm{C} 38 \mathrm{H} 57 \mathrm{~N} 1 \mathrm{O} 1$ & $2.30 \mathrm{E}-05$ \\
\hline
\end{tabular}




\begin{tabular}{|c|c|c|c|c|c|c|c|c|}
\hline Class & Formula & Mass & Class & Formula & Mass & Class & Formula & Mass \\
\hline S1 & $\mathrm{C} 28 \mathrm{H} 16 \mathrm{~S} 1$ & $2.19 \mathrm{E}-06$ & Non-basic N1O3 & $\mathrm{C} 26 \mathrm{H} 21 \mathrm{~N} 1 \mathrm{O} 3$ & 0.000158 & Basic N1O1 & C39H59N1O1 & $8.61 \mathrm{E}-05$ \\
\hline S1 & $\mathrm{C} 29 \mathrm{H} 18 \mathrm{~S} 1$ & $5.21 \mathrm{E}-05$ & Non-basic N1O3 & $\mathrm{C} 27 \mathrm{H} 23 \mathrm{~N} 1 \mathrm{O} 3$ & 0.000151 & Basic N1O1 & C40H61N1O1 & $1.77 \mathrm{E}-05$ \\
\hline S1 & $\mathrm{C} 30 \mathrm{H} 20 \mathrm{~S} 1$ & $8.46 \mathrm{E}-05$ & Non-basic N1O3 & $\mathrm{C} 28 \mathrm{H} 25 \mathrm{~N} 1 \mathrm{O} 3$ & 0.000132 & Basic N1O1 & C41H63N1O1 & $7.56 \mathrm{E}-06$ \\
\hline S1 & $\mathrm{C} 31 \mathrm{H} 22 \mathrm{~S} 1$ & 7.99E-05 & Non-basic N1O3 & $\mathrm{C} 29 \mathrm{H} 27 \mathrm{~N} 1 \mathrm{O} 3$ & 0.00011 & Basic N1O1 & $\mathrm{C} 42 \mathrm{H} 65 \mathrm{~N} 1 \mathrm{O} 1$ & $3.89 \mathrm{E}-06$ \\
\hline S1 & $\mathrm{C} 32 \mathrm{H} 24 \mathrm{~S} 1$ & $5.86 \mathrm{E}-05$ & Non-basic N1O3 & $\mathrm{C} 30 \mathrm{H} 29 \mathrm{~N} 1 \mathrm{O} 3$ & $6.30 \mathrm{E}-05$ & Basic N1O1 & C43H67N1O1 & $9.59 \mathrm{E}-06$ \\
\hline S1 & $\mathrm{C} 33 \mathrm{H} 26 \mathrm{~S} 1$ & $3.52 \mathrm{E}-05$ & Non-basic N1O3 & $\mathrm{C} 31 \mathrm{H} 31 \mathrm{~N} 1 \mathrm{O} 3$ & $4.68 \mathrm{E}-05$ & Basic N1O1 & C44H69N1O1 & $5.73 \mathrm{E}-06$ \\
\hline S1 & $\mathrm{C} 34 \mathrm{H} 28 \mathrm{~S} 1$ & $1.70 \mathrm{E}-05$ & Non-basic N1O3 & $\mathrm{C} 32 \mathrm{H} 33 \mathrm{~N} 1 \mathrm{O} 3$ & $3.11 \mathrm{E}-05$ & Basic N1O1 & $\mathrm{C} 45 \mathrm{H} 71 \mathrm{~N} 1 \mathrm{O} 1$ & $1.99 \mathrm{E}-06$ \\
\hline S1 & $\mathrm{C} 35 \mathrm{H} 30 \mathrm{~S} 1$ & $8.15 \mathrm{E}-06$ & Non-basic N1O3 & $\mathrm{C} 33 \mathrm{H} 35 \mathrm{~N} 1 \mathrm{O} 3$ & $3.31 \mathrm{E}-05$ & Basic N1O1 & C16H11N1O1 & $1.14 \mathrm{E}-05$ \\
\hline S1 & $\mathrm{C} 36 \mathrm{H} 32 \mathrm{~S} 1$ & $2.73 \mathrm{E}-06$ & Non-basic N1O3 & $\mathrm{C} 34 \mathrm{H} 37 \mathrm{~N} 1 \mathrm{O} 3$ & $1.83 \mathrm{E}-05$ & Basic N1O1 & $\mathrm{C} 17 \mathrm{H} 13 \mathrm{~N} 1 \mathrm{O} 1$ & $1.69 \mathrm{E}-05$ \\
\hline S1 & $\mathrm{C} 29 \mathrm{H} 16 \mathrm{~S} 1$ & $7.89 \mathrm{E}-06$ & Non-basic N1O3 & $\mathrm{C} 35 \mathrm{H} 39 \mathrm{~N} 1 \mathrm{O} 3$ & $1.76 \mathrm{E}-05$ & Basic N1O1 & C18H15N1O1 & $6.40 \mathrm{E}-06$ \\
\hline S1 & $\mathrm{C} 30 \mathrm{H} 18 \mathrm{~S} 1$ & $4.27 \mathrm{E}-05$ & Non-basic N1O3 & $\mathrm{C} 36 \mathrm{H} 41 \mathrm{~N} 1 \mathrm{O} 3$ & $1.10 \mathrm{E}-05$ & Basic N1O1 & C19H17N1O1 & $1.06 \mathrm{E}-05$ \\
\hline S1 & $\mathrm{C} 31 \mathrm{H} 20 \mathrm{~S} 1$ & $5.62 \mathrm{E}-05$ & Non-basic N1O3 & $\mathrm{C} 37 \mathrm{H} 43 \mathrm{~N} 1 \mathrm{O} 3$ & $1.41 \mathrm{E}-05$ & Basic N1O1 & C20H19N1O1 & $1.29 \mathrm{E}-05$ \\
\hline S1 & $\mathrm{C} 32 \mathrm{H} 22 \mathrm{~S} 1$ & $6.54 \mathrm{E}-05$ & Non-basic N1O3 & $\mathrm{C} 38 \mathrm{H} 45 \mathrm{~N} 1 \mathrm{O} 3$ & $1.21 \mathrm{E}-05$ & Basic N1O1 & $\mathrm{C} 21 \mathrm{H} 21 \mathrm{~N} 1 \mathrm{O} 1$ & $2.07 \mathrm{E}-05$ \\
\hline S1 & $\mathrm{C} 33 \mathrm{H} 24 \mathrm{~S} 1$ & $5.15 \mathrm{E}-05$ & Non-basic N1O3 & $\mathrm{C} 39 \mathrm{H} 47 \mathrm{~N} 1 \mathrm{O} 3$ & $2.17 \mathrm{E}-05$ & Basic N1O1 & $\mathrm{C} 22 \mathrm{H} 23 \mathrm{~N} 1 \mathrm{O} 1$ & $3.53 \mathrm{E}-05$ \\
\hline S1 & $\mathrm{C} 34 \mathrm{H} 26 \mathrm{~S} 1$ & $2.83 \mathrm{E}-05$ & Non-basic N1O3 & $\mathrm{C} 40 \mathrm{H} 49 \mathrm{~N} 1 \mathrm{O} 3$ & $2.79 \mathrm{E}-05$ & Basic N1O1 & $\mathrm{C} 23 \mathrm{H} 25 \mathrm{~N} 1 \mathrm{O} 1$ & $5.53 \mathrm{E}-05$ \\
\hline S1 & $\mathrm{C} 35 \mathrm{H} 28 \mathrm{~S} 1$ & $2.65 \mathrm{E}-05$ & Non-basic N1O3 & $\mathrm{C} 41 \mathrm{H} 51 \mathrm{~N} 1 \mathrm{O} 3$ & $3.29 \mathrm{E}-05$ & Basic N1O1 & $\mathrm{C} 24 \mathrm{H} 27 \mathrm{~N} 1 \mathrm{O} 1$ & $3.83 \mathrm{E}-05$ \\
\hline S1 & $\mathrm{C} 36 \mathrm{H} 30 \mathrm{~S} 1$ & $1.18 \mathrm{E}-05$ & Non-basic N1O3 & $\mathrm{C} 42 \mathrm{H} 53 \mathrm{~N} 1 \mathrm{O} 3$ & $1.65 \mathrm{E}-05$ & Basic N1O1 & $\mathrm{C} 25 \mathrm{H} 29 \mathrm{~N} 1 \mathrm{O} 1$ & $5.34 \mathrm{E}-05$ \\
\hline S1 & $\mathrm{C} 37 \mathrm{H} 32 \mathrm{~S} 1$ & $8.61 \mathrm{E}-06$ & Non-basic N1O3 & $\mathrm{C} 43 \mathrm{H} 55 \mathrm{~N} 1 \mathrm{O} 3$ & $1.43 \mathrm{E}-05$ & Basic N1O1 & $\mathrm{C} 26 \mathrm{H} 31 \mathrm{~N} 1 \mathrm{O} 1$ & $5.58 \mathrm{E}-05$ \\
\hline S1 & $\mathrm{C} 38 \mathrm{H} 34 \mathrm{~S} 1$ & $2.68 \mathrm{E}-06$ & Non-basic N1O3 & $\mathrm{C} 44 \mathrm{H} 57 \mathrm{~N} 1 \mathrm{O} 3$ & $1.90 \mathrm{E}-05$ & Basic N1O1 & $\mathrm{C} 27 \mathrm{H} 33 \mathrm{~N} 1 \mathrm{O} 1$ & $4.78 \mathrm{E}-05$ \\
\hline S1 & $\mathrm{C} 39 \mathrm{H} 36 \mathrm{~S} 1$ & $2.52 \mathrm{E}-06$ & Non-basic N1O3 & $\mathrm{C} 45 \mathrm{H} 59 \mathrm{~N} 1 \mathrm{O} 3$ & $1.08 \mathrm{E}-05$ & Basic N1O1 & $\mathrm{C} 28 \mathrm{H} 35 \mathrm{~N} 1 \mathrm{O} 1$ & 7.63E-05 \\
\hline S1 & $\mathrm{C} 31 \mathrm{H} 18 \mathrm{~S} 1$ & $1.05 \mathrm{E}-05$ & Non-basic N1O3 & C46H61N1O3 & 8.67E-06 & Basic N1O1 & $\mathrm{C} 29 \mathrm{H} 37 \mathrm{~N} 1 \mathrm{O} 1$ & $8.05 \mathrm{E}-05$ \\
\hline S1 & $\mathrm{C} 32 \mathrm{H} 20 \mathrm{~S} 1$ & $3.54 \mathrm{E}-05$ & Non-basic N1O3 & C47H63N1O3 & $5.27 \mathrm{E}-06$ & Basic N1O1 & $\mathrm{C} 30 \mathrm{H} 39 \mathrm{~N} 1 \mathrm{O} 1$ & $8.05 \mathrm{E}-05$ \\
\hline S1 & $\mathrm{C} 33 \mathrm{H} 22 \mathrm{~S} 1$ & $3.47 \mathrm{E}-05$ & Non-basic N1O3 & $\mathrm{C} 48 \mathrm{H} 65 \mathrm{~N} 1 \mathrm{O} 3$ & $1.42 \mathrm{E}-05$ & Basic N1O1 & $\mathrm{C} 31 \mathrm{H} 41 \mathrm{~N} 1 \mathrm{O} 1$ & $6.81 \mathrm{E}-05$ \\
\hline S1 & $\mathrm{C} 34 \mathrm{H} 24 \mathrm{~S} 1$ & $3.39 \mathrm{E}-05$ & Non-basic N1O3 & C49H67N1O3 & $1.00 \mathrm{E}-05$ & Basic N1O1 & $\mathrm{C} 32 \mathrm{H} 43 \mathrm{~N} 1 \mathrm{O} 1$ & $5.92 \mathrm{E}-05$ \\
\hline S1 & $\mathrm{C} 35 \mathrm{H} 26 \mathrm{~S} 1$ & $3.08 \mathrm{E}-05$ & Non-basic N1O3 & $\mathrm{C} 22 \mathrm{H} 11 \mathrm{~N} 1 \mathrm{O} 3$ & $2.93 \mathrm{E}-06$ & Basic N1O1 & $\mathrm{C} 33 \mathrm{H} 45 \mathrm{~N} 1 \mathrm{O} 1$ & $4.78 \mathrm{E}-05$ \\
\hline S1 & $\mathrm{C} 36 \mathrm{H} 28 \mathrm{~S} 1$ & $1.64 \mathrm{E}-05$ & Non-basic N1O3 & $\mathrm{C} 23 \mathrm{H} 13 \mathrm{~N} 1 \mathrm{O} 3$ & $1.12 \mathrm{E}-05$ & Basic N1O1 & $\mathrm{C} 34 \mathrm{H} 47 \mathrm{~N} 1 \mathrm{O} 1$ & $5.09 \mathrm{E}-05$ \\
\hline S1 & $\mathrm{C} 37 \mathrm{H} 30 \mathrm{~S} 1$ & $1.32 \mathrm{E}-05$ & Non-basic N1O3 & $\mathrm{C} 24 \mathrm{H} 15 \mathrm{~N} 1 \mathrm{O} 3$ & $3.44 \mathrm{E}-05$ & Basic N1O1 & $\mathrm{C} 35 \mathrm{H} 49 \mathrm{~N} 1 \mathrm{O} 1$ & $4.59 \mathrm{E}-05$ \\
\hline S1 & $\mathrm{C} 32 \mathrm{H} 18 \mathrm{~S} 1$ & $2.63 \mathrm{E}-06$ & Non-basic N1O3 & $\mathrm{C} 25 \mathrm{H} 17 \mathrm{~N} 1 \mathrm{O} 3$ & $5.90 \mathrm{E}-05$ & Basic N1O1 & $\mathrm{C} 36 \mathrm{H} 51 \mathrm{~N} 1 \mathrm{O} 1$ & $4.00 \mathrm{E}-05$ \\
\hline S1 & $\mathrm{C} 33 \mathrm{H} 20 \mathrm{~S} 1$ & $2.58 \mathrm{E}-06$ & Non-basic N1O3 & $\mathrm{C} 26 \mathrm{H} 19 \mathrm{~N} 1 \mathrm{O} 3$ & $8.88 \mathrm{E}-05$ & Basic N1O1 & C37H53N1O1 & $5.14 \mathrm{E}-05$ \\
\hline S1 & $\mathrm{C} 34 \mathrm{H} 22 \mathrm{~S} 1$ & $2.27 \mathrm{E}-06$ & Non-basic N1O3 & $\mathrm{C} 27 \mathrm{H} 21 \mathrm{~N} 1 \mathrm{O} 3$ & 0.000104 & Basic N1O1 & C38H55N1O1 & $3.03 \mathrm{E}-05$ \\
\hline S1 & $\mathrm{C} 35 \mathrm{H} 24 \mathrm{~S} 1$ & $8.74 \mathrm{E}-06$ & Non-basic N1O3 & $\mathrm{C} 28 \mathrm{H} 23 \mathrm{~N} 1 \mathrm{O} 3$ & $9.62 \mathrm{E}-05$ & Basic N1O1 & C39H57N1O1 & $3.01 \mathrm{E}-05$ \\
\hline S1 & $\mathrm{C} 36 \mathrm{H} 26 \mathrm{~S} 1$ & $1.08 \mathrm{E}-05$ & Non-basic N1O3 & $\mathrm{C} 29 \mathrm{H} 25 \mathrm{~N} 1 \mathrm{O} 3$ & $8.11 \mathrm{E}-05$ & Basic N1O1 & C40H59N1O1 & $1.93 \mathrm{E}-05$ \\
\hline S1 & $\mathrm{C} 37 \mathrm{H} 28 \mathrm{~S} 1$ & $6.15 \mathrm{E}-06$ & Non-basic N1O3 & $\mathrm{C} 30 \mathrm{H} 27 \mathrm{~N} 1 \mathrm{O} 3$ & 7.09E-05 & Basic N1O1 & $\mathrm{C} 41 \mathrm{H} 61 \mathrm{~N} 1 \mathrm{O} 1$ & $2.41 \mathrm{E}-05$ \\
\hline S1 & $\mathrm{C} 38 \mathrm{H} 30 \mathrm{~S} 1$ & $4.76 \mathrm{E}-06$ & Non-basic N1O3 & $\mathrm{C} 31 \mathrm{H} 29 \mathrm{~N} 1 \mathrm{O} 3$ & $5.07 \mathrm{E}-05$ & Basic N1O1 & $\mathrm{C} 42 \mathrm{H} 63 \mathrm{~N} 1 \mathrm{O} 1$ & $1.02 \mathrm{E}-05$ \\
\hline S1 & $\mathrm{C} 33 \mathrm{H} 18 \mathrm{~S} 1$ & $2.34 \mathrm{E}-06$ & Non-basic N1O3 & $\mathrm{C} 32 \mathrm{H} 31 \mathrm{~N} 1 \mathrm{O} 3$ & $3.69 \mathrm{E}-05$ & Basic N1O1 & $\mathrm{C} 43 \mathrm{H} 65 \mathrm{~N} 1 \mathrm{O} 1$ & $5.54 \mathrm{E}-06$ \\
\hline $\mathrm{S} 1$ & $\mathrm{C} 34 \mathrm{H} 20 \mathrm{~S} 1$ & $1.35 \mathrm{E}-05$ & Non-basic N1O3 & $\mathrm{C} 33 \mathrm{H} 33 \mathrm{~N} 1 \mathrm{O} 3$ & $3.24 \mathrm{E}-05$ & Basic N1O1 & C16H9N1O1 & $1.30 \mathrm{E}-07$ \\
\hline S1 & $\mathrm{C} 35 \mathrm{H} 22 \mathrm{~S} 1$ & 7.51E-06 & Non-basic N1O3 & $\mathrm{C} 34 \mathrm{H} 35 \mathrm{~N} 1 \mathrm{O} 3$ & $1.01 \mathrm{E}-05$ & Basic N1O1 & $\mathrm{C} 17 \mathrm{H} 11 \mathrm{~N} 1 \mathrm{O} 1$ & $1.74 \mathrm{E}-05$ \\
\hline $\mathrm{S} 1$ & $\mathrm{C} 36 \mathrm{H} 24 \mathrm{~S} 1$ & $9.33 \mathrm{E}-06$ & Non-basic N1O3 & $\mathrm{C} 35 \mathrm{H} 37 \mathrm{~N} 1 \mathrm{O} 3$ & $5.99 \mathrm{E}-06$ & Basic N1O1 & $\mathrm{C} 18 \mathrm{H} 13 \mathrm{~N} 1 \mathrm{O} 1$ & $2.65 \mathrm{E}-05$ \\
\hline
\end{tabular}




\begin{tabular}{|c|c|c|c|c|c|c|c|c|}
\hline Class & Formula & Mass & Class & Formula & Mass & Class & Formula & Mass \\
\hline S1 & $\mathrm{C} 37 \mathrm{H} 26 \mathrm{~S} 1$ & $1.29 \mathrm{E}-05$ & Non-basic N1O3 & $\mathrm{C} 36 \mathrm{H} 39 \mathrm{~N} 1 \mathrm{O} 3$ & $8.78 \mathrm{E}-06$ & Basic N1O1 & C19H15N1O1 & $2.64 \mathrm{E}-05$ \\
\hline S1 & $\mathrm{C} 38 \mathrm{H} 28 \mathrm{~S} 1$ & $3.64 \mathrm{E}-06$ & Non-basic N1O3 & $\mathrm{C} 37 \mathrm{H} 41 \mathrm{~N} 1 \mathrm{O} 3$ & $9.39 \mathrm{E}-06$ & Basic N1O1 & $\mathrm{C} 20 \mathrm{H} 17 \mathrm{~N} 1 \mathrm{O} 1$ & $3.81 \mathrm{E}-05$ \\
\hline S1 & $\mathrm{C} 39 \mathrm{H} 30 \mathrm{~S} 1$ & $3.32 \mathrm{E}-06$ & Non-basic N1O3 & $\mathrm{C} 38 \mathrm{H} 43 \mathrm{~N} 1 \mathrm{O} 3$ & $1.13 \mathrm{E}-05$ & Basic N1O1 & C21H19N1O1 & $2.76 \mathrm{E}-05$ \\
\hline S1 & $\mathrm{C} 40 \mathrm{H} 30 \mathrm{~S} 1$ & $2.67 \mathrm{E}-06$ & Non-basic N1O3 & C39H45N1O3 & 4.91E-06 & Basic N1O1 & $\mathrm{C} 22 \mathrm{H} 21 \mathrm{~N} 1 \mathrm{O} 1$ & $3.58 \mathrm{E}-05$ \\
\hline S1 & $\mathrm{C} 41 \mathrm{H} 32 \mathrm{~S} 1$ & $2.79 \mathrm{E}-06$ & Non-basic N1O3 & $\mathrm{C} 40 \mathrm{H} 47 \mathrm{~N} 1 \mathrm{O} 3$ & $9.14 \mathrm{E}-06$ & Basic N1O1 & $\mathrm{C} 23 \mathrm{H} 23 \mathrm{~N} 1 \mathrm{O} 1$ & $5.26 \mathrm{E}-05$ \\
\hline S1 & $\mathrm{C} 42 \mathrm{H} 34 \mathrm{~S} 1$ & $6.21 \mathrm{E}-06$ & Non-basic N1O3 & $\mathrm{C} 41 \mathrm{H} 49 \mathrm{~N} 1 \mathrm{O} 3$ & $1.88 \mathrm{E}-05$ & Basic N1O1 & $\mathrm{C} 24 \mathrm{H} 25 \mathrm{~N} 1 \mathrm{O} 1$ & $3.22 \mathrm{E}-05$ \\
\hline O1S1 & $\mathrm{C} 33 \mathrm{H} 64 \mathrm{O} 1 \mathrm{~S} 1$ & $1.43 \mathrm{E}-05$ & Non-basic N1O3 & $\mathrm{C} 42 \mathrm{H} 51 \mathrm{~N} 1 \mathrm{O} 3$ & $2.04 \mathrm{E}-05$ & Basic N1O1 & $\mathrm{C} 25 \mathrm{H} 27 \mathrm{~N} 1 \mathrm{O} 1$ & $4.82 \mathrm{E}-05$ \\
\hline O1S1 & $\mathrm{C} 34 \mathrm{H} 66 \mathrm{O} 1 \mathrm{~S} 1$ & $1.19 \mathrm{E}-05$ & Non-basic N1O3 & $\mathrm{C} 43 \mathrm{H} 53 \mathrm{~N} 1 \mathrm{O} 3$ & $7.10 \mathrm{E}-06$ & Basic N1O1 & $\mathrm{C} 26 \mathrm{H} 29 \mathrm{~N} 1 \mathrm{O} 1$ & $7.64 \mathrm{E}-05$ \\
\hline O1S1 & C35H68O1S1 & $4.48 \mathrm{E}-06$ & Non-basic N1O3 & $\mathrm{C} 44 \mathrm{H} 55 \mathrm{~N} 1 \mathrm{O} 3$ & $5.42 \mathrm{E}-06$ & Basic N1O1 & $\mathrm{C} 27 \mathrm{H} 31 \mathrm{~N} 1 \mathrm{O} 1$ & $6.10 \mathrm{E}-05$ \\
\hline O1S1 & $\mathrm{C} 24 \mathrm{H} 44 \mathrm{O} 1 \mathrm{~S} 1$ & $1.92 \mathrm{E}-05$ & Non-basic N1O3 & $\mathrm{C} 45 \mathrm{H} 57 \mathrm{~N} 1 \mathrm{O} 3$ & $6.15 \mathrm{E}-06$ & Basic N1O1 & $\mathrm{C} 28 \mathrm{H} 33 \mathrm{~N} 1 \mathrm{O} 1$ & $7.10 \mathrm{E}-05$ \\
\hline O1S1 & $\mathrm{C} 25 \mathrm{H} 46 \mathrm{O} 1 \mathrm{~S} 1$ & 7.92E-06 & Non-basic N1O3 & $\mathrm{C} 46 \mathrm{H} 59 \mathrm{~N} 1 \mathrm{O} 3$ & $1.03 \mathrm{E}-05$ & Basic N1O1 & $\mathrm{C} 29 \mathrm{H} 35 \mathrm{~N} 1 \mathrm{O} 1$ & $6.58 \mathrm{E}-05$ \\
\hline O1S1 & $\mathrm{C} 26 \mathrm{H} 48 \mathrm{O} 1 \mathrm{~S} 1$ & $1.15 \mathrm{E}-05$ & Non-basic N1O3 & C47H61N1O3 & $1.51 \mathrm{E}-05$ & Basic N1O1 & $\mathrm{C} 30 \mathrm{H} 37 \mathrm{~N} 1 \mathrm{O} 1$ & $7.95 \mathrm{E}-05$ \\
\hline O1S1 & $\mathrm{C} 27 \mathrm{H} 50 \mathrm{O} 1 \mathrm{~S} 1$ & $1.71 \mathrm{E}-05$ & Non-basic N1O3 & $\mathrm{C} 48 \mathrm{H} 63 \mathrm{~N} 1 \mathrm{O} 3$ & $7.28 \mathrm{E}-06$ & Basic N1O1 & $\mathrm{C} 31 \mathrm{H} 39 \mathrm{~N} 1 \mathrm{O} 1$ & $9.74 \mathrm{E}-05$ \\
\hline O1S1 & $\mathrm{C} 28 \mathrm{H} 52 \mathrm{O} 1 \mathrm{~S} 1$ & $2.57 \mathrm{E}-06$ & Non-basic N1O3 & C49H65N1O3 & $6.83 \mathrm{E}-06$ & Basic N1O1 & $\mathrm{C} 32 \mathrm{H} 41 \mathrm{~N} 1 \mathrm{O} 1$ & $7.85 \mathrm{E}-05$ \\
\hline O1S1 & C29H54O1S1 & $2.65 \mathrm{E}-05$ & Non-basic N1O3 & $\mathrm{C} 50 \mathrm{H} 67 \mathrm{~N} 1 \mathrm{O} 3$ & $4.31 \mathrm{E}-06$ & Basic N1O1 & $\mathrm{C} 33 \mathrm{H} 43 \mathrm{~N} 1 \mathrm{O} 1$ & $6.77 \mathrm{E}-05$ \\
\hline O1S1 & $\mathrm{C} 30 \mathrm{H} 56 \mathrm{O} 1 \mathrm{~S} 1$ & $2.30 \mathrm{E}-05$ & Non-basic N1O3 & C51H69N1O3 & $9.17 \mathrm{E}-06$ & Basic N1O1 & $\mathrm{C} 34 \mathrm{H} 45 \mathrm{~N} 1 \mathrm{O} 1$ & 7.97E-05 \\
\hline O1S1 & $\mathrm{C} 31 \mathrm{H} 58 \mathrm{O} 1 \mathrm{~S} 1$ & $1.95 \mathrm{E}-06$ & Non-basic N1O3 & $\mathrm{C} 52 \mathrm{H} 71 \mathrm{~N} 1 \mathrm{O} 3$ & $6.72 \mathrm{E}-06$ & Basic N1O1 & $\mathrm{C} 35 \mathrm{H} 47 \mathrm{~N} 1 \mathrm{O} 1$ & $6.00 \mathrm{E}-05$ \\
\hline O1S1 & $\mathrm{C} 20 \mathrm{H} 34 \mathrm{O} 1 \mathrm{~S} 1$ & $2.54 \mathrm{E}-05$ & Non-basic N1O3 & $\mathrm{C} 53 \mathrm{H} 73 \mathrm{~N} 1 \mathrm{O} 3$ & $3.69 \mathrm{E}-06$ & Basic N1O1 & $\mathrm{C} 36 \mathrm{H} 49 \mathrm{~N} 1 \mathrm{O} 1$ & $5.73 \mathrm{E}-05$ \\
\hline O1S1 & $\mathrm{C} 21 \mathrm{H} 36 \mathrm{O} 1 \mathrm{~S} 1$ & $2.79 \mathrm{E}-06$ & Non-basic N1O3 & $\mathrm{C} 25 \mathrm{H} 15 \mathrm{~N} 1 \mathrm{O} 3$ & $2.23 \mathrm{E}-05$ & Basic N1O1 & C37H51N1O1 & 4.44E-05 \\
\hline O1S1 & $\mathrm{C} 22 \mathrm{H} 38 \mathrm{O} 1 \mathrm{~S} 1$ & $8.84 \mathrm{E}-06$ & Non-basic N1O3 & $\mathrm{C} 26 \mathrm{H} 17 \mathrm{~N} 1 \mathrm{O} 3$ & $4.55 \mathrm{E}-05$ & Basic N1O1 & C38H53N1O1 & $3.00 \mathrm{E}-05$ \\
\hline O1S1 & $\mathrm{C} 23 \mathrm{H} 40 \mathrm{O} 1 \mathrm{~S} 1$ & $2.70 \mathrm{E}-05$ & Non-basic N1O3 & $\mathrm{C} 27 \mathrm{H} 19 \mathrm{~N} 1 \mathrm{O} 3$ & $5.21 \mathrm{E}-05$ & Basic N1O1 & C39H55N1O1 & $4.28 \mathrm{E}-05$ \\
\hline O1S1 & $\mathrm{C} 24 \mathrm{H} 42 \mathrm{O} 1 \mathrm{~S} 1$ & $1.93 \mathrm{E}-05$ & Non-basic N1O3 & $\mathrm{C} 28 \mathrm{H} 21 \mathrm{~N} 1 \mathrm{O} 3$ & $5.94 \mathrm{E}-05$ & Basic N1O1 & $\mathrm{C} 40 \mathrm{H} 57 \mathrm{~N} 1 \mathrm{O} 1$ & $3.27 \mathrm{E}-05$ \\
\hline O1S1 & $\mathrm{C} 25 \mathrm{H} 44 \mathrm{O} 1 \mathrm{~S} 1$ & $2.00 \mathrm{E}-05$ & Non-basic N1O3 & $\mathrm{C} 29 \mathrm{H} 23 \mathrm{~N} 1 \mathrm{O} 3$ & $6.29 \mathrm{E}-05$ & Basic N1O1 & C41H59N1O1 & $2.44 \mathrm{E}-05$ \\
\hline O1S1 & $\mathrm{C} 26 \mathrm{H} 46 \mathrm{O} 1 \mathrm{~S} 1$ & $2.21 \mathrm{E}-05$ & Non-basic N1O3 & $\mathrm{C} 30 \mathrm{H} 25 \mathrm{~N} 1 \mathrm{O} 3$ & $4.80 \mathrm{E}-05$ & Basic N1O1 & $\mathrm{C} 42 \mathrm{H} 61 \mathrm{~N} 1 \mathrm{O} 1$ & $1.18 \mathrm{E}-05$ \\
\hline O1S1 & $\mathrm{C} 27 \mathrm{H} 48 \mathrm{O} 1 \mathrm{~S} 1$ & 4.06E-06 & Non-basic N1O3 & $\mathrm{C} 31 \mathrm{H} 27 \mathrm{~N} 1 \mathrm{O} 3$ & $4.01 \mathrm{E}-05$ & Basic N1O1 & C19H13N1O1 & $1.61 \mathrm{E}-07$ \\
\hline O1S1 & $\mathrm{C} 28 \mathrm{H} 50 \mathrm{O} 1 \mathrm{~S} 1$ & $1.20 \mathrm{E}-05$ & Non-basic N1O3 & $\mathrm{C} 32 \mathrm{H} 29 \mathrm{~N} 1 \mathrm{O} 3$ & $3.18 \mathrm{E}-05$ & Basic N1O1 & $\mathrm{C} 20 \mathrm{H} 15 \mathrm{~N} 1 \mathrm{O} 1$ & $8.82 \mathrm{E}-06$ \\
\hline O1S1 & $\mathrm{C} 29 \mathrm{H} 52 \mathrm{O} 1 \mathrm{~S} 1$ & $5.12 \mathrm{E}-06$ & Non-basic N1O3 & $\mathrm{C} 33 \mathrm{H} 31 \mathrm{~N} 1 \mathrm{O} 3$ & $1.28 \mathrm{E}-05$ & Basic N1O1 & $\mathrm{C} 21 \mathrm{H} 17 \mathrm{~N} 1 \mathrm{O} 1$ & $8.89 \mathrm{E}-05$ \\
\hline O1S1 & $\mathrm{C} 30 \mathrm{H} 54 \mathrm{O} 1 \mathrm{~S} 1$ & $3.11 \mathrm{E}-05$ & Non-basic N1O3 & $\mathrm{C} 34 \mathrm{H} 33 \mathrm{~N} 1 \mathrm{O} 3$ & $1.19 \mathrm{E}-05$ & Basic N1O1 & $\mathrm{C} 22 \mathrm{H} 19 \mathrm{~N} 1 \mathrm{O} 1$ & 0.000104 \\
\hline O1S1 & $\mathrm{C} 31 \mathrm{H} 56 \mathrm{O} 1 \mathrm{~S} 1$ & $2.94 \mathrm{E}-05$ & Non-basic N1O3 & $\mathrm{C} 35 \mathrm{H} 35 \mathrm{~N} 1 \mathrm{O} 3$ & $5.78 \mathrm{E}-06$ & Basic N1O1 & $\mathrm{C} 23 \mathrm{H} 21 \mathrm{~N} 1 \mathrm{O} 1$ & $9.55 \mathrm{E}-05$ \\
\hline O1S1 & $\mathrm{C} 32 \mathrm{H} 58 \mathrm{O} 1 \mathrm{~S} 1$ & $1.31 \mathrm{E}-05$ & Non-basic N1O3 & $\mathrm{C} 36 \mathrm{H} 37 \mathrm{~N} 1 \mathrm{O} 3$ & $1.65 \mathrm{E}-06$ & Basic N1O1 & $\mathrm{C} 24 \mathrm{H} 23 \mathrm{~N} 1 \mathrm{O} 1$ & 7.94E-05 \\
\hline O1S1 & C33H60O1S1 & $1.29 \mathrm{E}-05$ & Non-basic N1O3 & $\mathrm{C} 38 \mathrm{H} 41 \mathrm{~N} 1 \mathrm{O} 3$ & $3.50 \mathrm{E}-06$ & Basic N1O1 & $\mathrm{C} 25 \mathrm{H} 25 \mathrm{~N} 1 \mathrm{O} 1$ & $9.14 \mathrm{E}-05$ \\
\hline O1S1 & $\mathrm{C} 34 \mathrm{H} 62 \mathrm{O} 1 \mathrm{~S} 1$ & $9.50 \mathrm{E}-06$ & Non-basic N1O3 & $\mathrm{C} 39 \mathrm{H} 43 \mathrm{~N} 1 \mathrm{O} 3$ & $6.52 \mathrm{E}-06$ & Basic N1O1 & $\mathrm{C} 26 \mathrm{H} 27 \mathrm{~N} 1 \mathrm{O} 1$ & $9.29 \mathrm{E}-05$ \\
\hline O1S1 & $\mathrm{C} 21 \mathrm{H} 34 \mathrm{O} 1 \mathrm{~S} 1$ & $1.54 \mathrm{E}-05$ & Non-basic N1O3 & $\mathrm{C} 40 \mathrm{H} 45 \mathrm{~N} 1 \mathrm{O} 3$ & $5.44 \mathrm{E}-06$ & Basic N1O1 & $\mathrm{C} 27 \mathrm{H} 29 \mathrm{~N} 1 \mathrm{O} 1$ & $8.90 \mathrm{E}-05$ \\
\hline O1S1 & $\mathrm{C} 22 \mathrm{H} 36 \mathrm{O} 1 \mathrm{~S} 1$ & $1.02 \mathrm{E}-05$ & Non-basic N1O3 & $\mathrm{C} 41 \mathrm{H} 47 \mathrm{~N} 1 \mathrm{O} 3$ & $8.75 \mathrm{E}-06$ & Basic N1O1 & $\mathrm{C} 28 \mathrm{H} 31 \mathrm{~N} 1 \mathrm{O} 1$ & 7.87E-05 \\
\hline O1S1 & $\mathrm{C} 23 \mathrm{H} 38 \mathrm{O} 1 \mathrm{~S} 1$ & $1.03 \mathrm{E}-05$ & Non-basic N1O3 & $\mathrm{C} 42 \mathrm{H} 49 \mathrm{~N} 1 \mathrm{O} 3$ & $8.95 \mathrm{E}-06$ & Basic N1O1 & $\mathrm{C} 29 \mathrm{H} 33 \mathrm{~N} 1 \mathrm{O} 1$ & $6.94 \mathrm{E}-05$ \\
\hline O1S1 & $\mathrm{C} 24 \mathrm{H} 40 \mathrm{O} 1 \mathrm{~S} 1$ & $1.82 \mathrm{E}-05$ & Non-basic N1O3 & $\mathrm{C} 43 \mathrm{H} 51 \mathrm{~N} 1 \mathrm{O} 3$ & $9.16 \mathrm{E}-06$ & Basic N1O1 & $\mathrm{C} 30 \mathrm{H} 35 \mathrm{~N} 1 \mathrm{O} 1$ & 0.000106 \\
\hline O1S1 & $\mathrm{C} 25 \mathrm{H} 42 \mathrm{O} 1 \mathrm{~S} 1$ & $1.45 \mathrm{E}-05$ & Non-basic N1O3 & $\mathrm{C} 44 \mathrm{H} 53 \mathrm{~N} 1 \mathrm{O} 3$ & $1.06 \mathrm{E}-05$ & Basic N1O1 & $\mathrm{C} 31 \mathrm{H} 37 \mathrm{~N} 1 \mathrm{O} 1$ & $8.28 \mathrm{E}-05$ \\
\hline O1S1 & $\mathrm{C} 26 \mathrm{H} 44 \mathrm{O} 1 \mathrm{~S} 1$ & $1.11 \mathrm{E}-05$ & Non-basic N1O3 & $\mathrm{C} 45 \mathrm{H} 55 \mathrm{~N} 1 \mathrm{O} 3$ & 7.11E-06 & Basic N1O1 & $\mathrm{C} 32 \mathrm{H} 39 \mathrm{~N} 1 \mathrm{O} 1$ & 0.0001 \\
\hline
\end{tabular}




\begin{tabular}{|c|c|c|c|c|c|c|c|c|}
\hline Class & Formula & Mass & Class & Formula & Mass & Class & Formula & Mass \\
\hline O1S1 & $\mathrm{C} 27 \mathrm{H} 46 \mathrm{O} 1 \mathrm{~S} 1$ & $1.12 \mathrm{E}-05$ & Non-basic N1O3 & $\mathrm{C} 46 \mathrm{H} 57 \mathrm{~N} 1 \mathrm{O} 3$ & 4.43E-06 & Basic N1O1 & C33H41N1O1 & $7.15 \mathrm{E}-05$ \\
\hline O1S1 & $\mathrm{C} 28 \mathrm{H} 48 \mathrm{O} 1 \mathrm{~S} 1$ & $2.37 \mathrm{E}-05$ & Non-basic N1O3 & C47H59N1O3 & $6.98 \mathrm{E}-06$ & Basic N1O1 & $\mathrm{C} 34 \mathrm{H} 43 \mathrm{~N} 1 \mathrm{O} 1$ & $8.38 \mathrm{E}-05$ \\
\hline O1S1 & $\mathrm{C} 29 \mathrm{H} 50 \mathrm{O} 1 \mathrm{~S} 1$ & $2.96 \mathrm{E}-05$ & Non-basic N1O3 & C48H61N1O3 & $7.32 \mathrm{E}-06$ & Basic N1O1 & $\mathrm{C} 35 \mathrm{H} 45 \mathrm{~N} 1 \mathrm{O} 1$ & $5.64 \mathrm{E}-05$ \\
\hline O1S1 & $\mathrm{C} 30 \mathrm{H} 52 \mathrm{O} 1 \mathrm{~S} 1$ & $2.41 \mathrm{E}-05$ & Non-basic N1O3 & C49H63N1O3 & $1.82 \mathrm{E}-05$ & Basic N1O1 & $\mathrm{C} 36 \mathrm{H} 47 \mathrm{~N} 1 \mathrm{O} 1$ & $7.25 \mathrm{E}-05$ \\
\hline O1S1 & $\mathrm{C} 31 \mathrm{H} 54 \mathrm{O} 1 \mathrm{~S} 1$ & $5.68 \mathrm{E}-06$ & Non-basic N1O3 & C50H65N1O3 & $8.44 \mathrm{E}-06$ & Basic N1O1 & C37H49N1O1 & $4.01 \mathrm{E}-05$ \\
\hline O1S1 & $\mathrm{C} 32 \mathrm{H} 56 \mathrm{O} 1 \mathrm{~S} 1$ & $3.81 \mathrm{E}-05$ & Non-basic N1O3 & C51H67N1O3 & $1.08 \mathrm{E}-05$ & Basic N1O1 & C38H51N1O1 & 4.33E-05 \\
\hline O1S1 & $\mathrm{C} 33 \mathrm{H} 58 \mathrm{O} 1 \mathrm{~S} 1$ & $5.30 \mathrm{E}-06$ & Non-basic N1O3 & C52H69N1O3 & 7.93E-06 & Basic N1O1 & C39H53N1O1 & $3.27 \mathrm{E}-05$ \\
\hline O1S1 & $\mathrm{C} 20 \mathrm{H} 30 \mathrm{O} 1 \mathrm{~S} 1$ & $2.78 \mathrm{E}-06$ & Non-basic N1O3 & C53H71N1O3 & $8.94 \mathrm{E}-06$ & Basic N1O1 & $\mathrm{C} 40 \mathrm{H} 55 \mathrm{~N} 1 \mathrm{O} 1$ & $2.57 \mathrm{E}-05$ \\
\hline O1S1 & $\mathrm{C} 21 \mathrm{H} 32 \mathrm{O} 1 \mathrm{~S} 1$ & $1.38 \mathrm{E}-05$ & Non-basic N1O3 & $\mathrm{C} 25 \mathrm{H} 13 \mathrm{~N} 1 \mathrm{O} 3$ & $5.23 \mathrm{E}-06$ & Basic N1O1 & $\mathrm{C} 41 \mathrm{H} 57 \mathrm{~N} 1 \mathrm{O} 1$ & $2.11 \mathrm{E}-05$ \\
\hline O1S1 & $\mathrm{C} 22 \mathrm{H} 34 \mathrm{O} 1 \mathrm{~S} 1$ & $1.08 \mathrm{E}-05$ & Non-basic N1O3 & $\mathrm{C} 26 \mathrm{H} 15 \mathrm{~N} 1 \mathrm{O} 3$ & $1.50 \mathrm{E}-05$ & Basic N1O1 & $\mathrm{C} 42 \mathrm{H} 59 \mathrm{~N} 1 \mathrm{O} 1$ & $8.17 \mathrm{E}-06$ \\
\hline O1S1 & $\mathrm{C} 23 \mathrm{H} 36 \mathrm{O} 1 \mathrm{~S} 1$ & $5.12 \mathrm{E}-06$ & Non-basic N1O3 & $\mathrm{C} 27 \mathrm{H} 17 \mathrm{~N} 1 \mathrm{O} 3$ & $3.28 \mathrm{E}-05$ & Basic N1O1 & C43H61N1O1 & $9.48 \mathrm{E}-06$ \\
\hline O1S1 & $\mathrm{C} 24 \mathrm{H} 38 \mathrm{O} 1 \mathrm{~S} 1$ & $6.16 \mathrm{E}-06$ & Non-basic N1O3 & $\mathrm{C} 28 \mathrm{H} 19 \mathrm{~N} 1 \mathrm{O} 3$ & $3.92 \mathrm{E}-05$ & Basic N1O1 & C44H63N1O1 & $4.69 \mathrm{E}-06$ \\
\hline O1S1 & $\mathrm{C} 25 \mathrm{H} 40 \mathrm{O} 1 \mathrm{~S} 1$ & $3.17 \mathrm{E}-05$ & Non-basic N1O3 & $\mathrm{C} 29 \mathrm{H} 21 \mathrm{~N} 1 \mathrm{O} 3$ & $4.05 \mathrm{E}-05$ & Basic N1O1 & C45H65N1O1 & $3.29 \mathrm{E}-06$ \\
\hline O1S1 & $\mathrm{C} 26 \mathrm{H} 42 \mathrm{O} 1 \mathrm{~S} 1$ & $3.23 \mathrm{E}-05$ & Non-basic N1O3 & $\mathrm{C} 30 \mathrm{H} 23 \mathrm{~N} 1 \mathrm{O} 3$ & $4.86 \mathrm{E}-05$ & Basic N1O1 & $\mathrm{C} 20 \mathrm{H} 13 \mathrm{~N} 1 \mathrm{O} 1$ & $1.21 \mathrm{E}-06$ \\
\hline O1S1 & $\mathrm{C} 27 \mathrm{H} 44 \mathrm{O} 1 \mathrm{~S} 1$ & $1.72 \mathrm{E}-05$ & Non-basic N1O3 & $\mathrm{C} 31 \mathrm{H} 25 \mathrm{~N} 1 \mathrm{O} 3$ & 4.47E-05 & Basic N1O1 & $\mathrm{C} 21 \mathrm{H} 15 \mathrm{~N} 1 \mathrm{O} 1$ & $4.56 \mathrm{E}-06$ \\
\hline O1S1 & $\mathrm{C} 28 \mathrm{H} 46 \mathrm{O} 1 \mathrm{~S} 1$ & $2.42 \mathrm{E}-05$ & Non-basic N1O3 & $\mathrm{C} 32 \mathrm{H} 27 \mathrm{~N} 1 \mathrm{O} 3$ & $5.07 \mathrm{E}-05$ & Basic N1O1 & $\mathrm{C} 22 \mathrm{H} 17 \mathrm{~N} 1 \mathrm{O} 1$ & $3.05 \mathrm{E}-05$ \\
\hline O1S1 & $\mathrm{C} 29 \mathrm{H} 48 \mathrm{O} 1 \mathrm{~S} 1$ & $3.70 \mathrm{E}-05$ & Non-basic N1O3 & $\mathrm{C} 33 \mathrm{H} 29 \mathrm{~N} 1 \mathrm{O} 3$ & $3.42 \mathrm{E}-05$ & Basic N1O1 & $\mathrm{C} 23 \mathrm{H} 19 \mathrm{~N} 1 \mathrm{O} 1$ & $6.56 \mathrm{E}-05$ \\
\hline O1S1 & $\mathrm{C} 30 \mathrm{H} 50 \mathrm{O} 1 \mathrm{~S} 1$ & $3.20 \mathrm{E}-05$ & Non-basic N1O3 & $\mathrm{C} 34 \mathrm{H} 31 \mathrm{~N} 1 \mathrm{O} 3$ & $1.96 \mathrm{E}-05$ & Basic N1O1 & $\mathrm{C} 24 \mathrm{H} 21 \mathrm{~N} 1 \mathrm{O} 1$ & 7.57E-05 \\
\hline O1S1 & $\mathrm{C} 31 \mathrm{H} 52 \mathrm{O} 1 \mathrm{~S} 1$ & $2.63 \mathrm{E}-05$ & Non-basic N1O3 & $\mathrm{C} 35 \mathrm{H} 33 \mathrm{~N} 1 \mathrm{O} 3$ & $4.27 \mathrm{E}-06$ & Basic N1O1 & $\mathrm{C} 25 \mathrm{H} 23 \mathrm{~N} 1 \mathrm{O} 1$ & $8.47 \mathrm{E}-05$ \\
\hline O1S1 & C15H18O1S1 & $1.04 \mathrm{E}-05$ & Non-basic N1O3 & $\mathrm{C} 37 \mathrm{H} 37 \mathrm{~N} 1 \mathrm{O} 3$ & $3.08 \mathrm{E}-06$ & Basic N1O1 & $\mathrm{C} 26 \mathrm{H} 25 \mathrm{~N} 1 \mathrm{O} 1$ & 0.000132 \\
\hline O1S1 & $\mathrm{C} 16 \mathrm{H} 20 \mathrm{O} 1 \mathrm{~S} 1$ & 0.000447 & Non-basic N1O3 & $\mathrm{C} 38 \mathrm{H} 39 \mathrm{~N} 1 \mathrm{O} 3$ & $6.04 \mathrm{E}-06$ & Basic N1O1 & $\mathrm{C} 27 \mathrm{H} 27 \mathrm{~N} 1 \mathrm{O} 1$ & $9.58 \mathrm{E}-05$ \\
\hline O1S1 & $\mathrm{C} 17 \mathrm{H} 22 \mathrm{O} 1 \mathrm{~S} 1$ & $6.26 \mathrm{E}-05$ & Non-basic N1O3 & C39H41N1O3 & $2.10 \mathrm{E}-06$ & Basic N1O1 & $\mathrm{C} 28 \mathrm{H} 29 \mathrm{~N} 1 \mathrm{O} 1$ & $9.62 \mathrm{E}-05$ \\
\hline O1S1 & $\mathrm{C} 18 \mathrm{H} 24 \mathrm{O} 1 \mathrm{~S} 1$ & 0.000114 & Non-basic N1O3 & $\mathrm{C} 40 \mathrm{H} 43 \mathrm{~N} 1 \mathrm{O} 3$ & $4.60 \mathrm{E}-06$ & Basic N1O1 & $\mathrm{C} 29 \mathrm{H} 31 \mathrm{~N} 1 \mathrm{O} 1$ & 0.000113 \\
\hline O1S1 & $\mathrm{C} 19 \mathrm{H} 26 \mathrm{O} 1 \mathrm{~S} 1$ & $1.56 \mathrm{E}-05$ & Non-basic N1O3 & $\mathrm{C} 41 \mathrm{H} 45 \mathrm{~N} 1 \mathrm{O} 3$ & $1.55 \mathrm{E}-06$ & Basic N1O1 & $\mathrm{C} 30 \mathrm{H} 33 \mathrm{~N} 1 \mathrm{O} 1$ & $8.60 \mathrm{E}-05$ \\
\hline O1S1 & $\mathrm{C} 20 \mathrm{H} 28 \mathrm{O} 1 \mathrm{~S} 1$ & $3.75 \mathrm{E}-05$ & Non-basic N1O3 & $\mathrm{C} 27 \mathrm{H} 15 \mathrm{~N} 1 \mathrm{O} 3$ & $2.40 \mathrm{E}-06$ & Basic N1O1 & $\mathrm{C} 31 \mathrm{H} 35 \mathrm{~N} 1 \mathrm{O} 1$ & $6.43 \mathrm{E}-05$ \\
\hline O1S1 & $\mathrm{C} 21 \mathrm{H} 30 \mathrm{O} 1 \mathrm{~S} 1$ & 0.000428 & Non-basic N1O3 & $\mathrm{C} 28 \mathrm{H} 17 \mathrm{~N} 1 \mathrm{O} 3$ & $3.85 \mathrm{E}-06$ & Basic N1O1 & $\mathrm{C} 32 \mathrm{H} 37 \mathrm{~N} 1 \mathrm{O} 1$ & 0.000104 \\
\hline O1S1 & $\mathrm{C} 22 \mathrm{H} 32 \mathrm{O} 1 \mathrm{~S} 1$ & $1.89 \mathrm{E}-05$ & Non-basic N1O3 & C29H19N1O3 & $1.15 \mathrm{E}-05$ & Basic N1O1 & $\mathrm{C} 33 \mathrm{H} 39 \mathrm{~N} 1 \mathrm{O} 1$ & $6.00 \mathrm{E}-05$ \\
\hline O1S1 & $\mathrm{C} 23 \mathrm{H} 34 \mathrm{O} 1 \mathrm{~S} 1$ & 0.00011 & Non-basic N1O3 & $\mathrm{C} 30 \mathrm{H} 21 \mathrm{~N} 1 \mathrm{O} 3$ & $2.96 \mathrm{E}-05$ & Basic N1O1 & $\mathrm{C} 34 \mathrm{H} 41 \mathrm{~N} 1 \mathrm{O} 1$ & $6.71 \mathrm{E}-05$ \\
\hline O1S1 & $\mathrm{C} 24 \mathrm{H} 36 \mathrm{O} 1 \mathrm{~S} 1$ & 0.001005 & Non-basic N1O3 & $\mathrm{C} 31 \mathrm{H} 23 \mathrm{~N} 1 \mathrm{O} 3$ & $2.63 \mathrm{E}-05$ & Basic N1O1 & $\mathrm{C} 35 \mathrm{H} 43 \mathrm{~N} 1 \mathrm{O} 1$ & $4.10 \mathrm{E}-05$ \\
\hline O1S1 & $\mathrm{C} 25 \mathrm{H} 38 \mathrm{O} 1 \mathrm{~S} 1$ & $5.18 \mathrm{E}-05$ & Non-basic N1O3 & $\mathrm{C} 32 \mathrm{H} 25 \mathrm{~N} 1 \mathrm{O} 3$ & $2.18 \mathrm{E}-05$ & Basic N1O1 & $\mathrm{C} 36 \mathrm{H} 45 \mathrm{~N} 1 \mathrm{O} 1$ & $3.74 \mathrm{E}-05$ \\
\hline O1S1 & $\mathrm{C} 26 \mathrm{H} 40 \mathrm{O} 1 \mathrm{~S} 1$ & 0.000142 & Non-basic N1O3 & $\mathrm{C} 33 \mathrm{H} 27 \mathrm{~N} 1 \mathrm{O} 3$ & $2.00 \mathrm{E}-05$ & Basic N1O1 & $\mathrm{C} 37 \mathrm{H} 47 \mathrm{~N} 1 \mathrm{O} 1$ & $6.69 \mathrm{E}-05$ \\
\hline O1S1 & $\mathrm{C} 27 \mathrm{H} 42 \mathrm{O} 1 \mathrm{~S} 1$ & $4.20 \mathrm{E}-05$ & Non-basic N1O3 & $\mathrm{C} 34 \mathrm{H} 29 \mathrm{~N} 1 \mathrm{O} 3$ & $1.53 \mathrm{E}-05$ & Basic N1O1 & $\mathrm{C} 38 \mathrm{H} 49 \mathrm{~N} 1 \mathrm{O} 1$ & 3.67E-05 \\
\hline O1S1 & $\mathrm{C} 28 \mathrm{H} 44 \mathrm{O} 1 \mathrm{~S} 1$ & 0.000793 & Non-basic N1O3 & $\mathrm{C} 35 \mathrm{H} 31 \mathrm{~N} 1 \mathrm{O} 3$ & $1.09 \mathrm{E}-05$ & Basic N1O1 & C39H51N1O1 & $2.46 \mathrm{E}-05$ \\
\hline O1S1 & $\mathrm{C} 29 \mathrm{H} 46 \mathrm{O} 1 \mathrm{~S} 1$ & 0.000176 & Non-basic N1O3 & $\mathrm{C} 36 \mathrm{H} 33 \mathrm{~N} 1 \mathrm{O} 3$ & $1.12 \mathrm{E}-05$ & Basic N1O1 & $\mathrm{C} 40 \mathrm{H} 53 \mathrm{~N} 1 \mathrm{O} 1$ & $2.05 \mathrm{E}-05$ \\
\hline O1S1 & $\mathrm{C} 30 \mathrm{H} 48 \mathrm{O} 1 \mathrm{~S} 1$ & 0.000258 & Non-basic N1O3 & $\mathrm{C} 29 \mathrm{H} 17 \mathrm{~N} 1 \mathrm{O} 3$ & $9.16 \mathrm{E}-06$ & Basic N1O1 & $\mathrm{C} 41 \mathrm{H} 55 \mathrm{~N} 1 \mathrm{O} 1$ & $1.78 \mathrm{E}-05$ \\
\hline O1S1 & $\mathrm{C} 31 \mathrm{H} 50 \mathrm{O} 1 \mathrm{~S} 1$ & 0.000121 & Non-basic N1O3 & $\mathrm{C} 30 \mathrm{H} 19 \mathrm{~N} 1 \mathrm{O} 3$ & $7.28 \mathrm{E}-06$ & Basic N1O1 & $\mathrm{C} 42 \mathrm{H} 57 \mathrm{~N} 1 \mathrm{O} 1$ & $1.13 \mathrm{E}-05$ \\
\hline O1S1 & C14H14O1S1 & $3.18 \mathrm{E}-05$ & Non-basic N1O3 & $\mathrm{C} 31 \mathrm{H} 21 \mathrm{~N} 1 \mathrm{O} 3$ & $9.42 \mathrm{E}-06$ & Basic N1O1 & C43H59N1O1 & $9.37 \mathrm{E}-06$ \\
\hline O1S1 & $\mathrm{C} 15 \mathrm{H} 16 \mathrm{O} 1 \mathrm{~S} 1$ & $4.33 \mathrm{E}-05$ & Non-basic N1O3 & $\mathrm{C} 32 \mathrm{H} 23 \mathrm{~N} 1 \mathrm{O} 3$ & $1.43 \mathrm{E}-05$ & Basic N1O1 & $\mathrm{C} 44 \mathrm{H} 61 \mathrm{~N} 1 \mathrm{O} 1$ & 4.97E-06 \\
\hline
\end{tabular}




\begin{tabular}{|c|c|c|c|c|c|c|c|c|}
\hline Class & Formula & Mass & Class & Formula & Mass & Class & Formula & Mass \\
\hline O1S1 & $\mathrm{C} 16 \mathrm{H} 18 \mathrm{O} 1 \mathrm{~S} 1$ & $5.46 \mathrm{E}-05$ & Non-basic N1O3 & $\mathrm{C} 33 \mathrm{H} 25 \mathrm{~N} 1 \mathrm{O} 3$ & $1.67 \mathrm{E}-05$ & Basic N1O1 & C20H11N1O1 & $1.78 \mathrm{E}-07$ \\
\hline O1S1 & $\mathrm{C} 17 \mathrm{H} 20 \mathrm{O} 1 \mathrm{~S} 1$ & $2.03 \mathrm{E}-05$ & Non-basic N1O3 & $\mathrm{C} 34 \mathrm{H} 27 \mathrm{~N} 1 \mathrm{O} 3$ & $1.16 \mathrm{E}-05$ & Basic N1O1 & C21H13N1O1 & $5.36 \mathrm{E}-06$ \\
\hline O1S1 & $\mathrm{C} 18 \mathrm{H} 22 \mathrm{O} 1 \mathrm{~S} 1$ & $6.23 \mathrm{E}-06$ & Non-basic N1O3 & $\mathrm{C} 35 \mathrm{H} 29 \mathrm{~N} 1 \mathrm{O} 3$ & $6.82 \mathrm{E}-06$ & Basic N1O1 & $\mathrm{C} 22 \mathrm{H} 15 \mathrm{~N} 1 \mathrm{O} 1$ & $1.88 \mathrm{E}-05$ \\
\hline O1S1 & $\mathrm{C} 19 \mathrm{H} 24 \mathrm{O} 1 \mathrm{~S} 1$ & $3.22 \mathrm{E}-05$ & Non-basic N1O3 & C36H31N1O3 & $5.37 \mathrm{E}-06$ & Basic N1O1 & $\mathrm{C} 23 \mathrm{H} 17 \mathrm{~N} 1 \mathrm{O} 1$ & $6.30 \mathrm{E}-05$ \\
\hline O1S1 & $\mathrm{C} 20 \mathrm{H} 26 \mathrm{O} 1 \mathrm{~S} 1$ & $6.21 \mathrm{E}-05$ & Non-basic N1O3 & C37H33N1O3 & $6.30 \mathrm{E}-06$ & Basic N1O1 & C24H19N1O1 & 0.000144 \\
\hline O1S1 & $\mathrm{C} 21 \mathrm{H} 28 \mathrm{O} 1 \mathrm{~S} 1$ & $7.55 \mathrm{E}-05$ & Non-basic N1O3 & $\mathrm{C} 38 \mathrm{H} 35 \mathrm{~N} 1 \mathrm{O} 3$ & 4.49E-06 & Basic N1O1 & $\mathrm{C} 25 \mathrm{H} 21 \mathrm{~N} 1 \mathrm{O} 1$ & $8.70 \mathrm{E}-05$ \\
\hline O1S1 & $\mathrm{C} 22 \mathrm{H} 30 \mathrm{O} 1 \mathrm{~S} 1$ & 0.000153 & Non-basic N1O3 & $\mathrm{C} 30 \mathrm{H} 17 \mathrm{~N} 1 \mathrm{O} 3$ & $2.59 \mathrm{E}-06$ & Basic N1O1 & $\mathrm{C} 26 \mathrm{H} 23 \mathrm{~N} 1 \mathrm{O} 1$ & $6.88 \mathrm{E}-05$ \\
\hline O1S1 & $\mathrm{C} 23 \mathrm{H} 32 \mathrm{O} 1 \mathrm{~S} 1$ & $3.52 \mathrm{E}-05$ & Non-basic N1O3 & C31H19N1O3 & $2.40 \mathrm{E}-06$ & Basic N1O1 & $\mathrm{C} 27 \mathrm{H} 25 \mathrm{~N} 1 \mathrm{O} 1$ & $7.00 \mathrm{E}-05$ \\
\hline O1S1 & $\mathrm{C} 24 \mathrm{H} 34 \mathrm{O} 1 \mathrm{~S} 1$ & 0.000184 & Non-basic N1O3 & $\mathrm{C} 32 \mathrm{H} 21 \mathrm{~N} 1 \mathrm{O} 3$ & $4.72 \mathrm{E}-06$ & Basic N1O1 & $\mathrm{C} 28 \mathrm{H} 27 \mathrm{~N} 1 \mathrm{O} 1$ & 7.67E-05 \\
\hline O1S1 & $\mathrm{C} 25 \mathrm{H} 36 \mathrm{O} 1 \mathrm{~S} 1$ & $2.51 \mathrm{E}-05$ & Non-basic N1O3 & $\mathrm{C} 33 \mathrm{H} 23 \mathrm{~N} 1 \mathrm{O} 3$ & 4.87E-06 & Basic N1O1 & $\mathrm{C} 29 \mathrm{H} 29 \mathrm{~N} 1 \mathrm{O} 1$ & $7.96 \mathrm{E}-05$ \\
\hline O1S1 & $\mathrm{C} 26 \mathrm{H} 38 \mathrm{O} 1 \mathrm{~S} 1$ & $4.68 \mathrm{E}-05$ & Non-basic N1O3 & $\mathrm{C} 34 \mathrm{H} 25 \mathrm{~N} 1 \mathrm{O} 3$ & $5.32 \mathrm{E}-06$ & Basic N1O1 & $\mathrm{C} 30 \mathrm{H} 31 \mathrm{~N} 1 \mathrm{O} 1$ & $5.88 \mathrm{E}-05$ \\
\hline O1S1 & $\mathrm{C} 27 \mathrm{H} 40 \mathrm{O} 1 \mathrm{~S} 1$ & $4.41 \mathrm{E}-05$ & Non-basic N1O3 & $\mathrm{C} 35 \mathrm{H} 27 \mathrm{~N} 1 \mathrm{O} 3$ & $1.67 \mathrm{E}-06$ & Basic N1O1 & C31H33N1O1 & $7.39 \mathrm{E}-05$ \\
\hline O1S1 & $\mathrm{C} 28 \mathrm{H} 42 \mathrm{O} 1 \mathrm{~S} 1$ & $3.91 \mathrm{E}-05$ & Non-basic N1O3 & $\mathrm{C} 37 \mathrm{H} 31 \mathrm{~N} 1 \mathrm{O} 3$ & $9.84 \mathrm{E}-06$ & Basic N1O1 & $\mathrm{C} 32 \mathrm{H} 35 \mathrm{~N} 1 \mathrm{O} 1$ & $6.27 \mathrm{E}-05$ \\
\hline O1S1 & $\mathrm{C} 29 \mathrm{H} 44 \mathrm{O} 1 \mathrm{~S} 1$ & $5.44 \mathrm{E}-05$ & Non-basic N1O3 & $\mathrm{C} 38 \mathrm{H} 33 \mathrm{~N} 1 \mathrm{O} 3$ & $1.01 \mathrm{E}-05$ & Basic N1O1 & $\mathrm{C} 33 \mathrm{H} 37 \mathrm{~N} 1 \mathrm{O} 1$ & 7.74E-05 \\
\hline O1S1 & $\mathrm{C} 30 \mathrm{H} 46 \mathrm{O} 1 \mathrm{~S} 1$ & $6.79 \mathrm{E}-05$ & Non-basic N1O3 & $\mathrm{C} 39 \mathrm{H} 35 \mathrm{~N} 1 \mathrm{O} 3$ & $5.07 \mathrm{E}-06$ & Basic N1O1 & $\mathrm{C} 34 \mathrm{H} 39 \mathrm{~N} 1 \mathrm{O} 1$ & $5.78 \mathrm{E}-05$ \\
\hline O1S1 & $\mathrm{C} 31 \mathrm{H} 48 \mathrm{O} 1 \mathrm{~S} 1$ & $2.92 \mathrm{E}-05$ & Non-basic N1O3 & $\mathrm{C} 33 \mathrm{H} 21 \mathrm{~N} 1 \mathrm{O} 3$ & $1.43 \mathrm{E}-06$ & Basic N1O1 & C35H41N1O1 & $4.68 \mathrm{E}-05$ \\
\hline O1S1 & $\mathrm{C} 14 \mathrm{H} 12 \mathrm{O} 1 \mathrm{~S} 1$ & $1.69 \mathrm{E}-05$ & Non-basic N1O3 & $\mathrm{C} 34 \mathrm{H} 23 \mathrm{~N} 1 \mathrm{O} 3$ & 4.44E-06 & Basic N1O1 & $\mathrm{C} 36 \mathrm{H} 43 \mathrm{~N} 1 \mathrm{O} 1$ & 3.93E-05 \\
\hline O1S1 & C15H14O1S1 & $4.14 \mathrm{E}-05$ & Non-basic N1O3 & $\mathrm{C} 35 \mathrm{H} 25 \mathrm{~N} 1 \mathrm{O} 3$ & $3.98 \mathrm{E}-06$ & Basic N1O1 & $\mathrm{C} 37 \mathrm{H} 45 \mathrm{~N} 1 \mathrm{O} 1$ & $2.80 \mathrm{E}-05$ \\
\hline O1S1 & $\mathrm{C} 16 \mathrm{H} 16 \mathrm{O} 1 \mathrm{~S} 1$ & $5.40 \mathrm{E}-05$ & Non-basic N1O3 & $\mathrm{C} 36 \mathrm{H} 27 \mathrm{~N} 1 \mathrm{O} 3$ & $6.47 \mathrm{E}-06$ & Basic N1O1 & C38H47N1O1 & $3.35 \mathrm{E}-05$ \\
\hline O1S1 & $\mathrm{C} 17 \mathrm{H} 18 \mathrm{O} 1 \mathrm{~S} 1$ & $3.64 \mathrm{E}-05$ & Non-basic N1O3 & $\mathrm{C} 37 \mathrm{H} 29 \mathrm{~N} 1 \mathrm{O} 3$ & $6.64 \mathrm{E}-06$ & Basic N1O1 & C39H49N1O1 & $1.77 \mathrm{E}-05$ \\
\hline O1S1 & $\mathrm{C} 18 \mathrm{H} 20 \mathrm{O} 1 \mathrm{~S} 1$ & 0.000174 & Non-basic N1O3 & $\mathrm{C} 38 \mathrm{H} 31 \mathrm{~N} 1 \mathrm{O} 3$ & $5.27 \mathrm{E}-06$ & Basic N1O1 & $\mathrm{C} 40 \mathrm{H} 51 \mathrm{~N} 1 \mathrm{O} 1$ & $1.20 \mathrm{E}-05$ \\
\hline O1S1 & $\mathrm{C} 19 \mathrm{H} 22 \mathrm{O} 1 \mathrm{~S} 1$ & $1.14 \mathrm{E}-05$ & Non-basic N1O3 & $\mathrm{C} 40 \mathrm{H} 35 \mathrm{~N} 1 \mathrm{O} 3$ & $5.10 \mathrm{E}-06$ & Basic N1O1 & $\mathrm{C} 41 \mathrm{H} 53 \mathrm{~N} 1 \mathrm{O} 1$ & $1.57 \mathrm{E}-05$ \\
\hline O1S1 & $\mathrm{C} 20 \mathrm{H} 24 \mathrm{O} 1 \mathrm{~S} 1$ & $8.00 \mathrm{E}-06$ & Non-basic N1O3 & $\mathrm{C} 41 \mathrm{H} 37 \mathrm{~N} 1 \mathrm{O} 3$ & $3.19 \mathrm{E}-06$ & Basic N1O1 & $\mathrm{C} 42 \mathrm{H} 55 \mathrm{~N} 1 \mathrm{O} 1$ & $1.05 \mathrm{E}-05$ \\
\hline O1S1 & $\mathrm{C} 21 \mathrm{H} 26 \mathrm{O} 1 \mathrm{~S} 1$ & $8.47 \mathrm{E}-06$ & Non-basic N1O3 & $\mathrm{C} 42 \mathrm{H} 39 \mathrm{~N} 1 \mathrm{O} 3$ & $1.84 \mathrm{E}-06$ & Basic N1O1 & $\mathrm{C} 43 \mathrm{H} 57 \mathrm{~N} 1 \mathrm{O} 1$ & $2.00 \mathrm{E}-06$ \\
\hline O1S1 & $\mathrm{C} 22 \mathrm{H} 28 \mathrm{O} 1 \mathrm{~S} 1$ & $1.03 \mathrm{E}-05$ & Non-basic N1O3 & $\mathrm{C} 37 \mathrm{H} 27 \mathrm{~N} 1 \mathrm{O} 3$ & $8.14 \mathrm{E}-06$ & Basic N1O1 & $\mathrm{C} 44 \mathrm{H} 59 \mathrm{~N} 1 \mathrm{O} 1$ & $7.15 \mathrm{E}-06$ \\
\hline O1S1 & $\mathrm{C} 23 \mathrm{H} 30 \mathrm{O} 1 \mathrm{~S} 1$ & $1.05 \mathrm{E}-05$ & Non-basic N1O3 & $\mathrm{C} 38 \mathrm{H} 29 \mathrm{~N} 1 \mathrm{O} 3$ & $1.06 \mathrm{E}-05$ & Basic N1O1 & C45H61N1O1 & $3.58 \mathrm{E}-06$ \\
\hline O1S1 & $\mathrm{C} 24 \mathrm{H} 32 \mathrm{O} 1 \mathrm{~S} 1$ & $1.62 \mathrm{E}-05$ & Non-basic N1O3 & C39H31N1O3 & 7.73E-06 & Basic N1O1 & C46H63N1O1 & $3.45 \mathrm{E}-06$ \\
\hline O1S1 & $\mathrm{C} 25 \mathrm{H} 34 \mathrm{O} 1 \mathrm{~S} 1$ & $3.65 \mathrm{E}-05$ & Non-basic N1O3 & $\mathrm{C} 40 \mathrm{H} 33 \mathrm{~N} 1 \mathrm{O} 3$ & $8.13 \mathrm{E}-06$ & Basic N1O1 & $\mathrm{C} 47 \mathrm{H} 65 \mathrm{~N} 1 \mathrm{O} 1$ & 3.67E-07 \\
\hline O1S1 & $\mathrm{C} 26 \mathrm{H} 36 \mathrm{O} 1 \mathrm{~S} 1$ & $2.27 \mathrm{E}-05$ & Non-basic N1O3 & $\mathrm{C} 41 \mathrm{H} 35 \mathrm{~N} 1 \mathrm{O} 3$ & $1.22 \mathrm{E}-05$ & Basic N1O1 & $\mathrm{C} 23 \mathrm{H} 15 \mathrm{~N} 1 \mathrm{O} 1$ & $1.95 \mathrm{E}-06$ \\
\hline O1S1 & $\mathrm{C} 27 \mathrm{H} 38 \mathrm{O} 1 \mathrm{~S} 1$ & $2.48 \mathrm{E}-05$ & Non-basic N1O3 & $\mathrm{C} 42 \mathrm{H} 37 \mathrm{~N} 1 \mathrm{O} 3$ & $2.84 \mathrm{E}-06$ & Basic N1O1 & $\mathrm{C} 24 \mathrm{H} 17 \mathrm{~N} 1 \mathrm{O} 1$ & $5.42 \mathrm{E}-06$ \\
\hline O1S1 & $\mathrm{C} 28 \mathrm{H} 40 \mathrm{O} 1 \mathrm{~S} 1$ & $2.65 \mathrm{E}-05$ & Non-basic N1O3 & $\mathrm{C} 38 \mathrm{H} 27 \mathrm{~N} 1 \mathrm{O} 3$ & $3.40 \mathrm{E}-06$ & Basic N1O1 & $\mathrm{C} 25 \mathrm{H} 19 \mathrm{~N} 1 \mathrm{O} 1$ & $2.77 \mathrm{E}-05$ \\
\hline O1S1 & $\mathrm{C} 29 \mathrm{H} 42 \mathrm{O} 1 \mathrm{~S} 1$ & $2.50 \mathrm{E}-05$ & Non-basic N1O3 & $\mathrm{C} 39 \mathrm{H} 29 \mathrm{~N} 1 \mathrm{O} 3$ & $1.15 \mathrm{E}-05$ & Basic N1O1 & $\mathrm{C} 26 \mathrm{H} 21 \mathrm{~N} 1 \mathrm{O} 1$ & $3.60 \mathrm{E}-05$ \\
\hline O1S1 & $\mathrm{C} 30 \mathrm{H} 44 \mathrm{O} 1 \mathrm{~S} 1$ & $6.00 \mathrm{E}-05$ & Non-basic N1O3 & $\mathrm{C} 40 \mathrm{H} 31 \mathrm{~N} 1 \mathrm{O} 3$ & $1.28 \mathrm{E}-05$ & Basic N1O1 & $\mathrm{C} 27 \mathrm{H} 23 \mathrm{~N} 1 \mathrm{O} 1$ & $5.58 \mathrm{E}-05$ \\
\hline O1S1 & $\mathrm{C} 31 \mathrm{H} 46 \mathrm{O} 1 \mathrm{~S} 1$ & $4.60 \mathrm{E}-05$ & Non-basic N1O3 & $\mathrm{C} 41 \mathrm{H} 33 \mathrm{~N} 1 \mathrm{O} 3$ & $1.70 \mathrm{E}-05$ & Basic N1O1 & $\mathrm{C} 28 \mathrm{H} 25 \mathrm{~N} 1 \mathrm{O} 1$ & $6.48 \mathrm{E}-05$ \\
\hline O1S1 & $\mathrm{C} 32 \mathrm{H} 48 \mathrm{O} 1 \mathrm{~S} 1$ & $3.96 \mathrm{E}-05$ & Non-basic N1O3 & $\mathrm{C} 42 \mathrm{H} 35 \mathrm{~N} 1 \mathrm{O} 3$ & $5.95 \mathrm{E}-06$ & Basic N1O1 & C29H27N1O1 & 0.000102 \\
\hline O1S1 & $\mathrm{C} 33 \mathrm{H} 50 \mathrm{O} 1 \mathrm{~S} 1$ & $6.57 \mathrm{E}-05$ & Non-basic N1O3 & $\mathrm{C} 43 \mathrm{H} 37 \mathrm{~N} 1 \mathrm{O} 3$ & $8.83 \mathrm{E}-06$ & Basic N1O1 & $\mathrm{C} 30 \mathrm{H} 29 \mathrm{~N} 1 \mathrm{O} 1$ & $9.64 \mathrm{E}-05$ \\
\hline O1S1 & $\mathrm{C} 35 \mathrm{H} 54 \mathrm{O} 1 \mathrm{~S} 1$ & $2.75 \mathrm{E}-05$ & Non-basic N1O3 & $\mathrm{C} 44 \mathrm{H} 39 \mathrm{~N} 1 \mathrm{O} 3$ & $1.32 \mathrm{E}-05$ & Basic N1O1 & $\mathrm{C} 31 \mathrm{H} 31 \mathrm{~N} 1 \mathrm{O} 1$ & $6.45 \mathrm{E}-05$ \\
\hline O1S1 & $\mathrm{C} 36 \mathrm{H} 56 \mathrm{O} 1 \mathrm{~S} 1$ & $4.24 \mathrm{E}-05$ & Non-basic N1O3 & $\mathrm{C} 39 \mathrm{H} 27 \mathrm{~N} 1 \mathrm{O} 3$ & $1.63 \mathrm{E}-06$ & Basic N1O1 & C32H33N1O1 & 4.60E-05 \\
\hline
\end{tabular}




\begin{tabular}{|c|c|c|c|c|c|c|c|c|}
\hline Class & Formula & Mass & Class & Formula & Mass & Class & Formula & Mass \\
\hline O1S1 & $\mathrm{C} 37 \mathrm{H} 58 \mathrm{O} 1 \mathrm{~S} 1$ & $3.85 \mathrm{E}-05$ & Non-basic N1O3 & $\mathrm{C} 40 \mathrm{H} 29 \mathrm{~N} 1 \mathrm{O} 3$ & $5.97 \mathrm{E}-06$ & Basic N1O1 & $\mathrm{C} 33 \mathrm{H} 35 \mathrm{~N} 1 \mathrm{O} 1$ & $4.61 \mathrm{E}-05$ \\
\hline O1S1 & $\mathrm{C} 16 \mathrm{H} 14 \mathrm{O} 1 \mathrm{~S} 1$ & $2.97 \mathrm{E}-06$ & Non-basic N1O3 & $\mathrm{C} 41 \mathrm{H} 31 \mathrm{~N} 1 \mathrm{O} 3$ & $1.00 \mathrm{E}-05$ & Basic N1O1 & $\mathrm{C} 34 \mathrm{H} 37 \mathrm{~N} 1 \mathrm{O} 1$ & $3.35 \mathrm{E}-05$ \\
\hline O1S1 & $\mathrm{C} 17 \mathrm{H} 16 \mathrm{O} 1 \mathrm{~S} 1$ & $3.36 \mathrm{E}-05$ & Non-basic N1O3 & $\mathrm{C} 42 \mathrm{H} 33 \mathrm{~N} 1 \mathrm{O} 3$ & $2.10 \mathrm{E}-05$ & Basic N1O1 & C35H39N1O1 & $3.52 \mathrm{E}-05$ \\
\hline O1S1 & C18H18O1S1 & $2.76 \mathrm{E}-05$ & Non-basic N1O3 & $\mathrm{C} 43 \mathrm{H} 35 \mathrm{~N} 1 \mathrm{O} 3$ & $1.59 \mathrm{E}-05$ & Basic N1O1 & C36H41N1O1 & $2.56 \mathrm{E}-05$ \\
\hline O1S1 & C19H20O1S1 & $1.91 \mathrm{E}-05$ & Non-basic N1O3 & $\mathrm{C} 44 \mathrm{H} 37 \mathrm{~N} 1 \mathrm{O} 3$ & $1.43 \mathrm{E}-05$ & Basic N1O1 & C37H43N1O1 & $1.87 \mathrm{E}-05$ \\
\hline O1S1 & $\mathrm{C} 20 \mathrm{H} 22 \mathrm{O} 1 \mathrm{~S} 1$ & 7.07E-06 & Non-basic N1O3 & $\mathrm{C} 45 \mathrm{H} 39 \mathrm{~N} 1 \mathrm{O} 3$ & $7.00 \mathrm{E}-06$ & Basic N1O1 & $\mathrm{C} 38 \mathrm{H} 45 \mathrm{~N} 1 \mathrm{O} 1$ & $2.69 \mathrm{E}-05$ \\
\hline O1S1 & $\mathrm{C} 21 \mathrm{H} 24 \mathrm{O} 1 \mathrm{~S} 1$ & $1.31 \mathrm{E}-05$ & Non-basic N1O3 & $\mathrm{C} 46 \mathrm{H} 41 \mathrm{~N} 1 \mathrm{O} 3$ & $1.18 \mathrm{E}-05$ & Basic N1O1 & C39H47N1O1 & $1.47 \mathrm{E}-05$ \\
\hline O1S1 & $\mathrm{C} 22 \mathrm{H} 26 \mathrm{O} 1 \mathrm{~S} 1$ & $1.80 \mathrm{E}-06$ & Non-basic N1O3 & $\mathrm{C} 47 \mathrm{H} 43 \mathrm{~N} 1 \mathrm{O} 3$ & $3.62 \mathrm{E}-06$ & Basic N1O1 & $\mathrm{C} 40 \mathrm{H} 49 \mathrm{~N} 1 \mathrm{O} 1$ & $1.10 \mathrm{E}-05$ \\
\hline O1S1 & $\mathrm{C} 23 \mathrm{H} 28 \mathrm{O} 1 \mathrm{~S} 1$ & $2.18 \mathrm{E}-05$ & Non-basic N1O3 & $\mathrm{C} 40 \mathrm{H} 27 \mathrm{~N} 1 \mathrm{O} 3$ & $3.84 \mathrm{E}-06$ & Basic N1O1 & $\mathrm{C} 41 \mathrm{H} 51 \mathrm{~N} 1 \mathrm{O} 1$ & $5.94 \mathrm{E}-06$ \\
\hline O1S1 & $\mathrm{C} 24 \mathrm{H} 30 \mathrm{O} 1 \mathrm{~S} 1$ & $7.88 \mathrm{E}-06$ & Non-basic N1O3 & $\mathrm{C} 41 \mathrm{H} 29 \mathrm{~N} 1 \mathrm{O} 3$ & $4.72 \mathrm{E}-06$ & Basic N1O1 & $\mathrm{C} 42 \mathrm{H} 53 \mathrm{~N} 1 \mathrm{O} 1$ & $5.40 \mathrm{E}-06$ \\
\hline O1S1 & $\mathrm{C} 25 \mathrm{H} 32 \mathrm{O} 1 \mathrm{~S} 1$ & $1.12 \mathrm{E}-05$ & Non-basic N1O3 & $\mathrm{C} 42 \mathrm{H} 31 \mathrm{~N} 1 \mathrm{O} 3$ & $1.53 \mathrm{E}-05$ & Basic N1O1 & $\mathrm{C} 43 \mathrm{H} 55 \mathrm{~N} 1 \mathrm{O} 1$ & $1.47 \mathrm{E}-06$ \\
\hline O1S1 & $\mathrm{C} 26 \mathrm{H} 34 \mathrm{O} 1 \mathrm{~S} 1$ & $1.83 \mathrm{E}-05$ & Non-basic N1O3 & $\mathrm{C} 43 \mathrm{H} 33 \mathrm{~N} 1 \mathrm{O} 3$ & $1.65 \mathrm{E}-05$ & Basic N1O1 & C44H57N1O1 & $3.71 \mathrm{E}-07$ \\
\hline O1S1 & $\mathrm{C} 27 \mathrm{H} 36 \mathrm{O} 1 \mathrm{~S} 1$ & $1.90 \mathrm{E}-05$ & Non-basic N1O3 & $\mathrm{C} 44 \mathrm{H} 35 \mathrm{~N} 1 \mathrm{O} 3$ & $2.20 \mathrm{E}-05$ & Basic N1O1 & C45H59N1O1 & $2.77 \mathrm{E}-07$ \\
\hline O1S1 & $\mathrm{C} 28 \mathrm{H} 38 \mathrm{O} 1 \mathrm{~S} 1$ & $2.84 \mathrm{E}-05$ & Non-basic N1O3 & $\mathrm{C} 45 \mathrm{H} 37 \mathrm{~N} 1 \mathrm{O} 3$ & $1.56 \mathrm{E}-05$ & Basic N1O1 & $\mathrm{C} 24 \mathrm{H} 15 \mathrm{~N} 1 \mathrm{O} 1$ & $1.55 \mathrm{E}-06$ \\
\hline O1S1 & $\mathrm{C} 29 \mathrm{H} 40 \mathrm{O} 1 \mathrm{~S} 1$ & $3.81 \mathrm{E}-05$ & Non-basic N1O3 & $\mathrm{C} 46 \mathrm{H} 39 \mathrm{~N} 1 \mathrm{O} 3$ & $1.39 \mathrm{E}-05$ & Basic N1O1 & $\mathrm{C} 25 \mathrm{H} 17 \mathrm{~N} 1 \mathrm{O} 1$ & $6.65 \mathrm{E}-06$ \\
\hline O1S1 & $\mathrm{C} 30 \mathrm{H} 42 \mathrm{O} 1 \mathrm{~S} 1$ & $2.29 \mathrm{E}-05$ & Non-basic N1O3 & $\mathrm{C} 47 \mathrm{H} 41 \mathrm{~N} 1 \mathrm{O} 3$ & $1.30 \mathrm{E}-05$ & Basic N1O1 & C26H19N1O1 & $2.08 \mathrm{E}-05$ \\
\hline O1S1 & $\mathrm{C} 31 \mathrm{H} 44 \mathrm{O} 1 \mathrm{~S} 1$ & $4.98 \mathrm{E}-05$ & Non-basic N1O3 & $\mathrm{C} 48 \mathrm{H} 43 \mathrm{~N} 1 \mathrm{O} 3$ & $7.88 \mathrm{E}-06$ & Basic N1O1 & $\mathrm{C} 27 \mathrm{H} 21 \mathrm{~N} 1 \mathrm{O} 1$ & $3.30 \mathrm{E}-05$ \\
\hline O1S1 & $\mathrm{C} 32 \mathrm{H} 46 \mathrm{O} 1 \mathrm{~S} 1$ & $5.25 \mathrm{E}-05$ & Non-basic N1O3 & $\mathrm{C} 49 \mathrm{H} 45 \mathrm{~N} 1 \mathrm{O} 3$ & $7.69 \mathrm{E}-06$ & Basic N1O1 & $\mathrm{C} 28 \mathrm{H} 23 \mathrm{~N} 1 \mathrm{O} 1$ & $4.12 \mathrm{E}-05$ \\
\hline O1S1 & C15H10O1S1 & $3.45 \mathrm{E}-06$ & Non-basic N1O3 & $\mathrm{C} 50 \mathrm{H} 47 \mathrm{~N} 1 \mathrm{O} 3$ & $2.79 \mathrm{E}-06$ & Basic N1O1 & $\mathrm{C} 29 \mathrm{H} 25 \mathrm{~N} 1 \mathrm{O} 1$ & $5.42 \mathrm{E}-05$ \\
\hline O1S1 & $\mathrm{C} 16 \mathrm{H} 12 \mathrm{O} 1 \mathrm{~S} 1$ & $3.22 \mathrm{E}-05$ & Non-basic N1O3 & $\mathrm{C} 41 \mathrm{H} 27 \mathrm{~N} 1 \mathrm{O} 3$ & $3.10 \mathrm{E}-06$ & Basic N1O1 & $\mathrm{C} 30 \mathrm{H} 27 \mathrm{~N} 1 \mathrm{O} 1$ & $5.70 \mathrm{E}-05$ \\
\hline O1S1 & $\mathrm{C} 17 \mathrm{H} 14 \mathrm{O} 1 \mathrm{~S} 1$ & 0.00016 & Non-basic N1O3 & $\mathrm{C} 42 \mathrm{H} 29 \mathrm{~N} 1 \mathrm{O} 3$ & $1.84 \mathrm{E}-06$ & Basic N1O1 & $\mathrm{C} 31 \mathrm{H} 29 \mathrm{~N} 1 \mathrm{O} 1$ & 3.97E-05 \\
\hline O1S1 & $\mathrm{C} 18 \mathrm{H} 16 \mathrm{O} 1 \mathrm{~S} 1$ & 0.000106 & Non-basic N1O3 & $\mathrm{C} 43 \mathrm{H} 31 \mathrm{~N} 1 \mathrm{O} 3$ & 7.91E-06 & Basic N1O1 & $\mathrm{C} 32 \mathrm{H} 31 \mathrm{~N} 1 \mathrm{O} 1$ & $3.50 \mathrm{E}-05$ \\
\hline O1S1 & $\mathrm{C} 19 \mathrm{H} 18 \mathrm{O} 1 \mathrm{~S} 1$ & $5.22 \mathrm{E}-05$ & Non-basic N1O3 & C44H33N1O3 & $1.02 \mathrm{E}-05$ & Basic N1O1 & $\mathrm{C} 33 \mathrm{H} 33 \mathrm{~N} 1 \mathrm{O} 1$ & $3.37 \mathrm{E}-05$ \\
\hline O1S1 & $\mathrm{C} 20 \mathrm{H} 20 \mathrm{O} 1 \mathrm{~S} 1$ & $2.05 \mathrm{E}-05$ & Non-basic N1O3 & $\mathrm{C} 45 \mathrm{H} 35 \mathrm{~N} 1 \mathrm{O} 3$ & $1.12 \mathrm{E}-05$ & Basic N1O1 & $\mathrm{C} 34 \mathrm{H} 35 \mathrm{~N} 1 \mathrm{O} 1$ & $2.66 \mathrm{E}-05$ \\
\hline O1S1 & $\mathrm{C} 21 \mathrm{H} 22 \mathrm{O} 1 \mathrm{~S} 1$ & $2.15 \mathrm{E}-05$ & Non-basic N1O3 & $\mathrm{C} 46 \mathrm{H} 37 \mathrm{~N} 1 \mathrm{O} 3$ & $2.11 \mathrm{E}-05$ & Basic N1O1 & $\mathrm{C} 35 \mathrm{H} 37 \mathrm{~N} 1 \mathrm{O} 1$ & $2.12 \mathrm{E}-05$ \\
\hline O1S1 & $\mathrm{C} 22 \mathrm{H} 24 \mathrm{O} 1 \mathrm{~S} 1$ & $3.06 \mathrm{E}-06$ & Non-basic N1O3 & $\mathrm{C} 47 \mathrm{H} 39 \mathrm{~N} 1 \mathrm{O} 3$ & $1.42 \mathrm{E}-05$ & Basic N1O1 & $\mathrm{C} 36 \mathrm{H} 39 \mathrm{~N} 1 \mathrm{O} 1$ & $2.41 \mathrm{E}-05$ \\
\hline O1S1 & $\mathrm{C} 23 \mathrm{H} 26 \mathrm{O} 1 \mathrm{~S} 1$ & $8.41 \mathrm{E}-06$ & Non-basic N1O3 & $\mathrm{C} 48 \mathrm{H} 41 \mathrm{~N} 1 \mathrm{O} 3$ & $9.05 \mathrm{E}-06$ & Basic N1O1 & $\mathrm{C} 37 \mathrm{H} 41 \mathrm{~N} 1 \mathrm{O} 1$ & $8.53 \mathrm{E}-06$ \\
\hline O1S1 & $\mathrm{C} 24 \mathrm{H} 28 \mathrm{O} 1 \mathrm{~S} 1$ & $1.35 \mathrm{E}-05$ & Non-basic N1O3 & $\mathrm{C} 49 \mathrm{H} 43 \mathrm{~N} 1 \mathrm{O} 3$ & $9.36 \mathrm{E}-06$ & Basic N1O1 & $\mathrm{C} 38 \mathrm{H} 43 \mathrm{~N} 1 \mathrm{O} 1$ & $1.42 \mathrm{E}-05$ \\
\hline O1S1 & $\mathrm{C} 25 \mathrm{H} 30 \mathrm{O} 1 \mathrm{~S} 1$ & $2.24 \mathrm{E}-05$ & Non-basic N1O3 & $\mathrm{C} 42 \mathrm{H} 27 \mathrm{~N} 1 \mathrm{O} 3$ & $2.40 \mathrm{E}-06$ & Basic N1O1 & C39H45N1O1 & 7.65E-06 \\
\hline O1S1 & $\mathrm{C} 26 \mathrm{H} 32 \mathrm{O} 1 \mathrm{~S} 1$ & $3.09 \mathrm{E}-05$ & Non-basic N1O3 & $\mathrm{C} 43 \mathrm{H} 29 \mathrm{~N} 1 \mathrm{O} 3$ & $2.16 \mathrm{E}-06$ & Basic N1O1 & $\mathrm{C} 40 \mathrm{H} 47 \mathrm{~N} 1 \mathrm{O} 1$ & $5.32 \mathrm{E}-06$ \\
\hline O1S1 & $\mathrm{C} 27 \mathrm{H} 34 \mathrm{O} 1 \mathrm{~S} 1$ & $5.02 \mathrm{E}-05$ & Non-basic N1O3 & $\mathrm{C} 44 \mathrm{H} 31 \mathrm{~N} 1 \mathrm{O} 3$ & $1.48 \mathrm{E}-05$ & Basic N1O1 & C41H49N1O1 & $5.79 \mathrm{E}-06$ \\
\hline O1S1 & $\mathrm{C} 28 \mathrm{H} 36 \mathrm{O} 1 \mathrm{~S} 1$ & $9.44 \mathrm{E}-06$ & Non-basic N1O3 & $\mathrm{C} 45 \mathrm{H} 33 \mathrm{~N} 1 \mathrm{O} 3$ & 7.42E-06 & Basic N1O1 & $\mathrm{C} 42 \mathrm{H} 51 \mathrm{~N} 1 \mathrm{O} 1$ & $8.50 \mathrm{E}-06$ \\
\hline O1S1 & C29H38O1S1 & 7.92E-05 & Non-basic N1O3 & $\mathrm{C} 46 \mathrm{H} 35 \mathrm{~N} 1 \mathrm{O} 3$ & $1.28 \mathrm{E}-05$ & Basic N1O1 & $\mathrm{C} 43 \mathrm{H} 53 \mathrm{~N} 1 \mathrm{O} 1$ & $4.74 \mathrm{E}-07$ \\
\hline O1S1 & $\mathrm{C} 30 \mathrm{H} 40 \mathrm{O} 1 \mathrm{~S} 1$ & 0.001697 & Non-basic N1O3 & $\mathrm{C} 47 \mathrm{H} 37 \mathrm{~N} 1 \mathrm{O} 3$ & $2.48 \mathrm{E}-05$ & Basic N1O1 & $\mathrm{C} 26 \mathrm{H} 17 \mathrm{~N} 1 \mathrm{O} 1$ & $4.01 \mathrm{E}-06$ \\
\hline O1S1 & $\mathrm{C} 31 \mathrm{H} 42 \mathrm{O} 1 \mathrm{~S} 1$ & 0.009688 & Non-basic N1O3 & $\mathrm{C} 48 \mathrm{H} 39 \mathrm{~N} 1 \mathrm{O} 3$ & $1.43 \mathrm{E}-05$ & Basic N1O1 & C27H19N1O1 & $3.28 \mathrm{E}-05$ \\
\hline O1S1 & $\mathrm{C} 32 \mathrm{H} 44 \mathrm{O} 1 \mathrm{~S} 1$ & 0.000561 & Non-basic N1O3 & $\mathrm{C} 49 \mathrm{H} 41 \mathrm{~N} 1 \mathrm{O} 3$ & $1.42 \mathrm{E}-05$ & Basic N1O1 & $\mathrm{C} 28 \mathrm{H} 21 \mathrm{~N} 1 \mathrm{O} 1$ & $5.44 \mathrm{E}-05$ \\
\hline O1S1 & $\mathrm{C} 33 \mathrm{H} 46 \mathrm{O} 1 \mathrm{~S} 1$ & 0.000487 & Non-basic N1O3 & $\mathrm{C} 50 \mathrm{H} 43 \mathrm{~N} 1 \mathrm{O} 3$ & $2.13 \mathrm{E}-05$ & Basic N1O1 & $\mathrm{C} 29 \mathrm{H} 23 \mathrm{~N} 1 \mathrm{O} 1$ & $3.79 \mathrm{E}-05$ \\
\hline O1S1 & $\mathrm{C} 34 \mathrm{H} 48 \mathrm{O} 1 \mathrm{~S} 1$ & 0.000189 & Non-basic N1O3 & $\mathrm{C} 51 \mathrm{H} 45 \mathrm{~N} 1 \mathrm{O} 3$ & $5.12 \mathrm{E}-06$ & Basic N1O1 & $\mathrm{C} 30 \mathrm{H} 25 \mathrm{~N} 1 \mathrm{O} 1$ & $3.44 \mathrm{E}-05$ \\
\hline
\end{tabular}




\begin{tabular}{|c|c|c|c|c|c|c|c|c|}
\hline Class & Formula & Mass & Class & Formula & Mass & Class & Formula & Mass \\
\hline O1S1 & $\mathrm{C} 35 \mathrm{H} 50 \mathrm{O} 1 \mathrm{~S} 1$ & $5.26 \mathrm{E}-05$ & Non-basic N1O3 & $\mathrm{C} 52 \mathrm{H} 47 \mathrm{~N} 1 \mathrm{O} 3$ & $8.05 \mathrm{E}-06$ & Basic N1O1 & $\mathrm{C} 31 \mathrm{H} 27 \mathrm{~N} 1 \mathrm{O} 1$ & $3.02 \mathrm{E}-05$ \\
\hline O1S1 & $\mathrm{C} 36 \mathrm{H} 52 \mathrm{O} 1 \mathrm{~S} 1$ & 0.000331 & Non-basic N1O3 & $\mathrm{C} 46 \mathrm{H} 33 \mathrm{~N} 1 \mathrm{O} 3$ & $5.11 \mathrm{E}-06$ & Basic N1O1 & $\mathrm{C} 32 \mathrm{H} 29 \mathrm{~N} 1 \mathrm{O} 1$ & $3.02 \mathrm{E}-05$ \\
\hline O1S1 & $\mathrm{C} 37 \mathrm{H} 54 \mathrm{O} 1 \mathrm{~S} 1$ & $2.83 \mathrm{E}-05$ & Non-basic N1O3 & $\mathrm{C} 47 \mathrm{H} 35 \mathrm{~N} 1 \mathrm{O} 3$ & $1.78 \mathrm{E}-05$ & Basic N1O1 & $\mathrm{C} 33 \mathrm{H} 31 \mathrm{~N} 1 \mathrm{O} 1$ & $2.36 \mathrm{E}-05$ \\
\hline O1S1 & $\mathrm{C} 16 \mathrm{H} 10 \mathrm{O} 1 \mathrm{~S} 1$ & $3.06 \mathrm{E}-06$ & Non-basic N1O3 & $\mathrm{C} 48 \mathrm{H} 37 \mathrm{~N} 1 \mathrm{O} 3$ & $5.45 \mathrm{E}-06$ & Basic N1O1 & $\mathrm{C} 34 \mathrm{H} 33 \mathrm{~N} 1 \mathrm{O} 1$ & $2.27 \mathrm{E}-05$ \\
\hline O1S1 & $\mathrm{C} 17 \mathrm{H} 12 \mathrm{O} 1 \mathrm{~S} 1$ & $2.13 \mathrm{E}-05$ & Non-basic N1O3 & C49H39N1O3 & $1.58 \mathrm{E}-05$ & Basic N1O1 & $\mathrm{C} 35 \mathrm{H} 35 \mathrm{~N} 1 \mathrm{O} 1$ & $1.01 \mathrm{E}-05$ \\
\hline O1S1 & $\mathrm{C} 18 \mathrm{H} 14 \mathrm{O} 1 \mathrm{~S} 1$ & 0.000104 & Non-basic N1O3 & $\mathrm{C} 50 \mathrm{H} 41 \mathrm{~N} 1 \mathrm{O} 3$ & $1.28 \mathrm{E}-05$ & Basic N1O1 & $\mathrm{C} 36 \mathrm{H} 37 \mathrm{~N} 1 \mathrm{O} 1$ & $1.40 \mathrm{E}-05$ \\
\hline O1S1 & $\mathrm{C} 19 \mathrm{H} 16 \mathrm{O} 1 \mathrm{~S} 1$ & 0.000137 & Non-basic N1O3 & $\mathrm{C} 47 \mathrm{H} 33 \mathrm{~N} 1 \mathrm{O} 3$ & $4.56 \mathrm{E}-06$ & Basic N1O1 & $\mathrm{C} 37 \mathrm{H} 39 \mathrm{~N} 1 \mathrm{O} 1$ & 4.97E-06 \\
\hline O1S1 & $\mathrm{C} 20 \mathrm{H} 18 \mathrm{O} 1 \mathrm{~S} 1$ & $7.00 \mathrm{E}-05$ & Non-basic N1O3 & $\mathrm{C} 48 \mathrm{H} 35 \mathrm{~N} 1 \mathrm{O} 3$ & $1.86 \mathrm{E}-06$ & Basic N1O1 & $\mathrm{C} 38 \mathrm{H} 41 \mathrm{~N} 1 \mathrm{O} 1$ & $8.54 \mathrm{E}-06$ \\
\hline O1S1 & $\mathrm{C} 21 \mathrm{H} 20 \mathrm{O} 1 \mathrm{~S} 1$ & 0.000215 & Non-basic N1O3 & $\mathrm{C} 49 \mathrm{H} 37 \mathrm{~N} 1 \mathrm{O} 3$ & $1.73 \mathrm{E}-05$ & Basic N1O1 & $\mathrm{C} 39 \mathrm{H} 43 \mathrm{~N} 1 \mathrm{O} 1$ & $5.56 \mathrm{E}-06$ \\
\hline O1S1 & $\mathrm{C} 22 \mathrm{H} 22 \mathrm{O} 1 \mathrm{~S} 1$ & $1.84 \mathrm{E}-05$ & Non-basic N1O3 & $\mathrm{C} 50 \mathrm{H} 39 \mathrm{~N} 1 \mathrm{O} 3$ & $1.89 \mathrm{E}-05$ & Basic N1O1 & $\mathrm{C} 26 \mathrm{H} 15 \mathrm{~N} 1 \mathrm{O} 1$ & $3.23 \mathrm{E}-07$ \\
\hline O1S1 & $\mathrm{C} 23 \mathrm{H} 24 \mathrm{O} 1 \mathrm{~S} 1$ & $2.41 \mathrm{E}-05$ & Non-basic N1O3 & $\mathrm{C} 51 \mathrm{H} 41 \mathrm{~N} 1 \mathrm{O} 3$ & $1.01 \mathrm{E}-05$ & Basic N1O1 & $\mathrm{C} 27 \mathrm{H} 17 \mathrm{~N} 1 \mathrm{O} 1$ & $3.14 \mathrm{E}-06$ \\
\hline O1S1 & $\mathrm{C} 24 \mathrm{H} 26 \mathrm{O} 1 \mathrm{~S} 1$ & $1.79 \mathrm{E}-05$ & Non-basic N1O3 & $\mathrm{C} 52 \mathrm{H} 43 \mathrm{~N} 1 \mathrm{O} 3$ & 7.74E-06 & Basic N1O1 & C28H19N1O1 & $1.02 \mathrm{E}-05$ \\
\hline O1S1 & $\mathrm{C} 25 \mathrm{H} 28 \mathrm{O} 1 \mathrm{~S} 1$ & $4.61 \mathrm{E}-05$ & Non-basic N1S1 & C16H17N1S1 & $4.80 \mathrm{E}-07$ & Basic N1O1 & $\mathrm{C} 29 \mathrm{H} 21 \mathrm{~N} 1 \mathrm{O} 1$ & $1.27 \mathrm{E}-05$ \\
\hline O1S1 & $\mathrm{C} 27 \mathrm{H} 32 \mathrm{O} 1 \mathrm{~S} 1$ & $3.81 \mathrm{E}-05$ & Non-basic N1S1 & C17H19N1S1 & $2.27 \mathrm{E}-06$ & Basic N1O1 & $\mathrm{C} 30 \mathrm{H} 23 \mathrm{~N} 1 \mathrm{O} 1$ & $1.77 \mathrm{E}-05$ \\
\hline O1S1 & $\mathrm{C} 28 \mathrm{H} 34 \mathrm{O} 1 \mathrm{~S} 1$ & $4.30 \mathrm{E}-05$ & Non-basic N1S1 & $\mathrm{C} 18 \mathrm{H} 21 \mathrm{~N} 1 \mathrm{~S} 1$ & $2.49 \mathrm{E}-06$ & Basic N1O1 & $\mathrm{C} 31 \mathrm{H} 25 \mathrm{~N} 1 \mathrm{O} 1$ & $2.33 \mathrm{E}-05$ \\
\hline O1S1 & $\mathrm{C} 29 \mathrm{H} 36 \mathrm{O} 1 \mathrm{~S} 1$ & $9.40 \mathrm{E}-05$ & Non-basic N1S1 & C19H23N1S1 & $2.33 \mathrm{E}-06$ & Basic N1O1 & $\mathrm{C} 32 \mathrm{H} 27 \mathrm{~N} 1 \mathrm{O} 1$ & $1.79 \mathrm{E}-05$ \\
\hline O1S1 & $\mathrm{C} 30 \mathrm{H} 38 \mathrm{O} 1 \mathrm{~S} 1$ & $8.80 \mathrm{E}-05$ & Non-basic N1S1 & C19H21N1S1 & $2.43 \mathrm{E}-05$ & Basic N1O1 & $\mathrm{C} 33 \mathrm{H} 29 \mathrm{~N} 1 \mathrm{O} 1$ & $1.74 \mathrm{E}-05$ \\
\hline O1S1 & $\mathrm{C} 31 \mathrm{H} 40 \mathrm{O} 1 \mathrm{~S} 1$ & $4.88 \mathrm{E}-05$ & Non-basic N1S1 & $\mathrm{C} 20 \mathrm{H} 23 \mathrm{~N} 1 \mathrm{~S} 1$ & $1.29 \mathrm{E}-05$ & Basic N1O1 & $\mathrm{C} 34 \mathrm{H} 31 \mathrm{~N} 1 \mathrm{O} 1$ & $1.21 \mathrm{E}-05$ \\
\hline O1S1 & C19H14O1S1 & $6.52 \mathrm{E}-06$ & Non-basic N1S1 & $\mathrm{C} 21 \mathrm{H} 25 \mathrm{~N} 1 \mathrm{~S} 1$ & $1.32 \mathrm{E}-05$ & Basic N1O1 & $\mathrm{C} 35 \mathrm{H} 33 \mathrm{~N} 1 \mathrm{O} 1$ & $1.35 \mathrm{E}-05$ \\
\hline O1S1 & $\mathrm{C} 20 \mathrm{H} 16 \mathrm{O} 1 \mathrm{~S} 1$ & $1.06 \mathrm{E}-05$ & Non-basic N1S1 & C14H9N1S1 & $1.21 \mathrm{E}-06$ & Basic N1O1 & $\mathrm{C} 36 \mathrm{H} 35 \mathrm{~N} 1 \mathrm{O} 1$ & $3.81 \mathrm{E}-06$ \\
\hline O1S1 & $\mathrm{C} 21 \mathrm{H} 18 \mathrm{O} 1 \mathrm{~S} 1$ & $4.34 \mathrm{E}-05$ & Non-basic N1S1 & C15H11N1S1 & $2.87 \mathrm{E}-05$ & Basic N1O1 & $\mathrm{C} 37 \mathrm{H} 37 \mathrm{~N} 1 \mathrm{O} 1$ & 7.33E-06 \\
\hline O1S1 & $\mathrm{C} 22 \mathrm{H} 20 \mathrm{O} 1 \mathrm{~S} 1$ & $2.35 \mathrm{E}-05$ & Non-basic N1S1 & C16H13N1S1 & $4.80 \mathrm{E}-05$ & Basic N1O1 & $\mathrm{C} 28 \mathrm{H} 17 \mathrm{~N} 1 \mathrm{O} 1$ & $1.17 \mathrm{E}-06$ \\
\hline O1S1 & $\mathrm{C} 23 \mathrm{H} 22 \mathrm{O} 1 \mathrm{~S} 1$ & $1.50 \mathrm{E}-05$ & Non-basic N1S1 & C17H15N1S1 & $3.29 \mathrm{E}-05$ & Basic N1O1 & $\mathrm{C} 29 \mathrm{H} 19 \mathrm{~N} 1 \mathrm{O} 1$ & $4.25 \mathrm{E}-06$ \\
\hline O1S1 & $\mathrm{C} 24 \mathrm{H} 24 \mathrm{O} 1 \mathrm{~S} 1$ & $1.43 \mathrm{E}-05$ & Non-basic N1S1 & C18H17N1S1 & $1.09 \mathrm{E}-05$ & Basic N1O1 & $\mathrm{C} 30 \mathrm{H} 21 \mathrm{~N} 1 \mathrm{O} 1$ & $1.29 \mathrm{E}-05$ \\
\hline O1S1 & $\mathrm{C} 25 \mathrm{H} 26 \mathrm{O} 1 \mathrm{~S} 1$ & $1.18 \mathrm{E}-05$ & Non-basic N1S1 & C19H19N1S1 & $2.23 \mathrm{E}-06$ & Basic N1O1 & $\mathrm{C} 31 \mathrm{H} 23 \mathrm{~N} 1 \mathrm{O} 1$ & $1.15 \mathrm{E}-05$ \\
\hline O1S1 & $\mathrm{C} 26 \mathrm{H} 28 \mathrm{O} 1 \mathrm{~S} 1$ & $3.08 \mathrm{E}-05$ & Non-basic N1S1 & $\mathrm{C} 20 \mathrm{H} 21 \mathrm{~N} 1 \mathrm{~S} 1$ & $1.96 \mathrm{E}-06$ & Basic N1O1 & $\mathrm{C} 32 \mathrm{H} 25 \mathrm{~N} 1 \mathrm{O} 1$ & $1.20 \mathrm{E}-05$ \\
\hline O1S1 & $\mathrm{C} 27 \mathrm{H} 30 \mathrm{O} 1 \mathrm{~S} 1$ & $5.06 \mathrm{E}-05$ & Non-basic N1S1 & C19H17N1S1 & $2.56 \mathrm{E}-06$ & Basic N1O1 & $\mathrm{C} 33 \mathrm{H} 27 \mathrm{~N} 1 \mathrm{O} 1$ & $1.31 \mathrm{E}-05$ \\
\hline O1S1 & $\mathrm{C} 28 \mathrm{H} 32 \mathrm{O} 1 \mathrm{~S} 1$ & $5.70 \mathrm{E}-05$ & Non-basic N1S1 & C20H19N1S1 & $9.13 \mathrm{E}-07$ & Basic N1O1 & $\mathrm{C} 34 \mathrm{H} 29 \mathrm{~N} 1 \mathrm{O} 1$ & 5.32E-06 \\
\hline O1S1 & $\mathrm{C} 29 \mathrm{H} 34 \mathrm{O} 1 \mathrm{~S} 1$ & $3.10 \mathrm{E}-05$ & Non-basic N1S1 & $\mathrm{C} 21 \mathrm{H} 21 \mathrm{~N} 1 \mathrm{~S} 1$ & $4.74 \mathrm{E}-06$ & Basic N1O1 & $\mathrm{C} 35 \mathrm{H} 31 \mathrm{~N} 1 \mathrm{O} 1$ & $1.09 \mathrm{E}-06$ \\
\hline O1S1 & $\mathrm{C} 30 \mathrm{H} 36 \mathrm{O} 1 \mathrm{~S} 1$ & $5.16 \mathrm{E}-05$ & Non-basic N1S1 & $\mathrm{C} 22 \mathrm{H} 23 \mathrm{~N} 1 \mathrm{~S} 1$ & $3.18 \mathrm{E}-06$ & Basic N1O1 & $\mathrm{C} 30 \mathrm{H} 19 \mathrm{~N} 1 \mathrm{O} 1$ & $2.75 \mathrm{E}-06$ \\
\hline O1S1 & $\mathrm{C} 31 \mathrm{H} 38 \mathrm{O} 1 \mathrm{~S} 1$ & $2.78 \mathrm{E}-05$ & Non-basic N1S1 & $\mathrm{C} 22 \mathrm{H} 21 \mathrm{~N} 1 \mathrm{~S} 1$ & 4.19E-06 & Basic N1O1 & $\mathrm{C} 31 \mathrm{H} 21 \mathrm{~N} 1 \mathrm{O} 1$ & $3.36 \mathrm{E}-06$ \\
\hline O1S1 & $\mathrm{C} 18 \mathrm{H} 10 \mathrm{O} 1 \mathrm{~S} 1$ & $5.06 \mathrm{E}-06$ & Non-basic N1S1 & $\mathrm{C} 23 \mathrm{H} 23 \mathrm{~N} 1 \mathrm{~S} 1$ & $4.52 \mathrm{E}-06$ & Basic N1O1 & $\mathrm{C} 32 \mathrm{H} 23 \mathrm{~N} 1 \mathrm{O} 1$ & $5.36 \mathrm{E}-06$ \\
\hline O1S1 & C19H12O1S1 & $2.47 \mathrm{E}-05$ & Non-basic N1S1 & $\mathrm{C} 24 \mathrm{H} 25 \mathrm{~N} 1 \mathrm{~S} 1$ & $4.53 \mathrm{E}-06$ & Basic N1O1 & $\mathrm{C} 33 \mathrm{H} 25 \mathrm{~N} 1 \mathrm{O} 1$ & $4.66 \mathrm{E}-06$ \\
\hline O1S1 & $\mathrm{C} 20 \mathrm{H} 14 \mathrm{O} 1 \mathrm{~S} 1$ & 0.00011 & Non-basic N1S1 & C18H11N1S1 & $6.81 \mathrm{E}-05$ & Basic N1O1 & $\mathrm{C} 34 \mathrm{H} 27 \mathrm{~N} 1 \mathrm{O} 1$ & $2.97 \mathrm{E}-06$ \\
\hline O1S1 & $\mathrm{C} 21 \mathrm{H} 16 \mathrm{O} 1 \mathrm{~S} 1$ & 0.000119 & Non-basic N1S1 & C19H13N1S1 & 0.000385 & Basic N1O1 & $\mathrm{C} 35 \mathrm{H} 29 \mathrm{~N} 1 \mathrm{O} 1$ & 4.42E-06 \\
\hline O1S1 & $\mathrm{C} 22 \mathrm{H} 18 \mathrm{O} 1 \mathrm{~S} 1$ & $3.70 \mathrm{E}-05$ & Non-basic N1S1 & C20H15N1S1 & 0.000651 & Basic N1O1 & $\mathrm{C} 34 \mathrm{H} 25 \mathrm{~N} 1 \mathrm{O} 1$ & $2.63 \mathrm{E}-06$ \\
\hline O1S1 & $\mathrm{C} 23 \mathrm{H} 20 \mathrm{O} 1 \mathrm{~S} 1$ & $8.63 \mathrm{E}-06$ & Non-basic N1S1 & $\mathrm{C} 21 \mathrm{H} 17 \mathrm{~N} 1 \mathrm{~S} 1$ & 0.000598 & Basic N1O1 & $\mathrm{C} 35 \mathrm{H} 27 \mathrm{~N} 1 \mathrm{O} 1$ & $1.17 \mathrm{E}-06$ \\
\hline O1S1 & $\mathrm{C} 24 \mathrm{H} 22 \mathrm{O} 1 \mathrm{~S} 1$ & $3.04 \mathrm{E}-05$ & Non-basic N1S1 & C22H19N1S1 & 0.000378 & Basic N1O1 & $\mathrm{C} 36 \mathrm{H} 29 \mathrm{~N} 1 \mathrm{O} 1$ & $2.90 \mathrm{E}-06$ \\
\hline
\end{tabular}




\begin{tabular}{|c|c|c|c|c|c|c|c|c|}
\hline Class & Formula & Mass & Class & Formula & Mass & Class & Formula & Mass \\
\hline O1S1 & $\mathrm{C} 26 \mathrm{H} 26 \mathrm{O} 1 \mathrm{~S} 1$ & $2.17 \mathrm{E}-05$ & Non-basic N1S1 & $\mathrm{C} 23 \mathrm{H} 21 \mathrm{~N} 1 \mathrm{~S} 1$ & 0.000204 & Basic N1O1 & $\mathrm{C} 37 \mathrm{H} 31 \mathrm{~N} 1 \mathrm{O} 1$ & $1.24 \mathrm{E}-06$ \\
\hline O1S1 & $\mathrm{C} 27 \mathrm{H} 28 \mathrm{O} 1 \mathrm{~S} 1$ & $2.17 \mathrm{E}-05$ & Non-basic N1S1 & C24H23N1S1 & 0.000102 & Basic N1O1 & C38H33N1O1 & $6.10 \mathrm{E}-07$ \\
\hline O1S1 & $\mathrm{C} 21 \mathrm{H} 14 \mathrm{O} 1 \mathrm{~S} 1$ & $1.82 \mathrm{E}-05$ & Non-basic N1S1 & $\mathrm{C} 25 \mathrm{H} 25 \mathrm{~N} 1 \mathrm{~S} 1$ & $4.69 \mathrm{E}-05$ & Basic N1O1 & C34H23N1O1 & $4.11 \mathrm{E}-07$ \\
\hline O1S1 & $\mathrm{C} 22 \mathrm{H} 16 \mathrm{O} 1 \mathrm{~S} 1$ & $1.69 \mathrm{E}-05$ & Non-basic N1S1 & $\mathrm{C} 26 \mathrm{H} 27 \mathrm{~N} 1 \mathrm{~S} 1$ & $2.00 \mathrm{E}-05$ & Basic N1O1 & $\mathrm{C} 35 \mathrm{H} 25 \mathrm{~N} 1 \mathrm{O} 1$ & 4.62E-07 \\
\hline O1S1 & $\mathrm{C} 23 \mathrm{H} 18 \mathrm{O} 1 \mathrm{~S} 1$ & $2.86 \mathrm{E}-05$ & Non-basic N1S1 & $\mathrm{C} 27 \mathrm{H} 29 \mathrm{~N} 1 \mathrm{~S} 1$ & $3.95 \mathrm{E}-06$ & Basic N1O1 & $\mathrm{C} 36 \mathrm{H} 27 \mathrm{~N} 1 \mathrm{O} 1$ & $2.44 \mathrm{E}-07$ \\
\hline O1S1 & $\mathrm{C} 24 \mathrm{H} 20 \mathrm{O} 1 \mathrm{~S} 1$ & $4.53 \mathrm{E}-05$ & Non-basic N1S1 & $\mathrm{C} 28 \mathrm{H} 31 \mathrm{~N} 1 \mathrm{~S} 1$ & $3.60 \mathrm{E}-06$ & Basic N1O1 & $\mathrm{C} 37 \mathrm{H} 29 \mathrm{~N} 1 \mathrm{O} 1$ & $2.86 \mathrm{E}-07$ \\
\hline O1S1 & $\mathrm{C} 25 \mathrm{H} 22 \mathrm{O} 1 \mathrm{~S} 1$ & $1.50 \mathrm{E}-05$ & Non-basic N1S1 & C29H33N1S1 & $3.72 \mathrm{E}-06$ & Basic N1O1 & C38H29N1O1 & $1.31 \mathrm{E}-06$ \\
\hline O1S1 & $\mathrm{C} 26 \mathrm{H} 24 \mathrm{O} 1 \mathrm{~S} 1$ & $1.16 \mathrm{E}-05$ & Non-basic N1S1 & $\mathrm{C} 30 \mathrm{H} 35 \mathrm{~N} 1 \mathrm{~S} 1$ & $2.41 \mathrm{E}-06$ & Basic N1O1 & C39H31N1O1 & $2.32 \mathrm{E}-06$ \\
\hline O1S1 & $\mathrm{C} 27 \mathrm{H} 26 \mathrm{O} 1 \mathrm{~S} 1$ & $1.81 \mathrm{E}-05$ & Non-basic N1S1 & $\mathrm{C} 21 \mathrm{H} 15 \mathrm{~N} 1 \mathrm{~S} 1$ & $3.77 \mathrm{E}-06$ & Basic N1O1 & C39H29N1O1 & $2.52 \mathrm{E}-07$ \\
\hline O1S1 & $\mathrm{C} 28 \mathrm{H} 28 \mathrm{O} 1 \mathrm{~S} 1$ & $3.95 \mathrm{E}-06$ & Non-basic N1S1 & $\mathrm{C} 22 \mathrm{H} 17 \mathrm{~N} 1 \mathrm{~S} 1$ & $2.65 \mathrm{E}-05$ & Basic N1O1 & $\mathrm{C} 40 \mathrm{H} 31 \mathrm{~N} 1 \mathrm{O} 1$ & $1.72 \mathrm{E}-06$ \\
\hline O1S1 & $\mathrm{C} 29 \mathrm{H} 30 \mathrm{O} 1 \mathrm{~S} 1$ & $2.42 \mathrm{E}-05$ & Non-basic N1S1 & C23H19N1S1 & $3.91 \mathrm{E}-05$ & Basic N1O1 & $\mathrm{C} 41 \mathrm{H} 33 \mathrm{~N} 1 \mathrm{O} 1$ & $3.38 \mathrm{E}-06$ \\
\hline O1S1 & $\mathrm{C} 30 \mathrm{H} 32 \mathrm{O} 1 \mathrm{~S} 1$ & $3.08 \mathrm{E}-05$ & Non-basic N1S1 & $\mathrm{C} 24 \mathrm{H} 21 \mathrm{~N} 1 \mathrm{~S} 1$ & $4.18 \mathrm{E}-05$ & Basic N1O1 & $\mathrm{C} 42 \mathrm{H} 35 \mathrm{~N} 1 \mathrm{O} 1$ & $1.87 \mathrm{E}-06$ \\
\hline O1S1 & $\mathrm{C} 25 \mathrm{H} 20 \mathrm{O} 1 \mathrm{~S} 1$ & $2.09 \mathrm{E}-06$ & Non-basic N1S1 & $\mathrm{C} 25 \mathrm{H} 23 \mathrm{~N} 1 \mathrm{~S} 1$ & $7.32 \mathrm{E}-06$ & Basic N1O1 & $\mathrm{C} 43 \mathrm{H} 37 \mathrm{~N} 1 \mathrm{O} 1$ & $6.21 \mathrm{E}-07$ \\
\hline O1S1 & $\mathrm{C} 26 \mathrm{H} 22 \mathrm{O} 1 \mathrm{~S} 1$ & $1.52 \mathrm{E}-05$ & Non-basic N1S1 & $\mathrm{C} 26 \mathrm{H} 25 \mathrm{~N} 1 \mathrm{~S} 1$ & $2.55 \mathrm{E}-06$ & Basic N1O1 & $\mathrm{C} 44 \mathrm{H} 39 \mathrm{~N} 1 \mathrm{O} 1$ & $9.50 \mathrm{E}-07$ \\
\hline O1S1 & $\mathrm{C} 27 \mathrm{H} 24 \mathrm{O} 1 \mathrm{~S} 1$ & $6.51 \mathrm{E}-06$ & Non-basic N1S1 & $\mathrm{C} 20 \mathrm{H} 11 \mathrm{~N} 1 \mathrm{~S} 1$ & $8.15 \mathrm{E}-06$ & Basic N1O1 & C39H27N1O1 & $2.76 \mathrm{E}-07$ \\
\hline O1S1 & $\mathrm{C} 28 \mathrm{H} 26 \mathrm{O} 1 \mathrm{~S} 1$ & $3.20 \mathrm{E}-06$ & Non-basic N1S1 & $\mathrm{C} 21 \mathrm{H} 13 \mathrm{~N} 1 \mathrm{~S} 1$ & 7.14E-05 & Basic N1O1 & $\mathrm{C} 40 \mathrm{H} 29 \mathrm{~N} 1 \mathrm{O} 1$ & $6.84 \mathrm{E}-07$ \\
\hline O1S1 & $\mathrm{C} 29 \mathrm{H} 28 \mathrm{O} 1 \mathrm{~S} 1$ & $3.31 \mathrm{E}-06$ & Non-basic N1S1 & $\mathrm{C} 22 \mathrm{H} 15 \mathrm{~N} 1 \mathrm{~S} 1$ & 0.000141 & Basic N1O1 & $\mathrm{C} 41 \mathrm{H} 31 \mathrm{~N} 1 \mathrm{O} 1$ & $5.71 \mathrm{E}-06$ \\
\hline O1S1 & $\mathrm{C} 30 \mathrm{H} 30 \mathrm{O} 1 \mathrm{~S} 1$ & $4.10 \mathrm{E}-05$ & Non-basic N1S1 & $\mathrm{C} 23 \mathrm{H} 17 \mathrm{~N} 1 \mathrm{~S} 1$ & 0.000136 & Basic N1O1 & $\mathrm{C} 42 \mathrm{H} 33 \mathrm{~N} 1 \mathrm{O} 1$ & $1.58 \mathrm{E}-06$ \\
\hline O1S1 & $\mathrm{C} 31 \mathrm{H} 32 \mathrm{O} 1 \mathrm{~S} 1$ & 4.61E-06 & Non-basic N1S1 & $\mathrm{C} 24 \mathrm{H} 19 \mathrm{~N} 1 \mathrm{~S} 1$ & $9.89 \mathrm{E}-05$ & Basic N1O1 & $\mathrm{C} 43 \mathrm{H} 35 \mathrm{~N} 1 \mathrm{O} 1$ & $1.32 \mathrm{E}-06$ \\
\hline O1S1 & $\mathrm{C} 32 \mathrm{H} 34 \mathrm{O} 1 \mathrm{~S} 1$ & $3.76 \mathrm{E}-06$ & Non-basic N1S1 & $\mathrm{C} 25 \mathrm{H} 21 \mathrm{~N} 1 \mathrm{~S} 1$ & $5.01 \mathrm{E}-05$ & Basic N1O1 & $\mathrm{C} 44 \mathrm{H} 37 \mathrm{~N} 1 \mathrm{O} 1$ & $5.34 \mathrm{E}-07$ \\
\hline O1S1 & $\mathrm{C} 33 \mathrm{H} 36 \mathrm{O} 1 \mathrm{~S} 1$ & $3.23 \mathrm{E}-05$ & Non-basic N1S1 & $\mathrm{C} 26 \mathrm{H} 23 \mathrm{~N} 1 \mathrm{~S} 1$ & $1.29 \mathrm{E}-05$ & Basic N1O1 & $\mathrm{C} 45 \mathrm{H} 39 \mathrm{~N} 1 \mathrm{O} 1$ & $4.34 \mathrm{E}-07$ \\
\hline O1S1 & $\mathrm{C} 34 \mathrm{H} 38 \mathrm{O} 1 \mathrm{~S} 1$ & $2.55 \mathrm{E}-05$ & Non-basic N1S1 & $\mathrm{C} 27 \mathrm{H} 25 \mathrm{~N} 1 \mathrm{~S} 1$ & $3.24 \mathrm{E}-06$ & Basic N1O1 & $\mathrm{C} 46 \mathrm{H} 41 \mathrm{~N} 1 \mathrm{O} 1$ & $6.81 \mathrm{E}-07$ \\
\hline O1S1 & $\mathrm{C} 35 \mathrm{H} 40 \mathrm{O} 1 \mathrm{~S} 1$ & $7.09 \mathrm{E}-05$ & Non-basic N1S1 & $\mathrm{C} 22 \mathrm{H} 13 \mathrm{~N} 1 \mathrm{~S} 1$ & $3.55 \mathrm{E}-05$ & Basic N1O1 & $\mathrm{C} 42 \mathrm{H} 31 \mathrm{~N} 1 \mathrm{O} 1$ & $1.11 \mathrm{E}-06$ \\
\hline O1S1 & $\mathrm{C} 36 \mathrm{H} 42 \mathrm{O} 1 \mathrm{~S} 1$ & 0.000361 & Non-basic N1S1 & $\mathrm{C} 23 \mathrm{H} 15 \mathrm{~N} 1 \mathrm{~S} 1$ & 0.000164 & Basic N1O1 & $\mathrm{C} 43 \mathrm{H} 33 \mathrm{~N} 1 \mathrm{O} 1$ & $2.84 \mathrm{E}-06$ \\
\hline $\mathrm{O} 1 \mathrm{~S} 1$ & $\mathrm{C} 24 \mathrm{H} 16 \mathrm{O} 1 \mathrm{~S} 1$ & $1.39 \mathrm{E}-05$ & Non-basic N1S1 & $\mathrm{C} 24 \mathrm{H} 17 \mathrm{~N} 1 \mathrm{~S} 1$ & 0.000294 & Basic N1O1 & $\mathrm{C} 44 \mathrm{H} 35 \mathrm{~N} 1 \mathrm{O} 1$ & $2.82 \mathrm{E}-07$ \\
\hline O1S1 & $\mathrm{C} 25 \mathrm{H} 18 \mathrm{O} 1 \mathrm{~S} 1$ & $2.28 \mathrm{E}-05$ & Non-basic N1S1 & $\mathrm{C} 25 \mathrm{H} 19 \mathrm{~N} 1 \mathrm{~S} 1$ & 0.000273 & Basic N1O1 & $\mathrm{C} 45 \mathrm{H} 37 \mathrm{~N} 1 \mathrm{O} 1$ & 4.99E-07 \\
\hline Non-basic N1 & C16H11N1 & 0.001288 & Non-basic N1S1 & $\mathrm{C} 26 \mathrm{H} 21 \mathrm{~N} 1 \mathrm{~S} 1$ & 0.000174 & Basic N1O2 & $\mathrm{C} 14 \mathrm{H} 23 \mathrm{~N} 1 \mathrm{O} 2$ & $3.64 \mathrm{E}-06$ \\
\hline Non-basic N1 & $\mathrm{C} 17 \mathrm{H} 13 \mathrm{~N} 1$ & 0.011988 & Non-basic N1S1 & $\mathrm{C} 27 \mathrm{H} 23 \mathrm{~N} 1 \mathrm{~S} 1$ & $9.47 \mathrm{E}-05$ & Basic N1O2 & $\mathrm{C} 15 \mathrm{H} 25 \mathrm{~N} 1 \mathrm{O} 2$ & $2.53 \mathrm{E}-07$ \\
\hline Non-basic N1 & $\mathrm{C} 18 \mathrm{H} 15 \mathrm{~N} 1$ & 0.018428 & Non-basic N1S1 & $\mathrm{C} 28 \mathrm{H} 25 \mathrm{~N} 1 \mathrm{~S} 1$ & $4.11 \mathrm{E}-05$ & Basic N1O2 & $\mathrm{C} 16 \mathrm{H} 27 \mathrm{~N} 1 \mathrm{O} 2$ & $6.78 \mathrm{E}-07$ \\
\hline Non-basic N1 & $\mathrm{C} 19 \mathrm{H} 17 \mathrm{~N} 1$ & 0.01326 & Non-basic N1S1 & $\mathrm{C} 29 \mathrm{H} 27 \mathrm{~N} 1 \mathrm{~S} 1$ & $1.81 \mathrm{E}-05$ & Basic N1O2 & $\mathrm{C} 17 \mathrm{H} 29 \mathrm{~N} 1 \mathrm{O} 2$ & $6.32 \mathrm{E}-06$ \\
\hline Non-basic N1 & $\mathrm{C} 20 \mathrm{H} 19 \mathrm{~N} 1$ & 0.005832 & Non-basic N1S1 & $\mathrm{C} 30 \mathrm{H} 29 \mathrm{~N} 1 \mathrm{~S} 1$ & $1.20 \mathrm{E}-05$ & Basic N1O2 & $\mathrm{C} 18 \mathrm{H} 31 \mathrm{~N} 1 \mathrm{O} 2$ & $1.78 \mathrm{E}-06$ \\
\hline Non-basic N1 & $\mathrm{C} 21 \mathrm{H} 21 \mathrm{~N} 1$ & 0.002172 & Non-basic N1S1 & $\mathrm{C} 31 \mathrm{H} 31 \mathrm{~N} 1 \mathrm{~S} 1$ & $4.55 \mathrm{E}-06$ & Basic N1O2 & $\mathrm{C} 13 \mathrm{H} 19 \mathrm{~N} 1 \mathrm{O} 2$ & $1.89 \mathrm{E}-06$ \\
\hline Non-basic N1 & $\mathrm{C} 22 \mathrm{H} 23 \mathrm{~N} 1$ & 0.000921 & Non-basic N1S1 & $\mathrm{C} 33 \mathrm{H} 35 \mathrm{~N} 1 \mathrm{~S} 1$ & $1.40 \mathrm{E}-06$ & Basic N1O2 & $\mathrm{C} 14 \mathrm{H} 21 \mathrm{~N} 1 \mathrm{O} 2$ & $8.81 \mathrm{E}-07$ \\
\hline Non-basic N1 & $\mathrm{C} 23 \mathrm{H} 25 \mathrm{~N} 1$ & 0.000503 & Non-basic N1S1 & $\mathrm{C} 22 \mathrm{H} 11 \mathrm{~N} 1 \mathrm{~S} 1$ & $1.80 \mathrm{E}-06$ & Basic N1O2 & $\mathrm{C} 15 \mathrm{H} 23 \mathrm{~N} 1 \mathrm{O} 2$ & $1.06 \mathrm{E}-06$ \\
\hline Non-basic N1 & $\mathrm{C} 24 \mathrm{H} 27 \mathrm{~N} 1$ & 0.000365 & Non-basic N1S1 & $\mathrm{C} 24 \mathrm{H} 15 \mathrm{~N} 1 \mathrm{~S} 1$ & $1.18 \mathrm{E}-05$ & Basic N1O2 & $\mathrm{C} 19 \mathrm{H} 29 \mathrm{~N} 1 \mathrm{O} 2$ & $1.04 \mathrm{E}-07$ \\
\hline Non-basic N1 & $\mathrm{C} 25 \mathrm{H} 29 \mathrm{~N} 1$ & 0.000314 & Non-basic N1S1 & $\mathrm{C} 25 \mathrm{H} 17 \mathrm{~N} 1 \mathrm{~S} 1$ & $3.08 \mathrm{E}-05$ & Basic N1O2 & $\mathrm{C} 20 \mathrm{H} 31 \mathrm{~N} 1 \mathrm{O} 2$ & $1.36 \mathrm{E}-06$ \\
\hline Non-basic N1 & $\mathrm{C} 26 \mathrm{H} 31 \mathrm{~N} 1$ & 0.000257 & Non-basic N1S1 & $\mathrm{C} 26 \mathrm{H} 19 \mathrm{~N} 1 \mathrm{~S} 1$ & $3.44 \mathrm{E}-05$ & Basic N1O2 & $\mathrm{C} 21 \mathrm{H} 33 \mathrm{~N} 1 \mathrm{O} 2$ & $1.18 \mathrm{E}-06$ \\
\hline Non-basic N1 & $\mathrm{C} 27 \mathrm{H} 33 \mathrm{~N} 1$ & 0.000233 & Non-basic N1S1 & $\mathrm{C} 27 \mathrm{H} 21 \mathrm{~N} 1 \mathrm{~S} 1$ & $3.03 \mathrm{E}-05$ & Basic N1O2 & $\mathrm{C} 14 \mathrm{H} 17 \mathrm{~N} 1 \mathrm{O} 2$ & 0.000125 \\
\hline
\end{tabular}




\begin{tabular}{|c|c|c|c|c|c|c|c|c|}
\hline Class & Formula & Mass & Class & Formula & Mass & Class & Formula & Mass \\
\hline Non-basic N1 & $\mathrm{C} 28 \mathrm{H} 35 \mathrm{~N} 1$ & 0.000192 & Non-basic N1S1 & C28H23N1S1 & $1.86 \mathrm{E}-05$ & Basic N1O2 & C15H19N1O2 & $2.78 \mathrm{E}-06$ \\
\hline Non-basic N1 & $\mathrm{C} 29 \mathrm{H} 37 \mathrm{~N} 1$ & 0.000156 & Non-basic N1S1 & C29H25N1S1 & $1.22 \mathrm{E}-05$ & Basic N1O2 & $\mathrm{C} 16 \mathrm{H} 21 \mathrm{~N} 1 \mathrm{O} 2$ & $1.84 \mathrm{E}-06$ \\
\hline Non-basic N1 & $\mathrm{C} 30 \mathrm{H} 39 \mathrm{~N} 1$ & 0.000123 & Non-basic N1S1 & $\mathrm{C} 30 \mathrm{H} 27 \mathrm{~N} 1 \mathrm{~S} 1$ & $6.57 \mathrm{E}-06$ & Basic N1O2 & $\mathrm{C} 17 \mathrm{H} 23 \mathrm{~N} 1 \mathrm{O} 2$ & $6.37 \mathrm{E}-07$ \\
\hline Non-basic N1 & $\mathrm{C} 31 \mathrm{H} 41 \mathrm{~N} 1$ & 0.000108 & Non-basic N1S1 & C31H29N1S1 & 4.73E-06 & Basic N1O2 & $\mathrm{C} 18 \mathrm{H} 25 \mathrm{~N} 1 \mathrm{O} 2$ & $3.79 \mathrm{E}-07$ \\
\hline Non-basic N1 & $\mathrm{C} 32 \mathrm{H} 43 \mathrm{~N} 1$ & $8.90 \mathrm{E}-05$ & Non-basic N1S1 & C24H13N1S1 & $2.38 \mathrm{E}-06$ & Basic N1O2 & C19H27N1O2 & $5.40 \mathrm{E}-07$ \\
\hline Non-basic N1 & $\mathrm{C} 33 \mathrm{H} 45 \mathrm{~N} 1$ & $5.65 \mathrm{E}-05$ & Non-basic N1S1 & $\mathrm{C} 25 \mathrm{H} 15 \mathrm{~N} 1 \mathrm{~S} 1$ & $2.39 \mathrm{E}-05$ & Basic N1O2 & $\mathrm{C} 20 \mathrm{H} 29 \mathrm{~N} 1 \mathrm{O} 2$ & $4.45 \mathrm{E}-07$ \\
\hline Non-basic N1 & $\mathrm{C} 34 \mathrm{H} 47 \mathrm{~N} 1$ & 3.69E-05 & Non-basic N1S1 & C26H17N1S1 & $4.37 \mathrm{E}-05$ & Basic N1O2 & $\mathrm{C} 21 \mathrm{H} 31 \mathrm{~N} 1 \mathrm{O} 2$ & $1.63 \mathrm{E}-06$ \\
\hline Non-basic N1 & $\mathrm{C} 35 \mathrm{H} 49 \mathrm{~N} 1$ & $2.12 \mathrm{E}-05$ & Non-basic N1S1 & C27H19N1S1 & $5.30 \mathrm{E}-05$ & Basic N1O2 & $\mathrm{C} 22 \mathrm{H} 33 \mathrm{~N} 1 \mathrm{O} 2$ & $1.02 \mathrm{E}-06$ \\
\hline Non-basic N1 & $\mathrm{C} 36 \mathrm{H} 51 \mathrm{~N} 1$ & $1.10 \mathrm{E}-05$ & Non-basic N1S1 & $\mathrm{C} 28 \mathrm{H} 21 \mathrm{~N} 1 \mathrm{~S} 1$ & $4.78 \mathrm{E}-05$ & Basic N1O2 & $\mathrm{C} 23 \mathrm{H} 35 \mathrm{~N} 1 \mathrm{O} 2$ & $1.41 \mathrm{E}-06$ \\
\hline Non-basic N1 & $\mathrm{C} 37 \mathrm{H} 53 \mathrm{~N} 1$ & $5.57 \mathrm{E}-06$ & Non-basic N1S1 & C29H23N1S1 & $2.05 \mathrm{E}-05$ & Basic N1O2 & $\mathrm{C} 24 \mathrm{H} 37 \mathrm{~N} 1 \mathrm{O} 2$ & $2.04 \mathrm{E}-06$ \\
\hline Non-basic N1 & $\mathrm{C} 38 \mathrm{H} 55 \mathrm{~N} 1$ & 4.93E-06 & Non-basic N1S1 & $\mathrm{C} 30 \mathrm{H} 25 \mathrm{~N} 1 \mathrm{~S} 1$ & $1.36 \mathrm{E}-05$ & Basic N1O2 & $\mathrm{C} 25 \mathrm{H} 39 \mathrm{~N} 1 \mathrm{O} 2$ & $2.65 \mathrm{E}-06$ \\
\hline Non-basic N1 & C16H9N1 & $3.46 \mathrm{E}-06$ & Non-basic N1S1 & C31H27N1S1 & $6.89 \mathrm{E}-06$ & Basic N1O2 & $\mathrm{C} 26 \mathrm{H} 41 \mathrm{~N} 1 \mathrm{O} 2$ & $3.01 \mathrm{E}-06$ \\
\hline Non-basic N1 & C17H11N1 & $3.91 \mathrm{E}-06$ & Non-basic N1S1 & C26H15N1S1 & $5.58 \mathrm{E}-06$ & Basic N1O2 & $\mathrm{C} 27 \mathrm{H} 43 \mathrm{~N} 1 \mathrm{O} 2$ & $8.99 \mathrm{E}-06$ \\
\hline Non-basic N1 & $\mathrm{C} 18 \mathrm{H} 13 \mathrm{~N} 1$ & 0.000188 & Non-basic N1S1 & C27H17N1S1 & $2.52 \mathrm{E}-05$ & Basic N1O2 & $\mathrm{C} 28 \mathrm{H} 45 \mathrm{~N} 1 \mathrm{O} 2$ & 8.32E-06 \\
\hline Non-basic N1 & C19H15N1 & 0.001028 & Non-basic N1S1 & C28H19N1S1 & $3.13 \mathrm{E}-05$ & Basic N1O2 & $\mathrm{C} 29 \mathrm{H} 47 \mathrm{~N} 1 \mathrm{O} 2$ & $9.61 \mathrm{E}-06$ \\
\hline Non-basic N1 & $\mathrm{C} 20 \mathrm{H} 17 \mathrm{~N} 1$ & 0.001701 & Non-basic N1S1 & $\mathrm{C} 29 \mathrm{H} 21 \mathrm{~N} 1 \mathrm{~S} 1$ & $3.67 \mathrm{E}-05$ & Basic N1O2 & $\mathrm{C} 30 \mathrm{H} 49 \mathrm{~N} 1 \mathrm{O} 2$ & $1.50 \mathrm{E}-05$ \\
\hline Non-basic N1 & $\mathrm{C} 21 \mathrm{H} 19 \mathrm{~N} 1$ & 0.001611 & Non-basic N1S1 & $\mathrm{C} 30 \mathrm{H} 23 \mathrm{~N} 1 \mathrm{~S} 1$ & $3.20 \mathrm{E}-05$ & Basic N1O2 & C31H51N1O2 & $9.25 \mathrm{E}-06$ \\
\hline Non-basic N1 & $\mathrm{C} 22 \mathrm{H} 21 \mathrm{~N} 1$ & 0.00096 & Non-basic N1S1 & $\mathrm{C} 31 \mathrm{H} 25 \mathrm{~N} 1 \mathrm{~S} 1$ & $1.07 \mathrm{E}-05$ & Basic N1O2 & C32H53N1O2 & $1.06 \mathrm{E}-05$ \\
\hline Non-basic N1 & $\mathrm{C} 23 \mathrm{H} 23 \mathrm{~N} 1$ & 0.000542 & Non-basic N1S1 & $\mathrm{C} 32 \mathrm{H} 27 \mathrm{~N} 1 \mathrm{~S} 1$ & 7.61E-06 & Basic N1O2 & C33H55N1O2 & $6.36 \mathrm{E}-06$ \\
\hline Non-basic N1 & $\mathrm{C} 24 \mathrm{H} 25 \mathrm{~N} 1$ & 0.000301 & Non-basic N1S1 & C28H17N1S1 & $1.90 \mathrm{E}-06$ & Basic N1O2 & $\mathrm{C} 34 \mathrm{H} 57 \mathrm{~N} 1 \mathrm{O} 2$ & $1.44 \mathrm{E}-05$ \\
\hline Non-basic N1 & $\mathrm{C} 25 \mathrm{H} 27 \mathrm{~N} 1$ & 0.000174 & Non-basic N1S1 & C29H19N1S1 & $1.01 \mathrm{E}-05$ & Basic N1O2 & C35H59N1O2 & $1.30 \mathrm{E}-05$ \\
\hline Non-basic N1 & $\mathrm{C} 26 \mathrm{H} 29 \mathrm{~N} 1$ & 0.000118 & Non-basic N1S1 & $\mathrm{C} 30 \mathrm{H} 21 \mathrm{~N} 1 \mathrm{~S} 1$ & $6.05 \mathrm{E}-06$ & Basic N1O2 & $\mathrm{C} 36 \mathrm{H} 61 \mathrm{~N} 1 \mathrm{O} 2$ & $1.12 \mathrm{E}-05$ \\
\hline Non-basic N1 & $\mathrm{C} 27 \mathrm{H} 31 \mathrm{~N} 1$ & 0.000111 & Non-basic N1S1 & C31H23N1S1 & $5.04 \mathrm{E}-06$ & Basic N1O2 & $\mathrm{C} 37 \mathrm{H} 63 \mathrm{~N} 1 \mathrm{O} 2$ & 4.77E-06 \\
\hline Non-basic N1 & $\mathrm{C} 28 \mathrm{H} 33 \mathrm{~N} 1$ & $7.90 \mathrm{E}-05$ & Non-basic N1S1 & $\mathrm{C} 32 \mathrm{H} 25 \mathrm{~N} 1 \mathrm{~S} 1$ & $9.40 \mathrm{E}-06$ & Basic N1O2 & $\mathrm{C} 38 \mathrm{H} 65 \mathrm{~N} 1 \mathrm{O} 2$ & $5.64 \mathrm{E}-06$ \\
\hline Non-basic N1 & $\mathrm{C} 29 \mathrm{H} 35 \mathrm{~N} 1$ & $6.96 \mathrm{E}-05$ & Non-basic N1S1 & $\mathrm{C} 33 \mathrm{H} 27 \mathrm{~N} 1 \mathrm{~S} 1$ & $2.52 \mathrm{E}-06$ & Basic N1O2 & C39H67N1O2 & $5.23 \mathrm{E}-06$ \\
\hline Non-basic N1 & $\mathrm{C} 30 \mathrm{H} 37 \mathrm{~N} 1$ & $4.92 \mathrm{E}-05$ & Non-basic N1S1 & C34H29N1S1 & $4.00 \mathrm{E}-06$ & Basic N1O2 & C40H69N1O2 & 4.53E-06 \\
\hline Non-basic N1 & $\mathrm{C} 31 \mathrm{H} 39 \mathrm{~N} 1$ & 4.47E-05 & Non-basic N1S1 & $\mathrm{C} 35 \mathrm{H} 31 \mathrm{~N} 1 \mathrm{~S} 1$ & $1.75 \mathrm{E}-06$ & Basic N1O2 & $\mathrm{C} 41 \mathrm{H} 71 \mathrm{~N} 1 \mathrm{O} 2$ & $6.58 \mathrm{E}-06$ \\
\hline Non-basic N1 & $\mathrm{C} 32 \mathrm{H} 41 \mathrm{~N} 1$ & $3.24 \mathrm{E}-05$ & Non-basic N1S1 & C29H17N1S1 & $6.91 \mathrm{E}-06$ & Basic N1O2 & C16H19N1O2 & $1.56 \mathrm{E}-06$ \\
\hline Non-basic N1 & $\mathrm{C} 33 \mathrm{H} 43 \mathrm{~N} 1$ & $2.19 \mathrm{E}-05$ & Non-basic N1S1 & C30H19N1S1 & $8.07 \mathrm{E}-06$ & Basic N1O2 & $\mathrm{C} 17 \mathrm{H} 21 \mathrm{~N} 1 \mathrm{O} 2$ & $2.16 \mathrm{E}-06$ \\
\hline Non-basic N1 & $\mathrm{C} 34 \mathrm{H} 45 \mathrm{~N} 1$ & $1.62 \mathrm{E}-05$ & Non-basic N1S1 & C31H21N1S1 & $1.56 \mathrm{E}-05$ & Basic N1O2 & $\mathrm{C} 18 \mathrm{H} 23 \mathrm{~N} 1 \mathrm{O} 2$ & $1.47 \mathrm{E}-06$ \\
\hline Non-basic N1 & $\mathrm{C} 35 \mathrm{H} 47 \mathrm{~N} 1$ & $3.05 \mathrm{E}-06$ & Non-basic N1S1 & C32H23N1S1 & $6.92 \mathrm{E}-06$ & Basic N1O2 & $\mathrm{C} 19 \mathrm{H} 25 \mathrm{~N} 1 \mathrm{O} 2$ & $7.90 \mathrm{E}-07$ \\
\hline Non-basic N1 & $\mathrm{C} 36 \mathrm{H} 49 \mathrm{~N} 1$ & $2.03 \mathrm{E}-06$ & Non-basic N1S1 & $\mathrm{C} 33 \mathrm{H} 25 \mathrm{~N} 1 \mathrm{~S} 1$ & 7.43E-06 & Basic N1O2 & $\mathrm{C} 20 \mathrm{H} 27 \mathrm{~N} 1 \mathrm{O} 2$ & $2.54 \mathrm{E}-06$ \\
\hline Non-basic N1 & $\mathrm{C} 37 \mathrm{H} 51 \mathrm{~N} 1$ & $1.51 \mathrm{E}-06$ & Non-basic N1S1 & C34H27N1S1 & 7.65E-06 & Basic N1O2 & $\mathrm{C} 21 \mathrm{H} 29 \mathrm{~N} 1 \mathrm{O} 2$ & $1.16 \mathrm{E}-06$ \\
\hline Non-basic N1 & $\mathrm{C} 18 \mathrm{H} 11 \mathrm{~N} 1$ & $2.44 \mathrm{E}-05$ & Non-basic N1S1 & C35H29N1S1 & $1.46 \mathrm{E}-06$ & Basic N1O2 & $\mathrm{C} 22 \mathrm{H} 31 \mathrm{~N} 1 \mathrm{O} 2$ & $1.44 \mathrm{E}-06$ \\
\hline Non-basic N1 & C19H13N1 & 0.000258 & Non-basic N1S1 & C32H21N1S1 & $5.85 \mathrm{E}-06$ & Basic N1O2 & $\mathrm{C} 23 \mathrm{H} 33 \mathrm{~N} 1 \mathrm{O} 2$ & $5.65 \mathrm{E}-06$ \\
\hline Non-basic N1 & $\mathrm{C} 20 \mathrm{H} 15 \mathrm{~N} 1$ & 0.000817 & Non-basic N1S1 & C33H23N1S1 & $3.74 \mathrm{E}-06$ & Basic N1O2 & $\mathrm{C} 24 \mathrm{H} 35 \mathrm{~N} 1 \mathrm{O} 2$ & $6.70 \mathrm{E}-06$ \\
\hline Non-basic N1 & $\mathrm{C} 21 \mathrm{H} 17 \mathrm{~N} 1$ & 0.001445 & Non-basic N1S1 & $\mathrm{C} 34 \mathrm{H} 25 \mathrm{~N} 1 \mathrm{~S} 1$ & $1.38 \mathrm{E}-06$ & Basic N1O2 & $\mathrm{C} 25 \mathrm{H} 37 \mathrm{~N} 1 \mathrm{O} 2$ & $5.24 \mathrm{E}-06$ \\
\hline Non-basic N1 & $\mathrm{C} 22 \mathrm{H} 19 \mathrm{~N} 1$ & 0.001532 & Non-basic N1S1 & $\mathrm{C} 35 \mathrm{H} 27 \mathrm{~N} 1 \mathrm{~S} 1$ & $1.42 \mathrm{E}-06$ & Basic N1O2 & $\mathrm{C} 26 \mathrm{H} 39 \mathrm{~N} 1 \mathrm{O} 2$ & $6.82 \mathrm{E}-06$ \\
\hline
\end{tabular}




\begin{tabular}{|c|c|c|c|c|c|c|c|c|}
\hline Class & Formula & Mass & Class & Formula & Mass & Class & Formula & Mass \\
\hline Non-basic N1 & $\mathrm{C} 23 \mathrm{H} 21 \mathrm{~N} 1$ & 0.00105 & Non-basic N1S1 & $\mathrm{C} 36 \mathrm{H} 29 \mathrm{~N} 1 \mathrm{~S} 1$ & $1.59 \mathrm{E}-06$ & Basic N1O2 & $\mathrm{C} 27 \mathrm{H} 41 \mathrm{~N} 1 \mathrm{O} 2$ & $8.57 \mathrm{E}-06$ \\
\hline Non-basic N1 & $\mathrm{C} 24 \mathrm{H} 23 \mathrm{~N} 1$ & 0.000668 & Non-basic N1S1 & C37H31N1S1 & $2.57 \mathrm{E}-06$ & Basic N1O2 & $\mathrm{C} 28 \mathrm{H} 43 \mathrm{~N} 1 \mathrm{O} 2$ & $8.87 \mathrm{E}-06$ \\
\hline Non-basic N1 & $\mathrm{C} 25 \mathrm{H} 25 \mathrm{~N} 1$ & 0.000366 & Non-basic N1S1 & $\mathrm{C} 38 \mathrm{H} 33 \mathrm{~N} 1 \mathrm{~S} 1$ & $2.85 \mathrm{E}-06$ & Basic N1O2 & $\mathrm{C} 29 \mathrm{H} 45 \mathrm{~N} 1 \mathrm{O} 2$ & $2.08 \mathrm{E}-05$ \\
\hline Non-basic N1 & $\mathrm{C} 26 \mathrm{H} 27 \mathrm{~N} 1$ & 0.000222 & Non-basic N2 & $\mathrm{C} 27 \mathrm{H} 46 \mathrm{~N} 2$ & $7.35 \mathrm{E}-06$ & Basic N1O2 & $\mathrm{C} 30 \mathrm{H} 47 \mathrm{~N} 1 \mathrm{O} 2$ & $1.72 \mathrm{E}-05$ \\
\hline Non-basic N1 & $\mathrm{C} 27 \mathrm{H} 29 \mathrm{~N} 1$ & 0.000116 & Non-basic N2 & $\mathrm{C} 28 \mathrm{H} 48 \mathrm{~N} 2$ & $1.45 \mathrm{E}-05$ & Basic N1O2 & $\mathrm{C} 31 \mathrm{H} 49 \mathrm{~N} 1 \mathrm{O} 2$ & $2.06 \mathrm{E}-05$ \\
\hline Non-basic N1 & $\mathrm{C} 28 \mathrm{H} 31 \mathrm{~N} 1$ & $7.50 \mathrm{E}-05$ & Non-basic N2 & $\mathrm{C} 29 \mathrm{H} 50 \mathrm{~N} 2$ & $1.58 \mathrm{E}-05$ & Basic N1O2 & $\mathrm{C} 32 \mathrm{H} 51 \mathrm{~N} 1 \mathrm{O} 2$ & $2.38 \mathrm{E}-05$ \\
\hline Non-basic N1 & $\mathrm{C} 29 \mathrm{H} 33 \mathrm{~N} 1$ & 4.96E-05 & Non-basic N2 & $\mathrm{C} 30 \mathrm{H} 52 \mathrm{~N} 2$ & $1.27 \mathrm{E}-05$ & Basic N1O2 & $\mathrm{C} 33 \mathrm{H} 53 \mathrm{~N} 1 \mathrm{O} 2$ & $1.74 \mathrm{E}-05$ \\
\hline Non-basic N1 & $\mathrm{C} 30 \mathrm{H} 35 \mathrm{~N} 1$ & $2.95 \mathrm{E}-05$ & Non-basic N2 & C31H54N2 & $1.33 \mathrm{E}-05$ & Basic N1O2 & $\mathrm{C} 34 \mathrm{H} 55 \mathrm{~N} 1 \mathrm{O} 2$ & $1.97 \mathrm{E}-05$ \\
\hline Non-basic N1 & $\mathrm{C} 31 \mathrm{H} 37 \mathrm{~N} 1$ & $2.91 \mathrm{E}-05$ & Non-basic N2 & $\mathrm{C} 32 \mathrm{H} 56 \mathrm{~N} 2$ & $1.99 \mathrm{E}-05$ & Basic N1O2 & $\mathrm{C} 35 \mathrm{H} 57 \mathrm{~N} 1 \mathrm{O} 2$ & $1.68 \mathrm{E}-05$ \\
\hline Non-basic N1 & $\mathrm{C} 32 \mathrm{H} 39 \mathrm{~N} 1$ & $2.56 \mathrm{E}-05$ & Non-basic N2 & $\mathrm{C} 33 \mathrm{H} 58 \mathrm{~N} 2$ & $2.62 \mathrm{E}-05$ & Basic N1O2 & $\mathrm{C} 36 \mathrm{H} 59 \mathrm{~N} 1 \mathrm{O} 2$ & $1.65 \mathrm{E}-05$ \\
\hline Non-basic N1 & $\mathrm{C} 33 \mathrm{H} 41 \mathrm{~N} 1$ & $1.25 \mathrm{E}-05$ & Non-basic N2 & $\mathrm{C} 34 \mathrm{H} 60 \mathrm{~N} 2$ & $1.22 \mathrm{E}-05$ & Basic N1O2 & $\mathrm{C} 37 \mathrm{H} 61 \mathrm{~N} 1 \mathrm{O} 2$ & $1.31 \mathrm{E}-05$ \\
\hline Non-basic N1 & $\mathrm{C} 34 \mathrm{H} 43 \mathrm{~N} 1$ & $2.32 \mathrm{E}-05$ & Non-basic N2 & $\mathrm{C} 35 \mathrm{H} 62 \mathrm{~N} 2$ & $2.39 \mathrm{E}-05$ & Basic N1O2 & $\mathrm{C} 38 \mathrm{H} 63 \mathrm{~N} 1 \mathrm{O} 2$ & $7.22 \mathrm{E}-06$ \\
\hline Non-basic N1 & $\mathrm{C} 35 \mathrm{H} 45 \mathrm{~N} 1$ & $2.67 \mathrm{E}-06$ & Non-basic N2 & $\mathrm{C} 36 \mathrm{H} 64 \mathrm{~N} 2$ & $9.75 \mathrm{E}-06$ & Basic N1O2 & $\mathrm{C} 15 \mathrm{H} 15 \mathrm{~N} 1 \mathrm{O} 2$ & $3.72 \mathrm{E}-06$ \\
\hline Non-basic N1 & $\mathrm{C} 36 \mathrm{H} 47 \mathrm{~N} 1$ & $3.20 \mathrm{E}-06$ & Non-basic N2 & $\mathrm{C} 37 \mathrm{H} 66 \mathrm{~N} 2$ & $1.00 \mathrm{E}-05$ & Basic N1O2 & $\mathrm{C} 16 \mathrm{H} 17 \mathrm{~N} 1 \mathrm{O} 2$ & $2.15 \mathrm{E}-06$ \\
\hline Non-basic N1 & $\mathrm{C} 20 \mathrm{H} 13 \mathrm{~N} 1$ & 0.005318 & Non-basic N2 & $\mathrm{C} 26 \mathrm{H} 42 \mathrm{~N} 2$ & 7.17E-06 & Basic N1O2 & $\mathrm{C} 17 \mathrm{H} 19 \mathrm{~N} 1 \mathrm{O} 2$ & $1.12 \mathrm{E}-06$ \\
\hline Non-basic N1 & $\mathrm{C} 21 \mathrm{H} 15 \mathrm{~N} 1$ & 0.014891 & Non-basic N2 & $\mathrm{C} 27 \mathrm{H} 44 \mathrm{~N} 2$ & $6.86 \mathrm{E}-06$ & Basic N1O2 & $\mathrm{C} 18 \mathrm{H} 21 \mathrm{~N} 1 \mathrm{O} 2$ & $1.25 \mathrm{E}-06$ \\
\hline Non-basic N1 & $\mathrm{C} 22 \mathrm{H} 17 \mathrm{~N} 1$ & 0.019099 & Non-basic N2 & $\mathrm{C} 28 \mathrm{H} 46 \mathrm{~N} 2$ & $1.05 \mathrm{E}-05$ & Basic N1O2 & $\mathrm{C} 19 \mathrm{H} 23 \mathrm{~N} 1 \mathrm{O} 2$ & $1.39 \mathrm{E}-06$ \\
\hline Non-basic N1 & $\mathrm{C} 23 \mathrm{H} 19 \mathrm{~N} 1$ & 0.014882 & Non-basic N2 & $\mathrm{C} 29 \mathrm{H} 48 \mathrm{~N} 2$ & $1.64 \mathrm{E}-05$ & Basic N1O2 & $\mathrm{C} 20 \mathrm{H} 25 \mathrm{~N} 1 \mathrm{O} 2$ & $1.55 \mathrm{E}-06$ \\
\hline Non-basic N1 & $\mathrm{C} 24 \mathrm{H} 21 \mathrm{~N} 1$ & 0.008208 & Non-basic N2 & $\mathrm{C} 30 \mathrm{H} 50 \mathrm{~N} 2$ & $1.77 \mathrm{E}-05$ & Basic N1O2 & $\mathrm{C} 21 \mathrm{H} 27 \mathrm{~N} 1 \mathrm{O} 2$ & 3.37E-06 \\
\hline Non-basic N1 & $\mathrm{C} 25 \mathrm{H} 23 \mathrm{~N} 1$ & 0.004006 & Non-basic N2 & $\mathrm{C} 31 \mathrm{H} 52 \mathrm{~N} 2$ & $2.12 \mathrm{E}-05$ & Basic N1O2 & $\mathrm{C} 22 \mathrm{H} 29 \mathrm{~N} 1 \mathrm{O} 2$ & $5.92 \mathrm{E}-06$ \\
\hline Non-basic N1 & $\mathrm{C} 26 \mathrm{H} 25 \mathrm{~N} 1$ & 0.002122 & Non-basic N2 & $\mathrm{C} 32 \mathrm{H} 54 \mathrm{~N} 2$ & $2.44 \mathrm{E}-05$ & Basic N1O2 & $\mathrm{C} 23 \mathrm{H} 31 \mathrm{~N} 1 \mathrm{O} 2$ & $5.08 \mathrm{E}-06$ \\
\hline Non-basic N1 & $\mathrm{C} 27 \mathrm{H} 27 \mathrm{~N} 1$ & 0.001127 & Non-basic N2 & $\mathrm{C} 33 \mathrm{H} 56 \mathrm{~N} 2$ & $3.34 \mathrm{E}-05$ & Basic N1O2 & $\mathrm{C} 24 \mathrm{H} 33 \mathrm{~N} 1 \mathrm{O} 2$ & $1.00 \mathrm{E}-05$ \\
\hline Non-basic N1 & $\mathrm{C} 28 \mathrm{H} 29 \mathrm{~N} 1$ & 0.000649 & Non-basic N2 & $\mathrm{C} 34 \mathrm{H} 58 \mathrm{~N} 2$ & $1.42 \mathrm{E}-05$ & Basic N1O2 & $\mathrm{C} 25 \mathrm{H} 35 \mathrm{~N} 1 \mathrm{O} 2$ & $1.01 \mathrm{E}-05$ \\
\hline Non-basic N1 & $\mathrm{C} 29 \mathrm{H} 31 \mathrm{~N} 1$ & 0.000408 & Non-basic N2 & $\mathrm{C} 35 \mathrm{H} 60 \mathrm{~N} 2$ & $2.25 \mathrm{E}-05$ & Basic N1O2 & $\mathrm{C} 26 \mathrm{H} 37 \mathrm{~N} 1 \mathrm{O} 2$ & $8.92 \mathrm{E}-06$ \\
\hline Non-basic N1 & $\mathrm{C} 30 \mathrm{H} 33 \mathrm{~N} 1$ & 0.000288 & Non-basic N2 & $\mathrm{C} 36 \mathrm{H} 62 \mathrm{~N} 2$ & $9.68 \mathrm{E}-06$ & Basic N1O2 & $\mathrm{C} 27 \mathrm{H} 39 \mathrm{~N} 1 \mathrm{O} 2$ & $1.40 \mathrm{E}-05$ \\
\hline Non-basic N1 & $\mathrm{C} 31 \mathrm{H} 35 \mathrm{~N} 1$ & 0.000213 & Non-basic N2 & C37H64N2 & $1.56 \mathrm{E}-05$ & Basic N1O2 & $\mathrm{C} 28 \mathrm{H} 41 \mathrm{~N} 1 \mathrm{O} 2$ & $2.10 \mathrm{E}-05$ \\
\hline Non-basic N1 & $\mathrm{C} 32 \mathrm{H} 37 \mathrm{~N} 1$ & 0.000153 & Non-basic N2 & $\mathrm{C} 38 \mathrm{H} 66 \mathrm{~N} 2$ & $1.39 \mathrm{E}-05$ & Basic N1O2 & $\mathrm{C} 29 \mathrm{H} 43 \mathrm{~N} 1 \mathrm{O} 2$ & $2.12 \mathrm{E}-05$ \\
\hline Non-basic N1 & $\mathrm{C} 33 \mathrm{H} 39 \mathrm{~N} 1$ & 0.000121 & Non-basic N2 & $\mathrm{C} 25 \mathrm{H} 38 \mathrm{~N} 2$ & $6.36 \mathrm{E}-06$ & Basic N1O2 & $\mathrm{C} 30 \mathrm{H} 45 \mathrm{~N} 1 \mathrm{O} 2$ & $2.60 \mathrm{E}-05$ \\
\hline Non-basic N1 & $\mathrm{C} 34 \mathrm{H} 41 \mathrm{~N} 1$ & $8.62 \mathrm{E}-05$ & Non-basic N2 & $\mathrm{C} 26 \mathrm{H} 40 \mathrm{~N} 2$ & $1.15 \mathrm{E}-05$ & Basic N1O2 & $\mathrm{C} 31 \mathrm{H} 47 \mathrm{~N} 1 \mathrm{O} 2$ & $2.48 \mathrm{E}-05$ \\
\hline Non-basic N1 & $\mathrm{C} 35 \mathrm{H} 43 \mathrm{~N} 1$ & $5.29 \mathrm{E}-05$ & Non-basic N2 & $\mathrm{C} 27 \mathrm{H} 42 \mathrm{~N} 2$ & $1.66 \mathrm{E}-05$ & Basic N1O2 & $\mathrm{C} 32 \mathrm{H} 49 \mathrm{~N} 1 \mathrm{O} 2$ & $2.41 \mathrm{E}-05$ \\
\hline Non-basic N1 & $\mathrm{C} 36 \mathrm{H} 45 \mathrm{~N} 1$ & $5.78 \mathrm{E}-05$ & Non-basic N2 & $\mathrm{C} 28 \mathrm{H} 44 \mathrm{~N} 2$ & $1.74 \mathrm{E}-05$ & Basic N1O2 & $\mathrm{C} 33 \mathrm{H} 51 \mathrm{~N} 1 \mathrm{O} 2$ & $3.21 \mathrm{E}-05$ \\
\hline Non-basic N1 & $\mathrm{C} 37 \mathrm{H} 47 \mathrm{~N} 1$ & $4.08 \mathrm{E}-05$ & Non-basic N2 & $\mathrm{C} 29 \mathrm{H} 46 \mathrm{~N} 2$ & $1.32 \mathrm{E}-05$ & Basic N1O2 & $\mathrm{C} 34 \mathrm{H} 53 \mathrm{~N} 1 \mathrm{O} 2$ & $2.65 \mathrm{E}-05$ \\
\hline Non-basic N1 & $\mathrm{C} 38 \mathrm{H} 49 \mathrm{~N} 1$ & $2.89 \mathrm{E}-05$ & Non-basic N2 & $\mathrm{C} 30 \mathrm{H} 48 \mathrm{~N} 2$ & $1.06 \mathrm{E}-05$ & Basic N1O2 & $\mathrm{C} 35 \mathrm{H} 55 \mathrm{~N} 1 \mathrm{O} 2$ & $2.85 \mathrm{E}-05$ \\
\hline Non-basic N1 & $\mathrm{C} 39 \mathrm{H} 51 \mathrm{~N} 1$ & $1.34 \mathrm{E}-05$ & Non-basic N2 & $\mathrm{C} 31 \mathrm{H} 50 \mathrm{~N} 2$ & $1.12 \mathrm{E}-05$ & Basic N1O2 & $\mathrm{C} 36 \mathrm{H} 57 \mathrm{~N} 1 \mathrm{O} 2$ & $2.50 \mathrm{E}-05$ \\
\hline Non-basic N1 & $\mathrm{C} 40 \mathrm{H} 53 \mathrm{~N} 1$ & $7.95 \mathrm{E}-06$ & Non-basic N2 & $\mathrm{C} 32 \mathrm{H} 50 \mathrm{~N} 2$ & $1.16 \mathrm{E}-05$ & Basic N1O2 & $\mathrm{C} 37 \mathrm{H} 59 \mathrm{~N} 1 \mathrm{O} 2$ & $1.97 \mathrm{E}-05$ \\
\hline Non-basic N1 & $\mathrm{C} 41 \mathrm{H} 55 \mathrm{~N} 1$ & $1.05 \mathrm{E}-05$ & Non-basic N2 & $\mathrm{C} 33 \mathrm{H} 54 \mathrm{~N} 2$ & $1.44 \mathrm{E}-05$ & Basic N1O2 & $\mathrm{C} 38 \mathrm{H} 61 \mathrm{~N} 1 \mathrm{O} 2$ & $1.58 \mathrm{E}-05$ \\
\hline Non-basic N1 & $\mathrm{C} 20 \mathrm{H} 11 \mathrm{~N} 1$ & $2.19 \mathrm{E}-06$ & Non-basic N2 & $\mathrm{C} 34 \mathrm{H} 56 \mathrm{~N} 2$ & $1.14 \mathrm{E}-05$ & Basic N1O2 & $\mathrm{C} 39 \mathrm{H} 63 \mathrm{~N} 1 \mathrm{O} 2$ & $1.23 \mathrm{E}-05$ \\
\hline Non-basic N1 & $\mathrm{C} 21 \mathrm{H} 13 \mathrm{~N} 1$ & $7.13 \mathrm{E}-05$ & Non-basic N2 & $\mathrm{C} 35 \mathrm{H} 58 \mathrm{~N} 2$ & $1.56 \mathrm{E}-05$ & Basic N1O2 & $\mathrm{C} 17 \mathrm{H} 17 \mathrm{~N} 1 \mathrm{O} 2$ & $2.53 \mathrm{E}-06$ \\
\hline
\end{tabular}




\begin{tabular}{|c|c|c|c|c|c|c|c|c|}
\hline Class & Formula & Mass & Class & Formula & Mass & Class & Formula & Mass \\
\hline Non-basic N1 & $\mathrm{C} 22 \mathrm{H} 15 \mathrm{~N} 1$ & 0.000889 & Non-basic N2 & $\mathrm{C} 23 \mathrm{H} 32 \mathrm{~N} 2$ & $8.36 \mathrm{E}-06$ & Basic N1O2 & $\mathrm{C} 18 \mathrm{H} 19 \mathrm{~N} 1 \mathrm{O} 2$ & $3.80 \mathrm{E}-06$ \\
\hline Non-basic N1 & $\mathrm{C} 23 \mathrm{H} 17 \mathrm{~N} 1$ & 0.002523 & Non-basic N2 & $\mathrm{C} 24 \mathrm{H} 34 \mathrm{~N} 2$ & $1.65 \mathrm{E}-05$ & Basic $\mathrm{N} 1 \mathrm{O} 2$ & $\mathrm{C} 19 \mathrm{H} 21 \mathrm{~N} 1 \mathrm{O} 2$ & $2.54 \mathrm{E}-06$ \\
\hline Non-basic N1 & C24H19N1 & 0.003443 & Non-basic N2 & $\mathrm{C} 25 \mathrm{H} 36 \mathrm{~N} 2$ & $2.15 \mathrm{E}-05$ & Basic N1O2 & $\mathrm{C} 20 \mathrm{H} 23 \mathrm{~N} 1 \mathrm{O} 2$ & $3.16 \mathrm{E}-06$ \\
\hline Non-basic N1 & $\mathrm{C} 25 \mathrm{H} 21 \mathrm{~N} 1$ & 0.002925 & Non-basic N2 & $\mathrm{C} 26 \mathrm{H} 38 \mathrm{~N} 2$ & $2.60 \mathrm{E}-05$ & Basic $\mathrm{N} 1 \mathrm{O} 2$ & $\mathrm{C} 21 \mathrm{H} 25 \mathrm{~N} 1 \mathrm{O} 2$ & $6.95 \mathrm{E}-06$ \\
\hline Non-basic N1 & $\mathrm{C} 26 \mathrm{H} 23 \mathrm{~N} 1$ & 0.001906 & Non-basic N2 & $\mathrm{C} 27 \mathrm{H} 40 \mathrm{~N} 2$ & $2.21 \mathrm{E}-05$ & Basic N1O2 & $\mathrm{C} 22 \mathrm{H} 27 \mathrm{~N} 1 \mathrm{O} 2$ & $1.09 \mathrm{E}-05$ \\
\hline Non-basic N1 & $\mathrm{C} 27 \mathrm{H} 25 \mathrm{~N} 1$ & 0.001007 & Non-basic N2 & $\mathrm{C} 28 \mathrm{H} 42 \mathrm{~N} 2$ & $3.29 \mathrm{E}-05$ & Basic N1O2 & $\mathrm{C} 23 \mathrm{H} 29 \mathrm{~N} 1 \mathrm{O} 2$ & $1.85 \mathrm{E}-05$ \\
\hline Non-basic N1 & $\mathrm{C} 28 \mathrm{H} 27 \mathrm{~N} 1$ & 0.000515 & Non-basic N2 & $\mathrm{C} 29 \mathrm{H} 44 \mathrm{~N} 2$ & $3.62 \mathrm{E}-05$ & Basic N1O2 & $\mathrm{C} 24 \mathrm{H} 31 \mathrm{~N} 1 \mathrm{O} 2$ & $1.74 \mathrm{E}-05$ \\
\hline Non-basic N1 & $\mathrm{C} 29 \mathrm{H} 29 \mathrm{~N} 1$ & 0.000263 & Non-basic N2 & $\mathrm{C} 30 \mathrm{H} 46 \mathrm{~N} 2$ & 4.07E-05 & Basic N1O2 & $\mathrm{C} 25 \mathrm{H} 33 \mathrm{~N} 1 \mathrm{O} 2$ & $2.30 \mathrm{E}-05$ \\
\hline Non-basic N1 & $\mathrm{C} 30 \mathrm{H} 31 \mathrm{~N} 1$ & 0.000153 & Non-basic N2 & $\mathrm{C} 31 \mathrm{H} 48 \mathrm{~N} 2$ & $5.44 \mathrm{E}-05$ & Basic N1O2 & $\mathrm{C} 26 \mathrm{H} 35 \mathrm{~N} 1 \mathrm{O} 2$ & $1.97 \mathrm{E}-05$ \\
\hline Non-basic N1 & $\mathrm{C} 31 \mathrm{H} 33 \mathrm{~N} 1$ & $9.37 \mathrm{E}-05$ & Non-basic N2 & $\mathrm{C} 32 \mathrm{H} 50 \mathrm{~N} 2$ & $2.91 \mathrm{E}-05$ & Basic N1O2 & $\mathrm{C} 27 \mathrm{H} 37 \mathrm{~N} 1 \mathrm{O} 2$ & $3.57 \mathrm{E}-05$ \\
\hline Non-basic N1 & $\mathrm{C} 32 \mathrm{H} 35 \mathrm{~N} 1$ & $6.54 \mathrm{E}-05$ & Non-basic N2 & $\mathrm{C} 33 \mathrm{H} 52 \mathrm{~N} 2$ & $4.66 \mathrm{E}-05$ & Basic N1O2 & $\mathrm{C} 28 \mathrm{H} 39 \mathrm{~N} 1 \mathrm{O} 2$ & $2.15 \mathrm{E}-05$ \\
\hline Non-basic N1 & $\mathrm{C} 33 \mathrm{H} 37 \mathrm{~N} 1$ & $4.74 \mathrm{E}-05$ & Non-basic N2 & C34H54N2 & $1.31 \mathrm{E}-05$ & Basic N1O2 & $\mathrm{C} 29 \mathrm{H} 41 \mathrm{~N} 1 \mathrm{O} 2$ & 4.93E-05 \\
\hline Non-basic N1 & $\mathrm{C} 34 \mathrm{H} 39 \mathrm{~N} 1$ & $4.21 \mathrm{E}-05$ & Non-basic N2 & $\mathrm{C} 35 \mathrm{H} 56 \mathrm{~N} 2$ & $2.72 \mathrm{E}-05$ & Basic N1O2 & $\mathrm{C} 30 \mathrm{H} 43 \mathrm{~N} 1 \mathrm{O} 2$ & $2.37 \mathrm{E}-05$ \\
\hline Non-basic N1 & $\mathrm{C} 35 \mathrm{H} 41 \mathrm{~N} 1$ & $2.82 \mathrm{E}-05$ & Non-basic N2 & $\mathrm{C} 36 \mathrm{H} 58 \mathrm{~N} 2$ & $3.06 \mathrm{E}-05$ & Basic $\mathrm{N} 1 \mathrm{O} 2$ & $\mathrm{C} 31 \mathrm{H} 45 \mathrm{~N} 1 \mathrm{O} 2$ & $3.23 \mathrm{E}-05$ \\
\hline Non-basic N1 & $\mathrm{C} 36 \mathrm{H} 43 \mathrm{~N} 1$ & $2.31 \mathrm{E}-05$ & Non-basic N2 & $\mathrm{C} 20 \mathrm{H} 24 \mathrm{~N} 2$ & 8.91E-06 & Basic N1O2 & $\mathrm{C} 32 \mathrm{H} 47 \mathrm{~N} 1 \mathrm{O} 2$ & $3.52 \mathrm{E}-05$ \\
\hline Non-basic N1 & $\mathrm{C} 37 \mathrm{H} 45 \mathrm{~N} 1$ & $2.30 \mathrm{E}-05$ & Non-basic N2 & $\mathrm{C} 21 \mathrm{H} 26 \mathrm{~N} 2$ & $6.93 \mathrm{E}-06$ & Basic N1O2 & $\mathrm{C} 33 \mathrm{H} 49 \mathrm{~N} 1 \mathrm{O} 2$ & $3.27 \mathrm{E}-05$ \\
\hline Non-basic N1 & $\mathrm{C} 38 \mathrm{H} 47 \mathrm{~N} 1$ & $1.04 \mathrm{E}-05$ & Non-basic N2 & $\mathrm{C} 22 \mathrm{H} 28 \mathrm{~N} 2$ & $1.38 \mathrm{E}-05$ & Basic N1O2 & $\mathrm{C} 34 \mathrm{H} 51 \mathrm{~N} 1 \mathrm{O} 2$ & $5.25 \mathrm{E}-05$ \\
\hline Non-basic N1 & $\mathrm{C} 39 \mathrm{H} 49 \mathrm{~N} 1$ & $5.78 \mathrm{E}-06$ & Non-basic N2 & $\mathrm{C} 23 \mathrm{H} 30 \mathrm{~N} 2$ & $1.38 \mathrm{E}-05$ & Basic N1O2 & $\mathrm{C} 35 \mathrm{H} 53 \mathrm{~N} 1 \mathrm{O} 2$ & $3.57 \mathrm{E}-05$ \\
\hline Non-basic N1 & $\mathrm{C} 40 \mathrm{H} 51 \mathrm{~N} 1$ & $4.82 \mathrm{E}-06$ & Non-basic N2 & $\mathrm{C} 24 \mathrm{H} 32 \mathrm{~N} 2$ & $1.22 \mathrm{E}-05$ & Basic N1O2 & $\mathrm{C} 36 \mathrm{H} 55 \mathrm{~N} 1 \mathrm{O} 2$ & $5.64 \mathrm{E}-05$ \\
\hline Non-basic N1 & $\mathrm{C} 22 \mathrm{H} 13 \mathrm{~N} 1$ & 0.000827 & Non-basic N2 & $\mathrm{C} 25 \mathrm{H} 34 \mathrm{~N} 2$ & $1.39 \mathrm{E}-05$ & Basic N1O2 & $\mathrm{C} 37 \mathrm{H} 57 \mathrm{~N} 1 \mathrm{O} 2$ & $3.08 \mathrm{E}-05$ \\
\hline Non-basic N1 & $\mathrm{C} 23 \mathrm{H} 15 \mathrm{~N} 1$ & 0.002824 & Non-basic N2 & $\mathrm{C} 26 \mathrm{H} 36 \mathrm{~N} 2$ & $3.07 \mathrm{E}-05$ & Basic N1O2 & $\mathrm{C} 38 \mathrm{H} 59 \mathrm{~N} 1 \mathrm{O} 2$ & $1.77 \mathrm{E}-05$ \\
\hline Non-basic N1 & $\mathrm{C} 24 \mathrm{H} 17 \mathrm{~N} 1$ & 0.00426 & Non-basic N2 & $\mathrm{C} 27 \mathrm{H} 38 \mathrm{~N} 2$ & $2.46 \mathrm{E}-05$ & Basic N1O2 & $\mathrm{C} 39 \mathrm{H} 61 \mathrm{~N} 1 \mathrm{O} 2$ & $3.12 \mathrm{E}-05$ \\
\hline Non-basic N1 & $\mathrm{C} 25 \mathrm{H} 19 \mathrm{~N} 1$ & 0.004137 & Non-basic N2 & $\mathrm{C} 28 \mathrm{H} 40 \mathrm{~N} 2$ & $2.18 \mathrm{E}-05$ & Basic N1O2 & $\mathrm{C} 40 \mathrm{H} 63 \mathrm{~N} 1 \mathrm{O} 2$ & $1.10 \mathrm{E}-05$ \\
\hline Non-basic N1 & $\mathrm{C} 26 \mathrm{H} 21 \mathrm{~N} 1$ & 0.003075 & Non-basic N2 & $\mathrm{C} 29 \mathrm{H} 42 \mathrm{~N} 2$ & $2.39 \mathrm{E}-05$ & Basic $\mathrm{N} 1 \mathrm{O} 2$ & $\mathrm{C} 41 \mathrm{H} 65 \mathrm{~N} 1 \mathrm{O} 2$ & 7.77E-06 \\
\hline Non-basic N1 & $\mathrm{C} 27 \mathrm{H} 23 \mathrm{~N} 1$ & 0.001817 & Non-basic N2 & $\mathrm{C} 30 \mathrm{H} 44 \mathrm{~N} 2$ & $3.35 \mathrm{E}-05$ & Basic N1O2 & $\mathrm{C} 42 \mathrm{H} 67 \mathrm{~N} 1 \mathrm{O} 2$ & $1.29 \mathrm{E}-05$ \\
\hline Non-basic N1 & $\mathrm{C} 28 \mathrm{H} 25 \mathrm{~N} 1$ & 0.000982 & Non-basic N2 & $\mathrm{C} 31 \mathrm{H} 46 \mathrm{~N} 2$ & $2.43 \mathrm{E}-05$ & Basic N1O2 & $\mathrm{C} 17 \mathrm{H} 15 \mathrm{~N} 1 \mathrm{O} 2$ & $1.45 \mathrm{E}-07$ \\
\hline Non-basic N1 & $\mathrm{C} 29 \mathrm{H} 27 \mathrm{~N} 1$ & 0.000505 & Non-basic N2 & $\mathrm{C} 32 \mathrm{H} 48 \mathrm{~N} 2$ & $4.14 \mathrm{E}-05$ & Basic N1O2 & $\mathrm{C} 18 \mathrm{H} 17 \mathrm{~N} 1 \mathrm{O} 2$ & $2.65 \mathrm{E}-07$ \\
\hline Non-basic N1 & $\mathrm{C} 30 \mathrm{H} 29 \mathrm{~N} 1$ & 0.000284 & Non-basic N2 & $\mathrm{C} 33 \mathrm{H} 50 \mathrm{~N} 2$ & $3.82 \mathrm{E}-05$ & Basic N1O2 & C19H19N1O2 & $3.35 \mathrm{E}-06$ \\
\hline Non-basic N1 & $\mathrm{C} 31 \mathrm{H} 31 \mathrm{~N} 1$ & 0.000144 & Non-basic N2 & $\mathrm{C} 34 \mathrm{H} 52 \mathrm{~N} 2$ & $3.20 \mathrm{E}-05$ & Basic N1O2 & $\mathrm{C} 20 \mathrm{H} 21 \mathrm{~N} 1 \mathrm{O} 2$ & $6.25 \mathrm{E}-06$ \\
\hline Non-basic N1 & $\mathrm{C} 32 \mathrm{H} 33 \mathrm{~N} 1$ & $9.75 \mathrm{E}-05$ & Non-basic N2 & $\mathrm{C} 35 \mathrm{H} 54 \mathrm{~N} 2$ & $2.43 \mathrm{E}-05$ & Basic N1O2 & $\mathrm{C} 21 \mathrm{H} 23 \mathrm{~N} 1 \mathrm{O} 2$ & $1.32 \mathrm{E}-05$ \\
\hline Non-basic N1 & $\mathrm{C} 33 \mathrm{H} 35 \mathrm{~N} 1$ & $6.81 \mathrm{E}-05$ & Non-basic N2 & $\mathrm{C} 36 \mathrm{H} 56 \mathrm{~N} 2$ & $1.26 \mathrm{E}-05$ & Basic N1O2 & $\mathrm{C} 22 \mathrm{H} 25 \mathrm{~N} 1 \mathrm{O} 2$ & $2.76 \mathrm{E}-05$ \\
\hline Non-basic N1 & $\mathrm{C} 34 \mathrm{H} 37 \mathrm{~N} 1$ & $4.20 \mathrm{E}-05$ & Non-basic N2 & $\mathrm{C} 37 \mathrm{H} 58 \mathrm{~N} 2$ & $1.39 \mathrm{E}-05$ & Basic N1O2 & $\mathrm{C} 23 \mathrm{H} 27 \mathrm{~N} 1 \mathrm{O} 2$ & $3.46 \mathrm{E}-05$ \\
\hline Non-basic N1 & $\mathrm{C} 35 \mathrm{H} 39 \mathrm{~N} 1$ & $3.31 \mathrm{E}-05$ & Non-basic N2 & $\mathrm{C} 38 \mathrm{H} 60 \mathrm{~N} 2$ & $1.29 \mathrm{E}-05$ & Basic N1O2 & $\mathrm{C} 24 \mathrm{H} 29 \mathrm{~N} 1 \mathrm{O} 2$ & $2.20 \mathrm{E}-05$ \\
\hline Non-basic N1 & $\mathrm{C} 36 \mathrm{H} 41 \mathrm{~N} 1$ & 3.67E-05 & Non-basic N2 & $\mathrm{C} 22 \mathrm{H} 26 \mathrm{~N} 2$ & $6.21 \mathrm{E}-06$ & Basic N1O2 & $\mathrm{C} 25 \mathrm{H} 31 \mathrm{~N} 1 \mathrm{O} 2$ & $3.09 \mathrm{E}-05$ \\
\hline Non-basic N1 & $\mathrm{C} 37 \mathrm{H} 43 \mathrm{~N} 1$ & $1.76 \mathrm{E}-05$ & Non-basic N2 & $\mathrm{C} 23 \mathrm{H} 28 \mathrm{~N} 2$ & $1.61 \mathrm{E}-05$ & Basic N1O2 & $\mathrm{C} 26 \mathrm{H} 33 \mathrm{~N} 1 \mathrm{O} 2$ & $3.08 \mathrm{E}-05$ \\
\hline Non-basic N1 & $\mathrm{C} 38 \mathrm{H} 45 \mathrm{~N} 1$ & 7.99E-06 & Non-basic N2 & $\mathrm{C} 24 \mathrm{H} 30 \mathrm{~N} 2$ & $1.79 \mathrm{E}-05$ & Basic N1O2 & $\mathrm{C} 27 \mathrm{H} 35 \mathrm{~N} 1 \mathrm{O} 2$ & $3.37 \mathrm{E}-05$ \\
\hline Non-basic N1 & $\mathrm{C} 39 \mathrm{H} 47 \mathrm{~N} 1$ & $9.99 \mathrm{E}-06$ & Non-basic N2 & $\mathrm{C} 25 \mathrm{H} 32 \mathrm{~N} 2$ & $8.08 \mathrm{E}-06$ & Basic N1O2 & $\mathrm{C} 28 \mathrm{H} 37 \mathrm{~N} 1 \mathrm{O} 2$ & $2.99 \mathrm{E}-05$ \\
\hline Non-basic N1 & $\mathrm{C} 40 \mathrm{H} 49 \mathrm{~N} 1$ & $3.35 \mathrm{E}-06$ & Non-basic N2 & $\mathrm{C} 26 \mathrm{H} 34 \mathrm{~N} 2$ & $1.51 \mathrm{E}-05$ & Basic N1O2 & $\mathrm{C} 29 \mathrm{H} 39 \mathrm{~N} 1 \mathrm{O} 2$ & 4.44E-05 \\
\hline
\end{tabular}




\begin{tabular}{|c|c|c|c|c|c|c|c|c|}
\hline Class & Formula & Mass & Class & Formula & Mass & Class & Formula & Mass \\
\hline Non-basic N1 & C41H51N1 & $4.20 \mathrm{E}-06$ & Non-basic N2 & $\mathrm{C} 27 \mathrm{H} 36 \mathrm{~N} 2$ & $2.10 \mathrm{E}-05$ & Basic N1O2 & $\mathrm{C} 30 \mathrm{H} 41 \mathrm{~N} 1 \mathrm{O} 2$ & $3.23 \mathrm{E}-05$ \\
\hline Non-basic N1 & $\mathrm{C} 42 \mathrm{H} 53 \mathrm{~N} 1$ & $6.11 \mathrm{E}-06$ & Non-basic N2 & $\mathrm{C} 28 \mathrm{H} 38 \mathrm{~N} 2$ & $2.82 \mathrm{E}-05$ & Basic N1O2 & $\mathrm{C} 31 \mathrm{H} 43 \mathrm{~N} 1 \mathrm{O} 2$ & $5.37 \mathrm{E}-05$ \\
\hline Non-basic N1 & $\mathrm{C} 23 \mathrm{H} 13 \mathrm{~N} 1$ & $5.17 \mathrm{E}-06$ & Non-basic N2 & $\mathrm{C} 29 \mathrm{H} 40 \mathrm{~N} 2$ & $2.04 \mathrm{E}-05$ & Basic N1O2 & $\mathrm{C} 32 \mathrm{H} 45 \mathrm{~N} 1 \mathrm{O} 2$ & $3.79 \mathrm{E}-05$ \\
\hline Non-basic N1 & $\mathrm{C} 24 \mathrm{H} 15 \mathrm{~N} 1$ & 0.001223 & Non-basic N2 & $\mathrm{C} 30 \mathrm{H} 42 \mathrm{~N} 2$ & $1.68 \mathrm{E}-05$ & Basic N1O2 & $\mathrm{C} 34 \mathrm{H} 49 \mathrm{~N} 1 \mathrm{O} 2$ & $3.32 \mathrm{E}-05$ \\
\hline Non-basic N1 & $\mathrm{C} 25 \mathrm{H} 17 \mathrm{~N} 1$ & 0.003152 & Non-basic N2 & $\mathrm{C} 31 \mathrm{H} 44 \mathrm{~N} 2$ & $2.30 \mathrm{E}-05$ & Basic N1O2 & $\mathrm{C} 35 \mathrm{H} 51 \mathrm{~N} 1 \mathrm{O} 2$ & $7.88 \mathrm{E}-05$ \\
\hline Non-basic N1 & $\mathrm{C} 26 \mathrm{H} 19 \mathrm{~N} 1$ & 0.004094 & Non-basic N2 & $\mathrm{C} 32 \mathrm{H} 46 \mathrm{~N} 2$ & $2.87 \mathrm{E}-05$ & Basic N1O2 & $\mathrm{C} 36 \mathrm{H} 53 \mathrm{~N} 1 \mathrm{O} 2$ & $2.53 \mathrm{E}-05$ \\
\hline Non-basic N1 & $\mathrm{C} 27 \mathrm{H} 21 \mathrm{~N} 1$ & 0.003304 & Non-basic N2 & $\mathrm{C} 33 \mathrm{H} 48 \mathrm{~N} 2$ & $1.94 \mathrm{E}-05$ & Basic N1O2 & $\mathrm{C} 37 \mathrm{H} 55 \mathrm{~N} 1 \mathrm{O} 2$ & $2.94 \mathrm{E}-05$ \\
\hline Non-basic N1 & $\mathrm{C} 28 \mathrm{H} 23 \mathrm{~N} 1$ & 0.001998 & Non-basic N2 & $\mathrm{C} 34 \mathrm{H} 50 \mathrm{~N} 2$ & $1.07 \mathrm{E}-05$ & Basic N1O2 & $\mathrm{C} 38 \mathrm{H} 57 \mathrm{~N} 1 \mathrm{O} 2$ & $2.26 \mathrm{E}-05$ \\
\hline Non-basic N1 & $\mathrm{C} 29 \mathrm{H} 25 \mathrm{~N} 1$ & 0.001015 & Non-basic N2 & $\mathrm{C} 35 \mathrm{H} 52 \mathrm{~N} 2$ & $2.03 \mathrm{E}-05$ & Basic N1O2 & C39H59N1O2 & $2.46 \mathrm{E}-05$ \\
\hline Non-basic N1 & $\mathrm{C} 30 \mathrm{H} 27 \mathrm{~N} 1$ & 0.000524 & Non-basic N2 & $\mathrm{C} 16 \mathrm{H} 12 \mathrm{~N} 2$ & $5.53 \mathrm{E}-06$ & Basic N1O2 & $\mathrm{C} 40 \mathrm{H} 61 \mathrm{~N} 1 \mathrm{O} 2$ & $1.88 \mathrm{E}-05$ \\
\hline Non-basic N1 & $\mathrm{C} 31 \mathrm{H} 29 \mathrm{~N} 1$ & 0.000266 & Non-basic N2 & $\mathrm{C} 17 \mathrm{H} 14 \mathrm{~N} 2$ & $1.29 \mathrm{E}-05$ & Basic N1O2 & $\mathrm{C} 17 \mathrm{H} 13 \mathrm{~N} 1 \mathrm{O} 2$ & $3.05 \mathrm{E}-07$ \\
\hline Non-basic N1 & $\mathrm{C} 32 \mathrm{H} 31 \mathrm{~N} 1$ & 0.000166 & Non-basic N2 & $\mathrm{C} 18 \mathrm{H} 16 \mathrm{~N} 2$ & $1.53 \mathrm{E}-05$ & Basic N1O2 & $\mathrm{C} 18 \mathrm{H} 15 \mathrm{~N} 1 \mathrm{O} 2$ & $6.09 \mathrm{E}-06$ \\
\hline Non-basic N1 & $\mathrm{C} 33 \mathrm{H} 33 \mathrm{~N} 1$ & $8.81 \mathrm{E}-05$ & Non-basic N2 & C19H18N2 & $1.48 \mathrm{E}-05$ & Basic N1O2 & C19H17N1O2 & $2.12 \mathrm{E}-05$ \\
\hline Non-basic N1 & $\mathrm{C} 34 \mathrm{H} 35 \mathrm{~N} 1$ & $6.43 \mathrm{E}-05$ & Non-basic N2 & $\mathrm{C} 20 \mathrm{H} 20 \mathrm{~N} 2$ & $1.90 \mathrm{E}-05$ & Basic N1O2 & $\mathrm{C} 20 \mathrm{H} 19 \mathrm{~N} 1 \mathrm{O} 2$ & $4.78 \mathrm{E}-05$ \\
\hline Non-basic N1 & $\mathrm{C} 35 \mathrm{H} 37 \mathrm{~N} 1$ & $3.37 \mathrm{E}-05$ & Non-basic N2 & $\mathrm{C} 21 \mathrm{H} 22 \mathrm{~N} 2$ & $1.67 \mathrm{E}-05$ & Basic N1O2 & $\mathrm{C} 21 \mathrm{H} 21 \mathrm{~N} 1 \mathrm{O} 2$ & $2.68 \mathrm{E}-05$ \\
\hline Non-basic N1 & $\mathrm{C} 36 \mathrm{H} 39 \mathrm{~N} 1$ & $3.67 \mathrm{E}-05$ & Non-basic N2 & $\mathrm{C} 22 \mathrm{H} 24 \mathrm{~N} 2$ & $2.96 \mathrm{E}-05$ & Basic N1O2 & $\mathrm{C} 22 \mathrm{H} 23 \mathrm{~N} 1 \mathrm{O} 2$ & $1.76 \mathrm{E}-05$ \\
\hline Non-basic N1 & $\mathrm{C} 37 \mathrm{H} 41 \mathrm{~N} 1$ & $2.86 \mathrm{E}-05$ & Non-basic N2 & $\mathrm{C} 23 \mathrm{H} 26 \mathrm{~N} 2$ & $2.40 \mathrm{E}-05$ & Basic N1O2 & $\mathrm{C} 23 \mathrm{H} 25 \mathrm{~N} 1 \mathrm{O} 2$ & $2.25 \mathrm{E}-05$ \\
\hline Non-basic N1 & $\mathrm{C} 38 \mathrm{H} 43 \mathrm{~N} 1$ & $2.07 \mathrm{E}-05$ & Non-basic N2 & $\mathrm{C} 24 \mathrm{H} 28 \mathrm{~N} 2$ & $3.46 \mathrm{E}-05$ & Basic N1O2 & $\mathrm{C} 24 \mathrm{H} 27 \mathrm{~N} 1 \mathrm{O} 2$ & $1.82 \mathrm{E}-05$ \\
\hline Non-basic N1 & $\mathrm{C} 39 \mathrm{H} 45 \mathrm{~N} 1$ & 4.61E-06 & Non-basic N2 & $\mathrm{C} 25 \mathrm{H} 30 \mathrm{~N} 2$ & $4.24 \mathrm{E}-05$ & Basic N1O2 & $\mathrm{C} 25 \mathrm{H} 29 \mathrm{~N} 1 \mathrm{O} 2$ & $3.98 \mathrm{E}-05$ \\
\hline Non-basic N1 & $\mathrm{C} 40 \mathrm{H} 47 \mathrm{~N} 1$ & $4.73 \mathrm{E}-06$ & Non-basic N2 & $\mathrm{C} 26 \mathrm{H} 32 \mathrm{~N} 2$ & $4.18 \mathrm{E}-05$ & Basic N1O2 & $\mathrm{C} 26 \mathrm{H} 31 \mathrm{~N} 1 \mathrm{O} 2$ & $3.75 \mathrm{E}-05$ \\
\hline Non-basic N1 & $\mathrm{C} 41 \mathrm{H} 49 \mathrm{~N} 1$ & $9.27 \mathrm{E}-06$ & Non-basic N2 & $\mathrm{C} 27 \mathrm{H} 34 \mathrm{~N} 2$ & $3.43 \mathrm{E}-05$ & Basic N1O2 & $\mathrm{C} 27 \mathrm{H} 33 \mathrm{~N} 1 \mathrm{O} 2$ & $4.86 \mathrm{E}-05$ \\
\hline Non-basic N1 & $\mathrm{C} 42 \mathrm{H} 51 \mathrm{~N} 1$ & $8.88 \mathrm{E}-06$ & Non-basic N2 & $\mathrm{C} 28 \mathrm{H} 36 \mathrm{~N} 2$ & $2.08 \mathrm{E}-05$ & Basic N1O2 & $\mathrm{C} 28 \mathrm{H} 35 \mathrm{~N} 1 \mathrm{O} 2$ & $5.69 \mathrm{E}-05$ \\
\hline Non-basic N1 & $\mathrm{C} 43 \mathrm{H} 53 \mathrm{~N} 1$ & $1.66 \mathrm{E}-06$ & Non-basic N2 & $\mathrm{C} 29 \mathrm{H} 38 \mathrm{~N} 2$ & $3.79 \mathrm{E}-05$ & Basic N1O2 & $\mathrm{C} 29 \mathrm{H} 37 \mathrm{~N} 1 \mathrm{O} 2$ & $4.38 \mathrm{E}-05$ \\
\hline Non-basic N1 & $\mathrm{C} 24 \mathrm{H} 13 \mathrm{~N} 1$ & $1.29 \mathrm{E}-05$ & Non-basic N2 & $\mathrm{C} 30 \mathrm{H} 40 \mathrm{~N} 2$ & $4.82 \mathrm{E}-05$ & Basic N1O2 & $\mathrm{C} 30 \mathrm{H} 39 \mathrm{~N} 1 \mathrm{O} 2$ & $4.55 \mathrm{E}-05$ \\
\hline Non-basic N1 & $\mathrm{C} 25 \mathrm{H} 15 \mathrm{~N} 1$ & 0.000113 & Non-basic N2 & $\mathrm{C} 31 \mathrm{H} 42 \mathrm{~N} 2$ & $2.89 \mathrm{E}-05$ & Basic N1O2 & $\mathrm{C} 31 \mathrm{H} 41 \mathrm{~N} 1 \mathrm{O} 2$ & $3.95 \mathrm{E}-05$ \\
\hline Non-basic N1 & $\mathrm{C} 26 \mathrm{H} 17 \mathrm{~N} 1$ & 0.000422 & Non-basic N2 & $\mathrm{C} 32 \mathrm{H} 44 \mathrm{~N} 2$ & $3.11 \mathrm{E}-05$ & Basic N1O2 & $\mathrm{C} 32 \mathrm{H} 43 \mathrm{~N} 1 \mathrm{O} 2$ & $6.98 \mathrm{E}-05$ \\
\hline Non-basic N1 & $\mathrm{C} 27 \mathrm{H} 19 \mathrm{~N} 1$ & 0.000819 & Non-basic N2 & $\mathrm{C} 33 \mathrm{H} 46 \mathrm{~N} 2$ & $2.63 \mathrm{E}-05$ & Basic N1O2 & $\mathrm{C} 33 \mathrm{H} 45 \mathrm{~N} 1 \mathrm{O} 2$ & 4.32E-05 \\
\hline Non-basic N1 & $\mathrm{C} 28 \mathrm{H} 21 \mathrm{~N} 1$ & 0.000983 & Non-basic N2 & $\mathrm{C} 34 \mathrm{H} 48 \mathrm{~N} 2$ & $2.28 \mathrm{E}-05$ & Basic N1O2 & $\mathrm{C} 34 \mathrm{H} 47 \mathrm{~N} 1 \mathrm{O} 2$ & $3.82 \mathrm{E}-05$ \\
\hline Non-basic N1 & $\mathrm{C} 29 \mathrm{H} 23 \mathrm{~N} 1$ & 0.000805 & Non-basic N2 & $\mathrm{C} 35 \mathrm{H} 50 \mathrm{~N} 2$ & $1.93 \mathrm{E}-05$ & Basic N1O2 & $\mathrm{C} 35 \mathrm{H} 49 \mathrm{~N} 1 \mathrm{O} 2$ & $3.76 \mathrm{E}-05$ \\
\hline Non-basic N1 & $\mathrm{C} 30 \mathrm{H} 25 \mathrm{~N} 1$ & 0.000539 & Non-basic N2 & $\mathrm{C} 36 \mathrm{H} 52 \mathrm{~N} 2$ & $1.42 \mathrm{E}-05$ & Basic N1O2 & $\mathrm{C} 36 \mathrm{H} 51 \mathrm{~N} 1 \mathrm{O} 2$ & $2.52 \mathrm{E}-05$ \\
\hline Non-basic N1 & $\mathrm{C} 31 \mathrm{H} 27 \mathrm{~N} 1$ & 0.000308 & Non-basic N2 & $\mathrm{C} 20 \mathrm{H} 18 \mathrm{~N} 2$ & $5.45 \mathrm{E}-06$ & Basic N1O2 & $\mathrm{C} 37 \mathrm{H} 53 \mathrm{~N} 1 \mathrm{O} 2$ & $2.82 \mathrm{E}-05$ \\
\hline Non-basic N1 & $\mathrm{C} 32 \mathrm{H} 29 \mathrm{~N} 1$ & 0.000174 & Non-basic N2 & $\mathrm{C} 21 \mathrm{H} 20 \mathrm{~N} 2$ & $1.31 \mathrm{E}-05$ & Basic N1O2 & $\mathrm{C} 38 \mathrm{H} 55 \mathrm{~N} 1 \mathrm{O} 2$ & $2.54 \mathrm{E}-05$ \\
\hline Non-basic N1 & $\mathrm{C} 33 \mathrm{H} 31 \mathrm{~N} 1$ & $9.43 \mathrm{E}-05$ & Non-basic N2 & $\mathrm{C} 22 \mathrm{H} 22 \mathrm{~N} 2$ & $2.26 \mathrm{E}-05$ & Basic N1O2 & $\mathrm{C} 39 \mathrm{H} 57 \mathrm{~N} 1 \mathrm{O} 2$ & $2.33 \mathrm{E}-05$ \\
\hline Non-basic N1 & $\mathrm{C} 34 \mathrm{H} 33 \mathrm{~N} 1$ & 4.84E-05 & Non-basic N2 & $\mathrm{C} 23 \mathrm{H} 24 \mathrm{~N} 2$ & $2.63 \mathrm{E}-05$ & Basic N1O2 & $\mathrm{C} 40 \mathrm{H} 59 \mathrm{~N} 1 \mathrm{O} 2$ & $1.37 \mathrm{E}-05$ \\
\hline Non-basic N1 & $\mathrm{C} 35 \mathrm{H} 35 \mathrm{~N} 1$ & $3.79 \mathrm{E}-05$ & Non-basic N2 & $\mathrm{C} 24 \mathrm{H} 26 \mathrm{~N} 2$ & 4.03E-05 & Basic N1O2 & $\mathrm{C} 41 \mathrm{H} 61 \mathrm{~N} 1 \mathrm{O} 2$ & $1.47 \mathrm{E}-05$ \\
\hline Non-basic N1 & $\mathrm{C} 36 \mathrm{H} 37 \mathrm{~N} 1$ & $2.49 \mathrm{E}-05$ & Non-basic N2 & $\mathrm{C} 25 \mathrm{H} 28 \mathrm{~N} 2$ & $4.83 \mathrm{E}-05$ & Basic N1O2 & $\mathrm{C} 42 \mathrm{H} 63 \mathrm{~N} 1 \mathrm{O} 2$ & $8.33 \mathrm{E}-06$ \\
\hline Non-basic N1 & $\mathrm{C} 37 \mathrm{H} 39 \mathrm{~N} 1$ & $8.79 \mathrm{E}-06$ & Non-basic N2 & $\mathrm{C} 26 \mathrm{H} 30 \mathrm{~N} 2$ & $4.28 \mathrm{E}-05$ & Basic N1O2 & $\mathrm{C} 43 \mathrm{H} 65 \mathrm{~N} 1 \mathrm{O} 2$ & $4.06 \mathrm{E}-07$ \\
\hline Non-basic N1 & $\mathrm{C} 38 \mathrm{H} 41 \mathrm{~N} 1$ & $5.35 \mathrm{E}-06$ & Non-basic N2 & $\mathrm{C} 27 \mathrm{H} 32 \mathrm{~N} 2$ & $4.41 \mathrm{E}-05$ & Basic N1O2 & $\mathrm{C} 44 \mathrm{H} 67 \mathrm{~N} 1 \mathrm{O} 2$ & $2.34 \mathrm{E}-06$ \\
\hline
\end{tabular}




\begin{tabular}{|c|c|c|c|c|c|c|c|c|}
\hline Class & Formula & Mass & Class & Formula & Mass & Class & Formula & Mass \\
\hline Non-basic N1 & $\mathrm{C} 39 \mathrm{H} 43 \mathrm{~N} 1$ & $6.84 \mathrm{E}-06$ & Non-basic N2 & $\mathrm{C} 28 \mathrm{H} 34 \mathrm{~N} 2$ & 4.47E-05 & Basic N1O2 & C45H69N1O2 & $5.75 \mathrm{E}-06$ \\
\hline Non-basic N1 & $\mathrm{C} 40 \mathrm{H} 45 \mathrm{~N} 1$ & $5.95 \mathrm{E}-06$ & Non-basic N2 & $\mathrm{C} 29 \mathrm{H} 36 \mathrm{~N} 2$ & $3.52 \mathrm{E}-05$ & Basic N1O2 & $\mathrm{C} 17 \mathrm{H} 11 \mathrm{~N} 1 \mathrm{O} 2$ & $4.42 \mathrm{E}-06$ \\
\hline Non-basic N1 & $\mathrm{C} 41 \mathrm{H} 47 \mathrm{~N} 1$ & $6.11 \mathrm{E}-06$ & Non-basic N2 & $\mathrm{C} 30 \mathrm{H} 38 \mathrm{~N} 2$ & 4.25E-05 & Basic N1O2 & $\mathrm{C} 18 \mathrm{H} 13 \mathrm{~N} 1 \mathrm{O} 2$ & $9.62 \mathrm{E}-06$ \\
\hline Non-basic N1 & $\mathrm{C} 42 \mathrm{H} 49 \mathrm{~N} 1$ & $2.74 \mathrm{E}-06$ & Non-basic N2 & $\mathrm{C} 31 \mathrm{H} 40 \mathrm{~N} 2$ & $3.69 \mathrm{E}-05$ & Basic N1O2 & C19H15N1O2 & $1.92 \mathrm{E}-05$ \\
\hline Non-basic N1 & $\mathrm{C} 43 \mathrm{H} 51 \mathrm{~N} 1$ & $2.65 \mathrm{E}-06$ & Non-basic N2 & $\mathrm{C} 32 \mathrm{H} 42 \mathrm{~N} 2$ & $1.38 \mathrm{E}-05$ & Basic N1O2 & $\mathrm{C} 20 \mathrm{H} 17 \mathrm{~N} 1 \mathrm{O} 2$ & $2.20 \mathrm{E}-05$ \\
\hline Non-basic N1 & $\mathrm{C} 26 \mathrm{H} 15 \mathrm{~N} 1$ & 0.000272 & Non-basic N2 & $\mathrm{C} 33 \mathrm{H} 44 \mathrm{~N} 2$ & $1.81 \mathrm{E}-05$ & Basic N1O2 & $\mathrm{C} 21 \mathrm{H} 19 \mathrm{~N} 1 \mathrm{O} 2$ & $2.81 \mathrm{E}-05$ \\
\hline Non-basic N1 & $\mathrm{C} 27 \mathrm{H} 17 \mathrm{~N} 1$ & 0.000849 & Non-basic N2 & $\mathrm{C} 19 \mathrm{H} 14 \mathrm{~N} 2$ & $6.95 \mathrm{E}-06$ & Basic N1O2 & $\mathrm{C} 22 \mathrm{H} 21 \mathrm{~N} 1 \mathrm{O} 2$ & $2.72 \mathrm{E}-05$ \\
\hline Non-basic N1 & $\mathrm{C} 28 \mathrm{H} 19 \mathrm{~N} 1$ & 0.001338 & Non-basic N2 & $\mathrm{C} 20 \mathrm{H} 16 \mathrm{~N} 2$ & $1.59 \mathrm{E}-05$ & Basic N1O2 & $\mathrm{C} 23 \mathrm{H} 23 \mathrm{~N} 1 \mathrm{O} 2$ & $3.02 \mathrm{E}-05$ \\
\hline Non-basic N1 & $\mathrm{C} 29 \mathrm{H} 21 \mathrm{~N} 1$ & 0.001172 & Non-basic N2 & $\mathrm{C} 21 \mathrm{H} 18 \mathrm{~N} 2$ & $2.20 \mathrm{E}-05$ & Basic N1O2 & $\mathrm{C} 24 \mathrm{H} 25 \mathrm{~N} 1 \mathrm{O} 2$ & $2.81 \mathrm{E}-05$ \\
\hline Non-basic N1 & $\mathrm{C} 30 \mathrm{H} 23 \mathrm{~N} 1$ & 0.000831 & Non-basic N2 & $\mathrm{C} 22 \mathrm{H} 20 \mathrm{~N} 2$ & $1.62 \mathrm{E}-05$ & Basic N1O2 & $\mathrm{C} 25 \mathrm{H} 27 \mathrm{~N} 1 \mathrm{O} 2$ & $3.30 \mathrm{E}-05$ \\
\hline Non-basic N1 & $\mathrm{C} 31 \mathrm{H} 25 \mathrm{~N} 1$ & 0.000483 & Non-basic N2 & $\mathrm{C} 23 \mathrm{H} 22 \mathrm{~N} 2$ & $7.25 \mathrm{E}-06$ & Basic N1O2 & $\mathrm{C} 26 \mathrm{H} 29 \mathrm{~N} 1 \mathrm{O} 2$ & $3.33 \mathrm{E}-05$ \\
\hline Non-basic N1 & $\mathrm{C} 32 \mathrm{H} 27 \mathrm{~N} 1$ & 0.000295 & Non-basic N2 & $\mathrm{C} 24 \mathrm{H} 24 \mathrm{~N} 2$ & $1.03 \mathrm{E}-05$ & Basic N1O2 & $\mathrm{C} 27 \mathrm{H} 31 \mathrm{~N} 1 \mathrm{O} 2$ & $3.84 \mathrm{E}-05$ \\
\hline Non-basic N1 & $\mathrm{C} 33 \mathrm{H} 29 \mathrm{~N} 1$ & 0.000174 & Non-basic N2 & $\mathrm{C} 25 \mathrm{H} 26 \mathrm{~N} 2$ & $2.81 \mathrm{E}-05$ & Basic N1O2 & $\mathrm{C} 28 \mathrm{H} 33 \mathrm{~N} 1 \mathrm{O} 2$ & $3.94 \mathrm{E}-05$ \\
\hline Non-basic N1 & $\mathrm{C} 34 \mathrm{H} 31 \mathrm{~N} 1$ & $9.41 \mathrm{E}-05$ & Non-basic N2 & $\mathrm{C} 26 \mathrm{H} 28 \mathrm{~N} 2$ & $2.27 \mathrm{E}-05$ & Basic N1O2 & $\mathrm{C} 29 \mathrm{H} 35 \mathrm{~N} 1 \mathrm{O} 2$ & $4.87 \mathrm{E}-05$ \\
\hline Non-basic N1 & $\mathrm{C} 35 \mathrm{H} 33 \mathrm{~N} 1$ & $4.26 \mathrm{E}-05$ & Non-basic N2 & $\mathrm{C} 27 \mathrm{H} 30 \mathrm{~N} 2$ & $2.68 \mathrm{E}-05$ & Basic N1O2 & $\mathrm{C} 30 \mathrm{H} 37 \mathrm{~N} 1 \mathrm{O} 2$ & $3.60 \mathrm{E}-05$ \\
\hline Non-basic N1 & $\mathrm{C} 36 \mathrm{H} 35 \mathrm{~N} 1$ & $3.08 \mathrm{E}-05$ & Non-basic N2 & $\mathrm{C} 28 \mathrm{H} 32 \mathrm{~N} 2$ & $1.02 \mathrm{E}-05$ & Basic N1O2 & $\mathrm{C} 31 \mathrm{H} 39 \mathrm{~N} 1 \mathrm{O} 2$ & $3.90 \mathrm{E}-05$ \\
\hline Non-basic N1 & $\mathrm{C} 37 \mathrm{H} 37 \mathrm{~N} 1$ & $1.65 \mathrm{E}-05$ & Non-basic N2 & $\mathrm{C} 29 \mathrm{H} 34 \mathrm{~N} 2$ & $1.91 \mathrm{E}-05$ & Basic N1O2 & $\mathrm{C} 32 \mathrm{H} 41 \mathrm{~N} 1 \mathrm{O} 2$ & $5.23 \mathrm{E}-05$ \\
\hline Non-basic N1 & $\mathrm{C} 38 \mathrm{H} 39 \mathrm{~N} 1$ & $1.80 \mathrm{E}-05$ & Non-basic N2 & $\mathrm{C} 30 \mathrm{H} 36 \mathrm{~N} 2$ & $2.40 \mathrm{E}-05$ & Basic N1O2 & $\mathrm{C} 33 \mathrm{H} 43 \mathrm{~N} 1 \mathrm{O} 2$ & $3.11 \mathrm{E}-05$ \\
\hline Non-basic N1 & $\mathrm{C} 39 \mathrm{H} 41 \mathrm{~N} 1$ & $3.39 \mathrm{E}-06$ & Non-basic N2 & $\mathrm{C} 31 \mathrm{H} 38 \mathrm{~N} 2$ & $9.46 \mathrm{E}-06$ & Basic N1O2 & $\mathrm{C} 34 \mathrm{H} 45 \mathrm{~N} 1 \mathrm{O} 2$ & $3.45 \mathrm{E}-05$ \\
\hline Non-basic N1 & $\mathrm{C} 40 \mathrm{H} 43 \mathrm{~N} 1$ & $2.72 \mathrm{E}-06$ & Non-basic N2 & $\mathrm{C} 32 \mathrm{H} 40 \mathrm{~N} 2$ & $9.97 \mathrm{E}-06$ & Basic N1O2 & $\mathrm{C} 35 \mathrm{H} 47 \mathrm{~N} 1 \mathrm{O} 2$ & $2.66 \mathrm{E}-05$ \\
\hline Non-basic N1 & $\mathrm{C} 26 \mathrm{H} 13 \mathrm{~N} 1$ & $1.71 \mathrm{E}-06$ & Non-basic N2 & $\mathrm{C} 33 \mathrm{H} 42 \mathrm{~N} 2$ & $1.38 \mathrm{E}-05$ & Basic N1O2 & $\mathrm{C} 36 \mathrm{H} 49 \mathrm{~N} 1 \mathrm{O} 2$ & $1.95 \mathrm{E}-05$ \\
\hline Non-basic N1 & $\mathrm{C} 27 \mathrm{H} 15 \mathrm{~N} 1$ & 4.47E-06 & Non-basic N2 & $\mathrm{C} 34 \mathrm{H} 44 \mathrm{~N} 2$ & $1.12 \mathrm{E}-05$ & Basic N1O2 & $\mathrm{C} 37 \mathrm{H} 51 \mathrm{~N} 1 \mathrm{O} 2$ & $2.43 \mathrm{E}-05$ \\
\hline Non-basic N1 & $\mathrm{C} 28 \mathrm{H} 17 \mathrm{~N} 1$ & 0.000138 & Non-basic N2 & $\mathrm{C} 20 \mathrm{H} 14 \mathrm{~N} 2$ & $1.48 \mathrm{E}-05$ & Basic N1O2 & $\mathrm{C} 38 \mathrm{H} 53 \mathrm{~N} 1 \mathrm{O} 2$ & $1.34 \mathrm{E}-05$ \\
\hline Non-basic N1 & $\mathrm{C} 29 \mathrm{H} 19 \mathrm{~N} 1$ & 0.000355 & Non-basic N2 & $\mathrm{C} 21 \mathrm{H} 16 \mathrm{~N} 2$ & $3.64 \mathrm{E}-05$ & Basic N1O2 & C39H55N1O2 & $1.27 \mathrm{E}-05$ \\
\hline Non-basic N1 & $\mathrm{C} 30 \mathrm{H} 21 \mathrm{~N} 1$ & 0.000546 & Non-basic N2 & $\mathrm{C} 22 \mathrm{H} 18 \mathrm{~N} 2$ & $4.61 \mathrm{E}-05$ & Basic N1O2 & $\mathrm{C} 40 \mathrm{H} 57 \mathrm{~N} 1 \mathrm{O} 2$ & $1.65 \mathrm{E}-05$ \\
\hline Non-basic N1 & $\mathrm{C} 31 \mathrm{H} 23 \mathrm{~N} 1$ & 0.000483 & Non-basic N2 & $\mathrm{C} 23 \mathrm{H} 20 \mathrm{~N} 2$ & $4.48 \mathrm{E}-05$ & Basic N1O2 & $\mathrm{C} 41 \mathrm{H} 59 \mathrm{~N} 1 \mathrm{O} 2$ & $8.34 \mathrm{E}-06$ \\
\hline Non-basic N1 & $\mathrm{C} 32 \mathrm{H} 25 \mathrm{~N} 1$ & 0.000355 & Non-basic N2 & $\mathrm{C} 24 \mathrm{H} 22 \mathrm{~N} 2$ & $3.80 \mathrm{E}-05$ & Basic N1O2 & $\mathrm{C} 43 \mathrm{H} 63 \mathrm{~N} 1 \mathrm{O} 2$ & $3.80 \mathrm{E}-06$ \\
\hline Non-basic N1 & $\mathrm{C} 33 \mathrm{H} 27 \mathrm{~N} 1$ & 0.00022 & Non-basic N2 & $\mathrm{C} 25 \mathrm{H} 24 \mathrm{~N} 2$ & $3.32 \mathrm{E}-05$ & Basic N1O2 & $\mathrm{C} 44 \mathrm{H} 65 \mathrm{~N} 1 \mathrm{O} 2$ & $4.36 \mathrm{E}-05$ \\
\hline Non-basic N1 & $\mathrm{C} 34 \mathrm{H} 29 \mathrm{~N} 1$ & 0.000139 & Non-basic N2 & $\mathrm{C} 26 \mathrm{H} 26 \mathrm{~N} 2$ & $2.64 \mathrm{E}-05$ & Basic N1O2 & $\mathrm{C} 45 \mathrm{H} 67 \mathrm{~N} 1 \mathrm{O} 2$ & $2.11 \mathrm{E}-06$ \\
\hline Non-basic N1 & $\mathrm{C} 35 \mathrm{H} 31 \mathrm{~N} 1$ & $6.76 \mathrm{E}-05$ & Non-basic N2 & $\mathrm{C} 27 \mathrm{H} 28 \mathrm{~N} 2$ & $1.75 \mathrm{E}-05$ & Basic N1O2 & C19H13N1O2 & $1.68 \mathrm{E}-07$ \\
\hline Non-basic N1 & $\mathrm{C} 36 \mathrm{H} 33 \mathrm{~N} 1$ & $3.30 \mathrm{E}-05$ & Non-basic N2 & $\mathrm{C} 28 \mathrm{H} 30 \mathrm{~N} 2$ & $9.78 \mathrm{E}-06$ & Basic N1O2 & $\mathrm{C} 20 \mathrm{H} 15 \mathrm{~N} 1 \mathrm{O} 2$ & $2.41 \mathrm{E}-06$ \\
\hline Non-basic N1 & $\mathrm{C} 37 \mathrm{H} 35 \mathrm{~N} 1$ & $2.38 \mathrm{E}-05$ & Non-basic N2 & $\mathrm{C} 29 \mathrm{H} 32 \mathrm{~N} 2$ & $1.07 \mathrm{E}-05$ & Basic N1O2 & $\mathrm{C} 21 \mathrm{H} 17 \mathrm{~N} 1 \mathrm{O} 2$ & $1.54 \mathrm{E}-05$ \\
\hline Non-basic N1 & $\mathrm{C} 38 \mathrm{H} 37 \mathrm{~N} 1$ & $7.98 \mathrm{E}-06$ & Non-basic N2 & $\mathrm{C} 30 \mathrm{H} 34 \mathrm{~N} 2$ & $1.89 \mathrm{E}-05$ & Basic N1O2 & $\mathrm{C} 22 \mathrm{H} 19 \mathrm{~N} 1 \mathrm{O} 2$ & $2.65 \mathrm{E}-05$ \\
\hline Non-basic N1 & $\mathrm{C} 39 \mathrm{H} 39 \mathrm{~N} 1$ & 7.88E-06 & Non-basic N2 & $\mathrm{C} 31 \mathrm{H} 36 \mathrm{~N} 2$ & $1.22 \mathrm{E}-05$ & Basic N1O2 & $\mathrm{C} 23 \mathrm{H} 21 \mathrm{~N} 1 \mathrm{O} 2$ & $6.21 \mathrm{E}-05$ \\
\hline Non-basic N1 & $\mathrm{C} 40 \mathrm{H} 41 \mathrm{~N} 1$ & $6.59 \mathrm{E}-06$ & Non-basic N2 & $\mathrm{C} 24 \mathrm{H} 20 \mathrm{~N} 2$ & $1.30 \mathrm{E}-05$ & Basic N1O2 & $\mathrm{C} 24 \mathrm{H} 23 \mathrm{~N} 1 \mathrm{O} 2$ & $4.88 \mathrm{E}-05$ \\
\hline Non-basic N1 & $\mathrm{C} 28 \mathrm{H} 15 \mathrm{~N} 1$ & $1.67 \mathrm{E}-05$ & Non-basic N2 & $\mathrm{C} 25 \mathrm{H} 22 \mathrm{~N} 2$ & $1.54 \mathrm{E}-05$ & Basic N1O2 & $\mathrm{C} 25 \mathrm{H} 25 \mathrm{~N} 1 \mathrm{O} 2$ & $3.59 \mathrm{E}-05$ \\
\hline Non-basic N1 & $\mathrm{C} 29 \mathrm{H} 17 \mathrm{~N} 1$ & $7.15 \mathrm{E}-05$ & Non-basic N2 & $\mathrm{C} 26 \mathrm{H} 24 \mathrm{~N} 2$ & $1.75 \mathrm{E}-05$ & Basic N1O2 & $\mathrm{C} 26 \mathrm{H} 27 \mathrm{~N} 1 \mathrm{O} 2$ & $3.80 \mathrm{E}-05$ \\
\hline Non-basic N1 & $\mathrm{C} 30 \mathrm{H} 19 \mathrm{~N} 1$ & 0.00015 & Non-basic N2 & $\mathrm{C} 27 \mathrm{H} 26 \mathrm{~N} 2$ & $1.65 \mathrm{E}-05$ & Basic N1O2 & $\mathrm{C} 27 \mathrm{H} 29 \mathrm{~N} 1 \mathrm{O} 2$ & $3.95 \mathrm{E}-05$ \\
\hline
\end{tabular}




\begin{tabular}{|c|c|c|c|c|c|c|c|c|}
\hline Class & Formula & Mass & Class & Formula & Mass & Class & Formula & Mass \\
\hline Non-basic N1 & $\mathrm{C} 31 \mathrm{H} 21 \mathrm{~N} 1$ & 0.000185 & Non-basic N2 & $\mathrm{C} 28 \mathrm{H} 28 \mathrm{~N} 2$ & $1.00 \mathrm{E}-05$ & Basic N1O2 & $\mathrm{C} 28 \mathrm{H} 31 \mathrm{~N} 1 \mathrm{O} 2$ & $3.98 \mathrm{E}-05$ \\
\hline Non-basic N1 & $\mathrm{C} 32 \mathrm{H} 23 \mathrm{~N} 1$ & 0.000216 & Non-basic N2 & $\mathrm{C} 23 \mathrm{H} 16 \mathrm{~N} 2$ & $1.36 \mathrm{E}-05$ & Basic N1O2 & $\mathrm{C} 29 \mathrm{H} 33 \mathrm{~N} 1 \mathrm{O} 2$ & $3.95 \mathrm{E}-05$ \\
\hline Non-basic N1 & $\mathrm{C} 33 \mathrm{H} 25 \mathrm{~N} 1$ & 0.00017 & Non-basic N2 & $\mathrm{C} 24 \mathrm{H} 18 \mathrm{~N} 2$ & $3.79 \mathrm{E}-05$ & Basic N1O2 & $\mathrm{C} 30 \mathrm{H} 35 \mathrm{~N} 1 \mathrm{O} 2$ & 4.97E-05 \\
\hline Non-basic N1 & $\mathrm{C} 34 \mathrm{H} 27 \mathrm{~N} 1$ & 0.000138 & Non-basic N2 & $\mathrm{C} 25 \mathrm{H} 20 \mathrm{~N} 2$ & $5.45 \mathrm{E}-05$ & Basic N1O2 & $\mathrm{C} 31 \mathrm{H} 37 \mathrm{~N} 1 \mathrm{O} 2$ & 4.94E-05 \\
\hline Non-basic N1 & $\mathrm{C} 35 \mathrm{H} 29 \mathrm{~N} 1$ & $8.08 \mathrm{E}-05$ & Non-basic N2 & $\mathrm{C} 26 \mathrm{H} 22 \mathrm{~N} 2$ & $4.34 \mathrm{E}-05$ & Basic N1O2 & $\mathrm{C} 32 \mathrm{H} 39 \mathrm{~N} 1 \mathrm{O} 2$ & $3.96 \mathrm{E}-05$ \\
\hline Non-basic N1 & $\mathrm{C} 36 \mathrm{H} 31 \mathrm{~N} 1$ & $5.89 \mathrm{E}-05$ & Non-basic N2 & $\mathrm{C} 27 \mathrm{H} 24 \mathrm{~N} 2$ & $2.80 \mathrm{E}-05$ & Basic N1O2 & $\mathrm{C} 33 \mathrm{H} 41 \mathrm{~N} 1 \mathrm{O} 2$ & $2.81 \mathrm{E}-05$ \\
\hline Non-basic N1 & $\mathrm{C} 37 \mathrm{H} 33 \mathrm{~N} 1$ & 3.39E-05 & Non-basic N2 & $\mathrm{C} 28 \mathrm{H} 26 \mathrm{~N} 2$ & $2.86 \mathrm{E}-05$ & Basic N1O2 & $\mathrm{C} 34 \mathrm{H} 43 \mathrm{~N} 1 \mathrm{O} 2$ & $4.66 \mathrm{E}-05$ \\
\hline Non-basic N1 & $\mathrm{C} 38 \mathrm{H} 35 \mathrm{~N} 1$ & $1.79 \mathrm{E}-05$ & Non-basic N2 & $\mathrm{C} 29 \mathrm{H} 28 \mathrm{~N} 2$ & $1.28 \mathrm{E}-05$ & Basic N1O2 & $\mathrm{C} 35 \mathrm{H} 45 \mathrm{~N} 1 \mathrm{O} 2$ & $2.30 \mathrm{E}-05$ \\
\hline Non-basic N1 & $\mathrm{C} 39 \mathrm{H} 37 \mathrm{~N} 1$ & $5.40 \mathrm{E}-06$ & Non-basic N2 & $\mathrm{C} 30 \mathrm{H} 30 \mathrm{~N} 2$ & $1.23 \mathrm{E}-05$ & Basic N1O2 & $\mathrm{C} 36 \mathrm{H} 47 \mathrm{~N} 1 \mathrm{O} 2$ & $1.43 \mathrm{E}-05$ \\
\hline Non-basic N1 & $\mathrm{C} 30 \mathrm{H} 17 \mathrm{~N} 1$ & $2.48 \mathrm{E}-05$ & Non-basic N2 & $\mathrm{C} 24 \mathrm{H} 16 \mathrm{~N} 2$ & $8.16 \mathrm{E}-06$ & Basic N1O2 & $\mathrm{C} 37 \mathrm{H} 49 \mathrm{~N} 1 \mathrm{O} 2$ & $1.91 \mathrm{E}-05$ \\
\hline Non-basic N1 & $\mathrm{C} 31 \mathrm{H} 19 \mathrm{~N} 1$ & $8.76 \mathrm{E}-05$ & Non-basic N2 & $\mathrm{C} 25 \mathrm{H} 18 \mathrm{~N} 2$ & $1.58 \mathrm{E}-05$ & Basic N1O2 & $\mathrm{C} 38 \mathrm{H} 51 \mathrm{~N} 1 \mathrm{O} 2$ & $1.15 \mathrm{E}-05$ \\
\hline Non-basic N1 & $\mathrm{C} 32 \mathrm{H} 21 \mathrm{~N} 1$ & 0.000154 & Non-basic N2 & $\mathrm{C} 26 \mathrm{H} 20 \mathrm{~N} 2$ & $9.60 \mathrm{E}-06$ & Basic N1O2 & C39H53N1O2 & $1.39 \mathrm{E}-05$ \\
\hline Non-basic N1 & $\mathrm{C} 33 \mathrm{H} 23 \mathrm{~N} 1$ & 0.000146 & Non-basic N2 & $\mathrm{C} 27 \mathrm{H} 22 \mathrm{~N} 2$ & $1.75 \mathrm{E}-05$ & Basic N1O2 & $\mathrm{C} 40 \mathrm{H} 55 \mathrm{~N} 1 \mathrm{O} 2$ & $5.81 \mathrm{E}-06$ \\
\hline Non-basic N1 & $\mathrm{C} 34 \mathrm{H} 25 \mathrm{~N} 1$ & 0.000133 & Non-basic N2 & $\mathrm{C} 28 \mathrm{H} 24 \mathrm{~N} 2$ & $9.48 \mathrm{E}-06$ & Basic N1O2 & $\mathrm{C} 41 \mathrm{H} 57 \mathrm{~N} 1 \mathrm{O} 2$ & $4.38 \mathrm{E}-05$ \\
\hline Non-basic N1 & $\mathrm{C} 35 \mathrm{H} 27 \mathrm{~N} 1$ & $8.98 \mathrm{E}-05$ & Non-basic N2 & $\mathrm{C} 29 \mathrm{H} 26 \mathrm{~N} 2$ & $1.50 \mathrm{E}-05$ & Basic N1O2 & $\mathrm{C} 42 \mathrm{H} 59 \mathrm{~N} 1 \mathrm{O} 2$ & $2.11 \mathrm{E}-06$ \\
\hline Non-basic N1 & $\mathrm{C} 36 \mathrm{H} 29 \mathrm{~N} 1$ & $6.05 \mathrm{E}-05$ & Acidic $\mathrm{O} 1$ & $\mathrm{C} 24 \mathrm{H} 42 \mathrm{O} 1$ & $4.83 \mathrm{E}-05$ & Basic N1O2 & $\mathrm{C} 20 \mathrm{H} 13 \mathrm{~N} 1 \mathrm{O} 2$ & $1.86 \mathrm{E}-07$ \\
\hline Non-basic N1 & $\mathrm{C} 37 \mathrm{H} 31 \mathrm{~N} 1$ & $5.49 \mathrm{E}-05$ & Acidic $\mathrm{O} 1$ & $\mathrm{C} 25 \mathrm{H} 44 \mathrm{O} 1$ & 4.44E-05 & Basic N1O2 & $\mathrm{C} 21 \mathrm{H} 15 \mathrm{~N} 1 \mathrm{O} 2$ & $1.39 \mathrm{E}-06$ \\
\hline Non-basic N1 & $\mathrm{C} 38 \mathrm{H} 33 \mathrm{~N} 1$ & $3.24 \mathrm{E}-05$ & Acidic $\mathrm{O} 1$ & $\mathrm{C} 26 \mathrm{H} 46 \mathrm{O} 1$ & 0.000156 & Basic N1O2 & $\mathrm{C} 22 \mathrm{H} 17 \mathrm{~N} 1 \mathrm{O} 2$ & 4.77E-06 \\
\hline Non-basic N1 & $\mathrm{C} 39 \mathrm{H} 35 \mathrm{~N} 1$ & $1.19 \mathrm{E}-05$ & Acidic $\mathrm{O} 1$ & $\mathrm{C} 27 \mathrm{H} 48 \mathrm{O} 1$ & 0.000269 & Basic N1O2 & $\mathrm{C} 23 \mathrm{H} 19 \mathrm{~N} 1 \mathrm{O} 2$ & $1.09 \mathrm{E}-05$ \\
\hline Non-basic N1 & $\mathrm{C} 40 \mathrm{H} 37 \mathrm{~N} 1$ & 7.91E-06 & Acidic $\mathrm{O} 1$ & $\mathrm{C} 28 \mathrm{H} 50 \mathrm{O} 1$ & 0.000466 & Basic N1O2 & $\mathrm{C} 24 \mathrm{H} 21 \mathrm{~N} 1 \mathrm{O} 2$ & $3.45 \mathrm{E}-05$ \\
\hline Non-basic N1 & $\mathrm{C} 41 \mathrm{H} 39 \mathrm{~N} 1$ & $2.72 \mathrm{E}-06$ & Acidic $\mathrm{O} 1$ & $\mathrm{C} 29 \mathrm{H} 52 \mathrm{O} 1$ & 0.000406 & Basic N1O2 & $\mathrm{C} 25 \mathrm{H} 23 \mathrm{~N} 1 \mathrm{O} 2$ & $3.69 \mathrm{E}-05$ \\
\hline Non-basic N1 & $\mathrm{C} 31 \mathrm{H} 17 \mathrm{~N} 1$ & $2.88 \mathrm{E}-06$ & Acidic $\mathrm{O} 1$ & $\mathrm{C} 30 \mathrm{H} 54 \mathrm{O} 1$ & 0.00048 & Basic N1O2 & $\mathrm{C} 26 \mathrm{H} 25 \mathrm{~N} 1 \mathrm{O} 2$ & $5.04 \mathrm{E}-05$ \\
\hline Non-basic N1 & $\mathrm{C} 32 \mathrm{H} 19 \mathrm{~N} 1$ & $6.36 \mathrm{E}-06$ & Acidic $\mathrm{O} 1$ & $\mathrm{C} 31 \mathrm{H} 56 \mathrm{O} 1$ & 0.00058 & Basic N1O2 & $\mathrm{C} 27 \mathrm{H} 27 \mathrm{~N} 1 \mathrm{O} 2$ & $3.36 \mathrm{E}-05$ \\
\hline Non-basic N1 & $\mathrm{C} 33 \mathrm{H} 21 \mathrm{~N} 1$ & $3.84 \mathrm{E}-05$ & Acidic $\mathrm{O} 1$ & $\mathrm{C} 32 \mathrm{H} 58 \mathrm{O} 1$ & 0.000668 & Basic N1O2 & $\mathrm{C} 28 \mathrm{H} 29 \mathrm{~N} 1 \mathrm{O} 2$ & $3.89 \mathrm{E}-05$ \\
\hline Non-basic N1 & $\mathrm{C} 34 \mathrm{H} 23 \mathrm{~N} 1$ & $5.75 \mathrm{E}-05$ & Acidic $\mathrm{O} 1$ & $\mathrm{C} 33 \mathrm{H} 60 \mathrm{O} 1$ & 0.000554 & Basic N1O2 & $\mathrm{C} 29 \mathrm{H} 31 \mathrm{~N} 1 \mathrm{O} 2$ & $3.14 \mathrm{E}-05$ \\
\hline Non-basic N1 & $\mathrm{C} 35 \mathrm{H} 25 \mathrm{~N} 1$ & $7.11 \mathrm{E}-05$ & Acidic $\mathrm{O} 1$ & C34H62O1 & 0.000556 & Basic N1O2 & $\mathrm{C} 30 \mathrm{H} 33 \mathrm{~N} 1 \mathrm{O} 2$ & $3.68 \mathrm{E}-05$ \\
\hline Non-basic N1 & $\mathrm{C} 36 \mathrm{H} 27 \mathrm{~N} 1$ & $6.64 \mathrm{E}-05$ & Acidic $\mathrm{O} 1$ & $\mathrm{C} 35 \mathrm{H} 64 \mathrm{O} 1$ & 0.000397 & Basic N1O2 & $\mathrm{C} 31 \mathrm{H} 35 \mathrm{~N} 1 \mathrm{O} 2$ & $4.16 \mathrm{E}-05$ \\
\hline Non-basic N1 & $\mathrm{C} 37 \mathrm{H} 29 \mathrm{~N} 1$ & $6.49 \mathrm{E}-05$ & Acidic $\mathrm{O} 1$ & $\mathrm{C} 36 \mathrm{H} 66 \mathrm{O} 1$ & 0.000482 & Basic N1O2 & $\mathrm{C} 32 \mathrm{H} 37 \mathrm{~N} 1 \mathrm{O} 2$ & $2.39 \mathrm{E}-05$ \\
\hline Non-basic N1 & $\mathrm{C} 38 \mathrm{H} 31 \mathrm{~N} 1$ & $3.51 \mathrm{E}-05$ & Acidic $\mathrm{O} 1$ & C37H68O1 & 0.000188 & Basic N1O2 & $\mathrm{C} 33 \mathrm{H} 39 \mathrm{~N} 1 \mathrm{O} 2$ & $2.08 \mathrm{E}-05$ \\
\hline Non-basic N1 & $\mathrm{C} 39 \mathrm{H} 33 \mathrm{~N} 1$ & $2.50 \mathrm{E}-05$ & Acidic $\mathrm{O} 1$ & $\mathrm{C} 38 \mathrm{H} 70 \mathrm{O} 1$ & 0.000113 & Basic N1O2 & $\mathrm{C} 34 \mathrm{H} 41 \mathrm{~N} 1 \mathrm{O} 2$ & $2.14 \mathrm{E}-05$ \\
\hline Non-basic N1 & $\mathrm{C} 40 \mathrm{H} 35 \mathrm{~N} 1$ & $8.13 \mathrm{E}-06$ & Acidic $\mathrm{O} 1$ & $\mathrm{C} 39 \mathrm{H} 72 \mathrm{O} 1$ & 0.000121 & Basic N1O2 & $\mathrm{C} 35 \mathrm{H} 43 \mathrm{~N} 1 \mathrm{O} 2$ & $3.90 \mathrm{E}-05$ \\
\hline Non-basic N1 & $\mathrm{C} 41 \mathrm{H} 37 \mathrm{~N} 1$ & $1.07 \mathrm{E}-05$ & Acidic $\mathrm{O} 1$ & $\mathrm{C} 40 \mathrm{H} 74 \mathrm{O} 1$ & 0.000145 & Basic N1O2 & $\mathrm{C} 36 \mathrm{H} 45 \mathrm{~N} 1 \mathrm{O} 2$ & $1.30 \mathrm{E}-05$ \\
\hline Non-basic N1 & $\mathrm{C} 42 \mathrm{H} 39 \mathrm{~N} 1$ & 4.12E-06 & Acidic $\mathrm{O} 1$ & $\mathrm{C} 41 \mathrm{H} 76 \mathrm{O} 1$ & $3.22 \mathrm{E}-05$ & Basic N1O2 & $\mathrm{C} 37 \mathrm{H} 47 \mathrm{~N} 1 \mathrm{O} 2$ & $1.57 \mathrm{E}-05$ \\
\hline Non-basic N1 & $\mathrm{C} 33 \mathrm{H} 19 \mathrm{~N} 1$ & $8.41 \mathrm{E}-06$ & Acidic $\mathrm{O} 1$ & $\mathrm{C} 42 \mathrm{H} 78 \mathrm{O} 1$ & $2.13 \mathrm{E}-05$ & Basic N1O2 & $\mathrm{C} 38 \mathrm{H} 49 \mathrm{~N} 1 \mathrm{O} 2$ & $9.77 \mathrm{E}-06$ \\
\hline Non-basic N1 & $\mathrm{C} 34 \mathrm{H} 21 \mathrm{~N} 1$ & $2.66 \mathrm{E}-05$ & Acidic O1 & $\mathrm{C} 26 \mathrm{H} 44 \mathrm{O} 1$ & $8.98 \mathrm{E}-05$ & Basic N1O2 & $\mathrm{C} 39 \mathrm{H} 51 \mathrm{~N} 1 \mathrm{O} 2$ & $9.51 \mathrm{E}-06$ \\
\hline Non-basic N1 & $\mathrm{C} 35 \mathrm{H} 23 \mathrm{~N} 1$ & $4.31 \mathrm{E}-05$ & Acidic O1 & $\mathrm{C} 27 \mathrm{H} 46 \mathrm{O} 1$ & 0.000293 & Basic N1O2 & $\mathrm{C} 40 \mathrm{H} 53 \mathrm{~N} 1 \mathrm{O} 2$ & $5.75 \mathrm{E}-06$ \\
\hline Non-basic N1 & $\mathrm{C} 36 \mathrm{H} 25 \mathrm{~N} 1$ & $3.93 \mathrm{E}-05$ & Acidic $\mathrm{O} 1$ & $\mathrm{C} 28 \mathrm{H} 48 \mathrm{O} 1$ & 0.000234 & Basic N1O2 & $\mathrm{C} 41 \mathrm{H} 55 \mathrm{~N} 1 \mathrm{O} 2$ & $6.33 \mathrm{E}-06$ \\
\hline Non-basic N1 & $\mathrm{C} 37 \mathrm{H} 27 \mathrm{~N} 1$ & $5.36 \mathrm{E}-05$ & Acidic $\mathrm{O} 1$ & $\mathrm{C} 29 \mathrm{H} 50 \mathrm{O} 1$ & 0.000191 & Basic N1O2 & $\mathrm{C} 22 \mathrm{H} 15 \mathrm{~N} 1 \mathrm{O} 2$ & $1.08 \mathrm{E}-06$ \\
\hline
\end{tabular}




\begin{tabular}{|c|c|c|c|c|c|c|c|c|}
\hline Class & Formula & Mass & Class & Formula & Mass & Class & Formula & Mass \\
\hline Non-basic N1 & $\mathrm{C} 38 \mathrm{H} 29 \mathrm{~N} 1$ & $4.18 \mathrm{E}-05$ & Acidic $\mathrm{O} 1$ & $\mathrm{C} 30 \mathrm{H} 52 \mathrm{O} 1$ & 0.000288 & Basic N1O2 & $\mathrm{C} 23 \mathrm{H} 17 \mathrm{~N} 1 \mathrm{O} 2$ & $2.50 \mathrm{E}-06$ \\
\hline Non-basic N1 & $\mathrm{C} 39 \mathrm{H} 31 \mathrm{~N} 1$ & $3.40 \mathrm{E}-05$ & Acidic $\mathrm{O} 1$ & $\mathrm{C} 31 \mathrm{H} 54 \mathrm{O} 1$ & 0.000368 & Basic $\mathrm{N} 1 \mathrm{O} 2$ & $\mathrm{C} 24 \mathrm{H} 19 \mathrm{~N} 1 \mathrm{O} 2$ & $5.22 \mathrm{E}-06$ \\
\hline Non-basic N1 & $\mathrm{C} 40 \mathrm{H} 33 \mathrm{~N} 1$ & $1.96 \mathrm{E}-05$ & Acidic $\mathrm{O} 1$ & $\mathrm{C} 32 \mathrm{H} 56 \mathrm{O} 1$ & 0.000441 & Basic N1O2 & $\mathrm{C} 25 \mathrm{H} 21 \mathrm{~N} 1 \mathrm{O} 2$ & $3.20 \mathrm{E}-05$ \\
\hline Non-basic N1 & $\mathrm{C} 41 \mathrm{H} 35 \mathrm{~N} 1$ & $1.02 \mathrm{E}-05$ & Acidic $\mathrm{O} 1$ & $\mathrm{C} 33 \mathrm{H} 58 \mathrm{O} 1$ & 0.000359 & Basic $\mathrm{N} 1 \mathrm{O} 2$ & $\mathrm{C} 26 \mathrm{H} 23 \mathrm{~N} 1 \mathrm{O} 2$ & $1.71 \mathrm{E}-05$ \\
\hline Non-basic N1 & $\mathrm{C} 42 \mathrm{H} 37 \mathrm{~N} 1$ & $1.11 \mathrm{E}-05$ & Acidic $\mathrm{O} 1$ & $\mathrm{C} 34 \mathrm{H} 60 \mathrm{O} 1$ & 0.000316 & Basic N1O2 & $\mathrm{C} 27 \mathrm{H} 25 \mathrm{~N} 1 \mathrm{O} 2$ & $2.35 \mathrm{E}-05$ \\
\hline Non-basic N1 & $\mathrm{C} 43 \mathrm{H} 39 \mathrm{~N} 1$ & $7.59 \mathrm{E}-06$ & Acidic O1 & $\mathrm{C} 35 \mathrm{H} 62 \mathrm{O} 1$ & 0.00038 & Basic N1O2 & $\mathrm{C} 28 \mathrm{H} 27 \mathrm{~N} 1 \mathrm{O} 2$ & $2.27 \mathrm{E}-05$ \\
\hline Non-basic N1 & $\mathrm{C} 34 \mathrm{H} 19 \mathrm{~N} 1$ & $2.15 \mathrm{E}-06$ & Acidic $\mathrm{O} 1$ & $\mathrm{C} 36 \mathrm{H} 64 \mathrm{O} 1$ & 0.00028 & Basic N1O2 & $\mathrm{C} 29 \mathrm{H} 29 \mathrm{~N} 1 \mathrm{O} 2$ & $3.29 \mathrm{E}-05$ \\
\hline Non-basic N1 & $\mathrm{C} 35 \mathrm{H} 21 \mathrm{~N} 1$ & $1.56 \mathrm{E}-06$ & Acidic $\mathrm{O} 1$ & $\mathrm{C} 37 \mathrm{H} 66 \mathrm{O} 1$ & 0.000237 & Basic N1O2 & $\mathrm{C} 30 \mathrm{H} 31 \mathrm{~N} 1 \mathrm{O} 2$ & $2.24 \mathrm{E}-05$ \\
\hline Non-basic N1 & $\mathrm{C} 36 \mathrm{H} 23 \mathrm{~N} 1$ & $1.67 \mathrm{E}-05$ & Acidic O1 & $\mathrm{C} 38 \mathrm{H} 68 \mathrm{O} 1$ & 0.000193 & Basic N1O2 & $\mathrm{C} 31 \mathrm{H} 33 \mathrm{~N} 1 \mathrm{O} 2$ & $1.66 \mathrm{E}-05$ \\
\hline Non-basic N1 & $\mathrm{C} 37 \mathrm{H} 25 \mathrm{~N} 1$ & $2.20 \mathrm{E}-05$ & Acidic $\mathrm{O} 1$ & $\mathrm{C} 27 \mathrm{H} 44 \mathrm{O} 1$ & $1.54 \mathrm{E}-05$ & Basic N1O2 & $\mathrm{C} 32 \mathrm{H} 35 \mathrm{~N} 1 \mathrm{O} 2$ & $2.33 \mathrm{E}-05$ \\
\hline Non-basic N1 & $\mathrm{C} 38 \mathrm{H} 27 \mathrm{~N} 1$ & $2.71 \mathrm{E}-05$ & Acidic $\mathrm{O} 1$ & $\mathrm{C} 28 \mathrm{H} 46 \mathrm{O} 1$ & $3.16 \mathrm{E}-05$ & Basic N1O2 & $\mathrm{C} 33 \mathrm{H} 37 \mathrm{~N} 1 \mathrm{O} 2$ & $2.44 \mathrm{E}-05$ \\
\hline Non-basic N1 & $\mathrm{C} 39 \mathrm{H} 29 \mathrm{~N} 1$ & $2.76 \mathrm{E}-05$ & Acidic $\mathrm{O} 1$ & $\mathrm{C} 29 \mathrm{H} 48 \mathrm{O} 1$ & $7.61 \mathrm{E}-05$ & Basic N1O2 & $\mathrm{C} 34 \mathrm{H} 39 \mathrm{~N} 1 \mathrm{O} 2$ & $1.33 \mathrm{E}-05$ \\
\hline Non-basic N1 & $\mathrm{C} 40 \mathrm{H} 31 \mathrm{~N} 1$ & $2.86 \mathrm{E}-05$ & Acidic $\mathrm{O} 1$ & $\mathrm{C} 30 \mathrm{H} 50 \mathrm{O} 1$ & 0.000118 & Basic N1O2 & $\mathrm{C} 35 \mathrm{H} 41 \mathrm{~N} 1 \mathrm{O} 2$ & $9.42 \mathrm{E}-06$ \\
\hline Non-basic N1 & C41H33N1 & $2.20 \mathrm{E}-05$ & Acidic $\mathrm{O} 1$ & $\mathrm{C} 31 \mathrm{H} 52 \mathrm{O} 1$ & 0.00015 & Basic $\mathrm{N} 1 \mathrm{O} 2$ & $\mathrm{C} 36 \mathrm{H} 43 \mathrm{~N} 1 \mathrm{O} 2$ & $1.68 \mathrm{E}-05$ \\
\hline Non-basic N1 & $\mathrm{C} 42 \mathrm{H} 35 \mathrm{~N} 1$ & $1.45 \mathrm{E}-05$ & Acidic $\mathrm{O} 1$ & $\mathrm{C} 32 \mathrm{H} 54 \mathrm{O} 1$ & 0.000143 & Basic N1O2 & $\mathrm{C} 37 \mathrm{H} 45 \mathrm{~N} 1 \mathrm{O} 2$ & $1.29 \mathrm{E}-05$ \\
\hline Non-basic N1 & $\mathrm{C} 37 \mathrm{H} 23 \mathrm{~N} 1$ & $4.59 \mathrm{E}-06$ & Acidic $\mathrm{O} 1$ & $\mathrm{C} 33 \mathrm{H} 56 \mathrm{O} 1$ & 0.00016 & Basic N1O2 & $\mathrm{C} 38 \mathrm{H} 47 \mathrm{~N} 1 \mathrm{O} 2$ & $5.25 \mathrm{E}-06$ \\
\hline Non-basic N1 & $\mathrm{C} 38 \mathrm{H} 25 \mathrm{~N} 1$ & $2.31 \mathrm{E}-05$ & Acidic $\mathrm{O} 1$ & $\mathrm{C} 34 \mathrm{H} 58 \mathrm{O} 1$ & 0.000156 & Basic N1O2 & $\mathrm{C} 23 \mathrm{H} 15 \mathrm{~N} 1 \mathrm{O} 2$ & $3.62 \mathrm{E}-06$ \\
\hline Non-basic N1 & $\mathrm{C} 39 \mathrm{H} 27 \mathrm{~N} 1$ & $2.56 \mathrm{E}-05$ & Acidic $\mathrm{O} 1$ & $\mathrm{C} 35 \mathrm{H} 60 \mathrm{O} 1$ & 0.000154 & Basic N1O2 & $\mathrm{C} 24 \mathrm{H} 17 \mathrm{~N} 1 \mathrm{O} 2$ & 4.37E-06 \\
\hline Non-basic N1 & $\mathrm{C} 40 \mathrm{H} 29 \mathrm{~N} 1$ & $2.64 \mathrm{E}-05$ & Acidic $\mathrm{O} 1$ & $\mathrm{C} 36 \mathrm{H} 62 \mathrm{O} 1$ & $8.85 \mathrm{E}-05$ & Basic N1O2 & $\mathrm{C} 25 \mathrm{H} 19 \mathrm{~N} 1 \mathrm{O} 2$ & $1.22 \mathrm{E}-05$ \\
\hline Non-basic N1 & $\mathrm{C} 41 \mathrm{H} 31 \mathrm{~N} 1$ & $1.84 \mathrm{E}-05$ & Acidic $\mathrm{O} 1$ & $\mathrm{C} 37 \mathrm{H} 64 \mathrm{O} 1$ & 0.000106 & Basic N1O2 & $\mathrm{C} 26 \mathrm{H} 21 \mathrm{~N} 1 \mathrm{O} 2$ & $1.15 \mathrm{E}-05$ \\
\hline Non-basic N1 & $\mathrm{C} 42 \mathrm{H} 33 \mathrm{~N} 1$ & $1.82 \mathrm{E}-05$ & Acidic $\mathrm{O} 1$ & $\mathrm{C} 27 \mathrm{H} 42 \mathrm{O} 1$ & $2.47 \mathrm{E}-05$ & Basic N1O2 & $\mathrm{C} 27 \mathrm{H} 23 \mathrm{~N} 1 \mathrm{O} 2$ & $1.24 \mathrm{E}-05$ \\
\hline Non-basic N1 & $\mathrm{C} 43 \mathrm{H} 35 \mathrm{~N} 1$ & $1.21 \mathrm{E}-05$ & Acidic $\mathrm{O} 1$ & $\mathrm{C} 28 \mathrm{H} 44 \mathrm{O} 1$ & $1.38 \mathrm{E}-05$ & Basic N1O2 & $\mathrm{C} 28 \mathrm{H} 25 \mathrm{~N} 1 \mathrm{O} 2$ & $1.88 \mathrm{E}-05$ \\
\hline Non-basic N1 & $\mathrm{C} 44 \mathrm{H} 37 \mathrm{~N} 1$ & $5.99 \mathrm{E}-06$ & Acidic $\mathrm{O} 1$ & $\mathrm{C} 29 \mathrm{H} 46 \mathrm{O} 1$ & $4.43 \mathrm{E}-05$ & Basic N1O2 & $\mathrm{C} 29 \mathrm{H} 27 \mathrm{~N} 1 \mathrm{O} 2$ & $2.83 \mathrm{E}-05$ \\
\hline Non-basic N1 & $\mathrm{C} 45 \mathrm{H} 39 \mathrm{~N} 1$ & $1.59 \mathrm{E}-06$ & Acidic $\mathrm{O} 1$ & $\mathrm{C} 30 \mathrm{H} 48 \mathrm{O} 1$ & $4.58 \mathrm{E}-05$ & Basic $\mathrm{N} 1 \mathrm{O} 2$ & $\mathrm{C} 30 \mathrm{H} 29 \mathrm{~N} 1 \mathrm{O} 2$ & $1.99 \mathrm{E}-05$ \\
\hline Non-basic N1 & $\mathrm{C} 39 \mathrm{H} 25 \mathrm{~N} 1$ & $1.47 \mathrm{E}-05$ & Acidic $\mathrm{O} 1$ & $\mathrm{C} 31 \mathrm{H} 50 \mathrm{O} 1$ & $6.85 \mathrm{E}-05$ & Basic N1O2 & $\mathrm{C} 31 \mathrm{H} 31 \mathrm{~N} 1 \mathrm{O} 2$ & $1.12 \mathrm{E}-05$ \\
\hline Non-basic N1 & $\mathrm{C} 40 \mathrm{H} 27 \mathrm{~N} 1$ & $1.71 \mathrm{E}-05$ & Acidic $\mathrm{O} 1$ & $\mathrm{C} 32 \mathrm{H} 52 \mathrm{O} 1$ & $8.64 \mathrm{E}-05$ & Basic N1O2 & $\mathrm{C} 32 \mathrm{H} 33 \mathrm{~N} 1 \mathrm{O} 2$ & $1.95 \mathrm{E}-05$ \\
\hline Non-basic N1 & $\mathrm{C} 41 \mathrm{H} 29 \mathrm{~N} 1$ & $2.43 \mathrm{E}-05$ & Acidic $\mathrm{O} 1$ & $\mathrm{C} 33 \mathrm{H} 54 \mathrm{O} 1$ & $6.19 \mathrm{E}-05$ & Basic N1O2 & $\mathrm{C} 33 \mathrm{H} 35 \mathrm{~N} 1 \mathrm{O} 2$ & $7.60 \mathrm{E}-06$ \\
\hline Non-basic N1 & $\mathrm{C} 42 \mathrm{H} 31 \mathrm{~N} 1$ & $1.55 \mathrm{E}-05$ & Acidic $\mathrm{O} 1$ & $\mathrm{C} 34 \mathrm{H} 56 \mathrm{O} 1$ & $9.07 \mathrm{E}-05$ & Basic N1O2 & $\mathrm{C} 34 \mathrm{H} 37 \mathrm{~N} 1 \mathrm{O} 2$ & $8.10 \mathrm{E}-06$ \\
\hline Non-basic N1 & $\mathrm{C} 43 \mathrm{H} 33 \mathrm{~N} 1$ & $1.88 \mathrm{E}-05$ & Acidic $\mathrm{O} 1$ & $\mathrm{C} 35 \mathrm{H} 58 \mathrm{O} 1$ & $1.79 \mathrm{E}-05$ & Basic N1O2 & $\mathrm{C} 35 \mathrm{H} 39 \mathrm{~N} 1 \mathrm{O} 2$ & $5.63 \mathrm{E}-06$ \\
\hline Non-basic N1 & $\mathrm{C} 44 \mathrm{H} 35 \mathrm{~N} 1$ & $1.21 \mathrm{E}-05$ & Acidic $\mathrm{O} 1$ & $\mathrm{C} 27 \mathrm{H} 40 \mathrm{O} 1$ & $3.14 \mathrm{E}-05$ & Basic N1O2 & $\mathrm{C} 36 \mathrm{H} 41 \mathrm{~N} 1 \mathrm{O} 2$ & 4.13E-06 \\
\hline Non-basic N1 & $\mathrm{C} 40 \mathrm{H} 25 \mathrm{~N} 1$ & $1.50 \mathrm{E}-06$ & Acidic $\mathrm{O} 1$ & $\mathrm{C} 28 \mathrm{H} 42 \mathrm{O} 1$ & $6.31 \mathrm{E}-05$ & Basic N1O2 & $\mathrm{C} 37 \mathrm{H} 43 \mathrm{~N} 1 \mathrm{O} 2$ & 4.17E-06 \\
\hline Non-basic N1 & $\mathrm{C} 41 \mathrm{H} 27 \mathrm{~N} 1$ & $3.89 \mathrm{E}-06$ & Acidic $\mathrm{O} 1$ & $\mathrm{C} 29 \mathrm{H} 44 \mathrm{O} 1$ & $5.99 \mathrm{E}-05$ & Basic N1O2 & $\mathrm{C} 38 \mathrm{H} 45 \mathrm{~N} 1 \mathrm{O} 2$ & $2.21 \mathrm{E}-06$ \\
\hline Non-basic N1 & $\mathrm{C} 42 \mathrm{H} 29 \mathrm{~N} 1$ & $9.10 \mathrm{E}-06$ & Acidic $\mathrm{O} 1$ & $\mathrm{C} 30 \mathrm{H} 46 \mathrm{O} 1$ & 0.000134 & Basic N1O2 & $\mathrm{C} 25 \mathrm{H} 17 \mathrm{~N} 1 \mathrm{O} 2$ & $1.08 \mathrm{E}-06$ \\
\hline Non-basic N1 & $\mathrm{C} 43 \mathrm{H} 31 \mathrm{~N} 1$ & $1.16 \mathrm{E}-05$ & Acidic $\mathrm{O} 1$ & $\mathrm{C} 31 \mathrm{H} 48 \mathrm{O} 1$ & 0.00011 & Basic N1O2 & $\mathrm{C} 26 \mathrm{H} 19 \mathrm{~N} 1 \mathrm{O} 2$ & $2.02 \mathrm{E}-05$ \\
\hline Non-basic N1 & $\mathrm{C} 44 \mathrm{H} 33 \mathrm{~N} 1$ & $8.90 \mathrm{E}-06$ & Acidic O1 & $\mathrm{C} 32 \mathrm{H} 50 \mathrm{O} 1$ & $7.29 \mathrm{E}-05$ & Basic N1O2 & $\mathrm{C} 27 \mathrm{H} 21 \mathrm{~N} 1 \mathrm{O} 2$ & $1.07 \mathrm{E}-05$ \\
\hline Non-basic N1 & $\mathrm{C} 45 \mathrm{H} 35 \mathrm{~N} 1$ & 7.99E-06 & Acidic O1 & $\mathrm{C} 33 \mathrm{H} 52 \mathrm{O} 1$ & 0.00021 & Basic N1O2 & $\mathrm{C} 28 \mathrm{H} 23 \mathrm{~N} 1 \mathrm{O} 2$ & $1.47 \mathrm{E}-05$ \\
\hline Non-basic N1 & $\mathrm{C} 46 \mathrm{H} 37 \mathrm{~N} 1$ & $1.74 \mathrm{E}-06$ & Acidic O1 & $\mathrm{C} 34 \mathrm{H} 54 \mathrm{O} 1$ & 0.000263 & Basic N1O2 & $\mathrm{C} 29 \mathrm{H} 25 \mathrm{~N} 1 \mathrm{O} 2$ & $9.52 \mathrm{E}-06$ \\
\hline Non-basic N1 & $\mathrm{C} 40 \mathrm{H} 23 \mathrm{~N} 1$ & $1.45 \mathrm{E}-06$ & Acidic $\mathrm{O} 1$ & C35H56O1 & 0.00017 & Basic N1O2 & $\mathrm{C} 30 \mathrm{H} 27 \mathrm{~N} 1 \mathrm{O} 2$ & $1.06 \mathrm{E}-05$ \\
\hline
\end{tabular}




\begin{tabular}{|c|c|c|c|c|c|c|c|c|}
\hline Class & Formula & Mass & Class & Formula & Mass & Class & Formula & Mass \\
\hline Non-basic N1 & $\mathrm{C} 41 \mathrm{H} 25 \mathrm{~N} 1$ & $3.06 \mathrm{E}-06$ & Acidic $\mathrm{O} 1$ & C36H58O1 & 0.000249 & Basic N1O2 & $\mathrm{C} 31 \mathrm{H} 29 \mathrm{~N} 1 \mathrm{O} 2$ & 8.67E-06 \\
\hline Non-basic N1 & $\mathrm{C} 42 \mathrm{H} 27 \mathrm{~N} 1$ & $8.27 \mathrm{E}-06$ & Acidic $\mathrm{O} 1$ & $\mathrm{C} 37 \mathrm{H} 60 \mathrm{O} 1$ & $5.50 \mathrm{E}-05$ & Basic N1O2 & $\mathrm{C} 32 \mathrm{H} 31 \mathrm{~N} 1 \mathrm{O} 2$ & $6.95 \mathrm{E}-06$ \\
\hline Non-basic N1 & $\mathrm{C} 43 \mathrm{H} 29 \mathrm{~N} 1$ & $5.18 \mathrm{E}-06$ & Acidic $\mathrm{O} 1$ & $\mathrm{C} 38 \mathrm{H} 62 \mathrm{O} 1$ & 0.000111 & Basic N1O2 & $\mathrm{C} 33 \mathrm{H} 33 \mathrm{~N} 1 \mathrm{O} 2$ & $5.10 \mathrm{E}-06$ \\
\hline Non-basic N1 & $\mathrm{C} 44 \mathrm{H} 31 \mathrm{~N} 1$ & 7.33E-06 & Acidic $\mathrm{O} 1$ & C39H64O1 & $2.89 \mathrm{E}-05$ & Basic N1O2 & $\mathrm{C} 34 \mathrm{H} 35 \mathrm{~N} 1 \mathrm{O} 2$ & 4.43E-06 \\
\hline Non-basic N1 & $\mathrm{C} 44 \mathrm{H} 29 \mathrm{~N} 1$ & $5.40 \mathrm{E}-06$ & Acidic $\mathrm{O} 1$ & $\mathrm{C} 40 \mathrm{H} 66 \mathrm{O} 1$ & $2.05 \mathrm{E}-05$ & Basic N1O2 & $\mathrm{C} 35 \mathrm{H} 37 \mathrm{~N} 1 \mathrm{O} 2$ & $7.18 \mathrm{E}-06$ \\
\hline Non-basic N1 & $\mathrm{C} 45 \mathrm{H} 31 \mathrm{~N} 1$ & $5.23 \mathrm{E}-06$ & Acidic $\mathrm{O} 1$ & $\mathrm{C} 25 \mathrm{H} 34 \mathrm{O} 1$ & $2.46 \mathrm{E}-05$ & Basic N1O2 & $\mathrm{C} 26 \mathrm{H} 17 \mathrm{~N} 1 \mathrm{O} 2$ & $7.45 \mathrm{E}-07$ \\
\hline Non-basic N1 & $\mathrm{C} 46 \mathrm{H} 33 \mathrm{~N} 1$ & $2.32 \mathrm{E}-06$ & Acidic $\mathrm{O} 1$ & $\mathrm{C} 26 \mathrm{H} 36 \mathrm{O} 1$ & $4.36 \mathrm{E}-05$ & Basic N1O2 & $\mathrm{C} 27 \mathrm{H} 19 \mathrm{~N} 1 \mathrm{O} 2$ & $1.43 \mathrm{E}-06$ \\
\hline Non-basic N1 & $\mathrm{C} 47 \mathrm{H} 35 \mathrm{~N} 1$ & $2.44 \mathrm{E}-06$ & Acidic $\mathrm{O} 1$ & $\mathrm{C} 27 \mathrm{H} 38 \mathrm{O} 1$ & $9.51 \mathrm{E}-05$ & Basic N1O2 & $\mathrm{C} 28 \mathrm{H} 21 \mathrm{~N} 1 \mathrm{O} 2$ & $2.31 \mathrm{E}-06$ \\
\hline Non-basic N1 & $\mathrm{C} 45 \mathrm{H} 29 \mathrm{~N} 1$ & $5.57 \mathrm{E}-06$ & Acidic $\mathrm{O} 1$ & $\mathrm{C} 28 \mathrm{H} 40 \mathrm{O} 1$ & 0.000143 & Basic N1O2 & $\mathrm{C} 29 \mathrm{H} 23 \mathrm{~N} 1 \mathrm{O} 2$ & $3.98 \mathrm{E}-06$ \\
\hline Non-basic N1 & $\mathrm{C} 46 \mathrm{H} 31 \mathrm{~N} 1$ & $1.79 \mathrm{E}-06$ & Acidic $\mathrm{O} 1$ & $\mathrm{C} 29 \mathrm{H} 42 \mathrm{O} 1$ & 0.000116 & Basic N1O2 & $\mathrm{C} 30 \mathrm{H} 25 \mathrm{~N} 1 \mathrm{O} 2$ & $2.25 \mathrm{E}-06$ \\
\hline Non-basic N1 & $\mathrm{C} 47 \mathrm{H} 33 \mathrm{~N} 1$ & $1.93 \mathrm{E}-06$ & Acidic $\mathrm{O} 1$ & $\mathrm{C} 30 \mathrm{H} 44 \mathrm{O} 1$ & 0.000141 & Basic N1O2 & $\mathrm{C} 31 \mathrm{H} 27 \mathrm{~N} 1 \mathrm{O} 2$ & 4.71E-06 \\
\hline Non-basic N1O1 & $\mathrm{C} 27 \mathrm{H} 43 \mathrm{~N} 1 \mathrm{O} 1$ & $5.16 \mathrm{E}-06$ & Acidic $\mathrm{O} 1$ & $\mathrm{C} 31 \mathrm{H} 46 \mathrm{O} 1$ & $6.72 \mathrm{E}-05$ & Basic N1O2 & $\mathrm{C} 32 \mathrm{H} 29 \mathrm{~N} 1 \mathrm{O} 2$ & $8.81 \mathrm{E}-06$ \\
\hline Non-basic N1O1 & $\mathrm{C} 28 \mathrm{H} 45 \mathrm{~N} 1 \mathrm{O} 1$ & $8.14 \mathrm{E}-06$ & Acidic $\mathrm{O} 1$ & $\mathrm{C} 32 \mathrm{H} 48 \mathrm{O} 1$ & 0.00021 & Basic N1O2 & $\mathrm{C} 33 \mathrm{H} 31 \mathrm{~N} 1 \mathrm{O} 2$ & $3.58 \mathrm{E}-06$ \\
\hline Non-basic N1O1 & $\mathrm{C} 29 \mathrm{H} 47 \mathrm{~N} 1 \mathrm{O} 1$ & $1.41 \mathrm{E}-05$ & Acidic $\mathrm{O} 1$ & $\mathrm{C} 33 \mathrm{H} 50 \mathrm{O} 1$ & 0.000263 & Basic N1O2 & $\mathrm{C} 34 \mathrm{H} 33 \mathrm{~N} 1 \mathrm{O} 2$ & $3.36 \mathrm{E}-06$ \\
\hline Non-basic N1O1 & $\mathrm{C} 30 \mathrm{H} 49 \mathrm{~N} 1 \mathrm{O} 1$ & $1.49 \mathrm{E}-05$ & Acidic $\mathrm{O} 1$ & $\mathrm{C} 34 \mathrm{H} 52 \mathrm{O} 1$ & $9.75 \mathrm{E}-05$ & Basic N1O2 & $\mathrm{C} 36 \mathrm{H} 37 \mathrm{~N} 1 \mathrm{O} 2$ & $2.48 \mathrm{E}-06$ \\
\hline Non-basic N1O1 & $\mathrm{C} 31 \mathrm{H} 51 \mathrm{~N} 1 \mathrm{O} 1$ & $1.73 \mathrm{E}-05$ & Acidic $\mathrm{O} 1$ & $\mathrm{C} 35 \mathrm{H} 54 \mathrm{O} 1$ & 0.000214 & Basic N1O2 & $\mathrm{C} 37 \mathrm{H} 39 \mathrm{~N} 1 \mathrm{O} 2$ & $1.64 \mathrm{E}-06$ \\
\hline Non-basic N1O1 & $\mathrm{C} 32 \mathrm{H} 53 \mathrm{~N} 1 \mathrm{O} 1$ & $1.53 \mathrm{E}-05$ & Acidic $\mathrm{O} 1$ & $\mathrm{C} 16 \mathrm{H} 14 \mathrm{O} 1$ & $7.12 \mathrm{E}-05$ & Basic N1O2 & $\mathrm{C} 38 \mathrm{H} 41 \mathrm{~N} 1 \mathrm{O} 2$ & $3.12 \mathrm{E}-06$ \\
\hline Non-basic N1O1 & $\mathrm{C} 33 \mathrm{H} 55 \mathrm{~N} 1 \mathrm{O} 1$ & $1.12 \mathrm{E}-05$ & Acidic $\mathrm{O} 1$ & $\mathrm{C} 17 \mathrm{H} 16 \mathrm{O} 1$ & 0.000103 & Basic N1O2 & $\mathrm{C} 27 \mathrm{H} 17 \mathrm{~N} 1 \mathrm{O} 2$ & $2.33 \mathrm{E}-06$ \\
\hline Non-basic N1O1 & $\mathrm{C} 34 \mathrm{H} 57 \mathrm{~N} 1 \mathrm{O} 1$ & $1.29 \mathrm{E}-05$ & Acidic $\mathrm{O} 1$ & $\mathrm{C} 18 \mathrm{H} 18 \mathrm{O} 1$ & $3.87 \mathrm{E}-05$ & Basic N1O2 & $\mathrm{C} 28 \mathrm{H} 19 \mathrm{~N} 1 \mathrm{O} 2$ & $1.78 \mathrm{E}-06$ \\
\hline Non-basic N1O1 & $\mathrm{C} 35 \mathrm{H} 59 \mathrm{~N} 1 \mathrm{O} 1$ & $3.50 \mathrm{E}-06$ & Acidic $\mathrm{O} 1$ & $\mathrm{C} 19 \mathrm{H} 20 \mathrm{O} 1$ & $1.11 \mathrm{E}-05$ & Basic N1O2 & $\mathrm{C} 29 \mathrm{H} 21 \mathrm{~N} 1 \mathrm{O} 2$ & $2.00 \mathrm{E}-06$ \\
\hline Non-basic N1O1 & $\mathrm{C} 36 \mathrm{H} 61 \mathrm{~N} 1 \mathrm{O} 1$ & $7.00 \mathrm{E}-06$ & Acidic $\mathrm{O} 1$ & $\mathrm{C} 20 \mathrm{H} 22 \mathrm{O} 1$ & $1.28 \mathrm{E}-05$ & Basic N1O2 & $\mathrm{C} 30 \mathrm{H} 23 \mathrm{~N} 1 \mathrm{O} 2$ & $6.45 \mathrm{E}-06$ \\
\hline Non-basic N1O1 & $\mathrm{C} 37 \mathrm{H} 63 \mathrm{~N} 1 \mathrm{O} 1$ & $1.98 \mathrm{E}-06$ & Acidic $\mathrm{O} 1$ & $\mathrm{C} 21 \mathrm{H} 24 \mathrm{O} 1$ & $3.20 \mathrm{E}-05$ & Basic N1O2 & $\mathrm{C} 31 \mathrm{H} 25 \mathrm{~N} 1 \mathrm{O} 2$ & 4.63E-06 \\
\hline Non-basic N1O1 & $\mathrm{C} 23 \mathrm{H} 33 \mathrm{~N} 1 \mathrm{O} 1$ & $1.71 \mathrm{E}-06$ & Acidic $\mathrm{O} 1$ & $\mathrm{C} 22 \mathrm{H} 26 \mathrm{O} 1$ & $3.36 \mathrm{E}-05$ & Basic N1O2 & $\mathrm{C} 32 \mathrm{H} 27 \mathrm{~N} 1 \mathrm{O} 2$ & $2.58 \mathrm{E}-06$ \\
\hline Non-basic N1O1 & $\mathrm{C} 24 \mathrm{H} 35 \mathrm{~N} 1 \mathrm{O} 1$ & 4.94E-06 & Acidic $\mathrm{O} 1$ & $\mathrm{C} 23 \mathrm{H} 28 \mathrm{O} 1$ & $3.51 \mathrm{E}-05$ & Basic N1O2 & $\mathrm{C} 33 \mathrm{H} 29 \mathrm{~N} 1 \mathrm{O} 2$ & $2.59 \mathrm{E}-06$ \\
\hline Non-basic N1O1 & $\mathrm{C} 25 \mathrm{H} 37 \mathrm{~N} 1 \mathrm{O} 1$ & $1.90 \mathrm{E}-06$ & Acidic $\mathrm{O} 1$ & $\mathrm{C} 24 \mathrm{H} 30 \mathrm{O} 1$ & $3.10 \mathrm{E}-05$ & Basic N1O2 & $\mathrm{C} 34 \mathrm{H} 31 \mathrm{~N} 1 \mathrm{O} 2$ & $1.77 \mathrm{E}-06$ \\
\hline Non-basic N1O1 & $\mathrm{C} 26 \mathrm{H} 39 \mathrm{~N} 1 \mathrm{O} 1$ & $5.99 \mathrm{E}-06$ & Acidic $\mathrm{O} 1$ & $\mathrm{C} 25 \mathrm{H} 32 \mathrm{O} 1$ & $1.61 \mathrm{E}-05$ & Basic N1O2 & $\mathrm{C} 29 \mathrm{H} 19 \mathrm{~N} 1 \mathrm{O} 2$ & $4.53 \mathrm{E}-07$ \\
\hline Non-basic N1O1 & $\mathrm{C} 27 \mathrm{H} 41 \mathrm{~N} 1 \mathrm{O} 1$ & $9.39 \mathrm{E}-06$ & Acidic $\mathrm{O} 1$ & $\mathrm{C} 26 \mathrm{H} 34 \mathrm{O} 1$ & $4.80 \mathrm{E}-05$ & Basic N1O2 & $\mathrm{C} 30 \mathrm{H} 21 \mathrm{~N} 1 \mathrm{O} 2$ & $1.38 \mathrm{E}-06$ \\
\hline Non-basic N1O1 & $\mathrm{C} 28 \mathrm{H} 43 \mathrm{~N} 1 \mathrm{O} 1$ & $5.10 \mathrm{E}-06$ & Acidic $\mathrm{O} 1$ & $\mathrm{C} 27 \mathrm{H} 36 \mathrm{O} 1$ & $2.99 \mathrm{E}-05$ & Basic N1O2 & $\mathrm{C} 31 \mathrm{H} 23 \mathrm{~N} 1 \mathrm{O} 2$ & $2.31 \mathrm{E}-06$ \\
\hline Non-basic N1O1 & $\mathrm{C} 29 \mathrm{H} 45 \mathrm{~N} 1 \mathrm{O} 1$ & $1.30 \mathrm{E}-05$ & Acidic $\mathrm{O} 1$ & $\mathrm{C} 28 \mathrm{H} 38 \mathrm{O} 1$ & 0.000107 & Basic N1O2 & $\mathrm{C} 32 \mathrm{H} 25 \mathrm{~N} 1 \mathrm{O} 2$ & $7.54 \mathrm{E}-07$ \\
\hline Non-basic N1O1 & $\mathrm{C} 30 \mathrm{H} 47 \mathrm{~N} 1 \mathrm{O} 1$ & $2.17 \mathrm{E}-05$ & Acidic $\mathrm{O} 1$ & $\mathrm{C} 29 \mathrm{H} 40 \mathrm{O} 1$ & $5.46 \mathrm{E}-05$ & Basic N1O2 & $\mathrm{C} 30 \mathrm{H} 19 \mathrm{~N} 1 \mathrm{O} 2$ & $3.77 \mathrm{E}-07$ \\
\hline Non-basic N1O1 & $\mathrm{C} 31 \mathrm{H} 49 \mathrm{~N} 1 \mathrm{O} 1$ & $1.55 \mathrm{E}-05$ & Acidic $\mathrm{O} 1$ & $\mathrm{C} 30 \mathrm{H} 42 \mathrm{O} 1$ & $1.53 \mathrm{E}-05$ & Basic N1O2 & $\mathrm{C} 31 \mathrm{H} 21 \mathrm{~N} 1 \mathrm{O} 2$ & $3.31 \mathrm{E}-07$ \\
\hline Non-basic N1O1 & $\mathrm{C} 32 \mathrm{H} 51 \mathrm{~N} 1 \mathrm{O} 1$ & $1.12 \mathrm{E}-05$ & Acidic $\mathrm{O} 1$ & $\mathrm{C} 31 \mathrm{H} 44 \mathrm{O} 1$ & 0.000106 & Basic N1O2 & $\mathrm{C} 32 \mathrm{H} 23 \mathrm{~N} 1 \mathrm{O} 2$ & $1.29 \mathrm{E}-06$ \\
\hline Non-basic N1O1 & C33H53N1O1 & $1.16 \mathrm{E}-05$ & Acidic $\mathrm{O} 1$ & $\mathrm{C} 32 \mathrm{H} 46 \mathrm{O} 1$ & $7.35 \mathrm{E}-05$ & Basic N1O2 & $\mathrm{C} 33 \mathrm{H} 25 \mathrm{~N} 1 \mathrm{O} 2$ & $3.78 \mathrm{E}-06$ \\
\hline Non-basic N1O1 & $\mathrm{C} 34 \mathrm{H} 55 \mathrm{~N} 1 \mathrm{O} 1$ & $1.10 \mathrm{E}-05$ & Acidic $\mathrm{O} 1$ & $\mathrm{C} 33 \mathrm{H} 48 \mathrm{O} 1$ & $7.58 \mathrm{E}-05$ & Basic N1O2 & $\mathrm{C} 34 \mathrm{H} 27 \mathrm{~N} 1 \mathrm{O} 2$ & $2.07 \mathrm{E}-07$ \\
\hline Non-basic N1O1 & $\mathrm{C} 35 \mathrm{H} 57 \mathrm{~N} 1 \mathrm{O} 1$ & $1.02 \mathrm{E}-05$ & Acidic $\mathrm{O} 1$ & $\mathrm{C} 34 \mathrm{H} 50 \mathrm{O} 1$ & $4.25 \mathrm{E}-05$ & Basic N1O2 & $\mathrm{C} 37 \mathrm{H} 31 \mathrm{~N} 1 \mathrm{O} 2$ & $5.94 \mathrm{E}-07$ \\
\hline Non-basic N1O1 & $\mathrm{C} 36 \mathrm{H} 59 \mathrm{~N} 1 \mathrm{O} 1$ & $1.88 \mathrm{E}-06$ & Acidic $\mathrm{O} 1$ & $\mathrm{C} 35 \mathrm{H} 52 \mathrm{O} 1$ & $1.89 \mathrm{E}-05$ & Basic N1O2 & $\mathrm{C} 38 \mathrm{H} 33 \mathrm{~N} 1 \mathrm{O} 2$ & $4.95 \mathrm{E}-07$ \\
\hline Non-basic N1O1 & $\mathrm{C} 14 \mathrm{H} 13 \mathrm{~N} 1 \mathrm{O} 1$ & $1.06 \mathrm{E}-06$ & Acidic $\mathrm{O} 1$ & $\mathrm{C} 36 \mathrm{H} 54 \mathrm{O} 1$ & $5.77 \mathrm{E}-05$ & Basic N1O2 & $\mathrm{C} 39 \mathrm{H} 35 \mathrm{~N} 1 \mathrm{O} 2$ & $1.21 \mathrm{E}-06$ \\
\hline Non-basic N1O1 & $\mathrm{C} 15 \mathrm{H} 15 \mathrm{~N} 1 \mathrm{O} 1$ & $3.51 \mathrm{E}-06$ & Acidic $\mathrm{O} 1$ & C37H56O1 & $4.54 \mathrm{E}-05$ & Basic N1O2 & $\mathrm{C} 40 \mathrm{H} 37 \mathrm{~N} 1 \mathrm{O} 2$ & $3.16 \mathrm{E}-06$ \\
\hline
\end{tabular}




\begin{tabular}{|c|c|c|c|c|c|c|c|c|}
\hline Class & Formula & Mass & Class & Formula & Mass & Class & Formula & Mass \\
\hline Non-basic N1O1 & C16H17N1O1 & $9.33 \mathrm{E}-06$ & Acidic $\mathrm{O} 1$ & $\mathrm{C} 17 \mathrm{H} 14 \mathrm{O} 1$ & 0.0002 & Basic N1O2 & $\mathrm{C} 35 \mathrm{H} 25 \mathrm{~N} 1 \mathrm{O} 2$ & $3.10 \mathrm{E}-07$ \\
\hline Non-basic N1O1 & C17H19N1O1 & $1.30 \mathrm{E}-06$ & Acidic $\mathrm{O} 1$ & $\mathrm{C} 18 \mathrm{H} 16 \mathrm{O} 1$ & 0.000358 & Basic N1O2 & $\mathrm{C} 36 \mathrm{H} 27 \mathrm{~N} 1 \mathrm{O} 2$ & $2.37 \mathrm{E}-07$ \\
\hline Non-basic N1O1 & $\mathrm{C} 18 \mathrm{H} 21 \mathrm{~N} 1 \mathrm{O} 1$ & $2.49 \mathrm{E}-06$ & Acidic $\mathrm{O} 1$ & $\mathrm{C} 19 \mathrm{H} 18 \mathrm{O} 1$ & 0.000291 & Basic N1O3 & $\mathrm{C} 25 \mathrm{H} 43 \mathrm{~N} 1 \mathrm{O} 3$ & $1.02 \mathrm{E}-05$ \\
\hline Non-basic N1O1 & C19H23N1O1 & $4.00 \mathrm{E}-06$ & Acidic O1 & $\mathrm{C} 20 \mathrm{H} 20 \mathrm{O} 1$ & 0.000106 & Basic N1O3 & $\mathrm{C} 26 \mathrm{H} 45 \mathrm{~N} 1 \mathrm{O} 3$ & $2.13 \mathrm{E}-05$ \\
\hline Non-basic N1O1 & $\mathrm{C} 20 \mathrm{H} 25 \mathrm{~N} 1 \mathrm{O} 1$ & $4.20 \mathrm{E}-06$ & Acidic $\mathrm{O} 1$ & $\mathrm{C} 21 \mathrm{H} 22 \mathrm{O} 1$ & $3.20 \mathrm{E}-05$ & Basic N1O3 & $\mathrm{C} 27 \mathrm{H} 47 \mathrm{~N} 1 \mathrm{O} 3$ & $2.32 \mathrm{E}-05$ \\
\hline Non-basic N1O1 & $\mathrm{C} 21 \mathrm{H} 27 \mathrm{~N} 1 \mathrm{O} 1$ & $2.52 \mathrm{E}-06$ & Acidic O1 & $\mathrm{C} 22 \mathrm{H} 24 \mathrm{O} 1$ & $2.60 \mathrm{E}-05$ & Basic N1O3 & $\mathrm{C} 28 \mathrm{H} 49 \mathrm{~N} 1 \mathrm{O} 3$ & $1.31 \mathrm{E}-05$ \\
\hline Non-basic N1O1 & $\mathrm{C} 22 \mathrm{H} 29 \mathrm{~N} 1 \mathrm{O} 1$ & $3.32 \mathrm{E}-06$ & Acidic $\mathrm{O} 1$ & $\mathrm{C} 23 \mathrm{H} 26 \mathrm{O} 1$ & $3.88 \mathrm{E}-05$ & Basic N1O3 & C29H51N1O3 & $1.98 \mathrm{E}-05$ \\
\hline Non-basic N1O1 & $\mathrm{C} 23 \mathrm{H} 31 \mathrm{~N} 1 \mathrm{O} 1$ & $4.83 \mathrm{E}-06$ & Acidic $\mathrm{O} 1$ & $\mathrm{C} 24 \mathrm{H} 28 \mathrm{O} 1$ & $3.54 \mathrm{E}-05$ & Basic N1O3 & $\mathrm{C} 30 \mathrm{H} 53 \mathrm{~N} 1 \mathrm{O} 3$ & $1.84 \mathrm{E}-05$ \\
\hline Non-basic N1O1 & $\mathrm{C} 24 \mathrm{H} 33 \mathrm{~N} 1 \mathrm{O} 1$ & $6.84 \mathrm{E}-06$ & Acidic O1 & $\mathrm{C} 26 \mathrm{H} 32 \mathrm{O} 1$ & $4.13 \mathrm{E}-05$ & Basic N1O3 & C31H55N1O3 & $1.63 \mathrm{E}-05$ \\
\hline Non-basic N1O1 & $\mathrm{C} 25 \mathrm{H} 35 \mathrm{~N} 1 \mathrm{O} 1$ & $5.50 \mathrm{E}-06$ & Acidic O1 & $\mathrm{C} 27 \mathrm{H} 34 \mathrm{O} 1$ & $6.72 \mathrm{E}-05$ & Basic N1O3 & $\mathrm{C} 32 \mathrm{H} 57 \mathrm{~N} 1 \mathrm{O} 3$ & $1.65 \mathrm{E}-05$ \\
\hline Non-basic N1O1 & $\mathrm{C} 26 \mathrm{H} 37 \mathrm{~N} 1 \mathrm{O} 1$ & $9.13 \mathrm{E}-06$ & Acidic O1 & $\mathrm{C} 28 \mathrm{H} 36 \mathrm{O} 1$ & 0.000137 & Basic N1O3 & C33H59N1O3 & $1.63 \mathrm{E}-05$ \\
\hline Non-basic N1O1 & $\mathrm{C} 27 \mathrm{H} 39 \mathrm{~N} 1 \mathrm{O} 1$ & $8.03 \mathrm{E}-06$ & Acidic $\mathrm{O} 1$ & $\mathrm{C} 29 \mathrm{H} 38 \mathrm{O} 1$ & $9.94 \mathrm{E}-05$ & Basic N1O3 & C34H61N1O3 & $1.26 \mathrm{E}-05$ \\
\hline Non-basic N1O1 & $\mathrm{C} 28 \mathrm{H} 41 \mathrm{~N} 1 \mathrm{O} 1$ & $1.26 \mathrm{E}-05$ & Acidic $\mathrm{O} 1$ & $\mathrm{C} 30 \mathrm{H} 40 \mathrm{O} 1$ & $4.62 \mathrm{E}-05$ & Basic N1O3 & C35H63N1O3 & $1.18 \mathrm{E}-05$ \\
\hline Non-basic N1O1 & $\mathrm{C} 29 \mathrm{H} 43 \mathrm{~N} 1 \mathrm{O} 1$ & $1.18 \mathrm{E}-05$ & Acidic O1 & $\mathrm{C} 31 \mathrm{H} 42 \mathrm{O} 1$ & 0.00011 & Basic N1O3 & C36H65N1O3 & $6.41 \mathrm{E}-06$ \\
\hline Non-basic N1O1 & $\mathrm{C} 30 \mathrm{H} 45 \mathrm{~N} 1 \mathrm{O} 1$ & $1.47 \mathrm{E}-05$ & Acidic $\mathrm{O} 1$ & $\mathrm{C} 32 \mathrm{H} 44 \mathrm{O} 1$ & $6.73 \mathrm{E}-05$ & Basic N1O3 & $\mathrm{C} 37 \mathrm{H} 67 \mathrm{~N} 1 \mathrm{O} 3$ & $8.90 \mathrm{E}-06$ \\
\hline Non-basic N1O1 & $\mathrm{C} 31 \mathrm{H} 47 \mathrm{~N} 1 \mathrm{O} 1$ & $1.01 \mathrm{E}-05$ & Acidic $\mathrm{O} 1$ & $\mathrm{C} 33 \mathrm{H} 46 \mathrm{O} 1$ & $8.13 \mathrm{E}-05$ & Basic N1O3 & C38H69N1O3 & 7.79E-06 \\
\hline Non-basic N1O1 & $\mathrm{C} 32 \mathrm{H} 49 \mathrm{~N} 1 \mathrm{O} 1$ & $1.35 \mathrm{E}-05$ & Acidic $\mathrm{O} 1$ & $\mathrm{C} 34 \mathrm{H} 48 \mathrm{O} 1$ & $3.06 \mathrm{E}-05$ & Basic N1O3 & C39H71N1O3 & $5.63 \mathrm{E}-06$ \\
\hline Non-basic N1O1 & C33H51N1O1 & $7.00 \mathrm{E}-06$ & Acidic $\mathrm{O} 1$ & $\mathrm{C} 35 \mathrm{H} 50 \mathrm{O} 1$ & 0.000103 & Basic N1O3 & $\mathrm{C} 40 \mathrm{H} 73 \mathrm{~N} 1 \mathrm{O} 3$ & $2.35 \mathrm{E}-06$ \\
\hline Non-basic N1O1 & C34H53N1O1 & $3.60 \mathrm{E}-06$ & Acidic $\mathrm{O} 1$ & $\mathrm{C} 16 \mathrm{H} 10 \mathrm{O} 1$ & $3.46 \mathrm{E}-05$ & Basic N1O3 & $\mathrm{C} 41 \mathrm{H} 75 \mathrm{~N} 1 \mathrm{O} 3$ & $2.87 \mathrm{E}-07$ \\
\hline Non-basic N1O1 & C15H13N1O1 & $1.88 \mathrm{E}-05$ & Acidic $\mathrm{O} 1$ & $\mathrm{C} 17 \mathrm{H} 12 \mathrm{O} 1$ & 0.000558 & Basic N1O3 & $\mathrm{C} 26 \mathrm{H} 43 \mathrm{~N} 1 \mathrm{O} 3$ & $2.00 \mathrm{E}-05$ \\
\hline Non-basic N1O1 & C16H15N1O1 & $2.56 \mathrm{E}-05$ & Acidic $\mathrm{O} 1$ & $\mathrm{C} 18 \mathrm{H} 14 \mathrm{O} 1$ & 0.00087 & Basic N1O3 & $\mathrm{C} 27 \mathrm{H} 45 \mathrm{~N} 1 \mathrm{O} 3$ & $1.87 \mathrm{E}-05$ \\
\hline Non-basic N1O1 & $\mathrm{C} 17 \mathrm{H} 17 \mathrm{~N} 1 \mathrm{O} 1$ & $1.97 \mathrm{E}-05$ & Acidic O1 & $\mathrm{C} 19 \mathrm{H} 16 \mathrm{O} 1$ & 0.000837 & Basic N1O3 & $\mathrm{C} 28 \mathrm{H} 47 \mathrm{~N} 1 \mathrm{O} 3$ & $1.28 \mathrm{E}-05$ \\
\hline Non-basic N1O1 & C18H19N1O1 & $1.03 \mathrm{E}-05$ & Acidic O1 & $\mathrm{C} 20 \mathrm{H} 18 \mathrm{O} 1$ & 0.000469 & Basic N1O3 & $\mathrm{C} 29 \mathrm{H} 49 \mathrm{~N} 1 \mathrm{O} 3$ & $5.73 \mathrm{E}-05$ \\
\hline Non-basic N1O1 & $\mathrm{C} 19 \mathrm{H} 21 \mathrm{~N} 1 \mathrm{O} 1$ & $4.89 \mathrm{E}-06$ & Acidic O1 & $\mathrm{C} 21 \mathrm{H} 20 \mathrm{O} 1$ & 0.000307 & Basic N1O3 & $\mathrm{C} 30 \mathrm{H} 51 \mathrm{~N} 1 \mathrm{O} 3$ & $2.04 \mathrm{E}-05$ \\
\hline Non-basic N1O1 & $\mathrm{C} 20 \mathrm{H} 23 \mathrm{~N} 1 \mathrm{O} 1$ & $6.70 \mathrm{E}-06$ & Acidic O1 & $\mathrm{C} 22 \mathrm{H} 22 \mathrm{O} 1$ & 0.000239 & Basic N1O3 & $\mathrm{C} 31 \mathrm{H} 53 \mathrm{~N} 1 \mathrm{O} 3$ & $1.48 \mathrm{E}-05$ \\
\hline Non-basic N1O1 & $\mathrm{C} 21 \mathrm{H} 25 \mathrm{~N} 1 \mathrm{O} 1$ & $8.05 \mathrm{E}-06$ & Acidic O1 & $\mathrm{C} 23 \mathrm{H} 24 \mathrm{O} 1$ & 0.00013 & Basic N1O3 & $\mathrm{C} 32 \mathrm{H} 55 \mathrm{~N} 1 \mathrm{O} 3$ & $1.47 \mathrm{E}-05$ \\
\hline Non-basic N1O1 & $\mathrm{C} 22 \mathrm{H} 27 \mathrm{~N} 1 \mathrm{O} 1$ & $9.38 \mathrm{E}-06$ & Acidic O1 & $\mathrm{C} 24 \mathrm{H} 26 \mathrm{O} 1$ & 0.000205 & Basic N1O3 & $\mathrm{C} 33 \mathrm{H} 57 \mathrm{~N} 1 \mathrm{O} 3$ & $1.36 \mathrm{E}-05$ \\
\hline Non-basic N1O1 & $\mathrm{C} 23 \mathrm{H} 29 \mathrm{~N} 1 \mathrm{O} 1$ & $1.17 \mathrm{E}-05$ & Acidic O1 & $\mathrm{C} 25 \mathrm{H} 28 \mathrm{O} 1$ & 0.000163 & Basic N1O3 & C34H59N1O3 & $1.39 \mathrm{E}-05$ \\
\hline Non-basic N1O1 & $\mathrm{C} 24 \mathrm{H} 31 \mathrm{~N} 1 \mathrm{O} 1$ & $1.65 \mathrm{E}-05$ & Acidic $\mathrm{O} 1$ & $\mathrm{C} 26 \mathrm{H} 30 \mathrm{O} 1$ & 0.000133 & Basic N1O3 & C35H61N1O3 & $1.10 \mathrm{E}-05$ \\
\hline Non-basic N1O1 & $\mathrm{C} 25 \mathrm{H} 33 \mathrm{~N} 1 \mathrm{O} 1$ & $1.54 \mathrm{E}-05$ & Acidic $\mathrm{O} 1$ & $\mathrm{C} 27 \mathrm{H} 32 \mathrm{O} 1$ & 0.000103 & Basic N1O3 & C36H63N1O3 & $1.24 \mathrm{E}-05$ \\
\hline Non-basic N1O1 & $\mathrm{C} 26 \mathrm{H} 35 \mathrm{~N} 1 \mathrm{O} 1$ & $1.70 \mathrm{E}-05$ & Acidic $\mathrm{O} 1$ & $\mathrm{C} 28 \mathrm{H} 34 \mathrm{O} 1$ & $6.39 \mathrm{E}-05$ & Basic N1O3 & C37H65N1O3 & $5.60 \mathrm{E}-06$ \\
\hline Non-basic N1O1 & $\mathrm{C} 27 \mathrm{H} 37 \mathrm{~N} 1 \mathrm{O} 1$ & $1.94 \mathrm{E}-05$ & Acidic O1 & $\mathrm{C} 29 \mathrm{H} 36 \mathrm{O} 1$ & 0.000126 & Basic N1O3 & C38H67N1O3 & $3.93 \mathrm{E}-06$ \\
\hline Non-basic N1O1 & C28H39N1O1 & $2.82 \mathrm{E}-05$ & Acidic O1 & $\mathrm{C} 30 \mathrm{H} 38 \mathrm{O} 1$ & 0.000116 & Basic N1O3 & C39H69N1O3 & $2.13 \mathrm{E}-06$ \\
\hline Non-basic N1O1 & $\mathrm{C} 29 \mathrm{H} 41 \mathrm{~N} 1 \mathrm{O} 1$ & $2.75 \mathrm{E}-05$ & Acidic $\mathrm{O} 1$ & $\mathrm{C} 31 \mathrm{H} 40 \mathrm{O} 1$ & $4.01 \mathrm{E}-05$ & Basic N1O3 & $\mathrm{C} 40 \mathrm{H} 71 \mathrm{~N} 1 \mathrm{O} 3$ & $5.21 \mathrm{E}-06$ \\
\hline Non-basic N1O1 & $\mathrm{C} 30 \mathrm{H} 43 \mathrm{~N} 1 \mathrm{O} 1$ & $1.84 \mathrm{E}-05$ & Acidic O1 & $\mathrm{C} 32 \mathrm{H} 43 \mathrm{O} 1$ & $4.14 \mathrm{E}-05$ & Basic N1O3 & $\mathrm{C} 41 \mathrm{H} 73 \mathrm{~N} 1 \mathrm{O} 3$ & $3.53 \mathrm{E}-06$ \\
\hline Non-basic N1O1 & $\mathrm{C} 31 \mathrm{H} 45 \mathrm{~N} 1 \mathrm{O} 1$ & $1.22 \mathrm{E}-05$ & Acidic $\mathrm{O} 1$ & $\mathrm{C} 33 \mathrm{H} 44 \mathrm{O} 1$ & 0.000125 & Basic N1O3 & $\mathrm{C} 42 \mathrm{H} 75 \mathrm{~N} 1 \mathrm{O} 3$ & $4.35 \mathrm{E}-06$ \\
\hline Non-basic N1O1 & $\mathrm{C} 32 \mathrm{H} 47 \mathrm{~N} 1 \mathrm{O} 1$ & $2.18 \mathrm{E}-05$ & Acidic O1 & $\mathrm{C} 34 \mathrm{H} 46 \mathrm{O} 1$ & $6.38 \mathrm{E}-05$ & Basic N1O3 & $\mathrm{C} 43 \mathrm{H} 77 \mathrm{~N} 1 \mathrm{O} 3$ & $3.73 \mathrm{E}-06$ \\
\hline Non-basic N1O1 & $\mathrm{C} 33 \mathrm{H} 49 \mathrm{~N} 1 \mathrm{O} 1$ & $1.23 \mathrm{E}-05$ & Acidic $\mathrm{O} 1$ & $\mathrm{C} 35 \mathrm{H} 48 \mathrm{O} 1$ & $3.69 \mathrm{E}-05$ & Basic N1O3 & $\mathrm{C} 15 \mathrm{H} 19 \mathrm{~N} 1 \mathrm{O} 3$ & $2.60 \mathrm{E}-07$ \\
\hline
\end{tabular}




\begin{tabular}{|c|c|c|c|c|c|c|c|c|}
\hline Class & Formula & Mass & Class & Formula & Mass & Class & Formula & Mass \\
\hline Non-basic N1O1 & C34H51N1O1 & $9.21 \mathrm{E}-06$ & Acidic $\mathrm{O} 1$ & $\mathrm{C} 36 \mathrm{H} 50 \mathrm{O} 1$ & $3.82 \mathrm{E}-05$ & Basic N1O3 & $\mathrm{C} 16 \mathrm{H} 21 \mathrm{~N} 1 \mathrm{O} 3$ & $2.64 \mathrm{E}-07$ \\
\hline Non-basic N1O1 & C35H53N1O1 & $3.94 \mathrm{E}-06$ & Acidic $\mathrm{O} 1$ & $\mathrm{C} 37 \mathrm{H} 52 \mathrm{O} 1$ & 7.77E-05 & Basic N1O3 & $\mathrm{C} 17 \mathrm{H} 23 \mathrm{~N} 1 \mathrm{O} 3$ & $1.19 \mathrm{E}-07$ \\
\hline Non-basic N1O1 & C15H11N1O1 & $7.56 \mathrm{E}-07$ & Acidic $\mathrm{O} 1$ & $\mathrm{C} 18 \mathrm{H} 12 \mathrm{O} 1$ & 0.000226 & Basic N1O3 & $\mathrm{C} 18 \mathrm{H} 25 \mathrm{~N} 1 \mathrm{O} 3$ & $1.65 \mathrm{E}-06$ \\
\hline Non-basic N1O1 & C16H13N1O1 & $3.25 \mathrm{E}-05$ & Acidic O1 & $\mathrm{C} 19 \mathrm{H} 14 \mathrm{O} 1$ & 0.000462 & Basic N1O3 & $\mathrm{C} 19 \mathrm{H} 27 \mathrm{~N} 1 \mathrm{O} 3$ & $2.95 \mathrm{E}-06$ \\
\hline Non-basic N1O1 & C17H15N1O1 & $5.49 \mathrm{E}-05$ & Acidic $\mathrm{O} 1$ & $\mathrm{C} 20 \mathrm{H} 16 \mathrm{O} 1$ & 0.000525 & Basic N1O3 & $\mathrm{C} 20 \mathrm{H} 29 \mathrm{~N} 1 \mathrm{O} 3$ & $2.77 \mathrm{E}-07$ \\
\hline Non-basic N1O1 & C18H17N1O1 & $4.78 \mathrm{E}-05$ & Acidic O1 & $\mathrm{C} 21 \mathrm{H} 18 \mathrm{O} 1$ & 0.000454 & Basic N1O3 & $\mathrm{C} 21 \mathrm{H} 31 \mathrm{~N} 1 \mathrm{O} 3$ & $8.27 \mathrm{E}-06$ \\
\hline Non-basic N1O1 & C19H19N1O1 & $3.47 \mathrm{E}-05$ & Acidic $\mathrm{O} 1$ & $\mathrm{C} 22 \mathrm{H} 20 \mathrm{O} 1$ & 0.000372 & Basic N1O3 & $\mathrm{C} 22 \mathrm{H} 33 \mathrm{~N} 1 \mathrm{O} 3$ & $8.91 \mathrm{E}-06$ \\
\hline Non-basic N1O1 & $\mathrm{C} 20 \mathrm{H} 21 \mathrm{~N} 1 \mathrm{O} 1$ & $2.28 \mathrm{E}-05$ & Acidic $\mathrm{O} 1$ & $\mathrm{C} 23 \mathrm{H} 22 \mathrm{O} 1$ & 0.000323 & Basic N1O3 & $\mathrm{C} 23 \mathrm{H} 35 \mathrm{~N} 1 \mathrm{O} 3$ & $9.36 \mathrm{E}-06$ \\
\hline Non-basic N1O1 & $\mathrm{C} 21 \mathrm{H} 23 \mathrm{~N} 1 \mathrm{O} 1$ & $2.62 \mathrm{E}-05$ & Acidic O1 & $\mathrm{C} 24 \mathrm{H} 24 \mathrm{O} 1$ & 0.000184 & Basic N1O3 & $\mathrm{C} 24 \mathrm{H} 37 \mathrm{~N} 1 \mathrm{O} 3$ & $1.52 \mathrm{E}-05$ \\
\hline Non-basic N1O1 & $\mathrm{C} 22 \mathrm{H} 25 \mathrm{~N} 1 \mathrm{O} 1$ & $2.60 \mathrm{E}-05$ & Acidic O1 & $\mathrm{C} 25 \mathrm{H} 26 \mathrm{O} 1$ & 0.000205 & Basic N1O3 & $\mathrm{C} 25 \mathrm{H} 39 \mathrm{~N} 1 \mathrm{O} 3$ & $9.88 \mathrm{E}-06$ \\
\hline Non-basic N1O1 & $\mathrm{C} 23 \mathrm{H} 27 \mathrm{~N} 1 \mathrm{O} 1$ & $1.61 \mathrm{E}-05$ & Acidic O1 & $\mathrm{C} 26 \mathrm{H} 28 \mathrm{O} 1$ & $7.74 \mathrm{E}-05$ & Basic N1O3 & $\mathrm{C} 26 \mathrm{H} 41 \mathrm{~N} 1 \mathrm{O} 3$ & $9.73 \mathrm{E}-06$ \\
\hline Non-basic N1O1 & C24H29N1O1 & $1.80 \mathrm{E}-05$ & Acidic $\mathrm{O} 1$ & $\mathrm{C} 27 \mathrm{H} 30 \mathrm{O} 1$ & 0.000146 & Basic N1O3 & $\mathrm{C} 27 \mathrm{H} 43 \mathrm{~N} 1 \mathrm{O} 3$ & $1.01 \mathrm{E}-05$ \\
\hline Non-basic N1O1 & $\mathrm{C} 25 \mathrm{H} 31 \mathrm{~N} 1 \mathrm{O} 1$ & $1.74 \mathrm{E}-05$ & Acidic $\mathrm{O} 1$ & $\mathrm{C} 28 \mathrm{H} 32 \mathrm{O} 1$ & 0.000107 & Basic N1O3 & $\mathrm{C} 28 \mathrm{H} 45 \mathrm{~N} 1 \mathrm{O} 3$ & $2.14 \mathrm{E}-05$ \\
\hline Non-basic N1O1 & $\mathrm{C} 26 \mathrm{H} 33 \mathrm{~N} 1 \mathrm{O} 1$ & $2.02 \mathrm{E}-05$ & Acidic O1 & $\mathrm{C} 29 \mathrm{H} 34 \mathrm{O} 1$ & $7.35 \mathrm{E}-05$ & Basic N1O3 & $\mathrm{C} 29 \mathrm{H} 47 \mathrm{~N} 1 \mathrm{O} 3$ & $1.60 \mathrm{E}-05$ \\
\hline Non-basic N1O1 & $\mathrm{C} 27 \mathrm{H} 35 \mathrm{~N} 1 \mathrm{O} 1$ & $1.45 \mathrm{E}-05$ & Acidic $\mathrm{O} 1$ & $\mathrm{C} 30 \mathrm{H} 36 \mathrm{O} 1$ & $3.15 \mathrm{E}-05$ & Basic N1O3 & $\mathrm{C} 30 \mathrm{H} 49 \mathrm{~N} 1 \mathrm{O} 3$ & $1.88 \mathrm{E}-05$ \\
\hline Non-basic N1O1 & $\mathrm{C} 28 \mathrm{H} 37 \mathrm{~N} 1 \mathrm{O} 1$ & $1.89 \mathrm{E}-05$ & Acidic $\mathrm{O} 1$ & $\mathrm{C} 31 \mathrm{H} 38 \mathrm{O} 1$ & $3.15 \mathrm{E}-05$ & Basic N1O3 & $\mathrm{C} 31 \mathrm{H} 51 \mathrm{~N} 1 \mathrm{O} 3$ & $7.91 \mathrm{E}-06$ \\
\hline Non-basic N1O1 & C29H39N1O1 & $2.30 \mathrm{E}-05$ & Acidic $\mathrm{O} 1$ & $\mathrm{C} 32 \mathrm{H} 40 \mathrm{O} 1$ & $6.90 \mathrm{E}-05$ & Basic N1O3 & $\mathrm{C} 32 \mathrm{H} 53 \mathrm{~N} 1 \mathrm{O} 3$ & $1.20 \mathrm{E}-05$ \\
\hline Non-basic N1O1 & $\mathrm{C} 30 \mathrm{H} 41 \mathrm{~N} 1 \mathrm{O} 1$ & $1.61 \mathrm{E}-05$ & Acidic $\mathrm{O} 1$ & $\mathrm{C} 33 \mathrm{H} 42 \mathrm{O} 1$ & $4.76 \mathrm{E}-05$ & Basic N1O3 & $\mathrm{C} 33 \mathrm{H} 55 \mathrm{~N} 1 \mathrm{O} 3$ & $9.54 \mathrm{E}-06$ \\
\hline Non-basic N1O1 & $\mathrm{C} 31 \mathrm{H} 43 \mathrm{~N} 1 \mathrm{O} 1$ & $1.12 \mathrm{E}-05$ & Acidic $\mathrm{O} 1$ & C19H12O1 & $9.47 \mathrm{E}-06$ & Basic N1O3 & $\mathrm{C} 34 \mathrm{H} 57 \mathrm{~N} 1 \mathrm{O} 3$ & $1.11 \mathrm{E}-05$ \\
\hline Non-basic N1O1 & $\mathrm{C} 32 \mathrm{H} 45 \mathrm{~N} 1 \mathrm{O} 1$ & $1.07 \mathrm{E}-05$ & Acidic $\mathrm{O} 1$ & $\mathrm{C} 20 \mathrm{H} 14 \mathrm{O} 1$ & 0.000165 & Basic N1O3 & C35H59N1O3 & $5.57 \mathrm{E}-06$ \\
\hline Non-basic N1O1 & $\mathrm{C} 33 \mathrm{H} 47 \mathrm{~N} 1 \mathrm{O} 1$ & $8.32 \mathrm{E}-06$ & Acidic $\mathrm{O} 1$ & $\mathrm{C} 21 \mathrm{H} 16 \mathrm{O} 1$ & 0.000235 & Basic N1O3 & $\mathrm{C} 36 \mathrm{H} 61 \mathrm{~N} 1 \mathrm{O} 3$ & $4.29 \mathrm{E}-06$ \\
\hline Non-basic N1O1 & C34H49N1O1 & $5.62 \mathrm{E}-06$ & Acidic O1 & $\mathrm{C} 22 \mathrm{H} 18 \mathrm{O} 1$ & 0.000408 & Basic N1O3 & C37H63N1O3 & $5.19 \mathrm{E}-06$ \\
\hline Non-basic N1O1 & C35H51N1O1 & $1.62 \mathrm{E}-06$ & Acidic O1 & $\mathrm{C} 23 \mathrm{H} 20 \mathrm{O} 1$ & 0.00031 & Basic N1O3 & C38H65N1O3 & $9.40 \mathrm{E}-06$ \\
\hline Non-basic N1O1 & C36H53N1O1 & $6.81 \mathrm{E}-06$ & Acidic O1 & $\mathrm{C} 24 \mathrm{H} 22 \mathrm{O} 1$ & 0.000307 & Basic N1O3 & $\mathrm{C} 18 \mathrm{H} 23 \mathrm{~N} 1 \mathrm{O} 3$ & 7.06E-07 \\
\hline Non-basic N1O1 & C15H9N1O1 & $1.06 \mathrm{E}-06$ & Acidic O1 & $\mathrm{C} 25 \mathrm{H} 24 \mathrm{O} 1$ & 0.000199 & Basic N1O3 & $\mathrm{C} 19 \mathrm{H} 25 \mathrm{~N} 1 \mathrm{O} 3$ & $2.24 \mathrm{E}-06$ \\
\hline Non-basic N1O1 & C16H11N1O1 & $1.70 \mathrm{E}-05$ & Acidic O1 & $\mathrm{C} 26 \mathrm{H} 26 \mathrm{O} 1$ & 0.000145 & Basic N1O3 & $\mathrm{C} 20 \mathrm{H} 27 \mathrm{~N} 1 \mathrm{O} 3$ & $1.70 \mathrm{E}-07$ \\
\hline Non-basic N1O1 & C17H13N1O1 & 0.000168 & Acidic O1 & $\mathrm{C} 27 \mathrm{H} 28 \mathrm{O} 1$ & $5.42 \mathrm{E}-05$ & Basic N1O3 & $\mathrm{C} 21 \mathrm{H} 29 \mathrm{~N} 1 \mathrm{O} 3$ & $4.48 \mathrm{E}-06$ \\
\hline Non-basic N1O1 & C18H15N1O1 & 0.000439 & Acidic O1 & $\mathrm{C} 28 \mathrm{H} 30 \mathrm{O} 1$ & 0.00011 & Basic N1O3 & $\mathrm{C} 22 \mathrm{H} 31 \mathrm{~N} 1 \mathrm{O} 3$ & $6.08 \mathrm{E}-06$ \\
\hline Non-basic N1O1 & C19H17N1O1 & 0.000436 & Acidic $\mathrm{O} 1$ & $\mathrm{C} 29 \mathrm{H} 32 \mathrm{O} 1$ & 0.000116 & Basic N1O3 & $\mathrm{C} 23 \mathrm{H} 33 \mathrm{~N} 1 \mathrm{O} 3$ & $9.15 \mathrm{E}-06$ \\
\hline Non-basic N1O1 & C20H19N1O1 & 0.00027 & Acidic $\mathrm{O} 1$ & $\mathrm{C} 30 \mathrm{H} 34 \mathrm{O} 1$ & $2.90 \mathrm{E}-05$ & Basic N1O3 & $\mathrm{C} 24 \mathrm{H} 35 \mathrm{~N} 1 \mathrm{O} 3$ & $1.24 \mathrm{E}-05$ \\
\hline Non-basic N1O1 & $\mathrm{C} 21 \mathrm{H} 21 \mathrm{~N} 1 \mathrm{O} 1$ & 0.000122 & Acidic $\mathrm{O} 1$ & $\mathrm{C} 31 \mathrm{H} 36 \mathrm{O} 1$ & $2.00 \mathrm{E}-05$ & Basic N1O3 & $\mathrm{C} 25 \mathrm{H} 37 \mathrm{~N} 1 \mathrm{O} 3$ & $7.90 \mathrm{E}-06$ \\
\hline Non-basic N1O1 & $\mathrm{C} 22 \mathrm{H} 23 \mathrm{~N} 1 \mathrm{O} 1$ & $6.34 \mathrm{E}-05$ & Acidic O1 & $\mathrm{C} 32 \mathrm{H} 38 \mathrm{O} 1$ & $1.51 \mathrm{E}-05$ & Basic N1O3 & $\mathrm{C} 26 \mathrm{H} 39 \mathrm{~N} 1 \mathrm{O} 3$ & $1.72 \mathrm{E}-05$ \\
\hline Non-basic N1O1 & $\mathrm{C} 23 \mathrm{H} 25 \mathrm{~N} 1 \mathrm{O} 1$ & 4.19E-05 & Acidic O1 & $\mathrm{C} 33 \mathrm{H} 40 \mathrm{O} 1$ & $1.79 \mathrm{E}-05$ & Basic N1O3 & $\mathrm{C} 27 \mathrm{H} 41 \mathrm{~N} 1 \mathrm{O} 3$ & $9.37 \mathrm{E}-06$ \\
\hline Non-basic N1O1 & $\mathrm{C} 24 \mathrm{H} 27 \mathrm{~N} 1 \mathrm{O} 1$ & $3.82 \mathrm{E}-05$ & Acidic $\mathrm{O} 1$ & $\mathrm{C} 20 \mathrm{H} 12 \mathrm{O} 1$ & 0.000337 & Basic N1O3 & $\mathrm{C} 28 \mathrm{H} 43 \mathrm{~N} 1 \mathrm{O} 3$ & $1.07 \mathrm{E}-05$ \\
\hline Non-basic N1O1 & $\mathrm{C} 25 \mathrm{H} 29 \mathrm{~N} 1 \mathrm{O} 1$ & $3.64 \mathrm{E}-05$ & Acidic $\mathrm{O} 1$ & $\mathrm{C} 21 \mathrm{H} 14 \mathrm{O} 1$ & 0.000476 & Basic N1O3 & $\mathrm{C} 29 \mathrm{H} 45 \mathrm{~N} 1 \mathrm{O} 3$ & $1.16 \mathrm{E}-05$ \\
\hline Non-basic N1O1 & $\mathrm{C} 26 \mathrm{H} 31 \mathrm{~N} 1 \mathrm{O} 1$ & $2.96 \mathrm{E}-05$ & Acidic $\mathrm{O} 1$ & $\mathrm{C} 22 \mathrm{H} 16 \mathrm{O} 1$ & 0.000692 & Basic N1O3 & $\mathrm{C} 30 \mathrm{H} 47 \mathrm{~N} 1 \mathrm{O} 3$ & $1.20 \mathrm{E}-05$ \\
\hline Non-basic N1O1 & $\mathrm{C} 27 \mathrm{H} 33 \mathrm{~N} 1 \mathrm{O} 1$ & $2.02 \mathrm{E}-05$ & Acidic $\mathrm{O} 1$ & $\mathrm{C} 23 \mathrm{H} 18 \mathrm{O} 1$ & 0.000544 & Basic N1O3 & $\mathrm{C} 31 \mathrm{H} 49 \mathrm{~N} 1 \mathrm{O} 3$ & $8.25 \mathrm{E}-06$ \\
\hline Non-basic N1O1 & $\mathrm{C} 28 \mathrm{H} 35 \mathrm{~N} 1 \mathrm{O} 1$ & $2.08 \mathrm{E}-05$ & Acidic $\mathrm{O} 1$ & $\mathrm{C} 24 \mathrm{H} 20 \mathrm{O} 1$ & 0.000403 & Basic N1O3 & $\mathrm{C} 32 \mathrm{H} 51 \mathrm{~N} 1 \mathrm{O} 3$ & $8.49 \mathrm{E}-06$ \\
\hline
\end{tabular}




\begin{tabular}{|c|c|c|c|c|c|c|c|c|}
\hline Class & Formula & Mass & Class & Formula & Mass & Class & Formula & Mass \\
\hline Non-basic N1O1 & $\mathrm{C} 29 \mathrm{H} 37 \mathrm{~N} 1 \mathrm{O} 1$ & $2.10 \mathrm{E}-05$ & Acidic $\mathrm{O} 1$ & $\mathrm{C} 25 \mathrm{H} 22 \mathrm{O} 1$ & 0.000334 & Basic N1O3 & $\mathrm{C} 33 \mathrm{H} 53 \mathrm{~N} 1 \mathrm{O} 3$ & 7.06E-06 \\
\hline Non-basic N1O1 & $\mathrm{C} 30 \mathrm{H} 39 \mathrm{~N} 1 \mathrm{O} 1$ & $2.08 \mathrm{E}-05$ & Acidic $\mathrm{O} 1$ & $\mathrm{C} 26 \mathrm{H} 24 \mathrm{O} 1$ & 0.000168 & Basic N1O3 & $\mathrm{C} 34 \mathrm{H} 55 \mathrm{~N} 1 \mathrm{O} 3$ & $1.39 \mathrm{E}-05$ \\
\hline Non-basic N1O1 & $\mathrm{C} 31 \mathrm{H} 41 \mathrm{~N} 1 \mathrm{O} 1$ & $1.33 \mathrm{E}-05$ & Acidic $\mathrm{O} 1$ & $\mathrm{C} 27 \mathrm{H} 26 \mathrm{O} 1$ & $9.06 \mathrm{E}-05$ & Basic N1O3 & $\mathrm{C} 36 \mathrm{H} 59 \mathrm{~N} 1 \mathrm{O} 3$ & 4.10E-06 \\
\hline Non-basic N1O1 & C32H43N1O1 & $1.19 \mathrm{E}-05$ & Acidic $\mathrm{O} 1$ & $\mathrm{C} 28 \mathrm{H} 28 \mathrm{O} 1$ & $2.78 \mathrm{E}-05$ & Basic N1O3 & C37H61N1O3 & $3.45 \mathrm{E}-06$ \\
\hline Non-basic N1O1 & $\mathrm{C} 33 \mathrm{H} 45 \mathrm{~N} 1 \mathrm{O} 1$ & $6.67 \mathrm{E}-06$ & Acidic $\mathrm{O} 1$ & $\mathrm{C} 22 \mathrm{H} 14 \mathrm{O} 1$ & $2.36 \mathrm{E}-05$ & Basic N1O3 & $\mathrm{C} 38 \mathrm{H} 63 \mathrm{~N} 1 \mathrm{O} 3$ & $6.76 \mathrm{E}-06$ \\
\hline Non-basic N1O1 & $\mathrm{C} 34 \mathrm{H} 47 \mathrm{~N} 1 \mathrm{O} 1$ & 4.27E-06 & Acidic $\mathrm{O} 1$ & $\mathrm{C} 23 \mathrm{H} 16 \mathrm{O} 1$ & 0.00021 & Basic N1O3 & C39H65N1O3 & $1.95 \mathrm{E}-06$ \\
\hline Non-basic N1O1 & $\mathrm{C} 35 \mathrm{H} 49 \mathrm{~N} 1 \mathrm{O} 1$ & $7.28 \mathrm{E}-06$ & Acidic $\mathrm{O} 1$ & $\mathrm{C} 24 \mathrm{H} 18 \mathrm{O} 1$ & 0.000341 & Basic N1O3 & $\mathrm{C} 40 \mathrm{H} 67 \mathrm{~N} 1 \mathrm{O} 3$ & $1.91 \mathrm{E}-06$ \\
\hline Non-basic N1O1 & C36H51N1O1 & $1.33 \mathrm{E}-06$ & Acidic $\mathrm{O} 1$ & $\mathrm{C} 25 \mathrm{H} 20 \mathrm{O} 1$ & 0.000311 & Basic N1O3 & C17H19N1O3 & $1.09 \mathrm{E}-06$ \\
\hline Non-basic N1O1 & C17H11N1O1 & 0.000181 & Acidic O1 & $\mathrm{C} 26 \mathrm{H} 22 \mathrm{O} 1$ & 0.000196 & Basic N1O3 & $\mathrm{C} 18 \mathrm{H} 21 \mathrm{~N} 1 \mathrm{O} 3$ & $3.02 \mathrm{E}-07$ \\
\hline Non-basic N1O1 & C18H13N1O1 & 0.000651 & Acidic $\mathrm{O} 1$ & $\mathrm{C} 27 \mathrm{H} 24 \mathrm{O} 1$ & 0.000134 & Basic N1O3 & $\mathrm{C} 19 \mathrm{H} 23 \mathrm{~N} 1 \mathrm{O} 3$ & $1.76 \mathrm{E}-06$ \\
\hline Non-basic N1O1 & C19H15N1O1 & 0.000823 & Acidic $\mathrm{O} 1$ & $\mathrm{C} 28 \mathrm{H} 26 \mathrm{O} 1$ & $9.36 \mathrm{E}-05$ & Basic N1O3 & $\mathrm{C} 20 \mathrm{H} 25 \mathrm{~N} 1 \mathrm{O} 3$ & $8.30 \mathrm{E}-07$ \\
\hline Non-basic N1O1 & C20H17N1O1 & 0.00063 & Acidic $\mathrm{O} 1$ & $\mathrm{C} 29 \mathrm{H} 28 \mathrm{O} 1$ & $3.16 \mathrm{E}-05$ & Basic N1O3 & $\mathrm{C} 21 \mathrm{H} 27 \mathrm{~N} 1 \mathrm{O} 3$ & $2.55 \mathrm{E}-06$ \\
\hline Non-basic N1O1 & C21H19N1O1 & 0.000366 & Acidic $\mathrm{O} 1$ & $\mathrm{C} 23 \mathrm{H} 14 \mathrm{O} 1$ & $2.95 \mathrm{E}-05$ & Basic N1O3 & $\mathrm{C} 22 \mathrm{H} 29 \mathrm{~N} 1 \mathrm{O} 3$ & $3.74 \mathrm{E}-06$ \\
\hline Non-basic N1O1 & $\mathrm{C} 22 \mathrm{H} 21 \mathrm{~N} 1 \mathrm{O} 1$ & 0.000216 & Acidic $\mathrm{O} 1$ & $\mathrm{C} 24 \mathrm{H} 16 \mathrm{O} 1$ & 0.000133 & Basic N1O3 & $\mathrm{C} 23 \mathrm{H} 31 \mathrm{~N} 1 \mathrm{O} 3$ & $5.70 \mathrm{E}-06$ \\
\hline Non-basic N1O1 & $\mathrm{C} 23 \mathrm{H} 23 \mathrm{~N} 1 \mathrm{O} 1$ & 0.000145 & Acidic $\mathrm{O} 1$ & $\mathrm{C} 25 \mathrm{H} 18 \mathrm{O} 1$ & 0.000111 & Basic N1O3 & $\mathrm{C} 24 \mathrm{H} 33 \mathrm{~N} 1 \mathrm{O} 3$ & $7.15 \mathrm{E}-06$ \\
\hline Non-basic N1O1 & $\mathrm{C} 24 \mathrm{H} 25 \mathrm{~N} 1 \mathrm{O} 1$ & 0.0001 & Acidic $\mathrm{O} 1$ & $\mathrm{C} 26 \mathrm{H} 20 \mathrm{O} 1$ & 0.000131 & Basic N1O3 & $\mathrm{C} 25 \mathrm{H} 35 \mathrm{~N} 1 \mathrm{O} 3$ & $8.38 \mathrm{E}-06$ \\
\hline Non-basic N1O1 & $\mathrm{C} 25 \mathrm{H} 27 \mathrm{~N} 1 \mathrm{O} 1$ & 7.41E-05 & Acidic $\mathrm{O} 1$ & $\mathrm{C} 27 \mathrm{H} 22 \mathrm{O} 1$ & $6.62 \mathrm{E}-05$ & Basic N1O3 & $\mathrm{C} 26 \mathrm{H} 37 \mathrm{~N} 1 \mathrm{O} 3$ & $1.33 \mathrm{E}-05$ \\
\hline Non-basic N1O1 & $\mathrm{C} 26 \mathrm{H} 29 \mathrm{~N} 1 \mathrm{O} 1$ & $5.04 \mathrm{E}-05$ & Acidic $\mathrm{O} 1$ & $\mathrm{C} 28 \mathrm{H} 24 \mathrm{O} 1$ & $5.40 \mathrm{E}-05$ & Basic N1O3 & $\mathrm{C} 27 \mathrm{H} 39 \mathrm{~N} 1 \mathrm{O} 3$ & $1.00 \mathrm{E}-05$ \\
\hline Non-basic N1O1 & $\mathrm{C} 27 \mathrm{H} 31 \mathrm{~N} 1 \mathrm{O} 1$ & $4.10 \mathrm{E}-05$ & Acidic $\mathrm{O} 1$ & $\mathrm{C} 25 \mathrm{H} 16 \mathrm{O} 1$ & $9.30 \mathrm{E}-05$ & Basic N1O3 & $\mathrm{C} 28 \mathrm{H} 41 \mathrm{~N} 1 \mathrm{O} 3$ & $9.07 \mathrm{E}-06$ \\
\hline Non-basic N1O1 & C28H33N1O1 & $3.81 \mathrm{E}-05$ & Acidic $\mathrm{O} 1$ & $\mathrm{C} 26 \mathrm{H} 18 \mathrm{O} 1$ & $8.90 \mathrm{E}-05$ & Basic N1O3 & $\mathrm{C} 29 \mathrm{H} 43 \mathrm{~N} 1 \mathrm{O} 3$ & $1.07 \mathrm{E}-05$ \\
\hline Non-basic N1O1 & $\mathrm{C} 29 \mathrm{H} 35 \mathrm{~N} 1 \mathrm{O} 1$ & $3.63 \mathrm{E}-05$ & Acidic $\mathrm{O} 1$ & $\mathrm{C} 27 \mathrm{H} 20 \mathrm{O} 1$ & $5.86 \mathrm{E}-05$ & Basic N1O3 & $\mathrm{C} 30 \mathrm{H} 45 \mathrm{~N} 1 \mathrm{O} 3$ & $1.16 \mathrm{E}-05$ \\
\hline Non-basic N1O1 & $\mathrm{C} 30 \mathrm{H} 37 \mathrm{~N} 1 \mathrm{O} 1$ & $3.94 \mathrm{E}-05$ & Acidic $\mathrm{O} 1$ & $\mathrm{C} 28 \mathrm{H} 22 \mathrm{O} 1$ & $3.18 \mathrm{E}-05$ & Basic N1O3 & $\mathrm{C} 31 \mathrm{H} 47 \mathrm{~N} 1 \mathrm{O} 3$ & $9.02 \mathrm{E}-06$ \\
\hline Non-basic N1O1 & C31H39N1O1 & $2.53 \mathrm{E}-05$ & Acidic $\mathrm{O} 1$ & $\mathrm{C} 29 \mathrm{H} 24 \mathrm{O} 1$ & $4.63 \mathrm{E}-05$ & Basic N1O3 & $\mathrm{C} 32 \mathrm{H} 49 \mathrm{~N} 1 \mathrm{O} 3$ & $1.45 \mathrm{E}-05$ \\
\hline Non-basic N1O1 & C32H41N1O1 & $2.65 \mathrm{E}-05$ & Acidic $\mathrm{O} 1$ & $\mathrm{C} 26 \mathrm{H} 16 \mathrm{O} 1$ & $1.33 \mathrm{E}-05$ & Basic N1O3 & C33H51N1O3 & $1.36 \mathrm{E}-05$ \\
\hline Non-basic N1O1 & C33H43N1O1 & $1.66 \mathrm{E}-05$ & Acidic $\mathrm{O} 1$ & C27H18O1 & $3.94 \mathrm{E}-05$ & Basic N1O3 & $\mathrm{C} 34 \mathrm{H} 53 \mathrm{~N} 1 \mathrm{O} 3$ & $1.13 \mathrm{E}-05$ \\
\hline Non-basic N1O1 & $\mathrm{C} 34 \mathrm{H} 45 \mathrm{~N} 1 \mathrm{O} 1$ & $1.02 \mathrm{E}-05$ & Acidic $\mathrm{O} 1$ & $\mathrm{C} 28 \mathrm{H} 20 \mathrm{O} 1$ & $6.38 \mathrm{E}-05$ & Basic N1O3 & $\mathrm{C} 35 \mathrm{H} 55 \mathrm{~N} 1 \mathrm{O} 3$ & $1.05 \mathrm{E}-05$ \\
\hline Non-basic N1O1 & $\mathrm{C} 35 \mathrm{H} 47 \mathrm{~N} 1 \mathrm{O} 1$ & $1.42 \mathrm{E}-05$ & Acidic $\mathrm{O} 1$ & $\mathrm{C} 29 \mathrm{H} 22 \mathrm{O} 1$ & $3.93 \mathrm{E}-05$ & Basic N1O3 & $\mathrm{C} 36 \mathrm{H} 57 \mathrm{~N} 1 \mathrm{O} 3$ & $5.36 \mathrm{E}-06$ \\
\hline Non-basic N1O1 & $\mathrm{C} 36 \mathrm{H} 49 \mathrm{~N} 1 \mathrm{O} 1$ & $1.20 \mathrm{E}-05$ & Acidic $\mathrm{O} 1$ & $\mathrm{C} 27 \mathrm{H} 16 \mathrm{O} 1$ & $4.86 \mathrm{E}-05$ & Basic N1O3 & C37H59N1O3 & $7.65 \mathrm{E}-06$ \\
\hline Non-basic N1O1 & C37H51N1O1 & $1.09 \mathrm{E}-05$ & Acidic $\mathrm{O} 1$ & $\mathrm{C} 28 \mathrm{H} 18 \mathrm{O} 1$ & $3.02 \mathrm{E}-05$ & Basic N1O3 & $\mathrm{C} 16 \mathrm{H} 15 \mathrm{~N} 1 \mathrm{O} 3$ & $1.01 \mathrm{E}-06$ \\
\hline Non-basic N1O1 & C38H53N1O1 & $2.63 \mathrm{E}-06$ & Acidic $\mathrm{O} 1$ & $\mathrm{C} 29 \mathrm{H} 20 \mathrm{O} 1$ & $3.05 \mathrm{E}-05$ & Basic N1O3 & $\mathrm{C} 17 \mathrm{H} 17 \mathrm{~N} 1 \mathrm{O} 3$ & $2.48 \mathrm{E}-07$ \\
\hline Non-basic N1O1 & C17H9N1O1 & $1.03 \mathrm{E}-06$ & Acidic $\mathrm{O} 2$ & $\mathrm{C} 13 \mathrm{H} 26 \mathrm{O} 2$ & $4.06 \mathrm{E}-05$ & Basic N1O3 & C18H19N1O3 & $1.46 \mathrm{E}-06$ \\
\hline Non-basic N1O1 & C18H11N1O1 & $3.83 \mathrm{E}-05$ & Acidic $\mathrm{O} 2$ & $\mathrm{C} 14 \mathrm{H} 28 \mathrm{O} 2$ & 0.003331 & Basic N1O3 & $\mathrm{C} 19 \mathrm{H} 21 \mathrm{~N} 1 \mathrm{O} 3$ & $9.99 \mathrm{E}-07$ \\
\hline Non-basic N1O1 & C19H13N1O1 & 0.000284 & Acidic $\mathrm{O} 2$ & $\mathrm{C} 15 \mathrm{H} 30 \mathrm{O} 2$ & 0.004418 & Basic N1O3 & $\mathrm{C} 20 \mathrm{H} 23 \mathrm{~N} 1 \mathrm{O} 3$ & $1.63 \mathrm{E}-06$ \\
\hline Non-basic N1O1 & C20H15N1O1 & 0.000692 & Acidic $\mathrm{O} 2$ & $\mathrm{C} 16 \mathrm{H} 32 \mathrm{O} 2$ & 0.004674 & Basic N1O3 & $\mathrm{C} 21 \mathrm{H} 25 \mathrm{~N} 1 \mathrm{O} 3$ & $2.99 \mathrm{E}-06$ \\
\hline Non-basic N1O1 & $\mathrm{C} 21 \mathrm{H} 17 \mathrm{~N} 1 \mathrm{O} 1$ & 0.000879 & Acidic $\mathrm{O} 2$ & $\mathrm{C} 17 \mathrm{H} 34 \mathrm{O} 2$ & 0.003246 & Basic N1O3 & $\mathrm{C} 22 \mathrm{H} 27 \mathrm{~N} 1 \mathrm{O} 3$ & $5.70 \mathrm{E}-06$ \\
\hline Non-basic N1O1 & $\mathrm{C} 22 \mathrm{H} 19 \mathrm{~N} 1 \mathrm{O} 1$ & 0.000745 & Acidic $\mathrm{O} 2$ & $\mathrm{C} 18 \mathrm{H} 36 \mathrm{O} 2$ & 0.003414 & Basic N1O3 & $\mathrm{C} 23 \mathrm{H} 29 \mathrm{~N} 1 \mathrm{O} 3$ & $7.82 \mathrm{E}-06$ \\
\hline Non-basic N1O1 & $\mathrm{C} 23 \mathrm{H} 21 \mathrm{~N} 1 \mathrm{O} 1$ & 0.000505 & Acidic $\mathrm{O} 2$ & $\mathrm{C} 19 \mathrm{H} 38 \mathrm{O} 2$ & 0.00053 & Basic N1O3 & $\mathrm{C} 24 \mathrm{H} 31 \mathrm{~N} 1 \mathrm{O} 3$ & $7.29 \mathrm{E}-06$ \\
\hline Non-basic N1O1 & $\mathrm{C} 24 \mathrm{H} 23 \mathrm{~N} 1 \mathrm{O} 1$ & 0.000257 & Acidic $\mathrm{O} 2$ & $\mathrm{C} 20 \mathrm{H} 40 \mathrm{O} 2$ & 0.001078 & Basic N1O3 & $\mathrm{C} 25 \mathrm{H} 33 \mathrm{~N} 1 \mathrm{O} 3$ & $8.22 \mathrm{E}-06$ \\
\hline
\end{tabular}




\begin{tabular}{|c|c|c|c|c|c|c|c|c|}
\hline Class & Formula & Mass & Class & Formula & Mass & Class & Formula & Mass \\
\hline Non-basic N1O1 & $\mathrm{C} 25 \mathrm{H} 25 \mathrm{~N} 1 \mathrm{O} 1$ & 0.000145 & Acidic $\mathrm{O} 2$ & $\mathrm{C} 21 \mathrm{H} 42 \mathrm{O} 2$ & 0.000521 & Basic N1O3 & $\mathrm{C} 26 \mathrm{H} 35 \mathrm{~N} 1 \mathrm{O} 3$ & $1.11 \mathrm{E}-05$ \\
\hline Non-basic N1O1 & $\mathrm{C} 26 \mathrm{H} 27 \mathrm{~N} 1 \mathrm{O} 1$ & $9.83 \mathrm{E}-05$ & Acidic $\mathrm{O} 2$ & $\mathrm{C} 22 \mathrm{H} 44 \mathrm{O} 2$ & 0.000845 & Basic N1O3 & $\mathrm{C} 27 \mathrm{H} 37 \mathrm{~N} 1 \mathrm{O} 3$ & $9.14 \mathrm{E}-06$ \\
\hline Non-basic N1O1 & $\mathrm{C} 27 \mathrm{H} 29 \mathrm{~N} 1 \mathrm{O} 1$ & $7.00 \mathrm{E}-05$ & Acidic $\mathrm{O} 2$ & $\mathrm{C} 23 \mathrm{H} 46 \mathrm{O} 2$ & 0.000673 & Basic N1O3 & $\mathrm{C} 28 \mathrm{H} 39 \mathrm{~N} 1 \mathrm{O} 3$ & $9.92 \mathrm{E}-06$ \\
\hline Non-basic N1O1 & $\mathrm{C} 28 \mathrm{H} 31 \mathrm{~N} 1 \mathrm{O} 1$ & $3.94 \mathrm{E}-05$ & Acidic $\mathrm{O} 2$ & $\mathrm{C} 24 \mathrm{H} 48 \mathrm{O} 2$ & 0.002078 & Basic N1O3 & $\mathrm{C} 29 \mathrm{H} 41 \mathrm{~N} 1 \mathrm{O} 3$ & $1.42 \mathrm{E}-05$ \\
\hline Non-basic N1O1 & $\mathrm{C} 29 \mathrm{H} 33 \mathrm{~N} 1 \mathrm{O} 1$ & 4.44E-05 & Acidic $\mathrm{O} 2$ & $\mathrm{C} 25 \mathrm{H} 50 \mathrm{O} 2$ & 0.001095 & Basic N1O3 & $\mathrm{C} 30 \mathrm{H} 43 \mathrm{~N} 1 \mathrm{O} 3$ & $1.33 \mathrm{E}-05$ \\
\hline Non-basic N1O1 & $\mathrm{C} 30 \mathrm{H} 35 \mathrm{~N} 1 \mathrm{O} 1$ & $2.29 \mathrm{E}-05$ & Acidic $\mathrm{O} 2$ & $\mathrm{C} 26 \mathrm{H} 52 \mathrm{O} 2$ & 0.001003 & Basic N1O3 & $\mathrm{C} 31 \mathrm{H} 45 \mathrm{~N} 1 \mathrm{O} 3$ & $1.07 \mathrm{E}-05$ \\
\hline Non-basic N1O1 & $\mathrm{C} 31 \mathrm{H} 37 \mathrm{~N} 1 \mathrm{O} 1$ & $2.25 \mathrm{E}-05$ & Acidic $\mathrm{O} 2$ & $\mathrm{C} 27 \mathrm{H} 54 \mathrm{O} 2$ & 0.000371 & Basic N1O3 & $\mathrm{C} 32 \mathrm{H} 47 \mathrm{~N} 1 \mathrm{O} 3$ & $7.62 \mathrm{E}-06$ \\
\hline Non-basic N1O1 & $\mathrm{C} 32 \mathrm{H} 39 \mathrm{~N} 1 \mathrm{O} 1$ & $1.97 \mathrm{E}-05$ & Acidic $\mathrm{O} 2$ & $\mathrm{C} 28 \mathrm{H} 56 \mathrm{O} 2$ & 0.000377 & Basic N1O3 & $\mathrm{C} 33 \mathrm{H} 49 \mathrm{~N} 1 \mathrm{O} 3$ & $1.54 \mathrm{E}-05$ \\
\hline Non-basic N1O1 & $\mathrm{C} 33 \mathrm{H} 41 \mathrm{~N} 1 \mathrm{O} 1$ & $1.54 \mathrm{E}-05$ & Acidic $\mathrm{O} 2$ & $\mathrm{C} 29 \mathrm{H} 58 \mathrm{O} 2$ & 0.0003 & Basic N1O3 & $\mathrm{C} 34 \mathrm{H} 51 \mathrm{~N} 1 \mathrm{O} 3$ & $1.64 \mathrm{E}-05$ \\
\hline Non-basic N1O1 & $\mathrm{C} 34 \mathrm{H} 43 \mathrm{~N} 1 \mathrm{O} 1$ & $9.97 \mathrm{E}-06$ & Acidic $\mathrm{O} 2$ & $\mathrm{C} 30 \mathrm{H} 60 \mathrm{O} 2$ & 0.000146 & Basic N1O3 & C35H53N1O3 & $6.91 \mathrm{E}-06$ \\
\hline Non-basic N1O1 & $\mathrm{C} 35 \mathrm{H} 45 \mathrm{~N} 1 \mathrm{O} 1$ & $4.61 \mathrm{E}-06$ & Acidic $\mathrm{O} 2$ & $\mathrm{C} 31 \mathrm{H} 62 \mathrm{O} 2$ & $7.58 \mathrm{E}-05$ & Basic N1O3 & $\mathrm{C} 36 \mathrm{H} 55 \mathrm{~N} 1 \mathrm{O} 3$ & 7.09E-06 \\
\hline Non-basic N1O1 & $\mathrm{C} 36 \mathrm{H} 47 \mathrm{~N} 1 \mathrm{O} 1$ & $6.17 \mathrm{E}-06$ & Acidic $\mathrm{O} 2$ & $\mathrm{C} 14 \mathrm{H} 26 \mathrm{O} 2$ & 0.00026 & Basic N1O3 & C37H57N1O3 & $1.28 \mathrm{E}-05$ \\
\hline Non-basic N1O1 & $\mathrm{C} 37 \mathrm{H} 49 \mathrm{~N} 1 \mathrm{O} 1$ & $3.18 \mathrm{E}-06$ & Acidic $\mathrm{O} 2$ & $\mathrm{C} 15 \mathrm{H} 28 \mathrm{O} 2$ & 0.000696 & Basic N1O3 & C38H59N1O3 & 7.16E-06 \\
\hline Non-basic N1O1 & C38H51N1O1 & $1.56 \mathrm{E}-06$ & Acidic $\mathrm{O} 2$ & $\mathrm{C} 16 \mathrm{H} 30 \mathrm{O} 2$ & 0.008734 & Basic N1O3 & C39H61N1O3 & $1.08 \mathrm{E}-05$ \\
\hline Non-basic N1O1 & C39H53N1O1 & $3.44 \mathrm{E}-06$ & Acidic $\mathrm{O} 2$ & $\mathrm{C} 17 \mathrm{H} 32 \mathrm{O} 2$ & 0.00158 & Basic N1O3 & $\mathrm{C} 40 \mathrm{H} 63 \mathrm{~N} 1 \mathrm{O} 3$ & $9.13 \mathrm{E}-06$ \\
\hline Non-basic N1O1 & C19H11N1O1 & 0.000237 & Acidic $\mathrm{O} 2$ & $\mathrm{C} 18 \mathrm{H} 34 \mathrm{O} 2$ & 0.007777 & Basic N1O3 & $\mathrm{C} 17 \mathrm{H} 15 \mathrm{~N} 1 \mathrm{O} 3$ & $8.41 \mathrm{E}-07$ \\
\hline Non-basic N1O1 & $\mathrm{C} 20 \mathrm{H} 13 \mathrm{~N} 1 \mathrm{O} 1$ & 0.00104 & Acidic $\mathrm{O} 2$ & $\mathrm{C} 19 \mathrm{H} 36 \mathrm{O} 2$ & 0.000188 & Basic N1O3 & $\mathrm{C} 18 \mathrm{H} 17 \mathrm{~N} 1 \mathrm{O} 3$ & $3.48 \mathrm{E}-07$ \\
\hline Non-basic N1O1 & $\mathrm{C} 21 \mathrm{H} 15 \mathrm{~N} 1 \mathrm{O} 1$ & 0.001882 & Acidic $\mathrm{O} 2$ & $\mathrm{C} 20 \mathrm{H} 38 \mathrm{O} 2$ & 0.000325 & Basic N1O3 & C19H19N1O3 & $7.83 \mathrm{E}-07$ \\
\hline Non-basic N1O1 & $\mathrm{C} 22 \mathrm{H} 17 \mathrm{~N} 1 \mathrm{O} 1$ & 0.001986 & Acidic $\mathrm{O} 2$ & $\mathrm{C} 21 \mathrm{H} 40 \mathrm{O} 2$ & 0.0001 & Basic N1O3 & $\mathrm{C} 20 \mathrm{H} 21 \mathrm{~N} 1 \mathrm{O} 3$ & $4.30 \mathrm{E}-06$ \\
\hline Non-basic N1O1 & $\mathrm{C} 23 \mathrm{H} 19 \mathrm{~N} 1 \mathrm{O} 1$ & 0.001427 & Acidic $\mathrm{O} 2$ & $\mathrm{C} 22 \mathrm{H} 42 \mathrm{O} 2$ & 0.000278 & Basic N1O3 & $\mathrm{C} 21 \mathrm{H} 23 \mathrm{~N} 1 \mathrm{O} 3$ & $2.77 \mathrm{E}-06$ \\
\hline Non-basic N1O1 & $\mathrm{C} 24 \mathrm{H} 21 \mathrm{~N} 1 \mathrm{O} 1$ & 0.000746 & Acidic $\mathrm{O} 2$ & $\mathrm{C} 23 \mathrm{H} 44 \mathrm{O} 2$ & $7.10 \mathrm{E}-05$ & Basic N1O3 & $\mathrm{C} 22 \mathrm{H} 25 \mathrm{~N} 1 \mathrm{O} 3$ & $1.02 \mathrm{E}-05$ \\
\hline Non-basic N1O1 & $\mathrm{C} 25 \mathrm{H} 23 \mathrm{~N} 1 \mathrm{O} 1$ & 0.000405 & Acidic $\mathrm{O} 2$ & $\mathrm{C} 24 \mathrm{H} 46 \mathrm{O} 2$ & 0.000176 & Basic N1O3 & $\mathrm{C} 23 \mathrm{H} 27 \mathrm{~N} 1 \mathrm{O} 3$ & $5.24 \mathrm{E}-06$ \\
\hline Non-basic N1O1 & $\mathrm{C} 26 \mathrm{H} 25 \mathrm{~N} 1 \mathrm{O} 1$ & 0.000224 & Acidic $\mathrm{O} 2$ & $\mathrm{C} 25 \mathrm{H} 48 \mathrm{O} 2$ & $4.30 \mathrm{E}-05$ & Basic N1O3 & $\mathrm{C} 24 \mathrm{H} 29 \mathrm{~N} 1 \mathrm{O} 3$ & $1.50 \mathrm{E}-05$ \\
\hline Non-basic N1O1 & $\mathrm{C} 27 \mathrm{H} 27 \mathrm{~N} 1 \mathrm{O} 1$ & 0.000127 & Acidic $\mathrm{O} 2$ & $\mathrm{C} 26 \mathrm{H} 50 \mathrm{O} 2$ & 0.000158 & Basic N1O3 & $\mathrm{C} 25 \mathrm{H} 31 \mathrm{~N} 1 \mathrm{O} 3$ & $1.24 \mathrm{E}-05$ \\
\hline Non-basic N1O1 & $\mathrm{C} 28 \mathrm{H} 29 \mathrm{~N} 1 \mathrm{O} 1$ & $8.88 \mathrm{E}-05$ & Acidic $\mathrm{O} 2$ & $\mathrm{C} 27 \mathrm{H} 52 \mathrm{O} 2$ & 0.000124 & Basic N1O3 & $\mathrm{C} 26 \mathrm{H} 33 \mathrm{~N} 1 \mathrm{O} 3$ & $7.20 \mathrm{E}-06$ \\
\hline Non-basic N1O1 & $\mathrm{C} 29 \mathrm{H} 31 \mathrm{~N} 1 \mathrm{O} 1$ & $5.97 \mathrm{E}-05$ & Acidic $\mathrm{O} 2$ & $\mathrm{C} 28 \mathrm{H} 54 \mathrm{O} 2$ & 0.000195 & Basic N1O3 & $\mathrm{C} 27 \mathrm{H} 35 \mathrm{~N} 1 \mathrm{O} 3$ & $1.76 \mathrm{E}-05$ \\
\hline Non-basic N1O1 & $\mathrm{C} 30 \mathrm{H} 33 \mathrm{~N} 1 \mathrm{O} 1$ & $3.82 \mathrm{E}-05$ & Acidic $\mathrm{O} 2$ & $\mathrm{C} 29 \mathrm{H} 56 \mathrm{O} 2$ & $4.70 \mathrm{E}-05$ & Basic N1O3 & $\mathrm{C} 28 \mathrm{H} 37 \mathrm{~N} 1 \mathrm{O} 3$ & $1.44 \mathrm{E}-05$ \\
\hline Non-basic N1O1 & $\mathrm{C} 31 \mathrm{H} 35 \mathrm{~N} 1 \mathrm{O} 1$ & $3.41 \mathrm{E}-05$ & Acidic $\mathrm{O} 2$ & $\mathrm{C} 30 \mathrm{H} 58 \mathrm{O} 2$ & 0.000188 & Basic N1O3 & C29H39N1O3 & $1.58 \mathrm{E}-05$ \\
\hline Non-basic N1O1 & $\mathrm{C} 32 \mathrm{H} 37 \mathrm{~N} 1 \mathrm{O} 1$ & $2.70 \mathrm{E}-05$ & Acidic $\mathrm{O} 2$ & $\mathrm{C} 31 \mathrm{H} 60 \mathrm{O} 2$ & 0.000194 & Basic N1O3 & $\mathrm{C} 30 \mathrm{H} 41 \mathrm{~N} 1 \mathrm{O} 3$ & $1.65 \mathrm{E}-05$ \\
\hline Non-basic N1O1 & C33H39N1O1 & $8.55 \mathrm{E}-06$ & Acidic $\mathrm{O} 2$ & $\mathrm{C} 32 \mathrm{H} 62 \mathrm{O} 2$ & 0.000232 & Basic N1O3 & $\mathrm{C} 31 \mathrm{H} 43 \mathrm{~N} 1 \mathrm{O} 3$ & $1.19 \mathrm{E}-05$ \\
\hline Non-basic N1O1 & $\mathrm{C} 34 \mathrm{H} 41 \mathrm{~N} 1 \mathrm{O} 1$ & $1.37 \mathrm{E}-05$ & Acidic $\mathrm{O} 2$ & $\mathrm{C} 14 \mathrm{H} 24 \mathrm{O} 2$ & $1.07 \mathrm{E}-05$ & Basic N1O3 & $\mathrm{C} 32 \mathrm{H} 45 \mathrm{~N} 1 \mathrm{O} 3$ & $1.43 \mathrm{E}-05$ \\
\hline Non-basic N1O1 & $\mathrm{C} 35 \mathrm{H} 43 \mathrm{~N} 1 \mathrm{O} 1$ & $3.64 \mathrm{E}-06$ & Acidic $\mathrm{O} 2$ & $\mathrm{C} 15 \mathrm{H} 26 \mathrm{O} 2$ & $1.78 \mathrm{E}-05$ & Basic N1O3 & $\mathrm{C} 33 \mathrm{H} 47 \mathrm{~N} 1 \mathrm{O} 3$ & $2.10 \mathrm{E}-05$ \\
\hline Non-basic N1O1 & $\mathrm{C} 36 \mathrm{H} 45 \mathrm{~N} 1 \mathrm{O} 1$ & $1.53 \mathrm{E}-06$ & Acidic $\mathrm{O} 2$ & $\mathrm{C} 16 \mathrm{H} 28 \mathrm{O} 2$ & 0.000129 & Basic N1O3 & $\mathrm{C} 34 \mathrm{H} 49 \mathrm{~N} 1 \mathrm{O} 3$ & $3.14 \mathrm{E}-05$ \\
\hline Non-basic N1O1 & $\mathrm{C} 37 \mathrm{H} 47 \mathrm{~N} 1 \mathrm{O} 1$ & $9.92 \mathrm{E}-06$ & Acidic $\mathrm{O} 2$ & $\mathrm{C} 17 \mathrm{H} 30 \mathrm{O} 2$ & $1.73 \mathrm{E}-05$ & Basic N1O3 & $\mathrm{C} 35 \mathrm{H} 51 \mathrm{~N} 1 \mathrm{O} 3$ & $1.37 \mathrm{E}-05$ \\
\hline Non-basic N1O1 & $\mathrm{C} 20 \mathrm{H} 11 \mathrm{~N} 1 \mathrm{O} 1$ & $2.71 \mathrm{E}-05$ & Acidic $\mathrm{O} 2$ & $\mathrm{C} 18 \mathrm{H} 32 \mathrm{O} 2$ & 0.001884 & Basic N1O3 & $\mathrm{C} 36 \mathrm{H} 53 \mathrm{~N} 1 \mathrm{O} 3$ & $1.22 \mathrm{E}-05$ \\
\hline Non-basic N1O1 & $\mathrm{C} 21 \mathrm{H} 13 \mathrm{~N} 1 \mathrm{O} 1$ & 0.000231 & Acidic $\mathrm{O} 2$ & $\mathrm{C} 19 \mathrm{H} 34 \mathrm{O} 2$ & $6.99 \mathrm{E}-05$ & Basic N1O3 & $\mathrm{C} 37 \mathrm{H} 55 \mathrm{~N} 1 \mathrm{O} 3$ & $1.56 \mathrm{E}-05$ \\
\hline Non-basic N1O1 & $\mathrm{C} 22 \mathrm{H} 15 \mathrm{~N} 1 \mathrm{O} 1$ & 0.000589 & Acidic $\mathrm{O} 2$ & $\mathrm{C} 20 \mathrm{H} 36 \mathrm{O} 2$ & 0.000306 & Basic N1O3 & $\mathrm{C} 38 \mathrm{H} 57 \mathrm{~N} 1 \mathrm{O} 3$ & 7.44E-06 \\
\hline Non-basic N1O1 & $\mathrm{C} 23 \mathrm{H} 17 \mathrm{~N} 1 \mathrm{O} 1$ & 0.000714 & Acidic $\mathrm{O} 2$ & $\mathrm{C} 21 \mathrm{H} 38 \mathrm{O} 2$ & $2.08 \mathrm{E}-05$ & Basic N1O3 & C39H59N1O3 & $1.17 \mathrm{E}-05$ \\
\hline
\end{tabular}




\begin{tabular}{|c|c|c|c|c|c|c|c|c|}
\hline Class & Formula & Mass & Class & Formula & Mass & Class & Formula & Mass \\
\hline Non-basic N1O1 & $\mathrm{C} 24 \mathrm{H} 19 \mathrm{~N} 1 \mathrm{O} 1$ & 0.000578 & Acidic $\mathrm{O} 2$ & $\mathrm{C} 22 \mathrm{H} 40 \mathrm{O} 2$ & $5.85 \mathrm{E}-05$ & Basic N1O3 & C40H61N1O3 & $1.68 \mathrm{E}-05$ \\
\hline Non-basic N1O1 & $\mathrm{C} 25 \mathrm{H} 21 \mathrm{~N} 1 \mathrm{O} 1$ & 0.000457 & Acidic $\mathrm{O} 2$ & $\mathrm{C} 23 \mathrm{H} 42 \mathrm{O} 2$ & $1.57 \mathrm{E}-05$ & Basic N1O3 & C41H63N1O3 & $9.09 \mathrm{E}-06$ \\
\hline Non-basic N1O1 & C26H23N1O1 & 0.00027 & Acidic $\mathrm{O} 2$ & $\mathrm{C} 27 \mathrm{H} 50 \mathrm{O} 2$ & $2.45 \mathrm{E}-05$ & Basic N1O3 & $\mathrm{C} 42 \mathrm{H} 65 \mathrm{~N} 1 \mathrm{O} 3$ & $9.30 \mathrm{E}-06$ \\
\hline Non-basic N1O1 & $\mathrm{C} 27 \mathrm{H} 25 \mathrm{~N} 1 \mathrm{O} 1$ & 0.000168 & Acidic $\mathrm{O} 2$ & $\mathrm{C} 28 \mathrm{H} 52 \mathrm{O} 2$ & $2.51 \mathrm{E}-05$ & Basic N1O3 & $\mathrm{C} 43 \mathrm{H} 67 \mathrm{~N} 1 \mathrm{O} 3$ & $3.08 \mathrm{E}-06$ \\
\hline Non-basic N1O1 & $\mathrm{C} 28 \mathrm{H} 27 \mathrm{~N} 1 \mathrm{O} 1$ & $9.62 \mathrm{E}-05$ & Acidic $\mathrm{O} 2$ & $\mathrm{C} 29 \mathrm{H} 54 \mathrm{O} 2$ & $4.82 \mathrm{E}-05$ & Basic N1O3 & C44H69N1O3 & $5.80 \mathrm{E}-06$ \\
\hline Non-basic N1O1 & $\mathrm{C} 29 \mathrm{H} 29 \mathrm{~N} 1 \mathrm{O} 1$ & $5.71 \mathrm{E}-05$ & Acidic $\mathrm{O} 2$ & $\mathrm{C} 30 \mathrm{H} 56 \mathrm{O} 2$ & 0.000109 & Basic N1O3 & $\mathrm{C} 45 \mathrm{H} 71 \mathrm{~N} 1 \mathrm{O} 3$ & 4.17E-06 \\
\hline Non-basic N1O1 & $\mathrm{C} 30 \mathrm{H} 31 \mathrm{~N} 1 \mathrm{O} 1$ & $3.63 \mathrm{E}-05$ & Acidic $\mathrm{O} 2$ & $\mathrm{C} 31 \mathrm{H} 58 \mathrm{O} 2$ & 4.09E-05 & Basic N1O3 & $\mathrm{C} 46 \mathrm{H} 73 \mathrm{~N} 1 \mathrm{O} 3$ & $6.14 \mathrm{E}-06$ \\
\hline Non-basic N1O1 & $\mathrm{C} 31 \mathrm{H} 33 \mathrm{~N} 1 \mathrm{O} 1$ & $2.17 \mathrm{E}-05$ & Acidic $\mathrm{O} 2$ & $\mathrm{C} 32 \mathrm{H} 60 \mathrm{O} 2$ & $3.44 \mathrm{E}-05$ & Basic N1O3 & $\mathrm{C} 47 \mathrm{H} 75 \mathrm{~N} 1 \mathrm{O} 3$ & $1.88 \mathrm{E}-06$ \\
\hline Non-basic N1O1 & $\mathrm{C} 32 \mathrm{H} 35 \mathrm{~N} 1 \mathrm{O} 1$ & $1.69 \mathrm{E}-05$ & Acidic $\mathrm{O} 2$ & $\mathrm{C} 33 \mathrm{H} 62 \mathrm{O} 2$ & $2.16 \mathrm{E}-05$ & Basic N1O3 & C48H77N1O3 & $1.16 \mathrm{E}-06$ \\
\hline Non-basic N1O1 & $\mathrm{C} 33 \mathrm{H} 37 \mathrm{~N} 1 \mathrm{O} 1$ & 4.63E-06 & Acidic $\mathrm{O} 2$ & $\mathrm{C} 34 \mathrm{H} 64 \mathrm{O} 2$ & 0.000206 & Basic N1O3 & C49H79N1O3 & $1.90 \mathrm{E}-06$ \\
\hline Non-basic N1O1 & $\mathrm{C} 21 \mathrm{H} 11 \mathrm{~N} 1 \mathrm{O} 1$ & 0.000158 & Acidic $\mathrm{O} 2$ & $\mathrm{C} 35 \mathrm{H} 66 \mathrm{O} 2$ & $6.25 \mathrm{E}-05$ & Basic N1O3 & $\mathrm{C} 50 \mathrm{H} 81 \mathrm{~N} 1 \mathrm{O} 3$ & 4.43E-06 \\
\hline Non-basic N1O1 & C22H13N1O1 & 0.000545 & Acidic $\mathrm{O} 2$ & $\mathrm{C} 25 \mathrm{H} 44 \mathrm{O} 2$ & $1.49 \mathrm{E}-05$ & Basic N1O3 & C51H83N1O3 & $2.03 \mathrm{E}-06$ \\
\hline Non-basic N1O1 & C23H15N1O1 & 0.00078 & Acidic $\mathrm{O} 2$ & $\mathrm{C} 26 \mathrm{H} 46 \mathrm{O} 2$ & $2.38 \mathrm{E}-05$ & Basic N1O3 & $\mathrm{C} 52 \mathrm{H} 85 \mathrm{~N} 1 \mathrm{O} 3$ & $1.68 \mathrm{E}-06$ \\
\hline Non-basic N1O1 & C24H17N1O1 & 0.00078 & Acidic $\mathrm{O} 2$ & $\mathrm{C} 27 \mathrm{H} 48 \mathrm{O} 2$ & $2.89 \mathrm{E}-05$ & Basic N1O3 & C17H13N1O3 & $1.32 \mathrm{E}-07$ \\
\hline Non-basic N1O1 & $\mathrm{C} 25 \mathrm{H} 19 \mathrm{~N} 1 \mathrm{O} 1$ & 0.000648 & Acidic $\mathrm{O} 2$ & $\mathrm{C} 28 \mathrm{H} 50 \mathrm{O} 2$ & $3.20 \mathrm{E}-05$ & Basic N1O3 & $\mathrm{C} 18 \mathrm{H} 15 \mathrm{~N} 1 \mathrm{O} 3$ & $1.01 \mathrm{E}-06$ \\
\hline Non-basic N1O1 & $\mathrm{C} 26 \mathrm{H} 21 \mathrm{~N} 1 \mathrm{O} 1$ & 0.000442 & Acidic $\mathrm{O} 2$ & $\mathrm{C} 29 \mathrm{H} 52 \mathrm{O} 2$ & 0.000103 & Basic N1O3 & $\mathrm{C} 19 \mathrm{H} 17 \mathrm{~N} 1 \mathrm{O} 3$ & $3.72 \mathrm{E}-06$ \\
\hline Non-basic N1O1 & $\mathrm{C} 27 \mathrm{H} 23 \mathrm{~N} 1 \mathrm{O} 1$ & 0.00026 & Acidic $\mathrm{O} 2$ & $\mathrm{C} 30 \mathrm{H} 54 \mathrm{O} 2$ & 0.000103 & Basic N1O3 & $\mathrm{C} 20 \mathrm{H} 19 \mathrm{~N} 1 \mathrm{O} 3$ & 4.19E-06 \\
\hline Non-basic N1O1 & $\mathrm{C} 28 \mathrm{H} 25 \mathrm{~N} 1 \mathrm{O} 1$ & 0.000151 & Acidic $\mathrm{O} 2$ & $\mathrm{C} 31 \mathrm{H} 56 \mathrm{O} 2$ & 0.000189 & Basic N1O3 & $\mathrm{C} 21 \mathrm{H} 21 \mathrm{~N} 1 \mathrm{O} 3$ & 4.17E-06 \\
\hline Non-basic N1O1 & $\mathrm{C} 29 \mathrm{H} 27 \mathrm{~N} 1 \mathrm{O} 1$ & $8.32 \mathrm{E}-05$ & Acidic $\mathrm{O} 2$ & $\mathrm{C} 32 \mathrm{H} 58 \mathrm{O} 2$ & 0.000196 & Basic N1O3 & $\mathrm{C} 22 \mathrm{H} 23 \mathrm{~N} 1 \mathrm{O} 3$ & $8.29 \mathrm{E}-06$ \\
\hline Non-basic N1O1 & $\mathrm{C} 30 \mathrm{H} 29 \mathrm{~N} 1 \mathrm{O} 1$ & $5.18 \mathrm{E}-05$ & Acidic $\mathrm{O} 2$ & $\mathrm{C} 33 \mathrm{H} 60 \mathrm{O} 2$ & 0.000126 & Basic N1O3 & $\mathrm{C} 23 \mathrm{H} 25 \mathrm{~N} 1 \mathrm{O} 3$ & $1.02 \mathrm{E}-05$ \\
\hline Non-basic N1O1 & $\mathrm{C} 31 \mathrm{H} 31 \mathrm{~N} 1 \mathrm{O} 1$ & $3.70 \mathrm{E}-05$ & Acidic $\mathrm{O} 2$ & $\mathrm{C} 34 \mathrm{H} 62 \mathrm{O} 2$ & 0.000147 & Basic N1O3 & $\mathrm{C} 24 \mathrm{H} 27 \mathrm{~N} 1 \mathrm{O} 3$ & $6.32 \mathrm{E}-06$ \\
\hline Non-basic N1O1 & C32H33N1O1 & $2.29 \mathrm{E}-05$ & Acidic $\mathrm{O} 2$ & $\mathrm{C} 35 \mathrm{H} 64 \mathrm{O} 2$ & $6.28 \mathrm{E}-05$ & Basic N1O3 & $\mathrm{C} 25 \mathrm{H} 29 \mathrm{~N} 1 \mathrm{O} 3$ & $1.40 \mathrm{E}-05$ \\
\hline Non-basic N1O1 & C33H35N1O1 & $1.35 \mathrm{E}-05$ & Acidic $\mathrm{O} 2$ & $\mathrm{C} 36 \mathrm{H} 66 \mathrm{O} 2$ & $6.00 \mathrm{E}-05$ & Basic N1O3 & $\mathrm{C} 26 \mathrm{H} 31 \mathrm{~N} 1 \mathrm{O} 3$ & $1.43 \mathrm{E}-05$ \\
\hline Non-basic N1O1 & C34H37N1O1 & $1.78 \mathrm{E}-05$ & Acidic $\mathrm{O} 2$ & $\mathrm{C} 18 \mathrm{H} 28 \mathrm{O} 2$ & $1.84 \mathrm{E}-05$ & Basic N1O3 & $\mathrm{C} 27 \mathrm{H} 33 \mathrm{~N} 1 \mathrm{O} 3$ & $1.80 \mathrm{E}-05$ \\
\hline Non-basic N1O1 & $\mathrm{C} 40 \mathrm{H} 49 \mathrm{~N} 1 \mathrm{O} 1$ & $1.74 \mathrm{E}-06$ & Acidic $\mathrm{O} 2$ & $\mathrm{C} 19 \mathrm{H} 30 \mathrm{O} 2$ & $4.83 \mathrm{E}-05$ & Basic N1O3 & $\mathrm{C} 28 \mathrm{H} 35 \mathrm{~N} 1 \mathrm{O} 3$ & $2.39 \mathrm{E}-05$ \\
\hline Non-basic N1O1 & $\mathrm{C} 42 \mathrm{H} 53 \mathrm{~N} 1 \mathrm{O} 1$ & $1.93 \mathrm{E}-06$ & Acidic $\mathrm{O} 2$ & $\mathrm{C} 20 \mathrm{H} 32 \mathrm{O} 2$ & 0.000139 & Basic N1O3 & $\mathrm{C} 29 \mathrm{H} 37 \mathrm{~N} 1 \mathrm{O} 3$ & 4.07E-05 \\
\hline Non-basic N1O1 & $\mathrm{C} 43 \mathrm{H} 55 \mathrm{~N} 1 \mathrm{O} 1$ & $2.99 \mathrm{E}-06$ & Acidic $\mathrm{O} 2$ & $\mathrm{C} 21 \mathrm{H} 34 \mathrm{O} 2$ & $1.17 \mathrm{E}-05$ & Basic N1O3 & $\mathrm{C} 30 \mathrm{H} 39 \mathrm{~N} 1 \mathrm{O} 3$ & $3.11 \mathrm{E}-05$ \\
\hline Non-basic N1O1 & C48H65N1O1 & $5.87 \mathrm{E}-06$ & Acidic $\mathrm{O} 2$ & $\mathrm{C} 22 \mathrm{H} 36 \mathrm{O} 2$ & $5.17 \mathrm{E}-05$ & Basic N1O3 & $\mathrm{C} 31 \mathrm{H} 41 \mathrm{~N} 1 \mathrm{O} 3$ & $2.89 \mathrm{E}-05$ \\
\hline Non-basic N1O1 & C49H67N1O1 & $1.08 \mathrm{E}-05$ & Acidic $\mathrm{O} 2$ & $\mathrm{C} 23 \mathrm{H} 38 \mathrm{O} 2$ & 4.42E-05 & Basic N1O3 & $\mathrm{C} 32 \mathrm{H} 43 \mathrm{~N} 1 \mathrm{O} 3$ & $2.67 \mathrm{E}-05$ \\
\hline Non-basic N1O1 & C51H71N1O1 & 4.19E-06 & Acidic $\mathrm{O} 2$ & $\mathrm{C} 24 \mathrm{H} 40 \mathrm{O} 2$ & $6.77 \mathrm{E}-05$ & Basic N1O3 & $\mathrm{C} 33 \mathrm{H} 45 \mathrm{~N} 1 \mathrm{O} 3$ & $1.96 \mathrm{E}-05$ \\
\hline Non-basic N1O1 & C52H73N1O1 & $6.23 \mathrm{E}-06$ & Acidic $\mathrm{O} 2$ & $\mathrm{C} 25 \mathrm{H} 42 \mathrm{O} 2$ & $9.49 \mathrm{E}-05$ & Basic N1O3 & $\mathrm{C} 34 \mathrm{H} 47 \mathrm{~N} 1 \mathrm{O} 3$ & $2.00 \mathrm{E}-05$ \\
\hline Non-basic N1O1 & C23H13N1O1 & 0.000153 & Acidic $\mathrm{O} 2$ & $\mathrm{C} 26 \mathrm{H} 44 \mathrm{O} 2$ & $3.49 \mathrm{E}-05$ & Basic N1O3 & $\mathrm{C} 35 \mathrm{H} 49 \mathrm{~N} 1 \mathrm{O} 3$ & $2.30 \mathrm{E}-05$ \\
\hline Non-basic N1O1 & C24H15N1O1 & 0.000611 & Acidic $\mathrm{O} 2$ & $\mathrm{C} 27 \mathrm{H} 46 \mathrm{O} 2$ & 0.000163 & Basic N1O3 & $\mathrm{C} 36 \mathrm{H} 51 \mathrm{~N} 1 \mathrm{O} 3$ & $8.76 \mathrm{E}-06$ \\
\hline Non-basic N1O1 & $\mathrm{C} 25 \mathrm{H} 17 \mathrm{~N} 1 \mathrm{O} 1$ & 0.000994 & Acidic $\mathrm{O} 2$ & $\mathrm{C} 28 \mathrm{H} 48 \mathrm{O} 2$ & 0.000228 & Basic N1O3 & C37H53N1O3 & $1.78 \mathrm{E}-05$ \\
\hline Non-basic N1O1 & $\mathrm{C} 26 \mathrm{H} 19 \mathrm{~N} 1 \mathrm{O} 1$ & 0.00087 & Acidic $\mathrm{O} 2$ & $\mathrm{C} 29 \mathrm{H} 50 \mathrm{O} 2$ & 0.000191 & Basic N1O3 & $\mathrm{C} 38 \mathrm{H} 55 \mathrm{~N} 1 \mathrm{O} 3$ & $1.65 \mathrm{E}-05$ \\
\hline Non-basic N1O1 & $\mathrm{C} 27 \mathrm{H} 21 \mathrm{~N} 1 \mathrm{O} 1$ & 0.000612 & Acidic $\mathrm{O} 2$ & $\mathrm{C} 30 \mathrm{H} 52 \mathrm{O} 2$ & 0.000358 & Basic N1O3 & $\mathrm{C} 39 \mathrm{H} 57 \mathrm{~N} 1 \mathrm{O} 3$ & $1.43 \mathrm{E}-05$ \\
\hline Non-basic N1O1 & $\mathrm{C} 28 \mathrm{H} 23 \mathrm{~N} 1 \mathrm{O} 1$ & 0.000346 & Acidic $\mathrm{O} 2$ & $\mathrm{C} 31 \mathrm{H} 54 \mathrm{O} 2$ & 0.000275 & Basic N1O3 & $\mathrm{C} 40 \mathrm{H} 59 \mathrm{~N} 1 \mathrm{O} 3$ & $1.47 \mathrm{E}-05$ \\
\hline Non-basic N1O1 & $\mathrm{C} 29 \mathrm{H} 25 \mathrm{~N} 1 \mathrm{O} 1$ & 0.000186 & Acidic $\mathrm{O} 2$ & $\mathrm{C} 32 \mathrm{H} 56 \mathrm{O} 2$ & 0.000392 & Basic N1O3 & $\mathrm{C} 41 \mathrm{H} 61 \mathrm{~N} 1 \mathrm{O} 3$ & $1.52 \mathrm{E}-05$ \\
\hline
\end{tabular}




\begin{tabular}{|c|c|c|c|c|c|c|c|c|}
\hline Class & Formula & Mass & Class & Formula & Mass & Class & Formula & Mass \\
\hline Non-basic N1O1 & $\mathrm{C} 30 \mathrm{H} 27 \mathrm{~N} 1 \mathrm{O} 1$ & $9.99 \mathrm{E}-05$ & Acidic $\mathrm{O} 2$ & $\mathrm{C} 33 \mathrm{H} 58 \mathrm{O} 2$ & 0.000394 & Basic N1O3 & $\mathrm{C} 42 \mathrm{H} 63 \mathrm{~N} 1 \mathrm{O} 3$ & $3.44 \mathrm{E}-06$ \\
\hline Non-basic N1O1 & $\mathrm{C} 31 \mathrm{H} 29 \mathrm{~N} 1 \mathrm{O} 1$ & $6.10 \mathrm{E}-05$ & Acidic $\mathrm{O} 2$ & $\mathrm{C} 34 \mathrm{H} 60 \mathrm{O} 2$ & 0.000253 & Basic N1O3 & $\mathrm{C} 43 \mathrm{H} 65 \mathrm{~N} 1 \mathrm{O} 3$ & $3.72 \mathrm{E}-06$ \\
\hline Non-basic N1O1 & $\mathrm{C} 32 \mathrm{H} 31 \mathrm{~N} 1 \mathrm{O} 1$ & $3.75 \mathrm{E}-05$ & Acidic $\mathrm{O} 2$ & $\mathrm{C} 35 \mathrm{H} 62 \mathrm{O} 2$ & 0.000258 & Basic N1O3 & $\mathrm{C} 19 \mathrm{H} 15 \mathrm{~N} 1 \mathrm{O} 3$ & 2.64E-07 \\
\hline Non-basic N1O1 & $\mathrm{C} 33 \mathrm{H} 33 \mathrm{~N} 1 \mathrm{O} 1$ & $1.93 \mathrm{E}-05$ & Acidic $\mathrm{O} 2$ & $\mathrm{C} 36 \mathrm{H} 64 \mathrm{O} 2$ & 0.000292 & Basic N1O3 & $\mathrm{C} 20 \mathrm{H} 17 \mathrm{~N} 1 \mathrm{O} 3$ & $2.27 \mathrm{E}-06$ \\
\hline Non-basic N1O1 & $\mathrm{C} 34 \mathrm{H} 35 \mathrm{~N} 1 \mathrm{O} 1$ & $9.32 \mathrm{E}-06$ & Acidic $\mathrm{O} 2$ & $\mathrm{C} 37 \mathrm{H} 66 \mathrm{O} 2$ & 0.000118 & Basic N1O3 & C21H19N1O3 & 4.79E-06 \\
\hline Non-basic N1O1 & $\mathrm{C} 35 \mathrm{H} 37 \mathrm{~N} 1 \mathrm{O} 1$ & $6.15 \mathrm{E}-06$ & Acidic $\mathrm{O} 2$ & $\mathrm{C} 38 \mathrm{H} 68 \mathrm{O} 2$ & 0.000132 & Basic N1O3 & $\mathrm{C} 22 \mathrm{H} 21 \mathrm{~N} 1 \mathrm{O} 3$ & $9.15 \mathrm{E}-06$ \\
\hline Non-basic N1O1 & $\mathrm{C} 36 \mathrm{H} 39 \mathrm{~N} 1 \mathrm{O} 1$ & $3.46 \mathrm{E}-06$ & Acidic $\mathrm{O} 2$ & $\mathrm{C} 39 \mathrm{H} 70 \mathrm{O} 2$ & $3.90 \mathrm{E}-05$ & Basic N1O3 & $\mathrm{C} 23 \mathrm{H} 23 \mathrm{~N} 1 \mathrm{O} 3$ & $1.37 \mathrm{E}-05$ \\
\hline Non-basic N1O1 & $\mathrm{C} 37 \mathrm{H} 41 \mathrm{~N} 1 \mathrm{O} 1$ & $2.35 \mathrm{E}-06$ & Acidic $\mathrm{O} 2$ & $\mathrm{C} 17 \mathrm{H} 24 \mathrm{O} 2$ & $9.40 \mathrm{E}-06$ & Basic N1O3 & $\mathrm{C} 24 \mathrm{H} 25 \mathrm{~N} 1 \mathrm{O} 3$ & $1.37 \mathrm{E}-05$ \\
\hline Non-basic N1O1 & $\mathrm{C} 23 \mathrm{H} 11 \mathrm{~N} 1 \mathrm{O} 1$ & $1.00 \mathrm{E}-05$ & Acidic $\mathrm{O} 2$ & $\mathrm{C} 18 \mathrm{H} 26 \mathrm{O} 2$ & $1.66 \mathrm{E}-05$ & Basic N1O3 & $\mathrm{C} 25 \mathrm{H} 27 \mathrm{~N} 1 \mathrm{O} 3$ & $1.83 \mathrm{E}-05$ \\
\hline Non-basic N1O1 & $\mathrm{C} 24 \mathrm{H} 13 \mathrm{~N} 1 \mathrm{O} 1$ & $7.67 \mathrm{E}-05$ & Acidic $\mathrm{O} 2$ & $\mathrm{C} 19 \mathrm{H} 28 \mathrm{O} 2$ & $2.32 \mathrm{E}-05$ & Basic N1O3 & $\mathrm{C} 26 \mathrm{H} 29 \mathrm{~N} 1 \mathrm{O} 3$ & $1.64 \mathrm{E}-05$ \\
\hline Non-basic N1O1 & $\mathrm{C} 25 \mathrm{H} 15 \mathrm{~N} 1 \mathrm{O} 1$ & 0.000209 & Acidic $\mathrm{O} 2$ & $\mathrm{C} 20 \mathrm{H} 30 \mathrm{O} 2$ & 0.000438 & Basic N1O3 & $\mathrm{C} 27 \mathrm{H} 31 \mathrm{~N} 1 \mathrm{O} 3$ & $1.91 \mathrm{E}-05$ \\
\hline Non-basic N1O1 & $\mathrm{C} 26 \mathrm{H} 17 \mathrm{~N} 1 \mathrm{O} 1$ & 0.000305 & Acidic $\mathrm{O} 2$ & $\mathrm{C} 21 \mathrm{H} 32 \mathrm{O} 2$ & $4.80 \mathrm{E}-05$ & Basic N1O3 & $\mathrm{C} 28 \mathrm{H} 33 \mathrm{~N} 1 \mathrm{O} 3$ & $2.07 \mathrm{E}-05$ \\
\hline Non-basic N1O1 & $\mathrm{C} 27 \mathrm{H} 19 \mathrm{~N} 1 \mathrm{O} 1$ & 0.000306 & Acidic $\mathrm{O} 2$ & $\mathrm{C} 21 \mathrm{H} 32 \mathrm{O} 2$ & $5.01 \mathrm{E}-05$ & Basic N1O3 & $\mathrm{C} 29 \mathrm{H} 35 \mathrm{~N} 1 \mathrm{O} 3$ & $2.18 \mathrm{E}-05$ \\
\hline Non-basic N1O1 & $\mathrm{C} 28 \mathrm{H} 21 \mathrm{~N} 1 \mathrm{O} 1$ & 0.000238 & Acidic $\mathrm{O} 2$ & $\mathrm{C} 23 \mathrm{H} 36 \mathrm{O} 2$ & 7.37E-06 & Basic N1O3 & $\mathrm{C} 30 \mathrm{H} 37 \mathrm{~N} 1 \mathrm{O} 3$ & $2.43 \mathrm{E}-05$ \\
\hline Non-basic N1O1 & $\mathrm{C} 29 \mathrm{H} 23 \mathrm{~N} 1 \mathrm{O} 1$ & 0.000178 & Acidic $\mathrm{O} 2$ & $\mathrm{C} 24 \mathrm{H} 38 \mathrm{O} 2$ & $5.17 \mathrm{E}-05$ & Basic N1O3 & $\mathrm{C} 31 \mathrm{H} 39 \mathrm{~N} 1 \mathrm{O} 3$ & $1.96 \mathrm{E}-05$ \\
\hline Non-basic N1O1 & $\mathrm{C} 30 \mathrm{H} 25 \mathrm{~N} 1 \mathrm{O} 1$ & 0.000103 & Acidic $\mathrm{O} 2$ & $\mathrm{C} 25 \mathrm{H} 40 \mathrm{O} 2$ & $5.00 \mathrm{E}-05$ & Basic N1O3 & $\mathrm{C} 32 \mathrm{H} 41 \mathrm{~N} 1 \mathrm{O} 3$ & $1.95 \mathrm{E}-05$ \\
\hline Non-basic N1O1 & $\mathrm{C} 31 \mathrm{H} 27 \mathrm{~N} 1 \mathrm{O} 1$ & $6.49 \mathrm{E}-05$ & Acidic $\mathrm{O} 2$ & $\mathrm{C} 26 \mathrm{H} 42 \mathrm{O} 2$ & $1.57 \mathrm{E}-05$ & Basic N1O3 & $\mathrm{C} 33 \mathrm{H} 43 \mathrm{~N} 1 \mathrm{O} 3$ & $1.66 \mathrm{E}-05$ \\
\hline Non-basic N1O1 & $\mathrm{C} 32 \mathrm{H} 29 \mathrm{~N} 1 \mathrm{O} 1$ & $4.16 \mathrm{E}-05$ & Acidic $\mathrm{O} 2$ & $\mathrm{C} 27 \mathrm{H} 44 \mathrm{O} 2$ & 0.00019 & Basic N1O3 & $\mathrm{C} 34 \mathrm{H} 45 \mathrm{~N} 1 \mathrm{O} 3$ & $5.75 \mathrm{E}-05$ \\
\hline Non-basic N1O1 & $\mathrm{C} 33 \mathrm{H} 31 \mathrm{~N} 1 \mathrm{O} 1$ & $2.84 \mathrm{E}-05$ & Acidic $\mathrm{O} 2$ & $\mathrm{C} 28 \mathrm{H} 46 \mathrm{O} 2$ & $9.79 \mathrm{E}-05$ & Basic N1O3 & $\mathrm{C} 35 \mathrm{H} 47 \mathrm{~N} 1 \mathrm{O} 3$ & $2.75 \mathrm{E}-05$ \\
\hline Non-basic N1O1 & $\mathrm{C} 34 \mathrm{H} 33 \mathrm{~N} 1 \mathrm{O} 1$ & $4.82 \mathrm{E}-06$ & Acidic $\mathrm{O} 2$ & $\mathrm{C} 29 \mathrm{H} 48 \mathrm{O} 2$ & 0.000156 & Basic N1O3 & $\mathrm{C} 36 \mathrm{H} 49 \mathrm{~N} 1 \mathrm{O} 3$ & $1.58 \mathrm{E}-05$ \\
\hline Non-basic N1O1 & $\mathrm{C} 35 \mathrm{H} 35 \mathrm{~N} 1 \mathrm{O} 1$ & $7.26 \mathrm{E}-06$ & Acidic $\mathrm{O} 2$ & $\mathrm{C} 30 \mathrm{H} 50 \mathrm{O} 2$ & 0.000316 & Basic N1O3 & C37H51N1O3 & $1.62 \mathrm{E}-05$ \\
\hline Non-basic N1O1 & $\mathrm{C} 36 \mathrm{H} 37 \mathrm{~N} 1 \mathrm{O} 1$ & $5.21 \mathrm{E}-06$ & Acidic $\mathrm{O} 2$ & $\mathrm{C} 31 \mathrm{H} 52 \mathrm{O} 2$ & 0.000298 & Basic N1O3 & C38H53N1O3 & $1.35 \mathrm{E}-05$ \\
\hline Non-basic N1O1 & $\mathrm{C} 37 \mathrm{H} 39 \mathrm{~N} 1 \mathrm{O} 1$ & $1.61 \mathrm{E}-06$ & Acidic $\mathrm{O} 2$ & $\mathrm{C} 32 \mathrm{H} 54 \mathrm{O} 2$ & 0.000201 & Basic N1O3 & C39H55N1O3 & $1.30 \mathrm{E}-05$ \\
\hline Non-basic N1O1 & $\mathrm{C} 38 \mathrm{H} 41 \mathrm{~N} 1 \mathrm{O} 1$ & $3.06 \mathrm{E}-06$ & Acidic $\mathrm{O} 2$ & $\mathrm{C} 33 \mathrm{H} 56 \mathrm{O} 2$ & 0.000269 & Basic N1O3 & $\mathrm{C} 40 \mathrm{H} 57 \mathrm{~N} 1 \mathrm{O} 3$ & $1.24 \mathrm{E}-05$ \\
\hline Non-basic N1O1 & $\mathrm{C} 25 \mathrm{H} 13 \mathrm{~N} 1 \mathrm{O} 1$ & $5.77 \mathrm{E}-05$ & Acidic $\mathrm{O} 2$ & $\mathrm{C} 34 \mathrm{H} 58 \mathrm{O} 2$ & 0.000175 & Basic N1O3 & $\mathrm{C} 41 \mathrm{H} 59 \mathrm{~N} 1 \mathrm{O} 3$ & $7.51 \mathrm{E}-06$ \\
\hline Non-basic N1O1 & $\mathrm{C} 26 \mathrm{H} 15 \mathrm{~N} 1 \mathrm{O} 1$ & 0.000173 & Acidic $\mathrm{O} 2$ & $\mathrm{C} 35 \mathrm{H} 60 \mathrm{O} 2$ & 0.000161 & Basic N1O3 & $\mathrm{C} 42 \mathrm{H} 61 \mathrm{~N} 1 \mathrm{O} 3$ & $1.05 \mathrm{E}-05$ \\
\hline Non-basic N1O1 & $\mathrm{C} 27 \mathrm{H} 17 \mathrm{~N} 1 \mathrm{O} 1$ & 0.000233 & Acidic $\mathrm{O} 2$ & $\mathrm{C} 36 \mathrm{H} 62 \mathrm{O} 2$ & 0.000103 & Basic N1O3 & $\mathrm{C} 43 \mathrm{H} 63 \mathrm{~N} 1 \mathrm{O} 3$ & $8.02 \mathrm{E}-06$ \\
\hline Non-basic N1O1 & C28H19N1O1 & 0.000212 & Acidic $\mathrm{O} 2$ & $\mathrm{C} 37 \mathrm{H} 64 \mathrm{O} 2$ & 0.000209 & Basic N1O3 & $\mathrm{C} 44 \mathrm{H} 65 \mathrm{~N} 1 \mathrm{O} 3$ & $3.36 \mathrm{E}-06$ \\
\hline Non-basic N1O1 & $\mathrm{C} 29 \mathrm{H} 21 \mathrm{~N} 1 \mathrm{O} 1$ & 0.000143 & Acidic $\mathrm{O} 2$ & $\mathrm{C} 38 \mathrm{H} 66 \mathrm{O} 2$ & $6.55 \mathrm{E}-05$ & Basic N1O3 & $\mathrm{C} 20 \mathrm{H} 15 \mathrm{~N} 1 \mathrm{O} 3$ & $3.00 \mathrm{E}-07$ \\
\hline Non-basic N1O1 & $\mathrm{C} 30 \mathrm{H} 23 \mathrm{~N} 1 \mathrm{O} 1$ & 0.000112 & Acidic $\mathrm{O} 2$ & $\mathrm{C} 39 \mathrm{H} 68 \mathrm{O} 2$ & $6.27 \mathrm{E}-05$ & Basic N1O3 & $\mathrm{C} 21 \mathrm{H} 17 \mathrm{~N} 1 \mathrm{O} 3$ & $1.19 \mathrm{E}-06$ \\
\hline Non-basic N1O1 & $\mathrm{C} 31 \mathrm{H} 25 \mathrm{~N} 1 \mathrm{O} 1$ & $7.00 \mathrm{E}-05$ & Acidic $\mathrm{O} 2$ & $\mathrm{C} 14 \mathrm{H} 16 \mathrm{O} 2$ & $1.20 \mathrm{E}-05$ & Basic N1O3 & $\mathrm{C} 22 \mathrm{H} 19 \mathrm{~N} 1 \mathrm{O} 3$ & $8.52 \mathrm{E}-06$ \\
\hline Non-basic N1O1 & $\mathrm{C} 32 \mathrm{H} 27 \mathrm{~N} 1 \mathrm{O} 1$ & $4.26 \mathrm{E}-05$ & Acidic $\mathrm{O} 2$ & $\mathrm{C} 15 \mathrm{H} 18 \mathrm{O} 2$ & $3.13 \mathrm{E}-05$ & Basic N1O3 & $\mathrm{C} 23 \mathrm{H} 21 \mathrm{~N} 1 \mathrm{O} 3$ & $5.93 \mathrm{E}-06$ \\
\hline Non-basic N1O1 & $\mathrm{C} 33 \mathrm{H} 29 \mathrm{~N} 1 \mathrm{O} 1$ & $2.77 \mathrm{E}-05$ & Acidic $\mathrm{O} 2$ & $\mathrm{C} 16 \mathrm{H} 20 \mathrm{O} 2$ & $1.53 \mathrm{E}-05$ & Basic N1O3 & $\mathrm{C} 24 \mathrm{H} 23 \mathrm{~N} 1 \mathrm{O} 3$ & $8.11 \mathrm{E}-06$ \\
\hline Non-basic N1O1 & $\mathrm{C} 34 \mathrm{H} 31 \mathrm{~N} 1 \mathrm{O} 1$ & 8.79E-06 & Acidic $\mathrm{O} 2$ & $\mathrm{C} 17 \mathrm{H} 22 \mathrm{O} 2$ & $5.49 \mathrm{E}-06$ & Basic N1O3 & $\mathrm{C} 25 \mathrm{H} 25 \mathrm{~N} 1 \mathrm{O} 3$ & $1.06 \mathrm{E}-05$ \\
\hline Non-basic N1O1 & $\mathrm{C} 35 \mathrm{H} 33 \mathrm{~N} 1 \mathrm{O} 1$ & $2.42 \mathrm{E}-06$ & Acidic $\mathrm{O} 2$ & $\mathrm{C} 18 \mathrm{H} 24 \mathrm{O} 2$ & $9.30 \mathrm{E}-06$ & Basic N1O3 & $\mathrm{C} 26 \mathrm{H} 27 \mathrm{~N} 1 \mathrm{O} 3$ & $1.92 \mathrm{E}-05$ \\
\hline Non-basic N1O1 & $\mathrm{C} 36 \mathrm{H} 35 \mathrm{~N} 1 \mathrm{O} 1$ & $1.46 \mathrm{E}-06$ & Acidic $\mathrm{O} 2$ & $\mathrm{C} 19 \mathrm{H} 26 \mathrm{O} 2$ & $6.35 \mathrm{E}-05$ & Basic N1O3 & $\mathrm{C} 27 \mathrm{H} 29 \mathrm{~N} 1 \mathrm{O} 3$ & $1.69 \mathrm{E}-05$ \\
\hline Non-basic N1O1 & $\mathrm{C} 25 \mathrm{H} 11 \mathrm{~N} 1 \mathrm{O} 1$ & $2.35 \mathrm{E}-06$ & Acidic $\mathrm{O} 2$ & $\mathrm{C} 20 \mathrm{H} 28 \mathrm{O} 2$ & 0.00088 & Basic N1O3 & $\mathrm{C} 28 \mathrm{H} 31 \mathrm{~N} 1 \mathrm{O} 3$ & $2.18 \mathrm{E}-05$ \\
\hline Non-basic N1O1 & $\mathrm{C} 26 \mathrm{H} 13 \mathrm{~N} 1 \mathrm{O} 1$ & $1.14 \mathrm{E}-05$ & Acidic $\mathrm{O} 2$ & $\mathrm{C} 21 \mathrm{H} 30 \mathrm{O} 2$ & 0.000921 & Basic N1O3 & $\mathrm{C} 29 \mathrm{H} 33 \mathrm{~N} 1 \mathrm{O} 3$ & $2.46 \mathrm{E}-05$ \\
\hline
\end{tabular}




\begin{tabular}{|c|c|c|c|c|c|c|c|c|}
\hline Class & Formula & Mass & Class & Formula & Mass & Class & Formula & Mass \\
\hline Non-basic N1O1 & C27H15N1O1 & $5.55 \mathrm{E}-05$ & Acidic $\mathrm{O} 2$ & $\mathrm{C} 22 \mathrm{H} 32 \mathrm{O} 2$ & $1.25 \mathrm{E}-05$ & Basic N1O3 & $\mathrm{C} 30 \mathrm{H} 35 \mathrm{~N} 1 \mathrm{O} 3$ & $2.01 \mathrm{E}-05$ \\
\hline Non-basic N1O1 & $\mathrm{C} 28 \mathrm{H} 17 \mathrm{~N} 1 \mathrm{O} 1$ & 0.000124 & Acidic $\mathrm{O} 2$ & $\mathrm{C} 23 \mathrm{H} 34 \mathrm{O} 2$ & $3.77 \mathrm{E}-05$ & Basic N1O3 & $\mathrm{C} 31 \mathrm{H} 37 \mathrm{~N} 1 \mathrm{O} 3$ & $1.78 \mathrm{E}-05$ \\
\hline Non-basic N1O1 & C29H19N1O1 & 0.000184 & Acidic $\mathrm{O} 2$ & $\mathrm{C} 24 \mathrm{H} 36 \mathrm{O} 2$ & $5.31 \mathrm{E}-05$ & Basic N1O3 & $\mathrm{C} 32 \mathrm{H} 39 \mathrm{~N} 1 \mathrm{O} 3$ & $2.26 \mathrm{E}-05$ \\
\hline Non-basic N1O1 & $\mathrm{C} 30 \mathrm{H} 21 \mathrm{~N} 1 \mathrm{O} 1$ & 0.000164 & Acidic $\mathrm{O} 2$ & $\mathrm{C} 25 \mathrm{H} 38 \mathrm{O} 2$ & $5.38 \mathrm{E}-05$ & Basic N1O3 & $\mathrm{C} 33 \mathrm{H} 41 \mathrm{~N} 1 \mathrm{O} 3$ & $1.72 \mathrm{E}-05$ \\
\hline Non-basic N1O1 & $\mathrm{C} 31 \mathrm{H} 23 \mathrm{~N} 1 \mathrm{O} 1$ & 0.000148 & Acidic $\mathrm{O} 2$ & $\mathrm{C} 26 \mathrm{H} 40 \mathrm{O} 2$ & $5.59 \mathrm{E}-05$ & Basic N1O3 & $\mathrm{C} 34 \mathrm{H} 43 \mathrm{~N} 1 \mathrm{O} 3$ & $2.23 \mathrm{E}-05$ \\
\hline Non-basic N1O1 & $\mathrm{C} 32 \mathrm{H} 25 \mathrm{~N} 1 \mathrm{O} 1$ & $9.97 \mathrm{E}-05$ & Acidic $\mathrm{O} 2$ & $\mathrm{C} 27 \mathrm{H} 42 \mathrm{O} 2$ & $1.22 \mathrm{E}-05$ & Basic N1O3 & $\mathrm{C} 35 \mathrm{H} 45 \mathrm{~N} 1 \mathrm{O} 3$ & $1.92 \mathrm{E}-05$ \\
\hline Non-basic N1O1 & $\mathrm{C} 33 \mathrm{H} 27 \mathrm{~N} 1 \mathrm{O} 1$ & $5.96 \mathrm{E}-05$ & Acidic $\mathrm{O} 2$ & $\mathrm{C} 28 \mathrm{H} 44 \mathrm{O} 2$ & $2.44 \mathrm{E}-05$ & Basic N1O3 & $\mathrm{C} 36 \mathrm{H} 47 \mathrm{~N} 1 \mathrm{O} 3$ & $2.04 \mathrm{E}-05$ \\
\hline Non-basic N1O1 & $\mathrm{C} 34 \mathrm{H} 29 \mathrm{~N} 1 \mathrm{O} 1$ & $2.59 \mathrm{E}-05$ & Acidic $\mathrm{O} 2$ & $\mathrm{C} 29 \mathrm{H} 46 \mathrm{O} 2$ & $7.88 \mathrm{E}-05$ & Basic N1O3 & $\mathrm{C} 37 \mathrm{H} 49 \mathrm{~N} 1 \mathrm{O} 3$ & $1.75 \mathrm{E}-05$ \\
\hline Non-basic N1O1 & $\mathrm{C} 35 \mathrm{H} 31 \mathrm{~N} 1 \mathrm{O} 1$ & $1.01 \mathrm{E}-05$ & Acidic $\mathrm{O} 2$ & $\mathrm{C} 30 \mathrm{H} 48 \mathrm{O} 2$ & $8.23 \mathrm{E}-05$ & Basic N1O3 & C38H51N1O3 & $1.29 \mathrm{E}-05$ \\
\hline Non-basic N1O1 & $\mathrm{C} 36 \mathrm{H} 33 \mathrm{~N} 1 \mathrm{O} 1$ & $5.86 \mathrm{E}-06$ & Acidic $\mathrm{O} 2$ & $\mathrm{C} 31 \mathrm{H} 50 \mathrm{O} 2$ & 0.000203 & Basic N1O3 & C39H53N1O3 & $1.10 \mathrm{E}-05$ \\
\hline Non-basic N1O1 & $\mathrm{C} 27 \mathrm{H} 13 \mathrm{~N} 1 \mathrm{O} 1$ & $3.56 \mathrm{E}-06$ & Acidic $\mathrm{O} 2$ & $\mathrm{C} 32 \mathrm{H} 52 \mathrm{O} 2$ & 0.000125 & Basic N1O3 & $\mathrm{C} 40 \mathrm{H} 55 \mathrm{~N} 1 \mathrm{O} 3$ & $1.44 \mathrm{E}-05$ \\
\hline Non-basic N1O1 & $\mathrm{C} 28 \mathrm{H} 15 \mathrm{~N} 1 \mathrm{O} 1$ & $2.85 \mathrm{E}-05$ & Acidic $\mathrm{O} 2$ & $\mathrm{C} 33 \mathrm{H} 54 \mathrm{O} 2$ & 0.000168 & Basic N1O3 & C41H57N1O3 & 4.42E-06 \\
\hline Non-basic N1O1 & $\mathrm{C} 29 \mathrm{H} 17 \mathrm{~N} 1 \mathrm{O} 1$ & $3.40 \mathrm{E}-05$ & Acidic $\mathrm{O} 2$ & $\mathrm{C} 34 \mathrm{H} 56 \mathrm{O} 2$ & $8.11 \mathrm{E}-05$ & Basic N1O3 & C43H61N1O3 & $3.69 \mathrm{E}-06$ \\
\hline Non-basic N1O1 & $\mathrm{C} 30 \mathrm{H} 19 \mathrm{~N} 1 \mathrm{O} 1$ & $6.24 \mathrm{E}-05$ & Acidic $\mathrm{O} 2$ & $\mathrm{C} 35 \mathrm{H} 58 \mathrm{O} 2$ & $5.89 \mathrm{E}-05$ & Basic N1O3 & C44H63N1O3 & $3.06 \mathrm{E}-07$ \\
\hline Non-basic N1O1 & $\mathrm{C} 31 \mathrm{H} 21 \mathrm{~N} 1 \mathrm{O} 1$ & $7.16 \mathrm{E}-05$ & Acidic $\mathrm{O} 2$ & $\mathrm{C} 36 \mathrm{H} 60 \mathrm{O} 2$ & $4.71 \mathrm{E}-05$ & Basic N1O3 & $\mathrm{C} 45 \mathrm{H} 65 \mathrm{~N} 1 \mathrm{O} 3$ & $3.69 \mathrm{E}-06$ \\
\hline Non-basic N1O1 & $\mathrm{C} 32 \mathrm{H} 23 \mathrm{~N} 1 \mathrm{O} 1$ & 7.99E-05 & Acidic $\mathrm{O} 2$ & $\mathrm{C} 37 \mathrm{H} 62 \mathrm{O} 2$ & $4.27 \mathrm{E}-05$ & Basic N1O3 & $\mathrm{C} 46 \mathrm{H} 67 \mathrm{~N} 1 \mathrm{O} 3$ & $3.01 \mathrm{E}-06$ \\
\hline Non-basic N1O1 & $\mathrm{C} 33 \mathrm{H} 25 \mathrm{~N} 1 \mathrm{O} 1$ & $6.25 \mathrm{E}-05$ & Acidic $\mathrm{O} 2$ & $\mathrm{C} 38 \mathrm{H} 64 \mathrm{O} 2$ & $3.94 \mathrm{E}-05$ & Basic N1O3 & $\mathrm{C} 48 \mathrm{H} 71 \mathrm{~N} 1 \mathrm{O} 3$ & $1.87 \mathrm{E}-06$ \\
\hline Non-basic N1O1 & $\mathrm{C} 34 \mathrm{H} 27 \mathrm{~N} 1 \mathrm{O} 1$ & $4.98 \mathrm{E}-05$ & Acidic $\mathrm{O} 2$ & $\mathrm{C} 19 \mathrm{H} 24 \mathrm{O} 2$ & $3.16 \mathrm{E}-05$ & Basic N1O3 & $\mathrm{C} 49 \mathrm{H} 73 \mathrm{~N} 1 \mathrm{O} 3$ & $4.55 \mathrm{E}-06$ \\
\hline Non-basic N1O1 & $\mathrm{C} 35 \mathrm{H} 29 \mathrm{~N} 1 \mathrm{O} 1$ & $2.91 \mathrm{E}-05$ & Acidic $\mathrm{O} 2$ & $\mathrm{C} 20 \mathrm{H} 26 \mathrm{O} 2$ & $7.02 \mathrm{E}-05$ & Basic N1O3 & $\mathrm{C} 50 \mathrm{H} 75 \mathrm{~N} 1 \mathrm{O} 3$ & 4.03E-06 \\
\hline Non-basic N1O1 & $\mathrm{C} 36 \mathrm{H} 31 \mathrm{~N} 1 \mathrm{O} 1$ & $2.27 \mathrm{E}-05$ & Acidic $\mathrm{O} 2$ & $\mathrm{C} 21 \mathrm{H} 28 \mathrm{O} 2$ & $2.31 \mathrm{E}-05$ & Basic N1O3 & C51H77N1O3 & $5.69 \mathrm{E}-06$ \\
\hline Non-basic N1O1 & $\mathrm{C} 37 \mathrm{H} 33 \mathrm{~N} 1 \mathrm{O} 1$ & $3.73 \mathrm{E}-06$ & Acidic $\mathrm{O} 2$ & $\mathrm{C} 22 \mathrm{H} 30 \mathrm{O} 2$ & $4.46 \mathrm{E}-05$ & Basic N1O3 & $\mathrm{C} 22 \mathrm{H} 17 \mathrm{~N} 1 \mathrm{O} 3$ & $1.58 \mathrm{E}-06$ \\
\hline Non-basic N1O1 & $\mathrm{C} 38 \mathrm{H} 35 \mathrm{~N} 1 \mathrm{O} 1$ & $2.48 \mathrm{E}-06$ & Acidic $\mathrm{O} 2$ & $\mathrm{C} 23 \mathrm{H} 32 \mathrm{O} 2$ & $2.08 \mathrm{E}-05$ & Basic N1O3 & $\mathrm{C} 23 \mathrm{H} 19 \mathrm{~N} 1 \mathrm{O} 3$ & $4.39 \mathrm{E}-06$ \\
\hline Non-basic N1O1 & $\mathrm{C} 39 \mathrm{H} 37 \mathrm{~N} 1 \mathrm{O} 1$ & $2.74 \mathrm{E}-06$ & Acidic $\mathrm{O} 2$ & $\mathrm{C} 24 \mathrm{H} 34 \mathrm{O} 2$ & $6.33 \mathrm{E}-05$ & Basic N1O3 & $\mathrm{C} 24 \mathrm{H} 21 \mathrm{~N} 1 \mathrm{O} 3$ & $5.27 \mathrm{E}-06$ \\
\hline Non-basic N1O1 & $\mathrm{C} 29 \mathrm{H} 15 \mathrm{~N} 1 \mathrm{O} 1$ & $3.05 \mathrm{E}-06$ & Acidic $\mathrm{O} 2$ & $\mathrm{C} 25 \mathrm{H} 36 \mathrm{O} 2$ & $3.52 \mathrm{E}-05$ & Basic N1O3 & $\mathrm{C} 25 \mathrm{H} 23 \mathrm{~N} 1 \mathrm{O} 3$ & $1.43 \mathrm{E}-05$ \\
\hline Non-basic N1O1 & $\mathrm{C} 30 \mathrm{H} 17 \mathrm{~N} 1 \mathrm{O} 1$ & $2.11 \mathrm{E}-05$ & Acidic $\mathrm{O} 2$ & $\mathrm{C} 26 \mathrm{H} 38 \mathrm{O} 2$ & $1.67 \mathrm{E}-05$ & Basic N1O3 & $\mathrm{C} 26 \mathrm{H} 25 \mathrm{~N} 1 \mathrm{O} 3$ & $1.62 \mathrm{E}-05$ \\
\hline Non-basic N1O1 & $\mathrm{C} 31 \mathrm{H} 19 \mathrm{~N} 1 \mathrm{O} 1$ & $3.63 \mathrm{E}-05$ & Acidic $\mathrm{O} 2$ & $\mathrm{C} 27 \mathrm{H} 40 \mathrm{O} 2$ & $6.51 \mathrm{E}-05$ & Basic N1O3 & $\mathrm{C} 27 \mathrm{H} 27 \mathrm{~N} 1 \mathrm{O} 3$ & $1.60 \mathrm{E}-05$ \\
\hline Non-basic N1O1 & $\mathrm{C} 32 \mathrm{H} 21 \mathrm{~N} 1 \mathrm{O} 1$ & $3.48 \mathrm{E}-05$ & Acidic $\mathrm{O} 2$ & $\mathrm{C} 28 \mathrm{H} 42 \mathrm{O} 2$ & 0.000139 & Basic N1O3 & $\mathrm{C} 28 \mathrm{H} 29 \mathrm{~N} 1 \mathrm{O} 3$ & 2.64E-05 \\
\hline Non-basic N1O1 & $\mathrm{C} 33 \mathrm{H} 23 \mathrm{~N} 1 \mathrm{O} 1$ & $2.15 \mathrm{E}-05$ & Acidic $\mathrm{O} 2$ & $\mathrm{C} 29 \mathrm{H} 44 \mathrm{O} 2$ & $3.43 \mathrm{E}-05$ & Basic N1O3 & $\mathrm{C} 29 \mathrm{H} 31 \mathrm{~N} 1 \mathrm{O} 3$ & $1.75 \mathrm{E}-05$ \\
\hline Non-basic N1O1 & $\mathrm{C} 34 \mathrm{H} 25 \mathrm{~N} 1 \mathrm{O} 1$ & $2.28 \mathrm{E}-05$ & Acidic $\mathrm{O} 2$ & $\mathrm{C} 30 \mathrm{H} 46 \mathrm{O} 2$ & 0.000176 & Basic N1O3 & $\mathrm{C} 30 \mathrm{H} 33 \mathrm{~N} 1 \mathrm{O} 3$ & $2.78 \mathrm{E}-05$ \\
\hline Non-basic N1O1 & $\mathrm{C} 35 \mathrm{H} 27 \mathrm{~N} 1 \mathrm{O} 1$ & $1.88 \mathrm{E}-05$ & Acidic $\mathrm{O} 2$ & $\mathrm{C} 31 \mathrm{H} 48 \mathrm{O} 2$ & $9.21 \mathrm{E}-05$ & Basic N1O3 & $\mathrm{C} 31 \mathrm{H} 35 \mathrm{~N} 1 \mathrm{O} 3$ & $1.95 \mathrm{E}-05$ \\
\hline Non-basic N1O1 & $\mathrm{C} 36 \mathrm{H} 29 \mathrm{~N} 1 \mathrm{O} 1$ & $1.93 \mathrm{E}-05$ & Acidic $\mathrm{O} 2$ & $\mathrm{C} 32 \mathrm{H} 50 \mathrm{O} 2$ & 0.000119 & Basic N1O3 & $\mathrm{C} 32 \mathrm{H} 37 \mathrm{~N} 1 \mathrm{O} 3$ & $1.57 \mathrm{E}-05$ \\
\hline Non-basic N1O1 & $\mathrm{C} 37 \mathrm{H} 31 \mathrm{~N} 1 \mathrm{O} 1$ & $9.68 \mathrm{E}-06$ & Acidic $\mathrm{O} 2$ & $\mathrm{C} 33 \mathrm{H} 52 \mathrm{O} 2$ & 0.000115 & Basic N1O3 & $\mathrm{C} 33 \mathrm{H} 39 \mathrm{~N} 1 \mathrm{O} 3$ & $1.99 \mathrm{E}-05$ \\
\hline Non-basic N1O1 & C38H33N1O1 & $5.89 \mathrm{E}-06$ & Acidic $\mathrm{O} 2$ & $\mathrm{C} 34 \mathrm{H} 54 \mathrm{O} 2$ & 0.000114 & Basic N1O3 & $\mathrm{C} 34 \mathrm{H} 41 \mathrm{~N} 1 \mathrm{O} 3$ & $1.05 \mathrm{E}-05$ \\
\hline Non-basic N1O1 & $\mathrm{C} 39 \mathrm{H} 35 \mathrm{~N} 1 \mathrm{O} 1$ & $1.07 \mathrm{E}-05$ & Acidic $\mathrm{O} 2$ & $\mathrm{C} 23 \mathrm{H} 30 \mathrm{O} 2$ & $1.49 \mathrm{E}-05$ & Basic N1O3 & $\mathrm{C} 35 \mathrm{H} 43 \mathrm{~N} 1 \mathrm{O} 3$ & $1.56 \mathrm{E}-05$ \\
\hline Non-basic N1O1 & $\mathrm{C} 40 \mathrm{H} 37 \mathrm{~N} 1 \mathrm{O} 1$ & $2.66 \mathrm{E}-06$ & Acidic $\mathrm{O} 2$ & $\mathrm{C} 24 \mathrm{H} 32 \mathrm{O} 2$ & 0.000111 & Basic N1O3 & $\mathrm{C} 36 \mathrm{H} 45 \mathrm{~N} 1 \mathrm{O} 3$ & $9.33 \mathrm{E}-06$ \\
\hline Non-basic N1O1 & $\mathrm{C} 31 \mathrm{H} 17 \mathrm{~N} 1 \mathrm{O} 1$ & $1.17 \mathrm{E}-06$ & Acidic $\mathrm{O} 2$ & $\mathrm{C} 25 \mathrm{H} 34 \mathrm{O} 2$ & 7.12E-05 & Basic N1O3 & $\mathrm{C} 37 \mathrm{H} 47 \mathrm{~N} 1 \mathrm{O} 3$ & $8.60 \mathrm{E}-06$ \\
\hline Non-basic N1O1 & $\mathrm{C} 32 \mathrm{H} 19 \mathrm{~N} 1 \mathrm{O} 1$ & $1.50 \mathrm{E}-05$ & Acidic $\mathrm{O} 2$ & $\mathrm{C} 26 \mathrm{H} 36 \mathrm{O} 2$ & $4.33 \mathrm{E}-05$ & Basic N1O3 & $\mathrm{C} 38 \mathrm{H} 49 \mathrm{~N} 1 \mathrm{O} 3$ & $1.16 \mathrm{E}-05$ \\
\hline Non-basic N1O1 & $\mathrm{C} 33 \mathrm{H} 21 \mathrm{~N} 1 \mathrm{O} 1$ & $2.81 \mathrm{E}-05$ & Acidic $\mathrm{O} 2$ & $\mathrm{C} 27 \mathrm{H} 38 \mathrm{O} 2$ & $6.60 \mathrm{E}-05$ & Basic N1O3 & C39H51N1O3 & 7.33E-06 \\
\hline
\end{tabular}




\begin{tabular}{|c|c|c|c|c|c|c|c|c|}
\hline Class & Formula & Mass & Class & Formula & Mass & Class & Formula & Mass \\
\hline Non-basic N1O1 & $\mathrm{C} 34 \mathrm{H} 23 \mathrm{~N} 1 \mathrm{O} 1$ & $4.82 \mathrm{E}-05$ & Acidic $\mathrm{O} 2$ & $\mathrm{C} 28 \mathrm{H} 40 \mathrm{O} 2$ & $5.99 \mathrm{E}-05$ & Basic N1O3 & $\mathrm{C} 40 \mathrm{H} 53 \mathrm{~N} 1 \mathrm{O} 3$ & $4.98 \mathrm{E}-06$ \\
\hline Non-basic N1O1 & $\mathrm{C} 35 \mathrm{H} 25 \mathrm{~N} 1 \mathrm{O} 1$ & $4.18 \mathrm{E}-05$ & Acidic $\mathrm{O} 2$ & $\mathrm{C} 29 \mathrm{H} 42 \mathrm{O} 2$ & 0.000114 & Basic N1O3 & $\mathrm{C} 41 \mathrm{H} 55 \mathrm{~N} 1 \mathrm{O} 3$ & $5.10 \mathrm{E}-06$ \\
\hline Non-basic N1O1 & $\mathrm{C} 36 \mathrm{H} 27 \mathrm{~N} 1 \mathrm{O} 1$ & $4.09 \mathrm{E}-05$ & Acidic $\mathrm{O} 2$ & $\mathrm{C} 30 \mathrm{H} 44 \mathrm{O} 2$ & 0.000102 & Basic N1O3 & $\mathrm{C} 42 \mathrm{H} 57 \mathrm{~N} 1 \mathrm{O} 3$ & $5.99 \mathrm{E}-06$ \\
\hline Non-basic N1O1 & $\mathrm{C} 37 \mathrm{H} 29 \mathrm{~N} 1 \mathrm{O} 1$ & $3.13 \mathrm{E}-05$ & Acidic $\mathrm{O} 2$ & $\mathrm{C} 31 \mathrm{H} 46 \mathrm{O} 2$ & 0.000126 & Basic N1O3 & $\mathrm{C} 43 \mathrm{H} 59 \mathrm{~N} 1 \mathrm{O} 3$ & $5.76 \mathrm{E}-06$ \\
\hline Non-basic N1O1 & $\mathrm{C} 38 \mathrm{H} 31 \mathrm{~N} 1 \mathrm{O} 1$ & $2.76 \mathrm{E}-05$ & Acidic $\mathrm{O} 2$ & $\mathrm{C} 32 \mathrm{H} 48 \mathrm{O} 2$ & 0.000199 & Basic N1O3 & C44H61N1O3 & $8.63 \mathrm{E}-06$ \\
\hline Non-basic N1O1 & $\mathrm{C} 39 \mathrm{H} 33 \mathrm{~N} 1 \mathrm{O} 1$ & $1.42 \mathrm{E}-05$ & Acidic $\mathrm{O} 2$ & $\mathrm{C} 33 \mathrm{H} 50 \mathrm{O} 2$ & 0.000101 & Basic N1O3 & $\mathrm{C} 45 \mathrm{H} 63 \mathrm{~N} 1 \mathrm{O} 3$ & $3.12 \mathrm{E}-07$ \\
\hline Non-basic N1O1 & $\mathrm{C} 40 \mathrm{H} 35 \mathrm{~N} 1 \mathrm{O} 1$ & $9.27 \mathrm{E}-06$ & Acidic $\mathrm{O} 2$ & $\mathrm{C} 34 \mathrm{H} 52 \mathrm{O} 2$ & $5.28 \mathrm{E}-05$ & Basic N1O3 & $\mathrm{C} 46 \mathrm{H} 65 \mathrm{~N} 1 \mathrm{O} 3$ & $4.80 \mathrm{E}-07$ \\
\hline Non-basic N1O1 & C33H19N1O1 & $3.41 \mathrm{E}-06$ & Acidic $\mathrm{O} 2$ & $\mathrm{C} 35 \mathrm{H} 54 \mathrm{O} 2$ & $5.65 \mathrm{E}-05$ & Basic N1O3 & $\mathrm{C} 24 \mathrm{H} 19 \mathrm{~N} 1 \mathrm{O} 3$ & 4.54E-07 \\
\hline Non-basic N1O1 & $\mathrm{C} 34 \mathrm{H} 21 \mathrm{~N} 1 \mathrm{O} 1$ & 4.79E-06 & Acidic $\mathrm{O} 2$ & $\mathrm{C} 36 \mathrm{H} 56 \mathrm{O} 2$ & 0.000125 & Basic N1O3 & $\mathrm{C} 25 \mathrm{H} 21 \mathrm{~N} 1 \mathrm{O} 3$ & $4.90 \mathrm{E}-06$ \\
\hline Non-basic N1O1 & $\mathrm{C} 35 \mathrm{H} 23 \mathrm{~N} 1 \mathrm{O} 1$ & $2.95 \mathrm{E}-05$ & Acidic $\mathrm{O} 2$ & $\mathrm{C} 37 \mathrm{H} 58 \mathrm{O} 2$ & $8.73 \mathrm{E}-05$ & Basic N1O3 & $\mathrm{C} 26 \mathrm{H} 23 \mathrm{~N} 1 \mathrm{O} 3$ & $1.19 \mathrm{E}-05$ \\
\hline Non-basic N1O1 & $\mathrm{C} 36 \mathrm{H} 25 \mathrm{~N} 1 \mathrm{O} 1$ & $3.64 \mathrm{E}-05$ & Acidic $\mathrm{O} 2$ & $\mathrm{C} 38 \mathrm{H} 60 \mathrm{O} 2$ & $6.17 \mathrm{E}-05$ & Basic N1O3 & $\mathrm{C} 27 \mathrm{H} 25 \mathrm{~N} 1 \mathrm{O} 3$ & $1.31 \mathrm{E}-05$ \\
\hline Non-basic N1O1 & $\mathrm{C} 37 \mathrm{H} 27 \mathrm{~N} 1 \mathrm{O} 1$ & $4.53 \mathrm{E}-05$ & Acidic $\mathrm{O} 2$ & $\mathrm{C} 15 \mathrm{H} 12 \mathrm{O} 2$ & 0.000197 & Basic N1O3 & $\mathrm{C} 28 \mathrm{H} 27 \mathrm{~N} 1 \mathrm{O} 3$ & $1.46 \mathrm{E}-05$ \\
\hline Non-basic N1O1 & $\mathrm{C} 38 \mathrm{H} 29 \mathrm{~N} 1 \mathrm{O} 1$ & 4.17E-05 & Acidic $\mathrm{O} 2$ & $\mathrm{C} 16 \mathrm{H} 14 \mathrm{O} 2$ & 0.000284 & Basic N1O3 & $\mathrm{C} 29 \mathrm{H} 29 \mathrm{~N} 1 \mathrm{O} 3$ & $1.01 \mathrm{E}-05$ \\
\hline Non-basic N1O1 & C39H31N1O1 & $2.93 \mathrm{E}-05$ & Acidic $\mathrm{O} 2$ & $\mathrm{C} 17 \mathrm{H} 16 \mathrm{O} 2$ & 0.000234 & Basic N1O3 & $\mathrm{C} 30 \mathrm{H} 31 \mathrm{~N} 1 \mathrm{O} 3$ & $1.53 \mathrm{E}-05$ \\
\hline Non-basic N1O1 & $\mathrm{C} 40 \mathrm{H} 33 \mathrm{~N} 1 \mathrm{O} 1$ & $2.25 \mathrm{E}-05$ & Acidic $\mathrm{O} 2$ & $\mathrm{C} 18 \mathrm{H} 18 \mathrm{O} 2$ & $7.87 \mathrm{E}-05$ & Basic N1O3 & $\mathrm{C} 31 \mathrm{H} 33 \mathrm{~N} 1 \mathrm{O} 3$ & $1.72 \mathrm{E}-05$ \\
\hline Non-basic N1O1 & $\mathrm{C} 41 \mathrm{H} 35 \mathrm{~N} 1 \mathrm{O} 1$ & $1.64 \mathrm{E}-05$ & Acidic $\mathrm{O} 2$ & $\mathrm{C} 19 \mathrm{H} 20 \mathrm{O} 2$ & $7.36 \mathrm{E}-05$ & Basic N1O3 & $\mathrm{C} 32 \mathrm{H} 35 \mathrm{~N} 1 \mathrm{O} 3$ & $1.33 \mathrm{E}-05$ \\
\hline Non-basic N1O1 & $\mathrm{C} 42 \mathrm{H} 37 \mathrm{~N} 1 \mathrm{O} 1$ & $8.24 \mathrm{E}-06$ & Acidic $\mathrm{O} 2$ & $\mathrm{C} 20 \mathrm{H} 22 \mathrm{O} 2$ & $5.58 \mathrm{E}-05$ & Basic N1O3 & $\mathrm{C} 33 \mathrm{H} 37 \mathrm{~N} 1 \mathrm{O} 3$ & $1.17 \mathrm{E}-05$ \\
\hline Non-basic N1O1 & $\mathrm{C} 43 \mathrm{H} 39 \mathrm{~N} 1 \mathrm{O} 1$ & 7.72E-06 & Acidic $\mathrm{O} 2$ & $\mathrm{C} 21 \mathrm{H} 24 \mathrm{O} 2$ & $5.13 \mathrm{E}-05$ & Basic N1O3 & C34H39N1O3 & $1.26 \mathrm{E}-05$ \\
\hline Non-basic N1O1 & $\mathrm{C} 37 \mathrm{H} 25 \mathrm{~N} 1 \mathrm{O} 1$ & $2.20 \mathrm{E}-05$ & Acidic $\mathrm{O} 2$ & $\mathrm{C} 22 \mathrm{H} 26 \mathrm{O} 2$ & 0.000116 & Basic N1O3 & $\mathrm{C} 35 \mathrm{H} 41 \mathrm{~N} 1 \mathrm{O} 3$ & $8.92 \mathrm{E}-06$ \\
\hline Non-basic N1O1 & $\mathrm{C} 38 \mathrm{H} 27 \mathrm{~N} 1 \mathrm{O} 1$ & $2.29 \mathrm{E}-05$ & Acidic $\mathrm{O} 2$ & $\mathrm{C} 23 \mathrm{H} 28 \mathrm{O} 2$ & $4.96 \mathrm{E}-05$ & Basic N1O3 & $\mathrm{C} 36 \mathrm{H} 43 \mathrm{~N} 1 \mathrm{O} 3$ & $8.69 \mathrm{E}-06$ \\
\hline Non-basic N1O1 & $\mathrm{C} 39 \mathrm{H} 29 \mathrm{~N} 1 \mathrm{O} 1$ & $2.18 \mathrm{E}-05$ & Acidic $\mathrm{O} 2$ & $\mathrm{C} 24 \mathrm{H} 30 \mathrm{O} 2$ & $8.83 \mathrm{E}-05$ & Basic N1O3 & $\mathrm{C} 37 \mathrm{H} 45 \mathrm{~N} 1 \mathrm{O} 3$ & $1.03 \mathrm{E}-05$ \\
\hline Non-basic N1O1 & $\mathrm{C} 40 \mathrm{H} 31 \mathrm{~N} 1 \mathrm{O} 1$ & $1.97 \mathrm{E}-05$ & Acidic $\mathrm{O} 2$ & $\mathrm{C} 25 \mathrm{H} 32 \mathrm{O} 2$ & 0.000112 & Basic N1O3 & $\mathrm{C} 38 \mathrm{H} 47 \mathrm{~N} 1 \mathrm{O} 3$ & $7.52 \mathrm{E}-06$ \\
\hline Non-basic N1O1 & C41H33N1O1 & $1.71 \mathrm{E}-05$ & Acidic $\mathrm{O} 2$ & $\mathrm{C} 26 \mathrm{H} 34 \mathrm{O} 2$ & 0.000103 & Basic N1O3 & $\mathrm{C} 24 \mathrm{H} 17 \mathrm{~N} 1 \mathrm{O} 3$ & $3.90 \mathrm{E}-07$ \\
\hline Non-basic N1O1 & $\mathrm{C} 42 \mathrm{H} 35 \mathrm{~N} 1 \mathrm{O} 1$ & $1.27 \mathrm{E}-05$ & Acidic $\mathrm{O} 2$ & $\mathrm{C} 27 \mathrm{H} 36 \mathrm{O} 2$ & 0.000198 & Basic N1O3 & $\mathrm{C} 25 \mathrm{H} 19 \mathrm{~N} 1 \mathrm{O} 3$ & $5.67 \mathrm{E}-06$ \\
\hline Non-basic N1O1 & $\mathrm{C} 43 \mathrm{H} 37 \mathrm{~N} 1 \mathrm{O} 1$ & $3.77 \mathrm{E}-06$ & Acidic $\mathrm{O} 2$ & $\mathrm{C} 28 \mathrm{H} 38 \mathrm{O} 2$ & 0.000229 & Basic N1O3 & $\mathrm{C} 26 \mathrm{H} 21 \mathrm{~N} 1 \mathrm{O} 3$ & 7.47E-06 \\
\hline Non-basic N1O1 & $\mathrm{C} 44 \mathrm{H} 39 \mathrm{~N} 1 \mathrm{O} 1$ & $9.40 \mathrm{E}-06$ & Acidic $\mathrm{O} 2$ & $\mathrm{C} 29 \mathrm{H} 40 \mathrm{O} 2$ & 0.000297 & Basic N1O3 & $\mathrm{C} 27 \mathrm{H} 23 \mathrm{~N} 1 \mathrm{O} 3$ & 7.44E-06 \\
\hline Non-basic N1O1 & $\mathrm{C} 46 \mathrm{H} 43 \mathrm{~N} 1 \mathrm{O} 1$ & $5.01 \mathrm{E}-06$ & Acidic $\mathrm{O} 2$ & $\mathrm{C} 30 \mathrm{H} 42 \mathrm{O} 2$ & 0.00022 & Basic N1O3 & $\mathrm{C} 28 \mathrm{H} 25 \mathrm{~N} 1 \mathrm{O} 3$ & $1.15 \mathrm{E}-05$ \\
\hline Non-basic N1O1 & $\mathrm{C} 47 \mathrm{H} 45 \mathrm{~N} 1 \mathrm{O} 1$ & $3.21 \mathrm{E}-06$ & Acidic $\mathrm{O} 2$ & $\mathrm{C} 31 \mathrm{H} 44 \mathrm{O} 2$ & 0.000255 & Basic N1O3 & $\mathrm{C} 29 \mathrm{H} 27 \mathrm{~N} 1 \mathrm{O} 3$ & $1.10 \mathrm{E}-05$ \\
\hline Non-basic N1O1 & $\mathrm{C} 48 \mathrm{H} 47 \mathrm{~N} 1 \mathrm{O} 1$ & 4.77E-06 & Acidic $\mathrm{O} 2$ & $\mathrm{C} 32 \mathrm{H} 46 \mathrm{O} 2$ & 0.000258 & Basic N1O3 & $\mathrm{C} 30 \mathrm{H} 29 \mathrm{~N} 1 \mathrm{O} 3$ & $1.00 \mathrm{E}-05$ \\
\hline Non-basic N1O1 & C49H49N1O1 & $3.27 \mathrm{E}-06$ & Acidic $\mathrm{O} 2$ & $\mathrm{C} 33 \mathrm{H} 48 \mathrm{O} 2$ & 0.000126 & Basic N1O3 & $\mathrm{C} 31 \mathrm{H} 31 \mathrm{~N} 1 \mathrm{O} 3$ & 4.36E-06 \\
\hline Non-basic N1O1 & $\mathrm{C} 37 \mathrm{H} 23 \mathrm{~N} 1 \mathrm{O} 1$ & $2.38 \mathrm{E}-05$ & Acidic $\mathrm{O} 2$ & $\mathrm{C} 34 \mathrm{H} 50 \mathrm{O} 2$ & $8.05 \mathrm{E}-05$ & Basic N1O3 & $\mathrm{C} 32 \mathrm{H} 33 \mathrm{~N} 1 \mathrm{O} 3$ & $1.31 \mathrm{E}-05$ \\
\hline Non-basic N1O1 & $\mathrm{C} 38 \mathrm{H} 25 \mathrm{~N} 1 \mathrm{O} 1$ & 4.19E-05 & Acidic $\mathrm{O} 2$ & $\mathrm{C} 35 \mathrm{H} 52 \mathrm{O} 2$ & $5.75 \mathrm{E}-05$ & Basic N1O3 & $\mathrm{C} 33 \mathrm{H} 35 \mathrm{~N} 1 \mathrm{O} 3$ & $6.36 \mathrm{E}-06$ \\
\hline Non-basic N1O1 & $\mathrm{C} 39 \mathrm{H} 27 \mathrm{~N} 1 \mathrm{O} 1$ & $4.87 \mathrm{E}-05$ & Acidic $\mathrm{O} 2$ & $\mathrm{C} 36 \mathrm{H} 54 \mathrm{O} 2$ & $6.96 \mathrm{E}-05$ & Basic N1O3 & $\mathrm{C} 34 \mathrm{H} 37 \mathrm{~N} 1 \mathrm{O} 3$ & $5.64 \mathrm{E}-06$ \\
\hline Non-basic N1O1 & $\mathrm{C} 40 \mathrm{H} 29 \mathrm{~N} 1 \mathrm{O} 1$ & $3.53 \mathrm{E}-05$ & Acidic $\mathrm{O} 2$ & $\mathrm{C} 37 \mathrm{H} 56 \mathrm{O} 2$ & $9.81 \mathrm{E}-05$ & Basic N1O3 & $\mathrm{C} 35 \mathrm{H} 39 \mathrm{~N} 1 \mathrm{O} 3$ & 4.23E-06 \\
\hline Non-basic N1O1 & $\mathrm{C} 41 \mathrm{H} 31 \mathrm{~N} 1 \mathrm{O} 1$ & $3.78 \mathrm{E}-05$ & Acidic $\mathrm{O} 2$ & $\mathrm{C} 38 \mathrm{H} 58 \mathrm{O} 2$ & $6.38 \mathrm{E}-05$ & Basic N1O3 & $\mathrm{C} 36 \mathrm{H} 41 \mathrm{~N} 1 \mathrm{O} 3$ & $5.57 \mathrm{E}-06$ \\
\hline Non-basic N1O1 & $\mathrm{C} 42 \mathrm{H} 33 \mathrm{~N} 1 \mathrm{O} 1$ & $2.58 \mathrm{E}-05$ & Acidic $\mathrm{O} 2$ & $\mathrm{C} 39 \mathrm{H} 60 \mathrm{O} 2$ & $4.93 \mathrm{E}-05$ & Basic N1O3 & $\mathrm{C} 37 \mathrm{H} 43 \mathrm{~N} 1 \mathrm{O} 3$ & 7.97E-06 \\
\hline Non-basic N1O1 & $\mathrm{C} 43 \mathrm{H} 35 \mathrm{~N} 1 \mathrm{O} 1$ & $8.15 \mathrm{E}-06$ & Acidic $\mathrm{O} 2$ & $\mathrm{C} 16 \mathrm{H} 12 \mathrm{O} 2$ & $1.49 \mathrm{E}-05$ & Basic N1O3 & $\mathrm{C} 38 \mathrm{H} 45 \mathrm{~N} 1 \mathrm{O} 3$ & $5.00 \mathrm{E}-06$ \\
\hline Non-basic N1O1 & $\mathrm{C} 44 \mathrm{H} 37 \mathrm{~N} 1 \mathrm{O} 1$ & $4.09 \mathrm{E}-06$ & Acidic $\mathrm{O} 2$ & $\mathrm{C} 17 \mathrm{H} 14 \mathrm{O} 2$ & 0.00011 & Basic N1O3 & $\mathrm{C} 25 \mathrm{H} 17 \mathrm{~N} 1 \mathrm{O} 3$ & 7.16E-07 \\
\hline
\end{tabular}




\begin{tabular}{|c|c|c|c|c|c|c|c|c|}
\hline Class & Formula & Mass & Class & Formula & Mass & Class & Formula & Mass \\
\hline Non-basic N1O1 & $\mathrm{C} 45 \mathrm{H} 39 \mathrm{~N} 1 \mathrm{O} 1$ & $6.55 \mathrm{E}-06$ & Acidic $\mathrm{O} 2$ & $\mathrm{C} 18 \mathrm{H} 16 \mathrm{O} 2$ & 0.000152 & Basic N1O3 & $\mathrm{C} 26 \mathrm{H} 19 \mathrm{~N} 1 \mathrm{O} 3$ & $1.38 \mathrm{E}-06$ \\
\hline Non-basic N1O1 & $\mathrm{C} 46 \mathrm{H} 41 \mathrm{~N} 1 \mathrm{O} 1$ & $3.16 \mathrm{E}-06$ & Acidic $\mathrm{O} 2$ & $\mathrm{C} 19 \mathrm{H} 18 \mathrm{O} 2$ & 0.000185 & Basic N1O3 & $\mathrm{C} 27 \mathrm{H} 21 \mathrm{~N} 1 \mathrm{O} 3$ & $1.97 \mathrm{E}-06$ \\
\hline Non-basic N1O1 & $\mathrm{C} 39 \mathrm{H} 25 \mathrm{~N} 1 \mathrm{O} 1$ & $7.94 \mathrm{E}-06$ & Acidic $\mathrm{O} 2$ & $\mathrm{C} 20 \mathrm{H} 20 \mathrm{O} 2$ & 0.000139 & Basic N1O3 & $\mathrm{C} 28 \mathrm{H} 23 \mathrm{~N} 1 \mathrm{O} 3$ & $5.99 \mathrm{E}-06$ \\
\hline Non-basic N1O1 & $\mathrm{C} 40 \mathrm{H} 27 \mathrm{~N} 1 \mathrm{O} 1$ & $1.54 \mathrm{E}-05$ & Acidic $\mathrm{O} 2$ & $\mathrm{C} 21 \mathrm{H} 22 \mathrm{O} 2$ & $9.86 \mathrm{E}-05$ & Basic N1O3 & $\mathrm{C} 29 \mathrm{H} 25 \mathrm{~N} 1 \mathrm{O} 3$ & $5.01 \mathrm{E}-06$ \\
\hline Non-basic N1O1 & C41H29N1O1 & $2.16 \mathrm{E}-05$ & Acidic $\mathrm{O} 2$ & $\mathrm{C} 22 \mathrm{H} 24 \mathrm{O} 2$ & 0.000108 & Basic N1O3 & $\mathrm{C} 30 \mathrm{H} 27 \mathrm{~N} 1 \mathrm{O} 3$ & $4.88 \mathrm{E}-06$ \\
\hline Non-basic N1O1 & $\mathrm{C} 42 \mathrm{H} 31 \mathrm{~N} 1 \mathrm{O} 1$ & $2.65 \mathrm{E}-05$ & Acidic $\mathrm{O} 2$ & $\mathrm{C} 23 \mathrm{H} 26 \mathrm{O} 2$ & 0.000187 & Basic N1O3 & $\mathrm{C} 31 \mathrm{H} 29 \mathrm{~N} 1 \mathrm{O} 3$ & $4.48 \mathrm{E}-06$ \\
\hline Non-basic N1O1 & $\mathrm{C} 43 \mathrm{H} 33 \mathrm{~N} 1 \mathrm{O} 1$ & $1.72 \mathrm{E}-05$ & Acidic $\mathrm{O} 2$ & $\mathrm{C} 24 \mathrm{H} 28 \mathrm{O} 2$ & 0.000216 & Basic N1O3 & $\mathrm{C} 32 \mathrm{H} 31 \mathrm{~N} 1 \mathrm{O} 3$ & $3.32 \mathrm{E}-06$ \\
\hline Non-basic N1O1 & $\mathrm{C} 44 \mathrm{H} 35 \mathrm{~N} 1 \mathrm{O} 1$ & $1.64 \mathrm{E}-05$ & Acidic $\mathrm{O} 2$ & $\mathrm{C} 25 \mathrm{H} 30 \mathrm{O} 2$ & $8.49 \mathrm{E}-05$ & Basic N1O3 & $\mathrm{C} 33 \mathrm{H} 33 \mathrm{~N} 1 \mathrm{O} 3$ & $4.59 \mathrm{E}-06$ \\
\hline Non-basic N1O1 & $\mathrm{C} 45 \mathrm{H} 37 \mathrm{~N} 1 \mathrm{O} 1$ & $3.54 \mathrm{E}-06$ & Acidic $\mathrm{O} 2$ & $\mathrm{C} 26 \mathrm{H} 32 \mathrm{O} 2$ & $6.33 \mathrm{E}-05$ & Basic N1O3 & $\mathrm{C} 34 \mathrm{H} 35 \mathrm{~N} 1 \mathrm{O} 3$ & $2.98 \mathrm{E}-06$ \\
\hline Non-basic N1O1 & $\mathrm{C} 46 \mathrm{H} 39 \mathrm{~N} 1 \mathrm{O} 1$ & $7.30 \mathrm{E}-06$ & Acidic $\mathrm{O} 2$ & $\mathrm{C} 27 \mathrm{H} 34 \mathrm{O} 2$ & $8.37 \mathrm{E}-05$ & Basic N1O3 & $\mathrm{C} 35 \mathrm{H} 37 \mathrm{~N} 1 \mathrm{O} 3$ & $2.49 \mathrm{E}-06$ \\
\hline Non-basic N1O1 & $\mathrm{C} 47 \mathrm{H} 41 \mathrm{~N} 1 \mathrm{O} 1$ & $2.15 \mathrm{E}-06$ & Acidic $\mathrm{O} 2$ & $\mathrm{C} 28 \mathrm{H} 36 \mathrm{O} 2$ & 0.000134 & Basic N1O3 & $\mathrm{C} 36 \mathrm{H} 39 \mathrm{~N} 1 \mathrm{O} 3$ & $1.75 \mathrm{E}-06$ \\
\hline Non-basic N1O1 & C39H23N1O1 & $7.41 \mathrm{E}-06$ & Acidic $\mathrm{O} 2$ & $\mathrm{C} 29 \mathrm{H} 38 \mathrm{O} 2$ & $9.60 \mathrm{E}-05$ & Basic N1O3 & $\mathrm{C} 37 \mathrm{H} 41 \mathrm{~N} 1 \mathrm{O} 3$ & 4.49E-06 \\
\hline Non-basic N1O1 & $\mathrm{C} 40 \mathrm{H} 25 \mathrm{~N} 1 \mathrm{O} 1$ & $1.52 \mathrm{E}-05$ & Acidic $\mathrm{O} 2$ & $\mathrm{C} 30 \mathrm{H} 40 \mathrm{O} 2$ & 0.000106 & Basic N1O3 & $\mathrm{C} 38 \mathrm{H} 43 \mathrm{~N} 1 \mathrm{O} 3$ & $1.35 \mathrm{E}-05$ \\
\hline Non-basic N1O1 & $\mathrm{C} 41 \mathrm{H} 27 \mathrm{~N} 1 \mathrm{O} 1$ & $1.12 \mathrm{E}-05$ & Acidic $\mathrm{O} 2$ & $\mathrm{C} 31 \mathrm{H} 42 \mathrm{O} 2$ & $6.94 \mathrm{E}-05$ & Basic N1O3 & $\mathrm{C} 39 \mathrm{H} 45 \mathrm{~N} 1 \mathrm{O} 3$ & $1.85 \mathrm{E}-06$ \\
\hline Non-basic N1O1 & $\mathrm{C} 42 \mathrm{H} 29 \mathrm{~N} 1 \mathrm{O} 1$ & $1.74 \mathrm{E}-05$ & Acidic $\mathrm{O} 2$ & $\mathrm{C} 32 \mathrm{H} 44 \mathrm{O} 2$ & 0.000235 & Basic N1O3 & $\mathrm{C} 29 \mathrm{H} 23 \mathrm{~N} 1 \mathrm{O} 3$ & $2.22 \mathrm{E}-06$ \\
\hline Non-basic N1O1 & $\mathrm{C} 43 \mathrm{H} 31 \mathrm{~N} 1 \mathrm{O} 1$ & $8.53 \mathrm{E}-06$ & Acidic $\mathrm{O} 2$ & $\mathrm{C} 33 \mathrm{H} 46 \mathrm{O} 2$ & $3.82 \mathrm{E}-05$ & Basic N1O3 & $\mathrm{C} 30 \mathrm{H} 25 \mathrm{~N} 1 \mathrm{O} 3$ & $1.37 \mathrm{E}-06$ \\
\hline Non-basic N1O1 & C44H33N1O1 & $1.08 \mathrm{E}-05$ & Acidic $\mathrm{O} 2$ & $\mathrm{C} 34 \mathrm{H} 48 \mathrm{O} 2$ & $5.35 \mathrm{E}-05$ & Basic N1O3 & $\mathrm{C} 31 \mathrm{H} 27 \mathrm{~N} 1 \mathrm{O} 3$ & $1.42 \mathrm{E}-06$ \\
\hline Non-basic N1O1 & $\mathrm{C} 45 \mathrm{H} 35 \mathrm{~N} 1 \mathrm{O} 1$ & $6.81 \mathrm{E}-06$ & Acidic $\mathrm{O} 2$ & $\mathrm{C} 35 \mathrm{H} 50 \mathrm{O} 2$ & $6.93 \mathrm{E}-05$ & Basic N1O3 & $\mathrm{C} 32 \mathrm{H} 29 \mathrm{~N} 1 \mathrm{O} 3$ & $1.18 \mathrm{E}-06$ \\
\hline Non-basic N1O1 & $\mathrm{C} 46 \mathrm{H} 37 \mathrm{~N} 1 \mathrm{O} 1$ & $9.41 \mathrm{E}-06$ & Acidic $\mathrm{O} 2$ & $\mathrm{C} 17 \mathrm{H} 12 \mathrm{O} 2$ & 0.000135 & Basic N1O3 & $\mathrm{C} 33 \mathrm{H} 31 \mathrm{~N} 1 \mathrm{O} 3$ & $2.44 \mathrm{E}-07$ \\
\hline Non-basic N1O1 & $\mathrm{C} 41 \mathrm{H} 25 \mathrm{~N} 1 \mathrm{O} 1$ & 4.97E-06 & Acidic $\mathrm{O} 2$ & $\mathrm{C} 18 \mathrm{H} 14 \mathrm{O} 2$ & 0.00026 & Basic N1O3 & $\mathrm{C} 34 \mathrm{H} 33 \mathrm{~N} 1 \mathrm{O} 3$ & $4.08 \mathrm{E}-06$ \\
\hline Non-basic N1O1 & $\mathrm{C} 42 \mathrm{H} 27 \mathrm{~N} 1 \mathrm{O} 1$ & $1.97 \mathrm{E}-05$ & Acidic $\mathrm{O} 2$ & $\mathrm{C} 19 \mathrm{H} 16 \mathrm{O} 2$ & 0.000321 & Basic N1O3 & $\mathrm{C} 35 \mathrm{H} 35 \mathrm{~N} 1 \mathrm{O} 3$ & $9.31 \mathrm{E}-07$ \\
\hline Non-basic N1O1 & $\mathrm{C} 43 \mathrm{H} 29 \mathrm{~N} 1 \mathrm{O} 1$ & $2.50 \mathrm{E}-05$ & Acidic $\mathrm{O} 2$ & $\mathrm{C} 20 \mathrm{H} 18 \mathrm{O} 2$ & 0.000271 & Basic N1O3 & C28H19N1O3 & $1.76 \mathrm{E}-06$ \\
\hline Non-basic N1O1 & C44H31N1O1 & $1.85 \mathrm{E}-06$ & Acidic $\mathrm{O} 2$ & $\mathrm{C} 21 \mathrm{H} 20 \mathrm{O} 2$ & 0.000228 & Basic N1O3 & $\mathrm{C} 29 \mathrm{H} 21 \mathrm{~N} 1 \mathrm{O} 3$ & $5.53 \mathrm{E}-07$ \\
\hline Non-basic N1O1 & C45H33N1O1 & $5.84 \mathrm{E}-06$ & Acidic $\mathrm{O} 2$ & $\mathrm{C} 22 \mathrm{H} 22 \mathrm{O} 2$ & 0.000191 & Basic N1O3 & $\mathrm{C} 30 \mathrm{H} 23 \mathrm{~N} 1 \mathrm{O} 3$ & $4.66 \mathrm{E}-07$ \\
\hline Non-basic N1O1 & $\mathrm{C} 46 \mathrm{H} 35 \mathrm{~N} 1 \mathrm{O} 1$ & $6.48 \mathrm{E}-06$ & Acidic $\mathrm{O} 2$ & $\mathrm{C} 23 \mathrm{H} 24 \mathrm{O} 2$ & 0.00028 & Basic N1O3 & $\mathrm{C} 31 \mathrm{H} 25 \mathrm{~N} 1 \mathrm{O} 3$ & $1.24 \mathrm{E}-06$ \\
\hline Non-basic N1O1 & $\mathrm{C} 47 \mathrm{H} 37 \mathrm{~N} 1 \mathrm{O} 1$ & $6.90 \mathrm{E}-06$ & Acidic $\mathrm{O} 2$ & $\mathrm{C} 24 \mathrm{H} 26 \mathrm{O} 2$ & 0.000225 & Basic N1O3 & $\mathrm{C} 32 \mathrm{H} 27 \mathrm{~N} 1 \mathrm{O} 3$ & $1.47 \mathrm{E}-06$ \\
\hline Non-basic N1O1 & C48H39N1O1 & $6.75 \mathrm{E}-06$ & Acidic $\mathrm{O} 2$ & $\mathrm{C} 25 \mathrm{H} 28 \mathrm{O} 2$ & 0.000216 & Basic N1O3 & $\mathrm{C} 33 \mathrm{H} 29 \mathrm{~N} 1 \mathrm{O} 3$ & $6.65 \mathrm{E}-07$ \\
\hline Non-basic N1O1 & C49H41N1O1 & $4.06 \mathrm{E}-06$ & Acidic $\mathrm{O} 2$ & $\mathrm{C} 26 \mathrm{H} 30 \mathrm{O} 2$ & 0.000172 & Basic N1O3 & $\mathrm{C} 34 \mathrm{H} 31 \mathrm{~N} 1 \mathrm{O} 3$ & $2.55 \mathrm{E}-07$ \\
\hline Non-basic N1O1 & $\mathrm{C} 43 \mathrm{H} 27 \mathrm{~N} 1 \mathrm{O} 1$ & $3.14 \mathrm{E}-06$ & Acidic $\mathrm{O} 2$ & $\mathrm{C} 27 \mathrm{H} 32 \mathrm{O} 2$ & 0.000148 & Basic N1O3 & $\mathrm{C} 35 \mathrm{H} 33 \mathrm{~N} 1 \mathrm{O} 3$ & $3.57 \mathrm{E}-07$ \\
\hline Non-basic N1O1 & $\mathrm{C} 44 \mathrm{H} 29 \mathrm{~N} 1 \mathrm{O} 1$ & $3.03 \mathrm{E}-06$ & Acidic $\mathrm{O} 2$ & $\mathrm{C} 28 \mathrm{H} 34 \mathrm{O} 2$ & 0.000156 & Basic N1O3 & $\mathrm{C} 36 \mathrm{H} 35 \mathrm{~N} 1 \mathrm{O} 3$ & $1.42 \mathrm{E}-06$ \\
\hline Non-basic N1O1 & $\mathrm{C} 45 \mathrm{H} 31 \mathrm{~N} 1 \mathrm{O} 1$ & $9.51 \mathrm{E}-06$ & Acidic $\mathrm{O} 2$ & $\mathrm{C} 29 \mathrm{H} 36 \mathrm{O} 2$ & 0.000139 & Basic N1O3 & $\mathrm{C} 35 \mathrm{H} 31 \mathrm{~N} 1 \mathrm{O} 3$ & $6.04 \mathrm{E}-07$ \\
\hline Non-basic N1O1 & C46H33N1O1 & $4.06 \mathrm{E}-06$ & Acidic $\mathrm{O} 2$ & $\mathrm{C} 30 \mathrm{H} 38 \mathrm{O} 2$ & 0.000158 & Basic N1O3 & $\mathrm{C} 36 \mathrm{H} 33 \mathrm{~N} 1 \mathrm{O} 3$ & $1.46 \mathrm{E}-06$ \\
\hline Non-basic N1O1 & $\mathrm{C} 47 \mathrm{H} 35 \mathrm{~N} 1 \mathrm{O} 1$ & $6.38 \mathrm{E}-06$ & Acidic $\mathrm{O} 2$ & $\mathrm{C} 31 \mathrm{H} 40 \mathrm{O} 2$ & 0.00014 & Basic N1O3 & $\mathrm{C} 37 \mathrm{H} 35 \mathrm{~N} 1 \mathrm{O} 3$ & $2.35 \mathrm{E}-07$ \\
\hline Non-basic N1O1 & C48H37N1O1 & $7.59 \mathrm{E}-06$ & Acidic $\mathrm{O} 2$ & $\mathrm{C} 32 \mathrm{H} 42 \mathrm{O} 2$ & $6.33 \mathrm{E}-05$ & Basic N1O3 & $\mathrm{C} 38 \mathrm{H} 37 \mathrm{~N} 1 \mathrm{O} 3$ & $1.64 \mathrm{E}-06$ \\
\hline Non-basic N1O1 & $\mathrm{C} 44 \mathrm{H} 27 \mathrm{~N} 1 \mathrm{O} 1$ & $2.13 \mathrm{E}-06$ & Acidic $\mathrm{O} 2$ & $\mathrm{C} 33 \mathrm{H} 44 \mathrm{O} 2$ & $1.65 \mathrm{E}-05$ & Basic N2 & $\mathrm{C} 25 \mathrm{H} 42 \mathrm{~N} 2$ & 7.44E-07 \\
\hline Non-basic N1O1 & $\mathrm{C} 45 \mathrm{H} 29 \mathrm{~N} 1 \mathrm{O} 1$ & $5.41 \mathrm{E}-06$ & Acidic $\mathrm{O} 2$ & $\mathrm{C} 34 \mathrm{H} 46 \mathrm{O} 2$ & $5.38 \mathrm{E}-05$ & Basic N2 & $\mathrm{C} 26 \mathrm{H} 44 \mathrm{~N} 2$ & $2.92 \mathrm{E}-06$ \\
\hline Non-basic N1O2 & $\mathrm{C} 30 \mathrm{H} 51 \mathrm{~N} 1 \mathrm{O} 2$ & $6.22 \mathrm{E}-06$ & Acidic $\mathrm{O} 2$ & $\mathrm{C} 17 \mathrm{H} 10 \mathrm{O} 2$ & 0.000271 & Basic N2 & $\mathrm{C} 27 \mathrm{H} 46 \mathrm{~N} 2$ & $5.69 \mathrm{E}-06$ \\
\hline Non-basic N1O2 & $\mathrm{C} 31 \mathrm{H} 53 \mathrm{~N} 1 \mathrm{O} 2$ & $1.50 \mathrm{E}-06$ & Acidic $\mathrm{O} 2$ & $\mathrm{C} 18 \mathrm{H} 12 \mathrm{O} 2$ & 0.001201 & Basic N2 & $\mathrm{C} 28 \mathrm{H} 48 \mathrm{~N} 2$ & 7.57E-06 \\
\hline
\end{tabular}




\begin{tabular}{|c|c|c|c|c|c|c|c|c|}
\hline Class & Formula & Mass & Class & Formula & Mass & Class & Formula & Mass \\
\hline Non-basic N1O2 & $\mathrm{C} 32 \mathrm{H} 55 \mathrm{~N} 1 \mathrm{O} 2$ & $1.68 \mathrm{E}-06$ & Acidic $\mathrm{O} 2$ & $\mathrm{C} 19 \mathrm{H} 14 \mathrm{O} 2$ & 0.001491 & Basic N2 & $\mathrm{C} 29 \mathrm{H} 50 \mathrm{~N} 2$ & $2.06 \mathrm{E}-05$ \\
\hline Non-basic N1O2 & $\mathrm{C} 33 \mathrm{H} 57 \mathrm{~N} 1 \mathrm{O} 2$ & $2.99 \mathrm{E}-06$ & Acidic $\mathrm{O} 2$ & $\mathrm{C} 20 \mathrm{H} 16 \mathrm{O} 2$ & 0.001269 & Basic N2 & $\mathrm{C} 30 \mathrm{H} 52 \mathrm{~N} 2$ & $2.12 \mathrm{E}-05$ \\
\hline Non-basic N1O2 & $\mathrm{C} 24 \mathrm{H} 37 \mathrm{~N} 1 \mathrm{O} 2$ & $1.76 \mathrm{E}-06$ & Acidic $\mathrm{O} 2$ & $\mathrm{C} 21 \mathrm{H} 18 \mathrm{O} 2$ & 0.000801 & Basic N2 & $\mathrm{C} 31 \mathrm{H} 54 \mathrm{~N} 2$ & $3.17 \mathrm{E}-05$ \\
\hline Non-basic N1O2 & $\mathrm{C} 25 \mathrm{H} 39 \mathrm{~N} 1 \mathrm{O} 2$ & $1.13 \mathrm{E}-06$ & Acidic $\mathrm{O} 2$ & $\mathrm{C} 22 \mathrm{H} 20 \mathrm{O} 2$ & 0.000556 & Basic N2 & $\mathrm{C} 32 \mathrm{H} 56 \mathrm{~N} 2$ & $2.15 \mathrm{E}-05$ \\
\hline Non-basic N1O2 & $\mathrm{C} 26 \mathrm{H} 41 \mathrm{~N} 1 \mathrm{O} 2$ & $1.19 \mathrm{E}-06$ & Acidic $\mathrm{O} 2$ & $\mathrm{C} 23 \mathrm{H} 22 \mathrm{O} 2$ & 0.000461 & Basic N2 & $\mathrm{C} 33 \mathrm{H} 58 \mathrm{~N} 2$ & $1.86 \mathrm{E}-05$ \\
\hline Non-basic N1O2 & $\mathrm{C} 27 \mathrm{H} 43 \mathrm{~N} 1 \mathrm{O} 2$ & $4.78 \mathrm{E}-06$ & Acidic $\mathrm{O} 2$ & $\mathrm{C} 24 \mathrm{H} 24 \mathrm{O} 2$ & 0.000367 & Basic N2 & $\mathrm{C} 34 \mathrm{H} 60 \mathrm{~N} 2$ & $1.97 \mathrm{E}-05$ \\
\hline Non-basic N1O2 & $\mathrm{C} 28 \mathrm{H} 45 \mathrm{~N} 1 \mathrm{O} 2$ & $3.71 \mathrm{E}-06$ & Acidic $\mathrm{O} 2$ & $\mathrm{C} 25 \mathrm{H} 26 \mathrm{O} 2$ & 0.000353 & Basic N2 & $\mathrm{C} 35 \mathrm{H} 62 \mathrm{~N} 2$ & $1.38 \mathrm{E}-05$ \\
\hline Non-basic N1O2 & $\mathrm{C} 29 \mathrm{H} 47 \mathrm{~N} 1 \mathrm{O} 2$ & $1.29 \mathrm{E}-05$ & Acidic $\mathrm{O} 2$ & $\mathrm{C} 26 \mathrm{H} 28 \mathrm{O} 2$ & 0.000319 & Basic N2 & $\mathrm{C} 36 \mathrm{H} 64 \mathrm{~N} 2$ & $1.22 \mathrm{E}-05$ \\
\hline Non-basic N1O2 & $\mathrm{C} 30 \mathrm{H} 49 \mathrm{~N} 1 \mathrm{O} 2$ & $9.00 \mathrm{E}-06$ & Acidic $\mathrm{O} 2$ & $\mathrm{C} 27 \mathrm{H} 30 \mathrm{O} 2$ & 0.000271 & Basic N2 & $\mathrm{C} 18 \mathrm{H} 26 \mathrm{~N} 2$ & $1.16 \mathrm{E}-07$ \\
\hline Non-basic N1O2 & $\mathrm{C} 31 \mathrm{H} 51 \mathrm{~N} 1 \mathrm{O} 2$ & $7.95 \mathrm{E}-06$ & Acidic $\mathrm{O} 2$ & $\mathrm{C} 28 \mathrm{H} 32 \mathrm{O} 2$ & 0.00017 & Basic N2 & $\mathrm{C} 19 \mathrm{H} 28 \mathrm{~N} 2$ & $7.98 \mathrm{E}-07$ \\
\hline Non-basic N1O2 & $\mathrm{C} 32 \mathrm{H} 53 \mathrm{~N} 1 \mathrm{O} 2$ & $9.42 \mathrm{E}-06$ & Acidic $\mathrm{O} 2$ & $\mathrm{C} 29 \mathrm{H} 34 \mathrm{O} 2$ & 0.000196 & Basic N2 & $\mathrm{C} 20 \mathrm{H} 30 \mathrm{~N} 2$ & $1.15 \mathrm{E}-07$ \\
\hline Non-basic N1O2 & $\mathrm{C} 33 \mathrm{H} 55 \mathrm{~N} 1 \mathrm{O} 2$ & $1.44 \mathrm{E}-05$ & Acidic $\mathrm{O} 2$ & $\mathrm{C} 30 \mathrm{H} 36 \mathrm{O} 2$ & 0.000187 & Basic N2 & $\mathrm{C} 21 \mathrm{H} 32 \mathrm{~N} 2$ & $1.17 \mathrm{E}-06$ \\
\hline Non-basic N1O2 & $\mathrm{C} 20 \mathrm{H} 27 \mathrm{~N} 1 \mathrm{O} 2$ & $1.57 \mathrm{E}-06$ & Acidic $\mathrm{O} 2$ & $\mathrm{C} 31 \mathrm{H} 38 \mathrm{O} 2$ & $5.22 \mathrm{E}-05$ & Basic N2 & $\mathrm{C} 22 \mathrm{H} 34 \mathrm{~N} 2$ & $1.42 \mathrm{E}-06$ \\
\hline Non-basic $\mathrm{N} 1 \mathrm{O} 2$ & $\mathrm{C} 21 \mathrm{H} 29 \mathrm{~N} 1 \mathrm{O} 2$ & $1.28 \mathrm{E}-06$ & Acidic $\mathrm{O} 2$ & $\mathrm{C} 32 \mathrm{H} 40 \mathrm{O} 2$ & 0.000128 & Basic N2 & $\mathrm{C} 23 \mathrm{H} 36 \mathrm{~N} 2$ & $5.44 \mathrm{E}-06$ \\
\hline Non-basic N1O2 & $\mathrm{C} 22 \mathrm{H} 31 \mathrm{~N} 1 \mathrm{O} 2$ & $4.00 \mathrm{E}-06$ & Acidic $\mathrm{O} 2$ & $\mathrm{C} 33 \mathrm{H} 42 \mathrm{O} 2$ & $5.70 \mathrm{E}-05$ & Basic N2 & $\mathrm{C} 24 \mathrm{H} 38 \mathrm{~N} 2$ & $6.84 \mathrm{E}-06$ \\
\hline Non-basic N1O2 & $\mathrm{C} 23 \mathrm{H} 33 \mathrm{~N} 1 \mathrm{O} 2$ & $1.00 \mathrm{E}-06$ & Acidic $\mathrm{O} 2$ & $\mathrm{C} 34 \mathrm{H} 44 \mathrm{O} 2$ & $6.10 \mathrm{E}-05$ & Basic N2 & $\mathrm{C} 25 \mathrm{H} 40 \mathrm{~N} 2$ & $1.23 \mathrm{E}-05$ \\
\hline Non-basic N1O2 & $\mathrm{C} 24 \mathrm{H} 35 \mathrm{~N} 1 \mathrm{O} 2$ & $6.06 \mathrm{E}-06$ & Acidic $\mathrm{O} 2$ & $\mathrm{C} 35 \mathrm{H} 46 \mathrm{O} 2$ & $1.92 \mathrm{E}-05$ & Basic N2 & $\mathrm{C} 26 \mathrm{H} 42 \mathrm{~N} 2$ & $1.56 \mathrm{E}-05$ \\
\hline Non-basic N1O2 & $\mathrm{C} 25 \mathrm{H} 37 \mathrm{~N} 1 \mathrm{O} 2$ & $6.29 \mathrm{E}-06$ & Acidic $\mathrm{O} 2$ & $\mathrm{C} 19 \mathrm{H} 12 \mathrm{O} 2$ & 0.000105 & Basic N2 & $\mathrm{C} 27 \mathrm{H} 44 \mathrm{~N} 2$ & $1.97 \mathrm{E}-05$ \\
\hline Non-basic N1O2 & $\mathrm{C} 26 \mathrm{H} 39 \mathrm{~N} 1 \mathrm{O} 2$ & $6.52 \mathrm{E}-06$ & Acidic $\mathrm{O} 2$ & $\mathrm{C} 20 \mathrm{H} 14 \mathrm{O} 2$ & 0.000377 & Basic N2 & $\mathrm{C} 28 \mathrm{H} 46 \mathrm{~N} 2$ & $2.48 \mathrm{E}-05$ \\
\hline Non-basic N1O2 & $\mathrm{C} 27 \mathrm{H} 41 \mathrm{~N} 1 \mathrm{O} 2$ & $7.58 \mathrm{E}-06$ & Acidic $\mathrm{O} 2$ & $\mathrm{C} 21 \mathrm{H} 16 \mathrm{O} 2$ & 0.0006 & Basic N2 & $\mathrm{C} 29 \mathrm{H} 48 \mathrm{~N} 2$ & $3.30 \mathrm{E}-05$ \\
\hline Non-basic N1O2 & $\mathrm{C} 29 \mathrm{H} 45 \mathrm{~N} 1 \mathrm{O} 2$ & $1.31 \mathrm{E}-05$ & Acidic $\mathrm{O} 2$ & $\mathrm{C} 22 \mathrm{H} 18 \mathrm{O} 2$ & 0.000588 & Basic N2 & $\mathrm{C} 30 \mathrm{H} 50 \mathrm{~N} 2$ & 4.23E-05 \\
\hline Non-basic N1O2 & $\mathrm{C} 30 \mathrm{H} 47 \mathrm{~N} 1 \mathrm{O} 2$ & $7.51 \mathrm{E}-06$ & Acidic $\mathrm{O} 2$ & $\mathrm{C} 23 \mathrm{H} 20 \mathrm{O} 2$ & 0.000588 & Basic N2 & $\mathrm{C} 31 \mathrm{H} 52 \mathrm{~N} 2$ & 7.04E-05 \\
\hline Non-basic N1O2 & $\mathrm{C} 31 \mathrm{H} 49 \mathrm{~N} 1 \mathrm{O} 2$ & $1.79 \mathrm{E}-05$ & Acidic $\mathrm{O} 2$ & $\mathrm{C} 24 \mathrm{H} 22 \mathrm{O} 2$ & 0.000484 & Basic N2 & $\mathrm{C} 32 \mathrm{H} 54 \mathrm{~N} 2$ & $5.35 \mathrm{E}-05$ \\
\hline Non-basic $\mathrm{N} 1 \mathrm{O} 2$ & C32H51N1O2 & $1.23 \mathrm{E}-05$ & Acidic $\mathrm{O} 2$ & $\mathrm{C} 25 \mathrm{H} 24 \mathrm{O} 2$ & 0.000367 & Basic N2 & $\mathrm{C} 33 \mathrm{H} 56 \mathrm{~N} 2$ & 4.87E-05 \\
\hline Non-basic N1O2 & $\mathrm{C} 33 \mathrm{H} 53 \mathrm{~N} 1 \mathrm{O} 2$ & $8.92 \mathrm{E}-06$ & Acidic $\mathrm{O} 2$ & $\mathrm{C} 26 \mathrm{H} 26 \mathrm{O} 2$ & 0.000249 & Basic N2 & $\mathrm{C} 34 \mathrm{H} 58 \mathrm{~N} 2$ & 4.04E-05 \\
\hline Non-basic N1O2 & $\mathrm{C} 34 \mathrm{H} 55 \mathrm{~N} 1 \mathrm{O} 2$ & $1.48 \mathrm{E}-05$ & Acidic $\mathrm{O} 2$ & $\mathrm{C} 27 \mathrm{H} 28 \mathrm{O} 2$ & 0.000164 & Basic N2 & $\mathrm{C} 35 \mathrm{H} 60 \mathrm{~N} 2$ & $5.88 \mathrm{E}-05$ \\
\hline Non-basic N1O2 & $\mathrm{C} 35 \mathrm{H} 57 \mathrm{~N} 1 \mathrm{O} 2$ & $1.12 \mathrm{E}-05$ & Acidic $\mathrm{O} 2$ & $\mathrm{C} 28 \mathrm{H} 30 \mathrm{O} 2$ & 0.000156 & Basic N2 & $\mathrm{C} 36 \mathrm{H} 62 \mathrm{~N} 2$ & $3.83 \mathrm{E}-05$ \\
\hline Non-basic N1O2 & $\mathrm{C} 36 \mathrm{H} 59 \mathrm{~N} 1 \mathrm{O} 2$ & $1.77 \mathrm{E}-06$ & Acidic $\mathrm{O} 2$ & $\mathrm{C} 29 \mathrm{H} 32 \mathrm{O} 2$ & 0.000112 & Basic N2 & $\mathrm{C} 37 \mathrm{H} 64 \mathrm{~N} 2$ & $2.78 \mathrm{E}-05$ \\
\hline Non-basic N1O2 & C37H61N1O2 & $2.58 \mathrm{E}-06$ & Acidic $\mathrm{O} 2$ & $\mathrm{C} 30 \mathrm{H} 34 \mathrm{O} 2$ & 0.000104 & Basic N2 & $\mathrm{C} 38 \mathrm{H} 66 \mathrm{~N} 2$ & $2.50 \mathrm{E}-05$ \\
\hline Non-basic N1O2 & C38H63N1O2 & $1.44 \mathrm{E}-06$ & Acidic $\mathrm{O} 2$ & $\mathrm{C} 31 \mathrm{H} 36 \mathrm{O} 2$ & 0.000101 & Basic N2 & $\mathrm{C} 39 \mathrm{H} 68 \mathrm{~N} 2$ & $2.69 \mathrm{E}-05$ \\
\hline Non-basic N1O2 & $\mathrm{C} 14 \mathrm{H} 13 \mathrm{~N} 1 \mathrm{O} 2$ & $8.07 \mathrm{E}-06$ & Acidic $\mathrm{O} 2$ & $\mathrm{C} 32 \mathrm{H} 38 \mathrm{O} 2$ & 0.0001 & Basic N2 & $\mathrm{C} 18 \mathrm{H} 24 \mathrm{~N} 2$ & $2.19 \mathrm{E}-07$ \\
\hline Non-basic N1O2 & $\mathrm{C} 15 \mathrm{H} 15 \mathrm{~N} 1 \mathrm{O} 2$ & $2.95 \mathrm{E}-05$ & Acidic $\mathrm{O} 2$ & $\mathrm{C} 19 \mathrm{H} 10 \mathrm{O} 2$ & 0.000293 & Basic N2 & $\mathrm{C} 19 \mathrm{H} 26 \mathrm{~N} 2$ & $1.28 \mathrm{E}-06$ \\
\hline Non-basic N1O2 & $\mathrm{C} 16 \mathrm{H} 17 \mathrm{~N} 1 \mathrm{O} 2$ & $2.65 \mathrm{E}-05$ & Acidic $\mathrm{O} 2$ & $\mathrm{C} 20 \mathrm{H} 12 \mathrm{O} 2$ & 0.000735 & Basic N2 & $\mathrm{C} 20 \mathrm{H} 28 \mathrm{~N} 2$ & $4.75 \mathrm{E}-06$ \\
\hline Non-basic N1O2 & $\mathrm{C} 17 \mathrm{H} 19 \mathrm{~N} 1 \mathrm{O} 2$ & $1.43 \mathrm{E}-05$ & Acidic $\mathrm{O} 2$ & $\mathrm{C} 21 \mathrm{H} 14 \mathrm{O} 2$ & 0.000621 & Basic N2 & $\mathrm{C} 21 \mathrm{H} 30 \mathrm{~N} 2$ & $1.29 \mathrm{E}-05$ \\
\hline Non-basic N1O2 & $\mathrm{C} 18 \mathrm{H} 21 \mathrm{~N} 1 \mathrm{O} 2$ & $9.11 \mathrm{E}-06$ & Acidic $\mathrm{O} 2$ & $\mathrm{C} 22 \mathrm{H} 16 \mathrm{O} 2$ & 0.000604 & Basic N2 & $\mathrm{C} 22 \mathrm{H} 32 \mathrm{~N} 2$ & 7.64E-06 \\
\hline Non-basic N1O2 & $\mathrm{C} 19 \mathrm{H} 23 \mathrm{~N} 1 \mathrm{O} 2$ & $1.26 \mathrm{E}-06$ & Acidic $\mathrm{O} 2$ & $\mathrm{C} 23 \mathrm{H} 18 \mathrm{O} 2$ & 0.000575 & Basic N2 & $\mathrm{C} 23 \mathrm{H} 34 \mathrm{~N} 2$ & $2.43 \mathrm{E}-05$ \\
\hline Non-basic N1O2 & $\mathrm{C} 20 \mathrm{H} 25 \mathrm{~N} 1 \mathrm{O} 2$ & $3.94 \mathrm{E}-06$ & Acidic $\mathrm{O} 2$ & $\mathrm{C} 24 \mathrm{H} 20 \mathrm{O} 2$ & 0.000503 & Basic N2 & $\mathrm{C} 24 \mathrm{H} 36 \mathrm{~N} 2$ & $2.07 \mathrm{E}-05$ \\
\hline Non-basic N1O2 & $\mathrm{C} 21 \mathrm{H} 27 \mathrm{~N} 1 \mathrm{O} 2$ & $9.35 \mathrm{E}-07$ & Acidic $\mathrm{O} 2$ & $\mathrm{C} 25 \mathrm{H} 22 \mathrm{O} 2$ & 0.000284 & Basic N2 & $\mathrm{C} 25 \mathrm{H} 38 \mathrm{~N} 2$ & $2.03 \mathrm{E}-05$ \\
\hline
\end{tabular}




\begin{tabular}{|c|c|c|c|c|c|c|c|c|}
\hline Class & Formula & Mass & Class & Formula & Mass & Class & Formula & Mass \\
\hline Non-basic N1O2 & $\mathrm{C} 22 \mathrm{H} 29 \mathrm{~N} 1 \mathrm{O} 2$ & $6.15 \mathrm{E}-06$ & Acidic $\mathrm{O} 2$ & $\mathrm{C} 26 \mathrm{H} 24 \mathrm{O} 2$ & 0.000153 & Basic N2 & $\mathrm{C} 26 \mathrm{H} 40 \mathrm{~N} 2$ & $2.60 \mathrm{E}-05$ \\
\hline Non-basic N1O2 & $\mathrm{C} 23 \mathrm{H} 31 \mathrm{~N} 1 \mathrm{O} 2$ & $5.53 \mathrm{E}-06$ & Acidic $\mathrm{O} 2$ & $\mathrm{C} 27 \mathrm{H} 26 \mathrm{O} 2$ & 0.000194 & Basic N2 & $\mathrm{C} 27 \mathrm{H} 42 \mathrm{~N} 2$ & $3.57 \mathrm{E}-05$ \\
\hline Non-basic N1O2 & $\mathrm{C} 24 \mathrm{H} 33 \mathrm{~N} 1 \mathrm{O} 2$ & $9.02 \mathrm{E}-06$ & Acidic $\mathrm{O} 2$ & $\mathrm{C} 28 \mathrm{H} 28 \mathrm{O} 2$ & 0.000146 & Basic N2 & $\mathrm{C} 28 \mathrm{H} 44 \mathrm{~N} 2$ & $4.29 \mathrm{E}-05$ \\
\hline Non-basic N1O2 & $\mathrm{C} 25 \mathrm{H} 35 \mathrm{~N} 1 \mathrm{O} 2$ & $9.18 \mathrm{E}-06$ & Acidic $\mathrm{O} 2$ & $\mathrm{C} 29 \mathrm{H} 30 \mathrm{O} 2$ & $6.30 \mathrm{E}-05$ & Basic N2 & $\mathrm{C} 29 \mathrm{H} 46 \mathrm{~N} 2$ & $6.86 \mathrm{E}-05$ \\
\hline Non-basic N1O2 & $\mathrm{C} 26 \mathrm{H} 37 \mathrm{~N} 1 \mathrm{O} 2$ & $1.29 \mathrm{E}-05$ & Acidic $\mathrm{O} 2$ & $\mathrm{C} 30 \mathrm{H} 32 \mathrm{O} 2$ & $5.21 \mathrm{E}-05$ & Basic N2 & $\mathrm{C} 30 \mathrm{H} 48 \mathrm{~N} 2$ & $5.81 \mathrm{E}-05$ \\
\hline Non-basic N1O2 & $\mathrm{C} 27 \mathrm{H} 39 \mathrm{~N} 1 \mathrm{O} 2$ & $1.46 \mathrm{E}-05$ & Acidic $\mathrm{O} 2$ & $\mathrm{C} 31 \mathrm{H} 34 \mathrm{O} 2$ & $2.06 \mathrm{E}-05$ & Basic N2 & $\mathrm{C} 31 \mathrm{H} 50 \mathrm{~N} 2$ & 4.95E-05 \\
\hline Non-basic N1O2 & $\mathrm{C} 28 \mathrm{H} 41 \mathrm{~N} 1 \mathrm{O} 2$ & $1.83 \mathrm{E}-05$ & Acidic $\mathrm{O} 2$ & $\mathrm{C} 33 \mathrm{H} 38 \mathrm{O} 2$ & $2.18 \mathrm{E}-05$ & Basic N2 & $\mathrm{C} 32 \mathrm{H} 52 \mathrm{~N} 2$ & $5.05 \mathrm{E}-05$ \\
\hline Non-basic N1O2 & $\mathrm{C} 29 \mathrm{H} 43 \mathrm{~N} 1 \mathrm{O} 2$ & $2.53 \mathrm{E}-05$ & Acidic $\mathrm{O} 2$ & $\mathrm{C} 21 \mathrm{H} 12 \mathrm{O} 2$ & 0.000119 & Basic N2 & $\mathrm{C} 33 \mathrm{H} 54 \mathrm{~N} 2$ & $6.02 \mathrm{E}-05$ \\
\hline Non-basic N1O2 & $\mathrm{C} 30 \mathrm{H} 45 \mathrm{~N} 1 \mathrm{O} 2$ & $3.28 \mathrm{E}-05$ & Acidic $\mathrm{O} 2$ & $\mathrm{C} 22 \mathrm{H} 14 \mathrm{O} 2$ & 0.000729 & Basic N2 & $\mathrm{C} 34 \mathrm{H} 56 \mathrm{~N} 2$ & $5.15 \mathrm{E}-05$ \\
\hline Non-basic N1O2 & $\mathrm{C} 31 \mathrm{H} 47 \mathrm{~N} 1 \mathrm{O} 2$ & $3.39 \mathrm{E}-05$ & Acidic $\mathrm{O} 2$ & $\mathrm{C} 23 \mathrm{H} 16 \mathrm{O} 2$ & 0.00095 & Basic N2 & $\mathrm{C} 35 \mathrm{H} 58 \mathrm{~N} 2$ & 4.99E-05 \\
\hline Non-basic N1O2 & $\mathrm{C} 32 \mathrm{H} 49 \mathrm{~N} 1 \mathrm{O} 2$ & $2.65 \mathrm{E}-05$ & Acidic $\mathrm{O} 2$ & $\mathrm{C} 24 \mathrm{H} 18 \mathrm{O} 2$ & 0.000756 & Basic N2 & $\mathrm{C} 36 \mathrm{H} 60 \mathrm{~N} 2$ & $5.04 \mathrm{E}-05$ \\
\hline Non-basic N1O2 & $\mathrm{C} 33 \mathrm{H} 51 \mathrm{~N} 1 \mathrm{O} 2$ & $2.62 \mathrm{E}-05$ & Acidic $\mathrm{O} 2$ & $\mathrm{C} 25 \mathrm{H} 20 \mathrm{O} 2$ & 0.000562 & Basic N2 & $\mathrm{C} 37 \mathrm{H} 62 \mathrm{~N} 2$ & $3.21 \mathrm{E}-05$ \\
\hline Non-basic N1O2 & $\mathrm{C} 34 \mathrm{H} 53 \mathrm{~N} 1 \mathrm{O} 2$ & $2.32 \mathrm{E}-05$ & Acidic $\mathrm{O} 2$ & $\mathrm{C} 26 \mathrm{H} 22 \mathrm{O} 2$ & 0.000274 & Basic N2 & $\mathrm{C} 38 \mathrm{H} 64 \mathrm{~N} 2$ & $3.26 \mathrm{E}-05$ \\
\hline Non-basic N1O2 & $\mathrm{C} 35 \mathrm{H} 55 \mathrm{~N} 1 \mathrm{O} 2$ & $1.09 \mathrm{E}-05$ & Acidic $\mathrm{O} 2$ & $\mathrm{C} 27 \mathrm{H} 24 \mathrm{O} 2$ & 0.000167 & Basic N2 & $\mathrm{C} 39 \mathrm{H} 66 \mathrm{~N} 2$ & $3.25 \mathrm{E}-05$ \\
\hline Non-basic N1O2 & $\mathrm{C} 36 \mathrm{H} 57 \mathrm{~N} 1 \mathrm{O} 2$ & $1.17 \mathrm{E}-05$ & Acidic $\mathrm{O} 2$ & $\mathrm{C} 28 \mathrm{H} 26 \mathrm{O} 2$ & 0.000128 & Basic N2 & $\mathrm{C} 40 \mathrm{H} 68 \mathrm{~N} 2$ & $1.76 \mathrm{E}-05$ \\
\hline Non-basic N1O2 & $\mathrm{C} 37 \mathrm{H} 59 \mathrm{~N} 1 \mathrm{O} 2$ & $2.76 \mathrm{E}-06$ & Acidic $\mathrm{O} 2$ & $\mathrm{C} 29 \mathrm{H} 28 \mathrm{O} 2$ & 0.000106 & Basic N2 & $\mathrm{C} 41 \mathrm{H} 70 \mathrm{~N} 2$ & $1.80 \mathrm{E}-05$ \\
\hline Non-basic N1O2 & C13H9N1O2 & $1.88 \mathrm{E}-06$ & Acidic $\mathrm{O} 2$ & $\mathrm{C} 30 \mathrm{H} 30 \mathrm{O} 2$ & $6.85 \mathrm{E}-05$ & Basic N2 & $\mathrm{C} 42 \mathrm{H} 72 \mathrm{~N} 2$ & $9.83 \mathrm{E}-06$ \\
\hline Non-basic N1O2 & $\mathrm{C} 14 \mathrm{H} 11 \mathrm{~N} 1 \mathrm{O} 2$ & $2.56 \mathrm{E}-05$ & Acidic $\mathrm{O} 2$ & $\mathrm{C} 31 \mathrm{H} 32 \mathrm{O} 2$ & $1.64 \mathrm{E}-05$ & Basic N2 & $\mathrm{C} 43 \mathrm{H} 74 \mathrm{~N} 2$ & $1.84 \mathrm{E}-05$ \\
\hline Non-basic N1O2 & $\mathrm{C} 15 \mathrm{H} 13 \mathrm{~N} 1 \mathrm{O} 2$ & $3.56 \mathrm{E}-05$ & Acidic $\mathrm{O} 2$ & $\mathrm{C} 22 \mathrm{H} 12 \mathrm{O} 2$ & $2.35 \mathrm{E}-05$ & Basic N2 & $\mathrm{C} 44 \mathrm{H} 76 \mathrm{~N} 2$ & $4.61 \mathrm{E}-06$ \\
\hline Non-basic N1O2 & $\mathrm{C} 16 \mathrm{H} 15 \mathrm{~N} 1 \mathrm{O} 2$ & $2.95 \mathrm{E}-05$ & Acidic $\mathrm{O} 2$ & $\mathrm{C} 23 \mathrm{H} 14 \mathrm{O} 2$ & 0.000104 & Basic N2 & $\mathrm{C} 17 \mathrm{H} 20 \mathrm{~N} 2$ & $6.91 \mathrm{E}-07$ \\
\hline Non-basic N1O2 & $\mathrm{C} 17 \mathrm{H} 17 \mathrm{~N} 1 \mathrm{O} 2$ & $3.00 \mathrm{E}-05$ & Acidic $\mathrm{O} 2$ & $\mathrm{C} 24 \mathrm{H} 16 \mathrm{O} 2$ & 0.000347 & Basic N2 & $\mathrm{C} 18 \mathrm{H} 22 \mathrm{~N} 2$ & $2.29 \mathrm{E}-07$ \\
\hline Non-basic N1O2 & $\mathrm{C} 18 \mathrm{H} 19 \mathrm{~N} 1 \mathrm{O} 2$ & $2.68 \mathrm{E}-05$ & Acidic $\mathrm{O} 2$ & $\mathrm{C} 25 \mathrm{H} 18 \mathrm{O} 2$ & 0.000574 & Basic N2 & $\mathrm{C} 19 \mathrm{H} 24 \mathrm{~N} 2$ & $6.25 \mathrm{E}-07$ \\
\hline Non-basic N1O2 & $\mathrm{C} 19 \mathrm{H} 21 \mathrm{~N} 1 \mathrm{O} 2$ & $1.47 \mathrm{E}-05$ & Acidic $\mathrm{O} 2$ & $\mathrm{C} 26 \mathrm{H} 20 \mathrm{O} 2$ & 0.000453 & Basic N2 & $\mathrm{C} 20 \mathrm{H} 26 \mathrm{~N} 2$ & $6.61 \mathrm{E}-06$ \\
\hline Non-basic N1O2 & $\mathrm{C} 20 \mathrm{H} 23 \mathrm{~N} 1 \mathrm{O} 2$ & $1.88 \mathrm{E}-05$ & Acidic $\mathrm{O} 2$ & $\mathrm{C} 27 \mathrm{H} 22 \mathrm{O} 2$ & 0.000269 & Basic N2 & $\mathrm{C} 21 \mathrm{H} 28 \mathrm{~N} 2$ & $9.99 \mathrm{E}-06$ \\
\hline Non-basic $\mathrm{N} 1 \mathrm{O} 2$ & $\mathrm{C} 21 \mathrm{H} 25 \mathrm{~N} 1 \mathrm{O} 2$ & $1.09 \mathrm{E}-05$ & Acidic $\mathrm{O} 2$ & $\mathrm{C} 28 \mathrm{H} 24 \mathrm{O} 2$ & 0.000141 & Basic N2 & $\mathrm{C} 22 \mathrm{H} 30 \mathrm{~N} 2$ & $2.17 \mathrm{E}-05$ \\
\hline Non-basic N1O2 & $\mathrm{C} 22 \mathrm{H} 27 \mathrm{~N} 1 \mathrm{O} 2$ & $2.02 \mathrm{E}-05$ & Acidic $\mathrm{O} 2$ & $\mathrm{C} 29 \mathrm{H} 26 \mathrm{O} 2$ & $9.09 \mathrm{E}-05$ & Basic N2 & $\mathrm{C} 23 \mathrm{H} 32 \mathrm{~N} 2$ & $2.86 \mathrm{E}-05$ \\
\hline Non-basic N1O2 & $\mathrm{C} 23 \mathrm{H} 29 \mathrm{~N} 1 \mathrm{O} 2$ & $1.45 \mathrm{E}-05$ & Acidic $\mathrm{O} 2$ & $\mathrm{C} 30 \mathrm{H} 28 \mathrm{O} 2$ & $5.45 \mathrm{E}-05$ & Basic N2 & $\mathrm{C} 24 \mathrm{H} 34 \mathrm{~N} 2$ & $5.38 \mathrm{E}-05$ \\
\hline Non-basic N1O2 & $\mathrm{C} 24 \mathrm{H} 31 \mathrm{~N} 1 \mathrm{O} 2$ & $1.94 \mathrm{E}-05$ & Acidic $\mathrm{O} 2$ & $\mathrm{C} 23 \mathrm{H} 12 \mathrm{O} 2$ & 0.00027 & Basic N2 & $\mathrm{C} 25 \mathrm{H} 36 \mathrm{~N} 2$ & $4.95 \mathrm{E}-05$ \\
\hline Non-basic N1O2 & $\mathrm{C} 25 \mathrm{H} 33 \mathrm{~N} 1 \mathrm{O} 2$ & $2.08 \mathrm{E}-05$ & Acidic $\mathrm{O} 2$ & $\mathrm{C} 24 \mathrm{H} 14 \mathrm{O} 2$ & 0.000713 & Basic N2 & $\mathrm{C} 26 \mathrm{H} 38 \mathrm{~N} 2$ & $5.67 \mathrm{E}-05$ \\
\hline Non-basic N1O2 & $\mathrm{C} 26 \mathrm{H} 35 \mathrm{~N} 1 \mathrm{O} 2$ & $1.98 \mathrm{E}-05$ & Acidic $\mathrm{O} 2$ & $\mathrm{C} 25 \mathrm{H} 16 \mathrm{O} 2$ & 0.000816 & Basic N2 & $\mathrm{C} 27 \mathrm{H} 40 \mathrm{~N} 2$ & $8.09 \mathrm{E}-05$ \\
\hline Non-basic N1O2 & $\mathrm{C} 27 \mathrm{H} 37 \mathrm{~N} 1 \mathrm{O} 2$ & $2.38 \mathrm{E}-05$ & Acidic $\mathrm{O} 2$ & $\mathrm{C} 26 \mathrm{H} 18 \mathrm{O} 2$ & 0.000519 & Basic N2 & $\mathrm{C} 28 \mathrm{H} 42 \mathrm{~N} 2$ & $6.61 \mathrm{E}-05$ \\
\hline Non-basic N1O2 & $\mathrm{C} 28 \mathrm{H} 39 \mathrm{~N} 1 \mathrm{O} 2$ & $2.84 \mathrm{E}-05$ & Acidic $\mathrm{O} 2$ & $\mathrm{C} 27 \mathrm{H} 20 \mathrm{O} 2$ & 0.000372 & Basic N2 & $\mathrm{C} 29 \mathrm{H} 44 \mathrm{~N} 2$ & 7.83E-05 \\
\hline Non-basic N1O2 & $\mathrm{C} 29 \mathrm{H} 41 \mathrm{~N} 1 \mathrm{O} 2$ & $3.26 \mathrm{E}-05$ & Acidic $\mathrm{O} 2$ & $\mathrm{C} 28 \mathrm{H} 22 \mathrm{O} 2$ & 0.000239 & Basic N2 & $\mathrm{C} 30 \mathrm{H} 46 \mathrm{~N} 2$ & 0.000101 \\
\hline Non-basic N1O2 & $\mathrm{C} 30 \mathrm{H} 43 \mathrm{~N} 1 \mathrm{O} 2$ & $2.58 \mathrm{E}-05$ & Acidic $\mathrm{O} 2$ & $\mathrm{C} 29 \mathrm{H} 24 \mathrm{O} 2$ & 0.000151 & Basic N2 & $\mathrm{C} 31 \mathrm{H} 48 \mathrm{~N} 2$ & 0.000107 \\
\hline Non-basic $\mathrm{N} 1 \mathrm{O} 2$ & $\mathrm{C} 31 \mathrm{H} 45 \mathrm{~N} 1 \mathrm{O} 2$ & $2.02 \mathrm{E}-05$ & Acidic $\mathrm{O} 2$ & $\mathrm{C} 25 \mathrm{H} 14 \mathrm{O} 2$ & $1.31 \mathrm{E}-05$ & Basic N2 & $\mathrm{C} 32 \mathrm{H} 50 \mathrm{~N} 2$ & $8.49 \mathrm{E}-05$ \\
\hline Non-basic $\mathrm{N} 1 \mathrm{O} 2$ & $\mathrm{C} 32 \mathrm{H} 47 \mathrm{~N} 1 \mathrm{O} 2$ & $2.87 \mathrm{E}-05$ & Acidic $\mathrm{O} 2$ & $\mathrm{C} 26 \mathrm{H} 16 \mathrm{O} 2$ & 0.000305 & Basic N2 & $\mathrm{C} 33 \mathrm{H} 52 \mathrm{~N} 2$ & $6.58 \mathrm{E}-05$ \\
\hline Non-basic $\mathrm{N} 1 \mathrm{O} 2$ & $\mathrm{C} 33 \mathrm{H} 49 \mathrm{~N} 1 \mathrm{O} 2$ & $2.01 \mathrm{E}-05$ & Acidic $\mathrm{O} 2$ & $\mathrm{C} 27 \mathrm{H} 18 \mathrm{O} 2$ & 0.000304 & Basic N2 & $\mathrm{C} 34 \mathrm{H} 54 \mathrm{~N} 2$ & $5.13 \mathrm{E}-05$ \\
\hline Non-basic $\mathrm{N} 1 \mathrm{O} 2$ & $\mathrm{C} 34 \mathrm{H} 51 \mathrm{~N} 1 \mathrm{O} 2$ & $2.24 \mathrm{E}-05$ & Acidic $\mathrm{O} 2$ & $\mathrm{C} 28 \mathrm{H} 20 \mathrm{O} 2$ & 0.00037 & Basic N2 & $\mathrm{C} 35 \mathrm{H} 56 \mathrm{~N} 2$ & $5.95 \mathrm{E}-05$ \\
\hline
\end{tabular}




\begin{tabular}{|c|c|c|c|c|c|c|c|c|}
\hline Class & Formula & Mass & Class & Formula & Mass & Class & Formula & Mass \\
\hline Non-basic N1O2 & $\mathrm{C} 35 \mathrm{H} 53 \mathrm{~N} 1 \mathrm{O} 2$ & $2.11 \mathrm{E}-05$ & Acidic $\mathrm{O} 2$ & $\mathrm{C} 29 \mathrm{H} 22 \mathrm{O} 2$ & 0.000124 & Basic N2 & $\mathrm{C} 36 \mathrm{H} 58 \mathrm{~N} 2$ & $3.74 \mathrm{E}-05$ \\
\hline Non-basic N1O2 & $\mathrm{C} 36 \mathrm{H} 55 \mathrm{~N} 1 \mathrm{O} 2$ & $1.60 \mathrm{E}-05$ & Acidic $\mathrm{O} 2$ & $\mathrm{C} 30 \mathrm{H} 24 \mathrm{O} 2$ & $8.86 \mathrm{E}-05$ & Basic N2 & $\mathrm{C} 37 \mathrm{H} 60 \mathrm{~N} 2$ & $5.13 \mathrm{E}-05$ \\
\hline Non-basic N1O2 & $\mathrm{C} 37 \mathrm{H} 57 \mathrm{~N} 1 \mathrm{O} 2$ & $6.72 \mathrm{E}-06$ & Acidic $\mathrm{O} 2$ & $\mathrm{C} 31 \mathrm{H} 26 \mathrm{O} 2$ & $2.85 \mathrm{E}-05$ & Basic N2 & $\mathrm{C} 38 \mathrm{H} 62 \mathrm{~N} 2$ & $3.98 \mathrm{E}-05$ \\
\hline Non-basic N1O2 & $\mathrm{C} 38 \mathrm{H} 59 \mathrm{~N} 1 \mathrm{O} 2$ & $2.72 \mathrm{E}-06$ & Acidic $\mathrm{O} 2$ & $\mathrm{C} 32 \mathrm{H} 28 \mathrm{O} 2$ & $5.64 \mathrm{E}-05$ & Basic N2 & C39H64N2 & $4.28 \mathrm{E}-05$ \\
\hline Non-basic N1O2 & $\mathrm{C} 15 \mathrm{H} 11 \mathrm{~N} 1 \mathrm{O} 2$ & $2.26 \mathrm{E}-05$ & Acidic $\mathrm{O} 2$ & $\mathrm{C} 26 \mathrm{H} 14 \mathrm{O} 2$ & $4.84 \mathrm{E}-05$ & Basic N2 & $\mathrm{C} 40 \mathrm{H} 66 \mathrm{~N} 2$ & $2.31 \mathrm{E}-05$ \\
\hline Non-basic N1O2 & $\mathrm{C} 16 \mathrm{H} 13 \mathrm{~N} 1 \mathrm{O} 2$ & $4.59 \mathrm{E}-05$ & Acidic $\mathrm{O} 2$ & $\mathrm{C} 27 \mathrm{H} 16 \mathrm{O} 2$ & 0.000139 & Basic N2 & $\mathrm{C} 41 \mathrm{H} 68 \mathrm{~N} 2$ & $2.65 \mathrm{E}-05$ \\
\hline Non-basic N1O2 & $\mathrm{C} 17 \mathrm{H} 15 \mathrm{~N} 1 \mathrm{O} 2$ & 0.000106 & Acidic $\mathrm{O} 2$ & $\mathrm{C} 28 \mathrm{H} 18 \mathrm{O} 2$ & 0.000104 & Basic N2 & $\mathrm{C} 42 \mathrm{H} 70 \mathrm{~N} 2$ & $1.17 \mathrm{E}-05$ \\
\hline Non-basic N1O2 & $\mathrm{C} 18 \mathrm{H} 17 \mathrm{~N} 1 \mathrm{O} 2$ & 0.000165 & Acidic $\mathrm{O} 2$ & $\mathrm{C} 29 \mathrm{H} 20 \mathrm{O} 2$ & 0.00021 & Basic N2 & $\mathrm{C} 43 \mathrm{H} 72 \mathrm{~N} 2$ & $8.98 \mathrm{E}-06$ \\
\hline Non-basic N1O2 & C19H19N1O2 & 0.000174 & Acidic $\mathrm{O} 2$ & $\mathrm{C} 30 \mathrm{H} 22 \mathrm{O} 2$ & 0.000138 & Basic N2 & $\mathrm{C} 44 \mathrm{H} 74 \mathrm{~N} 2$ & $4.61 \mathrm{E}-06$ \\
\hline Non-basic N1O2 & $\mathrm{C} 20 \mathrm{H} 21 \mathrm{~N} 1 \mathrm{O} 2$ & 0.000133 & Acidic $\mathrm{O} 2$ & $\mathrm{C} 31 \mathrm{H} 24 \mathrm{O} 2$ & $8.39 \mathrm{E}-05$ & Basic N2 & $\mathrm{C} 45 \mathrm{H} 76 \mathrm{~N} 2$ & 4.94E-06 \\
\hline Non-basic N1O2 & $\mathrm{C} 21 \mathrm{H} 23 \mathrm{~N} 1 \mathrm{O} 2$ & $8.06 \mathrm{E}-05$ & Acidic $\mathrm{O} 2$ & $\mathrm{C} 32 \mathrm{H} 26 \mathrm{O} 2$ & $1.72 \mathrm{E}-05$ & Basic N2 & $\mathrm{C} 16 \mathrm{H} 16 \mathrm{~N} 2$ & $9.20 \mathrm{E}-07$ \\
\hline Non-basic N1O2 & $\mathrm{C} 22 \mathrm{H} 25 \mathrm{~N} 1 \mathrm{O} 2$ & $5.08 \mathrm{E}-05$ & Acidic $\mathrm{O} 2$ & $\mathrm{C} 27 \mathrm{H} 14 \mathrm{O} 2$ & $1.46 \mathrm{E}-05$ & Basic N2 & $\mathrm{C} 17 \mathrm{H} 18 \mathrm{~N} 2$ & $1.30 \mathrm{E}-06$ \\
\hline Non-basic N1O2 & $\mathrm{C} 23 \mathrm{H} 27 \mathrm{~N} 1 \mathrm{O} 2$ & 4.33E-05 & Acidic $\mathrm{O} 2$ & $\mathrm{C} 28 \mathrm{H} 16 \mathrm{O} 2$ & $9.52 \mathrm{E}-05$ & Basic N2 & $\mathrm{C} 18 \mathrm{H} 20 \mathrm{~N} 2$ & $1.77 \mathrm{E}-06$ \\
\hline Non-basic N1O2 & $\mathrm{C} 24 \mathrm{H} 29 \mathrm{~N} 1 \mathrm{O} 2$ & $4.21 \mathrm{E}-05$ & Acidic $\mathrm{O} 2$ & $\mathrm{C} 29 \mathrm{H} 18 \mathrm{O} 2$ & $8.65 \mathrm{E}-05$ & Basic N2 & $\mathrm{C} 19 \mathrm{H} 22 \mathrm{~N} 2$ & $3.94 \mathrm{E}-06$ \\
\hline Non-basic N1O2 & $\mathrm{C} 25 \mathrm{H} 31 \mathrm{~N} 1 \mathrm{O} 2$ & $3.79 \mathrm{E}-05$ & Acidic $\mathrm{O} 2$ & $\mathrm{C} 30 \mathrm{H} 20 \mathrm{O} 2$ & 0.000101 & Basic N2 & $\mathrm{C} 20 \mathrm{H} 24 \mathrm{~N} 2$ & $5.21 \mathrm{E}-06$ \\
\hline Non-basic N1O2 & $\mathrm{C} 26 \mathrm{H} 33 \mathrm{~N} 1 \mathrm{O} 2$ & $3.32 \mathrm{E}-05$ & Acidic $\mathrm{O} 2$ & $\mathrm{C} 31 \mathrm{H} 22 \mathrm{O} 2$ & $9.48 \mathrm{E}-05$ & Basic N2 & $\mathrm{C} 21 \mathrm{H} 26 \mathrm{~N} 2$ & $1.43 \mathrm{E}-05$ \\
\hline Non-basic N1O2 & $\mathrm{C} 27 \mathrm{H} 35 \mathrm{~N} 1 \mathrm{O} 2$ & $3.38 \mathrm{E}-05$ & Acidic $\mathrm{O} 2$ & $\mathrm{C} 32 \mathrm{H} 24 \mathrm{O} 2$ & 0.000102 & Basic N2 & $\mathrm{C} 22 \mathrm{H} 28 \mathrm{~N} 2$ & $1.65 \mathrm{E}-05$ \\
\hline Non-basic N1O2 & $\mathrm{C} 28 \mathrm{H} 37 \mathrm{~N} 1 \mathrm{O} 2$ & $4.02 \mathrm{E}-05$ & Acidic $\mathrm{O} 2$ & $\mathrm{C} 33 \mathrm{H} 26 \mathrm{O} 2$ & $1.79 \mathrm{E}-05$ & Basic N2 & $\mathrm{C} 23 \mathrm{H} 30 \mathrm{~N} 2$ & $4.34 \mathrm{E}-05$ \\
\hline Non-basic N1O2 & $\mathrm{C} 29 \mathrm{H} 39 \mathrm{~N} 1 \mathrm{O} 2$ & $3.59 \mathrm{E}-05$ & Acidic $\mathrm{O} 2$ & $\mathrm{C} 31 \mathrm{H} 20 \mathrm{O} 2$ & $8.20 \mathrm{E}-05$ & Basic N2 & $\mathrm{C} 24 \mathrm{H} 32 \mathrm{~N} 2$ & $4.90 \mathrm{E}-05$ \\
\hline Non-basic N1O2 & $\mathrm{C} 30 \mathrm{H} 41 \mathrm{~N} 1 \mathrm{O} 2$ & $3.29 \mathrm{E}-05$ & Acidic $\mathrm{O} 2$ & $\mathrm{C} 32 \mathrm{H} 22 \mathrm{O} 2$ & $6.13 \mathrm{E}-05$ & Basic N2 & $\mathrm{C} 25 \mathrm{H} 34 \mathrm{~N} 2$ & $5.45 \mathrm{E}-05$ \\
\hline Non-basic N1O2 & $\mathrm{C} 31 \mathrm{H} 43 \mathrm{~N} 1 \mathrm{O} 2$ & $3.07 \mathrm{E}-05$ & Acidic $\mathrm{O} 2$ & $\mathrm{C} 33 \mathrm{H} 24 \mathrm{O} 2$ & $2.83 \mathrm{E}-05$ & Basic N2 & $\mathrm{C} 26 \mathrm{H} 36 \mathrm{~N} 2$ & 7.44E- 05 \\
\hline Non-basic N1O2 & $\mathrm{C} 32 \mathrm{H} 45 \mathrm{~N} 1 \mathrm{O} 2$ & $3.39 \mathrm{E}-05$ & Acidic $\mathrm{O} 2$ & $\mathrm{C} 34 \mathrm{H} 26 \mathrm{O} 2$ & $3.40 \mathrm{E}-05$ & Basic N2 & $\mathrm{C} 27 \mathrm{H} 38 \mathrm{~N} 2$ & 7.22E- 05 \\
\hline Non-basic N1O2 & $\mathrm{C} 33 \mathrm{H} 47 \mathrm{~N} 1 \mathrm{O} 2$ & $2.06 \mathrm{E}-05$ & Acidic $\mathrm{O} 2$ & $\mathrm{C} 35 \mathrm{H} 28 \mathrm{O} 2$ & $1.87 \mathrm{E}-05$ & Basic N2 & $\mathrm{C} 28 \mathrm{H} 40 \mathrm{~N} 2$ & $8.53 \mathrm{E}-05$ \\
\hline Non-basic N1O2 & $\mathrm{C} 34 \mathrm{H} 49 \mathrm{~N} 1 \mathrm{O} 2$ & $1.39 \mathrm{E}-05$ & Acidic $\mathrm{O} 2$ & $\mathrm{C} 31 \mathrm{H} 18 \mathrm{O} 2$ & 7.92E-05 & Basic N2 & $\mathrm{C} 29 \mathrm{H} 42 \mathrm{~N} 2$ & 0.000115 \\
\hline Non-basic $\mathrm{N} 1 \mathrm{O} 2$ & $\mathrm{C} 35 \mathrm{H} 51 \mathrm{~N} 1 \mathrm{O} 2$ & $1.35 \mathrm{E}-05$ & Acidic $\mathrm{O} 2$ & $\mathrm{C} 32 \mathrm{H} 20 \mathrm{O} 2$ & 4.32E-05 & Basic N2 & $\mathrm{C} 30 \mathrm{H} 44 \mathrm{~N} 2$ & $8.75 \mathrm{E}-05$ \\
\hline Non-basic $\mathrm{N} 1 \mathrm{O} 2$ & $\mathrm{C} 36 \mathrm{H} 53 \mathrm{~N} 1 \mathrm{O} 2$ & $1.43 \mathrm{E}-05$ & Acidic $\mathrm{O} 2$ & $\mathrm{C} 33 \mathrm{H} 22 \mathrm{O} 2$ & 4.13E-05 & Basic N2 & $\mathrm{C} 31 \mathrm{H} 46 \mathrm{~N} 2$ & $8.18 \mathrm{E}-05$ \\
\hline Non-basic N1O2 & $\mathrm{C} 37 \mathrm{H} 55 \mathrm{~N} 1 \mathrm{O} 2$ & $1.25 \mathrm{E}-05$ & Acidic $\mathrm{O} 2$ & $\mathrm{C} 34 \mathrm{H} 24 \mathrm{O} 2$ & $3.02 \mathrm{E}-05$ & Basic N2 & $\mathrm{C} 32 \mathrm{H} 48 \mathrm{~N} 2$ & $8.40 \mathrm{E}-05$ \\
\hline Non-basic N1O2 & $\mathrm{C} 38 \mathrm{H} 57 \mathrm{~N} 1 \mathrm{O} 2$ & $3.92 \mathrm{E}-06$ & Basic N1 & $\mathrm{C} 16 \mathrm{H} 27 \mathrm{~N} 1$ & $3.54 \mathrm{E}-07$ & Basic N2 & $\mathrm{C} 33 \mathrm{H} 50 \mathrm{~N} 2$ & $8.20 \mathrm{E}-05$ \\
\hline Non-basic N1O2 & C39H59N1O2 & $1.48 \mathrm{E}-06$ & Basic N1 & $\mathrm{C} 17 \mathrm{H} 29 \mathrm{~N} 1$ & $1.53 \mathrm{E}-06$ & Basic N2 & $\mathrm{C} 34 \mathrm{H} 52 \mathrm{~N} 2$ & 0.000119 \\
\hline Non-basic N1O2 & $\mathrm{C} 40 \mathrm{H} 61 \mathrm{~N} 1 \mathrm{O} 2$ & $5.04 \mathrm{E}-06$ & Basic N1 & $\mathrm{C} 18 \mathrm{H} 31 \mathrm{~N} 1$ & $5.78 \mathrm{E}-06$ & Basic N2 & $\mathrm{C} 35 \mathrm{H} 54 \mathrm{~N} 2$ & $8.94 \mathrm{E}-05$ \\
\hline Non-basic N1O2 & C15H9N1O2 & $5.30 \mathrm{E}-06$ & Basic N1 & $\mathrm{C} 19 \mathrm{H} 33 \mathrm{~N} 1$ & $1.11 \mathrm{E}-06$ & Basic N2 & $\mathrm{C} 36 \mathrm{H} 56 \mathrm{~N} 2$ & $6.80 \mathrm{E}-05$ \\
\hline Non-basic N1O2 & $\mathrm{C} 16 \mathrm{H} 11 \mathrm{~N} 1 \mathrm{O} 2$ & $9.48 \mathrm{E}-05$ & Basic N1 & $\mathrm{C} 20 \mathrm{H} 35 \mathrm{~N} 1$ & $2.12 \mathrm{E}-06$ & Basic N2 & $\mathrm{C} 37 \mathrm{H} 58 \mathrm{~N} 2$ & $5.84 \mathrm{E}-05$ \\
\hline Non-basic N1O2 & $\mathrm{C} 17 \mathrm{H} 13 \mathrm{~N} 1 \mathrm{O} 2$ & 0.001076 & Basic N1 & $\mathrm{C} 21 \mathrm{H} 37 \mathrm{~N} 1$ & 4.92E-06 & Basic N2 & $\mathrm{C} 38 \mathrm{H} 60 \mathrm{~N} 2$ & $5.60 \mathrm{E}-05$ \\
\hline Non-basic N1O2 & $\mathrm{C} 18 \mathrm{H} 15 \mathrm{~N} 1 \mathrm{O} 2$ & 0.003001 & Basic N1 & $\mathrm{C} 22 \mathrm{H} 39 \mathrm{~N} 1$ & $3.02 \mathrm{E}-06$ & Basic N2 & $\mathrm{C} 39 \mathrm{H} 62 \mathrm{~N} 2$ & $3.80 \mathrm{E}-05$ \\
\hline Non-basic $\mathrm{N} 1 \mathrm{O} 2$ & $\mathrm{C} 19 \mathrm{H} 17 \mathrm{~N} 1 \mathrm{O} 2$ & 0.004023 & Basic N1 & $\mathrm{C} 23 \mathrm{H} 41 \mathrm{~N} 1$ & $6.52 \mathrm{E}-06$ & Basic N2 & $\mathrm{C} 40 \mathrm{H} 64 \mathrm{~N} 2$ & $3.06 \mathrm{E}-05$ \\
\hline Non-basic $\mathrm{N} 1 \mathrm{O} 2$ & $\mathrm{C} 20 \mathrm{H} 19 \mathrm{~N} 1 \mathrm{O} 2$ & 0.003167 & Basic N1 & $\mathrm{C} 24 \mathrm{H} 43 \mathrm{~N} 1$ & $1.08 \mathrm{E}-05$ & Basic N2 & $\mathrm{C} 41 \mathrm{H} 66 \mathrm{~N} 2$ & $2.84 \mathrm{E}-05$ \\
\hline Non-basic $\mathrm{N} 1 \mathrm{O} 2$ & $\mathrm{C} 21 \mathrm{H} 21 \mathrm{~N} 1 \mathrm{O} 2$ & 0.001469 & Basic N1 & $\mathrm{C} 25 \mathrm{H} 45 \mathrm{~N} 1$ & $1.22 \mathrm{E}-05$ & Basic N2 & $\mathrm{C} 42 \mathrm{H} 68 \mathrm{~N} 2$ & $1.63 \mathrm{E}-05$ \\
\hline Non-basic $\mathrm{N} 1 \mathrm{O} 2$ & $\mathrm{C} 22 \mathrm{H} 23 \mathrm{~N} 1 \mathrm{O} 2$ & 0.000613 & Basic N1 & $\mathrm{C} 26 \mathrm{H} 47 \mathrm{~N} 1$ & $2.19 \mathrm{E}-05$ & Basic N2 & $\mathrm{C} 43 \mathrm{H} 70 \mathrm{~N} 2$ & $9.30 \mathrm{E}-06$ \\
\hline
\end{tabular}




\begin{tabular}{|c|c|c|c|c|c|c|c|c|}
\hline Class & Formula & Mass & Class & Formula & Mass & Class & Formula & Mass \\
\hline Non-basic N1O2 & $\mathrm{C} 23 \mathrm{H} 25 \mathrm{~N} 1 \mathrm{O} 2$ & 0.000292 & Basic N1 & $\mathrm{C} 27 \mathrm{H} 49 \mathrm{~N} 1$ & $2.29 \mathrm{E}-05$ & Basic N2 & $\mathrm{C} 44 \mathrm{H} 72 \mathrm{~N} 2$ & $5.81 \mathrm{E}-06$ \\
\hline Non-basic N1O2 & $\mathrm{C} 24 \mathrm{H} 27 \mathrm{~N} 1 \mathrm{O} 2$ & 0.000167 & Basic N1 & $\mathrm{C} 28 \mathrm{H} 51 \mathrm{~N} 1$ & $2.09 \mathrm{E}-05$ & Basic N2 & $\mathrm{C} 17 \mathrm{H} 16 \mathrm{~N} 2$ & $3.84 \mathrm{E}-07$ \\
\hline Non-basic N1O2 & $\mathrm{C} 25 \mathrm{H} 29 \mathrm{~N} 1 \mathrm{O} 2$ & 0.000134 & Basic N1 & $\mathrm{C} 29 \mathrm{H} 53 \mathrm{~N} 1$ & $3.11 \mathrm{E}-05$ & Basic N2 & $\mathrm{C} 18 \mathrm{H} 18 \mathrm{~N} 2$ & $2.73 \mathrm{E}-07$ \\
\hline Non-basic N1O2 & $\mathrm{C} 26 \mathrm{H} 31 \mathrm{~N} 1 \mathrm{O} 2$ & 0.000113 & Basic N1 & $\mathrm{C} 30 \mathrm{H} 55 \mathrm{~N} 1$ & $4.24 \mathrm{E}-05$ & Basic N2 & $\mathrm{C} 19 \mathrm{H} 20 \mathrm{~N} 2$ & $1.56 \mathrm{E}-06$ \\
\hline Non-basic N1O2 & $\mathrm{C} 27 \mathrm{H} 33 \mathrm{~N} 1 \mathrm{O} 2$ & 0.000109 & Basic N1 & $\mathrm{C} 31 \mathrm{H} 57 \mathrm{~N} 1$ & $3.33 \mathrm{E}-05$ & Basic N2 & $\mathrm{C} 20 \mathrm{H} 22 \mathrm{~N} 2$ & $1.09 \mathrm{E}-05$ \\
\hline Non-basic N1O2 & $\mathrm{C} 28 \mathrm{H} 35 \mathrm{~N} 1 \mathrm{O} 2$ & $9.21 \mathrm{E}-05$ & Basic N1 & $\mathrm{C} 32 \mathrm{H} 59 \mathrm{~N} 1$ & $4.04 \mathrm{E}-05$ & Basic N2 & $\mathrm{C} 21 \mathrm{H} 24 \mathrm{~N} 2$ & $1.51 \mathrm{E}-05$ \\
\hline Non-basic N1O2 & $\mathrm{C} 29 \mathrm{H} 37 \mathrm{~N} 1 \mathrm{O} 2$ & $8.44 \mathrm{E}-05$ & Basic N1 & $\mathrm{C} 33 \mathrm{H} 61 \mathrm{~N} 1$ & $4.26 \mathrm{E}-05$ & Basic N2 & $\mathrm{C} 22 \mathrm{H} 26 \mathrm{~N} 2$ & $3.59 \mathrm{E}-05$ \\
\hline Non-basic N1O2 & $\mathrm{C} 30 \mathrm{H} 39 \mathrm{~N} 1 \mathrm{O} 2$ & $9.48 \mathrm{E}-05$ & Basic N1 & $\mathrm{C} 34 \mathrm{H} 63 \mathrm{~N} 1$ & $4.22 \mathrm{E}-05$ & Basic N2 & $\mathrm{C} 23 \mathrm{H} 28 \mathrm{~N} 2$ & $3.86 \mathrm{E}-05$ \\
\hline Non-basic N1O2 & $\mathrm{C} 31 \mathrm{H} 41 \mathrm{~N} 1 \mathrm{O} 2$ & 0.000112 & Basic N1 & $\mathrm{C} 35 \mathrm{H} 65 \mathrm{~N} 1$ & $3.72 \mathrm{E}-05$ & Basic N2 & $\mathrm{C} 24 \mathrm{H} 30 \mathrm{~N} 2$ & $6.73 \mathrm{E}-05$ \\
\hline Non-basic N1O2 & $\mathrm{C} 32 \mathrm{H} 43 \mathrm{~N} 1 \mathrm{O} 2$ & 0.000191 & Basic N1 & $\mathrm{C} 36 \mathrm{H} 67 \mathrm{~N} 1$ & $4.38 \mathrm{E}-05$ & Basic N2 & $\mathrm{C} 25 \mathrm{H} 32 \mathrm{~N} 2$ & $5.64 \mathrm{E}-05$ \\
\hline Non-basic N1O2 & $\mathrm{C} 33 \mathrm{H} 45 \mathrm{~N} 1 \mathrm{O} 2$ & 0.000415 & Basic N1 & $\mathrm{C} 37 \mathrm{H} 69 \mathrm{~N} 1$ & $3.49 \mathrm{E}-05$ & Basic N2 & $\mathrm{C} 26 \mathrm{H} 34 \mathrm{~N} 2$ & $6.62 \mathrm{E}-05$ \\
\hline Non-basic N1O2 & $\mathrm{C} 34 \mathrm{H} 47 \mathrm{~N} 1 \mathrm{O} 2$ & 0.000606 & Basic N1 & $\mathrm{C} 38 \mathrm{H} 71 \mathrm{~N} 1$ & $2.87 \mathrm{E}-05$ & Basic N2 & $\mathrm{C} 27 \mathrm{H} 36 \mathrm{~N} 2$ & 7.63E-05 \\
\hline Non-basic N1O2 & $\mathrm{C} 35 \mathrm{H} 49 \mathrm{~N} 1 \mathrm{O} 2$ & 0.000607 & Basic N1 & $\mathrm{C} 39 \mathrm{H} 73 \mathrm{~N} 1$ & $3.15 \mathrm{E}-05$ & Basic N2 & $\mathrm{C} 28 \mathrm{H} 38 \mathrm{~N} 2$ & $8.18 \mathrm{E}-05$ \\
\hline Non-basic $\mathrm{N} 1 \mathrm{O} 2$ & C36H51N1O2 & 0.000436 & Basic N1 & $\mathrm{C} 40 \mathrm{H} 75 \mathrm{~N} 1$ & $2.39 \mathrm{E}-05$ & Basic N2 & $\mathrm{C} 29 \mathrm{H} 40 \mathrm{~N} 2$ & $9.34 \mathrm{E}-05$ \\
\hline Non-basic N1O2 & $\mathrm{C} 37 \mathrm{H} 53 \mathrm{~N} 1 \mathrm{O} 2$ & 0.000255 & Basic N1 & $\mathrm{C} 16 \mathrm{H} 25 \mathrm{~N} 1$ & $1.41 \mathrm{E}-07$ & Basic N2 & $\mathrm{C} 30 \mathrm{H} 42 \mathrm{~N} 2$ & $9.43 \mathrm{E}-05$ \\
\hline Non-basic N1O2 & $\mathrm{C} 38 \mathrm{H} 55 \mathrm{~N} 1 \mathrm{O} 2$ & 0.000115 & Basic N1 & $\mathrm{C} 17 \mathrm{H} 27 \mathrm{~N} 1$ & $1.86 \mathrm{E}-06$ & Basic N2 & $\mathrm{C} 31 \mathrm{H} 44 \mathrm{~N} 2$ & $9.24 \mathrm{E}-05$ \\
\hline Non-basic N1O2 & C39H57N1O2 & $7.48 \mathrm{E}-05$ & Basic N1 & $\mathrm{C} 18 \mathrm{H} 29 \mathrm{~N} 1$ & $2.00 \mathrm{E}-06$ & Basic N2 & $\mathrm{C} 32 \mathrm{H} 46 \mathrm{~N} 2$ & 8.06E-05 \\
\hline Non-basic N1O2 & $\mathrm{C} 40 \mathrm{H} 59 \mathrm{~N} 1 \mathrm{O} 2$ & $6.45 \mathrm{E}-05$ & Basic N1 & $\mathrm{C} 19 \mathrm{H} 31 \mathrm{~N} 1$ & $4.70 \mathrm{E}-06$ & Basic N2 & $\mathrm{C} 33 \mathrm{H} 48 \mathrm{~N} 2$ & $7.78 \mathrm{E}-05$ \\
\hline Non-basic N1O2 & C41H61N1O2 & $5.96 \mathrm{E}-05$ & Basic N1 & $\mathrm{C} 20 \mathrm{H} 33 \mathrm{~N} 1$ & 7.61E-06 & Basic N2 & $\mathrm{C} 34 \mathrm{H} 50 \mathrm{~N} 2$ & 0.000102 \\
\hline Non-basic N1O2 & $\mathrm{C} 42 \mathrm{H} 63 \mathrm{~N} 1 \mathrm{O} 2$ & $6.29 \mathrm{E}-05$ & Basic N1 & $\mathrm{C} 21 \mathrm{H} 35 \mathrm{~N} 1$ & $1.46 \mathrm{E}-05$ & Basic N2 & $\mathrm{C} 35 \mathrm{H} 52 \mathrm{~N} 2$ & $6.43 \mathrm{E}-05$ \\
\hline Non-basic N1O2 & $\mathrm{C} 43 \mathrm{H} 65 \mathrm{~N} 1 \mathrm{O} 2$ & $5.24 \mathrm{E}-05$ & Basic N1 & $\mathrm{C} 22 \mathrm{H} 37 \mathrm{~N} 1$ & $3.77 \mathrm{E}-05$ & Basic N2 & $\mathrm{C} 36 \mathrm{H} 54 \mathrm{~N} 2$ & $6.26 \mathrm{E}-05$ \\
\hline Non-basic N1O2 & C44H67N1O2 & $4.40 \mathrm{E}-05$ & Basic N1 & $\mathrm{C} 23 \mathrm{H} 39 \mathrm{~N} 1$ & $5.94 \mathrm{E}-05$ & Basic N2 & $\mathrm{C} 37 \mathrm{H} 56 \mathrm{~N} 2$ & $7.21 \mathrm{E}-05$ \\
\hline Non-basic N1O2 & $\mathrm{C} 45 \mathrm{H} 69 \mathrm{~N} 1 \mathrm{O} 2$ & $3.15 \mathrm{E}-05$ & Basic N1 & $\mathrm{C} 24 \mathrm{H} 41 \mathrm{~N} 1$ & $7.52 \mathrm{E}-05$ & Basic N2 & $\mathrm{C} 38 \mathrm{H} 58 \mathrm{~N} 2$ & $4.62 \mathrm{E}-05$ \\
\hline Non-basic $\mathrm{N} 1 \mathrm{O} 2$ & $\mathrm{C} 46 \mathrm{H} 71 \mathrm{~N} 1 \mathrm{O} 2$ & $1.07 \mathrm{E}-05$ & Basic N1 & $\mathrm{C} 25 \mathrm{H} 43 \mathrm{~N} 1$ & $8.86 \mathrm{E}-05$ & Basic N2 & $\mathrm{C} 39 \mathrm{H} 60 \mathrm{~N} 2$ & $3.21 \mathrm{E}-05$ \\
\hline Non-basic N1O2 & $\mathrm{C} 47 \mathrm{H} 73 \mathrm{~N} 1 \mathrm{O} 2$ & $7.62 \mathrm{E}-06$ & Basic N1 & $\mathrm{C} 26 \mathrm{H} 45 \mathrm{~N} 1$ & 0.000148 & Basic N2 & $\mathrm{C} 40 \mathrm{H} 62 \mathrm{~N} 2$ & $3.14 \mathrm{E}-05$ \\
\hline Non-basic N1O2 & $\mathrm{C} 48 \mathrm{H} 75 \mathrm{~N} 1 \mathrm{O} 2$ & $9.08 \mathrm{E}-06$ & Basic N1 & $\mathrm{C} 27 \mathrm{H} 47 \mathrm{~N} 1$ & 0.000169 & Basic N2 & $\mathrm{C} 41 \mathrm{H} 64 \mathrm{~N} 2$ & $2.39 \mathrm{E}-05$ \\
\hline Non-basic N1O2 & $\mathrm{C} 49 \mathrm{H} 77 \mathrm{~N} 1 \mathrm{O} 2$ & $1.79 \mathrm{E}-06$ & Basic N1 & $\mathrm{C} 28 \mathrm{H} 49 \mathrm{~N} 1$ & 0.000194 & Basic N2 & $\mathrm{C} 42 \mathrm{H} 66 \mathrm{~N} 2$ & $2.20 \mathrm{E}-05$ \\
\hline Non-basic N1O2 & $\mathrm{C} 50 \mathrm{H} 79 \mathrm{~N} 1 \mathrm{O} 2$ & $2.19 \mathrm{E}-06$ & Basic N1 & $\mathrm{C} 29 \mathrm{H} 51 \mathrm{~N} 1$ & 0.000241 & Basic N2 & $\mathrm{C} 43 \mathrm{H} 68 \mathrm{~N} 2$ & $6.51 \mathrm{E}-06$ \\
\hline Non-basic N1O2 & $\mathrm{C} 16 \mathrm{H} 9 \mathrm{~N} 1 \mathrm{O} 2$ & 0.000253 & Basic N1 & $\mathrm{C} 30 \mathrm{H} 53 \mathrm{~N} 1$ & 0.000238 & Basic N2 & $\mathrm{C} 17 \mathrm{H} 14 \mathrm{~N} 2$ & $1.68 \mathrm{E}-06$ \\
\hline Non-basic N1O2 & $\mathrm{C} 17 \mathrm{H} 11 \mathrm{~N} 1 \mathrm{O} 2$ & 0.000652 & Basic N1 & $\mathrm{C} 31 \mathrm{H} 55 \mathrm{~N} 1$ & 0.000233 & Basic N2 & $\mathrm{C} 18 \mathrm{H} 16 \mathrm{~N} 2$ & $1.18 \mathrm{E}-06$ \\
\hline Non-basic N1O2 & $\mathrm{C} 18 \mathrm{H} 13 \mathrm{~N} 1 \mathrm{O} 2$ & 0.000908 & Basic N1 & $\mathrm{C} 32 \mathrm{H} 57 \mathrm{~N} 1$ & 0.000319 & Basic N2 & C19H18N2 & $8.10 \mathrm{E}-06$ \\
\hline Non-basic N1O2 & C19H15N1O2 & 0.001361 & Basic N1 & $\mathrm{C} 33 \mathrm{H} 59 \mathrm{~N} 1$ & 0.000241 & Basic N2 & $\mathrm{C} 20 \mathrm{H} 20 \mathrm{~N} 2$ & $1.29 \mathrm{E}-05$ \\
\hline Non-basic N1O2 & $\mathrm{C} 20 \mathrm{H} 17 \mathrm{~N} 1 \mathrm{O} 2$ & 0.002076 & Basic N1 & $\mathrm{C} 34 \mathrm{H} 61 \mathrm{~N} 1$ & 0.000292 & Basic N2 & $\mathrm{C} 21 \mathrm{H} 22 \mathrm{~N} 2$ & $1.71 \mathrm{E}-05$ \\
\hline Non-basic N1O2 & $\mathrm{C} 21 \mathrm{H} 19 \mathrm{~N} 1 \mathrm{O} 2$ & 0.002269 & Basic N1 & $\mathrm{C} 35 \mathrm{H} 63 \mathrm{~N} 1$ & 0.000283 & Basic N2 & $\mathrm{C} 22 \mathrm{H} 24 \mathrm{~N} 2$ & $2.40 \mathrm{E}-05$ \\
\hline Non-basic N1O2 & $\mathrm{C} 22 \mathrm{H} 21 \mathrm{~N} 1 \mathrm{O} 2$ & 0.001678 & Basic N1 & $\mathrm{C} 36 \mathrm{H} 65 \mathrm{~N} 1$ & 0.000283 & Basic N2 & $\mathrm{C} 23 \mathrm{H} 26 \mathrm{~N} 2$ & $3.60 \mathrm{E}-05$ \\
\hline Non-basic N1O2 & $\mathrm{C} 23 \mathrm{H} 23 \mathrm{~N} 1 \mathrm{O} 2$ & 0.000956 & Basic N1 & $\mathrm{C} 37 \mathrm{H} 67 \mathrm{~N} 1$ & 0.000237 & Basic N2 & $\mathrm{C} 24 \mathrm{H} 28 \mathrm{~N} 2$ & $4.50 \mathrm{E}-05$ \\
\hline Non-basic N1O2 & $\mathrm{C} 24 \mathrm{H} 25 \mathrm{~N} 1 \mathrm{O} 2$ & 0.000493 & Basic N1 & $\mathrm{C} 38 \mathrm{H} 69 \mathrm{~N} 1$ & 0.000211 & Basic N2 & $\mathrm{C} 25 \mathrm{H} 30 \mathrm{~N} 2$ & $5.89 \mathrm{E}-05$ \\
\hline Non-basic N1O2 & $\mathrm{C} 25 \mathrm{H} 27 \mathrm{~N} 1 \mathrm{O} 2$ & 0.000296 & Basic N1 & $\mathrm{C} 39 \mathrm{H} 71 \mathrm{~N} 1$ & 0.000188 & Basic N2 & $\mathrm{C} 26 \mathrm{H} 32 \mathrm{~N} 2$ & $7.56 \mathrm{E}-05$ \\
\hline
\end{tabular}




\begin{tabular}{|c|c|c|c|c|c|c|c|c|}
\hline Class & Formula & Mass & Class & Formula & Mass & Class & Formula & Mass \\
\hline Non-basic N1O2 & $\mathrm{C} 26 \mathrm{H} 29 \mathrm{~N} 1 \mathrm{O} 2$ & 0.000166 & Basic N1 & $\mathrm{C} 40 \mathrm{H} 73 \mathrm{~N} 1$ & 0.00016 & Basic N2 & $\mathrm{C} 27 \mathrm{H} 34 \mathrm{~N} 2$ & $8.50 \mathrm{E}-05$ \\
\hline Non-basic N1O2 & $\mathrm{C} 27 \mathrm{H} 31 \mathrm{~N} 1 \mathrm{O} 2$ & 0.000142 & Basic N1 & $\mathrm{C} 41 \mathrm{H} 75 \mathrm{~N} 1$ & 0.000114 & Basic N2 & $\mathrm{C} 28 \mathrm{H} 36 \mathrm{~N} 2$ & 0.000118 \\
\hline Non-basic N1O2 & $\mathrm{C} 28 \mathrm{H} 33 \mathrm{~N} 1 \mathrm{O} 2$ & $9.66 \mathrm{E}-05$ & Basic N1 & $\mathrm{C} 42 \mathrm{H} 77 \mathrm{~N} 1$ & $7.81 \mathrm{E}-05$ & Basic N2 & $\mathrm{C} 29 \mathrm{H} 38 \mathrm{~N} 2$ & 7.63E-05 \\
\hline Non-basic N1O2 & $\mathrm{C} 29 \mathrm{H} 35 \mathrm{~N} 1 \mathrm{O} 2$ & $9.13 \mathrm{E}-05$ & Basic N1 & $\mathrm{C} 43 \mathrm{H} 79 \mathrm{~N} 1$ & $8.46 \mathrm{E}-05$ & Basic N2 & $\mathrm{C} 30 \mathrm{H} 40 \mathrm{~N} 2$ & 0.000125 \\
\hline Non-basic N1O2 & $\mathrm{C} 30 \mathrm{H} 37 \mathrm{~N} 1 \mathrm{O} 2$ & $8.07 \mathrm{E}-05$ & Basic N1 & $\mathrm{C} 44 \mathrm{H} 81 \mathrm{~N} 1$ & 4.13E-05 & Basic N2 & $\mathrm{C} 31 \mathrm{H} 42 \mathrm{~N} 2$ & $8.62 \mathrm{E}-05$ \\
\hline Non-basic N1O2 & $\mathrm{C} 31 \mathrm{H} 39 \mathrm{~N} 1 \mathrm{O} 2$ & $7.61 \mathrm{E}-05$ & Basic N1 & $\mathrm{C} 45 \mathrm{H} 83 \mathrm{~N} 1$ & $2.32 \mathrm{E}-05$ & Basic N2 & $\mathrm{C} 32 \mathrm{H} 44 \mathrm{~N} 2$ & $8.24 \mathrm{E}-05$ \\
\hline Non-basic N1O2 & $\mathrm{C} 32 \mathrm{H} 41 \mathrm{~N} 1 \mathrm{O} 2$ & $7.96 \mathrm{E}-05$ & Basic N1 & $\mathrm{C} 46 \mathrm{H} 85 \mathrm{~N} 1$ & $2.20 \mathrm{E}-05$ & Basic N2 & $\mathrm{C} 33 \mathrm{H} 46 \mathrm{~N} 2$ & 7.07E-05 \\
\hline Non-basic N1O2 & $\mathrm{C} 33 \mathrm{H} 43 \mathrm{~N} 1 \mathrm{O} 2$ & 0.000121 & Basic N1 & $\mathrm{C} 47 \mathrm{H} 87 \mathrm{~N} 1$ & $8.48 \mathrm{E}-06$ & Basic N2 & $\mathrm{C} 34 \mathrm{H} 48 \mathrm{~N} 2$ & $6.90 \mathrm{E}-05$ \\
\hline Non-basic N1O2 & $\mathrm{C} 34 \mathrm{H} 45 \mathrm{~N} 1 \mathrm{O} 2$ & 0.000177 & Basic N1 & $\mathrm{C} 48 \mathrm{H} 89 \mathrm{~N} 1$ & $8.72 \mathrm{E}-06$ & Basic N2 & $\mathrm{C} 35 \mathrm{H} 50 \mathrm{~N} 2$ & $5.72 \mathrm{E}-05$ \\
\hline Non-basic N1O2 & $\mathrm{C} 35 \mathrm{H} 47 \mathrm{~N} 1 \mathrm{O} 2$ & 0.000217 & Basic N1 & $\mathrm{C} 16 \mathrm{H} 23 \mathrm{~N} 1$ & $3.57 \mathrm{E}-06$ & Basic N2 & $\mathrm{C} 36 \mathrm{H} 52 \mathrm{~N} 2$ & 4.46E-05 \\
\hline Non-basic N1O2 & $\mathrm{C} 36 \mathrm{H} 49 \mathrm{~N} 1 \mathrm{O} 2$ & 0.000232 & Basic N1 & $\mathrm{C} 17 \mathrm{H} 25 \mathrm{~N} 1$ & $3.50 \mathrm{E}-06$ & Basic N2 & $\mathrm{C} 37 \mathrm{H} 54 \mathrm{~N} 2$ & $4.58 \mathrm{E}-05$ \\
\hline Non-basic N1O2 & C37H51N1O2 & 0.000186 & Basic N1 & $\mathrm{C} 18 \mathrm{H} 27 \mathrm{~N} 1$ & $1.32 \mathrm{E}-05$ & Basic N2 & $\mathrm{C} 38 \mathrm{H} 56 \mathrm{~N} 2$ & $3.83 \mathrm{E}-05$ \\
\hline Non-basic N1O2 & $\mathrm{C} 38 \mathrm{H} 53 \mathrm{~N} 1 \mathrm{O} 2$ & 0.000104 & Basic N1 & $\mathrm{C} 19 \mathrm{H} 29 \mathrm{~N} 1$ & $2.14 \mathrm{E}-05$ & Basic N2 & $\mathrm{C} 39 \mathrm{H} 58 \mathrm{~N} 2$ & $2.74 \mathrm{E}-05$ \\
\hline Non-basic $\mathrm{N} 1 \mathrm{O} 2$ & C39H55N1O2 & $7.31 \mathrm{E}-05$ & Basic N1 & $\mathrm{C} 20 \mathrm{H} 31 \mathrm{~N} 1$ & $3.71 \mathrm{E}-05$ & Basic N2 & $\mathrm{C} 40 \mathrm{H} 60 \mathrm{~N} 2$ & $2.24 \mathrm{E}-05$ \\
\hline Non-basic N1O2 & $\mathrm{C} 40 \mathrm{H} 57 \mathrm{~N} 1 \mathrm{O} 2$ & $5.08 \mathrm{E}-05$ & Basic N1 & $\mathrm{C} 21 \mathrm{H} 33 \mathrm{~N} 1$ & $4.24 \mathrm{E}-05$ & Basic N2 & $\mathrm{C} 41 \mathrm{H} 62 \mathrm{~N} 2$ & $2.31 \mathrm{E}-05$ \\
\hline Non-basic N1O2 & $\mathrm{C} 41 \mathrm{H} 59 \mathrm{~N} 1 \mathrm{O} 2$ & $3.44 \mathrm{E}-05$ & Basic N1 & $\mathrm{C} 22 \mathrm{H} 35 \mathrm{~N} 1$ & $9.93 \mathrm{E}-05$ & Basic N2 & $\mathrm{C} 42 \mathrm{H} 64 \mathrm{~N} 2$ & $1.82 \mathrm{E}-05$ \\
\hline Non-basic N1O2 & $\mathrm{C} 42 \mathrm{H} 61 \mathrm{~N} 1 \mathrm{O} 2$ & $2.00 \mathrm{E}-05$ & Basic N1 & $\mathrm{C} 23 \mathrm{H} 37 \mathrm{~N} 1$ & 0.000111 & Basic N2 & $\mathrm{C} 43 \mathrm{H} 66 \mathrm{~N} 2$ & 7.26E-06 \\
\hline Non-basic N1O2 & $\mathrm{C} 43 \mathrm{H} 63 \mathrm{~N} 1 \mathrm{O} 2$ & $1.29 \mathrm{E}-05$ & Basic N1 & $\mathrm{C} 24 \mathrm{H} 39 \mathrm{~N} 1$ & 0.000191 & Basic N2 & $\mathrm{C} 44 \mathrm{H} 68 \mathrm{~N} 2$ & $5.43 \mathrm{E}-06$ \\
\hline Non-basic N1O2 & $\mathrm{C} 44 \mathrm{H} 65 \mathrm{~N} 1 \mathrm{O} 2$ & $1.62 \mathrm{E}-05$ & Basic N1 & $\mathrm{C} 25 \mathrm{H} 41 \mathrm{~N} 1$ & 0.000231 & Basic N2 & $\mathrm{C} 45 \mathrm{H} 70 \mathrm{~N} 2$ & 4.63E-06 \\
\hline Non-basic N1O2 & $\mathrm{C} 45 \mathrm{H} 67 \mathrm{~N} 1 \mathrm{O} 2$ & $1.14 \mathrm{E}-05$ & Basic N1 & $\mathrm{C} 26 \mathrm{H} 43 \mathrm{~N} 1$ & 0.000278 & Basic N2 & C19H16N2 & $8.86 \mathrm{E}-07$ \\
\hline Non-basic N1O2 & C46H69N1O2 & $7.98 \mathrm{E}-06$ & Basic N1 & $\mathrm{C} 27 \mathrm{H} 45 \mathrm{~N} 1$ & 0.000298 & Basic N2 & $\mathrm{C} 20 \mathrm{H} 18 \mathrm{~N} 2$ & $2.56 \mathrm{E}-06$ \\
\hline Non-basic N1O2 & $\mathrm{C} 47 \mathrm{H} 71 \mathrm{~N} 1 \mathrm{O} 2$ & $9.52 \mathrm{E}-06$ & Basic N1 & $\mathrm{C} 28 \mathrm{H} 47 \mathrm{~N} 1$ & 0.000326 & Basic N2 & $\mathrm{C} 21 \mathrm{H} 20 \mathrm{~N} 2$ & $9.35 \mathrm{E}-06$ \\
\hline Non-basic N1O2 & $\mathrm{C} 48 \mathrm{H} 73 \mathrm{~N} 1 \mathrm{O} 2$ & $4.03 \mathrm{E}-06$ & Basic N1 & $\mathrm{C} 29 \mathrm{H} 49 \mathrm{~N} 1$ & 0.0004 & Basic N2 & $\mathrm{C} 22 \mathrm{H} 22 \mathrm{~N} 2$ & $2.47 \mathrm{E}-05$ \\
\hline Non-basic $\mathrm{N} 1 \mathrm{O} 2$ & $\mathrm{C} 49 \mathrm{H} 75 \mathrm{~N} 1 \mathrm{O} 2$ & $9.12 \mathrm{E}-06$ & Basic N1 & $\mathrm{C} 30 \mathrm{H} 51 \mathrm{~N} 1$ & 0.00041 & Basic N2 & $\mathrm{C} 23 \mathrm{H} 24 \mathrm{~N} 2$ & $5.02 \mathrm{E}-05$ \\
\hline Non-basic N1O2 & $\mathrm{C} 50 \mathrm{H} 77 \mathrm{~N} 1 \mathrm{O} 2$ & $2.25 \mathrm{E}-06$ & Basic N1 & $\mathrm{C} 31 \mathrm{H} 53 \mathrm{~N} 1$ & 0.000446 & Basic N2 & $\mathrm{C} 24 \mathrm{H} 26 \mathrm{~N} 2$ & 4.93E-05 \\
\hline Non-basic N1O2 & $\mathrm{C} 51 \mathrm{H} 79 \mathrm{~N} 1 \mathrm{O} 2$ & $4.78 \mathrm{E}-06$ & Basic N1 & $\mathrm{C} 32 \mathrm{H} 55 \mathrm{~N} 1$ & 0.000425 & Basic N2 & $\mathrm{C} 25 \mathrm{H} 28 \mathrm{~N} 2$ & $9.79 \mathrm{E}-05$ \\
\hline Non-basic N1O2 & $\mathrm{C} 18 \mathrm{H} 11 \mathrm{~N} 1 \mathrm{O} 2$ & $1.49 \mathrm{E}-05$ & Basic N1 & $\mathrm{C} 33 \mathrm{H} 57 \mathrm{~N} 1$ & 0.000456 & Basic N2 & $\mathrm{C} 26 \mathrm{H} 30 \mathrm{~N} 2$ & 8.03E-05 \\
\hline Non-basic N1O2 & C19H13N1O2 & 0.000172 & Basic N1 & $\mathrm{C} 34 \mathrm{H} 59 \mathrm{~N} 1$ & 0.000381 & Basic N2 & $\mathrm{C} 27 \mathrm{H} 32 \mathrm{~N} 2$ & 8.06E-05 \\
\hline Non-basic N1O2 & $\mathrm{C} 20 \mathrm{H} 15 \mathrm{~N} 1 \mathrm{O} 2$ & 0.000413 & Basic N1 & $\mathrm{C} 35 \mathrm{H} 61 \mathrm{~N} 1$ & 0.000427 & Basic N2 & $\mathrm{C} 28 \mathrm{H} 34 \mathrm{~N} 2$ & $9.36 \mathrm{E}-05$ \\
\hline Non-basic N1O2 & $\mathrm{C} 21 \mathrm{H} 17 \mathrm{~N} 1 \mathrm{O} 2$ & 0.000593 & Basic N1 & $\mathrm{C} 36 \mathrm{H} 63 \mathrm{~N} 1$ & 0.000412 & Basic N2 & $\mathrm{C} 29 \mathrm{H} 36 \mathrm{~N} 2$ & $9.79 \mathrm{E}-05$ \\
\hline Non-basic N1O2 & $\mathrm{C} 22 \mathrm{H} 19 \mathrm{~N} 1 \mathrm{O} 2$ & 0.000745 & Basic N1 & $\mathrm{C} 37 \mathrm{H} 65 \mathrm{~N} 1$ & 0.000326 & Basic N2 & $\mathrm{C} 30 \mathrm{H} 38 \mathrm{~N} 2$ & 0.000102 \\
\hline Non-basic N1O2 & $\mathrm{C} 23 \mathrm{H} 21 \mathrm{~N} 1 \mathrm{O} 2$ & 0.000726 & Basic N1 & $\mathrm{C} 38 \mathrm{H} 67 \mathrm{~N} 1$ & 0.000407 & Basic N2 & $\mathrm{C} 31 \mathrm{H} 40 \mathrm{~N} 2$ & $6.41 \mathrm{E}-05$ \\
\hline Non-basic N1O2 & $\mathrm{C} 24 \mathrm{H} 23 \mathrm{~N} 1 \mathrm{O} 2$ & 0.00056 & Basic N1 & $\mathrm{C} 39 \mathrm{H} 69 \mathrm{~N} 1$ & 0.000285 & Basic N2 & $\mathrm{C} 32 \mathrm{H} 42 \mathrm{~N} 2$ & $6.78 \mathrm{E}-05$ \\
\hline Non-basic N1O2 & $\mathrm{C} 25 \mathrm{H} 25 \mathrm{~N} 1 \mathrm{O} 2$ & 0.000347 & Basic N1 & $\mathrm{C} 40 \mathrm{H} 71 \mathrm{~N} 1$ & 0.000283 & Basic N2 & $\mathrm{C} 33 \mathrm{H} 44 \mathrm{~N} 2$ & $5.31 \mathrm{E}-05$ \\
\hline Non-basic N1O2 & $\mathrm{C} 26 \mathrm{H} 27 \mathrm{~N} 1 \mathrm{O} 2$ & 0.0002 & Basic N1 & $\mathrm{C} 41 \mathrm{H} 73 \mathrm{~N} 1$ & 0.000143 & Basic N2 & $\mathrm{C} 34 \mathrm{H} 46 \mathrm{~N} 2$ & $5.31 \mathrm{E}-05$ \\
\hline Non-basic N1O2 & $\mathrm{C} 27 \mathrm{H} 29 \mathrm{~N} 1 \mathrm{O} 2$ & 0.000134 & Basic N1 & $\mathrm{C} 42 \mathrm{H} 75 \mathrm{~N} 1$ & 0.000112 & Basic N2 & $\mathrm{C} 35 \mathrm{H} 48 \mathrm{~N} 2$ & $4.95 \mathrm{E}-05$ \\
\hline Non-basic N1O2 & $\mathrm{C} 28 \mathrm{H} 31 \mathrm{~N} 1 \mathrm{O} 2$ & $8.95 \mathrm{E}-05$ & Basic N1 & $\mathrm{C} 43 \mathrm{H} 77 \mathrm{~N} 1$ & $8.04 \mathrm{E}-05$ & Basic N2 & $\mathrm{C} 36 \mathrm{H} 50 \mathrm{~N} 2$ & $4.98 \mathrm{E}-05$ \\
\hline Non-basic N1O2 & $\mathrm{C} 29 \mathrm{H} 33 \mathrm{~N} 1 \mathrm{O} 2$ & $6.48 \mathrm{E}-05$ & Basic N1 & $\mathrm{C} 44 \mathrm{H} 79 \mathrm{~N} 1$ & $6.86 \mathrm{E}-05$ & Basic N2 & $\mathrm{C} 37 \mathrm{H} 52 \mathrm{~N} 2$ & $3.19 \mathrm{E}-05$ \\
\hline
\end{tabular}




\begin{tabular}{|c|c|c|c|c|c|c|c|c|}
\hline Class & Formula & Mass & Class & Formula & Mass & Class & Formula & Mass \\
\hline Non-basic N1O2 & $\mathrm{C} 30 \mathrm{H} 35 \mathrm{~N} 1 \mathrm{O} 2$ & $6.18 \mathrm{E}-05$ & Basic N1 & $\mathrm{C} 45 \mathrm{H} 81 \mathrm{~N} 1$ & $9.01 \mathrm{E}-05$ & Basic N2 & $\mathrm{C} 38 \mathrm{H} 54 \mathrm{~N} 2$ & $2.87 \mathrm{E}-05$ \\
\hline Non-basic N1O2 & $\mathrm{C} 31 \mathrm{H} 37 \mathrm{~N} 1 \mathrm{O} 2$ & $5.87 \mathrm{E}-05$ & Basic N1 & $\mathrm{C} 46 \mathrm{H} 83 \mathrm{~N} 1$ & $4.13 \mathrm{E}-05$ & Basic N2 & $\mathrm{C} 39 \mathrm{H} 56 \mathrm{~N} 2$ & $2.21 \mathrm{E}-05$ \\
\hline Non-basic N1O2 & $\mathrm{C} 32 \mathrm{H} 39 \mathrm{~N} 1 \mathrm{O} 2$ & $4.39 \mathrm{E}-05$ & Basic N1 & $\mathrm{C} 47 \mathrm{H} 85 \mathrm{~N} 1$ & $2.31 \mathrm{E}-05$ & Basic N2 & $\mathrm{C} 40 \mathrm{H} 58 \mathrm{~N} 2$ & $2.54 \mathrm{E}-05$ \\
\hline Non-basic N1O2 & $\mathrm{C} 33 \mathrm{H} 41 \mathrm{~N} 1 \mathrm{O} 2$ & $3.62 \mathrm{E}-05$ & Basic N1 & $\mathrm{C} 48 \mathrm{H} 87 \mathrm{~N} 1$ & $1.63 \mathrm{E}-05$ & Basic N2 & C41H60N2 & $9.58 \mathrm{E}-06$ \\
\hline Non-basic N1O2 & $\mathrm{C} 34 \mathrm{H} 43 \mathrm{~N} 1 \mathrm{O} 2$ & $4.96 \mathrm{E}-05$ & Basic N1 & $\mathrm{C} 49 \mathrm{H} 89 \mathrm{~N} 1$ & $1.61 \mathrm{E}-05$ & Basic N2 & $\mathrm{C} 42 \mathrm{H} 62 \mathrm{~N} 2$ & $7.75 \mathrm{E}-06$ \\
\hline Non-basic N1O2 & $\mathrm{C} 35 \mathrm{H} 45 \mathrm{~N} 1 \mathrm{O} 2$ & $5.01 \mathrm{E}-05$ & Basic N1 & C15H19N1 & $1.62 \mathrm{E}-07$ & Basic N2 & $\mathrm{C} 43 \mathrm{H} 64 \mathrm{~N} 2$ & $1.06 \mathrm{E}-05$ \\
\hline Non-basic N1O2 & $\mathrm{C} 36 \mathrm{H} 47 \mathrm{~N} 1 \mathrm{O} 2$ & $8.34 \mathrm{E}-05$ & Basic N1 & $\mathrm{C} 16 \mathrm{H} 21 \mathrm{~N} 1$ & $1.87 \mathrm{E}-06$ & Basic N2 & $\mathrm{C} 44 \mathrm{H} 66 \mathrm{~N} 2$ & $9.36 \mathrm{E}-06$ \\
\hline Non-basic N1O2 & $\mathrm{C} 37 \mathrm{H} 49 \mathrm{~N} 1 \mathrm{O} 2$ & $9.15 \mathrm{E}-05$ & Basic N1 & $\mathrm{C} 17 \mathrm{H} 23 \mathrm{~N} 1$ & $2.60 \mathrm{E}-06$ & Basic N2 & C19H14N2 & $6.95 \mathrm{E}-07$ \\
\hline Non-basic N1O2 & $\mathrm{C} 38 \mathrm{H} 51 \mathrm{~N} 1 \mathrm{O} 2$ & $8.82 \mathrm{E}-05$ & Basic N1 & $\mathrm{C} 18 \mathrm{H} 25 \mathrm{~N} 1$ & $1.33 \mathrm{E}-05$ & Basic N2 & $\mathrm{C} 20 \mathrm{H} 16 \mathrm{~N} 2$ & $1.26 \mathrm{E}-06$ \\
\hline Non-basic N1O2 & C39H53N1O2 & $8.45 \mathrm{E}-05$ & Basic N1 & $\mathrm{C} 19 \mathrm{H} 27 \mathrm{~N} 1$ & $2.86 \mathrm{E}-05$ & Basic N2 & $\mathrm{C} 21 \mathrm{H} 18 \mathrm{~N} 2$ & $1.06 \mathrm{E}-06$ \\
\hline Non-basic N1O2 & $\mathrm{C} 40 \mathrm{H} 55 \mathrm{~N} 1 \mathrm{O} 2$ & $4.86 \mathrm{E}-05$ & Basic N1 & $\mathrm{C} 20 \mathrm{H} 29 \mathrm{~N} 1$ & $8.86 \mathrm{E}-05$ & Basic N2 & $\mathrm{C} 22 \mathrm{H} 20 \mathrm{~N} 2$ & $2.56 \mathrm{E}-06$ \\
\hline Non-basic N1O2 & $\mathrm{C} 41 \mathrm{H} 57 \mathrm{~N} 1 \mathrm{O} 2$ & $4.36 \mathrm{E}-05$ & Basic N1 & $\mathrm{C} 21 \mathrm{H} 31 \mathrm{~N} 1$ & 0.000135 & Basic N2 & $\mathrm{C} 23 \mathrm{H} 22 \mathrm{~N} 2$ & $1.06 \mathrm{E}-05$ \\
\hline Non-basic N1O2 & $\mathrm{C} 42 \mathrm{H} 59 \mathrm{~N} 1 \mathrm{O} 2$ & $2.34 \mathrm{E}-05$ & Basic N1 & $\mathrm{C} 22 \mathrm{H} 33 \mathrm{~N} 1$ & 0.000186 & Basic N2 & $\mathrm{C} 24 \mathrm{H} 24 \mathrm{~N} 2$ & $3.05 \mathrm{E}-05$ \\
\hline Non-basic N1O2 & $\mathrm{C} 43 \mathrm{H} 61 \mathrm{~N} 1 \mathrm{O} 2$ & $1.31 \mathrm{E}-05$ & Basic N1 & $\mathrm{C} 23 \mathrm{H} 35 \mathrm{~N} 1$ & 0.000267 & Basic N2 & $\mathrm{C} 25 \mathrm{H} 26 \mathrm{~N} 2$ & 4.10E-05 \\
\hline Non-basic N1O2 & $\mathrm{C} 44 \mathrm{H} 63 \mathrm{~N} 1 \mathrm{O} 2$ & $9.67 \mathrm{E}-06$ & Basic N1 & $\mathrm{C} 24 \mathrm{H} 37 \mathrm{~N} 1$ & 0.000304 & Basic N2 & $\mathrm{C} 26 \mathrm{H} 28 \mathrm{~N} 2$ & $5.10 \mathrm{E}-05$ \\
\hline Non-basic N1O2 & $\mathrm{C} 45 \mathrm{H} 65 \mathrm{~N} 1 \mathrm{O} 2$ & $1.40 \mathrm{E}-05$ & Basic N1 & $\mathrm{C} 25 \mathrm{H} 39 \mathrm{~N} 1$ & 0.000307 & Basic N2 & $\mathrm{C} 27 \mathrm{H} 30 \mathrm{~N} 2$ & $5.51 \mathrm{E}-05$ \\
\hline Non-basic N1O2 & $\mathrm{C} 46 \mathrm{H} 67 \mathrm{~N} 1 \mathrm{O} 2$ & $1.14 \mathrm{E}-05$ & Basic N1 & $\mathrm{C} 26 \mathrm{H} 41 \mathrm{~N} 1$ & 0.000342 & Basic N2 & $\mathrm{C} 28 \mathrm{H} 32 \mathrm{~N} 2$ & $5.42 \mathrm{E}-05$ \\
\hline Non-basic N1O2 & $\mathrm{C} 47 \mathrm{H} 69 \mathrm{~N} 1 \mathrm{O} 2$ & $4.03 \mathrm{E}-06$ & Basic N1 & $\mathrm{C} 27 \mathrm{H} 43 \mathrm{~N} 1$ & 0.000396 & Basic N2 & $\mathrm{C} 29 \mathrm{H} 34 \mathrm{~N} 2$ & $6.02 \mathrm{E}-05$ \\
\hline Non-basic N1O2 & $\mathrm{C} 48 \mathrm{H} 71 \mathrm{~N} 1 \mathrm{O} 2$ & $1.03 \mathrm{E}-05$ & Basic N1 & $\mathrm{C} 28 \mathrm{H} 45 \mathrm{~N} 1$ & 0.000476 & Basic N2 & $\mathrm{C} 30 \mathrm{H} 36 \mathrm{~N} 2$ & 7.64E-05 \\
\hline Non-basic N1O2 & $\mathrm{C} 18 \mathrm{H} 9 \mathrm{~N} 1 \mathrm{O} 2$ & $3.56 \mathrm{E}-05$ & Basic N1 & $\mathrm{C} 29 \mathrm{H} 47 \mathrm{~N} 1$ & 0.000412 & Basic N2 & $\mathrm{C} 31 \mathrm{H} 38 \mathrm{~N} 2$ & $5.52 \mathrm{E}-05$ \\
\hline Non-basic N1O2 & C19H11N1O2 & $7.53 \mathrm{E}-05$ & Basic N1 & $\mathrm{C} 30 \mathrm{H} 49 \mathrm{~N} 1$ & 0.000477 & Basic N2 & $\mathrm{C} 32 \mathrm{H} 40 \mathrm{~N} 2$ & $5.06 \mathrm{E}-05$ \\
\hline Non-basic N1O2 & $\mathrm{C} 20 \mathrm{H} 13 \mathrm{~N} 1 \mathrm{O} 2$ & 0.000154 & Basic N1 & $\mathrm{C} 31 \mathrm{H} 51 \mathrm{~N} 1$ & 0.000443 & Basic N2 & $\mathrm{C} 33 \mathrm{H} 42 \mathrm{~N} 2$ & 7.01E-05 \\
\hline Non-basic N1O2 & $\mathrm{C} 21 \mathrm{H} 15 \mathrm{~N} 1 \mathrm{O} 2$ & 0.00063 & Basic N1 & $\mathrm{C} 32 \mathrm{H} 53 \mathrm{~N} 1$ & 0.00046 & Basic N2 & $\mathrm{C} 34 \mathrm{H} 44 \mathrm{~N} 2$ & 4.17E-05 \\
\hline Non-basic $\mathrm{N} 1 \mathrm{O} 2$ & $\mathrm{C} 22 \mathrm{H} 17 \mathrm{~N} 1 \mathrm{O} 2$ & 0.001626 & Basic N1 & $\mathrm{C} 33 \mathrm{H} 55 \mathrm{~N} 1$ & 0.000429 & Basic N2 & $\mathrm{C} 35 \mathrm{H} 46 \mathrm{~N} 2$ & $3.34 \mathrm{E}-05$ \\
\hline Non-basic N1O2 & $\mathrm{C} 23 \mathrm{H} 19 \mathrm{~N} 1 \mathrm{O} 2$ & 0.002202 & Basic N1 & $\mathrm{C} 34 \mathrm{H} 57 \mathrm{~N} 1$ & 0.000458 & Basic N2 & $\mathrm{C} 36 \mathrm{H} 48 \mathrm{~N} 2$ & $2.94 \mathrm{E}-05$ \\
\hline Non-basic N1O2 & $\mathrm{C} 24 \mathrm{H} 21 \mathrm{~N} 1 \mathrm{O} 2$ & 0.001808 & Basic N1 & $\mathrm{C} 35 \mathrm{H} 59 \mathrm{~N} 1$ & 0.000469 & Basic N2 & $\mathrm{C} 37 \mathrm{H} 50 \mathrm{~N} 2$ & $2.73 \mathrm{E}-05$ \\
\hline Non-basic N1O2 & $\mathrm{C} 25 \mathrm{H} 23 \mathrm{~N} 1 \mathrm{O} 2$ & 0.001106 & Basic N1 & $\mathrm{C} 36 \mathrm{H} 61 \mathrm{~N} 1$ & 0.000424 & Basic N2 & $\mathrm{C} 38 \mathrm{H} 52 \mathrm{~N} 2$ & $1.16 \mathrm{E}-05$ \\
\hline Non-basic N1O2 & $\mathrm{C} 26 \mathrm{H} 25 \mathrm{~N} 1 \mathrm{O} 2$ & 0.000566 & Basic N1 & $\mathrm{C} 37 \mathrm{H} 63 \mathrm{~N} 1$ & 0.000328 & Basic N2 & C39H54N2 & $1.19 \mathrm{E}-05$ \\
\hline Non-basic N1O2 & $\mathrm{C} 27 \mathrm{H} 27 \mathrm{~N} 1 \mathrm{O} 2$ & 0.000302 & Basic N1 & $\mathrm{C} 38 \mathrm{H} 65 \mathrm{~N} 1$ & 0.000304 & Basic N2 & $\mathrm{C} 40 \mathrm{H} 56 \mathrm{~N} 2$ & $1.61 \mathrm{E}-05$ \\
\hline Non-basic N1O2 & $\mathrm{C} 28 \mathrm{H} 29 \mathrm{~N} 1 \mathrm{O} 2$ & 0.000184 & Basic N1 & $\mathrm{C} 39 \mathrm{H} 67 \mathrm{~N} 1$ & 0.000225 & Basic N2 & $\mathrm{C} 41 \mathrm{H} 58 \mathrm{~N} 2$ & $3.19 \mathrm{E}-05$ \\
\hline Non-basic N1O2 & $\mathrm{C} 29 \mathrm{H} 31 \mathrm{~N} 1 \mathrm{O} 2$ & 0.000141 & Basic N1 & $\mathrm{C} 40 \mathrm{H} 69 \mathrm{~N} 1$ & 0.000204 & Basic N2 & $\mathrm{C} 42 \mathrm{H} 60 \mathrm{~N} 2$ & 7.42E-06 \\
\hline Non-basic N1O2 & $\mathrm{C} 30 \mathrm{H} 33 \mathrm{~N} 1 \mathrm{O} 2$ & $9.41 \mathrm{E}-05$ & Basic N1 & $\mathrm{C} 41 \mathrm{H} 71 \mathrm{~N} 1$ & 0.000161 & Basic N2 & $\mathrm{C} 43 \mathrm{H} 62 \mathrm{~N} 2$ & $3.97 \mathrm{E}-06$ \\
\hline Non-basic N1O2 & $\mathrm{C} 31 \mathrm{H} 35 \mathrm{~N} 1 \mathrm{O} 2$ & $6.37 \mathrm{E}-05$ & Basic N1 & $\mathrm{C} 42 \mathrm{H} 73 \mathrm{~N} 1$ & 0.000115 & Basic N2 & $\mathrm{C} 44 \mathrm{H} 64 \mathrm{~N} 2$ & $1.61 \mathrm{E}-06$ \\
\hline Non-basic N1O2 & $\mathrm{C} 32 \mathrm{H} 37 \mathrm{~N} 1 \mathrm{O} 2$ & $5.77 \mathrm{E}-05$ & Basic N1 & $\mathrm{C} 43 \mathrm{H} 75 \mathrm{~N} 1$ & $9.37 \mathrm{E}-05$ & Basic N2 & $\mathrm{C} 45 \mathrm{H} 66 \mathrm{~N} 2$ & $6.46 \mathrm{E}-06$ \\
\hline Non-basic N1O2 & $\mathrm{C} 33 \mathrm{H} 39 \mathrm{~N} 1 \mathrm{O} 2$ & $4.01 \mathrm{E}-05$ & Basic N1 & $\mathrm{C} 44 \mathrm{H} 77 \mathrm{~N} 1$ & $7.27 \mathrm{E}-05$ & Basic N2 & $\mathrm{C} 21 \mathrm{H} 16 \mathrm{~N} 2$ & $5.36 \mathrm{E}-07$ \\
\hline Non-basic N1O2 & $\mathrm{C} 34 \mathrm{H} 41 \mathrm{~N} 1 \mathrm{O} 2$ & $2.86 \mathrm{E}-05$ & Basic N1 & $\mathrm{C} 45 \mathrm{H} 79 \mathrm{~N} 1$ & $9.67 \mathrm{E}-05$ & Basic N2 & $\mathrm{C} 22 \mathrm{H} 18 \mathrm{~N} 2$ & $9.52 \mathrm{E}-07$ \\
\hline Non-basic N1O2 & $\mathrm{C} 35 \mathrm{H} 43 \mathrm{~N} 1 \mathrm{O} 2$ & $4.18 \mathrm{E}-05$ & Basic N1 & $\mathrm{C} 46 \mathrm{H} 81 \mathrm{~N} 1$ & $3.48 \mathrm{E}-05$ & Basic N2 & $\mathrm{C} 23 \mathrm{H} 20 \mathrm{~N} 2$ & $6.50 \mathrm{E}-06$ \\
\hline Non-basic N1O2 & $\mathrm{C} 36 \mathrm{H} 45 \mathrm{~N} 1 \mathrm{O} 2$ & $8.37 \mathrm{E}-05$ & Basic N1 & C47H83N1 & $3.12 \mathrm{E}-05$ & Basic N2 & $\mathrm{C} 24 \mathrm{H} 22 \mathrm{~N} 2$ & $6.35 \mathrm{E}-06$ \\
\hline
\end{tabular}




\begin{tabular}{|c|c|c|c|c|c|c|c|c|}
\hline Class & Formula & Mass & Class & Formula & Mass & Class & Formula & Mass \\
\hline Non-basic N1O2 & $\mathrm{C} 37 \mathrm{H} 47 \mathrm{~N} 1 \mathrm{O} 2$ & 0.000179 & Basic N1 & $\mathrm{C} 15 \mathrm{H} 17 \mathrm{~N} 1$ & $1.86 \mathrm{E}-05$ & Basic N2 & $\mathrm{C} 25 \mathrm{H} 24 \mathrm{~N} 2$ & $1.06 \mathrm{E}-05$ \\
\hline Non-basic N1O2 & $\mathrm{C} 38 \mathrm{H} 49 \mathrm{~N} 1 \mathrm{O} 2$ & 0.000233 & Basic N1 & $\mathrm{C} 16 \mathrm{H} 19 \mathrm{~N} 1$ & $8.35 \mathrm{E}-06$ & Basic N2 & $\mathrm{C} 26 \mathrm{H} 26 \mathrm{~N} 2$ & $1.73 \mathrm{E}-05$ \\
\hline Non-basic N1O2 & C39H51N1O2 & 0.000265 & Basic N1 & $\mathrm{C} 17 \mathrm{H} 21 \mathrm{~N} 1$ & $1.50 \mathrm{E}-05$ & Basic N2 & $\mathrm{C} 27 \mathrm{H} 28 \mathrm{~N} 2$ & $2.82 \mathrm{E}-05$ \\
\hline Non-basic N1O2 & $\mathrm{C} 40 \mathrm{H} 53 \mathrm{~N} 1 \mathrm{O} 2$ & 0.000229 & Basic N1 & $\mathrm{C} 18 \mathrm{H} 23 \mathrm{~N} 1$ & $2.94 \mathrm{E}-05$ & Basic N2 & $\mathrm{C} 28 \mathrm{H} 30 \mathrm{~N} 2$ & $2.96 \mathrm{E}-05$ \\
\hline Non-basic N1O2 & $\mathrm{C} 41 \mathrm{H} 55 \mathrm{~N} 1 \mathrm{O} 2$ & 0.000169 & Basic N1 & $\mathrm{C} 19 \mathrm{H} 25 \mathrm{~N} 1$ & 4.30E-05 & Basic N2 & $\mathrm{C} 29 \mathrm{H} 32 \mathrm{~N} 2$ & $3.33 \mathrm{E}-05$ \\
\hline Non-basic N1O2 & $\mathrm{C} 42 \mathrm{H} 57 \mathrm{~N} 1 \mathrm{O} 2$ & 0.000125 & Basic N1 & $\mathrm{C} 20 \mathrm{H} 27 \mathrm{~N} 1$ & $7.75 \mathrm{E}-05$ & Basic N2 & $\mathrm{C} 30 \mathrm{H} 34 \mathrm{~N} 2$ & $3.26 \mathrm{E}-05$ \\
\hline Non-basic N1O2 & $\mathrm{C} 43 \mathrm{H} 59 \mathrm{~N} 1 \mathrm{O} 2$ & $7.62 \mathrm{E}-05$ & Basic N1 & $\mathrm{C} 21 \mathrm{H} 29 \mathrm{~N} 1$ & 0.000149 & Basic N2 & $\mathrm{C} 31 \mathrm{H} 36 \mathrm{~N} 2$ & $3.16 \mathrm{E}-05$ \\
\hline Non-basic N1O2 & $\mathrm{C} 44 \mathrm{H} 61 \mathrm{~N} 1 \mathrm{O} 2$ & $5.69 \mathrm{E}-05$ & Basic N1 & $\mathrm{C} 22 \mathrm{H} 31 \mathrm{~N} 1$ & 0.000214 & Basic N2 & $\mathrm{C} 32 \mathrm{H} 38 \mathrm{~N} 2$ & $5.61 \mathrm{E}-05$ \\
\hline Non-basic N1O2 & $\mathrm{C} 45 \mathrm{H} 63 \mathrm{~N} 1 \mathrm{O} 2$ & $4.30 \mathrm{E}-05$ & Basic N1 & $\mathrm{C} 23 \mathrm{H} 33 \mathrm{~N} 1$ & 0.000323 & Basic N2 & $\mathrm{C} 33 \mathrm{H} 40 \mathrm{~N} 2$ & $3.74 \mathrm{E}-05$ \\
\hline Non-basic N1O2 & $\mathrm{C} 46 \mathrm{H} 65 \mathrm{~N} 1 \mathrm{O} 2$ & $4.57 \mathrm{E}-05$ & Basic N1 & $\mathrm{C} 24 \mathrm{H} 35 \mathrm{~N} 1$ & 0.00036 & Basic N2 & $\mathrm{C} 34 \mathrm{H} 42 \mathrm{~N} 2$ & $3.92 \mathrm{E}-05$ \\
\hline Non-basic N1O2 & $\mathrm{C} 47 \mathrm{H} 67 \mathrm{~N} 1 \mathrm{O} 2$ & $5.81 \mathrm{E}-05$ & Basic N1 & $\mathrm{C} 25 \mathrm{H} 37 \mathrm{~N} 1$ & 0.000454 & Basic N2 & $\mathrm{C} 35 \mathrm{H} 44 \mathrm{~N} 2$ & $2.95 \mathrm{E}-05$ \\
\hline Non-basic N1O2 & C48H69N1O2 & $2.75 \mathrm{E}-05$ & Basic N1 & $\mathrm{C} 26 \mathrm{H} 39 \mathrm{~N} 1$ & 0.000529 & Basic N2 & $\mathrm{C} 36 \mathrm{H} 46 \mathrm{~N} 2$ & $3.08 \mathrm{E}-05$ \\
\hline Non-basic N1O2 & $\mathrm{C} 49 \mathrm{H} 71 \mathrm{~N} 1 \mathrm{O} 2$ & $1.91 \mathrm{E}-05$ & Basic N1 & $\mathrm{C} 27 \mathrm{H} 41 \mathrm{~N} 1$ & 0.000546 & Basic N2 & $\mathrm{C} 37 \mathrm{H} 48 \mathrm{~N} 2$ & $1.94 \mathrm{E}-05$ \\
\hline Non-basic $\mathrm{N} 1 \mathrm{O} 2$ & $\mathrm{C} 50 \mathrm{H} 73 \mathrm{~N} 1 \mathrm{O} 2$ & $2.90 \mathrm{E}-05$ & Basic N1 & $\mathrm{C} 28 \mathrm{H} 43 \mathrm{~N} 1$ & 0.000616 & Basic N2 & $\mathrm{C} 38 \mathrm{H} 50 \mathrm{~N} 2$ & $1.30 \mathrm{E}-05$ \\
\hline Non-basic N1O2 & $\mathrm{C} 51 \mathrm{H} 75 \mathrm{~N} 1 \mathrm{O} 2$ & $9.98 \mathrm{E}-06$ & Basic N1 & $\mathrm{C} 29 \mathrm{H} 45 \mathrm{~N} 1$ & 0.000634 & Basic N2 & $\mathrm{C} 39 \mathrm{H} 52 \mathrm{~N} 2$ & $8.00 \mathrm{E}-06$ \\
\hline Non-basic N1O2 & $\mathrm{C} 52 \mathrm{H} 77 \mathrm{~N} 1 \mathrm{O} 2$ & $1.47 \mathrm{E}-05$ & Basic N1 & $\mathrm{C} 30 \mathrm{H} 47 \mathrm{~N} 1$ & 0.000656 & Basic N2 & $\mathrm{C} 40 \mathrm{H} 54 \mathrm{~N} 2$ & $1.04 \mathrm{E}-05$ \\
\hline Non-basic N1O2 & $\mathrm{C} 53 \mathrm{H} 79 \mathrm{~N} 1 \mathrm{O} 2$ & $1.32 \mathrm{E}-05$ & Basic N1 & $\mathrm{C} 31 \mathrm{H} 49 \mathrm{~N} 1$ & 0.000703 & Basic N2 & $\mathrm{C} 41 \mathrm{H} 56 \mathrm{~N} 2$ & $4.83 \mathrm{E}-06$ \\
\hline Non-basic N1O2 & $\mathrm{C} 54 \mathrm{H} 81 \mathrm{~N} 1 \mathrm{O} 2$ & $6.46 \mathrm{E}-06$ & Basic N1 & $\mathrm{C} 32 \mathrm{H} 51 \mathrm{~N} 1$ & 0.000967 & Basic N2 & $\mathrm{C} 22 \mathrm{H} 16 \mathrm{~N} 2$ & $2.04 \mathrm{E}-08$ \\
\hline Non-basic N1O2 & $\mathrm{C} 19 \mathrm{H} 9 \mathrm{~N} 1 \mathrm{O} 2$ & $1.33 \mathrm{E}-06$ & Basic N1 & $\mathrm{C} 33 \mathrm{H} 53 \mathrm{~N} 1$ & 0.001049 & Basic N2 & $\mathrm{C} 23 \mathrm{H} 18 \mathrm{~N} 2$ & $1.48 \mathrm{E}-06$ \\
\hline Non-basic N1O2 & $\mathrm{C} 20 \mathrm{H} 11 \mathrm{~N} 1 \mathrm{O} 2$ & $5.82 \mathrm{E}-05$ & Basic N1 & $\mathrm{C} 34 \mathrm{H} 55 \mathrm{~N} 1$ & 0.001282 & Basic N2 & $\mathrm{C} 24 \mathrm{H} 20 \mathrm{~N} 2$ & $2.83 \mathrm{E}-06$ \\
\hline Non-basic N1O2 & $\mathrm{C} 21 \mathrm{H} 13 \mathrm{~N} 1 \mathrm{O} 2$ & 0.000284 & Basic N1 & $\mathrm{C} 35 \mathrm{H} 57 \mathrm{~N} 1$ & 0.00083 & Basic N2 & $\mathrm{C} 25 \mathrm{H} 22 \mathrm{~N} 2$ & $6.37 \mathrm{E}-06$ \\
\hline Non-basic N1O2 & $\mathrm{C} 22 \mathrm{H} 15 \mathrm{~N} 1 \mathrm{O} 2$ & 0.000648 & Basic N1 & $\mathrm{C} 36 \mathrm{H} 59 \mathrm{~N} 1$ & 0.000654 & Basic N2 & $\mathrm{C} 26 \mathrm{H} 24 \mathrm{~N} 2$ & $1.00 \mathrm{E}-05$ \\
\hline Non-basic N1O2 & $\mathrm{C} 23 \mathrm{H} 17 \mathrm{~N} 1 \mathrm{O} 2$ & 0.00088 & Basic N1 & $\mathrm{C} 37 \mathrm{H} 61 \mathrm{~N} 1$ & 0.000413 & Basic N2 & $\mathrm{C} 27 \mathrm{H} 26 \mathrm{~N} 2$ & $1.68 \mathrm{E}-05$ \\
\hline Non-basic $\mathrm{N} 1 \mathrm{O} 2$ & $\mathrm{C} 24 \mathrm{H} 19 \mathrm{~N} 1 \mathrm{O} 2$ & 0.001049 & Basic N1 & $\mathrm{C} 38 \mathrm{H} 63 \mathrm{~N} 1$ & 0.000403 & Basic N2 & $\mathrm{C} 28 \mathrm{H} 28 \mathrm{~N} 2$ & $2.35 \mathrm{E}-05$ \\
\hline Non-basic N1O2 & $\mathrm{C} 25 \mathrm{H} 21 \mathrm{~N} 1 \mathrm{O} 2$ & 0.001038 & Basic N1 & $\mathrm{C} 39 \mathrm{H} 65 \mathrm{~N} 1$ & 0.000355 & Basic N2 & $\mathrm{C} 29 \mathrm{H} 30 \mathrm{~N} 2$ & $2.12 \mathrm{E}-05$ \\
\hline Non-basic N1O2 & $\mathrm{C} 26 \mathrm{H} 23 \mathrm{~N} 1 \mathrm{O} 2$ & 0.000756 & Basic N1 & $\mathrm{C} 40 \mathrm{H} 67 \mathrm{~N} 1$ & 0.000301 & Basic N2 & $\mathrm{C} 30 \mathrm{H} 32 \mathrm{~N} 2$ & $2.17 \mathrm{E}-05$ \\
\hline Non-basic N1O2 & $\mathrm{C} 27 \mathrm{H} 25 \mathrm{~N} 1 \mathrm{O} 2$ & 0.000495 & Basic N1 & $\mathrm{C} 41 \mathrm{H} 69 \mathrm{~N} 1$ & 0.000152 & Basic N2 & $\mathrm{C} 31 \mathrm{H} 34 \mathrm{~N} 2$ & $1.97 \mathrm{E}-05$ \\
\hline Non-basic N1O2 & $\mathrm{C} 28 \mathrm{H} 27 \mathrm{~N} 1 \mathrm{O} 2$ & 0.000277 & Basic N1 & $\mathrm{C} 42 \mathrm{H} 71 \mathrm{~N} 1$ & 0.000151 & Basic N2 & $\mathrm{C} 32 \mathrm{H} 36 \mathrm{~N} 2$ & $2.52 \mathrm{E}-05$ \\
\hline Non-basic N1O2 & $\mathrm{C} 29 \mathrm{H} 29 \mathrm{~N} 1 \mathrm{O} 2$ & 0.000159 & Basic N1 & $\mathrm{C} 43 \mathrm{H} 73 \mathrm{~N} 1$ & 0.000129 & Basic N2 & $\mathrm{C} 33 \mathrm{H} 38 \mathrm{~N} 2$ & $2.85 \mathrm{E}-05$ \\
\hline Non-basic N1O2 & $\mathrm{C} 30 \mathrm{H} 31 \mathrm{~N} 1 \mathrm{O} 2$ & 0.000104 & Basic N1 & $\mathrm{C} 44 \mathrm{H} 75 \mathrm{~N} 1$ & $9.51 \mathrm{E}-05$ & Basic N2 & $\mathrm{C} 34 \mathrm{H} 40 \mathrm{~N} 2$ & $2.17 \mathrm{E}-05$ \\
\hline Non-basic N1O2 & $\mathrm{C} 31 \mathrm{H} 33 \mathrm{~N} 1 \mathrm{O} 2$ & $6.34 \mathrm{E}-05$ & Basic N1 & $\mathrm{C} 45 \mathrm{H} 77 \mathrm{~N} 1$ & 4.44E-05 & Basic N2 & $\mathrm{C} 35 \mathrm{H} 42 \mathrm{~N} 2$ & $1.90 \mathrm{E}-05$ \\
\hline Non-basic N1O2 & $\mathrm{C} 32 \mathrm{H} 35 \mathrm{~N} 1 \mathrm{O} 2$ & $4.70 \mathrm{E}-05$ & Basic N1 & $\mathrm{C} 46 \mathrm{H} 79 \mathrm{~N} 1$ & $5.21 \mathrm{E}-05$ & Basic N2 & $\mathrm{C} 36 \mathrm{H} 44 \mathrm{~N} 2$ & $4.28 \mathrm{E}-05$ \\
\hline Non-basic N1O2 & $\mathrm{C} 33 \mathrm{H} 37 \mathrm{~N} 1 \mathrm{O} 2$ & $2.32 \mathrm{E}-05$ & Basic N1 & $\mathrm{C} 47 \mathrm{H} 81 \mathrm{~N} 1$ & $2.61 \mathrm{E}-05$ & Basic N2 & $\mathrm{C} 37 \mathrm{H} 46 \mathrm{~N} 2$ & $1.32 \mathrm{E}-05$ \\
\hline Non-basic N1O2 & $\mathrm{C} 34 \mathrm{H} 39 \mathrm{~N} 1 \mathrm{O} 2$ & $2.61 \mathrm{E}-05$ & Basic N1 & C48H83N1 & $2.27 \mathrm{E}-05$ & Basic N2 & $\mathrm{C} 38 \mathrm{H} 48 \mathrm{~N} 2$ & $1.74 \mathrm{E}-05$ \\
\hline Non-basic N1O2 & $\mathrm{C} 35 \mathrm{H} 41 \mathrm{~N} 1 \mathrm{O} 2$ & $1.96 \mathrm{E}-05$ & Basic N1 & $\mathrm{C} 49 \mathrm{H} 85 \mathrm{~N} 1$ & $1.98 \mathrm{E}-05$ & Basic N2 & $\mathrm{C} 39 \mathrm{H} 50 \mathrm{~N} 2$ & $1.35 \mathrm{E}-05$ \\
\hline Non-basic N1O2 & $\mathrm{C} 36 \mathrm{H} 43 \mathrm{~N} 1 \mathrm{O} 2$ & $4.32 \mathrm{E}-05$ & Basic N1 & $\mathrm{C} 50 \mathrm{H} 87 \mathrm{~N} 1$ & $3.93 \mathrm{E}-05$ & Basic N2 & $\mathrm{C} 40 \mathrm{H} 52 \mathrm{~N} 2$ & $1.38 \mathrm{E}-05$ \\
\hline Non-basic N1O2 & $\mathrm{C} 37 \mathrm{H} 45 \mathrm{~N} 1 \mathrm{O} 2$ & $5.35 \mathrm{E}-05$ & Basic N1 & $\mathrm{C} 16 \mathrm{H} 17 \mathrm{~N} 1$ & $3.41 \mathrm{E}-06$ & Basic N2 & $\mathrm{C} 41 \mathrm{H} 54 \mathrm{~N} 2$ & $2.53 \mathrm{E}-05$ \\
\hline Non-basic N1O2 & $\mathrm{C} 38 \mathrm{H} 47 \mathrm{~N} 1 \mathrm{O} 2$ & $8.39 \mathrm{E}-05$ & Basic N1 & C17H19N1 & $1.65 \mathrm{E}-05$ & Basic N2 & $\mathrm{C} 42 \mathrm{H} 56 \mathrm{~N} 2$ & $2.64 \mathrm{E}-05$ \\
\hline
\end{tabular}




\begin{tabular}{|c|c|c|c|c|c|c|c|c|}
\hline Class & Formula & Mass & Class & Formula & Mass & Class & Formula & Mass \\
\hline Non-basic N1O2 & C39H49N1O2 & 0.000119 & Basic N1 & $\mathrm{C} 18 \mathrm{H} 21 \mathrm{~N} 1$ & $3.11 \mathrm{E}-05$ & Basic N2 & $\mathrm{C} 43 \mathrm{H} 58 \mathrm{~N} 2$ & $6.36 \mathrm{E}-05$ \\
\hline Non-basic N1O2 & $\mathrm{C} 40 \mathrm{H} 51 \mathrm{~N} 1 \mathrm{O} 2$ & 0.000133 & Basic N1 & $\mathrm{C} 19 \mathrm{H} 23 \mathrm{~N} 1$ & 0.000102 & Basic N2 & $\mathrm{C} 44 \mathrm{H} 60 \mathrm{~N} 2$ & $3.84 \mathrm{E}-05$ \\
\hline Non-basic N1O2 & $\mathrm{C} 41 \mathrm{H} 53 \mathrm{~N} 1 \mathrm{O} 2$ & 0.000138 & Basic N1 & $\mathrm{C} 20 \mathrm{H} 25 \mathrm{~N} 1$ & 0.000133 & Basic N2 & $\mathrm{C} 45 \mathrm{H} 62 \mathrm{~N} 2$ & $5.89 \mathrm{E}-06$ \\
\hline Non-basic N1O2 & $\mathrm{C} 42 \mathrm{H} 55 \mathrm{~N} 1 \mathrm{O} 2$ & $9.54 \mathrm{E}-05$ & Basic N1 & $\mathrm{C} 21 \mathrm{H} 27 \mathrm{~N} 1$ & 0.000204 & Basic N2 & C46H64N2 & $2.51 \mathrm{E}-05$ \\
\hline Non-basic N1O2 & $\mathrm{C} 43 \mathrm{H} 57 \mathrm{~N} 1 \mathrm{O} 2$ & $7.34 \mathrm{E}-05$ & Basic N1 & $\mathrm{C} 22 \mathrm{H} 29 \mathrm{~N} 1$ & 0.000339 & Basic N2 & $\mathrm{C} 47 \mathrm{H} 66 \mathrm{~N} 2$ & $4.53 \mathrm{E}-05$ \\
\hline Non-basic N1O2 & C44H59N1O2 & 4.91E-05 & Basic N1 & $\mathrm{C} 23 \mathrm{H} 31 \mathrm{~N} 1$ & 0.000321 & Basic N2 & C48H68N2 & $7.69 \mathrm{E}-05$ \\
\hline Non-basic N1O2 & $\mathrm{C} 45 \mathrm{H} 61 \mathrm{~N} 1 \mathrm{O} 2$ & $3.35 \mathrm{E}-05$ & Basic N1 & $\mathrm{C} 24 \mathrm{H} 33 \mathrm{~N} 1$ & 0.000382 & Basic N2 & $\mathrm{C} 49 \mathrm{H} 70 \mathrm{~N} 2$ & $1.38 \mathrm{E}-05$ \\
\hline Non-basic N1O2 & $\mathrm{C} 46 \mathrm{H} 63 \mathrm{~N} 1 \mathrm{O} 2$ & $3.39 \mathrm{E}-05$ & Basic N1 & $\mathrm{C} 25 \mathrm{H} 35 \mathrm{~N} 1$ & 0.000449 & Basic N2 & $\mathrm{C} 23 \mathrm{H} 16 \mathrm{~N} 2$ & $1.51 \mathrm{E}-07$ \\
\hline Non-basic N1O2 & $\mathrm{C} 47 \mathrm{H} 65 \mathrm{~N} 1 \mathrm{O} 2$ & $1.89 \mathrm{E}-05$ & Basic N1 & $\mathrm{C} 26 \mathrm{H} 37 \mathrm{~N} 1$ & 0.000473 & Basic N2 & $\mathrm{C} 24 \mathrm{H} 18 \mathrm{~N} 2$ & $2.39 \mathrm{E}-07$ \\
\hline Non-basic N1O2 & $\mathrm{C} 48 \mathrm{H} 67 \mathrm{~N} 1 \mathrm{O} 2$ & $2.99 \mathrm{E}-05$ & Basic N1 & $\mathrm{C} 27 \mathrm{H} 39 \mathrm{~N} 1$ & 0.00064 & Basic N2 & $\mathrm{C} 25 \mathrm{H} 20 \mathrm{~N} 2$ & $1.70 \mathrm{E}-06$ \\
\hline Non-basic N1O2 & C49H69N1O2 & $3.28 \mathrm{E}-05$ & Basic N1 & $\mathrm{C} 28 \mathrm{H} 41 \mathrm{~N} 1$ & 0.000649 & Basic N2 & $\mathrm{C} 26 \mathrm{H} 22 \mathrm{~N} 2$ & $3.96 \mathrm{E}-06$ \\
\hline Non-basic N1O2 & C50H71N1O2 & $2.65 \mathrm{E}-05$ & Basic N1 & $\mathrm{C} 29 \mathrm{H} 43 \mathrm{~N} 1$ & 0.000941 & Basic N2 & $\mathrm{C} 27 \mathrm{H} 24 \mathrm{~N} 2$ & $6.79 \mathrm{E}-06$ \\
\hline Non-basic N1O2 & C51H73N1O2 & $1.69 \mathrm{E}-05$ & Basic N1 & $\mathrm{C} 30 \mathrm{H} 45 \mathrm{~N} 1$ & 0.000725 & Basic N2 & $\mathrm{C} 28 \mathrm{H} 26 \mathrm{~N} 2$ & $6.15 \mathrm{E}-06$ \\
\hline Non-basic N1O2 & $\mathrm{C} 52 \mathrm{H} 75 \mathrm{~N} 1 \mathrm{O} 2$ & $1.58 \mathrm{E}-05$ & Basic N1 & $\mathrm{C} 31 \mathrm{H} 47 \mathrm{~N} 1$ & 0.000841 & Basic N2 & $\mathrm{C} 29 \mathrm{H} 28 \mathrm{~N} 2$ & $6.96 \mathrm{E}-06$ \\
\hline Non-basic N1O2 & $\mathrm{C} 53 \mathrm{H} 77 \mathrm{~N} 1 \mathrm{O} 2$ & $1.97 \mathrm{E}-05$ & Basic N1 & $\mathrm{C} 32 \mathrm{H} 49 \mathrm{~N} 1$ & 0.000815 & Basic N2 & $\mathrm{C} 30 \mathrm{H} 30 \mathrm{~N} 2$ & $9.72 \mathrm{E}-06$ \\
\hline Non-basic N1O2 & $\mathrm{C} 20 \mathrm{H} 9 \mathrm{~N} 1 \mathrm{O} 2$ & $1.53 \mathrm{E}-05$ & Basic N1 & $\mathrm{C} 33 \mathrm{H} 51 \mathrm{~N} 1$ & 0.000809 & Basic N2 & $\mathrm{C} 31 \mathrm{H} 32 \mathrm{~N} 2$ & $6.34 \mathrm{E}-06$ \\
\hline Non-basic N1O2 & $\mathrm{C} 21 \mathrm{H} 11 \mathrm{~N} 1 \mathrm{O} 2$ & $5.58 \mathrm{E}-05$ & Basic N1 & $\mathrm{C} 34 \mathrm{H} 53 \mathrm{~N} 1$ & 0.000966 & Basic N2 & $\mathrm{C} 32 \mathrm{H} 34 \mathrm{~N} 2$ & $7.28 \mathrm{E}-06$ \\
\hline Non-basic N1O2 & $\mathrm{C} 22 \mathrm{H} 13 \mathrm{~N} 1 \mathrm{O} 2$ & 0.000101 & Basic N1 & $\mathrm{C} 35 \mathrm{H} 55 \mathrm{~N} 1$ & 0.001329 & Basic N2 & $\mathrm{C} 33 \mathrm{H} 36 \mathrm{~N} 2$ & $1.17 \mathrm{E}-05$ \\
\hline Non-basic N1O2 & $\mathrm{C} 23 \mathrm{H} 15 \mathrm{~N} 1 \mathrm{O} 2$ & 0.000179 & Basic N1 & $\mathrm{C} 36 \mathrm{H} 57 \mathrm{~N} 1$ & 0.000924 & Basic N2 & $\mathrm{C} 34 \mathrm{H} 38 \mathrm{~N} 2$ & $7.53 \mathrm{E}-06$ \\
\hline Non-basic N1O2 & $\mathrm{C} 24 \mathrm{H} 17 \mathrm{~N} 1 \mathrm{O} 2$ & 0.000365 & Basic N1 & $\mathrm{C} 37 \mathrm{H} 59 \mathrm{~N} 1$ & 0.000667 & Basic N2 & $\mathrm{C} 35 \mathrm{H} 40 \mathrm{~N} 2$ & 7.67E-06 \\
\hline Non-basic N1O2 & $\mathrm{C} 25 \mathrm{H} 19 \mathrm{~N} 1 \mathrm{O} 2$ & 0.000545 & Basic N1 & $\mathrm{C} 38 \mathrm{H} 61 \mathrm{~N} 1$ & 0.000565 & Basic N2 & $\mathrm{C} 36 \mathrm{H} 42 \mathrm{~N} 2$ & $9.17 \mathrm{E}-06$ \\
\hline Non-basic N1O2 & $\mathrm{C} 26 \mathrm{H} 21 \mathrm{~N} 1 \mathrm{O} 2$ & 0.000602 & Basic N1 & $\mathrm{C} 39 \mathrm{H} 63 \mathrm{~N} 1$ & 0.000442 & Basic N2 & $\mathrm{C} 37 \mathrm{H} 44 \mathrm{~N} 2$ & $1.19 \mathrm{E}-05$ \\
\hline Non-basic N1O2 & $\mathrm{C} 27 \mathrm{H} 23 \mathrm{~N} 1 \mathrm{O} 2$ & 0.000605 & Basic N1 & $\mathrm{C} 40 \mathrm{H} 65 \mathrm{~N} 1$ & 0.000402 & Basic N2 & $\mathrm{C} 38 \mathrm{H} 46 \mathrm{~N} 2$ & $1.09 \mathrm{E}-05$ \\
\hline Non-basic $\mathrm{N} 1 \mathrm{O} 2$ & $\mathrm{C} 28 \mathrm{H} 25 \mathrm{~N} 1 \mathrm{O} 2$ & 0.000593 & Basic N1 & $\mathrm{C} 41 \mathrm{H} 67 \mathrm{~N} 1$ & 0.000253 & Basic N2 & $\mathrm{C} 39 \mathrm{H} 48 \mathrm{~N} 2$ & $9.98 \mathrm{E}-06$ \\
\hline Non-basic N1O2 & $\mathrm{C} 29 \mathrm{H} 27 \mathrm{~N} 1 \mathrm{O} 2$ & 0.00041 & Basic N1 & $\mathrm{C} 42 \mathrm{H} 69 \mathrm{~N} 1$ & 0.000172 & Basic N2 & $\mathrm{C} 40 \mathrm{H} 50 \mathrm{~N} 2$ & $1.48 \mathrm{E}-05$ \\
\hline Non-basic N1O2 & $\mathrm{C} 30 \mathrm{H} 29 \mathrm{~N} 1 \mathrm{O} 2$ & 0.000205 & Basic N1 & $\mathrm{C} 43 \mathrm{H} 71 \mathrm{~N} 1$ & $8.74 \mathrm{E}-05$ & Basic N2 & $\mathrm{C} 41 \mathrm{H} 52 \mathrm{~N} 2$ & $1.93 \mathrm{E}-05$ \\
\hline Non-basic N1O2 & $\mathrm{C} 31 \mathrm{H} 31 \mathrm{~N} 1 \mathrm{O} 2$ & 0.000108 & Basic N1 & $\mathrm{C} 44 \mathrm{H} 73 \mathrm{~N} 1$ & $8.49 \mathrm{E}-05$ & Basic N2 & $\mathrm{C} 42 \mathrm{H} 54 \mathrm{~N} 2$ & $1.86 \mathrm{E}-05$ \\
\hline Non-basic N1O2 & $\mathrm{C} 32 \mathrm{H} 33 \mathrm{~N} 1 \mathrm{O} 2$ & $6.71 \mathrm{E}-05$ & Basic N1 & $\mathrm{C} 45 \mathrm{H} 75 \mathrm{~N} 1$ & $6.96 \mathrm{E}-05$ & Basic N2 & $\mathrm{C} 43 \mathrm{H} 56 \mathrm{~N} 2$ & $3.56 \mathrm{E}-05$ \\
\hline Non-basic N1O2 & $\mathrm{C} 33 \mathrm{H} 35 \mathrm{~N} 1 \mathrm{O} 2$ & $3.50 \mathrm{E}-05$ & Basic N1 & $\mathrm{C} 47 \mathrm{H} 79 \mathrm{~N} 1$ & $2.09 \mathrm{E}-05$ & Basic N2 & $\mathrm{C} 44 \mathrm{H} 58 \mathrm{~N} 2$ & $1.91 \mathrm{E}-05$ \\
\hline Non-basic N1O2 & $\mathrm{C} 34 \mathrm{H} 37 \mathrm{~N} 1 \mathrm{O} 2$ & $2.70 \mathrm{E}-05$ & Basic N1 & $\mathrm{C} 48 \mathrm{H} 81 \mathrm{~N} 1$ & $2.21 \mathrm{E}-05$ & Basic N2 & $\mathrm{C} 46 \mathrm{H} 62 \mathrm{~N} 2$ & $1.90 \mathrm{E}-05$ \\
\hline Non-basic N1O2 & $\mathrm{C} 35 \mathrm{H} 39 \mathrm{~N} 1 \mathrm{O} 2$ & $2.06 \mathrm{E}-05$ & Basic N1 & $\mathrm{C} 50 \mathrm{H} 85 \mathrm{~N} 1$ & $2.35 \mathrm{E}-05$ & Basic N2 & C47H64N2 & $1.28 \mathrm{E}-05$ \\
\hline Non-basic N1O2 & $\mathrm{C} 36 \mathrm{H} 41 \mathrm{~N} 1 \mathrm{O} 2$ & $2.37 \mathrm{E}-05$ & Basic N1 & $\mathrm{C} 51 \mathrm{H} 87 \mathrm{~N} 1$ & $1.05 \mathrm{E}-05$ & Basic N2 & C48H66N2 & $1.95 \mathrm{E}-05$ \\
\hline Non-basic N1O2 & $\mathrm{C} 37 \mathrm{H} 43 \mathrm{~N} 1 \mathrm{O} 2$ & $2.20 \mathrm{E}-05$ & Basic N1 & $\mathrm{C} 15 \mathrm{H} 13 \mathrm{~N} 1$ & $1.29 \mathrm{E}-06$ & Basic N2 & C49H68N2 & $6.73 \mathrm{E}-06$ \\
\hline Non-basic N1O2 & $\mathrm{C} 38 \mathrm{H} 45 \mathrm{~N} 1 \mathrm{O} 2$ & $1.92 \mathrm{E}-05$ & Basic N1 & $\mathrm{C} 16 \mathrm{H} 15 \mathrm{~N} 1$ & $1.89 \mathrm{E}-05$ & Basic N2 & $\mathrm{C} 50 \mathrm{H} 70 \mathrm{~N} 2$ & 4.46E-06 \\
\hline Non-basic N1O2 & $\mathrm{C} 39 \mathrm{H} 47 \mathrm{~N} 1 \mathrm{O} 2$ & $4.54 \mathrm{E}-05$ & Basic N1 & $\mathrm{C} 17 \mathrm{H} 17 \mathrm{~N} 1$ & $2.48 \mathrm{E}-05$ & Basic N2 & $\mathrm{C} 51 \mathrm{H} 72 \mathrm{~N} 2$ & $8.79 \mathrm{E}-06$ \\
\hline Non-basic N1O2 & $\mathrm{C} 40 \mathrm{H} 49 \mathrm{~N} 1 \mathrm{O} 2$ & $8.03 \mathrm{E}-05$ & Basic N1 & $\mathrm{C} 18 \mathrm{H} 19 \mathrm{~N} 1$ & $6.23 \mathrm{E}-05$ & Basic N2 & $\mathrm{C} 52 \mathrm{H} 74 \mathrm{~N} 2$ & $8.39 \mathrm{E}-06$ \\
\hline Non-basic N1O2 & $\mathrm{C} 41 \mathrm{H} 51 \mathrm{~N} 1 \mathrm{O} 2$ & $8.34 \mathrm{E}-05$ & Basic N1 & $\mathrm{C} 19 \mathrm{H} 21 \mathrm{~N} 1$ & $9.73 \mathrm{E}-05$ & Basic N2 & $\mathrm{C} 53 \mathrm{H} 76 \mathrm{~N} 2$ & $2.66 \mathrm{E}-05$ \\
\hline Non-basic N1O2 & $\mathrm{C} 42 \mathrm{H} 53 \mathrm{~N} 1 \mathrm{O} 2$ & $9.28 \mathrm{E}-05$ & Basic N1 & $\mathrm{C} 20 \mathrm{H} 23 \mathrm{~N} 1$ & 0.000152 & Basic N2 & $\mathrm{C} 54 \mathrm{H} 78 \mathrm{~N} 2$ & $2.60 \mathrm{E}-06$ \\
\hline
\end{tabular}




\begin{tabular}{|c|c|c|c|c|c|c|c|c|}
\hline Class & Formula & Mass & Class & Formula & Mass & Class & Formula & Mass \\
\hline Non-basic N1O2 & $\mathrm{C} 43 \mathrm{H} 55 \mathrm{~N} 1 \mathrm{O} 2$ & $7.82 \mathrm{E}-05$ & Basic N1 & $\mathrm{C} 21 \mathrm{H} 25 \mathrm{~N} 1$ & 0.0002 & Basic N2 & $\mathrm{C} 55 \mathrm{H} 80 \mathrm{~N} 2$ & $2.52 \mathrm{E}-06$ \\
\hline Non-basic N1O2 & $\mathrm{C} 44 \mathrm{H} 57 \mathrm{~N} 1 \mathrm{O} 2$ & $6.21 \mathrm{E}-05$ & Basic N1 & $\mathrm{C} 22 \mathrm{H} 27 \mathrm{~N} 1$ & 0.000314 & Basic N2 & $\mathrm{C} 26 \mathrm{H} 20 \mathrm{~N} 2$ & 4.14E-07 \\
\hline Non-basic N1O2 & $\mathrm{C} 45 \mathrm{H} 59 \mathrm{~N} 1 \mathrm{O} 2$ & $3.38 \mathrm{E}-05$ & Basic N1 & $\mathrm{C} 23 \mathrm{H} 29 \mathrm{~N} 1$ & 0.000431 & Basic N2 & $\mathrm{C} 27 \mathrm{H} 22 \mathrm{~N} 2$ & $1.72 \mathrm{E}-06$ \\
\hline Non-basic N1O2 & C46H61N1O2 & $2.99 \mathrm{E}-05$ & Basic N1 & $\mathrm{C} 24 \mathrm{H} 31 \mathrm{~N} 1$ & 0.00047 & Basic N2 & $\mathrm{C} 28 \mathrm{H} 24 \mathrm{~N} 2$ & $2.97 \mathrm{E}-07$ \\
\hline Non-basic N1O2 & C47H63N1O2 & $1.96 \mathrm{E}-05$ & Basic N1 & $\mathrm{C} 25 \mathrm{H} 33 \mathrm{~N} 1$ & 0.000574 & Basic N2 & $\mathrm{C} 29 \mathrm{H} 26 \mathrm{~N} 2$ & $2.21 \mathrm{E}-06$ \\
\hline Non-basic N1O2 & C48H65N1O2 & $3.14 \mathrm{E}-05$ & Basic N1 & $\mathrm{C} 26 \mathrm{H} 35 \mathrm{~N} 1$ & 0.000601 & Basic N2 & $\mathrm{C} 30 \mathrm{H} 28 \mathrm{~N} 2$ & $2.23 \mathrm{E}-06$ \\
\hline Non-basic N1O2 & $\mathrm{C} 49 \mathrm{H} 67 \mathrm{~N} 1 \mathrm{O} 2$ & $1.95 \mathrm{E}-05$ & Basic N1 & $\mathrm{C} 27 \mathrm{H} 37 \mathrm{~N} 1$ & 0.000606 & Basic N2 & $\mathrm{C} 31 \mathrm{H} 30 \mathrm{~N} 2$ & $2.43 \mathrm{E}-06$ \\
\hline Non-basic N1O2 & $\mathrm{C} 50 \mathrm{H} 69 \mathrm{~N} 1 \mathrm{O} 2$ & $2.20 \mathrm{E}-05$ & Basic N1 & $\mathrm{C} 28 \mathrm{H} 39 \mathrm{~N} 1$ & 0.000629 & Basic N2 & $\mathrm{C} 32 \mathrm{H} 32 \mathrm{~N} 2$ & $3.33 \mathrm{E}-06$ \\
\hline Non-basic N1O2 & $\mathrm{C} 51 \mathrm{H} 71 \mathrm{~N} 1 \mathrm{O} 2$ & $2.48 \mathrm{E}-05$ & Basic N1 & $\mathrm{C} 29 \mathrm{H} 41 \mathrm{~N} 1$ & 0.000683 & Basic N2 & $\mathrm{C} 33 \mathrm{H} 34 \mathrm{~N} 2$ & $2.37 \mathrm{E}-06$ \\
\hline Non-basic N1O2 & C52H73N1O2 & $1.69 \mathrm{E}-05$ & Basic N1 & $\mathrm{C} 30 \mathrm{H} 43 \mathrm{~N} 1$ & 0.000824 & Basic N2 & $\mathrm{C} 34 \mathrm{H} 36 \mathrm{~N} 2$ & $6.84 \mathrm{E}-07$ \\
\hline Non-basic N1O2 & $\mathrm{C} 53 \mathrm{H} 75 \mathrm{~N} 1 \mathrm{O} 2$ & $1.46 \mathrm{E}-05$ & Basic N1 & $\mathrm{C} 31 \mathrm{H} 45 \mathrm{~N} 1$ & 0.000794 & Basic N2 & $\mathrm{C} 35 \mathrm{H} 38 \mathrm{~N} 2$ & $2.37 \mathrm{E}-05$ \\
\hline Non-basic N1O2 & C54H77N1O2 & $9.10 \mathrm{E}-06$ & Basic N1 & $\mathrm{C} 32 \mathrm{H} 47 \mathrm{~N} 1$ & 0.000717 & Basic N2 & $\mathrm{C} 36 \mathrm{H} 40 \mathrm{~N} 2$ & $2.28 \mathrm{E}-06$ \\
\hline Non-basic N1O2 & $\mathrm{C} 22 \mathrm{H} 11 \mathrm{~N} 1 \mathrm{O} 2$ & $1.37 \mathrm{E}-05$ & Basic N1 & $\mathrm{C} 33 \mathrm{H} 49 \mathrm{~N} 1$ & 0.000755 & Basic N2 & $\mathrm{C} 37 \mathrm{H} 42 \mathrm{~N} 2$ & $2.70 \mathrm{E}-06$ \\
\hline Non-basic $\mathrm{N} 1 \mathrm{O} 2$ & $\mathrm{C} 23 \mathrm{H} 13 \mathrm{~N} 1 \mathrm{O} 2$ & $5.19 \mathrm{E}-05$ & Basic N1 & $\mathrm{C} 34 \mathrm{H} 51 \mathrm{~N} 1$ & 0.000601 & Basic N2 & $\mathrm{C} 38 \mathrm{H} 44 \mathrm{~N} 2$ & 4.47E-06 \\
\hline Non-basic N1O2 & $\mathrm{C} 24 \mathrm{H} 15 \mathrm{~N} 1 \mathrm{O} 2$ & $9.78 \mathrm{E}-05$ & Basic N1 & $\mathrm{C} 35 \mathrm{H} 53 \mathrm{~N} 1$ & 0.000523 & Basic N2 & $\mathrm{C} 39 \mathrm{H} 46 \mathrm{~N} 2$ & $5.84 \mathrm{E}-06$ \\
\hline Non-basic N1O2 & $\mathrm{C} 25 \mathrm{H} 17 \mathrm{~N} 1 \mathrm{O} 2$ & 0.000215 & Basic N1 & $\mathrm{C} 36 \mathrm{H} 55 \mathrm{~N} 1$ & 0.000577 & Basic N2 & $\mathrm{C} 40 \mathrm{H} 48 \mathrm{~N} 2$ & $5.55 \mathrm{E}-06$ \\
\hline Non-basic N1O2 & $\mathrm{C} 26 \mathrm{H} 19 \mathrm{~N} 1 \mathrm{O} 2$ & 0.000371 & Basic N1 & $\mathrm{C} 37 \mathrm{H} 57 \mathrm{~N} 1$ & 0.000494 & Basic N2 & $\mathrm{C} 41 \mathrm{H} 50 \mathrm{~N} 2$ & $1.34 \mathrm{E}-06$ \\
\hline Non-basic N1O2 & $\mathrm{C} 27 \mathrm{H} 21 \mathrm{~N} 1 \mathrm{O} 2$ & 0.000451 & Basic N1 & $\mathrm{C} 38 \mathrm{H} 59 \mathrm{~N} 1$ & 0.000479 & Basic N2 & $\mathrm{C} 42 \mathrm{H} 52 \mathrm{~N} 2$ & 7.47E-06 \\
\hline Non-basic N1O2 & $\mathrm{C} 28 \mathrm{H} 23 \mathrm{~N} 1 \mathrm{O} 2$ & 0.000387 & Basic N1 & $\mathrm{C} 39 \mathrm{H} 61 \mathrm{~N} 1$ & 0.000362 & Basic N2 & $\mathrm{C} 43 \mathrm{H} 54 \mathrm{~N} 2$ & $9.84 \mathrm{E}-06$ \\
\hline Non-basic N1O2 & $\mathrm{C} 29 \mathrm{H} 25 \mathrm{~N} 1 \mathrm{O} 2$ & 0.000271 & Basic N1 & $\mathrm{C} 40 \mathrm{H} 63 \mathrm{~N} 1$ & 0.000303 & Basic N2 & $\mathrm{C} 44 \mathrm{H} 56 \mathrm{~N} 2$ & $5.17 \mathrm{E}-06$ \\
\hline Non-basic N1O2 & $\mathrm{C} 30 \mathrm{H} 27 \mathrm{~N} 1 \mathrm{O} 2$ & 0.000191 & Basic N1 & $\mathrm{C} 41 \mathrm{H} 65 \mathrm{~N} 1$ & 0.000191 & Basic N2 & $\mathrm{C} 46 \mathrm{H} 60 \mathrm{~N} 2$ & $1.03 \mathrm{E}-05$ \\
\hline Non-basic N1O2 & $\mathrm{C} 31 \mathrm{H} 29 \mathrm{~N} 1 \mathrm{O} 2$ & 0.000136 & Basic N1 & $\mathrm{C} 42 \mathrm{H} 67 \mathrm{~N} 1$ & 0.000146 & Basic N2 & $\mathrm{C} 47 \mathrm{H} 62 \mathrm{~N} 2$ & $7.81 \mathrm{E}-06$ \\
\hline Non-basic N1O2 & $\mathrm{C} 32 \mathrm{H} 31 \mathrm{~N} 1 \mathrm{O} 2$ & $7.92 \mathrm{E}-05$ & Basic N1 & $\mathrm{C} 43 \mathrm{H} 69 \mathrm{~N} 1$ & 0.000118 & Basic N2 & $\mathrm{C} 49 \mathrm{H} 66 \mathrm{~N} 2$ & $2.30 \mathrm{E}-06$ \\
\hline Non-basic $\mathrm{N} 1 \mathrm{O} 2$ & C33H33N1O2 & $4.85 \mathrm{E}-05$ & Basic N1 & $\mathrm{C} 44 \mathrm{H} 71 \mathrm{~N} 1$ & $8.00 \mathrm{E}-05$ & Basic N2 & $\mathrm{C} 50 \mathrm{H} 68 \mathrm{~N} 2$ & 4.42E-06 \\
\hline Non-basic N1O2 & $\mathrm{C} 34 \mathrm{H} 35 \mathrm{~N} 1 \mathrm{O} 2$ & $3.07 \mathrm{E}-05$ & Basic N1 & $\mathrm{C} 45 \mathrm{H} 73 \mathrm{~N} 1$ & $6.35 \mathrm{E}-05$ & Basic N2 & $\mathrm{C} 26 \mathrm{H} 18 \mathrm{~N} 2$ & $2.24 \mathrm{E}-06$ \\
\hline Non-basic N1O2 & $\mathrm{C} 35 \mathrm{H} 37 \mathrm{~N} 1 \mathrm{O} 2$ & $1.18 \mathrm{E}-05$ & Basic N1 & $\mathrm{C} 46 \mathrm{H} 75 \mathrm{~N} 1$ & $7.41 \mathrm{E}-05$ & Basic N2 & $\mathrm{C} 27 \mathrm{H} 20 \mathrm{~N} 2$ & $8.89 \mathrm{E}-06$ \\
\hline Non-basic N1O2 & $\mathrm{C} 36 \mathrm{H} 39 \mathrm{~N} 1 \mathrm{O} 2$ & 4.92E-06 & Basic N1 & $\mathrm{C} 47 \mathrm{H} 77 \mathrm{~N} 1$ & $5.83 \mathrm{E}-05$ & Basic N2 & $\mathrm{C} 28 \mathrm{H} 22 \mathrm{~N} 2$ & $1.88 \mathrm{E}-05$ \\
\hline Non-basic N1O2 & $\mathrm{C} 37 \mathrm{H} 41 \mathrm{~N} 1 \mathrm{O} 2$ & $1.67 \mathrm{E}-05$ & Basic N1 & C49H81N1 & $1.74 \mathrm{E}-05$ & Basic N2 & $\mathrm{C} 29 \mathrm{H} 24 \mathrm{~N} 2$ & $2.24 \mathrm{E}-05$ \\
\hline Non-basic N1O2 & $\mathrm{C} 38 \mathrm{H} 43 \mathrm{~N} 1 \mathrm{O} 2$ & $3.70 \mathrm{E}-06$ & Basic N1 & $\mathrm{C} 50 \mathrm{H} 83 \mathrm{~N} 1$ & $1.28 \mathrm{E}-05$ & Basic N2 & $\mathrm{C} 30 \mathrm{H} 26 \mathrm{~N} 2$ & $8.46 \mathrm{E}-06$ \\
\hline Non-basic N1O2 & $\mathrm{C} 39 \mathrm{H} 45 \mathrm{~N} 1 \mathrm{O} 2$ & $1.64 \mathrm{E}-05$ & Basic N1 & $\mathrm{C} 51 \mathrm{H} 85 \mathrm{~N} 1$ & $9.67 \mathrm{E}-06$ & Basic N2 & $\mathrm{C} 31 \mathrm{H} 28 \mathrm{~N} 2$ & $3.97 \mathrm{E}-06$ \\
\hline Non-basic N1O2 & $\mathrm{C} 40 \mathrm{H} 47 \mathrm{~N} 1 \mathrm{O} 2$ & $2.46 \mathrm{E}-05$ & Basic N1 & $\mathrm{C} 52 \mathrm{H} 87 \mathrm{~N} 1$ & $1.08 \mathrm{E}-05$ & Basic N2 & $\mathrm{C} 32 \mathrm{H} 30 \mathrm{~N} 2$ & $7.90 \mathrm{E}-07$ \\
\hline Non-basic N1O2 & $\mathrm{C} 41 \mathrm{H} 49 \mathrm{~N} 1 \mathrm{O} 2$ & $3.91 \mathrm{E}-05$ & Basic N1 & $\mathrm{C} 53 \mathrm{H} 89 \mathrm{~N} 1$ & $2.63 \mathrm{E}-06$ & Basic N2 & $\mathrm{C} 33 \mathrm{H} 32 \mathrm{~N} 2$ & $2.92 \mathrm{E}-06$ \\
\hline Non-basic N1O2 & $\mathrm{C} 42 \mathrm{H} 51 \mathrm{~N} 1 \mathrm{O} 2$ & $5.78 \mathrm{E}-05$ & Basic N1 & $\mathrm{C} 54 \mathrm{H} 91 \mathrm{~N} 1$ & 4.22E-06 & Basic N2 & $\mathrm{C} 34 \mathrm{H} 34 \mathrm{~N} 2$ & $3.23 \mathrm{E}-06$ \\
\hline Non-basic N1O2 & $\mathrm{C} 43 \mathrm{H} 53 \mathrm{~N} 1 \mathrm{O} 2$ & $7.60 \mathrm{E}-05$ & Basic N1 & $\mathrm{C} 16 \mathrm{H} 13 \mathrm{~N} 1$ & $4.14 \mathrm{E}-06$ & Basic N2 & $\mathrm{C} 35 \mathrm{H} 36 \mathrm{~N} 2$ & $8.30 \mathrm{E}-07$ \\
\hline Non-basic N1O2 & $\mathrm{C} 44 \mathrm{H} 55 \mathrm{~N} 1 \mathrm{O} 2$ & $6.94 \mathrm{E}-05$ & Basic N1 & $\mathrm{C} 17 \mathrm{H} 15 \mathrm{~N} 1$ & $2.17 \mathrm{E}-05$ & Basic N2 & $\mathrm{C} 36 \mathrm{H} 38 \mathrm{~N} 2$ & $1.54 \mathrm{E}-06$ \\
\hline Non-basic N1O2 & $\mathrm{C} 45 \mathrm{H} 57 \mathrm{~N} 1 \mathrm{O} 2$ & $3.08 \mathrm{E}-05$ & Basic N1 & $\mathrm{C} 18 \mathrm{H} 17 \mathrm{~N} 1$ & $6.85 \mathrm{E}-05$ & Basic N2 & $\mathrm{C} 37 \mathrm{H} 40 \mathrm{~N} 2$ & $3.46 \mathrm{E}-06$ \\
\hline Non-basic N1O2 & $\mathrm{C} 46 \mathrm{H} 59 \mathrm{~N} 1 \mathrm{O} 2$ & $2.99 \mathrm{E}-05$ & Basic N1 & C19H19N1 & 0.000118 & Basic N2 & $\mathrm{C} 38 \mathrm{H} 42 \mathrm{~N} 2$ & $3.11 \mathrm{E}-06$ \\
\hline Non-basic N1O2 & $\mathrm{C} 47 \mathrm{H} 61 \mathrm{~N} 1 \mathrm{O} 2$ & $2.38 \mathrm{E}-05$ & Basic N1 & $\mathrm{C} 20 \mathrm{H} 21 \mathrm{~N} 1$ & 0.000167 & Basic N2 & $\mathrm{C} 39 \mathrm{H} 44 \mathrm{~N} 2$ & $4.28 \mathrm{E}-06$ \\
\hline
\end{tabular}




\begin{tabular}{|c|c|c|c|c|c|c|c|c|}
\hline Class & Formula & Mass & Class & Formula & Mass & Class & Formula & Mass \\
\hline Non-basic N1O2 & C48H63N1O2 & $1.98 \mathrm{E}-05$ & Basic N1 & $\mathrm{C} 21 \mathrm{H} 23 \mathrm{~N} 1$ & 0.000192 & Basic N2 & $\mathrm{C} 40 \mathrm{H} 46 \mathrm{~N} 2$ & $2.38 \mathrm{E}-06$ \\
\hline Non-basic N1O2 & $\mathrm{C} 49 \mathrm{H} 65 \mathrm{~N} 1 \mathrm{O} 2$ & $1.56 \mathrm{E}-05$ & Basic N1 & $\mathrm{C} 22 \mathrm{H} 25 \mathrm{~N} 1$ & 0.000243 & Basic N2 & $\mathrm{C} 41 \mathrm{H} 48 \mathrm{~N} 2$ & $2.18 \mathrm{E}-06$ \\
\hline Non-basic N1O2 & $\mathrm{C} 50 \mathrm{H} 67 \mathrm{~N} 1 \mathrm{O} 2$ & $1.61 \mathrm{E}-05$ & Basic N1 & $\mathrm{C} 23 \mathrm{H} 27 \mathrm{~N} 1$ & 0.000365 & Basic N2 & $\mathrm{C} 42 \mathrm{H} 50 \mathrm{~N} 2$ & $6.75 \mathrm{E}-06$ \\
\hline Non-basic N1O2 & C51H69N1O2 & $1.59 \mathrm{E}-05$ & Basic N1 & $\mathrm{C} 24 \mathrm{H} 29 \mathrm{~N} 1$ & 0.000438 & Basic N2 & $\mathrm{C} 43 \mathrm{H} 52 \mathrm{~N} 2$ & 4.27E-06 \\
\hline Non-basic N1O2 & $\mathrm{C} 52 \mathrm{H} 71 \mathrm{~N} 1 \mathrm{O} 2$ & 4.76E-06 & Basic N1 & $\mathrm{C} 25 \mathrm{H} 31 \mathrm{~N} 1$ & 0.000524 & Basic N2 & $\mathrm{C} 44 \mathrm{H} 54 \mathrm{~N} 2$ & 4.37E-06 \\
\hline Non-basic N1O2 & C54H75N1O2 & 4.89E-06 & Basic N1 & $\mathrm{C} 26 \mathrm{H} 33 \mathrm{~N} 1$ & 0.000655 & Basic N2 & $\mathrm{C} 45 \mathrm{H} 56 \mathrm{~N} 2$ & 4.64E-06 \\
\hline Non-basic N1O2 & $\mathrm{C} 24 \mathrm{H} 13 \mathrm{~N} 1 \mathrm{O} 2$ & $2.02 \mathrm{E}-05$ & Basic N1 & $\mathrm{C} 27 \mathrm{H} 35 \mathrm{~N} 1$ & 0.000668 & Basic N2 & $\mathrm{C} 46 \mathrm{H} 58 \mathrm{~N} 2$ & $5.56 \mathrm{E}-06$ \\
\hline Non-basic N1O2 & $\mathrm{C} 25 \mathrm{H} 15 \mathrm{~N} 1 \mathrm{O} 2$ & $9.62 \mathrm{E}-05$ & Basic N1 & $\mathrm{C} 28 \mathrm{H} 37 \mathrm{~N} 1$ & 0.000712 & Basic N2 & $\mathrm{C} 47 \mathrm{H} 60 \mathrm{~N} 2$ & $2.28 \mathrm{E}-06$ \\
\hline Non-basic N1O2 & $\mathrm{C} 26 \mathrm{H} 17 \mathrm{~N} 1 \mathrm{O} 2$ & 0.000206 & Basic N1 & $\mathrm{C} 29 \mathrm{H} 39 \mathrm{~N} 1$ & 0.000735 & Basic N2 & $\mathrm{C} 29 \mathrm{H} 22 \mathrm{~N} 2$ & $1.63 \mathrm{E}-07$ \\
\hline Non-basic N1O2 & $\mathrm{C} 27 \mathrm{H} 19 \mathrm{~N} 1 \mathrm{O} 2$ & 0.000212 & Basic N1 & $\mathrm{C} 30 \mathrm{H} 41 \mathrm{~N} 1$ & 0.000657 & Basic N2 & $\mathrm{C} 30 \mathrm{H} 24 \mathrm{~N} 2$ & $1.65 \mathrm{E}-06$ \\
\hline Non-basic N1O2 & $\mathrm{C} 28 \mathrm{H} 21 \mathrm{~N} 1 \mathrm{O} 2$ & 0.000235 & Basic N1 & $\mathrm{C} 31 \mathrm{H} 43 \mathrm{~N} 1$ & 0.000706 & Basic N2 & $\mathrm{C} 31 \mathrm{H} 26 \mathrm{~N} 2$ & $2.74 \mathrm{E}-06$ \\
\hline Non-basic N1O2 & $\mathrm{C} 29 \mathrm{H} 23 \mathrm{~N} 1 \mathrm{O} 2$ & 0.000193 & Basic N1 & $\mathrm{C} 32 \mathrm{H} 45 \mathrm{~N} 1$ & 0.000591 & Basic N2 & $\mathrm{C} 32 \mathrm{H} 28 \mathrm{~N} 2$ & $3.73 \mathrm{E}-06$ \\
\hline Non-basic N1O2 & $\mathrm{C} 30 \mathrm{H} 25 \mathrm{~N} 1 \mathrm{O} 2$ & 0.00016 & Basic N1 & $\mathrm{C} 33 \mathrm{H} 47 \mathrm{~N} 1$ & 0.000563 & Basic N2 & $\mathrm{C} 33 \mathrm{H} 30 \mathrm{~N} 2$ & $9.92 \mathrm{E}-07$ \\
\hline Non-basic $\mathrm{N} 1 \mathrm{O} 2$ & $\mathrm{C} 31 \mathrm{H} 27 \mathrm{~N} 1 \mathrm{O} 2$ & 0.000125 & Basic N1 & $\mathrm{C} 34 \mathrm{H} 49 \mathrm{~N} 1$ & 0.000501 & Basic N2 & $\mathrm{C} 34 \mathrm{H} 32 \mathrm{~N} 2$ & $1.73 \mathrm{E}-06$ \\
\hline Non-basic N1O2 & $\mathrm{C} 32 \mathrm{H} 29 \mathrm{~N} 1 \mathrm{O} 2$ & 7.89E-05 & Basic N1 & $\mathrm{C} 35 \mathrm{H} 51 \mathrm{~N} 1$ & 0.000445 & Basic N2 & $\mathrm{C} 35 \mathrm{H} 34 \mathrm{~N} 2$ & $7.83 \mathrm{E}-07$ \\
\hline Non-basic N1O2 & $\mathrm{C} 33 \mathrm{H} 31 \mathrm{~N} 1 \mathrm{O} 2$ & $4.66 \mathrm{E}-05$ & Basic N1 & $\mathrm{C} 36 \mathrm{H} 53 \mathrm{~N} 1$ & 0.000456 & Basic N2 & $\mathrm{C} 36 \mathrm{H} 36 \mathrm{~N} 2$ & $1.24 \mathrm{E}-06$ \\
\hline Non-basic N1O2 & $\mathrm{C} 34 \mathrm{H} 33 \mathrm{~N} 1 \mathrm{O} 2$ & $2.61 \mathrm{E}-05$ & Basic N1 & $\mathrm{C} 37 \mathrm{H} 55 \mathrm{~N} 1$ & 0.000289 & Basic N2 & $\mathrm{C} 37 \mathrm{H} 38 \mathrm{~N} 2$ & $2.98 \mathrm{E}-07$ \\
\hline Non-basic N1O2 & $\mathrm{C} 35 \mathrm{H} 35 \mathrm{~N} 1 \mathrm{O} 2$ & $1.80 \mathrm{E}-05$ & Basic N1 & $\mathrm{C} 38 \mathrm{H} 57 \mathrm{~N} 1$ & 0.000292 & Basic N2 & $\mathrm{C} 38 \mathrm{H} 40 \mathrm{~N} 2$ & $1.61 \mathrm{E}-06$ \\
\hline Non-basic N1O2 & $\mathrm{C} 36 \mathrm{H} 37 \mathrm{~N} 1 \mathrm{O} 2$ & $1.17 \mathrm{E}-05$ & Basic N1 & $\mathrm{C} 39 \mathrm{H} 59 \mathrm{~N} 1$ & 0.000282 & Basic N2 & $\mathrm{C} 39 \mathrm{H} 42 \mathrm{~N} 2$ & $6.49 \mathrm{E}-07$ \\
\hline Non-basic N1O2 & $\mathrm{C} 38 \mathrm{H} 41 \mathrm{~N} 1 \mathrm{O} 2$ & $2.97 \mathrm{E}-06$ & Basic N1 & $\mathrm{C} 40 \mathrm{H} 61 \mathrm{~N} 1$ & 0.000256 & Basic N2 & $\mathrm{C} 29 \mathrm{H} 20 \mathrm{~N} 2$ & $3.58 \mathrm{E}-07$ \\
\hline Non-basic N1O2 & $\mathrm{C} 41 \mathrm{H} 47 \mathrm{~N} 1 \mathrm{O} 2$ & $1.88 \mathrm{E}-06$ & Basic N1 & $\mathrm{C} 41 \mathrm{H} 63 \mathrm{~N} 1$ & 0.000171 & Basic N2 & $\mathrm{C} 30 \mathrm{H} 22 \mathrm{~N} 2$ & $3.18 \mathrm{E}-06$ \\
\hline Non-basic N1O2 & $\mathrm{C} 42 \mathrm{H} 49 \mathrm{~N} 1 \mathrm{O} 2$ & 7.54E-06 & Basic N1 & $\mathrm{C} 42 \mathrm{H} 65 \mathrm{~N} 1$ & 0.000105 & Basic N2 & $\mathrm{C} 31 \mathrm{H} 24 \mathrm{~N} 2$ & $2.45 \mathrm{E}-06$ \\
\hline Non-basic N1O2 & $\mathrm{C} 43 \mathrm{H} 51 \mathrm{~N} 1 \mathrm{O} 2$ & 7.01E-06 & Basic N1 & $\mathrm{C} 43 \mathrm{H} 67 \mathrm{~N} 1$ & 0.000115 & Basic N2 & $\mathrm{C} 32 \mathrm{H} 26 \mathrm{~N} 2$ & $1.15 \mathrm{E}-06$ \\
\hline Non-basic $\mathrm{N} 1 \mathrm{O} 2$ & C44H53N1O2 & $2.89 \mathrm{E}-05$ & Basic N1 & C44H69N1 & $6.79 \mathrm{E}-05$ & Basic N2 & $\mathrm{C} 33 \mathrm{H} 28 \mathrm{~N} 2$ & $3.11 \mathrm{E}-07$ \\
\hline Non-basic N1O2 & $\mathrm{C} 45 \mathrm{H} 55 \mathrm{~N} 1 \mathrm{O} 2$ & $3.59 \mathrm{E}-05$ & Basic N1 & $\mathrm{C} 45 \mathrm{H} 71 \mathrm{~N} 1$ & 0.0001 & Netural O1 & $\mathrm{C} 17 \mathrm{H} 10 \mathrm{O} 1$ & $4.25 \mathrm{E}-05$ \\
\hline Non-basic N1O2 & $\mathrm{C} 46 \mathrm{H} 57 \mathrm{~N} 1 \mathrm{O} 2$ & $1.84 \mathrm{E}-05$ & Basic N1 & $\mathrm{C} 46 \mathrm{H} 73 \mathrm{~N} 1$ & $3.55 \mathrm{E}-05$ & Netural O1 & $\mathrm{C} 18 \mathrm{H} 12 \mathrm{O} 1$ & 0.002653 \\
\hline Non-basic N1O2 & $\mathrm{C} 47 \mathrm{H} 59 \mathrm{~N} 1 \mathrm{O} 2$ & $1.73 \mathrm{E}-05$ & Basic N1 & $\mathrm{C} 47 \mathrm{H} 75 \mathrm{~N} 1$ & $2.86 \mathrm{E}-05$ & Netural O1 & C19H14O1 & 0.008614 \\
\hline Non-basic N1O2 & $\mathrm{C} 48 \mathrm{H} 61 \mathrm{~N} 1 \mathrm{O} 2$ & $1.17 \mathrm{E}-05$ & Basic N1 & $\mathrm{C} 48 \mathrm{H} 77 \mathrm{~N} 1$ & $1.27 \mathrm{E}-05$ & Netural O1 & $\mathrm{C} 20 \mathrm{H} 16 \mathrm{O} 1$ & 0.010804 \\
\hline Non-basic N1O2 & $\mathrm{C} 49 \mathrm{H} 63 \mathrm{~N} 1 \mathrm{O} 2$ & $8.04 \mathrm{E}-06$ & Basic N1 & $\mathrm{C} 49 \mathrm{H} 79 \mathrm{~N} 1$ & $1.02 \mathrm{E}-05$ & Netural O1 & $\mathrm{C} 21 \mathrm{H} 18 \mathrm{O} 1$ & 0.002781 \\
\hline Non-basic N1O2 & C50H65N1O2 & $3.72 \mathrm{E}-06$ & Basic N1 & $\mathrm{C} 50 \mathrm{H} 81 \mathrm{~N} 1$ & $8.44 \mathrm{E}-06$ & Netural O1 & $\mathrm{C} 22 \mathrm{H} 20 \mathrm{O} 1$ & 0.001128 \\
\hline Non-basic N1O2 & C51H67N1O2 & $1.69 \mathrm{E}-05$ & Basic N1 & C15H9N1 & $3.18 \mathrm{E}-07$ & Netural O1 & $\mathrm{C} 23 \mathrm{H} 22 \mathrm{O} 1$ & 0.000667 \\
\hline Non-basic N1O2 & C52H69N1O2 & $6.58 \mathrm{E}-06$ & Basic N1 & $\mathrm{C} 16 \mathrm{H} 11 \mathrm{~N} 1$ & $1.51 \mathrm{E}-06$ & Netural O1 & $\mathrm{C} 19 \mathrm{H} 12 \mathrm{O} 1$ & 0.001076 \\
\hline Non-basic N1O2 & C53H71N1O2 & $9.46 \mathrm{E}-06$ & Basic N1 & $\mathrm{C} 17 \mathrm{H} 13 \mathrm{~N} 1$ & $1.36 \mathrm{E}-05$ & Netural O1 & $\mathrm{C} 20 \mathrm{H} 14 \mathrm{O} 1$ & 7.23E-05 \\
\hline Non-basic N1O2 & C54H73N1O2 & $1.14 \mathrm{E}-05$ & Basic N1 & $\mathrm{C} 18 \mathrm{H} 15 \mathrm{~N} 1$ & $4.12 \mathrm{E}-05$ & Netural O1 & $\mathrm{C} 21 \mathrm{H} 16 \mathrm{O} 1$ & 0.000839 \\
\hline Non-basic N1O2 & $\mathrm{C} 55 \mathrm{H} 75 \mathrm{~N} 1 \mathrm{O} 2$ & $6.81 \mathrm{E}-06$ & Basic N1 & $\mathrm{C} 19 \mathrm{H} 17 \mathrm{~N} 1$ & 0.000101 & Netural O1 & $\mathrm{C} 22 \mathrm{H} 18 \mathrm{O} 1$ & 0.000948 \\
\hline Non-basic N1O2 & $\mathrm{C} 24 \mathrm{H} 11 \mathrm{~N} 1 \mathrm{O} 2$ & $6.80 \mathrm{E}-06$ & Basic N1 & $\mathrm{C} 20 \mathrm{H} 19 \mathrm{~N} 1$ & 0.000161 & Netural O1 & $\mathrm{C} 23 \mathrm{H} 20 \mathrm{O} 1$ & 0.000826 \\
\hline Non-basic N1O2 & $\mathrm{C} 25 \mathrm{H} 13 \mathrm{~N} 1 \mathrm{O} 2$ & $2.81 \mathrm{E}-05$ & Basic N1 & $\mathrm{C} 21 \mathrm{H} 21 \mathrm{~N} 1$ & 0.000223 & Netural O1 & $\mathrm{C} 24 \mathrm{H} 22 \mathrm{O} 1$ & 0.000273 \\
\hline Non-basic N1O2 & $\mathrm{C} 26 \mathrm{H} 15 \mathrm{~N} 1 \mathrm{O} 2$ & $4.18 \mathrm{E}-05$ & Basic N1 & $\mathrm{C} 22 \mathrm{H} 23 \mathrm{~N} 1$ & 0.000306 & Netural O1 & C19H10O1 & 0.001023 \\
\hline
\end{tabular}




\begin{tabular}{|c|c|c|c|c|c|c|c|c|}
\hline Class & Formula & Mass & Class & Formula & Mass & Class & Formula & Mass \\
\hline Non-basic N1O2 & $\mathrm{C} 27 \mathrm{H} 17 \mathrm{~N} 1 \mathrm{O} 2$ & $5.88 \mathrm{E}-05$ & Basic N1 & $\mathrm{C} 23 \mathrm{H} 25 \mathrm{~N} 1$ & 0.000391 & Netural O1 & $\mathrm{C} 20 \mathrm{H} 12 \mathrm{O} 1$ & 0.004408 \\
\hline Non-basic N1O2 & $\mathrm{C} 28 \mathrm{H} 19 \mathrm{~N} 1 \mathrm{O} 2$ & $8.59 \mathrm{E}-05$ & Basic N1 & $\mathrm{C} 24 \mathrm{H} 27 \mathrm{~N} 1$ & 0.000424 & Netural O1 & $\mathrm{C} 21 \mathrm{H} 14 \mathrm{O} 1$ & 0.007081 \\
\hline Non-basic N1O2 & $\mathrm{C} 29 \mathrm{H} 21 \mathrm{~N} 1 \mathrm{O} 2$ & 0.000136 & Basic N1 & $\mathrm{C} 25 \mathrm{H} 29 \mathrm{~N} 1$ & 0.000493 & Netural O1 & $\mathrm{C} 22 \mathrm{H} 16 \mathrm{O} 1$ & 0.002747 \\
\hline Non-basic N1O2 & $\mathrm{C} 30 \mathrm{H} 23 \mathrm{~N} 1 \mathrm{O} 2$ & 0.000151 & Basic N1 & $\mathrm{C} 26 \mathrm{H} 31 \mathrm{~N} 1$ & 0.000632 & Netural O1 & $\mathrm{C} 23 \mathrm{H} 18 \mathrm{O} 1$ & 0.001356 \\
\hline Non-basic N1O2 & $\mathrm{C} 31 \mathrm{H} 25 \mathrm{~N} 1 \mathrm{O} 2$ & 0.000189 & Basic N1 & $\mathrm{C} 27 \mathrm{H} 33 \mathrm{~N} 1$ & 0.00052 & Netural O1 & $\mathrm{C} 24 \mathrm{H} 20 \mathrm{O} 1$ & 0.000249 \\
\hline Non-basic N1O2 & $\mathrm{C} 32 \mathrm{H} 27 \mathrm{~N} 1 \mathrm{O} 2$ & 0.000171 & Basic N1 & $\mathrm{C} 28 \mathrm{H} 35 \mathrm{~N} 1$ & 0.000532 & Netural O1 & $\mathrm{C} 25 \mathrm{H} 22 \mathrm{O} 1$ & 8.77E-05 \\
\hline Non-basic N1O2 & $\mathrm{C} 33 \mathrm{H} 29 \mathrm{~N} 1 \mathrm{O} 2$ & 0.000121 & Basic N1 & $\mathrm{C} 29 \mathrm{H} 37 \mathrm{~N} 1$ & 0.000656 & Netural O1 & $\mathrm{C} 26 \mathrm{H} 24 \mathrm{O} 1$ & 0.000267 \\
\hline Non-basic N1O2 & $\mathrm{C} 34 \mathrm{H} 31 \mathrm{~N} 1 \mathrm{O} 2$ & $6.91 \mathrm{E}-05$ & Basic N1 & $\mathrm{C} 30 \mathrm{H} 39 \mathrm{~N} 1$ & 0.000665 & Netural O1 & $\mathrm{C} 27 \mathrm{H} 26 \mathrm{O} 1$ & 4.62E-05 \\
\hline Non-basic N1O2 & $\mathrm{C} 35 \mathrm{H} 33 \mathrm{~N} 1 \mathrm{O} 2$ & $4.39 \mathrm{E}-05$ & Basic N1 & $\mathrm{C} 31 \mathrm{H} 41 \mathrm{~N} 1$ & 0.000676 & Netural O1 & $\mathrm{C} 22 \mathrm{H} 14 \mathrm{O} 1$ & 0.002269 \\
\hline Non-basic N1O2 & $\mathrm{C} 36 \mathrm{H} 35 \mathrm{~N} 1 \mathrm{O} 2$ & $1.11 \mathrm{E}-05$ & Basic N1 & $\mathrm{C} 32 \mathrm{H} 43 \mathrm{~N} 1$ & 0.000507 & Netural O1 & $\mathrm{C} 23 \mathrm{H} 16 \mathrm{O} 1$ & 0.003593 \\
\hline Non-basic N1O2 & $\mathrm{C} 37 \mathrm{H} 37 \mathrm{~N} 1 \mathrm{O} 2$ & $4.65 \mathrm{E}-06$ & Basic N1 & $\mathrm{C} 33 \mathrm{H} 45 \mathrm{~N} 1$ & 0.000487 & Netural O1 & $\mathrm{C} 24 \mathrm{H} 18 \mathrm{O} 1$ & 0.003418 \\
\hline Non-basic N1O2 & $\mathrm{C} 38 \mathrm{H} 39 \mathrm{~N} 1 \mathrm{O} 2$ & $5.43 \mathrm{E}-06$ & Basic N1 & $\mathrm{C} 34 \mathrm{H} 47 \mathrm{~N} 1$ & 0.000396 & Netural O1 & $\mathrm{C} 25 \mathrm{H} 20 \mathrm{O} 1$ & 0.001589 \\
\hline Non-basic N1O2 & $\mathrm{C} 39 \mathrm{H} 41 \mathrm{~N} 1 \mathrm{O} 2$ & $2.17 \mathrm{E}-06$ & Basic N1 & $\mathrm{C} 35 \mathrm{H} 49 \mathrm{~N} 1$ & 0.000362 & Netural O1 & $\mathrm{C} 26 \mathrm{H} 22 \mathrm{O} 1$ & 0.000545 \\
\hline Non-basic N1O2 & $\mathrm{C} 40 \mathrm{H} 43 \mathrm{~N} 1 \mathrm{O} 2$ & $2.23 \mathrm{E}-06$ & Basic N1 & $\mathrm{C} 36 \mathrm{H} 51 \mathrm{~N} 1$ & 0.000353 & Netural O1 & $\mathrm{C} 27 \mathrm{H} 24 \mathrm{O} 1$ & 0.000312 \\
\hline Non-basic N1O2 & $\mathrm{C} 41 \mathrm{H} 45 \mathrm{~N} 1 \mathrm{O} 2$ & $2.28 \mathrm{E}-06$ & Basic N1 & $\mathrm{C} 37 \mathrm{H} 53 \mathrm{~N} 1$ & 0.000294 & Netural O1 & $\mathrm{C} 24 \mathrm{H} 16 \mathrm{O} 1$ & 0.00031 \\
\hline Non-basic N1O2 & $\mathrm{C} 42 \mathrm{H} 47 \mathrm{~N} 1 \mathrm{O} 2$ & $2.01 \mathrm{E}-06$ & Basic N1 & $\mathrm{C} 38 \mathrm{H} 55 \mathrm{~N} 1$ & 0.000248 & Netural O1 & $\mathrm{C} 25 \mathrm{H} 18 \mathrm{O} 1$ & 0.000195 \\
\hline Non-basic N1O2 & $\mathrm{C} 43 \mathrm{H} 49 \mathrm{~N} 1 \mathrm{O} 2$ & $3.27 \mathrm{E}-06$ & Basic N1 & $\mathrm{C} 39 \mathrm{H} 57 \mathrm{~N} 1$ & 0.000209 & Netural O1 & $\mathrm{C} 26 \mathrm{H} 20 \mathrm{O} 1$ & 0.000203 \\
\hline Non-basic N1O2 & $\mathrm{C} 44 \mathrm{H} 51 \mathrm{~N} 1 \mathrm{O} 2$ & $1.15 \mathrm{E}-05$ & Basic N1 & $\mathrm{C} 40 \mathrm{H} 59 \mathrm{~N} 1$ & 0.000205 & Netural O1 & $\mathrm{C} 27 \mathrm{H} 22 \mathrm{O} 1$ & 0.000572 \\
\hline Non-basic N1O2 & $\mathrm{C} 45 \mathrm{H} 53 \mathrm{~N} 1 \mathrm{O} 2$ & $1.55 \mathrm{E}-05$ & Basic N1 & $\mathrm{C} 41 \mathrm{H} 61 \mathrm{~N} 1$ & 0.000146 & Netural O1 & $\mathrm{C} 23 \mathrm{H} 12 \mathrm{O} 1$ & 0.000188 \\
\hline Non-basic N1O2 & $\mathrm{C} 46 \mathrm{H} 55 \mathrm{~N} 1 \mathrm{O} 2$ & $2.22 \mathrm{E}-05$ & Basic N1 & $\mathrm{C} 42 \mathrm{H} 63 \mathrm{~N} 1$ & 0.000106 & Netural O1 & $\mathrm{C} 24 \mathrm{H} 14 \mathrm{O} 1$ & 0.001857 \\
\hline Non-basic N1O2 & $\mathrm{C} 47 \mathrm{H} 57 \mathrm{~N} 1 \mathrm{O} 2$ & $1.69 \mathrm{E}-05$ & Basic N1 & $\mathrm{C} 43 \mathrm{H} 65 \mathrm{~N} 1$ & $7.69 \mathrm{E}-05$ & Netural O1 & $\mathrm{C} 25 \mathrm{H} 16 \mathrm{O} 1$ & 0.004295 \\
\hline Non-basic N1O2 & $\mathrm{C} 48 \mathrm{H} 59 \mathrm{~N} 1 \mathrm{O} 2$ & $1.43 \mathrm{E}-05$ & Basic N1 & $\mathrm{C} 44 \mathrm{H} 67 \mathrm{~N} 1$ & $6.12 \mathrm{E}-05$ & Netural O1 & $\mathrm{C} 26 \mathrm{H} 18 \mathrm{O} 1$ & 0.001201 \\
\hline Non-basic N1O2 & $\mathrm{C} 49 \mathrm{H} 61 \mathrm{~N} 1 \mathrm{O} 2$ & $1.78 \mathrm{E}-05$ & Basic N1 & $\mathrm{C} 45 \mathrm{H} 69 \mathrm{~N} 1$ & $4.75 \mathrm{E}-05$ & Netural O1 & $\mathrm{C} 27 \mathrm{H} 20 \mathrm{O} 1$ & 0.001064 \\
\hline Non-basic N1O2 & $\mathrm{C} 50 \mathrm{H} 63 \mathrm{~N} 1 \mathrm{O} 2$ & $3.04 \mathrm{E}-06$ & Basic N1 & $\mathrm{C} 46 \mathrm{H} 71 \mathrm{~N} 1$ & $2.92 \mathrm{E}-05$ & Netural O1 & $\mathrm{C} 28 \mathrm{H} 22 \mathrm{O} 1$ & 0.000224 \\
\hline Non-basic $\mathrm{N} 1 \mathrm{O} 2$ & $\mathrm{C} 51 \mathrm{H} 65 \mathrm{~N} 1 \mathrm{O} 2$ & $5.48 \mathrm{E}-06$ & Basic N1 & $\mathrm{C} 47 \mathrm{H} 73 \mathrm{~N} 1$ & $2.06 \mathrm{E}-05$ & Netural O1 & $\mathrm{C} 29 \mathrm{H} 24 \mathrm{O} 1$ & 0.000107 \\
\hline Non-basic $\mathrm{N} 1 \mathrm{O} 2$ & $\mathrm{C} 52 \mathrm{H} 67 \mathrm{~N} 1 \mathrm{O} 2$ & $6.51 \mathrm{E}-06$ & Basic N1 & $\mathrm{C} 48 \mathrm{H} 75 \mathrm{~N} 1$ & $1.31 \mathrm{E}-05$ & Netural O1 & $\mathrm{C} 26 \mathrm{H} 16 \mathrm{O} 1$ & 0.000183 \\
\hline Non-basic N1O2 & $\mathrm{C} 27 \mathrm{H} 15 \mathrm{~N} 1 \mathrm{O} 2$ & $2.00 \mathrm{E}-05$ & Basic N1 & $\mathrm{C} 49 \mathrm{H} 77 \mathrm{~N} 1$ & $1.80 \mathrm{E}-05$ & Netural O1 & $\mathrm{C} 27 \mathrm{H} 18 \mathrm{O} 1$ & $6.65 \mathrm{E}-05$ \\
\hline Non-basic N1O2 & $\mathrm{C} 28 \mathrm{H} 17 \mathrm{~N} 1 \mathrm{O} 2$ & $3.97 \mathrm{E}-05$ & Basic N1 & $\mathrm{C} 50 \mathrm{H} 79 \mathrm{~N} 1$ & $1.83 \mathrm{E}-05$ & Netural O1 & $\mathrm{C} 28 \mathrm{H} 20 \mathrm{O} 1$ & 0.000461 \\
\hline Non-basic $\mathrm{N} 1 \mathrm{O} 2$ & C29H19N1O2 & $6.04 \mathrm{E}-05$ & Basic N1 & $\mathrm{C} 51 \mathrm{H} 81 \mathrm{~N} 1$ & $5.21 \mathrm{E}-06$ & Netural O1 & $\mathrm{C} 29 \mathrm{H} 22 \mathrm{O} 1$ & 0.000438 \\
\hline Non-basic N1O2 & $\mathrm{C} 30 \mathrm{H} 21 \mathrm{~N} 1 \mathrm{O} 2$ & $6.85 \mathrm{E}-05$ & Basic N1 & $\mathrm{C} 52 \mathrm{H} 83 \mathrm{~N} 1$ & 7.49E-06 & Netural O1 & $\mathrm{C} 30 \mathrm{H} 24 \mathrm{O} 1$ & $4.30 \mathrm{E}-05$ \\
\hline Non-basic N1O2 & $\mathrm{C} 31 \mathrm{H} 23 \mathrm{~N} 1 \mathrm{O} 2$ & 7.99E-05 & Basic N1 & $\mathrm{C} 53 \mathrm{H} 85 \mathrm{~N} 1$ & $3.56 \mathrm{E}-06$ & Netural O1 & $\mathrm{C} 27 \mathrm{H} 16 \mathrm{O} 1$ & 0.000412 \\
\hline Non-basic N1O2 & $\mathrm{C} 32 \mathrm{H} 25 \mathrm{~N} 1 \mathrm{O} 2$ & $7.21 \mathrm{E}-05$ & Basic N1 & $\mathrm{C} 54 \mathrm{H} 87 \mathrm{~N} 1$ & $5.06 \mathrm{E}-06$ & Netural O1 & $\mathrm{C} 28 \mathrm{H} 18 \mathrm{O} 1$ & $8.76 \mathrm{E}-05$ \\
\hline Non-basic N1O2 & $\mathrm{C} 33 \mathrm{H} 27 \mathrm{~N} 1 \mathrm{O} 2$ & $6.02 \mathrm{E}-05$ & Basic N1 & $\mathrm{C} 17 \mathrm{H} 11 \mathrm{~N} 1$ & $7.68 \mathrm{E}-07$ & Netural O1 & $\mathrm{C} 29 \mathrm{H} 20 \mathrm{O} 1$ & 0.000306 \\
\hline Non-basic N1O2 & $\mathrm{C} 34 \mathrm{H} 29 \mathrm{~N} 1 \mathrm{O} 2$ & $5.68 \mathrm{E}-05$ & Basic N1 & $\mathrm{C} 18 \mathrm{H} 13 \mathrm{~N} 1$ & $2.72 \mathrm{E}-05$ & Netural O1 & $\mathrm{C} 30 \mathrm{H} 22 \mathrm{O} 1$ & 0.000628 \\
\hline Non-basic $\mathrm{N} 1 \mathrm{O} 2$ & $\mathrm{C} 35 \mathrm{H} 31 \mathrm{~N} 1 \mathrm{O} 2$ & $3.17 \mathrm{E}-05$ & Basic N1 & $\mathrm{C} 19 \mathrm{H} 15 \mathrm{~N} 1$ & $5.24 \mathrm{E}-05$ & Netural O1 & $\mathrm{C} 31 \mathrm{H} 24 \mathrm{O} 1$ & 0.000221 \\
\hline Non-basic $\mathrm{N} 1 \mathrm{O} 2$ & $\mathrm{C} 36 \mathrm{H} 33 \mathrm{~N} 1 \mathrm{O} 2$ & $1.08 \mathrm{E}-05$ & Basic N1 & $\mathrm{C} 20 \mathrm{H} 17 \mathrm{~N} 1$ & $8.45 \mathrm{E}-05$ & Netural O1 & $\mathrm{C} 32 \mathrm{H} 26 \mathrm{O} 1$ & 0.000555 \\
\hline Non-basic $\mathrm{N} 1 \mathrm{O} 2$ & $\mathrm{C} 37 \mathrm{H} 35 \mathrm{~N} 1 \mathrm{O} 2$ & $7.68 \mathrm{E}-06$ & Basic N1 & $\mathrm{C} 21 \mathrm{H} 19 \mathrm{~N} 1$ & 0.000116 & Netural O1 & C29H18O1 & $6.35 \mathrm{E}-05$ \\
\hline Non-basic $\mathrm{N} 1 \mathrm{O} 2$ & $\mathrm{C} 38 \mathrm{H} 37 \mathrm{~N} 1 \mathrm{O} 2$ & $1.49 \mathrm{E}-06$ & Basic N1 & $\mathrm{C} 22 \mathrm{H} 21 \mathrm{~N} 1$ & 0.00019 & Netural O1 & $\mathrm{C} 30 \mathrm{H} 20 \mathrm{O} 1$ & 0.000102 \\
\hline
\end{tabular}




\begin{tabular}{ccccccccc}
\hline Class & Formula & Mass & Class & Formula & Mass & Class & Formula & Mass \\
\hline Non-basic N1O2 & C40H41N1O2 & $2.53 \mathrm{E}-06$ & Basic N1 & C23H23N1 & 0.000258 & Netural O1 & C31H22O1 & $7.24 \mathrm{E}-05$ \\
& & & & & & Netural O1 & C32H24O1 & 0.000329 \\
\hline
\end{tabular}

